UNIVERSIDAD POLITÉCNICA DE MADRID

ESCUELA TÉCNICA SUPERIOR DE INGENIEROS EN TOPOGRAFÍA, GEODESIA Y CARTOGRAFÍA

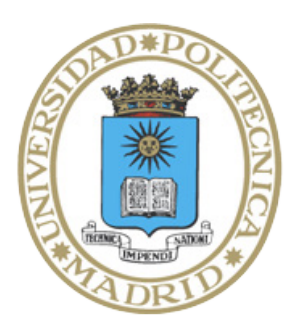

SIMULACIÓN DE ESCENARIOS SÍSMICOS MEDIANTE UN SISTEMA DE INFORMACIÓN GEOGRÁFICA PARA LA PENÍNSULA IBÉRICA, LAS ISLAS BALEARES Y LAS ISLAS CANARIAS, CONSIDERANDO EL EFECTO DE SITIO Y LAS DIMENSIONES Y CARACTERÍSTICAS DE LA FUENTE SÍSMICA

\author{
Tesis Doctoral
}

ALBERTO NÚÑNEZ MURILLO

Ingeniero Geólogo 
DEPARTAMENTO DE INGENIERÍA TOPOGRÁFICA Y CARTOGRAFÍA ESCUELA TÉCNICA SUPERIOR DE INGENIEROS EN TOPOGRAFÍA, GEODESIA Y CARTOGRAFÍA

\title{
SIMULACIÓN DE ESCENARIOS SÍSMICOS MEDIANTE UN SISTEMA DE INFORMACIÓN GEOGRÁFICA PARA LA PENÍNSULA IBÉRICA, LAS ISLAS BALEARES Y LAS ISLAS CANARIAS, CONSIDERANDO EL EFECTO DE SITIO Y LAS DIMENSIONES Y CARACTERÍSTICAS DE LA FUENTE SÍSMICA
}

\author{
ALBERTO NÚÑEZ MURILLO \\ Ingeniero Geólogo
}

Directores:

Juan José Rueda Núñez, Doctor Ingeniero en Geodesia y Cartografía Rosa María García Blanco, Doctora en Ciencias Geológicas 
El Tribunal de la Tesis Doctoral, nombrado por el Magnífico y Excelentísimo Sr. Rector de la Universidad Politécnica de Madrid el día de de 2017, integrado por:

Presidente:

Secretario:

Vocal $1^{\circ}$ :

Vocal $2^{\circ}$ :

Vocal $3^{\circ}$ :

Vocal suplente $1^{\circ}$ :

Vocal suplente $2^{\circ}$ :

Realizado el acto de defensa y lectura de tesis el día de de 2017, decide otorgar a la tesis doctoral presentada la calificación de: 


\section{AGRADECIMIENTOS}

En el momento de terminar esta tesis doctoral, me gustaría expresar mi más sincero agradecimiento a todas las personas que me han ayudado a completar mi investigación con éxito.

Primeramente, le agradezco a mi director, Juan Rueda, su ofrecimiento a dirigir mi tesis, en un momento en el que terminaba mi beca de cuatro años en el Instituto Geográfico Nacional y tenía un panorama profesional incierto por delante. En este sentido, me permitió extender a la tesis el trabajo desarrollado en el Instituto bajo su supervisión, en cuanto a la producción del mapa de amplificación de la Península Ibérica y las Islas Baleares, y su utilización como herramienta de caracterización del efecto de sitio para la simulación de terremotos históricos en España. De esta forma, puedo decir que comencé la tesis con una base de investigación sólida, que al poco tiempo fue publicada en la revista Natural Hazards, y con ello pude obtener el artículo en publicación indexada en el Journal Citation Reports, preceptiva para poder defender la tesis. Juan también me abrió la posibilidad de desarrollar esta investigación en el Centro GEOI+D, en la Escuela Técnica Superior de Ingenieros en Topografía, Geodesia y Cartografía, y con ello la opción de trabajar en un entorno adecuado y con los medios disponibles. Por supuesto, también me ha facilitado el acceso a los recursos del Instituto Geográfico Nacional en todo momento. A nivel científico, él me ha hecho analizar, cuestionar y profundizar en mis conocimientos de sismología, haciéndome madurar poco a poco en este aspecto, y también me ha llevado a valorar la gran importancia de recurrir constantemente a la literatura sismológica como medio para orientar un trabajo riguroso de investigación. Le agradezco también su ánimo constante para seguir hacia delante con esta tesis doctoral, y su disponibilidad permanente para resolver cuantas dudas me iban surgiendo y para enseñarme a analizarlas de una forma metódica para probar yo también a solucionarlas.

Mi codirectora, Rosa García, ha estado constantemente a mi disposición en la Escuela de Topografía, siempre que lo he necesitado. Ha sido la primera persona a la que recurrir cuando he tenido problemas de índole informático y logístico, incluso de cambio de lugar de trabajo, facilitándome enseguida el contacto de la persona que me podía prestar la ayuda precisa, en cada momento. También me ha ayudado en primera instancia a agilizar las gestiones necesarias para poder tramitar la tesis, una vez concluida. Valoro y agradezco sus aportaciones y visión sobre el trabajo de investigación, para cuestionarme algunos aspectos que quizá yo no había profundizado lo suficiente, y sus observaciones en cuanto al interés de dotar de un hilo conductor a la tesis que permitiera al lector saber claramente el objetivo y alcance del trabajo. Le doy las gracias igualmente por su empuje y ánimo para terminar la tesis.

A Julio Mezcua, hoy en la Fundación José García Siñeriz, debo agradecerle en primer lugar el haberse ofrecido a dirigir mi trabajo de investigación tutelado, auténtico embrión de esta tesis doctoral, y que me permitió obtener el Certificado-Diploma de Estudios Avanzados. En segundo lugar, tengo que decir que he aprendido mucho de él en todos los trabajos que hemos publicado juntos y en relación a esta tesis, 
dándome su visión del rumbo que iba tomando cada artículo, nuevas posibilidades de enfocar algunas etapas del mismo, y referencias a tener en cuenta. En el contexto de esta tesis, no olvido su ayuda decisiva para enfocar y llevar a cabo la calibración del algoritmo de simulación EXSIM en El Hierro y sus aplicaciones posteriores en la simulación de los escenarios sísmicos en la isla. Le agradezco también su confianza y cercanía, especialmente en las últimas etapas del desarrollo de la tesis.

Le doy las gracias a Stefania Bartolini por toda su ayuda para poder desarrollar el plugin de QGIS para el cálculo de los mapas de sacudidas y de intensidades sintéticas, en el marco del proyecto del Consejo Superior de Investigaciones Científicas para dotar a la plataforma VOLCANBOX de una herramienta de simulación de aceleraciones máximas del terreno. Su ayuda en cuanto a la programación de la herramienta ha tenido un valor incalculable para mí, y me ha permitido abordar con garantías la aplicación de los resultados obtenidos en la tesis en la simulación automática de los escenarios sísmicos. También me ha permitido tener la tranquilidad de poder culminar el conjunto de la tesis en los plazos previstos. Le agradezco su buenísima disposición a profundizar en los objetivos que debía alcanzar el plugin y en la complejidad que planteaba la aplicación de la metodología a utilizar. Por supuesto, también valoro enormemente su disponibilidad cuando hemos tenido la oportunidad de trabajar juntos en Madrid, y muy especialmente cuando decidimos culminar el trabajo por medio de videoconferencia.

He podido contar también con la ayuda de Marta García, doctoranda en la Universidad Politécnica de Madrid, para afrontar el análisis de las relaciones empíricas de atenuación en términos de aceleración máxima del terreno, especialmente en el ámbito de la Península Ibérica y las Islas Baleares. Gracias al tiempo dedicado por su parte y a sus frecuentes consejos, pude desarrollar esta parte de la investigación, especialmente amplia, con criterios y objetivos muy definidos.

Quiero agradecer a continuación toda la ayuda que me ha prestado Antonio Vázquez desde el primer día que entré en la Escuela de Topografía. Le agradezco el haberme brindado la oportunidad de desarrollar la tesis en el Centro GEOI $+\mathrm{D}$ y también su apoyo constante en el desarrollo de mi investigación. Le doy las gracias igualmente por su gran disposición a resolver cuantas dudas de carácter burocrático y académico se me han planteado para poder cumplir las exigencias del programa de doctorado en cuanto al seguimiento del trabajo y, en última instancia, a la tramitación de la tesis para su defensa.

Le agradezco también a Miguel Ángel Manso y Mayte Manrique todas las facilidades para desarrollar mi investigación en el Centro GEOI+D durante estos 6 años, especialmente en los primeros momentos de establecimiento en un puesto nuevo y de necesidad de conocer los recursos del Centro. También recuerdo con afecto su interés en mi investigación y en su evolución.

También le doy las gracias al Instituto Geográfico Nacional, y en particular al Observatorio Geofísico Central, por su gran ayuda en el transcurso de esta tesis. A pesar de que, conforme han ido pasando los años, se ha ido alejando el recuerdo de mi etapa de becario en el Instituto, para mí siempre ha sido un placer volver, reencontrarme con antiguos compañeros, conocer a los nuevos integrantes, e incluso pasar alguna temporada analizando datos de campo, como los de ReMi, en la casa. Le agradezco a todo el grupo humano del Observatorio su ánimo y apoyo constante en el devenir de mi tesis, y en particular a su directora Carmen López. En cuestiones 
concernientes a la elaboración del trabajo, Rafael Abella me ayudó a obtener los datos de ruido sísmico ambiental de las estaciones de registro de velocidad y las estaciones centrales de los arrays sísmicos de El Hierro para la aplicación del método H/V. Laura García, por su parte, me proporcionó las reseñas de las estaciones GPS que fueron necesarias para diseñar la campaña de adquisición de medidas ReMi en El Hierro. A todo el equipo, mi agradecimiento con afecto.

Aún en el Instituto Geográfico Nacional, aunque en la Red Sísmica Nacional, valoro enormemente la ayuda proporcionada por Juan Manuel Alcalde, quien me proporcionó el catálogo de acelerogramas y los registros acelerométricos de los terremotos de El Hierro ocurridos entre 2011 y 2013, y que han sido utilizados en la calibración de las relaciones de atenuación aplicables en Canarias. Le agradezco sus frecuentes envíos de registros para actualizar el catálogo, y su disposición a resolverme las dudas sobre la utilización de los acelerogramas. Quiero agradecerle a Resurrección Antón su disponibilidad para proporcionarme vía e-mail las formas de onda de las estaciones de Canarias que en un primer momento analicé con el objetivo de calcular la caída de esfuerzos de algunos terremotos de alta magnitud, y así comenzar a probar el algoritmo EXSIM para simular escenarios sísmicos en el archipiélago. En este contexto, Juan Vicente Cantavella me prestó su ayuda en varias ocasiones para entender correctamente las especificidades de la respuesta instrumental de las estaciones sísmicas, necesaria para estudiar adecuadamente la caída de esfuerzos.

En el momento de viajar a El Hierro y tomar las medidas ReMi en los puntos de la campaña de adquisición de medidas en la isla, mis directores y yo contamos con la ayuda inestimable del Ayuntamiento de El Pinar de El Hierro, en cuanto a la organización logística, y muy especialmente la de D. Jesús García Quintero. También le agradezco al Ayuntamiento de La Frontera su disposición en este sentido. Y, cómo no, les agradezco a Ernesto, Gregory y Yoel sus "marronazos" para generar ondas superficiales Rayleigh en cada uno de los 25 puntos de medida, y así poder obtener los modelos de Vs experimentales.

Durante todos estos años he podido compartir mucho tiempo de trabajo y de descanso con mis compañeros en el Centro GEOI + D. Me siento muy afortunado de haber coincidido con tan buenos amigos como Ayar, Víctor, Adolfo, Xavier, Eladio, Vladimir e Isa. Espero que nos queden aún muchas charlas y derivas por hacer, pues he disfrutado muchísimo de todas las historias, anécdotas, puntos de vista. En fin, ha sido un intercambio constante de cultura, compañerismo, amistad, con unas personas increíbles en todos los aspectos. No me quiero olvidar de otros compañeros que también estuvieron conmigo en una primera época en el Centro, y a los que ya veo menos, en especial me acuerdo con afecto de Arancha, Watse, Javi, Iván, Alba, Lola, Willington y Alberto, esperando que sigan sus respectivos caminos con éxito.

Les agradezco también a mis compañeros y amigos de la Escuela de Minas todo su apoyo y aliento en la terminación de esta tesis. Gracias por estar siempre cerca y por recordarme siempre nuestra época de estudiantes. Ahora como ingenieros geólogos, me alegra ver que cada uno está cumpliendo sus objetivos y también que nos seguimos viendo para compartirlos.

A mis grandes amigos de Santa Rita, quiero agradecerles tantos y tan buenos momentos juntos, tantos años, y el apoyo que me han dado para continuar adelante. 
Ahora que termino esta etapa, espero poder recuperar algo del tiempo que no he podido compartir últimamente con ellos.

Por último, les agradezco a mis padres y a mi hermana su aliento, apoyo y confianza en que iba a sacar esta tesis adelante. Sin ellos no hubiera sido posible llegar a donde lo he hecho, y siempre recordaré su apoyo decidido en las épocas más inciertas para mí. Pero más aún su convencimiento de que era capaz de terminar la tesis y su confianza para llegar al final. 


\section{Índice general}

RESUMEN

XLVII

ABSTRACT

XLIX

1. INTRODUCCIÓN

2. METODOLOGÍA

2.1. Generación de cartografía de amplificación sísmica . . . . . . . . . 9

2.1.1. Clasificaciones de suelos y rocas según su capacidad de amplificación sísmica . . . . . . . . . . . . 13

2.1.2. Clasificación de las unidades geológicas según su capacidad de amplificación sísmica . . . . . . . . . . . 17

2.1.3. Cálculo de factores de amplificación en función de la frecuencia del movimiento del terreno a partir de Vs30 . . . . . . . . 19

2.1.4. Estudios que aplican la metodología de Borcherdt (1994) para obtener cartografía de amplificación sísmica . . . . . . . 22

2.2. Cálculo de la distribución de la velocidad de onda de corte (Vs) en los primeros $30 \mathrm{~m}$ de profundidad por medio del método ReMi . . . . 25

2.2.1. Antecedentes del método ReMi: SASW, MASW . . . . . . 27

2.2.2. Fundamentos teóricos del método ReMi . . . . . . . . 28

2.2.3. Adquisición de medidas ReMi . . . . . . . . . . . 30

2.2.4. Análisis espectral para la construcción del diagrama de la transformada lentitud $(\mathrm{p})$-frecuencia $(\mathrm{f}) \ldots \ldots . \ldots 30$

2.2.5. Construcción de la curva de dispersión de las ondas Rayleigh . 32

2.2.6. Modelización de la curva de dispersión y obtención del modelo de Vs en los primeros $30 \mathrm{~m}$ de profundidad . . . . . . . 33

2.3. Determinación de la amplificación sísmica en función de la frecuencia a partir del modelo de Vs en los primeros $30 \mathrm{~m}$ de profundidad . . . . 33

2.3.1. Cálculo de la amplificación teórica de una onda $\mathrm{SH}$ que se propaga en una estructura de capas con velocidad constante . 34 
2.3.2. Implementación de la solución completa en algoritmos de cálculo de la amplificación sísmica en función de la frecuencia:

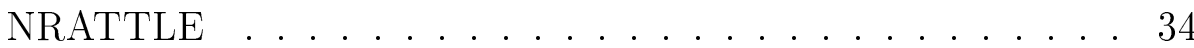

2.3.3. Cálculo del periodo fundamental del suelo a partir de la curva de amplificación sísmica . . . . . . . . . . 35

2.4. Determinación experimental del periodo fundamental del suelo mediante el método $\mathrm{H} / \mathrm{V} \ldots \ldots . \ldots . \ldots . \ldots . . \ldots 35$

2.4.1. Fundamentos del método $\mathrm{H} / \mathrm{V} \ldots \ldots . \ldots . . . . . .35$

2.4.2. Obtención y análisis de la curva de la relación $\mathrm{H} / \mathrm{V}$. . . . . 36

2.4.3. Determinación del periodo fundamental . . . . . . . . . 37

2.5. Cálculo de la aceleración máxima y de la intensidad sintéticas considerando una fuente sísmica puntual . . . . . . . . . . . 37

2.5.1. Leyes de atenuación obtenidas a partir del ajuste empírico de registros de aceleración . . . . . . . . . . . 38

2.5.2. Calibración de relaciones de atenuación a partir de la comparación entre las aceleraciones máximas sintéticas y las aceleraciones máximas registradas . . . . . . . . . . . 41

2.5.3. Selección de leyes de atenuación óptimas para distintas escalas de trabajo y en función de la magnitud del terremoto . . . . . 42

2.5.4. Correlaciones entre los parámetros del movimiento fuerte y la intensidad sísmica . . . . . . . . . . . . . . 43

2.6. Estimación de la atenuación anelástica de la litosfera . . . . . . . . 43

2.6.1. Metodologías de estimación del factor de calidad Q a partir del análisis de las ondas de coda . . . . . . . . . . . 43

2.6.2. Metodología de Havskov et al. (1989) para la determinación de Q en función de la frecuencia de filtrado de la señal . . . . 45

2.6.3. Regionalización de los parámetros $Q_{0}$ y $\nu \ldots . . . . .45$

2.7. Simulación estocástica de acelerogramas sintéticos considerando una fuente sísmica extensa . . . . . . . . . . . . 46

2.7.1. Modelo estocástico de fuente puntual . . . . . . . . . . 47

2.7.2. Modelo estocástico de fuente extensa . . . . . . . . . . . 51

2.7.3. Calibración del algoritmo de simulación . . . . . . . . . . . 53

2.8. Generación de mapas de aceleraciones máximas sintéticas y de intensidades sintéticas . . . . . . . . . . . . . . 54

2.8.1. Antecedentes ... . . . . . . . . . . . . . 54

2.8.2. Cálculo de aceleraciones máximas sintéticas en la condición del terreno de referencia . . . . . . . . . . . . 55

2.8.3. Corrección de aceleraciones máximas registradas a la condición del terreno de referencia . . . . . . . . . 57

2.8.4. Interpolación de aceleraciones máximas sintéticas y registradas y amplificación a suelo . . . . . . . . . . 5 58 
2.8.5. Producción de mapas de sacudidas y de intensidades sintéticas 58

\section{MAPA DE AMPLIFICACIÓN SÍSMICA DE LA PENÍNSULA} IBÉRICA, LAS ISLAS BALEARES Y LAS ISLAS CANARIAS

3.1. Base de partida 1: Clasificaciones de suelos y rocas según su capacidad de amplificación sísmica . . . . . . . . . . . . . 62

3.1.1. Clasificación de síntesis . . . . . . . . . . . . 63

3.2. Base de partida 2: Cartografía geológica 1:1.000.000 de la Península Ibérica y las Islas Baleares y cartografía geológica 1:50.000 de las Islas Canarias ........................ 69

3.3. Clasificación de las unidades geológicas según la clasificación de síntesis 80 3.3.1. Unidades geológicas de la Península Ibérica y las Islas Baleares 80

3.3.2. Unidades geológicas de las Islas Canarias . . . . . . . . . 96

3.4. Obtención del mapa de clases de emplazamiento y Vs30 . . . . . . . . 188

3.5. Cálculo de factores de amplificación en función de la frecuencia del movimiento del terreno para cada clase de emplazamiento (Borcherdt, 1994) . . . . . . . . . . . . . . . . 191

3.6. Obtención del mapa de amplificación sísmica . . . . . . . . . . . 192

4. DETERMINACIÓN DE LA AMPLIFICACIÓN SÍSMICA EN FUNCIÓN DE LA FRECUENCIA EN EL HIERRO (ISLAS CANARIAS) MEDIANTE LA DISTRIBUCIÓN DE LA VELOCIDAD DE LA ONDA DE CORTE EN LOS PRIMEROS 30 M DE PROFUNDIDAD $\quad 195$

4.1. Campaña de adquisición de medidas ReMi . . . . . . . . . . . . 196

4.2. Procesado de datos . . . . . . . . . . . . . . . . 199

4.3. Valores de Vs30 en los emplazamientos de medida . . . . . . . . . . 203

4.3.1. Comparación de los valores de Vs30 en los emplazamientos de medida con los valores de Vs30 del mapa . . . . . . . . . 203

4.4. Curvas de amplificación sísmica espectral en los emplazamientos de medida . . . . . . . . . . . . . . 206

4.4.1. Comparación de los valores máximos de amplificación en los emplazamientos de medida con los factores de amplificación del mapa . . . . . . . . . . . . . . . . . . 209

5. DETERMINACIÓN EXPERIMENTAL DEL PERIODO FUNDAMENTAL DEL SUELO EN EL HIERRO MEDIANTE EL MÉTO$\mathrm{DO} \mathrm{H} / \mathrm{V}$

5.1. Adquisición de muestras de ruido sísmico ambiental a partir del registro continuo de las estaciones de registro de velocidad del Instituto Geográfico Nacional (IGN) en El Hierro . . . . . . . . . . . . 211

5.2. Análisis de las curvas de la relación $\mathrm{H} / \mathrm{V}$ y determinación del periodo fundamental . . . . . . . . . . . . . . . . . . . . 212 


\section{ANÁLISIS Y SELECCIÓN DE LEYES DE ATENUACIÓN EN TÉRMINOS DE ACELERACIÓN MÁXIMA Y DETERMINA- CIÓN DE LA INTENSIDAD SINTÉTICA}

6.1. Leyes de atenuación aplicables en la Península Ibérica y las Islas Baleares . . . . . . . . . . . . . . . . . 2 217

6.1.1. Análisis de leyes de atenuación aplicables en el ámbito de la Península Ibérica y las Islas Baleares . . . . . . . . . . . 219

6.1.2. Calibración de las leyes de atenuación . . . . . . . . . 225

6.1.3. Selección de leyes de atenuación óptimas para distintas escalas de trabajo y en función de la magnitud del terremoto . . . . . 228

6.2. Leyes de atenuación aplicables en las Islas Canarias . . . . . . . . . 230

6.2.1. Investigación bibliográfica de leyes de atenuación susceptibles de aplicación en las Islas Canarias . . . . . . . . . . . . 230

6.2.2. Preparación de datos de aceleración máxima registrada de los terremotos de $\mathrm{Mw}>4,0$ localizados en El Hierro . . . . . . 244

6.2.3. Calibración de las relaciones de atenuación en aceleración máxima . . . . . . . . . . . . . . 246

6.2.4. Selección de leyes de atenuación óptimas para distintas escalas de trabajo y en función de la magnitud del terremoto . . . . . 248

6.3. Selección de leyes de atenuación de alcance mundial . . . . . . . . . 251

6.3.1. Regiones continentales estables: Atkinson y Boore (2006), modificada por Atkinson y Boore (2011) . . . . . . . . . . . 252

6.3.2. Regiones corticales activas: Chiou y Youngs (2008) . . . . 253

6.3.3. Zonas de subducción: Zhao et al. (2006) . . . . . . . . . . 254

6.4. Cálculo de intensidades sintéticas a partir de parámetros del movimiento fuerte . . . . . . . . . . . . . . . 255

7. ESTIMACIÓN DE LA ATENUACIÓN ANELÁSTICA DE LA LITOSFERA EN CANARIAS

7.1. Determinación de Q y de su dependencia frecuencial en las Islas Ca-

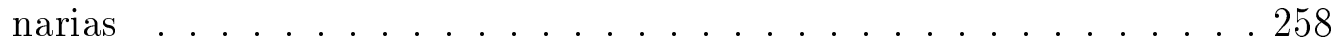

7.2. Variación temporal de $Q_{0}$ y $\nu$ en la isla de El Hierro durante la crisis sísmica y el proceso eruptivo de 2011-2012 . . . . . . . . . . . 265

7.3. Discusión . . . . . . . . . . . . . . . . . 272

8. CALIBRACIÓN DEL ALGORITMO DE SIMULACIÓN ESTOCÁSTICA DE ACELEROGRAMAS SINTÉTICOS EN EL HIERRO

8.1. Selección del terremoto de calibración y obtención de acelerogramas registrados . . . . . . . . . . . . . . 2 276

8.2. Parámetros del modelo . . . . . . . . . . . . . . . 276 
8.2.1. Mecanismo focal . . . . . . . . . . . . 276

8.2.2. Partición en subfallas . . . . . . . . . . . . . 277

8.2.3. Parámetro de esfuerzo . . . . . . . . . . . . 277

8.2.4. Parámetros elásticos . . . . . . . . . . . . 277

8.2.5. Atenuación geométrica y anelástica . . . . . . . . 278

8.2.6. Kappa . . . . . . . . . . . . . . 278

8.2.7. Porcentaje de solapamiento . . . . . . . . . . 278

8.2.8. Duración . . . . . . . . . . . . . . . 278

8.2.9. Corrección por corteza y efecto de sitio . . . . . . . . . 279

8.3. Calibración del algoritmo en los acelerógrafos ATIG y AROQ . . . . . 279

9. DESARROLLO DEL SISTEMA QGIS PARA LA GENERACIÓN DE MAPAS DE ACELERACIONES MÁXIMAS SINTÉTICAS Y DE INTENSIDADES SINTÉTICAS 285

9.1. Estructura del plugin . . . . . . . . . . . . . . . . . . 286

9.1.1. Implementación de resultados obtenidos $\ldots . . .289$

9.1.2. Variables de entrada . . . . . . . . . . . . . 294

9.2. Generación de mapas de aceleraciones máximas sintéticas (mapas de

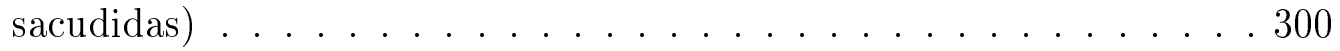

9.2.1. Producción de capas de trabajo . . . . . . . . . . . . . 301

9.2.2. Salidas del plugin . . . . . . . . . . . . . . . . 313

9.3. Generación de mapas de intensidades sintéticas . . . . . . . 315

10.SIMULACIÓN DE ESCENARIOS SÍSMICOS 319

10.1. Definición de zonas geográficas según el nivel de aproximación a valores sintéticos realistas de la aceleración e intensidad sísmicas . . . . 319

10.1.1. Utilización de las leyes de atenuación y del mapa de amplificación, por medio del plugin . . . . . . . . . . . . 320

10.1.2. Utilización del algoritmo de simulación estocástica EXSIM . 321

10.2. Simulación con el plugin . . . . . . . . . . . . . . . . 321

10.2.1. Selección de terremotos de $\mathrm{Mw}>4,5$ con registros de aceleración 322

10.2.2. Producción de mapas de sacudidas y de intensidades sintéticas 324

10.2.3. Comparación de aceleraciones máximas sintéticas y aceleraciones máximas registradas . . . . . . . . . . . 337

10.3. Simulación con EXSIM . . . . . . . . . . . . . . . . . 340

10.3.1. Obtención de acelerogramas sintéticos para el terremoto de calibración . . . . . . . . . . . . . . 341

10.3.2. Obtención de intensidades sintéticas . . . . . . . . . . . . 342

10.3.3. Comparación de intensidades sintéticas e intensidades observadas . . . . . . . . . . . . . . . . . 343 
11.1. Determinación de la amplificación sísmica en función de la frecuencia 348

11.2. Determinación experimental del periodo fundamental del suelo . . . . 351

11.3. Estimación de la atenuación anelástica de la litosfera . . . . . . . . . 351

11.4. Simulación de escenarios sísmicos con relaciones empíricas de atenuación 353

11.5. Simulación estocástica de acelerogramas sintéticos . . . . . . . . . 357

12. CONCLUSIONES FINALES

13.LÍNEAS FUTURAS DE TRABAJO

A. UNIDADES DE LA CARTOGRAFÍA GEOLÓGICA CONTINUA GEODE DE LAS ISLAS CANARIAS

B. COORDENADAS GEOGRÁFICAS WGS84 Y ALTITUD DE LOS EMPLAZAMIENTOS DE MEDIDA REMI

C. RESEÑA GEOLÓGICA DE LOS EMPLAZAMIENTOS DE MEDIDA REMI

D. DIAGRAMAS DE LA TRANSFORMADA LENTITUD-FRECUENCIA (P-F) DEFINITIVA PARA LOS 25 EMPLAZAMIENTOS DE MEDIDA REMI

E. PROCESADO COMPLETO DE LOS 25 EMPLAZAMIENTOS DE MEDIDA REMI: PUNTOS DE DISPERSIÓN EXPERIMENTALES, AJUSTE DE LA CURVA DE DISPERSIÓN TEÓRICA Y MODELO DE VELOCIDADES VS PROPUESTO, Y CURVA DE AMPLIFICACIÓN ESPECTRAL OBTENIDA PARA EL MODELO

F. CURVAS DEL RATIO ESPECTRAL H/V EN LOS 6 EMPLAZAMIENTOS DE APLICACIÓN DEL MÉTODO H/V

G. CALIBRACIÓN DE LAS RELACIONES DE ATENUACIÓN EN ACELERACIÓN MÁXIMA EN FUNCIÓN DE LAS ACELERACIONES MÁXIMAS REGISTRADAS PARA LOS TERREMOTOS DE EL HIERRO CON Mw > 4,0 EN EL PERIODO 20112013

H. ARCHIVOS SHAPEFILE PARA LA EJECUCIÓN DE HERRAMIENTA PLUGIN EN LA SIMULACIÓN DE ESCENARIOS SÍSMICOS EN LORCA Y EL HIERRO (CAPÍTULOS 9 Y 10) 


\section{Índice de figuras}

2.1. Representación esquemática del efecto de sitio producido por distitintos materiales geológicos (roca y sedimentos blandos), emplazados en las capas más superficiales. La amplitud de las ondas sísmicas que atraviesan estos materiales en su camino hacia la superficie es modificada en distinto grado en función del tipo de material. . . . . . . . 9

2.2. Mapa de la distribución de Vs30 en Japón, obtenido a partir de la geología superficial y las condiciones geomorfológicas. Tomado de Geological Survey of Japan (2014). . . . . . . . . . . . . 11

2.3. Mapa de Vs30 en los Estados Unidos continentales situados al este de las Montañas Rocosas, obtenido a partir de la pendiente topográfica y de los coeficientes de predicción de Vs30 para regiones estables. Tomado de Allen y Wald (2007). . . . . . . . . . . . . . . . . . 12

2.4. Mapa de la distribución de Vs30 (m/s) en Torreperogil y Sabiote (Jaén), obtenido a partir de la estimación del modelo de Vs en los primeros $30 \mathrm{~m}$ de profundidad en 68 emplazamientos de medida de ruido sísmico ambiental. Las medidas han sido procesadas por medio del método ReMi para obtener la estructura de Vs en cada emplazamiento. Tomado de Rueda et al. (2015). . . . . . . . . . . . . . . . 14

2.5. Proceso de producción de una clasificación de síntesis a partir de $\mathrm{N}$ clasificaciones de suelos y rocas en función de su capacidad de amplificación sísmica. Cada clasificación puede presentar un número determinado de clases de emplazamiento (A-Z), que están caracterizadas por determinadas propiedades geotécnicas (por ejemplo, Vs30). Las clases de emplazamiento que se caracterizan por valores similares de estas propiedades (Vs30) y que se presentan en todas las clasificaciones pueden integrarse en la clasificación de síntesis. . . . . . . . . .

2.6. Proceso de asignación de una clase de emplazamiento (A), y por tanto, de un valor de Vs30 (Vs30A ), a una unidad geológica (2), de acuerdo con la metodología de Borcherdt (1994). Esta asignación se lleva a cabo considerando la coherencia y similitud entre determinadas propiedades geotécnicas cualitativas (resistencia, compacidad y consistencia) de la clase y las mismas propiedades de la unidad, estimadas a partir de su descripción litológica. . . . . . . . . . . . 18

2.7. Estaciones de la red del proyecto TriNet y clases de emplazamiento (Quaternary, Tertiary, Mesozoic) en el sur de California. Los cuadrados representan las estaciones pertenecientes al CDMG y los triángulos las estaciones del USGS-Caltech. Tomado de Wald et al. (1999b). 
2.8. Mapa de amplificación sísmica de la Región de Murcia a escala 1:200.000. Tomado de Tsige y García Flores (2006). . . . . . . . . . . . . . . 24

2.9. Relación entre la velocidad de fase $C_{R}$ de las ondas Rayleigh y los cambios verticales en la distribución de Vs en profundidad. Tomado de Granda et al. (2005). . . . . . . . . . . . . . . . . . 29

2.10. Curva de dispersión experimental de la onda Rayleigh y curva teórica (izquierda), la cual ha sido generada a partir del modelo de Vs local

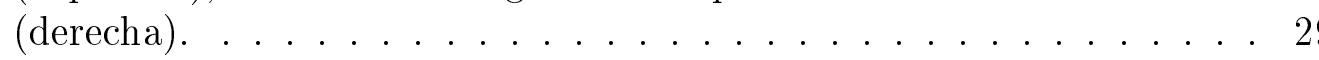

2.11. Procesado completo de las medidas ReMi: Diagrama de la transformada lentitud-frecuencia $(p-f)$, curva de dispersión experimental de las ondas Rayleigh y modelización por medio un modelo de velocidad Vs. Tomado de Granda et al. (2005). . . . . . . . . . . . . . . . . . 31

2.12. Proceso de simulación de movimientos sísmicos fuertes en el dominio del tiempo, por medio del método estocástico, con obtención del registro en aceleración. Tomado de Boore (2003). . . . . . . . . . . 52

3.1. Mapa Geológico de España a escala 1:1.000.000. La leyenda indica el número de referencia o sigla asignada a cada unidad geológica. La descripción litológica de cada unidad se encuentra en las Tablas 3.8 a 3.24. Tomado de Instituto Geológico y Minero de España (1994). . 70

3.2. Mapa geológico continuo GEODE a escala 1:50.000 de El Hierro (Zona 2916). La leyenda indica el código alfanumérico asignado a cada unidad geológica. La descripción de cada unidad en cuanto a su litología se encuentra en la Tabla A.1. Tomado de GEODE (2014). . . . 73

3.3. Mapa geológico continuo GEODE a escala 1:50.000 de Fuerteventura (Zona 2911). La leyenda indica el código alfanumérico asignado a cada unidad geológica. La descripción de cada unidad en cuanto a su litología se encuentra en la Tabla A.2. Tomado de GEODE (2014). . . 74

3.4. Mapa geológico continuo GEODE a escala 1:50.000 de Gran Canaria (Zona 2912). La leyenda indica el código alfanumérico asignado a cada unidad geológica. La descripción de cada unidad en cuanto a su litología se encuentra en la Tabla A.3. Tomado de GEODE (2014). . . 75

3.5. Mapa geológico continuo GEODE a escala 1:50.000 de La Gomera (Zona 2914). La leyenda indica el código alfanumérico asignado a cada unidad geológica. La descripción de cada unidad en cuanto a su litología se encuentra en la Tabla A.4. Tomado de GEODE (2014). . . 76

3.6. Mapa geológico continuo GEODE a escala 1:50.000 de La Palma (Zona 2915). La leyenda indica el código alfanumérico asignado a cada unidad geológica. La descripción de cada unidad en cuanto a su litología se encuentra en la Tabla A.5. Tomado de GEODE (2014). . . 77

3.7. Mapa geológico continuo GEODE a escala 1:50.000 de Lanzarote (Zona 2910). La leyenda indica el código alfanumérico asignado a cada unidad geológica. La descripción de cada unidad en cuanto a su litología se encuentra en la Tabla A.6. Tomado de GEODE (2014). . . 78 
3.8. Mapa geológico continuo GEODE a escala 1:50.000 de Tenerife (Zona 2912). La leyenda indica el código alfanumérico asignado a cada unidad geológica. La descripción de cada unidad en cuanto a su litología se encuentra en la Tabla A.7. Tomado de GEODE (2014). . . . . . . 79

3.9. Mapa de clases de emplazamiento y Vs30 de la Península Ibérica y las Islas Baleares. . . . . . . . . . . . . . . . . . . . 189

3.10. Mapa de clases de emplazamiento y Vs30 de las Islas Canarias. . . . . 190

3.11. Mapa de amplificación sísmica de la Península Ibérica y las Islas Baleares, en términos de los factores de amplificación de Borcherdt (1994) para altas frecuencias $(2-10 \mathrm{~Hz})$ y para bajas frecuencias $(0,5-$ $2,5 \mathrm{~Hz}) . \ldots \ldots \ldots . \ldots \ldots . \ldots \ldots$

3.12. Mapa de amplificación sísmica de las Islas Canarias, en términos de los factores de amplificación de Borcherdt (1994) para altas frecuencias $(2-10 \mathrm{~Hz})$ y para bajas frecuencias $(0,5-2,5 \mathrm{~Hz}) . \ldots 194$

4.1. Emplazamientos correspondientes a la campaña de adquisición de medidas ReMi en El Hierro en noviembre de 2013. La instrumentación sísmica y geodésica dispuesta por el IGN en la isla aparece también representada, incluyendo las estaciones de registro de velocidad de corto periodo (SP) y de banda ancha (BB), en función de su operatividad (Instituto Geográfico Nacional, 2015a); las estaciones centrales de arrays sísmicos, los acelerómetros en función de su operatividad (Instituto Geográfico Nacional, 2017b), y las estaciones de GPS. Se presenta asimismo el mapa de clases de emplazamiento y de Vs30 de El Hierro, de acuerdo con el mapa general para las Islas Canarias de la Figura 3.10. La información relativa a los arrays sísmicos y a las estaciones de GPS ha sido proporcionada directamente por el Instituto Geográfico Nacional, previa petición motivada. . . . . . . . . . . 197

4.2. Geófono perfectamente instalado sobre el terreno por medio de su acoplamiento previo a una placa de duraluminio, durante la toma de medidas de ruido sísmico ambiental por medio del método ReMi, en el emplazamiento $n^{\circ} 2$ (Array sísmico de Tacorón, en el municipio de El Pinar). . . . . . . . . . . . . . . . . . . 198

4.3. Líneas sísmicas con 24 geófonos equiespaciados $3 \mathrm{~m}$, desplegadas en el emplazamiento $n^{\circ} 5$ (municipio de Valverde) y preparadas para la toma de medidas con el método ReMi. Se observa, en primer plano, la conexión de las líneas al equipo digitalizador. Al fondo, el ayudante golpea la placa metálica dispuesta en el extremo del dispositivo con la maza de $6 \mathrm{~kg}$. . . . . . . . . . . . . . . . . . 199

4.4. Diagrama de la transformada lentitud-frecuencia $(p-f)$ definitiva para el emplazamiento $n^{\circ} 23$, resultado de la suma de las diez transformadas individuales obtenidas, una por cada ventana de registro. Los puntos cuadrados blancos son los puntos de dispersión experimentales que se han seleccionado en este emplazamiento. . . . . . . 200 
4.5. Observaciones que constituyen la curva de dispersión experimental del emplazamiento $n^{\circ} 23$, a las que se ajusta la curva de dispersión teórica que se ha obtenido a partir del modelo de velocidad Vs. El error cuadrático medio (error RMS) del ajuste es de $16 \mathrm{~m} / \mathrm{s}$, y el valor de Vs30 que corresponde al modelo Vs es de $306 \mathrm{~m} / \mathrm{s}$. . . . . . . 201

4.6. Modelos de Vs de los emplazamientos $n^{\circ} 1$ y 13 , en los que se ha introducido una capa de baja velocidad de acuerdo con las consideraciones realizadas en el texto. Se muestra, asimismo, el valor de Vs30 que corresponde a cada modelo de Vs. . . . . . . . . . . . . 202

4.7. Distribución espacial de los valores de Vs30 obtenidos en El Hierro por medio del método ReMi. Se presenta igualmente el mapa de clases de emplazamiento y de Vs30 de la isla, de acuerdo con el mapa general para las Islas Canarias de la Figura 3.10 . . . . . . . . . . . . 205

4.8. Curvas de amplificación espectral obtenidas a partir de los modelos de velocidad Vs en los emplazamientos $n^{\circ} 4,17$ y $22 \ldots 208$

5.1. Curvas de la relación $\mathrm{H} / \mathrm{V}$ en función de la frecuencia (izquierda) obtenidas con ventana de ruido sísmico de 30 minutos en los emplazamientos $n^{\circ}$ 4, 17 y 22. En la parte derecha, análisis del ratio $\mathrm{H} / \mathrm{V}$ en función de la frecuencia para los acimutes $0^{\circ}$ a $180^{\circ}$. Se señala la frecuencia fundamental $\left(f_{0}\right)$ y su desviación estándar para el emplazamiento 17 , único en el que el máximo ratio $\mathrm{H} / \mathrm{V}$ no se encuentra focalizado. . . . . . . . . . . . . . . . . . 214

6.1. Terremotos de magnitud $4,1 \leq M w \leq 4,9$ ocurridos en El Hierro durante las crisis sísmicas de julio-octubre de 2011, junio de 2012 y marzo de 2013, para los cuales se han registrado acelerogramas en las estaciones acelerométricas del IGN. Estos eventos han sido utilizados en el análisis y calibración de las relaciones de atenuación en aceleración máxima que son aplicables en la isla. . . . . . . . . . 231

6.2. PGA registradas y corregidas a roca dura para los terremotos de calibración de El Hierro (2011-2013) con 4,0<Mw $\leq$ 5,0, curva de atenuación de la PGA para un terremoto de $\mathrm{Mw} 4,5$, de acuerdo al modelo de atenuación de Pétursson y Vogfjörd (2009), y curvas de atenuación para la PGA que incluyen la desviación estándar $\sigma$ del modelo y que han sido obtenidas a partir de $\log (P G A) \pm 2 \sigma)$. . . 247

6.3. PGA registradas y corregidas a roca dura para los terremotos de calibración de El Hierro (2011-2013) con Mw > 4,5, PGA sintéticas obtenidas con el modelo de atenuación de Pétursson y Vogfjörd (2009) y PGA sintéticas obtenidas a partir de $\log (P G A) \pm 2 \sigma$, siendo $\sigma$ la desviación estándar del modelo. . . . . . . . . . . . . . 248

7.1. Distribución espacial de las estaciones de registro de velocidad del IGN en las Islas Canarias y que han sido utilizadas en esta tesis. Se muestra también una ampliación específica para la isla de El Hierro (esquina inferior derecha). La información sobre el estado operativo de cada estación está actualizada a fecha de octubre de 2015 . . . . . 259 
7.2. Terremotos de magnitud $M_{L} \geq 3$ para los que se ha obtenido al menos una observación de Q, y que han sido registrados por el IGN entre mayo de 2003 y agosto de 2013. En la esquina inferior derecha se muestra una ampliación específica para la isla de El Hierro. . . . . . . 260

7.3. Traza original del registro de la componente vertical de la estación CTIG (arriba) y ventanas de coda filtradas con filtro paso-banda (abajo), para el terremoto de $M_{L} 3,3$ ocurrido el 26-03-2013 a las 05:52:11 UTC en la isla de El Hierro, a $23 \mathrm{~km}$ de distancia, tal y como son presentadas por CODAQ. En la traza original (arriba), las flechas verticales indican (de izquierda a derecha), el tiempo origen del terremoto, el inicio de la ventana de coda y el final de la misma. Encima de esta traza, $\mathrm{H}$ es la profundidad del hipocentro $(\mathrm{km}), \mathrm{M}$ es la magnitud $M_{L}$, TP es el tiempo de viaje de la onda P (s), TC es el tiempo inicial de la ventana de coda medido desde el origen (s), WIN es la longitud de la ventana de coda (s) y ST el tiempo inicial de la ventana de coda en términos del tiempo de viaje de la onda S. En cada ventana de coda filtrada se dan los valores Q: observación de Q de coda, CO: coeficiente de correlación, S/N: relación señal/ruido. El ajuste de la envolvente a la señal filtrada se representa como una curva decreciente con el tiempo. UTC es Tiempo Universal Coordinado.261

7.4. Mapa de isolíneas de $Q_{0}$ para las trayectorias evento-estación con profundidad focal $>15 \mathrm{~km}$. Los puntos medios de las trayectorias se encuentran también representados. . . . . . . . . . . 263

7.5. Mapa de isolíneas de $\nu$ para las trayectorias evento-estación con profundidad focal $>15 \mathrm{~km}$. Los puntos medios de las trayectorias se encuentran también representados. . . . . . . . . . . 263

7.6. Mapa de isolíneas de $Q_{0}$ para las trayectorias evento-estación con profundidad focal $\leq 15 \mathrm{~km}$. Los puntos medios de las trayectorias se encuentran también representados. . . . . . . . . . . . 264

7.7. Mapa de isolíneas de $\nu$ para las trayectorias evento-estación con profundidad focal $\leq 15 \mathrm{~km}$. Los puntos medios de las trayectorias se encuentran también representados. . . . . . . . . . 264

7.8. Sismicidad con $M_{L} \geq 3$ registrada por el IGN y localizada en una zona fuente constreñida entre $27,55^{\circ} \mathrm{N}$ y $27,85^{\circ} \mathrm{N}$, y entre $17,85^{\circ} \mathrm{W}$ y $18,20^{\circ} \mathrm{W}$. Los terremotos ocurrieron en una ventana temporal que se extiende desde el 1 de enero de 2011 al 31 de mayo de 2012. También se muestra la situación de la estación CTIG y la erupción de El Hierro

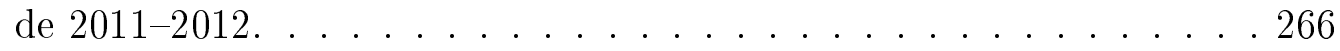

7.9. Evolución temporal de la sismicidad representada en la Figura 7.8, mostrando las magnitudes $M_{L}$ de los terremotos y el número de sismos por día. Se señala también el comienzo de la erupción de 2011-2012 en El Hierro. . . . . . . . . . . . . . . . . . 266 
7.10. Evolución temporal de los valores de $Q_{0}$ (parte superior) y $\nu$ (parte inferior), obtenidos en la estación CTIG (El Hierro), para cada uno de los días de la Tabla 7.1 (círculos sin relleno). Las desviaciones estándar de cada parámetro son representadas por medio de barras de error. Se simbolizan también los valores de $Q_{0}$ y $\nu$ calculados en esta estación para cada una de las fases de López et al. (2012), así como los valores calculados durante el periodo del tremor registrado por el IGN (líneas continuas o discontinuas), ver la Tabla 7.2. Se indica también el comienzo de la erupción de 2011-2012 en El Hierro. 268

8.1. Transformadas de Fourier del acelerograma registrado y del acelerograma simulado en ATIG para el terremoto de 31-03-2013 al W de El Hierro. . . . . . . . . . . . . . . . . . . . 281

8.2. Funciones empíricas de la amplificación de corteza, obtenidas como resultado del ajuste de los acelerogramas observados y simulados en ATIG y AROQ, y también a partir del ajuste de las transformadas de Fourier y los espectros de respuesta en pseudoaceleración correspondientes. También se presenta el modelo de amplificación de la corteza publicado por Kamai et al. (2014) para el proyecto NGA-West2. . . . 281

8.3. Acelerogramas sintético y registrado en AROQ para el terremoto de 31-03-2013 al W de El Hierro. . . . . . . . . . . . . . . . . 282

8.4. Transformadas de Fourier del acelerograma registrado y del acelerograma simulado en AROQ para el terremoto de 31-03-2013 al W de El Hierro. . . . . . . . . . . . . . . . . . . . . . 283

8.5. Espectros de respuesta en pseudoaceleración, para un amortiguamiento del 5\%, obtenidos en ATIG para el terremoto de 31-03-2013 al W de El Hierro, a partir del acelerograma observado y del acelerograma sintético. . . . . . . . . . . . . . . . 283

8.6. Espectros de respuesta en pseudoaceleración, para un amortiguamiento del 5\%, obtenidos en AROQ para el terremoto de 31-03-2013 al W de El Hierro, a partir del acelerograma observado y del acelerograma sintético. . . . . . . . . . . . . . . . . . 284

9.1. Pestaña INPUT DATA del plugin. . . . . . . . . . . . . . . 287

9.2. Pestaña Canary Islands, correspondiente a la pestaña principal $A T$ TENUATION RELATIONSHIPS. . . . . . . . . . . . . 288

9.3. Pestaña Iberian Peninsula and Balearic Islands, correspondiente a la pestaña principal ATTENUATION RELATIONSHIPS. . . . . . . . 289

9.4. Pestaña World, correspondiente a la pestaña principal ATTENUATION RELATIONSHIPS. . . . . . . . . . . . . . . 290

9.5. Vista de la capa Peninsula_amplificacion.shp, descargada en un proyecto QGIS. . . . . . . . . . . . . . . . 291

9.6. Vista de la capa El_Hierro_amplificacion_170317.shp, descargada en un proyecto QGIS. . . . . . . . . . . . . . . . . 292 
9.7. Vista de la capa PLUGIN_GRID_EVID_1060340_EXTENDIDA.shp sobre el mapa de amplificación sísmica de la Península Ibérica y las Islas Baleares. . . . . . . . . . . . . . . . . . . 295

9.8. Vista de la capa PLUGIN_GRID_EL_HIERRO_E2.shp sobre el mapa de amplificación sísmica de la isla de El Hierro. . . . . . . . . 297

9.9. Vista de la capa Aceleracion_Estacion_1060340.shp sobre la malla de cálculo seleccionada para simular el terremoto de 11-05-2011 en Lorca $(\mathrm{Mw}$ 5,1) y el mapa de amplificación sísmica de la Península Ibérica y las Islas Baleares. Los puntos de esta capa, con la aceleración registrada, están simbolizados por triángulos rojos. . . . . . . . . 298

9.10. Vista de la capa Aceleracion_Estacion_1202861_v010417.shp sobre la malla de cálculo seleccionada para simular el terremoto de 31-032013 en el W de El Hierro (Mw 4,9) y el mapa de amplificación sísmica de la isla de El Hierro. Los puntos de esta capa, con la aceleración registrada, están simbolizados por triángulos amarillos. . . . . . . . 300

9.11. Flujo de trabajo seguido por la herramienta plugin para la generación de los mapas de sacudidas correspondientes a un terremoto determinado y en dos casos diferenciados, según se implementen o no las aceleraciones máximas del terreno (PGA) que han sido registradas por los acelerómetros que han detectado el terremoto. Se muestran los sucesivos archivos creados por la herramienta, archivos shapefile (.shp) y archivos de texto delimitado (.csv), así como las tres salidas proporcionadas, en archivos con extensión .tif. . . . . . . . . . 302

9.12. Selección de variables de entrada para la simulación del terremoto de Lorca de 11-05-2011: Parámetros de la fuente, malla de cálculo y mapa de amplificación. . . . . . . . . . . . . 303

9.13. Selección de variables de entrada para la simulación del terremoto de Lorca de 11-05-2011: Magnitud momento y mecanismo focal. . . . . . 303

9.14. Tabla de atributos de la capa grid_amplification.shp, creada a partir de la capa PLUGIN_GRID_EVID_1060340_EXTENDIDA.shp (malla de cálculo) y de la capa Peninsula_amplificacion.shp (mapa de amplificación). . . . . . . . . . . . . . . . 304

9.15. Selección de campos en la creación de la capa con las PGA amplificadas, a partir del fichero de texto delimitado final_amplification.csv. 305

9.16. Selección del sistema de referencia de coordenadas en la creación de la capa con las PGA amplificadas, a partir del fichero de texto delimitado final_amplification.csv. . . . . . . . . . 306

9.17. Capa final_amplification, con las PGA amplificadas y simbolizadas de acuerdo con su valor en $\mathrm{cm} / \mathrm{s}^{2}$, creada a partir del fichero de texto delimitado final_amplification.csv. . . . . . . . . 306

9.18. Mapa de sacudidas obtenido para el terremoto de Lorca de 11-05-2011 (evid 1060340), por medio de la relación de atenuación de Akkar y Bommer (2010), y sin tener en cuenta las aceleraciones registradas en tiempo real. Constituye uno de los archivos de salida del plugin. . . . 307 
9.19. Selección de variables de entrada para la simulación del terremoto de Lorca de 11-05-2011: Parámetros de la fuente, malla de cálculo, mapa de amplificación, capa de aceleraciones máximas horizontales registradas en estaciones acelerométricas en tiempo real y paso de malla de interpolación. . . . . . . . . . . . . . . . 308

9.20. Tabla de atributos de la capa stations_amplification.shp. . . . . . . . 309

9.21. Vista de la capa buffer.shp sobre la capa stations_amplification.shp. . 309

9.22. Vista de la capa difference.shp. . . . . . . . . . . . . . . . 310

9.23. Vista de la capa merge.shp. . . . . . . . . . . . . . . 311

9.24. Tabla de atributos la capa merge.shp. Las filas seleccionadas corresponden a los puntos de la malla de cálculo en los que se ha obtenido la PGA sintética, previamente almacenados en difference.shp. . . . . 311

9.25. Vista de la capa ráster a_rock_sum_2.tif, constituyendo uno de los archivos de salida del plugin. . . . . . . . . . . . . . . 312

9.26. Vista de la capa regular_grid_fa.shp. . . . . . . . . . . 313

9.27. Capa a_int_final, con las aceleraciones máximas interpoladas y amplificadas, siendo simbolizadas de acuerdo con su valor en $\mathrm{cm} / \mathrm{s}^{2}$. La capa se ha creado a partir del fichero de texto delimitado a_int_final.csv.314

9.28. Mapa de sacudidas obtenido para el terremoto de Lorca de 11-052011 (evid 1060340), por medio de la relación de atenuación de Akkar y Bommer (2010) y de las aceleraciones registradas en tiempo real. Constituye uno de los archivos de salida del plugin. . . . . . . . . . 314

9.29. Parte superior: Detalle del mapa de sacudidas obtenido en la Figura 9.18. Parte inferior: Detalle del mapa de sacudidas obtenido en la Figura 9.28. . . . . . . . . . . . . . . . . . . 316

9.30. Capa intensity, con los valores de Intensidad Modificada de Mercalli en los puntos de la malla de interpolación. La capa se ha creado a partir del fichero de texto delimitado intensity.csv. . . . . . . . . . . 317

10.1. Situación del epicentro del terremoto de 11-05-2011 a las 16:47:26 UTC, al NE de Lorca (Murcia), con Mw 5,1. También se muestran los acelerómetros del IGN que han registrado el citado evento. . . . . 322

10.2. Situación de los epicentros de los terremotos de 08-10-2011 a las 20:34:48 UTC, al SW de El Hierro, con Mw 4,0, y de 31-03-2013 a las 10:59:54 UTC, al W de El Hierro, con Mw 4,9. También se muestran los acelerómetros del IGN que han registrado el evento de 31-03-2013. 323

10.3. Mapa de sacudidas del terremoto de 11-05-2011 en Lorca (Mw 5,1), producido a partir de las aceleraciones máximas sintéticas calculadas por medio de la relación de atenuación de Instituto Geográfico Nacional (2013) y de las aceleraciones máximas registradas en los acelerómetros del IGN. . . . . . . . . . . . . . . . . . 326 
10.4. Mapa de sacudidas del terremoto de 11-05-2011 en Lorca (Mw 5,1), producido a partir de las aceleraciones máximas sintéticas calculadas por medio de la relación de atenuación de Akkar y Bommer (2010), y sin implementar las aceleraciones máximas registradas en los acelerómetros del IGN. . . . . . . . . . . . . . . . . . . . 326

10.5. Mapa de contornos de los valores interpolados de las aceleraciones máximas registradas y corregidas a la condición del terreno de referencia, y de las aceleraciones máximas calculadas en la condición del terreno de referencia por medio de la ley de Akkar y Bommer (2010), correspondiente al terremoto de 11-05-2011 en Lorca (Mw 5,1). . . . . 327

10.6. Mapa de sacudidas del terremoto de 11-05-2011 en Lorca (Mw 5,1), producido a partir de las aceleraciones máximas sintéticas calculadas por medio de la relación de atenuación de Akkar y Bommer (2010) y de las aceleraciones máximas registradas en los acelerómetros del IGN.327

10.7. Mapa de intensidades sintéticas (EMS-98) del terremoto de 11-052011 en Lorca (Mw 5,1), producido a partir de las aceleraciones máximas sintéticas proporcionadas por el mapa de sacudidas de la Figura 10.6, y por medio de la utilización de las correlaciones entre la aceleración máxima del terreno y la intensidad sísmica de Wald et al. (1999a). . . . . . . . . . . . . . . . . . . . . . . . . . . . . .

10.8. Mapa de sacudidas del terremoto de 11-05-2011 en Lorca (Mw 5,1), producido a partir de las aceleraciones máximas sintéticas calculadas por medio de la relación de atenuación de García Blanco (2009) y de las aceleraciones máximas registradas en los acelerómetros del IGN. . 328

10.9. Mapa de sacudidas del terremoto de 11-05-2011 en Lorca (Mw 5,1), producido a partir de las aceleraciones máximas sintéticas calculadas por medio de la relación de atenuación de Boore y Atkinson (2008) y de las aceleraciones máximas registradas en los acelerómetros del IGN.329

10.10Mapa de sacudidas del terremoto de 11-05-2011 en Lorca (Mw 5,1), producido a partir de las aceleraciones máximas sintéticas calculadas por medio de la relación de atenuación de Mezcua et al. (2008) y de las aceleraciones máximas registradas en los acelerómetros del IGN. 329

10.11Mapa de sacudidas del terremoto de 31-03-2013 al W de El Hierro (Mw 4,9), producido a partir de las aceleraciones máximas sintéticas calculadas por medio de la relación de atenuación de Pétursson y Vogfjörd (2009), y sin implementar las aceleraciones máximas registradas en los acelerómetros del IGN. . . . . . . . . . . . . . . 333

10.12Mapa de contornos de los valores interpolados de las aceleraciones máximas registradas y corregidas a la condición del terreno de referencia, y de las aceleraciones máximas calculadas en la condición del terreno de referencia por medio de la ley de Pétursson y Vogfjörd (2009), correspondiente al terremoto de 31-03-2013 al W de El Hierro

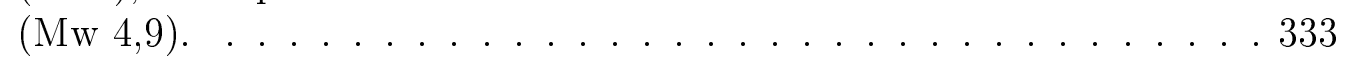


10.13Mapa de sacudidas del terremoto de 31-03-2013 al W de El Hierro (Mw 4,9), producido a partir de las aceleraciones máximas sintéticas calculadas por medio de la relación de atenuación de Pétursson y Vogfjörd (2009) y de las aceleraciones máximas registradas en los acelerómetros del IGN. . . . . . . . . . . . . . . . . . . . 334

10.14Mapa de intensidades sintéticas (EMS-98) del terremoto de 31-032013 al W de El Hierro (Mw 4,9), producido a partir de las aceleraciones máximas sintéticas proporcionadas por el mapa de sacudidas de la Figura 10.13, y por medio de la utilización de las correlaciones entre la aceleración máxima del terreno y la intensidad sísmica de Wald et al. (1999a). . . . . . . . . . . . . . . . . . . 334

10.15Mapa de sacudidas del terremoto de 31-03-2013 al W de El Hierro (Mw 4,9), producido a partir de las aceleraciones máximas sintéticas calculadas por medio de la relación de atenuación de Ágústsson et al. (2008) y de las aceleraciones máximas registradas en los acelerómetros

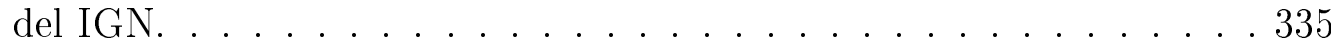

10.16Mapa de sacudidas del terremoto de 31-03-2013 al W de El Hierro (Mw 4,9), producido a partir de las aceleraciones máximas sintéticas calculadas por medio de la relación de atenuación de Beauducel et al. (2004) y de las aceleraciones máximas registradas en los acelerómetros del IGN. . . . . . . . . . . . . . . . . . . . . . . . . . . . . . . . . . . . . . . .

10.17Mapa de sacudidas del terremoto de 31-03-2013 al W de El Hierro (Mw 4,9), producido a partir de las aceleraciones máximas sintéticas calculadas por medio de la relación de atenuación de Chiou y Youngs (2008) y de las aceleraciones máximas registradas en los acelerómetros del IGN. Los ángulos de buzamiento y deslizamiento (rake) del plano de la falla que se han considerado en la relación de Chiou y Youngs (2008) corresponden al plano focal A del mecanismo focal del terremoto de 08-10-2011 ( Mw 4,0) (Tabla 10.1) . . . . . . . . . 336

10.18Mapa de sacudidas del terremoto de 31-03-2013 al W de El Hierro (Mw 4,9), producido a partir de las aceleraciones máximas sintéticas calculadas por medio de la relación de atenuación de Chiou y Youngs (2008) y de las aceleraciones máximas registradas en los acelerómetros del IGN. Los ángulos de buzamiento y deslizamiento (rake) del plano de la falla que se han considerado en la relación de Chiou y Youngs (2008) corresponden al plano focal B del mecanismo focal del terremoto de 08-10-2011 ( Mw 4,0) (Tabla 10.1) . . . . . . . . . 336

10.19Mapa de sacudidas del terremoto de 31-03-2013 al W de El Hierro (Mw 4,9), producido a partir de las aceleraciones máximas sintéticas calculadas por medio de la relación de atenuación de Zhao et al. (2006) y de las aceleraciones máximas registradas en los acelerómetros del IGN . . . . . . . . . . . . . . . . . . . . . . . . . . . . . . . . . .

10.20Intensidades calculadas y observadas para el terremoto de 31-03-2013 al W de El Hierro, con Mw 4,9, en función de la distancia hipocentral, junto con las respectivas rectas de regresión logarítmica. . . . . . . 345 
D.1. Diagrama de la transformada lentitud-frecuencia $(p-f)$ definitiva para el emplazamiento $n^{\circ} 1$. Los puntos de dispersión experimentales se representan como cuadrados blancos. . . . . . . . . . . 406

D.2. Diagrama de la transformada lentitud-frecuencia $(p-f)$ definitiva para el emplazamiento $n^{\circ} 2$. Los puntos de dispersión experimentales se representan como cuadrados blancos. . . . . . . . . . . . 406

D.3. Diagrama de la transformada lentitud-frecuencia $(p-f)$ definitiva para el emplazamiento $n^{\circ} 3$. Los puntos de dispersión experimentales se representan como cuadrados blancos. . . . . . . . . . . . 407

D.4. Diagrama de la transformada lentitud-frecuencia $(p-f)$ definitiva para el emplazamiento $n^{\circ} 4$. Los puntos de dispersión experimentales se representan como cuadrados blancos. . . . . . . . . . . . 407

D.5. Diagrama de la transformada lentitud-frecuencia $(p-f)$ definitiva para el emplazamiento $n^{\circ} 5$. Los puntos de dispersión experimentales se representan como cuadrados blancos. . . . . . . . . . . . . 408

D.6. Diagrama de la transformada lentitud-frecuencia $(p-f)$ definitiva para el emplazamiento $n^{\circ} 6$. Los puntos de dispersión experimentales se representan como cuadrados blancos. . . . . . . . . . . . . 408

D.7. Diagrama de la transformada lentitud-frecuencia $(p-f)$ definitiva para el emplazamiento $n^{\circ}$ 7. Los puntos de dispersión experimentales se representan como cuadrados blancos. . . . . . . . . . . . 409

D.8. Diagrama de la transformada lentitud-frecuencia $(p-f)$ definitiva para el emplazamiento $n^{\circ} 8$. Los puntos de dispersión experimentales se representan como cuadrados blancos. . . . . . . . . . . . . 409

D.9. Diagrama de la transformada lentitud-frecuencia $(p-f)$ definitiva para el emplazamiento $n^{\circ}$ 9. Los puntos de dispersión experimentales se representan como cuadrados blancos. . . . . . . . . . . . . 410

D.10.Diagrama de la transformada lentitud-frecuencia $(p-f)$ definitiva para el emplazamiento $n^{\circ} 10$. Los puntos de dispersión experimentales se representan como cuadrados blancos. . . . . . . . . . . . . . . 410

D.11.Diagrama de la transformada lentitud-frecuencia $(p-f)$ definitiva para el emplazamiento $n^{\circ} 11$. Los puntos de dispersión experimentales se representan como cuadrados blancos. . . . . . . . . . . . . 411

D.12.Diagrama de la transformada lentitud-frecuencia $(p-f)$ definitiva para el emplazamiento $n^{\circ} 12$. Los puntos de dispersión experimentales se representan como cuadrados blancos. . . . . . . . . . . . . . 411

D.13.Diagrama de la transformada lentitud-frecuencia $(p-f)$ definitiva para el emplazamiento $n^{\circ} 13$. Los puntos de dispersión experimentales se representan como cuadrados blancos. . . . . . . . . . . . . . 412

D.14.Diagrama de la transformada lentitud-frecuencia $(p-f)$ definitiva para el emplazamiento $n^{\circ} 14$. Los puntos de dispersión experimentales se representan como cuadrados blancos. . . . . . . . . . . . . 412 
D.15.Diagrama de la transformada lentitud-frecuencia $(p-f)$ definitiva para el emplazamiento $n^{\circ} 15$. Los puntos de dispersión experimentales se representan como cuadrados blancos. . . . . . . . . . . . . . 413

D.16.Diagrama de la transformada lentitud-frecuencia $(p-f)$ definitiva para el emplazamiento $n^{\circ} 16$. Los puntos de dispersión experimentales se representan como cuadrados blancos. . . . . . . . . . . . . 413

D.17.Diagrama de la transformada lentitud-frecuencia $(p-f)$ definitiva para el emplazamiento $n^{\circ} 17$. Los puntos de dispersión experimentales se representan como cuadrados blancos. . . . . . . . . . . . . . . . 414

D.18.Diagrama de la transformada lentitud-frecuencia $(p-f)$ definitiva para el emplazamiento $n^{\circ} 18$. Los puntos de dispersión experimentales se representan como cuadrados blancos. . . . . . . . . . . . . . . 414

D.19.Diagrama de la transformada lentitud-frecuencia $(p-f)$ definitiva para el emplazamiento $n^{\circ} 19$. Los puntos de dispersión experimentales se representan como cuadrados blancos. . . . . . . . . . . . . 415

D.20.Diagrama de la transformada lentitud-frecuencia $(p-f)$ definitiva para el emplazamiento $n^{\circ} 20$. Los puntos de dispersión experimentales se representan como cuadrados blancos. . . . . . . . . . . 415

D.21.Diagrama de la transformada lentitud-frecuencia $(p-f)$ definitiva para el emplazamiento $n^{\circ} 21$. Los puntos de dispersión experimentales se representan como cuadrados blancos. . . . . . . . . . . . 416

D.22.Diagrama de la transformada lentitud-frecuencia $(p-f)$ definitiva para el emplazamiento $n^{\circ} 22$. Los puntos de dispersión experimentales se representan como cuadrados blancos. . . . . . . . . . . 4 416

D.23.Diagrama de la transformada lentitud-frecuencia $(p-f)$ definitiva para el emplazamiento $n^{\circ} 23$. Los puntos de dispersión experimentales se representan como cuadrados blancos. . . . . . . . . . . . . 417

D.24.Diagrama de la transformada lentitud-frecuencia $(p-f)$ definitiva para el emplazamiento $n^{\circ} 24$. Los puntos de dispersión experimentales se representan como cuadrados blancos. . . . . . . . . . . . 417

D.25.Diagrama de la transformada lentitud-frecuencia $(p-f)$ definitiva para el emplazamiento $n^{\circ} 25$. Los puntos de dispersión experimentales se representan como cuadrados blancos. . . . . . . . . . . . . . 4418

E.1. Puntos de dispersión observados en el emplazamiento $n^{\circ} 1$ y ajuste de la curva de dispersión teórica. Modelo de velocidad Vs obtenido y curva de amplificación espectral correspondiente. . . . . . . . . . . 420

E.2. Puntos de dispersión observados en el emplazamiento $n^{\circ} 2$ y ajuste de la curva de dispersión teórica. Modelo de velocidad Vs obtenido y curva de amplificación espectral correspondiente. . . . . . . . . . 420

E.3. Puntos de dispersión observados en el emplazamiento $n^{\circ} 3$ y ajuste de la curva de dispersión teórica. Modelo de velocidad Vs obtenido y curva de amplificación espectral correspondiente. . . . . . . . . . . 421 
E.4. Puntos de dispersión observados en el emplazamiento $n^{\circ} 4$ y ajuste de la curva de dispersión teórica. Modelo de velocidad Vs obtenido y curva de amplificación espectral correspondiente. . . . . . . . . . .

E.5. Puntos de dispersión observados en el emplazamiento $n^{\circ} 5$ y ajuste de la curva de dispersión teórica. Modelo de velocidad Vs obtenido y curva de amplificación espectral correspondiente. . . . . . . . . . . . . 422

E.6. Puntos de dispersión observados en el emplazamiento $n^{\circ} 6$ y ajuste de la curva de dispersión teórica. Modelo de velocidad Vs obtenido y curva de amplificación espectral correspondiente. . . . . . . . . . . . 422

E.7. Puntos de dispersión observados en el emplazamiento $n^{\circ} 7$ y ajuste de la curva de dispersión teórica. Modelo de velocidad Vs obtenido y curva de amplificación espectral correspondiente. . . . . . . . . 423

E.8. Puntos de dispersión observados en el emplazamiento $n^{\circ} 8$ y ajuste de la curva de dispersión teórica. Modelo de velocidad Vs obtenido y curva de amplificación espectral correspondiente. . . . . . . . . 423

E.9. Puntos de dispersión observados en el emplazamiento $n^{\circ} 9$ y ajuste de la curva de dispersión teórica. Modelo de velocidad Vs obtenido y curva de amplificación espectral correspondiente. . . . . . . . . . . . . 424

E.10.Puntos de dispersión observados en el emplazamiento $n^{\circ} 10$ y ajuste de la curva de dispersión teórica. Modelo de velocidad Vs obtenido y curva de amplificación espectral correspondiente. . . . . . . . . . 424

E.11.Puntos de dispersión observados en el emplazamiento $n^{\circ} 11$ y ajuste de la curva de dispersión teórica. Modelo de velocidad Vs obtenido y curva de amplificación espectral correspondiente. . . . . . . . . . . 425

E.12.Puntos de dispersión observados en el emplazamiento $n^{\circ} 12$ y ajuste de la curva de dispersión teórica. Modelo de velocidad Vs obtenido y curva de amplificación espectral correspondiente. . . . . . . . . . 4 425

E.13.Puntos de dispersión observados en el emplazamiento $n^{\circ} 13$ y ajuste de la curva de dispersión teórica. Modelo de velocidad Vs obtenido y curva de amplificación espectral correspondiente. . . . . . . . . . 426

E.14.Puntos de dispersión observados en el emplazamiento $n^{\circ} 14$ y ajuste de la curva de dispersión teórica. Modelo de velocidad Vs obtenido y curva de amplificación espectral correspondiente. . . . . . . . . 426

E.15.Puntos de dispersión observados en el emplazamiento $n^{\circ} 15$ y ajuste de la curva de dispersión teórica. Modelo de velocidad Vs obtenido y curva de amplificación espectral correspondiente. . . . . . . . . . . 427

E.16.Puntos de dispersión observados en el emplazamiento $n^{\circ} 16$ y ajuste de la curva de dispersión teórica. Modelo de velocidad Vs obtenido y curva de amplificación espectral correspondiente. . . . . . . . . . . 427

E.17.Puntos de dispersión observados en el emplazamiento $n^{\circ} 17$ y ajuste de la curva de dispersión teórica. Modelo de velocidad Vs obtenido y curva de amplificación espectral correspondiente. . . . . . . . . 428 
E.18.Puntos de dispersión observados en el emplazamiento $n^{\circ} 18$ y ajuste de la curva de dispersión teórica. Modelo de velocidad Vs obtenido y curva de amplificación espectral correspondiente. . . . . . . . . . . 428

E.19.Puntos de dispersión observados en el emplazamiento $n^{\circ} 19$ y ajuste de la curva de dispersión teórica. Modelo de velocidad Vs obtenido y curva de amplificación espectral correspondiente. . . . . . . . . . . 4

E.20.Puntos de dispersión observados en el emplazamiento $n^{\circ} 20$ y ajuste de la curva de dispersión teórica. Modelo de velocidad Vs obtenido y curva de amplificación espectral correspondiente. . . . . . . . . . .

E.21.Puntos de dispersión observados en el emplazamiento $n^{\circ} 21$ y ajuste de la curva de dispersión teórica. Modelo de velocidad Vs obtenido y curva de amplificación espectral correspondiente. . . . . . . . . . . 430

E.22.Puntos de dispersión observados en el emplazamiento $n^{\circ} 22$ y ajuste de la curva de dispersión teórica. Modelo de velocidad Vs obtenido y curva de amplificación espectral correspondiente. . . . . . . . . . . 430

E.23.Puntos de dispersión observados en el emplazamiento $n^{\circ} 23$ y ajuste de la curva de dispersión teórica. Modelo de velocidad Vs obtenido y curva de amplificación espectral correspondiente. . . . . . . . . . . 431

E.24.Puntos de dispersión observados en el emplazamiento $n^{\circ} 24$ y ajuste de la curva de dispersión teórica. Modelo de velocidad Vs obtenido y curva de amplificación espectral correspondiente. . . . . . . . . . . . 431

E.25.Puntos de dispersión observados en el emplazamiento $n^{\circ} 25$ y ajuste de la curva de dispersión teórica. Modelo de velocidad Vs obtenido y curva de amplificación espectral correspondiente. . . . . . . . . . . 432

F.1. Curva de la relación $\mathrm{H} / \mathrm{V}$ en función de la frecuencia, obtenida con ventana de ruido sísmico de 30 minutos en el emplazamiento 1 (izquierda), y análisis del ratio $\mathrm{H} / \mathrm{V}$ en función de la frecuencia y del acimut, para los acimutes de $0^{\circ}$ a $180^{\circ}$ (derecha). . . . . . . . . 434

F.2. Curva de la relación $\mathrm{H} / \mathrm{V}$ en función de la frecuencia, obtenida con ventana de ruido sísmico de 30 minutos en el emplazamiento 2 (izquierda), y análisis del ratio $\mathrm{H} / \mathrm{V}$ en función de la frecuencia y del acimut, para los acimutes de $0^{\circ}$ a $180^{\circ}$ (derecha). . . . . . . . . . 444

F.3. Curva de la relación $\mathrm{H} / \mathrm{V}$ en función de la frecuencia, obtenida con ventana de ruido sísmico de 30 minutos en el emplazamiento 4 (izquierda), y análisis del ratio $\mathrm{H} / \mathrm{V}$ en función de la frecuencia y del acimut, para los acimutes de $0^{\circ}$ a $180^{\circ}$ (derecha). . . . . . . . . 435

F.4. Curva de la relación $\mathrm{H} / \mathrm{V}$ en función de la frecuencia, obtenida con ventana de ruido sísmico de 30 minutos en el emplazamiento 17 (izquierda), y análisis del ratio $\mathrm{H} / \mathrm{V}$ en función de la frecuencia y del acimut, para los acimutes de $0^{\circ}$ a $180^{\circ}$ (derecha). Se señala la frecuencia fundamental $\left(f_{0}\right)$ y su desviación estándar. . . . . . . 4 435 
F.5. Curvas de la relación $\mathrm{H} / \mathrm{V}$ en función de la frecuencia, obtenidas con ventana de ruido sísmico de 30 minutos en las estaciones de registro de velocidad CTAN y CTAB, próximas al emplazamiento 19 (izquierda), y análisis correspondientes del ratio $\mathrm{H} / \mathrm{V}$ en función de la frecuencia y del acimut, para los acimutes de $0^{\circ}$ a $180^{\circ}$ (derecha) . . . . . . 436

F.6. Curva de la relación $\mathrm{H} / \mathrm{V}$ en función de la frecuencia, obtenida con ventana de ruido sísmico de 30 minutos en el emplazamiento 22 (izquierda), y análisis del ratio $\mathrm{H} / \mathrm{V}$ en función de la frecuencia y del acimut, para los acimutes de $0^{\circ}$ a $180^{\circ}$ (derecha) . . . . . . . . 4477

G.1. Parte superior: PGA registradas y corregidas a roca dura para los terremotos de calibración de El Hierro (2011-2013) con 4,0<Mw $\leq$ 5,0, curva de atenuación de la PGA para un terremoto de Mw 4,5, de acuerdo al modelo de atenuación de García Blanco (2009), y curvas de atenuación para la PGA que incluyen la desviación estándar $\sigma$ del modelo y que han sido obtenidas a partir de $\log (P G A) \pm 2 \sigma)$. Parte inferior: PGA registradas y corregidas a roca dura para los terremotos con con $\mathrm{Mw}>4,5$, PGA sintéticas obtenidas con el modelo de atenuación de García Blanco (2009) y PGA sintéticas obtenidas a

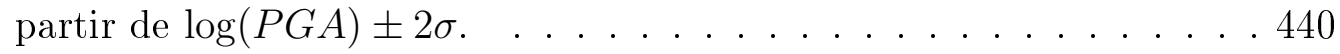

G.2. Parte superior: PGA registradas y corregidas a roca dura para los terremotos de calibración de El Hierro (2011-2013) con 4,0<Mw $\leq$ 5,0 , curva de atenuación de la PGA para un terremoto de $\mathrm{Mw} 4,5$, de acuerdo al modelo de atenuación de Instituto Geográfico Nacional (2013), y curvas de atenuación para la PGA que incluyen la desviación estándar $\sigma$ del modelo y que han sido obtenidas a partir de $\log (P G A) \pm 2 \sigma)$. Parte inferior: PGA registradas y corregidas a roca dura para los terremotos con $\mathrm{Mw}>4,5$, PGA sintéticas obtenidas con el modelo de atenuación de Instituto Geográfico Nacional (2013) y PGA sintéticas obtenidas a partir de $\log (P G A) \pm 2 \sigma$. . . . . . 441

G.3. Parte superior: PGA registradas y corregidas a roca dura para los terremotos de calibración de El Hierro (2011-2013) con 4,0<Mw $\leq$ 5,0, curva de atenuación de la PGA para un terremoto de Mw 4,5, de acuerdo al modelo de atenuación de Sigbjörnsson y Baldvinsson (1992) con PGA definida en función de la componente máxima horizontal, y curvas de atenuación para la PGA que incluyen la desviación estándar $\sigma$ del modelo y que han sido obtenidas a partir de $\log (P G A) \pm 2 \sigma)$. Parte inferior: PGA registradas y corregidas a roca dura para los terremotos con $\mathrm{Mw}>4,5$, PGA sintéticas obtenidas con el modelo de atenuación de Sigbjörnsson y Baldvinsson (1992) con PGA definida en función de la componente máxima horizontal y PGA sintéticas obtenidas a partir de $\log (P G A) \pm 2 \sigma$. . . . . . 442 
G.4. Parte superior: PGA registradas y corregidas a roca dura para los terremotos de calibración de El Hierro (2011-2013) con 4,0<Mw $\leq$ 5,0, curva de atenuación de la PGA para un terremoto de Mw 4,5, de acuerdo al modelo de atenuación de Sigbjörnsson y Baldvinsson (1992) con PGA definida en función de la media de las componentes horizontales, y curvas de atenuación para la PGA que incluyen la desviación estándar $\sigma$ del modelo y que han sido obtenidas a partir de $\log (P G A) \pm 2 \sigma)$. Parte inferior: PGA registradas y corregidas a roca dura para los terremotos con $\mathrm{Mw}>4,5$, PGA sintéticas obtenidas con el modelo de atenuación de Sigbjörnsson y Baldvinsson (1992) con PGA definida en función de la media de las componentes horizontales, y PGA sintéticas obtenidas a partir de $\log (P G A) \pm 2 \sigma \ldots . . . . .443$

G.5. Parte superior: PGA registradas y corregidas a roca dura para los terremotos de calibración de El Hierro (2011-2013) con 4,0<Mw $\leq$ 5,0 , curva de atenuación de la PGA para un terremoto de $\mathrm{Mw} 4,5$, de acuerdo al modelo de atenuación de Sigbjörnsson y Baldvinsson (1992) con PGA definida en función de la componente horizontal E-W, y curvas de atenuación para la PGA que incluyen la desviación estándar $\sigma$ del modelo y que han sido obtenidas a partir de $\log (P G A) \pm 2 \sigma)$. Parte inferior: PGA registradas y corregidas a roca dura para los terremotos con $\mathrm{Mw}>4,5$, PGA sintéticas obtenidas con el modelo de atenuación de Sigbjörnsson y Baldvinsson (1992) con PGA definida en función de la componente horizontal E-W, y PGA sintéticas obtenidas a partir de $\log (P G A) \pm 2 \sigma . \ldots . . . . .444$

G.6. Parte superior: PGA registradas y corregidas a roca dura para los terremotos de calibración de El Hierro (2011-2013) con 4,0<Mw 5,0, curva de atenuación de la PGA para un terremoto de Mw 4,5, de acuerdo al modelo de atenuación de Sigbjörnsson y Baldvinsson (1992) con PGA definida en función de la componente horizontal N-S, y curvas de atenuación para la PGA que incluyen la desviación estándar $\sigma$ del modelo y que han sido obtenidas a partir de $\log (P G A) \pm 2 \sigma)$. Parte inferior: PGA registradas y corregidas a roca dura para los terremotos con $\mathrm{Mw}>4,5$, PGA sintéticas obtenidas con el modelo de atenuación de Sigbjörnsson y Baldvinsson (1992) con PGA definida en función de la componente horizontal N-S, y PGA sintéticas obtenidas a partir de $\log (P G A) \pm 2 \sigma \ldots \ldots . \ldots . \ldots . \ldots 445$

G.7. Parte superior: PGA registradas y corregidas a roca dura para los terremotos de calibración de El Hierro (2011-2013) con 4,0<Mw $\leq$ 5,0 , curva de atenuación de la PGA para un terremoto de Mw 4,5, de acuerdo al modelo de atenuación de Ólafsson y Sigbjörnsson (1999), y curvas de atenuación para la PGA que incluyen la desviación estándar $\sigma$ del modelo y que han sido obtenidas a partir $\operatorname{de} \log (P G A) \pm 2 \sigma)$. Parte inferior: PGA registradas y corregidas a roca dura para los terremotos con $\mathrm{Mw}>4,5$, PGA sintéticas obtenidas con el modelo de atenuación de Ólafsson y Sigbjörnsson (1999) y PGA sintéticas obtenidas a partir de $\log (P G A) \pm 2 \sigma \ldots . . . . . . . . . .446$ 
G.8. Parte superior: PGA registradas y corregidas a roca dura para los terremotos de calibración de El Hierro (2011-2013) con 4,0<Mw $\leq$ 5,0, curva de atenuación de la PGA para un terremoto de $\mathrm{Mw} 4,5$, de acuerdo al modelo de atenuación de Ágústsson et al. (2008) con dependencia del logaritmo de la magnitud $\mathrm{M}$, y curvas de atenuación para la PGA que incluyen la desviación estándar $\sigma$ del modelo y que han sido obtenidas a partir de $\log (P G A) \pm 2 \sigma)$. Parte inferior: PGA registradas y corregidas a roca dura para los terremotos con $\mathrm{Mw}>4,5$, PGA sintéticas obtenidas con el modelo de atenuación de Ágústsson et al. (2008) con dependencia del logaritmo de la magnitud M, y PGA sintéticas obtenidas a partir de $\log (P G A) \pm 2 \sigma \ldots . . .447$

G.9. Parte superior: PGA registradas y corregidas a roca dura para los terremotos de calibración de El Hierro (2011-2013) con 4,0<Mw $\leq$ 5,0 , curva de atenuación de la PGA para un terremoto de Mw 4,5, de acuerdo al modelo de atenuación de Ágústsson et al. (2008) con dependencia lineal con la magnitud M, y curvas de atenuación para la PGA que incluyen la desviación estándar $\sigma$ del modelo y que han sido obtenidas a partir de $\log (P G A) \pm 2 \sigma)$. Parte inferior: PGA registradas y corregidas a roca dura para los terremotos con $\mathrm{Mw}>4,5$, PGA sintéticas obtenidas con el modelo de atenuación de Ágústsson et al. (2008) con dependencia lineal con la magnitud M, y PGA sintéticas obtenidas a partir de $\log (P G A) \pm 2 \sigma \ldots \ldots . \ldots . \ldots . \ldots 448$

G.10.Parte superior: PGA registradas y corregidas a roca dura para los terremotos de calibración de El Hierro (2011-2013) con 4,0<Mw $\leq$ 5,0 , curva de atenuación de la PGA para un terremoto de Mw 4,5, de acuerdo al modelo de atenuación de Pétursson y Vogfjörd (2009), y curvas de atenuación para la PGA que incluyen la desviación estándar $\sigma$ del modelo y que han sido obtenidas a partir de $\log (P G A) \pm 2 \sigma)$. Parte inferior: PGA registradas y corregidas a roca dura para los terremotos con $\mathrm{Mw}>4,5$, PGA sintéticas obtenidas con el modelo de atenuación de Pétursson y Vogfjörd (2009) y PGA sintéticas obtenidas

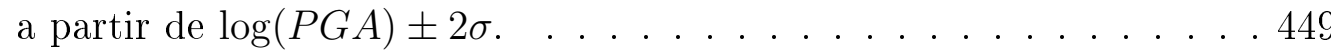

G.11.Parte superior: PGA registradas y corregidas a roca dura para los terremotos de calibración de El Hierro (2011-2013) con 4,0<Mw $\leq$ 5,0 , curva de atenuación de la PGA para un terremoto de $\mathrm{Mw} 4,5$, de acuerdo al modelo de atenuación de Tento et al. (1992), y curvas de atenuación para la PGA que incluyen la desviación estándar $\sigma$ del modelo y que han sido obtenidas a partir de $\log (P G A) \pm 2 \sigma)$. Parte inferior: PGA registradas y corregidas a roca dura para los terremotos de calibración con $\mathrm{Mw}>4,5$, PGA sintéticas obtenidas con el modelo de atenuación de Tento et al. (1992), y PGA sintéticas obtenidas a

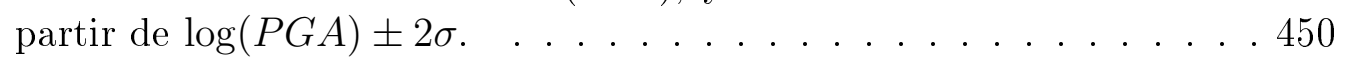


G.12.Parte superior: PGA registradas y corregidas a roca dura para los terremotos de calibración de El Hierro (2011-2013) con 4,0<Mw $\leq$ 5,0, curva de atenuación de la PGA para un terremoto de Mw 4,5, de acuerdo al modelo de atenuación de Bindi et al. (2009a) con PGA definida en función de la componente máxima horizontal, y curvas de atenuación para la PGA que incluyen la desviación estándar $\sigma$ del modelo y que han sido obtenidas a partir de $\log (P G A) \pm 2 \sigma)$. Parte inferior: PGA registradas y corregidas a roca dura para los terremotos con $\mathrm{Mw}>4,5$, PGA sintéticas obtenidas con el modelo de atenuación de Bindi et al. (2009a) con PGA definida en función de la componente máxima horizontal, y PGA sintéticas obtenidas a

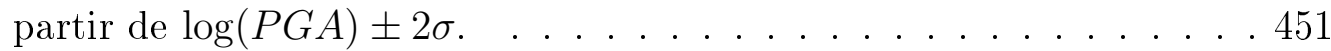

G.13.Parte superior: PGA registradas y corregidas a roca dura para los terremotos de calibración de El Hierro (2011-2013) con 4,0<Mw $\leq$ 5,0, curva de atenuación de la PGA para un terremoto de $\mathrm{Mw} 4,5$, de acuerdo al modelo de atenuación de Bindi et al. (2009a) con PGA definida en función de la media geométrica de las componentes horizontales, y curvas de atenuación para la PGA que incluyen la desviación estándar $\sigma$ del modelo y que han sido obtenidas a partir de $\log (P G A) \pm 2 \sigma)$. Parte inferior: PGA registradas y corregidas a roca dura para los terremotos con $\mathrm{Mw}>4,5$, PGA sintéticas obtenidas con el modelo de atenuación de Bindi et al. (2009a) con PGA definida en función de la media geométrica de las componentes horizontales, y PGA sintéticas obtenidas a partir de $\log (P G A) \pm 2 \sigma$. . . . . . . 452

G.14.Parte superior: PGA registradas y corregidas a roca dura para los terremotos de calibración de El Hierro (2011-2013) con 4,0<Mw $\leq$ 5,0 , curva de atenuación de la PGA para un terremoto de Mw 4,5, de acuerdo al modelo de atenuación de Bindi et al. (2009a) con PGA definida en función de la componente vertical, y curvas de atenuación para la PGA que incluyen la desviación estándar $\sigma$ del modelo y que han sido obtenidas a partir de $\log (P G A) \pm 2 \sigma)$. Parte inferior: PGA registradas y corregidas a roca dura para los terremotos con $\mathrm{Mw}>4,5$, PGA sintéticas obtenidas con el modelo de atenuación de Bindi et al. (2009a) con PGA definida en función de la componente vertical, y PGA sintéticas obtenidas a partir de $\log (P G A) \pm 2 \sigma$. . . 453 
G.15.Parte superior: PGA registradas y corregidas a roca dura para los terremotos de calibración de El Hierro (2011-2013) con 4,0<Mw $\leq$ 5,0, curva de atenuación de la PGA para un terremoto de Mw 4,5, de acuerdo al modelo de atenuación de Bindi et al. (2009a) con PGA definida en función de la componente máxima horizontal y con dependencia de la distancia hipocentral (D-hipo.), y curvas de atenuación para la PGA que incluyen la desviación estándar $\sigma$ del modelo y que han sido obtenidas a partir de $\log (P G A) \pm 2 \sigma)$. Parte inferior: PGA registradas y corregidas a roca dura para los terremotos con $\mathrm{Mw}>4,5$, PGA sintéticas obtenidas con el modelo de atenuación de Bindi et al. (2009a) con PGA definida en función de la componente máxima horizontal y con dependencia de la distancia hipocentral (D-hipo.), y PGA sintéticas obtenidas a partir de $\log (P G A) \pm 2 \sigma . \ldots . . . .454$

G.16.Parte superior: PGA registradas y corregidas a roca dura para los terremotos de calibración de El Hierro (2011-2013) con 4,0<Mw $\leq$ 5,0 , curva de atenuación de la PGA para un terremoto de $\mathrm{Mw} 4,5$, de acuerdo al modelo de atenuación de Bindi et al. (2009b), y curvas de atenuación para la PGA que incluyen la desviación estándar $\sigma$ del modelo y que han sido obtenidas a partir de $\log (P G A) \pm 2 \sigma)$. Parte inferior: PGA registradas y corregidas a roca dura para los terremotos con $\mathrm{Mw}>4,5$, PGA sintéticas obtenidas con el modelo de atenuación de Bindi et al. (2009b), y PGA sintéticas obtenidas a partir de $\log (P G A) \pm 2 \sigma$.

G.17.Parte superior: PGA registradas y corregidas a roca dura para los terremotos de calibración de El Hierro (2011-2013) con 4,0<Mw $\leq$ 5,0, curva de atenuación de la PGA para un terremoto de Mw 4,5, de acuerdo al modelo de atenuación de Bindi et al. (2010) con PGA definida en función de la componente máxima horizontal, y curvas de atenuación para la PGA que incluyen la desviación estándar $\sigma$ del modelo y que han sido obtenidas a partir de $\log (P G A) \pm 2 \sigma)$. Parte inferior: PGA registradas y corregidas a roca dura para los terremotos con $\mathrm{Mw}>4,5$, PGA sintéticas obtenidas con el modelo de atenuación de Bindi et al. (2010) con PGA definida en función de la componente máxima horizontal, y PGA sintéticas obtenidas a

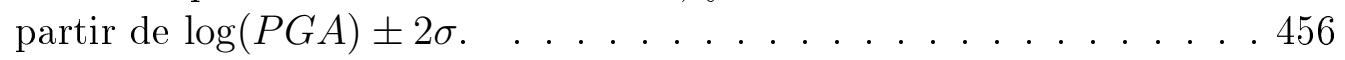

G.18.Parte superior: PGA registradas y corregidas a roca dura para los terremotos de calibración de El Hierro (2011-2013) con 4,0<Mw $\leq$ 5,0 , curva de atenuación de la PGA para un terremoto de Mw 4,5, de acuerdo al modelo de atenuación de Bindi et al. (2010) con PGA definida en función de la componente vertical, y curvas de atenuación para la PGA que incluyen la desviación estándar $\sigma$ del modelo y que han sido obtenidas a partir de $\log (P G A) \pm 2 \sigma)$. Parte inferior: PGA registradas y corregidas a roca dura para los terremotos con $\mathrm{Mw}>4,5$, PGA sintéticas obtenidas con el modelo de atenuación de Bindi et al. (2010) con PGA definida en función de la componente vertical, y PGA sintéticas obtenidas a partir de $\log (P G A) \pm 2 \sigma$. . . 457 
G.19.Parte superior: PGA registradas y corregidas a roca dura para los terremotos de calibración de El Hierro (2011-2013) con 4,0<Mw $\leq$ 5,0, curva de atenuación de la PGA para un terremoto de $\mathrm{Mw} 4,5$, de acuerdo al modelo de atenuación de Munson y Thurber (1997), y curvas de atenuación para la PGA que incluyen la desviación estándar $\sigma$ del modelo y que han sido obtenidas a partir de $\log (P G A) \pm 2 \sigma)$. Parte inferior: PGA registradas y corregidas a roca dura para los terremotos con $\mathrm{Mw}>4,5$, PGA sintéticas obtenidas con el modelo de atenuación de Munson y Thurber (1997) y PGA sintéticas obtenidas a partir de $\log (P G A) \pm 2 \sigma . \ldots \ldots . \ldots . \ldots . \ldots 458$

G.20.Parte superior: PGA registradas y corregidas a roca dura para los terremotos de calibración de El Hierro (2011-2013) con 4,0<Mw 5,0 , curva de atenuación de la PGA para un terremoto de $\mathrm{Mw} 4,5$, de acuerdo al modelo de atenuación de Beauducel et al. (2004), y curvas de atenuación para la PGA que incluyen la desviación estándar $\sigma$ del modelo y que han sido obtenidas a partir de $\log (P G A) \pm 2 \sigma)$. Parte inferior: PGA registradas y corregidas a roca dura para los terremotos con $\mathrm{Mw}>4,5$, PGA sintéticas obtenidas con el modelo de atenuación de Beauducel et al. (2004) y PGA sintéticas obtenidas

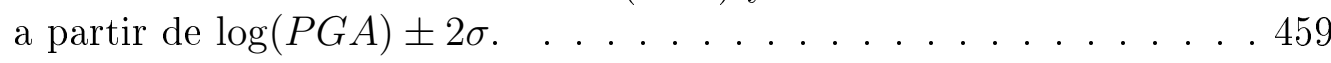




\section{Îndice de tablas}

2.1. Clases de emplazamiento según Borcherdt (1994). PI es el índice de plasticidad y N la resistencia según el ensayo SPT (Standard Penetration Test). . . . . . . . . . . . . . 16

2.2. Factores de amplificación para periodos cortos $\left(F_{a}\right)$ y periodos medios $\left(F_{v}\right)$ para las clases de emplazamiento de Borcherdt (1994) (Tabla $2.1)$, con respecto a las condiciones del terreno de referencia SC-Ib y SC-(II+III). $m_{a}$ y $m_{v}$ son parámetros definidos en función de los factores de amplificación $F_{a}$ y $F_{v}$ correspondientes a la clase de emplazamiento SC-IV (Tabla 2.1), como se explica en el texto. . . . . . 21

3.1. Tipos de perfil de suelo según el Uniform Building Code de 1997 (International Council of Building Officials, 1997) . . . . . . . . 65

3.2. Definiciones de clases de emplazamiento según la normativa de construcción sismorresistente del NEHRP de 1997 (Building Seismic Safety Council, 1997). PI es el Índice de Plasticidad, $w$ es el contenido en humedad, $s_{u}$ es la resistencia a cortante y sin drenar y $H$ la potencia del suelo. . . . . . . . . . . . . . . 66 66

3.3. Definiciones de clases de emplazamiento según la normativa de construcción sismorresistente del NEHRP de 2003 (Building Seismic Safety Council, 2004). PI es el Índice de Plasticidad, $w$ es el contenido en humedad, $s_{u}$ es la resistencia a cortante y sin drenar y $H$ la potencia del suelo. . . . . . . . . . . . . . . . .

3.4. Clasificación de síntesis de suelos y rocas en función de su capacidad de amplificación. Vs30 es la velocidad media de onda de corte en los primeros $30 \mathrm{~m}$ de profundidad, $\bar{N}$ la resistencia media según el ensayo SPT (Standard Penetration Test) en los primeros $30 \mathrm{~m}$ de profundidad, PI el Índice de Plasticidad, $w$ el contenido en humedad, $s_{u}$ la resistencia a cortante y sin drenar, $\bar{s}_{u}$ la resistencia media a cortante y sin drenar en los primeros $30 \mathrm{~m}$ de profundidad y $H$ la potencia del suelo.

3.5. Litologías genéricas correspondientes a las unidades geológicas de la Península Ibérica y las Islas Baleares, de acuerdo con el Mapa Geológico de España a escala 1:1.000.000 (Instituto Geológico y Minero de España, 1994). C indica litofacies de conglomerados, CA litofacies de conglomerados y areniscas, CB litofacies carbonatada y S litofacies sulfatada. . . . . . . . . . . . . . . . 
3.6. Regiones GEODE que corresponden a las Islas Canarias, según la cartografía geológica continua GEODE a escala 1:50.000 (GEODE, 2014).

3.7. Tablas DESC_LITOLOGIA y LIT_DESC, y atributos correspondientes, de acuerdo a la base de datos de la cartografía geológica continua GEODE a escala 1:50.000 (GEODE, 2014).

3.8. Unidades del dominio geológico "Rocas plutónicas prehercínicas". Tomado de Instituto Geológico y Minero de España (1994), elaboración propia. ..................... 81

3.9. Unidades del dominio geológico "Rocas plutónicas hercínicas". La abreviatura s.l. (sensu lato) indica que la referencia a la que acompaña se entiende en un sentido amplio. Tomado de Instituto Geológico y Minero de España (1994), elaboración propia. . . . . . . . . . . . 83

3.10. Unidades del dominio geológico "Rocas plutónicas alpinas". Tomado de Instituto Geológico y Minero de España (1994), elaboración propia. 83

3.11. Unidades del dominio geológico "Unidades alóctonas del Macizo Hespérico". Tomado de Instituto Geológico y Minero de España (1994), elaboración propia. . . . . . . . . . . . 83

3.12. Unidades del dominio geológico "Bloque de Alborán". Tomado de Instituto Geológico y Minero de España (1994), elaboración propia. . . . 86

3.13. Unidades de la litología genérica "Gneises; esquistos; mármoles y vulcanitas". Tomado de Instituto Geológico y Minero de España (1994), elaboración propia. . . . . . . . . . . . 86

3.14. Unidades de la litología genérica "Cuarcitas; pizarras; areniscas; calizas y vulcanitas". Tomado de Instituto Geológico y Minero de España (1994), elaboración propia. . . . . . . . . . . . 88

3.15. Unidades de la litología genérica "Areniscas; pizarras y calizas". Tomado de Instituto Geológico y Minero de España (1994), elaboración propia. .................... 88

3.16. Unidades de la litología genérica "Conglomerados; areniscas; pizarras; calizas y vulcanitas; carbón". Tomado de Instituto Geológico y Minero de España (1994), elaboración propia. . . . . . . . . . . . . . 88

3.17. Unidades de la litología genérica "Conglomerados; areniscas y lutitas; vulcanitas". Tomado de Instituto Geológico y Minero de España (1994), elaboración propia. . . . . . . . . . . 89

3.18. Unidades de la litología genérica "Conglomerados; areniscas; calizas; yesos y arcillas versicolores". Tomado de Instituto Geológico y Minero de España (1994), elaboración propia. . . . . . . . . . . . . 90

3.19. Unidades de la litología genérica "Calizas; dolomías y margas; conglomerados y areniscas". Tomado de Instituto Geológico y Minero de España (1994), elaboración propia. . . . . . . . . . . . . .

3.20. Unidades de la litología genérica "Calizas; dolomías y margas; areniscas". Tomado de Instituto Geológico y Minero de España (1994), elaboración propia. 
3.21. Unidades de la litología genérica "Conglomerados; areniscas; arcillas y calizas; evaporitas". Tomado de Instituto Geológico y Minero de España (1994), elaboración propia. . . . . . . . . . . . .

3.22. Unidades de la litología genérica "Turbiditas calcáreas". Tomado de Instituto Geológico y Minero de España (1994), elaboración propia. 93

3.23. Unidades de la litología genérica "Conglomerados; areniscas; arcillas; calizas y evaporitas; vulcanitas básicas". Tomado de Instituto Geológico y Minero de España (1994), elaboración propia. . . . . . . . . . . 95

3.24. Unidades de la litología genérica "Conglomerados; gravas; arenas y limos". Tomado de Instituto Geológico y Minero de España (1994), elaboración propia. . . . . . . . . . . . 95

3.25. División de las unidades del mapa geológico continuo GEODE a escala 1:50.000 de El Hierro (Zona 2916), de acuerdo a la leyenda cronoestratigráfica. La abreviatura s.l. (sensu lato) indica que la referencia a la que acompaña se entiende en un sentido amplio. La abreviatura s.s. (sensu stricto) indica que la referencia se entiende en un sentido estricto. Tomado de GEODE (2014). . . . . . . . . . . . . . . 96

3.26. Resumen de la clasificación de las unidades del mapa geológico continuo GEODE a escala 1:50.000 de El Hierro (Zona 2916), de acuerdo a la clasificación de síntesis. La abreviatura s.l. (sensu lato) indica que la referencia a la que acompaña se entiende en un sentido amplio. La abreviatura s.s. (sensu stricto) indica que la referencia se entiende en un sentido estricto. Tomado de GEODE (2014), elaboración propia. . 102

3.27. División de las unidades del mapa geológico continuo GEODE a escala 1:50.000 de Fuerteventura (Zona 2911), de acuerdo a la leyenda cronoestratigráfica. Tomado de GEODE (2014). . . . . . . . . . . 103

3.28. Resumen de la clasificación de las unidades del mapa geológico continuo GEODE a escala 1:50.000 de Fuerteventura (Zona 2911), de acuerdo a la clasificación de síntesis. Tomado de GEODE (2014), elaboración propia. . . . . . . . . . . . . . 115

3.29. División de las unidades del mapa geológico continuo GEODE a escala 1:50.000 de Gran Canaria (Zona 2912), de acuerdo con la leyenda cronoestratigráfica. Tomado de GEODE (2014). . . . . . . . . . . 119

3.30. Resumen de la clasificación de las unidades del mapa geológico continuo GEODE a escala 1:50.000 de Gran Canaria (Zona 2912), de acuerdo a la clasificación de síntesis. Tomado de GEODE (2014), elaboración propia. . . . . . . . . . . . . 130

3.31. División de las unidades del mapa geológico continuo GEODE a escala 1:50.000 de La Gomera (Zona 2914), de acuerdo a la leyenda cronoestratigráfica. Tomado de GEODE (2014). . . . . . . . . . . . 132

3.32. Resumen de la clasificación de las unidades del mapa geológico continuo GEODE a escala 1:50.000 de La Gomera (Zona 2914), de acuerdo a la clasificación de síntesis. Tomado de GEODE (2014), elaboración propia. . . . . . . . . . . . . . . . . . . 140 
3.33. División de las unidades del mapa geológico continuo GEODE a escala 1:50.000 de La Palma (Zona 2915), de acuerdo a la leyenda cronoestratigráfica. Tomado de GEODE (2014). . . . . . . . . . . . . . 141

3.34. Resumen de la clasificación de las unidades del mapa geológico continuo GEODE a escala 1:50.000 de La Palma (Zona 2915), de acuerdo a la clasificación de síntesis. Tomado de GEODE (2014), elaboración propia. . . . . . . . . . . . . . . . . 148

3.35. División de las unidades del mapa geológico continuo GEODE a escala 1:50.000 de Lanzarote (Zona 2910), de acuerdo a la leyenda cronoestratigráfica. Tomado de GEODE (2014) . . . . . . . . . . . . 150

3.36. Resumen de la clasificación de las unidades del mapa geológico continuo GEODE a escala 1:50.000 de la Región 2910 (Isla de Lanzarote), de acuerdo a la clasificación de síntesis. Tomado de GEODE (2014), elaboración propia. . . . . . . . . . . . . . 162

3.37. Resumen de la clasificación de las unidades del mapa geológico continuo GEODE a escala 1:50.000 de la Región 2910 (Archipiélago Chinijo (islas de La Graciosa, Montaña Clara, Alegranza, Roque del Este, y Roque del Oeste)), de acuerdo a la clasificación de síntesis. Tomado de GEODE (2014), elaboración propia. . . . . . . . . . . . 164

3.38. División de las unidades del mapa geológico continuo GEODE a escala 1:50.000 de Tenerife (Zona 2913), de acuerdo con la leyenda cronoestratigráfica. Tomado de GEODE (2014) . . . . . . . . . . . . . . . 164

3.39. Resumen de la clasificación de las unidades del mapa geológico continuo GEODE a escala 1:50.000 de Tenerife (Zona 2913), de acuerdo a la clasificación de síntesis. Tomado de GEODE (2014), elaboración propia. . . . . . . . . . . . . . . . . 185

3.40. Valores medios del intervalo de valores de Vs30 $(v)$, factor de amplificación para periodos cortos $\left(F_{a}\right)$ y factor de amplificación para periodos medios $\left(F_{v}\right)$ de cada clase de emplazamiento de la clasificación de síntesis (Tabla 3.4). . . . . . . . . . . . . . . . 192

4.1. Valores obtenidos para Vs30 y para el error RMS del ajuste $(\mathrm{m} / \mathrm{s})$ en los 25 emplazamientos de medida ReMi en El Hierro, e intervalos de valores de Vs30 proporcionados por el mapa de clases de emplazamiento y Vs30 de las Islas Canarias (Figuras 3.10) y 4.1) . . . . . . . 204

4.2. Valores de la máxima amplificacíon y del periodo fundamental (s) en los 25 emplazamientos de medida ReMi en El Hierro, obtenidos a partir de las curvas de amplificación espectral calculadas con los respectivos modelos de velocidad Vs. También se presentan los factores de amplificación $F_{a}$ para altas frecuencias $(2-10 \mathrm{~Hz})$ que corresponden a los emplazamientos, de acuerdo con el mapa de amplificación sísmica de las Islas Canarias (Figura 3.12) . . . . . . . . . . . 207 
5.1. Comienzo de las ventanas de 30 minutos de duración y con ruido sísmico ambiental, procedentes de las estaciones de registro de velocidad CCUM, CJUL, CTAB, CTAN y CRST. Los tiempos están expresados en UTC (Universal Time Coordinated) . . . . . . . . . . . . . . . . . 212

5.2. Comienzo de las ventanas de 30 minutos de duración y con ruido sísmico ambiental, procedentes de la estación central HI01 del array de Tacorón, y de la estación central HV01 del array de El Verodal. Los tiempos están expresados en UTC (Universal Time Coordinated). 213

6.1. Resumen de las características de las relaciones empíricas de atenuación analizadas para valorar su aplicación en la Península Ibérica. . 226

6.2. Parámetros focales de los terremotos ocurridos en El Hierro que se han utilizado en la calibración de las relaciones de atenuación en aceleración máxima que son aplicables en la isla. Se indican también la distancia epicentral de cada acelerómetro con acelerograma registrado, el factor de amplificación $F_{a}$ para periodos cortos (Borcherdt, 1994) que le corresponde en función de su emplazamiento, y las aceleraciones pico de cada componente del acelerograma. La hora origen de cada terremoto está referida a UTC. La magnitud Mw de cada terremoto ha sido obtenida a partir de la $M_{L}$ por medio de la conversión de Rueda y Mezcua (2002) y de acuerdo con las consideraciones realizadas en el texto. $D_{e p i}$ es la distancia epicentral. PGA es la aceleración pico del registro de cada componente (N-S, V, E-W) del acelerograma. Fuente: IGN, elaboración propia. . . . . . . . . 245

6.3. Análisis de los resultados de la calibración de las relaciones de atenuación aplicables en El Hierro (sección 6.2.3 y Apéndice G). Se presenta la estimación cualitativa de la bondad del ajuste de cada relación a las PGA registradas y reducidas al terreno de referencia correspondiente para los terremotos con 4,0<Mw $\leq 5,0$, el número de PGA registradas que coinciden con las correspondientes PGA sintéticas ( ${ }^{\circ}$ PGA reg. $=$ PGA sint.), la confirmación o no de la condición del terreno de referencia para cada modelo de atenuación (Terr. ref. confirmado) y el factor de amplificación $F_{a}$ finalmente considerado. En el caso del modelo de atenuación de Ágústsson et al. (2008), Log(R)-lin(M) se refiere a la expresión de la PGA que depende linealmente de la magnitud $M_{L w}$ (ecuación 6.23), y $\log (\mathrm{R})-\log (\mathrm{M})$ a la expresión de la PGA que depende del logaritmo de la magnitud $M_{L w}$ (ecuación 6.22). . . . 250

6.4. Rangos de distancias epicentrales $\left(D_{\text {epi }}\right)$ o hipocentrales $\left(D_{\text {hipo }}\right)$ y rangos de magnitudes $\mathrm{Mw}$ de las relaciones de atenuación seleccionadas en el análisis de los resultados de la calibración (Tabla 6.3). En la relación de Sigbjörnsson y Baldvinsson (1992), se ha supuesto que el rango de magnitudes se expresa en Mw. . . . . . . . . . . . . . 251 
7.1. Parámetros $Q_{0}$ y $\nu$ obtenidos en la estación CTIG (El Hierro) desde el 24 de septiembre hasta el 5 de noviembre de 2011, según se explica en el texto. Se presentan también las desviaciones estándar de $Q_{0} \mathrm{y}$ $\nu$ (SD), el número d eventos por día, el número de observaciones de Q para cada intervalo de frecuencias y el total de las observaciones que han sido analizadas. El valor medio de Q para cada intervalo de frecuencias de filtrado ha sido calculado tras seleccionar las observaciones de Q correspondientes por medio del criterio de Chauvenet. . . 271

7.2. Parámetros $Q_{0}$ y $\nu$ obtenidos en la estación CTIG (El Hierro) para cada fase pre-eruptiva de López et al. (2012), y para el periodo de registro de la señal de tremor (Instituto Geográfico Nacional, 2016b), según se explica en el texto. Se presentan también las desviaciones estándar de $Q_{0}$ y $\nu$ (SD), el número de eventos para cada periodo, el intervalo de tiempo en el cual ocurrieron los eventos, el número de observaciones de $\mathrm{Q}$ para cada intervalo de frecuencias y el total de las observaciones que han sido analizadas. El valor medio de $\mathrm{Q}$ para cada intervalo de frecuencias de filtrado ha sido calculado tras seleccionar las observaciones de Q correspondientes por medio del criterio de Chauvenet. . . . . . . . . . . . . . . 271

8.1. Parámetros de localización y magnitud del terremoto de 31-03-2013 al W de El Hierro. Prof. es profundidad. . . . . . . . . . . . . . 276

8.2. Parámetros de localización de los acelerógrafos ATIG y AROQ, y registros de aceleración pico por componentes (Comp.) para el terremoto de 31-03-2013 al W de El Hierro. Lat. es latitud, Long. es longitud y Dist. Epi. es distancia epicentral. . . . . . . . . . . 276

8.3. Planos focales del mecanismo focal obtenido por medio del cálculo del Tensor Momento Sísmico del terremoto de 08-10-2011, localizado a $1 \mathrm{~km}$ de la costa SW de El Hierro (evid 1103959). Fuente: Instituto Geográfico Nacional (2016c) . . . . . . . . . . . . 277

8.4. Duración (DUR) proporcionada por el programa Next Generation Attenuation (NGA)-West 2 para regiones continentales estables, la cual se ha considerado en la simulación de escenarios sísmicos en El Hierro. $R_{P S}$ es distancia de fuente puntual, aproximada por la distancia hipocentral con el fin de simular los escenarios. . . . . . . . . 279

9.1. Campos de la tabla de atributos de la capa shapefile de polígonos Peninsula_amplificacion.shp, junto con los valores para 7 polígonos de unidades geológicas en la Península Ibérica y las Islas Baleares. . . 291

9.2. Campos de la tabla de atributos de la capa shapefile de polígonos El_Hierro_amplificacion_170317.shp, junto con los valores para 7 polígonos de unidades geológicas en la isla de El Hierro. . . . . . . . . 292

9.3. Variables predictivas y factores de amplificación $F_{a} *$ para periodos cortos (Borcherdt, 1994) de la condición del terreno de referencia, para las relaciones de atenuación óptimas en las Islas Canarias. Mw es la magnitud momento, $D_{e p i}$ la distancia epicentral y h la profundidad.293 
9.4. Variables predictivas y factores de amplificación $F_{a} *$ para periodos cortos (Borcherdt, 1994) de la condición del terreno de referencia, para las relaciones de atenuación óptimas en la Península Ibérica y las Islas Baleares. Mw es magnitud momento, $D_{e p i}$ la distancia epicentral y h la profundidad. Focal mechanism se refiere al mecanismo focal. . . 293

9.5. Variables predictivas y factores de amplificación $F_{a} *$ para periodos cortos (Borcherdt, 1994) de la condición del terreno de referencia, para las relaciones de atenuación de alcance mundial. Mw es la magnitud momento, $D_{e p i}$ la distancia epicentral, h la profundidad, d el ángulo de buzamiento (Fault dip angle) y r el ángulo de deslizamiento (Rake angle) de la falla. Event type hace referencia al tipo de evento, Focal mechanism al mecanismo focal y Source type al tipo de fuente. . . . . 293

9.6. Capa shapefile PLUGIN_GRID_EVID_1060340_EXTENDIDA.shp: Campos de la tabla de atributos y valores para los 7 primeros puntos de la malla de cálculo empleada en la Península Ibérica. . . . . . . . . 296

9.7. Capa shapefile PLUGIN_GRID_EL_HIERRO_E2.shp: Campos de la tabla de atributos y valores para los 7 primeros puntos de la malla de cálculo empleada en la isla de El Hierro. . . . . . . . . . . . . 297

9.8. Capa shapefile Aceleracion_Estacion_1060340.shp: Tabla de atributos.299

9.9. Capa shapefile Aceleracion_Estacion_1202861_v010417.shp: Tabla de atributos. . . . . . . . . . . . . . . . . 299

10.1. Planos focales del mecanismo focal obtenido por medio del cálculo del Tensor Momento Sísmico del terremoto de 08-10-2011, localizado a $1 \mathrm{~km}$ de la costa SW de El Hierro. Fuente: Instituto Geográfico Nacional (2016c). . . . . . . . . . . . . . . . . . 332

10.2. Aceleración máxima (PGA) sintética $\left(\mathrm{cm} / \mathrm{s}^{2}\right)$ en cada uno de los acelerómetros que han registrado el terremoto de 11-05-2011 en Lorca, proporcionada, para cada relación de atenuación, por los mapas de sacudidas de las Figuras 10.3, 10.6 y 10.8 a 10.10. AMH es la aceleración máxima horizontal registrada $\left(\mathrm{cm} / \mathrm{s}^{2}\right)$ en cada acelerómetro, proporcionada por la Tabla 9.8. Diff. es la diferencia entre la AMH registrada en cada acelerómetro y la correspondiente PGA sintética. . 338

10.3. Aceleración máxima (PGA) sintética $\left(\mathrm{cm} / \mathrm{s}^{2}\right)$ en cada uno de los acelerómetros que han registrado el terremoto de 31-03-2013 al W de El Hierro, proporcionada, para cada relación de atenuación, por los mapas de sacudidas de las Figuras 10.13 y 10.15 a 10.19 . AMH es la aceleración máxima horizontal registrada $\left(\mathrm{cm} / \mathrm{s}^{2}\right)$ en cada acelerómetro, proporcionada por la Tabla 9.9. Diff. es la diferencia entre la AMH registrada en cada acelerómetro y la correspondiente PGA sintética. . . . . . . . . . . . . . . . . . 339

10.4. Valores de intensidad observada en las poblaciones de El Hierro donde fue sentido el terremoto de 31-03-2013, con Mw 4,9. . . . . . . . . 341

10.5. Constantes de aplicación de las correlaciones de Atkinson y Kaka (2007). $\sigma_{M M I}$ es la desviación estándar total de las ecuaciones 10.1

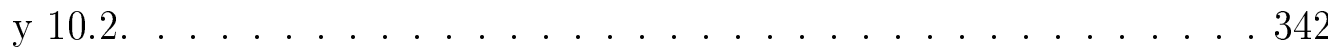


10.6. Valores de intensidad calculada, intensidad observada, PGA y PGV en las poblaciones de El Hierro donde fue sentido el terremoto de 3103-2013, con Mw 4,9. $F_{a}$ es el factor de amplificación para periodos cortos y $F_{v}$ el factor de amplificación para periodos medios, calculados de acuerdo con Borcherdt (1994). Dist. Hipo. es distancia hipocentral. Vs30 es el valor medio del intervalo de valores de Vs30 de la clase de emplazamiento donde se sitúa cada población, de acuerdo con el mapa de amplificación de El Hierro (subsección 10.2.2). Dif. es la diferencia entre la intensidad observada en cada población y la correspondiente intensidad calculada. . . . . . . . . . . . . . . . . 344

A.1. Unidades del mapa geológico continuo GEODE a escala 1:50.000 de El Hierro (Zona 2916). El código alfanumérico de cada unidad hace referencia a la leyenda cronoestratigráfica del mapa. La descripción de cada unidad está referida a sus características litológicas. La abreviatura s.l. (sensu lato) indica que la referencia a la que acompaña se entiende en un sentido amplio. Tomado de GEODE (2014). . . . . . . 381

A.2. Unidades del mapa geológico continuo GEODE a escala 1:50.000 de Fuerteventura (Zona 2911). El código alfanumérico de cada unidad hace referencia a la leyenda cronoestratigráfica del mapa. La descripción de cada unidad está referida a sus características litológicas. Tomado de GEODE (2014). . . . . . . . . . . . . . . . 382

A.3. Unidades del mapa geológico continuo GEODE a escala 1:50.000 de Gran Canaria (Zona 2912). El código alfanumérico de cada unidad hace referencia a la leyenda cronoestratigráfica del mapa. La descripción de cada unidad está referida a sus características litológicas. Tomado de GEODE (2014) . . . . . . . . . . . . . . . . . 386

A.4. Unidades del mapa geológico continuo GEODE a escala 1:50.000 de La Gomera (Zona 2914). El código alfanumérico de cada unidad hace referencia a la leyenda cronoestratigráfica del mapa. La descripción de cada unidad está referida a sus características litológicas. Tomado de GEODE (2014). . . . . . . . . . . . . . . . . . 390

A.5. Unidades del mapa geológico continuo GEODE a escala 1:50.000 de La Palma (Zona 2915). El código alfanumérico de cada unidad hace referencia a la leyenda cronoestratigráfica del mapa. La descripción de cada unidad está referida a sus características litológicas. Tomado de GEODE (2014) . . . . . . . . . . . . . . . . . . 391

A.6. Unidades del mapa geológico continuo GEODE a escala 1:50.000 de Lanzarote (Zona 2910). El código alfanumérico de cada unidad hace referencia a la leyenda cronoestratigráfica del mapa. La descripción de cada unidad está referida a sus características litológicas. Tomado de GEODE (2014) . . . . . . . . . . . . . . . . . . 392 
A.7. Unidades del mapa geológico continuo GEODE a escala 1:50.000 de Tenerife (Zona 2913). El código alfanumérico de cada unidad hace referencia a la leyenda cronoestratigráfica del mapa. La descripción de cada unidad está referida a sus características litológicas. La abreviatura s.l. (sensu lato) indica que la referencia a la que acompaña se entiende en un sentido amplio. Tomado de GEODE (2014). . . . . . . 395

B.1. Coordenadas geográficas WGS84 y altitud de los emplazamientos ReMi. Se indica el nombre y tipo de estación del IGN, situada en las proximidades del emplazamiento, en su caso: Estación de registro de velocidad (Instituto Geográfico Nacional, 2015a), estación central de array sísmico, acelerómetro (Instituto Geográfico Nacional, 2017b), o estación de GPS. Alternativamente, se indica el nombre de la localidad o entidad de población donde se ha obtenido la medida, o bien, para los puntos de máxima amplificación, la clase de emplazamiento de la Tabla 3.4), de acuerdo con la Figura 4.1. En marzo de 2017, sólo los acelerómetros AROQ, ASAB y ATIG se encuentran operativos, estando AFRO, AMIR y ARES fuera de servicio, de acuerdo con Instituto Geográfico Nacional (2017b). En cuanto a los arrays sísmicos y a las estaciones de GPS, la información correspondiente ha sido proporcionada directamente por el Instituto Geográfico Nacional, previa petición motivada. . . . . . . . . . . . . . . 402

C.1. Observaciones de campo para los emplazamientos de ReMi, relativas a la secuencia de capas situadas encima y debajo del dispositivo de medida. Las capas se listan desde techo hasta muro de la secuencia. También se presentan las unidades geológicas que corresponden a los emplazamientos, de acuerdo con el mapa geológico continuo GEODE a escala 1:50.000 de El Hierro (Zona 2916) (Figura 3.2) . . . . . . . . 403 


\section{RESUMEN}

En esta tesis doctoral se presentan los resultados obtenidos en las simulaciones de escenarios sísmicos en la Península Ibérica, las Islas Baleares y las Islas Canarias, para terremotos de alta magnitud $(>4,5)$, en base a dos aproximaciones diferenciadas a los valores sintéticos realistas de la aceleración sísmica y de la intensidad sísmica. En el cálculo de estos valores, se han considerando tanto las características de la fuente sísmica como la amplificación sísmica del terreno, o efecto de sitio, con diferentes grados de precisión en función del nivel de aproximación.

La primera aproximación al cálculo del escenario sísmico de un terremoto considera una fuente sísmica puntual y la capacidad de amplificación sísmica de los materiales aflorantes, expresada en un mapa de amplificación sísmica del territorio. Se ha utilizado la metodología de Borcherdt (1994) para generar un mapa de amplificación de la Península Ibérica y las Islas Baleares y un mapa de amplificación de las Islas Canarias, a partir de la clasificación de las unidades geológicas en 6 clases de emplazamiento caracterizadas por un factor de amplificación para altas frecuencias y un factor de amplificación para bajas frecuencias. En el contexto de la simulación de escenarios en la Península Ibérica y las Islas Baleares, se han seleccionado 5 relaciones de atenuación que permiten calcular la aceleración pico del terreno (Peak Ground Acceleration, PGA). En las Islas Canarias, se han utilizado las PGA registradas por el Instituto Geográfico Nacional (IGN) en la isla de El Hierro en tres crisis sísmicas ocurridas entre 2011 y 2013, como consecuencia del reciente proceso de reactivación volcánica en la isla, con objeto de comprobar el ajuste proporcionado por 13 modelos de atenuación en PGA. Como resultado, se han seleccionado 3 relaciones de atenuación óptimas en función de la magnitud del terremoto y de la escala de trabajo.

La simulación de escenarios sísmicos en primera aproximación se ha realizado automáticamente por medio de una herramienta (plugin) de desarrollo del Sistema de Información Geográfica QGIS, para producir mapas de sacudidas con los valores sintéticos realistas de la PGA. Los cálculos realizados con cada modelo de atenuación incorporan el factor de amplificación para altas frecuencias procedente de los mapas de amplificación y, opcionalmente, las aceleraciones máximas horizontales (AMH) registradas $\left(\mathrm{cm} / \mathrm{s}^{2}\right)$ en los acelerómetros con registro para el terremoto en estudio. De acuerdo con la metodología de Wald et al. (1999b), la herramienta corrige las AMH observadas al terreno de referencia en el que se expresan las PGA calculadas con la relación de atenuación elegida, y realiza una interpolación conjunta de los valores de AMH y de PGA sintéticas, correspondientes al mismo nivel de referencia. Los valores interpolados son multiplicados por los factores de amplificación del mapa de amplificación, generándose como resultado el mapa de PGA sintéticas que constituye el mapa de sacudidas. 
Se ha utilizado el plugin para simular los escenarios sísmicos de los terremotos de 11-05-2011 en Lorca, con Mw 5,1, y de 31-03-2013 al W de El Hierro, con Mw 4,9. Utilizando cada modelo de atenuación, se han producido mapas de sacudidas que se han utilizado posteriormente para comprobar la bondad de los valores de la PGA sintética, a partir de su comparación con los registros de AMH disponibles. En ambos casos se observa que, en general, los valores sintéticos de PGA se aproximan a las observaciones de AMH conforme el nivel del movimiento del terreno disminuye hasta valores del orden de $10 \mathrm{~cm} / \mathrm{s}^{2}$. En Lorca, se concluye que los modelos de atenuación con una definición de PGA acorde con la definición de la AMH consiguen un ajuste a las observaciones que es sensiblemente mejor que el ajuste proporcionado por el resto de los modelos. En este sentido, para el citado orden de magnitud del movimiento del terreno, se obtienen diferencias menores que $4,9 \mathrm{~cm} / \mathrm{s}^{2}$.

En un segundo nivel de aproximación, la simulación de escenarios sísmicos se ha realizado por medio de la simulación estocástica de acelerogramas sintéticos, bajo la consideración de una fuente sísmica extensa. Esta simulación, que se ha realizado por medio del algoritmo EXSIM (Motazedian y Atkinson, 2005), requiere un conocimiento más preciso de las características de la atenuación del medio de propagación de las ondas sísmicas y de la amplificación sísmica de las capas más superficiales.

La reactivación volcánica ocurrida en la isla de El Hierro ha proporcionado un gran número de registros acelerométricos que permiten la calibración del algoritmo de simulación estocástica. Con objeto de preparar las variables de entrada del mismo, se ha estimado la amplificación sísmica local en 25 emplazamientos distribuidos en la isla, a partir de modelos de velocidad de onda de corte (Vs) en los primeros 30 $m$ de profundidad, obtenidos en cada punto por medio del procesado de medidas de ruido sísmico ambiental por medio del método Refraction Microtremor (ReMi). Además, se ha estimado la atenuación anelástica de la litosfera regional en las Islas Canarias a partir del análisis de las ondas de coda registradas por la red sísmica del IGN, incluyendo los datos digitales proporcionados por las nuevas estaciones de banda ancha desplegadas en las últimas décadas. Como resultado, se ha obtenido la regionalización de los parámetros $Q_{0}$ y $\nu$ que expresan la dependencia frecuencial del factor de calidad Q.

Por medio del algoritmo EXSIM, se han simulado los acelerogramas sintéticos del terremoto de 31-03-2013 en las dos estaciones acelerométricas de El Hierro que obtuvieron registro para este terremoto. El ajuste de los espectros de respuesta en pseudoaceleración simulados a los espectros observados en las estaciones ha permitido obtener una función empírica de la amplificación de la corteza para la isla. Esta función se ha implementado en el algoritmo con el fin de calcular el movimiento fuerte en términos de la velocidad máxima del terreno, PGV, en las poblaciones en las que el terremoto ha sido sentido con intensidad EMS-98 superior a II. Por medio de la correlación de Atkinson y Kaka (2007), se han obtenido las intensidades sintéticas en la escala EMS-98 a partir de los valores de PGV. La semejanza encontrada entre estas intensidades y las correspondientes intensidades observadas permite plantear la validez de esta simulación en el cálculo de escenarios sísmicos en El Hierro. 


\section{ABSTRACT}

This $\mathrm{PhD}$ thesis presents the results obtained after carrying out several simulations for seismic scenarios for $\mathrm{Mw}>4.5$ earthquakes occurred in the Iberian Peninsula, the Balearic Islands and the Canary Islands, following two different levels of approximation to realistic synthetic values of both the seismic acceleration and the seismic intensity. Seismic source characteristics, as well as the site amplification (site effect), have been considered with different degrees of precision depending on the approximation level sought.

First approximation to the computation of a single earthquake seismic scenario considers a seismic point source and the seismic amplification capacity of outcropping materials, which is given by a site amplification map fo the area. The methodology of Borcherdt (1994) has been used to produce a site amplification map of the Iberian Peninsula and the Balearic Islands and a site amplification map of the Canary Islands. The production of these maps have implied the classification of geological units into six site classes characterized by both high-frequency and lowfrequency amplification factors. In the context of the seismic scenario simulations in the Iberian Peninsula and the Balearic Islands, 5 attenuation relationships, suitable to compute the Peak Ground Acceleration (PGA) for a particular earthquake, have been selected. A set of PGA recorded by the Geographical Institute of Spain (IGN) in El Hierro Island (Canary Islands), during three seismic crisis occurred between 2011 and 2013 due to the recent volcanic reactivation process on the island, has been used to analize 13 PGA attenuation models, testing the data fit provided by the synthetics from each model. As a result, 3 attenuation relationships have been selected for use in the archipelago, depending on the earthquake magnitude and working scale.

Seismic scenario simulation under first approximation has been automatically carried out by means of a developing tool (plugin) of the QGIS Geographical Information System, with the aim of producing maps of realistic synthetic values of the Peak Ground Acceleration. Acceleration calculations for each attenuation model consider the high-frequency site amplification factor, given by the site amplification maps, and, optionally, the peak horizontal acceleration values (in $\mathrm{cm} / \mathrm{s}^{2}$ ) which have been yet recorded at ground motion stations detecting the study eathquake. According to the methodology of Wald et al. (1999b), the plugin corrects the recorded accelerations to the reference site characterizing the PGA calculated by the attenuation relationship selected. Afterwards, it interpolates the corrected data jointly with the synthetic accelerations, both corresponding to the same reference level. These interpolated accelerations are subsequently multiplied by the suitable site amplification factors, in order to generate the final synthetic Peak Ground Acceleration map. 
The plugin has been utilized in order to simulate the seismic scenarios for the Mw 5.1 May 11, 2011 Lorca earthquake and the Mw 4.9 March 31, 2013 earthquake, located offshore the W coast of El Hierro Island. By means of each attenuation model, PGA maps have been produced and later used to test the goodness of fit of synthetic values, when compared with the available peak horizontal acceleration data. In both cases, it is realized that, in general, synthetic PGA values approach the horizontal data as ground motion level decrease to values of an order equal to $10 \mathrm{~cm} / \mathrm{s}^{2}$. At Lorca, a conclusion has been drawn on the sensibly better fit to the observations that is reached by the attenuation relationships which define a PGA coherent with the horizontal peak acceleration. Thus, for the referred ground motion level, differences minor than $4.9 \mathrm{~cm} / \mathrm{s}^{2}$ are obtained.

At a second level of approximation, simulations of seismic scenarios have been performed by means of a stochastic finite-fault simulation of strong ground motions. A more precise knowledge of both the attenuation of the propagation medium of seismic waves and the seismic amplification introduced by the shallower layers is required, in order to carry out this simulation, which has been finally done using the EXSIM code (Motazedian y Atkinson, 2005).

The volcanic reactivation on El Hierro Island has supplied with a great number of accelerometric records which let us test the parameters of the stochastic simulation code. With the purpose of preparing its input variables, the local site amplification at 25 points distributed accross the island has been estimated from the shear-wave velocity (Vs) model to a depth of $30 \mathrm{~m}$, which has been computed by processing ambient noise measures by means of the Refraction Microtremor (ReMi) method. Additionally, the anelastic attenuation has been estimated for the regional lithosphere in the Canary Islands by means of the analysis of the coda waves recorded by the IGN seismic stations, including digital data from new broad-band stations deployed during the last decades. As a result, regionalizations have been obtained for parameters $Q_{0}$ and $\nu$, which describe the frequency-dependent quality factor $\mathrm{Q}$.

Using the EXSIM code, synthetic accelerograms have been calculated for the March 31, 2013 earthquake at two accelerometric stations at El Hierro which recorded this event. Fitting simulated pseudoacceleration response spectra to the observed ones at these stations have let us obtained an empirical function for the crust amplification on the island. This function has been implemented in the code in order to calculate the ground motion in terms of Peak Ground Velocity, PGV, at the towns where the shock was felt with greater than EMS-98 II intensity. By means of the Atkinson y Kaka (2007) relationship, synthetic intensities at the EMS-98 scale have been computed from the PGV values. The resemblance found between these intensities and those observed allow us to propose the validity of this simulation for the seismic scenario calculation on El Hierro. 


\section{Capítulo 1}

\section{INTRODUCCIÓN}

La simulación de un escenario sísmico consiste en la determinación de los parámetros del movimiento del terreno producido por un terremoto en un punto determinado (aceleración y velocidad), o bien de la intensidad sísmica experimentada en dicho punto. La aceleración sísmica en un emplazamiento depende fundamentalmente de las características de la fuente sísmica, la atenuación del medio y de la capacidad de amplificación sísmica de los materiales de las capas más superficiales. Distintos trabajos publicados sobre el cálculo de la amplificación sísmica local y de la atenuación tratan de determinar las posibles correlaciones entre ambas variables, y ponen de relieve su infuencia específica en los valores que toman los parámetros del movimiento del terreno ante un terremoto ocurrido en un área determinada. A este respecto, se pueden citar los trabajos de Seale y Archuleta (1989), Jemberie y Langston (2005) y Assimaki et al. (2008), entre otros.

Seale y Archuleta (1989) presentan la amplificación y la atenuación de los parámetros del movimiento del terreno en un emplazamiento como efectos locales simultáneos introducidos por los materiales superficiales. Los autores destacan que estos efectos pueden dominar en una determinada banda frecuencial de la señal sísmica. De acuerdo con Assimaki et al. (2008), los sedimentos no consolidados pueden causar amplificaciones significativas del movimiento del terreno, a pesar de contribuir también a la disminución de sus amplitudes, por medio de una alta capacidad de atenuación. Consecuentemente, los autores sugieren que las estimaciones fiables de la atenuación y de la amplificación local son fundamentales para la predicción de valores realistas de la aceleración sísmica en este tipo de suelo, ante la ocurrencia de un terremoto futuro. Estos valores también son de gran importancia en el análisis y evaluación de la peligrosidad sísmica.

Uno de los factores que gobiernan la aceleración sísmica del terreno en un emplazamiento determinado es la amplificación sísmica local, también denominada respuesta sísmica o efecto de sitio. De acuerdo con la literatura sismológica, el efecto de sitio se describe como la modificación de la amplitud de las ondas sísmicas producida por los distintos materiales geológicos que, emplazados en los metros más superficiales, son atravesados por las ondas en su camino hacia la superficie. No obstante, los estudios sobre el efecto local en un emplazamiento determinado también abarcan la determinación de la frecuencia fundamental de resonancia de los suelos o rocas existentes. El estudio de la frecuencia o periodo fundamental de un suelo tiene bastante interés, pues si es del orden de la frecuencia predominante del movimiento 
del terreno producido por un terremoto, se producen fenómenos de resonancia en la aceleración del movimiento del terreno. Además, existe una relación entre la frecuencia fundamental del suelo y la profundidad a la que se encuentra el basamento rocoso, que permite conocer la morfología del basamento sin necesidad de medidas directas (Lermo y Chávez-García, 1993).

La microzonación sísmica abarca la determinación de la capacidad de amplificación sísmica y del periodo fundamental en una región determinada. En el marco de las metodologías que permiten llevar a cabo la microzonación sísmica, existen metodologías que se sirven de mapas de amplificación obtenidos en base a las características generales de tipo geológico y geotécnico de los materiales aflorantes, y a la topografía del terreno. En cambio, otras metodologías de microzonación utilizan estimaciones directas de los parámetros mecánicos del material, como la densidad o la velocidad de propagación de las ondas sísmicas, a partir de medidas de campo, con objeto de determinar a partir de estos datos la amplificación sísmica y la frecuencia fundamental correspondientes.

Los mapas de amplificación muestran la distribución espacial de la capacidad de amplificación en una región determinada. Son producidos generalmente a escalas pequeñas (Wald et al., 1999b; Tsige y García Flores, 2006), en las cuales constituyen quizá el instrumento más rápido para tratar de estimar, al menos en una primera aproximación, el potencial de amplificación del terreno. Los mapas de amplificación son generados por medio de la clasificación de los materiales geológicos aflorantes en clases o tipologías de emplazamiento específicas. Cada una de estas clases tiene una capacidad de amplificación determinada, que se establece a partir de la consideración de una serie de parámetros geotécnicos (Borcherdt, 1994; International Council of Building Officials, 1997). Uno de los parámetros geotécnicos fundamentales es el valor medio de la velocidad de propagación de la onda de corte (Vs) en los primeros $30 \mathrm{~m}$ de profundidad (Vs30), de forma que, a cada clase de emplazamiento, le corresponde un valor o intervalo de valores de Vs30 determinado.

Borcherdt (1994) desarrolla una metodología de clasificación de los materiales geológicos de acuerdo con su capacidad de amplificación, con objeto de encuadrarlos en un conjunto de 6 clases de emplazamiento, que se definen en función de determinadas propiedades geotécnicas, como su compacidad y cohesión, y un intervalo concreto de valores de Vs30. De acuerdo con esta metodología, el procedimiento de clasificación de los materiales geológicos se basa en la comparación entre las propiedades geotécnicas que se derivan de la composición litológica de cada material y las características geotécnicas de cada clase de emplazamiento. Borcherdt (1994) utiliza el valor el Vs30 de cada una de las 6 clases de emplazamiento con objeto de cuantificar la capacidad de amplificación de cada clase, por medio de factores de amplificación para periodos cortos $(0,1-0,5 \mathrm{~s})$ y medios $(0,4-2,0 \mathrm{~s})$, que dependen del periodo (o frecuencia) del movimiento del terreno. La metodología de Borcherdt (1994) es utilizada por diversos autores para generar mapas de amplificación sísmica a partir de la geología local. Wald et al. (1999b) incorpora los factores de amplificación de Borcherdt (1994) en el proceso de obtención de mapas de parámetros máximos del movimiento del terreno (ShakeMaps) en la región sur de California, en particular mapas de la aceleración pico y de la velocidad pico del movimiento del terreno. En España, Tsige y García Flores (2006) obtienen un mapa de la amplificación sísmica de la Región de Murcia a partir de la clasificación de los materiales geoló- 
gicos aflorantes según su respuesta sísmica, en el contexto de los objetivos previstos por el proyecto RISMUR, llevado a cabo por el Instituto Geográfico Nacional (IGN) y la Dirección General de Protección Civil en la Región de Murcia para evaluar el riesgo sísmico en esta región.

Un método alternativo para abordar la microzonación sísmica mediante mapas de amplificación se basa en la determinación de Vs30 a partir de la pendiente topográfica del terreno. De acuerdo con Allen y Wald (2007), a partir de la correlación de diversas medidas de Vs30 y de pendientes topográficas se han obtenido dos grupos de coeficientes de predicción de esta velocidad: uno para regiones tectónicas activas, con un relieve dinámico y cambiante, y otro para regiones estables, con cambios topográficos más dilatados en el tiempo. De acuerdo con Allen y Wald (2007), estos coeficientes han permitido obtener mapas de amplificación sísmica de similar o superior calidad a los obtenidos a partir de la información geológica local o de medidas de Vs30 realizadas en campo.

La microzonación sísmica también se ha desarrollado en base a estimaciones directas de los parámetros geotécnicos del terreno, por medio de medidas en campo. Con respecto a la velocidad Vs, distintos métodos geofísicos permiten estimarla en emplazamientos determinados. Entre estos métodos destaca el método de la Sísmica Pasiva Refraction Microtremor (ReMi), pues determina Vs por medio de medidas del ruido sísmico ambiental que es producido por actividades humanas comunes y por el tráfico, sin necesidad de una fuente artificial, con lo cual permite una utilización muy flexible en entornos urbanos (Louie, 2001). También debe hacerse referencia al método SASW (Spectral Analysis of Surface Waves), que utiliza una fuente sísmica artificial para determinar Vs (Nazarian y Stokoe II, 1984), y al método MASW (Multichannel Analysis of Surface Waves), que mejora las limitaciones del método SASW mediante medidas de ruido sísmico ambiental Park et al. (1999). A partir de los datos de Vs obtenidos en campo, distintos algoritmos permiten obtener la amplificación sísmica espectral (Kanai, 1951; Haskell, 1960; Joyner et al., 1981; Idriss y Sun, 1992), y por tanto conocer el valor de la amplificación para cada frecuencia de la señal. En cuanto a la determinación de la frecuencia o periodo fundamental, Nogoshi e Igarashi (1971) y Nakamura (1989) desarrollan el método $\mathrm{H} / \mathrm{V}$ para derivar esta frecuencia a partir de los espectros de amplitud de Fourier de las componentes horizontal y vertical de un microtremor, entendido como ruido ambiental de alta frecuencia.

En España, se han realizado recientemente un número significativo de trabajos sobre microzonación sísmica, como los de Alfaro et al. $(1998,2001)$ sobre la determinación de frecuencia fundamental de diferentes suelos de la ciudad de Barcelona. Iniesto et al. (2002) comparan diferentes metodologías de cartografía de microzonación, y Macau et al. $(2007,2009)$ y Schmidt et al. (2009) llevan a cabo diferentes estudios a partir de medidas de ruido sísmico ambiental procesadas por medio del método $\mathrm{H} / \mathrm{V}$ en emplazamientos localizados en el Pirineo Oriental y en la bahía de Cádiz, respectivamente. Algunas de estas publicaciones han dado como resultado la defensa de varias tesis doctorales en esta temática. En este sentido, puede destacarse el trabajo de Coral (2002), en el que se utiliza el método H/V en Andorra; la tesis de Macau (2008), a partir de la aplicación del método $\mathrm{H} / \mathrm{V}$ en la cubeta de Andorra la Vella, el valle de la Cerdanya y la ciudad de Málaga; la tesis de Goded (2010), quien también utiliza el método $\mathrm{H} / \mathrm{V}$ en la ciudad de Málaga; y la investigación doctoral 
de Pérez Santisteban (2012) a partir de la aplicación del método ReMi en el área metropolitana de Madrid.

Además de la amplificación sísmica de las capas superficiales, la atenuación del medio de propagación de las ondas sísmicas en su camino hacia la superficie influye decisivamente en el valor de la aceleración sísmica del terreno. La atenuación del medio se define como el decaimiento de la amplitud de estas ondas conforme se propagan en el medio. Se distinguen dos causas de la atenuación, una de carácter geométrico y otra definida como atenuación anelástica. Usualmente, la atenuación anelástica total se representa por medio del factor de calidad Q, que depende de la frecuencia y se define en términos de la fracción de energía perdida durante el ciclo de una onda. La dependencia frecuencial de Q es explicitada por Mitchell (1981) en función de los parámetros $Q_{0}$ y $\nu$, que toman un valor constante en función de las frecuencias de estudio.

A partir del desarrollo de la teoría de las ondas de coda por parte de Aki (1969), estas ondas se han incorporado a la estimación de la atenuación anelástica. Los métodos empleados para calcular Q a partir de las ondas de coda asumen un medio dispersivo, que introduce cambios en la trayectoria seguida por las ondas sísmicas, y la mayor parte de los mismos se basa en la distribución aleatoria de las heterogeneidades que introducen esta dispersión en el medio. En este sentido, Aki y Chouet (1975) proponen que las ondas de coda de terremotos locales de baja magnitud proceden de la dispersión de las ondas sísmicas por heterogeneidades uniformemente distribuidas en la corteza terrestre. Su trabajo es utilizado por Havskov et al. (1989) como punto de partida para su metodología de estimación del factor de calidad Q a partir del ajuste de una envolvente decreciente con el tiempo a la señal de la onda de coda. Herrmann (1980) y Pujades et al. (1990) se basan en el trabajo de Aki (1969) para estimar el factor de calidad por medio de observaciones de la frecuencia predominante en la onda de coda.

Ante el problema de la simulación de un escenario sísmico, y con objeto de obtener un valor sintético realista de la aceleración del movimiento del terreno, debe establecerse el nivel de aproximación que se va a utilizar para modelizar la fuente sísmica. En este sentido, es posible realizar una primera aproximación, considerando una fuente puntual, y llevar a cabo la simulación a partir de relaciones empíricas de atenuación, en términos de aceleración máxima del terreno. Estas relaciones se obtienen generalmente a partir del ajuste de determinados valores de aceleración pico del terreno, registrados en determinados tipos de suelos y rocas y en regiones específicas (Campbell, 1997; Munson y Thurber, 1997; Zhao et al., 2006; Chiou y Youngs, 2008; Mezcua et al., 2008; Instituto Geográfico Nacional, 2013, entre otros). Los modelos de atenuación así determinados comportan la ventaja de considerar un número relativamente reducido de variables, comúnmente la magnitud del terremoto y la distancia a la fuente sísmica. Sin embargo, se encuentran influenciados por los datos de aceleración empleados en su construcción, y hacen necesario conocer, al menos, la capacidad de amplificación del material geológico en el que se emplazan los acelerómetros que han proporcionado estos datos, pues las aceleraciones calculadas con el modelo también se encuentran referidas a este material. Con respecto a la simulación de escenarios sísmicos en términos de intensidad, diversas relaciones de atenuación permiten calcular este parámetro, como las de Muñoz (1974), Martín Martín (1983, 1984) y López Casado et al. (2000), entre otros. 
Una segunda aproximación a la fuente sísmica implica la consideración de una falla finita. En este caso, la simulación del movimiento sísmico fuerte se realiza por medio la aproximación estocástica propuesta inicialmente por Hanks (1979) y McGuire y Hanks (1980), a partir del modelo de Brune (1970) para el espectro en la fuente. Posteriormente, Boore (1983) generaliza esta aproximación a otros modelos más complejos y la extiende a la simulación de registros temporales. Beresnev y Atkinson (1997) desarrollan el modelo estocástico de falla finita, el cual se fundamenta en la división de la falla en pequeñas subfallas, de forma que se calcula el parámetro del movimiento fuerte del terreno generado por cada subfalla a partir de una aproximación a fuente puntual. El movimiento fuerte es obtenido, por tanto, como la suma de las contribuciones de cada subfalla, en función del tiempo. Motazedian y Atkinson (2005) desarrollan una aproximación al espectro de amplitudes de la fuente finita, el cual depende del decaimiento del espectro en las altas frecuencias $(\kappa)$, del momento sísmico y de la frecuencia esquina (dependiente del tiempo) para cada subfalla, de la atenuación geométrica y anelástica, y de la distancia entre la subfalla y el punto de observación.

La simulación de escenarios sísmicos en términos de aceleraciones e intensidades sintéticas ha sido objeto de varios trabajos publicados en las últimas décadas. Sirovich $(1996 b, a)$ desarrolla un algoritmo que, partiendo de las características de la fuente sísmica, da como resultado intensidades sintéticas similares a las observadas para determinados terremotos en California. Panza et al. (1991) y Moratto et al. (2011) también han publicado trabajos relacionados con el cálculo de las intensidades sintéticas.

El trabajo de Wald et al. (1999b) introduce el efecto de sitio en la determinación de los escenarios sísmicos, con objeto de generar automáticamente mapas de aceleraciones y velocidades máximas del movimiento del terreno, ShakeMaps, producidos por terremotos del sur de California. Para la generación de los ShakeMaps, los autores siguen una metodología concreta que exige calcular la aceleración o velocidad sintética en roca y en una malla de puntos equiespaciados, por medio de relaciones de atenuación que consideran una aproximación a una fuente puntual. También consideran las aceleraciones y velocidades ya observadas en estaciones de registro, que deben corregirse a condiciones de roca por medio de los factores de amplificación sísmica en función de la frecuencia, determinados a partir de la geología local siguiendo la metodología de Borcherdt (1994). A continuación, tanto los valores observados como las aceleraciones y velocidades sintéticas se interpolan sobre una malla de puntos más densa. Por último, los valores interpolados son corregidos por medio de los factores de amplificación correspondientes a las características geológicas de cada emplazamiento, de forma que se recuperan las aceleraciones y velocidades registradas inicialmente.

En otras partes del mundo también se han obtenido mapas similares a los ShakeMaps, siguiendo la metodología de Wald et al. (1999b). Por ejemplo, en Japón se han generado de forma rápida mapas de la distribución de la intensidad sintética y de la velocidad máxima del movimiento del terreno, QuakeMaps, a partir de los registros de determinadas estaciones sísmicas y de un mapa de la capacidad de amplificación sísmica del terreno para todo el territorio (Geological Survey of Japan, 2014). Este mapa se presenta en términos de Vs30, y se ha obtenido a partir de datos sobre geología superficial y condiciones geomorfológicas. 
En el ámbito de la Península Ibérica, la Dirección General de Protección Civil de España ha desarollado el programa SES 2002, que permite simular escenarios sísmicos en términos de intensidad sísmica en cualquier municipio, con el fin de estimar los efectos sobre la población y las construcciones ante la ocurrencia de un terremoto (Dirección General de Protección Civil y Dirección General del Instituto Geográfico Nacional, 2002). Este algoritmo puede seleccionar la relación de atenuación más adecuada en función de la zona sismogenética donde se localiza el epicentro del terremoto, a partir de las relaciones obtenidas por Martín Martín (1983), pero no realiza ninguna estimación de la amplificación sísmica del terreno que pueda corregir las intensidades calculadas. La ausencia de una estimación razonable del efecto de sitio en la simulación puede llevar a una subestimación frecuente de las intensidades sintéticas. Con carácter previo a la realización de esta tesis doctoral, se han llevado a cabo varias simulaciones del terremoto de Mw 5,1, de 11-05-2011, en el NE de Lorca (Murcia), último terremoto catastrófico sucedido en España. Como resultado, el programa SES 2002 ha obtenido intensidades sintéticas que son, por lo general, menores que las intensidades observadas en los municipios para los que se cuenta con información macrosísmica.

Desde el comienzo y desarrollo de la sismología instrumental, en España no se ha contado con un número amplio de registros de aceleración, debido a la moderada sismicidad del territorio. Sin embargo, la reactivación volcánica experimentada por la isla de El Hierro, en las Islas Canarias, entre los años 2011 y 2012 (López et al., 2012) ha proporcionado a la comunidad científica nacional un número inusualmente elevado de acelerogramas, en un orden de 200, obtenidos en una región tan específica como la isla, que implica una escala regional, y en un periodo de tiempo determinado del orden de 2 años (2011-2013). Este gran número de registros acelerométricos convierte a la isla en un lugar propicio para simular escenarios sísmicos, en términos de aceleración, pues permite la calibración de los métodos y algoritmos de simulación en función de estos registros.

Las Islas Canarias se encuentran situadas en una franja de 200 por $500 \mathrm{~km}$ aproximadamente, en el margen continental pasivo de África, y sobre litosfera oceánica de edad jurásica. Las formación del archipiélago se relaciona con un punto caliente (hotspot), cuyo movimiento preciso es aún materia de debate (Carracedo et al., 2001). En coherencia con la actividad del punto caliente, la formación de las islas ha seguido una progresión continua hacia el oeste, de acuerdo con la datación radiométrica del volcanismo subáereo más antiguo (Guillou et al., 2001).

La actividad sísmica del archipiélago es moderada, y presenta terremotos de magnitudes bajas, normalmente iguales o inferiores a 5. Antes de 2011, en el periodo instrumental se puede destacar el terremoto del 9 de mayo de 1989 con magnitud momento (Mw) 5,2, ocurrido entre las islas de Tenerife y Gran Canaria. El estudio de este evento y de sus réplicas llevó a Mezcua et al. (1992) a establecer la existencia de una falla inversa con dirección NE-SW entre las dos islas. Entre julio y octubre de 2011, casi 10000 terremotos con una magnitud Mw máxima de 4,0 fueron localizados en El Hierro, precediendo al comienzo de una erupción volcánica submarina el día 10 de octubre de 2011 (López et al., 2012). La erupción comenzó a $2 \mathrm{~km}$ de la costa sur de la isla, y se desarrolló hasta el mes de marzo de 2012. Desde entonces, seis crisis sísmicas adicionales han tenido lugar. En cuanto a las crisis sísmicas de junio de 2012 y de marzo de 2013, los eventos registrados han alcanzado magnitudes de 
hasta 5,0 .

El IGN monitoriza de forma permanente la sismicidad de las Islas Canarias por medio de una red desplegada en las siete islas del archipiélago, constituida actualmente por 32 estaciones de registro de velocidad, tanto de banda ancha como de corto periodo (Instituto Geográfico Nacional, 2017c). Adicionalmente, el Instituto realiza medidas constantes de otros parámetros geofísicos (intensidad del campo geomagnético total), geoquímicos (concentración de $\mathrm{CO}_{2} \mathrm{y}{ }^{222} \mathrm{Rn}$ en aire) y geodésicos (deformaciones superficiales y desplazamientos temporales), especialmente en El Hierro (López et al., 2012), con un concepto de red multiparamétrica que permite la comparación de los distintos tipos de datos, de forma continua y en el contexto de las funciones de vigilancia volcánica que le corresponden legalmente.

En Canarias, se han llevado a cabo estimaciones previas de la atenuación anelástica de la litosfera. Canas et al. (1995) siguen el método de Pujades et al. (1990) para estimar los valores de los parámetros que definen la dependencia frecuencial del factor de calidad $\mathrm{Q}, Q_{0}$ y $\nu$, a partir de los datos de las 6 estaciones analógicas de corto periodo ya instaladas por el IGN en 1995. A partir de estos valores, los autores generan un mapa de la distribución de $Q_{0}$ en el archipiélago. También se debe citar el trabajo posterior de Canas et al. (1998), quienes determinan $Q_{0}$ y $\nu$ para distintas distancias hipocentrales, obteniendo resultados coherentes con los menores valores de estos parámetros, proporcionados por Canas et al. (1995). En el contexto de una simulación de escenarios sísmicos en Canarias, y teniendo en cuenta que el IGN ha instalado nuevas estaciones de registro de velocidad de banda ancha en los últimos 20 años, resulta conveniente llevar a cabo un estudio de la atenuación anelástica que incorpore los registros digitales de las nuevas estaciones, para poder obtener valores de $Q_{0}$ y $\nu$ con mejor resolución, y de esta forma realizar una regionalización de ambos parámetros que supere las limitaciones de los trabajos previos citados, sobre todo en cuanto a número de datos utilizado.

El objetivo principal de esta tesis doctoral consiste en obtener valores sintéticos realistas de la aceleración sísmica y de la intensidad sísmica en la Península Ibérica, las Islas Baleares y las Islas Canarias, ante un terremoto predeterminado y en base a dos niveles de aproximación. En un primer nivel, la simulación de escenarios sísmicos considera una fuente sísmica puntual, una relación de atenuación y la amplificación sísmica de los materiales geológicos aflorantes en el territorio, proporcionada por un mapa de amplificación sísmica. Esta simulación se realiza automáticamente por medio de una herramienta de desarrollo del Sistema de Información Geográfica QGIS, producto del proyecto oficial del Open Source Geospatial Foundation (OSGeo). La herramienta permite incorporar a los cálculos las aceleraciones observadas en las estaciones acelerométricas que han registrado el terremoto. En un segundo nivel de aproximación, se utiliza un algoritmo de simulación estocástica de acelerogramas sintéticos para simular escenarios sísmicos en la isla de El Hierro, dadas las grandes posibilidades de calibrar el algoritmo en función de los registros de aceleración disponibles. Este algoritmo considera los parámetros de una fuente sísmica extensa, un modelo de atenuación de la litosfera regional en las Islas Canarias y la amplificación sísmica espectral en 25 emplazamientos distribuidos en la isla. Esta amplificación se obtiene a partir de los modelos de velocidad de onda de corte que son determinados en cada emplazamiento por medio del método ReMi. En los dos niveles de aproximación, las aceleraciones sintéticas son utilizadas para producir los correspondientes 
mapas de sacudidas, y también para calcular las intensidades sintéticas por medio de una correlación previamente establecida.

Para lograr el objetivo principal, se plantean una serie de objetivos intermedios, de forma que los resultados parciales conseguidos con cada uno permitan su implementación en la simulación de los escenarios sísmicos:

1. Generar cartografía que cuantifique la amplificación sísmica de los materiales geológicos aflorantes en la Península Ibérica, las Islas Baleares y las Islas Canarias por medio de factores de amplificación dependientes de la frecuencia del movimiento del terreno.

2. Determinar la amplificación sísmica en función de la frecuencia del movimiento del terreno en 25 emplazamientos distribuidos en la isla de El Hierro, a partir de la obtención, mediante el método ReMi, de la distribución de la velocidad de la onda de corte en los primeros $30 \mathrm{~m}$ de profundidad, y calcular el periodo fundamental del suelo.

3. Determinar el periodo fundamental del suelo en los emplazamientos seleccionados en el objetivo intermedio $n^{\circ} 2$, utilizando con este fin el método $\mathrm{H} / \mathrm{V}$, y comparar el resultado obtenido con el periodo calculado a partir del método ReMi.

4. Analizar el comportamiento de distintas relaciones de atenuación en términos de aceleración sintética en la Península Ibérica, las Islas Baleares y las Islas Canarias.

5. Estimar la atenuación anelástica de la litosfera regional en las Islas Canarias, por medio del análisis de las ondas de coda registradas por las estaciones sísmicas de corto periodo y de banda ancha emplazadas en el archipiélago, y regionalizar espacial y temporalmente los parámetros $Q_{0}$ y $\nu$, con el fin de mejorar las regionalizaciones efectuadas en trabajos previos (Canas et al., 1995).

6. Calibrar un algoritmo de simulación estocástica de acelerogramas sintéticos en El Hierro, por medio de los acelerogramas registrados en los acelerógrafos emplazados en la isla. 


\section{Capítulo 2}

\section{METODOLOGÍA}

\subsection{Generación de cartografía de amplificación sís- mica}

La aceleración del movimiento del terreno que produce un terremoto en un punto determinado depende fundamentalmente de las características de la fuente sísmica, la atenuación del medio con la distancia y la capacidad de amplificación sísmica (o respuesta sísmica) de los materiales geológicos emplazados en los metros más superficiales. La capacidad de amplificación sísmica se conoce en la literatura sismológica como efecto de sitio, o efecto local, y consiste en la modificación de la amplitud de las ondas sísmicas que producen los materiales geológicos que son atravesados por las ondas en su camino hacia la superficie (Figura 2.1).

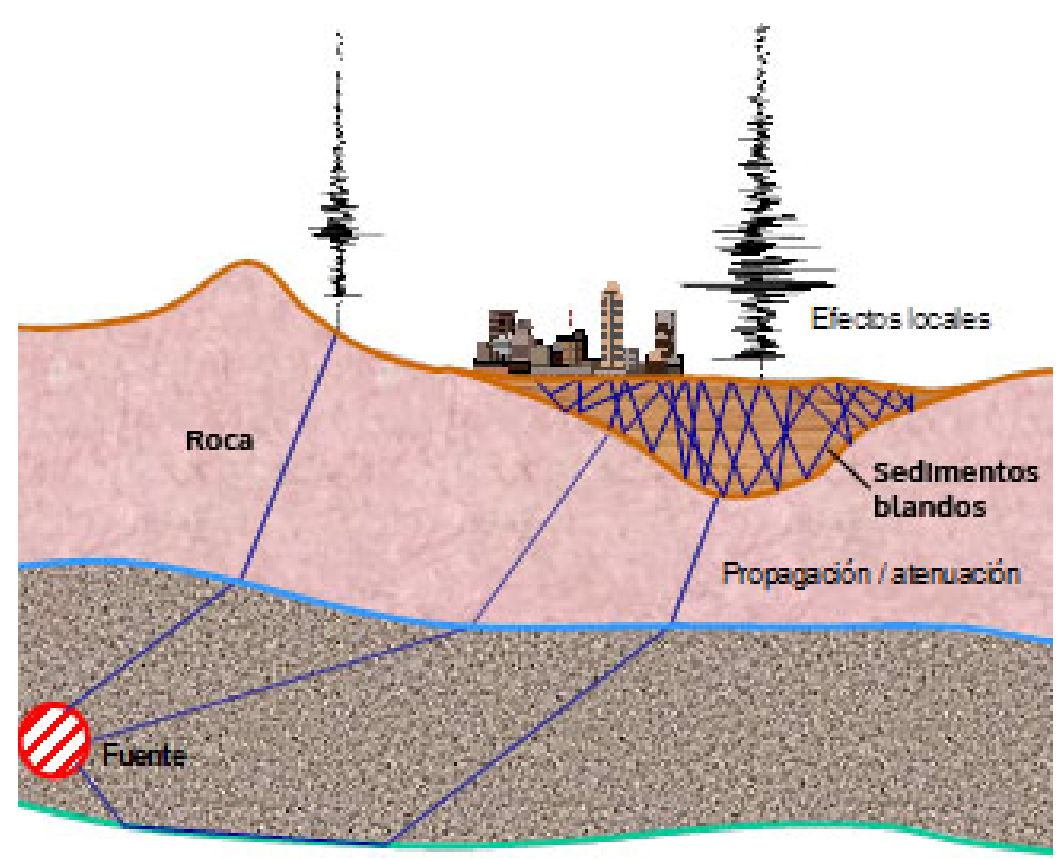

Figura 2.1: Representación esquemática del efecto de sitio producido por distitintos materiales geológicos (roca y sedimentos blandos), emplazados en las capas más superficiales. La amplitud de las ondas sísmicas que atraviesan estos materiales en su camino hacia la superficie es modificada en distinto grado en función del tipo de material. 
Los estudios de amplificación sísmica en un determinado emplazamiento también abarcan la determinación de la frecuencia o periodo fundamental de resonancia del suelo. El análisis del periodo fundamental de un suelo presenta interés debido a la posibilidad de que, si la frecuencia fundamental correspondiente es del orden de la frecuencia predominante del movimiento del terreno producido por un terremoto, tengan lugar fenómenos de resonancia en la aceleración del movimiento del terreno. Por otra parte, y según Lermo y Chávez-García (1993), existe una relación entre el periodo fundamental del suelo y la profundidad del basamento rocoso, lo cual permite conocer la morfología del basamento sin necesidad de recurrir a medidas directas.

La estimación de la capacidad de amplificación sísmica y del periodo fundamental de los materiales aflorantes en un determinado territorio constituye el problema de la microzonación sísmica de dicho territorio. La microzonación sísmica puede abordarse de acuerdo a distintas metodologías. A este respecto, existen métodos de microzonación que proporcionan una primera aproximación a la distribución espacial de la amplificación sísmica por medio de mapas de amplificación sísmica. Alternativamente, otras metodologías de microzonación recurren a estimaciones directas de los parámetros mecánicos de los materiales para determinar tanto la amplificación sísmica como la frecuencia fundamental.

Los mapas de amplificación sísmica pueden generarse por medio de la clasificación de los materiales aflorantes en distintas clases de emplazamiento, las cuales presentan una determinada respuesta sísmica. Con este fin, se utilizan diversas clasificaciones de suelos y rocas en función de su respuesta sísmica, que se basan en criterios geotécnicos. Uno de los criterios geotécnicos más utilizados es el valor medio de la velocidad de propagación de la onda de corte (Vs) en los primeros $30 \mathrm{~m}$ de profundidad (Vs30), el cual es incluido en una gran parte de las clasificaciones (Borcherdt, 1994; International Council of Building Officials, 1997; Building Seismic Safety Council, 2004; European Committee for Standardization, 2004). Estas clasificaciones permiten asignar una clase de emplazamiento a un determinado material sin necesidad de realizar medidas in situ, sino a partir de la información de la cartografía geológica oficial de la zona.

En la Figura 2.2 se observa un mapa de la distribución de Vs30 en Japón, utilizado como herramienta en la generación de mapas de la distribución de la intensidad sintética y de la velocidad máxima del movimiento del terreno ante un terremoto (QuakeMaps) (Geological Survey of Japan, 2014). El mapa de Vs30 se ha obtenido a partir de los datos relativos a la geología superficial y las condiciones geomorfológicas del territorio, contenidos en un mapa de clasificación geomorfológica de Japón ( $J a-$ pan Engineering Geomorphologic Classification Map) (Geological Survey of Japan, 2014). Este mapa, por tanto, permite distinguir distintos grados de la capacidad de amplificación sísmica en superficie, que vendrán determinados por Vs30.

Por otra parte, los mapas de amplificación sísmica pueden obtenerse también mediante valores de Vs30 estimados a partir de la pendiente topográfica del terreno. De acuerdo a Allen y Wald (2007), la correlación de distintas medidas de Vs30 y pendientes topográficas ha permitido obtener coeficientes de predicción de Vs30 para regiones tectónicas activas y para regiones estables. Por medio de estos coeficientes, los autores han generado mapas de amplificación sísmica de similar calidad a otros mapas producidos a partir de la información geológica local y de las estimaciones 

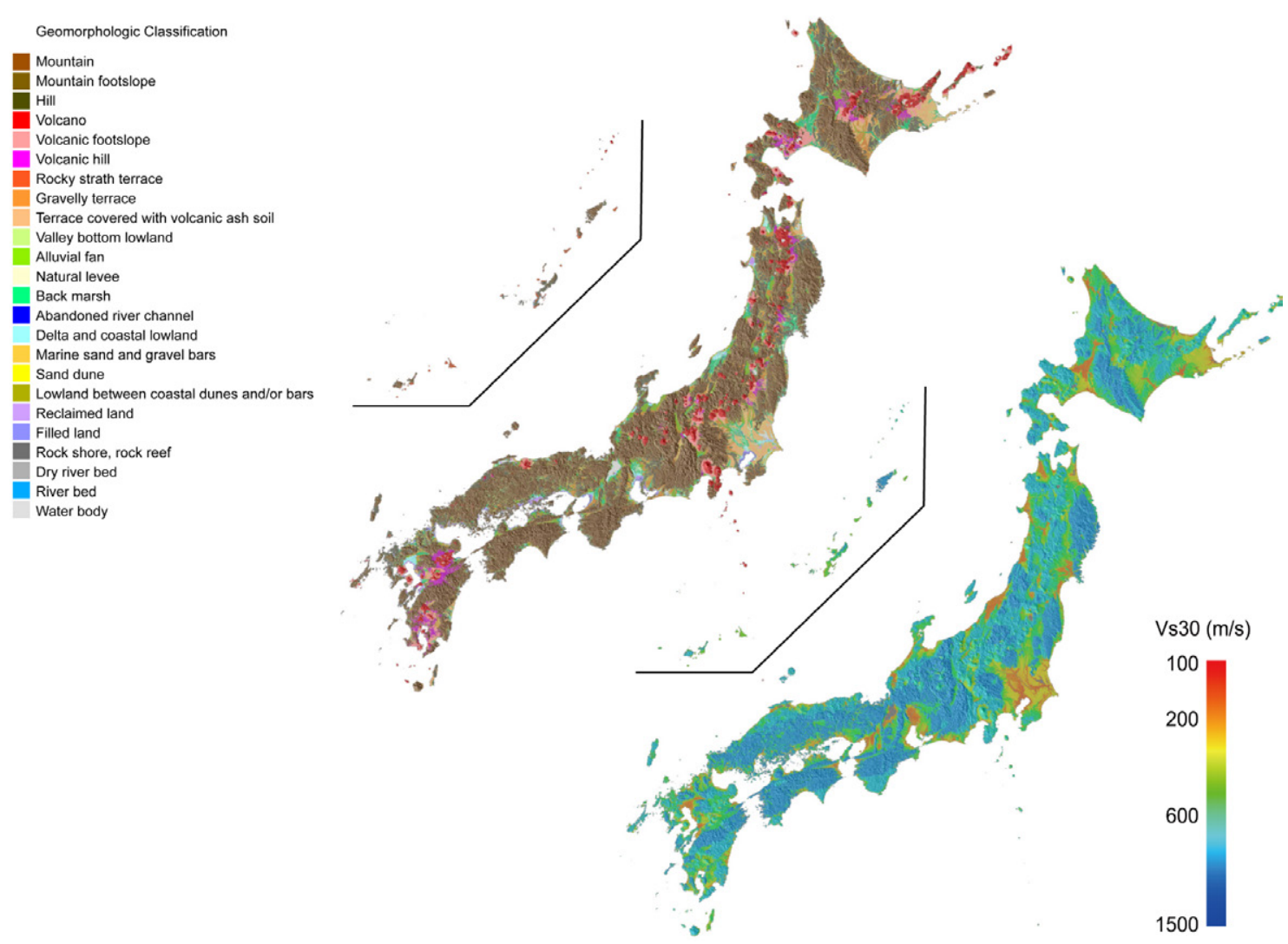

Figura 2.2: Mapa de la distribución de Vs30 en Japón, obtenido a partir de la geología superficial y las condiciones geomorfológicas. Tomado de Geological Survey of Japan (2014). 
directas de la velocidad de onda de corte. Como ejemplo, en la Figura 2.3 se muestra el mapa de la distribución de Vs30 en los Estados Unidos continentales situados al este de las Montañas Rocosas, obtenido por Allen y Wald (2007) a partir de la pendiente topográfica.

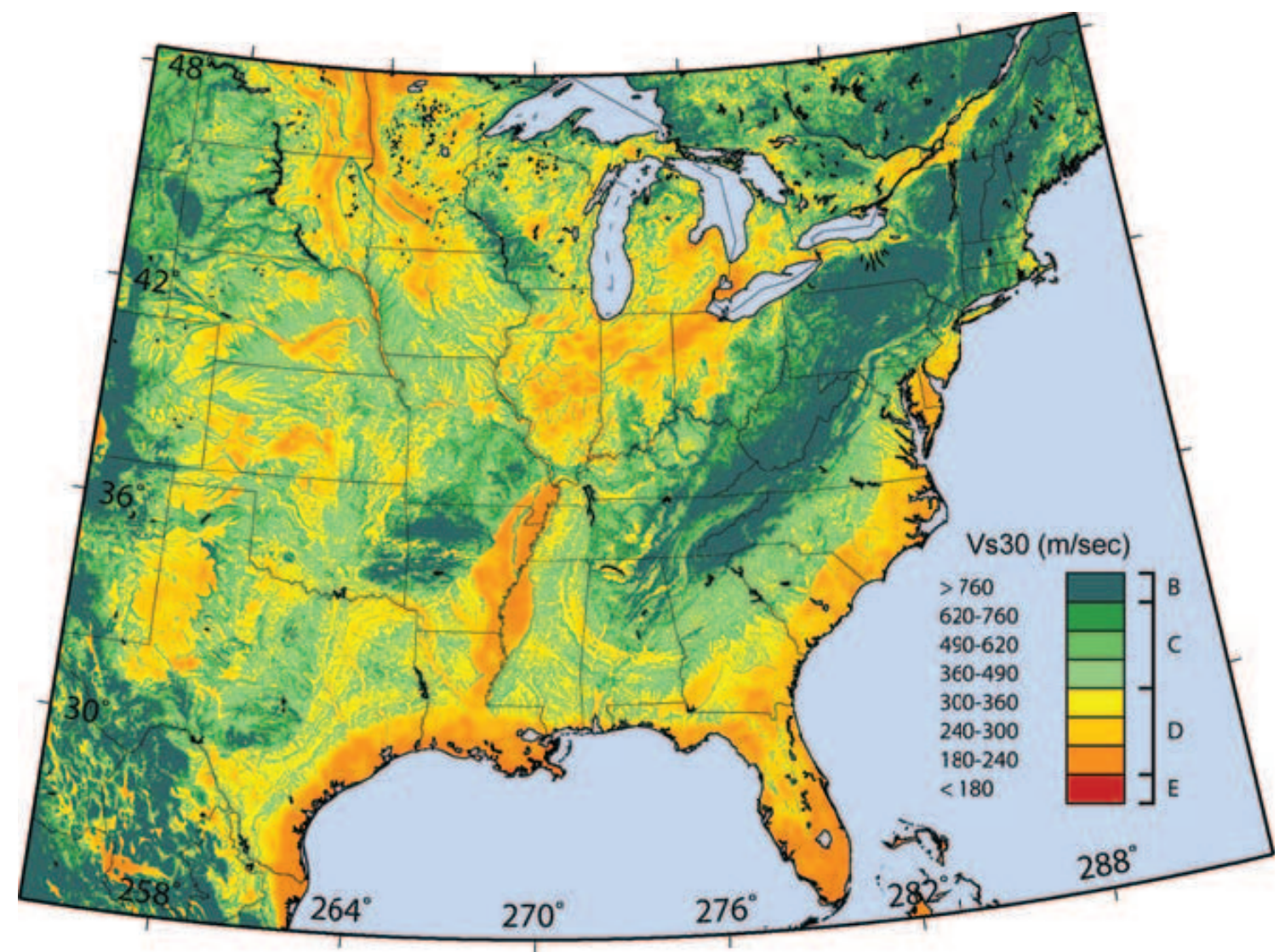

Figura 2.3: Mapa de Vs30 en los Estados Unidos continentales situados al este de las Montañas Rocosas, obtenido a partir de la pendiente topográfica y de los coeficientes de predicción de Vs30 para regiones estables. Tomado de Allen y Wald (2007).

Si se pretende calcular la amplificación sísmica del territorio con mayor exactitud y a escalas mayores que las de los mapas de amplificación sísmica, es preciso utilizar metodologías de microzonación basadas en estimación directa de los parámetros mecánicos de los materiales por medio de medidas de campo. Ejemplos de estos parámetros son la densidad y la distribución de Vs en los primeros $30 \mathrm{~m}$ de profundidad. Respecto a la estimación de la estructura de Vs, puede citarse el método ReMi (Refraction Microtremor), que permite obtener este parámetro a partir de medidas de ruido sísmico ambiental (Louie, 2001); también puede hacerse referencia al método SASW (Spectral Analysis of Surface Waves), que determina Vs utilizando una fuente sísmica artificial (Nazarian y Stokoe II, 1984), y al método MASW (Multichannel Analysis of Surface Waves), que mejora las limitaciones del SASW con medidas de ruido sísmico ambiental (Park et al., 1999).

En la Figura 2.4 se observa el mapa de distribución del parámetro Vs30 en las localidades de Torreperogil y Sabiote (Jaén), obtenido por Rueda et al. (2015). Para generar este mapa, se ha estimado el modelo de Vs en los primeros $30 \mathrm{~m}$ de profundidad en determinados emplazamientos, a partir de la toma de medidas de ruido sísmico ambiental en los mismos y su procesado posterior por medio del método 
ReMi. La toma de medidas se ha realizado en 33 emplazamientos situados en Torreperogil y en 35 emplazamientos pertenecientes a Sabiote. En cada emplazamiento de medida, se ha obtenido un valor de Vs30 a partir de la estructura de Vs que proporciona el método ReMi. A partir de todos los valores de Vs30 procedentes de todos los emplazamientos de medida se han trazado las isolíneas que describen la distribución de este parámetro en ambas localidades.

En cuanto a la estimación del periodo fundamental, Nogoshi e Igarashi (1971) y Nakamura (1989) desarrollan el método H/V. Este método permite obtener este parámetro de forma experimental a partir de los espectros de amplitud de Fourier de las componentes horizontal y vertical del ruido ambiental de alta frecuencia, originado predominantemente por actividades antrópicas.

\subsubsection{Clasificaciones de suelos y rocas según su capacidad de amplificación sísmica}

Con el fin de obtener una primera aproximación a la capacidad de amplificación sísmica en el territorio de la Península Ibérica, las Islas Baleares y las Islas Canarias, se pretende obtener un mapa de amplificación sísmica que abarque la totalidad del territorio en estudio. Por tanto, se requiere utilizar una clasificación de suelos y rocas según su capacidad de amplificación sísmica, para poder agrupar los distintos tipos de materiales geológicos aflorantes del territorio en determinadas clases de emplazamiento.

La literatura sismológica ofrece diversos ejemplos de clasificaciones de suelos y rocas en función de su capacidad de amplificación sísmica. A estos efectos, puede citarse la clasificación de Borcherdt (1994), obtenida de forma empírica a partir de datos geotécnicos y registros de movimientos sísmicos fuertes ocurridos en California. También cabe mencionar las clasificaciones incluidas en códigos de edificación y normativas de construcción sismorresistente que han sido utilizados en Estados Unidos. En relación a los códigos de edificación, puede hacerse referencia al Uniform Building Code (International Council of Building Officials, 1997) y al International Building Code (International Code Council, 2006); también deben mencionarse las normativas de construcción sismorresistente desarrolladas por el National Earthquake Hazards Reduction Program (NEHRP) (Building Seismic Safety Council, 1997, 2004). En el ámbito de la Unión Europea, el Eurocódigo-8 presenta una clasificación de tipos de terreno (European Committee for Standardization, 2004), y a nivel de España puede citarse la clasificación incluida en la Norma de Construcción Sismorresistente NCSE-02 (Ministerio de Fomento, 2003).

Generalmente, las clasificaciones establecen clases de emplazamiento cuya capacidad de amplificación sísmica depende de sus propiedades geotécnicas. Como se ha mencionado, una de las propiedades geotécnicas utilizadas es el parámetro Vs30. En general, cada clase se caracteriza por un intervalo de valores de Vs30, aunque también puede presentar un valor medio para el intervalo (Borcherdt, 1994).

Además de Vs30, la clasificaciones presentan otros criterios geotécnicos como la descripción geotécnica en términos de compacidad y consistencia (Borcherdt, 1994; Building Seismic Safety Council, 1997, 2004; International Council of Building Officials, 1997; Ministerio de Fomento, 2003; European Committee for Standardization, 


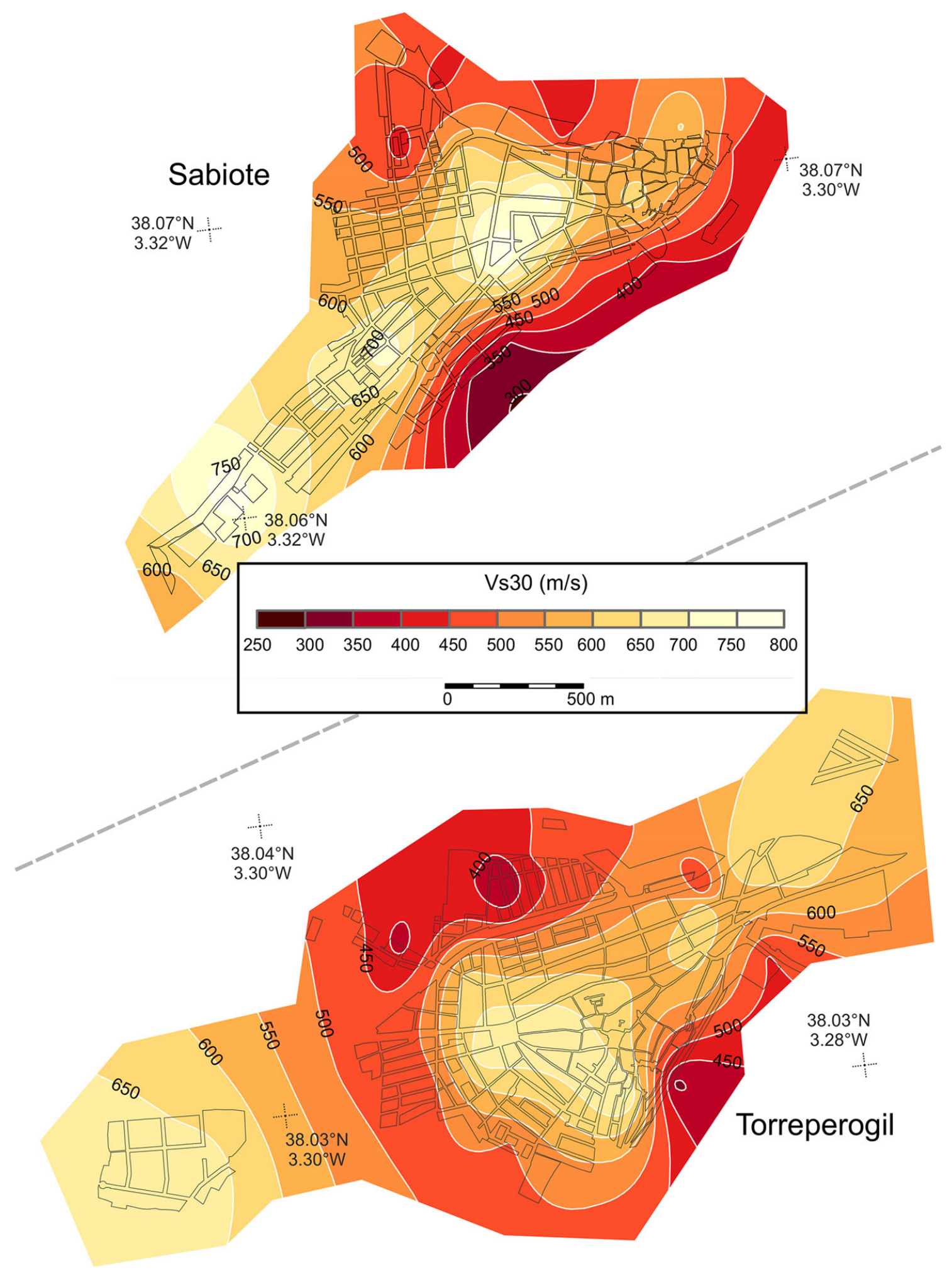

Figura 2.4: Mapa de la distribución de Vs30 (m/s) en Torreperogil y Sabiote (Jaén), obtenido a partir de la estimación del modelo de Vs en los primeros $30 \mathrm{~m}$ de profundidad en 68 emplazamientos de medida de ruido sísmico ambiental. Las medidas han sido procesadas por medio del método ReMi para obtener la estructura de Vs en cada emplazamiento. Tomado de Rueda et al. (2015). 
2004; International Code Council, 2006), y la resistencia media según el ensayo SPT (Standard Penetration Test) en los primeros $30 \mathrm{~m}$ de profundidad (International Council of Building Officials, 1997; Building Seismic Safety Council, 1997, 2004). Otras propiedades geotécnicas que caracterizan a las clases de emplazamiento son la resistencia media a cortante y sin drenar en los primeros $30 \mathrm{~m}$ de profundidad (International Council of Building Officials, 1997; Building Seismic Safety Council, 1997, 2004) y el espesor mínimo (Borcherdt, 1994).

La descripción geotécnica de un emplazamiento en términos de compacidad y consistencia puede considerarse como una descripción cualitativa de la resistencia del emplazamiento. Bajo esta consideración, puede decirse que un suelo más compacto o consistente que otro tiene mayor resistencia.

En la Tabla 2.1 se presentan las clases de emplazamiento que constituyen la clasificación de Borcherdt (1994) (Tabla 1 en Borcherdt (1994)). Cada clase de emplazamiento se caracteriza por una descripción general en la que se hace referencia a la compacidad y consistencia de los materiales característicos de la clase, e incluso a su tamaño de grano. Por ejemplo, la clase de emplazamiento SC-III se describe como arcillas consistentes y suelos arenosos. Por tanto, esta clasificación proporciona, para cada clase, la descripción geotécnica. La clase de emplazamiento también es definida según los valores mínimo, máximo y promedio de la velocidad media de corte en los primeros $30 \mathrm{~m}$ de profundidad (Vs30), y también según el espesor mínimo.

Debe tenerse en cuenta que la capacidad de amplificación también depende de la magnitud del movimiento del terreno producido a consecuencia de un terremoto. Si este movimiento excede un determinado nivel, el terreno, y en particular los suelos blandos, puede presentar un comportamiento no lineal. Por tanto, al considerar que la amplificación sísmica del terreno depende sólo de sus propiedades geotécnicas se está restrigiendo su estudio al dominio lineal.

Por otra parte, diversos autores, como Wald y Mori (2000), Mucciarelli y Gallipolli (2006) y Castellaro et al. (2008), han cuestionado recientemente que la distribución de Vs en profundidad, y el mismo parámetro Vs30, sean suficientes para cuantificar la amplificación sísmica local en un emplazamiento. A este respecto, Harmsen (1997) sugiere la influencia de la topografía local en el valor de la amplificación.

La producción del mapa de amplificación propuesto puede llevarse a cabo por medio de una clasificación de las ya publicadas. No obstante, resulta preferible realizar una síntesis entre distintas clasificaciones, pues de este modo se pueden tener en cuenta los criterios geotécnicos empleados en cada una y abarcar un mayor número de clases de emplazamiento. Para producir una clasificación de síntesis, se comienza con la selección de las clasificaciones que se van a analizar (Figura 2.5). Para cada una de estas clasificaciones, debe analizarse el número de clases de emplazamiento que describe y los tipos de propiedades geotécnicas que presenta. A continuación, y para cada clase de emplazamiento, es preciso estudiar el rango de valores o descripción de cada propiedad geotécnica. Por ejemplo, de acuerdo con Borcherdt (1994), la clase de emplazamiento SC-II se describe como suelos pedregosos y roca blanda a firme, tiene una Vs30 comprendida entre 375 y $700 \mathrm{~m} / \mathrm{s}$, con un valor promedio de $540 \mathrm{~m} / \mathrm{s}$, y un espesor mínimo de $10 \mathrm{~m}$. Una vez se han sistematizado las propiedades geotécnicas de cada clase de emplazamiento en cada clasificación, se debe comprobar que haya clases de emplazamiento con propiedades parecidas o con rangos de 
Tabla 2.1: Clases de emplazamiento según Borcherdt (1994). PI es el índice de plasticidad y N la resistencia según el ensayo SPT (Standard Penetration Test).

\begin{tabular}{|c|c|c|c|c|c|}
\hline CLASE DE EMPLA- & \multicolumn{5}{|c|}{ CRITERIOS DE CLASIFICACIÓN } \\
\hline \multirow[t]{2}{*}{ Nombre } & Descripción general & \multicolumn{3}{|c|}{ Vs30 (m/s) } & \multirow{2}{*}{$\begin{array}{l}\text { Espesor } \\
\text { mínimo } \\
(\mathrm{m})\end{array}$} \\
\hline & & Mínima & Promedio & Máxima & \\
\hline SC-I & $\begin{array}{l}\text { ROCA FIRME Y DU- } \\
\text { RA }\end{array}$ & & & & \\
\hline SC-Ia & $\begin{array}{l}\text { ROCA DURA (ej. roca } \\
\text { metamórfica con fracturas } \\
\text { muy espaciadas) }\end{array}$ & 1400 & 1620 & & \\
\hline SC-Ib & $\begin{array}{l}\text { ROCA FIRME A DURA } \\
\text { (ej. granito, roca ígnea, } \\
\text { conglomerados, arenisca, y } \\
\text { esquistos con fracturas pró- } \\
\text { ximas a muy espaciadas) }\end{array}$ & 700 & 1050 & 1400 & \\
\hline SC-II & $\begin{array}{l}\text { SUELOS PEDREGO- } \\
\text { SOS Y ROCA BLAN- } \\
\text { DA A FIRME (ej. ro- } \\
\text { ca blanda sedimentaria } \\
\text { ígnea, areniscas, y es- } \\
\text { quistos, gravilla, y sue- } \\
\text { los con más del } 20 \% \text { de } \\
\text { gravilla) }\end{array}$ & 375 & 540 & 700 & 10 \\
\hline SC-III & $\begin{array}{l}\text { ARCILLAS CONSIS- } \\
\text { TENTES Y SUELOS } \\
\text { ARENOSOS (ej. are- } \\
\text { nas sueltas a muy den- } \\
\text { sas, margas limosas y } \\
\text { arcilla arenosa, y arci- } \\
\text { llas de consistencia me- } \\
\text { dio dura a dura y arci- } \\
\text { llas limosas (N }>5 \text { gol- } \\
\text { pes/pie)) }\end{array}$ & 200 & 290 & 375 & 5 \\
\hline SC-IV & SUELOS BLANDOS & 100 & 150 & 200 & \\
\hline SC-IVa & $\begin{array}{l}\text { SUELOS BLANDOS DE } \\
\text { ESTUDIO NO ESPECIIFI- } \\
\text { CO (ej. relleno suelto su- } \\
\text { mergido, arcillas muy blan- } \\
\text { das a blandas ( } \mathrm{N}<5 \text { gol- } \\
\text { pes/pie) y arcillas limosas } \\
\text { de espesor menor que } 37 \\
\text { m) }\end{array}$ & & & & 3 \\
\hline SC-IVb & $\begin{array}{l}\text { SUELOS BLANDOS DE } \\
\text { ESTUDIO ESPECÍFICO } \\
\text { (ej. suelos licuefactibles, } \\
\text { arcillas deformables (quick } \\
\text { and highly sensitive clays), } \\
\text { turbas, arcillas altamente } \\
\text { orgánicas, arcillas de un } \\
\text { alto grado de plasticidad } \\
\text { (PI }>75 \% \text { ) y suelos blan- } \\
\text { dos con más de } 37 \text { m de es- } \\
\text { pesor) }\end{array}$ & & & & 3 \\
\hline
\end{tabular}


valores de intervalos similares, y que estas clases se presentan en todas las clasificaciones. Por tanto, se busca asegurar que cada clase de emplazamiento perteneciente a una clasificación equivale a una sola clase en cada una de las restantes clasificaciones. Estas clases de emplazamiento equivalentes son las que pueden constituir la nueva clasificación de síntesis. Por tanto, la clasificación de síntesis presenta las clases de emplazamiento equivalentes que proceden de las clasificaciones de partida, e incorpora los criterios geotécnicos utilizados por estas clasificaciones.

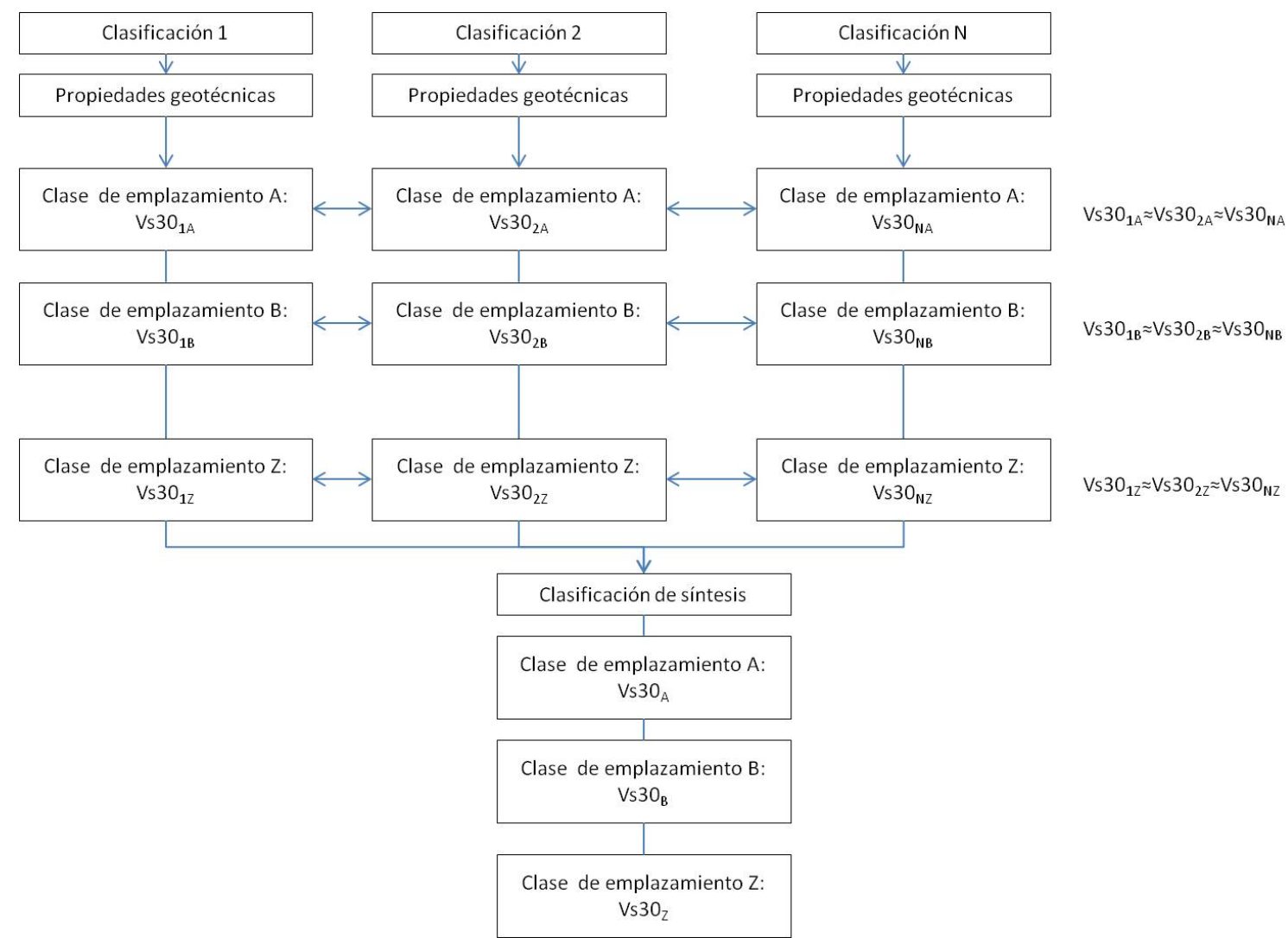

Figura 2.5: Proceso de producción de una clasificación de síntesis a partir de N clasificaciones de suelos y rocas en función de su capacidad de amplificación sísmica. Cada clasificación puede presentar un número determinado de clases de emplazamiento (A-Z), que están caracterizadas por determinadas propiedades geotécnicas (por ejemplo, Vs30). Las clases de emplazamiento que se caracterizan por valores similares de estas propiedades (Vs30) y que se presentan en todas las clasificaciones pueden integrarse en la clasificación de síntesis.

\subsubsection{Clasificación de las unidades geológicas según su capa- cidad de amplificación sísmica}

Con el propósito de producir el mapa de amplificación sísmica del territorio en estudio, es necesario analizar las características de los materiales geológicos que afloran en el mismo, con el fin de clasificar estos materiales de acuerdo a las clases de emplazamiento de la clasificación de síntesis. El análisis de los materiales geológicos aflorantes puede realizarse en un plazo de tiempo razonable por medio de la cartografía geológica oficial, a una escala que cubra la totalidad del territorio, obteniendo de esta forma las unidades geológicas presentes.

De acuerdo a la metodología propuesta por Borcherdt (1994), a partir de las características de las unidades geológicas, fundamentalmente su litología, es posible 
obtener una primera aproximación al valor de Vs30 de la unidad y, en última instancia, a su capacidad de amplificación. La estimación del valor de Vs30 se realiza a partir de la asignación de una clase de emplazamiento, previamente definida, a la unidad en cuestión. Esta clase presenta determinadas propiedades geotécnicas de tipo cualitativo, como el grado de resistencia, la compacidad y la consistencia, y también un valor de Vs30. Por tanto, esta metodología (Figura 2.6) propone estimar la resistencia, compacidad y consistencia de la unidad a partir de su litología, comparar estas propiedades con las de las clases de emplazamiento previamente establecidas, para asignar finalmente a la unidad aquella clase de emplazamiento cuyas propiedades sean coherentes con las de la unidad geológica. A partir de esta asignación, la unidad queda caracterizada con la Vs30 de la clase a la que pertenece.

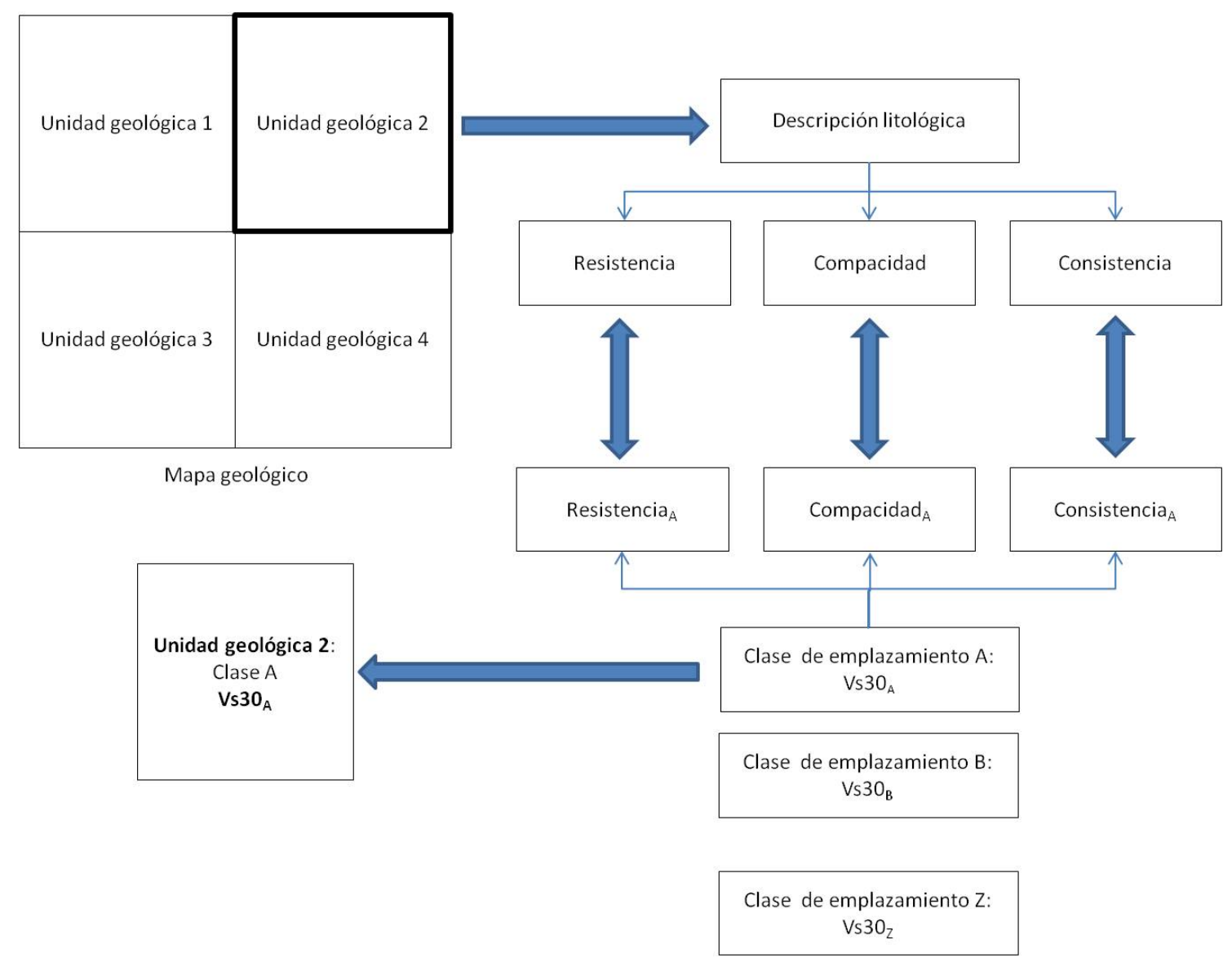

Figura 2.6: Proceso de asignación de una clase de emplazamiento (A), y por tanto, de un valor de Vs30 (Vs30 A), a una unidad geológica (2), de acuerdo con la metodología de Borcherdt (1994). Esta asignación se lleva a cabo considerando la coherencia y similitud entre determinadas propiedades geotécnicas cualitativas (resistencia, compacidad y consistencia) de la clase y las mismas propiedades de la unidad, estimadas a partir de su descripción litológica.

En el trabajo de Wills et al. (2000) se efectúa una clasificación de las unidades geológicas cartografiadas en California en categorías definidas por un valor de Vs30, la cual se desarrolla de acuerdo con las mismas pautas que observa Borcherdt (1994). Partiendo de medidas de Vs30 obtenidas en campo, las unidades geológicas son asociadas a determinados valores de Vs30 en función de su edad y propiedades físicas (tamaño de grano, dureza y fracturación). Esta asociación se basa en las similitudes encontradas entre las unidades donde no se conoce el valor de Vs30 y las unidades donde se han obtenido las medidas. 
Utilizando la cartografía geológica y siguiendo la metodología de Borcherdt (1994), para cada una de las unidades geológicas del territorio en estudio, se propone analizar las características de tipo geológico que permiten estimar las propiedades geotécnicas cualitativas de la resistencia, la compacidad y la consistencia. El Mapa Geológico de España a escala 1:1.000.000 (Instituto Geológico y Minero de España, 1994) permite ejemplificar estas características. Cada una de las unidades geológicas que lo componen se describe en términos de litología y edad cronoestratigráfica, detallada hasta el nivel de serie. Es claro que una unidad cuya descripción litológica consiste en rocas ígneas, como las rocas volcánicas o las rocas plutónicas, tiene una resistencia mayor que otra unidad compuesta por sedimentos de arcillas o de limos. En cuanto a la edad cronoestratigráfica, es posible afirmar que una unidad compuesta por rocas sedimentarias extremadamente antiguas, como las areniscas de edad cretácica, tiene una compacidad alta. En cambio, una unidad consistente en arenas sueltas de edad cuaternaria tiene una compacidad bastante menor.

Las clases de emplazamiento de la clasificación de síntesis se definen de acuerdo con los criterios geotécnicos de las clasificaciones que se utilizan en su elaboración. Estos criterios abarcan la Vs30 y la descripción geotécnica de cada clase en términos de compacidad y consistencia, la cual también permite estimar cualitativamente su resistencia. Por tanto, se puede asignar a cada unidad geológica aquella clase de emplazamiento que presente características coherentes con las propiedades geotécnicas cualitativas que se han inferido para la unidad. En consecuencia, por medio de este proceso se llevará a cabo la clasificación de las unidades geológicas de acuerdo a las clases de emplazamiento de la clasificación de síntesis.

\subsubsection{Cálculo de factores de amplificación en función de la frecuencia del movimiento del terreno a partir de Vs30}

El siguiente paso en el proceso de producción del mapa de amplificación sísmica consiste en la cuantificación de la capacidad de amplificación de cada clase de emplazamiento de la clasificación de síntesis. Obtenidos los valores de la capacidad de amplificación de las distintas clases de emplazamiento, se puede asignar a cada unidad geológica el valor de la amplificación de la clase en la cual se ha agrupado previamente.

Borcherdt (1994) propone en su metodología de trabajo la determinación de la capacidad de amplificación sísmica de cada clase de emplazamiento por medio de dos factores de amplificación, que dependen de la frecuencia del movimiento del terreno debido a un terremoto. En este sentido, se calcula un factor de amplificación $\left(F_{a}\right)$ para altas frecuencias $(2-10 \mathrm{~Hz})$ o periodos cortos $(0,1-0,5 \mathrm{~s})$, y otro factor $\left(F_{v}\right)$ para bajas frecuencias $(0,5-2,5 \mathrm{~Hz})$ o periodos medios $(0,4-2,0 \mathrm{~s})$.

Antes de calcular ambos factores de amplificación, es preciso determinar dos parámetros: el valor de Vs30 para una condición del terreno de referencia y un nivel espectral inicial del movimiento del terreno (I) (Borcherdt, 1994). Como condición del terreno de referencia se selecciona la clase de emplazamiento SC-Ib o la combinación de las clases SC-II y SC-III (SC-(II+III)), pertenecientes a la clasificación de Borcherdt (1994), con valores de Vs30 iguales a $1050 \mathrm{~m} / \mathrm{s}$ y $450 \mathrm{~m} / \mathrm{s}$, respectivamente (Tabla 2.1). El nivel espectral inicial del movimiento del terreno está relacionado con 
la aceleración máxima del terreno que puede experimentar el emplazamiento en caso de que ocurra un terremoto. A este respecto, Borcherdt (1994) realiza los cálculos de $F_{a}$ y $F_{v}$ para determinados valores de $I$, comprendidos entre 0,1 y $0,5 \mathrm{~g}$, siendo $\mathrm{g}=$ $9,8 \mathrm{~m} / \mathrm{s}^{2}$. Estos valores se muestran en la Tabla 2.2 (Tabla 2 en Borcherdt (1994)). Asimismo, se presentan los valores de $F_{a}$ y $F_{v}$ obtenidos por Borcherdt (1994) para cada $I$ con respecto a las condiciones del terreno de referencia $\mathrm{SC}-\mathrm{Ib}$ y $\mathrm{SC}-(\mathrm{II}+\mathrm{III})$.

Los factores de amplificación $F_{a}$ y $F_{v}$ de cada clase de emplazamiento se calculan a partir de un valor medio del intervalo de valores de Vs30 de la clase, de la Vs30 de la condición del terreno de referencia y del valor de $I$, por medio de las ecuaciones 7a y 7 b de Borcherdt (1994):

$$
\begin{aligned}
& F_{a}=\left(v_{0} / v\right)^{m_{a}} \\
& F_{v}=\left(v_{0} / v\right)^{m_{v}}
\end{aligned}
$$

donde $v$ es el valor medio del intervalo de valores de Vs30 de la clase de emplazamiento, $v_{0}$ es el valor de la Vs30 de la condición del terreno de referencia, y $m_{a}$ y $m_{v}$ son dos parámetros que se definen en función de los factores de amplificación para periodos cortos y periodos medios correspondientes a la clase de emplazamiento SCIV (Tabla 2.1), y que se expresan de acuerdo a las ecuaciones 2c y $2 \mathrm{~b}$ de Borcherdt (1994):

$$
\begin{aligned}
& m_{a}=\log \left[F_{a}\left(v_{S C-I V}, I\right)\right] / \log \left[v_{0} / v_{S C-I V}\right] \\
& m_{v}=\log \left[F_{v}\left(v_{S C-I V}, I\right)\right] / \log \left[v_{0} / v_{S C-I V}\right]
\end{aligned}
$$

Para calcular $m_{a}$ y $m_{v}$ es preciso conocer:

- $v_{S C-I V}$ : Valor medio del intervalo de valores de Vs30 para la clase SC-IV

- $F_{a}\left(v_{S C-I V}, I\right):$ Factor de amplificación para periodos cortos para la clase SCIV, tabulado en función de I y de la condición del terreno de referencia en la Tabla 2.2

- $F_{v}\left(v_{S C-I V}, I\right)$ : Factor de amplificación para periodos medios para la clase SCIV, tabulado en función de I y de la condición del terreno de referencia en la Tabla 2.2

La Tabla 2.2 también permite obtener los valores de $m_{a}$ y $m_{v}$ de entre determinados valores tabulados, que se seleccionan en función del valor de $I$ y de la condición del terreno de referencia (SC-Ib o SC-(II+III)).

Siguiendo esta metodología y partiendo de las clases de emplazamiento que constituyen la clasificación de síntesis, la cuantificación de la capacidad de amplificación de cada clase se realiza por medio del cálculo de los correspondientes factores de amplificación $F_{a}$ y $F_{v}$. Para ello, será preciso escoger una de las dos condiciones del terreno de referencia propuestas por Borcherdt (1994) y determinar así el parámetro $v_{0}$, seleccionar un valor para el nivel espectral inicial del movimiento del terreno $(I)$ y calcular el valor medio del intervalo de Vs30 que caracteriza a la clase de emplazamiento $(v)$. Con la condición del terreno de referencia y el valor de $I$ se seleccionan los valores de $m_{a}$ y $m_{v}$ a partir de la Tabla 2.2. A continuación, se pueden calcular los factores de amplificación $F_{a}$ y $F_{v}$ utilizando las ecuaciones 2.1 y 2.2 y los valores de $v_{0}, v, m_{a}$ y $m_{v}$. 


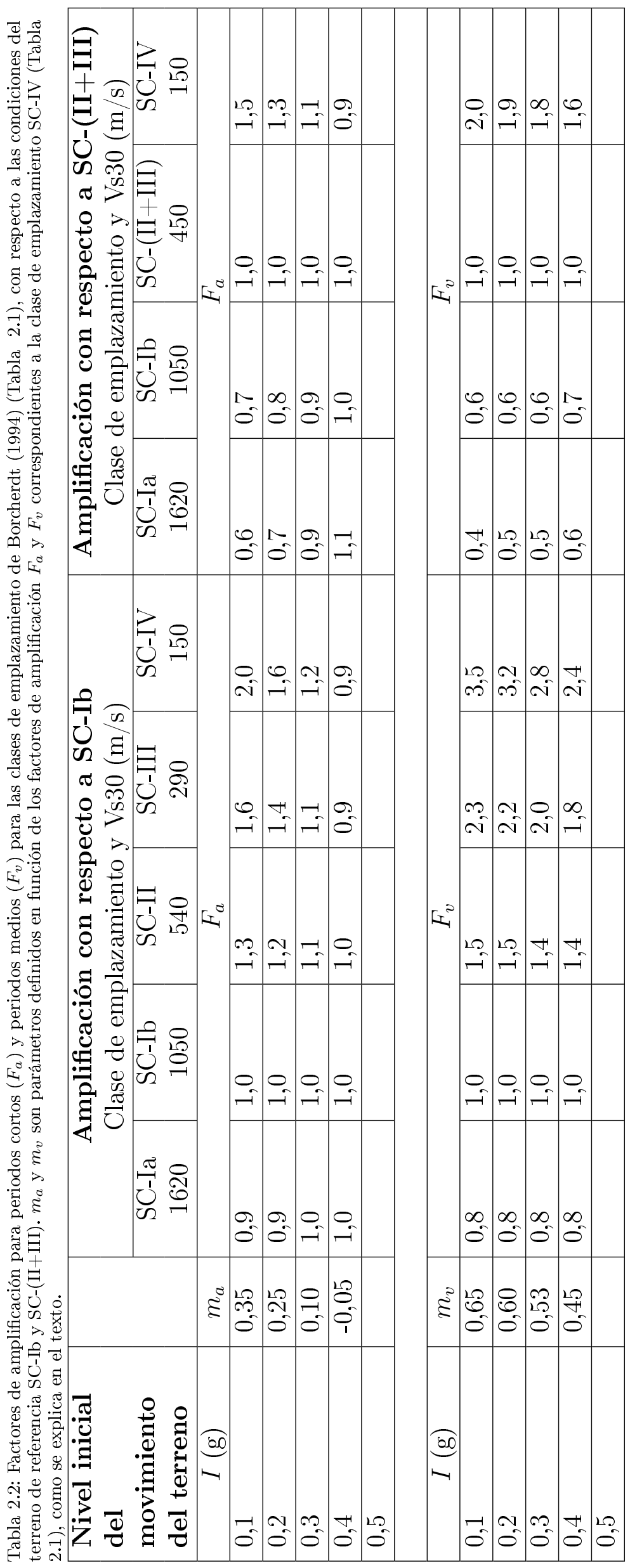


Finalmente, el mapa de amplificación se obtiene por medio de la asignación de los factores de amplificación de cada clase de emplazamiento a todas las unidades geológicas que pertenecen a la citada clase. Dado que se conoce la distribución espacial de las unidades a partir de la cartografía geológica, este proceso permite generar el mapa de amplificación buscado, el cual cuantifica en cada punto el valor de $F_{a}$ y el valor de $F_{v}$.

\subsubsection{Estudios que aplican la metodología de Borcherdt (1994) para obtener cartografía de amplificación sísmica}

La metodología de Borcherdt (1994) ha sido utilizada en los últimos años en diversos estudios, con objeto de calcular la capacidad de amplificación sísmica en una determinada región y, de esta forma, poder obtener el correspondiente mapa de amplificación sísmica.

Wald et al. (1999b) incorporan los factores de amplificación en el proceso de generación de mapas de parámetros máximos del movimiento del terreno (ShakeMaps) en el sur de California. Respecto a estos parámetros, los ShakeMaps representan la distribución espacial de la aceleración máxima del terreno (Peak Ground Acceleration, PGA), la velocidad máxima del terreno (Peak Ground Velocity, PGV) y la amplitud máxima del espectro de respuesta de aceleración para periodos de 0,3 s, 1 s y $3 \mathrm{~s}$.

El proceso de producción de los ShakeMaps determina los parámetros del movimiento del terreno en el territorio en estudio, haciendo uso de observaciones de aceleración, velocidad y aceleración espectral obtenidas en las estaciones de registro pertenecientes a tres instituciones encuadradas en el proyecto TriNet (Wald et al., 1999b): California Institute of Technology (Caltech), California Division of Mines and Geology (CDMG) y United States Geological Survey (USGS). Por otra parte, también se utilizan determinadas relaciones de atenuación válidas en emplazamientos en roca. Por medio de estas leyes de atenuación, en primer lugar se calculan los valores sintéticos de los parámetros pico en roca, en los puntos equiespaciados de una malla que abarca el territorio.

En segundo lugar, los valores máximos de aceleración, velocidad y aceleración espectral que han sido registrados en las estaciones deben ser corregidos por el tipo de suelo en el que éstas están instaladas. De esta forma, se pretende obtener los correspondientes valores referidos a condiciones de roca. Es en este punto en el que Wald et al. (1999b) recurren a la clasificación de los materiales aflorantes en el territorio, con el fin de asignar al emplazamiento de cada estación una clase de emplazamiento caracterizada por su valor de Vs30. Como se observa en la Figura 2.7, los materiales son agrupados según tres clases de emplazamiento (Quaternary, Tertiary, Mesozoic). De acuerdo a la Tabla 1 en Wald et al. (1999b), estas clases a su vez corresponden a 3 valores de Vs30 $(589 \mathrm{~m} / \mathrm{s}, 406 \mathrm{~m} / \mathrm{s}$ y $333 \mathrm{~m} / \mathrm{s})$.

A continuación, los autores siguen la metodología de Borcherdt (1994) para obtener los factores de amplificación $F_{a}$ y $F_{v}$ de cada clase de emplazamiento a partir del valor correspondiente de Vs30. Conocidos estos factores de amplificación, las aceleraciones máximas observadas son corregidas por medio del factor $F_{a}$, mientras que $F_{v}$ es utilizado para calcular las velocidades máximas observadas referidas a roca. 
En el caso de los valores pico de la aceleración espectral, éstos son modificados por $F_{a}$ y $F_{v}$ en función de la frecuencia que les corresponde.

El proceso continúa con la interpolación de los valores sintéticos de los parámetros máximos en roca y de las observaciones, también referidas a roca, sobre los puntos de una malla más densa. Los valores interpolados deben ser corregidos de acuerdo con la clase de emplazamiento correspondiente a cada punto de interpolación (Wald et al., 1999b), para lo cual se utilizan los factores de amplificación de Borcherdt que corresponden a la clase de emplazamiento donde se sitúa el punto.

En España, Tsige y García Flores (2006) obtienen un mapa de la amplificación sísmica en la Región de Murcia a partir de la clasificación de los materiales aflorantes de acuerdo a su capacidad de amplificación, agrupándolos en clases de emplazamiento definidas por su correspondiente valor de Vs30 (Figura 2.8). Siguiendo la metodología de Borcherdt (1994), los autores calculan para cada clase los factores de amplificación $F_{a}$ y $F_{v}$, con el fin de obtener su distribución espacial en el territorio de la Región de Murcia y cuantificar de esta forma la amplificación en cada punto. El mapa de amplificación sísmica obtenido para la Región de Murcia por Tsige y García Flores (2006) es uno de los resultados del proyecto RISMUR, llevado a cabo por el Instituto Geográfico Nacional (IGN) y la Dirección General de Protección Civil en la Región de Murcia con el objetivo de evaluar el riesgo sísmico en la región y asesorar a las autoridades competentes en Protección Civil.

Dada la falta de cartografía de amplificación sísmica referida a la totalidad de la región de la Península Ibérica, Núñez et al. (2013) presentan el mapa de amplificación sísmica de la Península y las Islas Baleares que constituye uno de los resultados de esta tesis doctoral. Como parte del proceso de producción del mapa, se ha establecido una clasificación de síntesis que incorpora los criterios utilizados por las clasificaciones de Borcherdt (1994) y del Uniform Building Code de 1997 (International Council of Building Officials, 1997), así como los criterios de las clasificaciones incluidas en las normativas de construcción sismorresistente del NEHRP de 1997 y 2003 (Building Seismic Safety Council, 1997, 2004). La clasificación de síntesis ha permitido agrupar posteriormente las 134 unidades geológicas del Mapa Geológico de España a escala 1:1.000.000 (Instituto Geológico y Minero de España, 1994) en 6 clases de emplazamiento que se caracterizan por determinados intervalos de valores de Vs30. El proceso de clasificación se ha desarrollado de acuerdo a la metodología de Borcherdt (1994). Se ha utilizado la descripción litológica y edad cronoestratigráfica de cada unidad para tratar de estimar su resistencia, compacidad y consistencia, las cuales han sido comparadas con las propiedades geotécnicas de cada clase. De esta forma, se ha asignado a cada unidad la clase de emplazamiento que presenta propiedades geotécnicas coherentes con las propiedades geotécnicas de la unidad.

En el caso de cada clase de emplazamiento, Núñez et al. (2013) han calculado los factores de amplificación $F_{a}$ y $F_{v}$ de acuerdo a la metodología de Borcherdt (1994), tomando como condición del terreno de referencia la clase SC-Ib y como nivel espectral inicial del movimiento del terreno el valor $I=0,1 \mathrm{~g}$. A continuación, se han asignado los factores de amplificación de cada clase de emplazamiento a las unidades agrupadas en la clase, y se ha cuantificado por tanto la amplificación de cada unidad. La representación de la distribución espacial de las unidades ha permitido, finalmente, obtener el mapa de amplificación sísmica.

La aplicabilidad y validez del mapa ha sido comprobada mediantes la incor- 


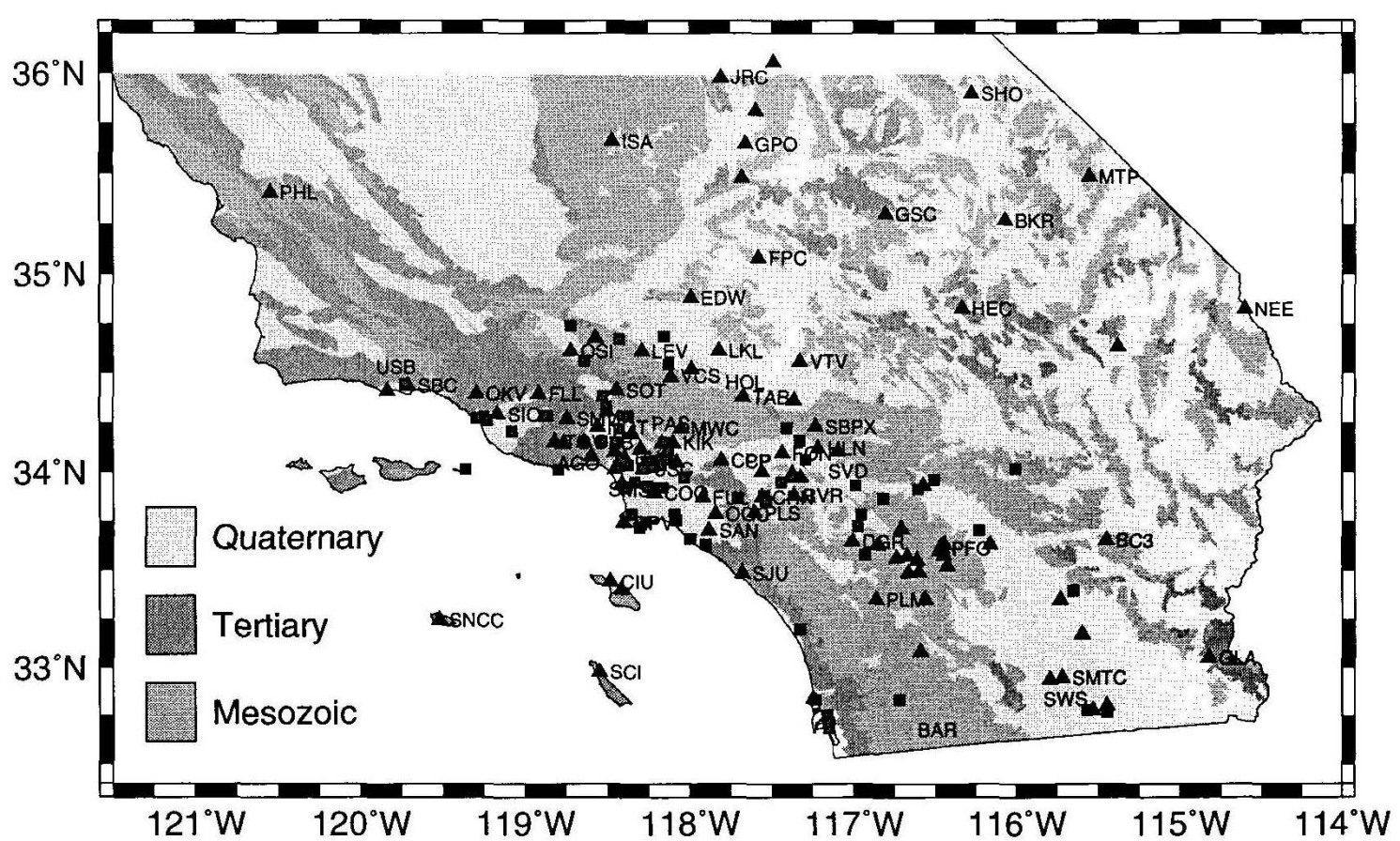

Figura 2.7: Estaciones de la red del proyecto TriNet y clases de emplazamiento (Quaternary, Tertiary, Mesozoic) en el sur de California. Los cuadrados representan las estaciones pertenecientes al CDMG y los triángulos las estaciones del USGS-Caltech. Tomado de Wald et al. (1999b).

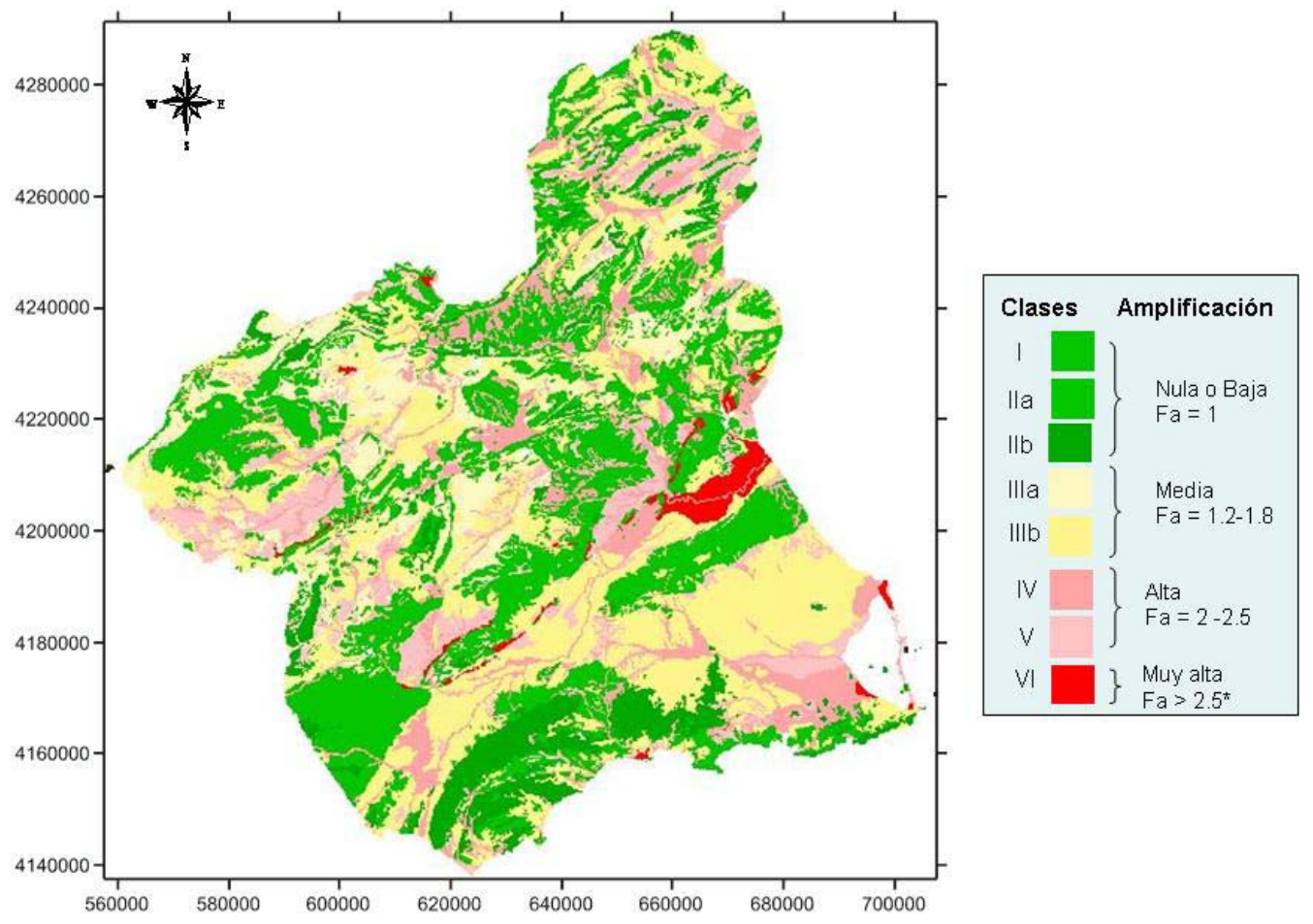

Figura 2.8: Mapa de amplificación sísmica de la Región de Murcia a escala 1:200.000. Tomado de Tsige y García Flores (2006). 
poración de los factores de amplificación al cálculo de las isosistas sintéticas de determinados terremotos históricos, cuyas intensidades máximas observadas están comprendidas entre VIII y X en la escala EMS-98 (European Macroseismic Scale 1998) (Grüntal y Levret, 2001). Los autores han comparado las isosistas sintéticas de cada terremoto con las correspondientes isosistas observadas, concluyendo que los factores de amplificación proporcionados por el mapa permiten obtener isosistas razonablemente similares a las observadas, siempre que la intensidades sean menores que VIII y, por tanto, permanezcan dentro del dominio lineal.

Núñez et al. (2014a) utilizan la clasificación de síntesis publicada por Núñez et al. (2013) para agrupar 759 unidades geológicas de las Islas Canarias de acuerdo con las 6 clases de emplazamiento, siguiendo la metodología de Borcherdt (1994). Estas unidades han sido analizadas a partir de la cartografía geológica continua GEODE de las Islas Canarias, a escala 1:50.000 (GEODE, 2014). Los factores de amplificación $F_{a}$ y $F_{v}$ de cada clase de emplazamiento coinciden con los obtenidos por Núñez et al. (2013), y son asignados a las unidades pertenecientes a cada categoría. De esta forma, se ha generado el mapa de amplificación sísmica de las Islas Canarias que se presenta en esta tesis doctoral como uno de sus resultados.

Respecto a la isla de El Hierro, y ante las recientes crisis sísmicas acaecidas en 2011 y 2012, Núñez et al. (2014b) han llevado a cabo un estudio con el fin de simular las aceleraciones máximas del terreno ante distintos escenarios sísmicos que pudieran tener lugar en la isla, considerando la capacidad de amplificación sísmica de los materiales aflorantes. Con este fin, se ha obtenido un mapa de amplificación sísmica de la isla de El Hierro, para lo cual se han clasificado las unidades geológicas de la isla, proporcionadas por la cartografía GEODE, en función de las clases de emplazamiento de Núñez et al. (2013), siguiendo la misma metodología de trabajo que Borcherdt (1994). A continuación, se han asignado los factores de amplificación de cada clase de emplazamiento, también obtenidos por Núñez et al. (2013), a las unidades agrupadas en cada clase, generando de esta forma el mapa de amplificación de El Hierro.

\subsection{Cálculo de la distribución de la velocidad de onda de corte $(\mathrm{Vs})$ en los primeros $30 \mathrm{~m}$ de profundidad por medio del método ReMi}

Con objeto de calcular la amplificación sísmica y el periodo fundamental de un emplazamiento con una exactitud mayor que la proporcionada por las metodologías de microzonación que emplean mapas de amplificación sísmica, se requiere la utilización de metodologías de microzonación que buscan estimar de forma directa los parámetros mecánicos de los materiales por medio de medidas de campo. Estas metodologías constituyen una segunda aproximación a la estimación de la amplificación sísmica y el periodo fundamental, así como a la estimación de su distribución espacial. En este sentido, permiten obtener esta distribución a escalas mayores que las permitidas por los mapas de amplificación sísmica basados únicamente en criterios geológicos o topográficos.

La distribución de la velocidad de onda de corte (Vs) en los primeros $30 \mathrm{~m}$ 
de profundidad es uno de los parámetros mecánicos de los materiales geológicos que permiten la obtención de la amplificación sísmica de los citados materiales. Esta estructura o modelo de Vs, hasta los $30 \mathrm{~m}$ de profundidad, puede estimarse directamente en un emplazamiento, por medio de mediciones in situ. A este respecto, existen distintos métodos de prospección que proporcionan la estructura de Vs en profundidad. En esta tesis doctoral se ha recurrido al método de la Sísmica Pasiva Refraction Microtremor (ReMi), que emplea medidas de ruido sísmico ambiental (Louie, 2001), con el fin de poder obtener de una forma relativamente rápida los modelos de Vs en los primeros $30 \mathrm{~m}$ de profundidad para un número suficiente de emplazamientos situados en una región, con el fin de estimar en estos emplazamientos el valor medio de Vs en los primeros $30 \mathrm{~m}$ de profundidad (Vs30), la amplificación sísmica y periodo fundamental. Por otra parte, la estimación de estos resultados por cada emplazamiento hace posible la regionalización de los mismos a una escala regional o local.

La elección del método ReMi se fundamenta en su condición de metodología no invasiva, al requerir medidas realizadas en superficie, en contraposición a las técnicas de sondeos y de toma de muestras en profundidad para su análisis en laboratorio, que requieren la perforación del terreno. Por este motivo, ReMi aporta una gran flexibilidad para tomar medidas de ruido ambiental en emplazamientos urbanos, de una forma rápida y económica. Además, no comporta la necesidad de utilizar una fuente artificial. El método ReMi permite realizar del orden de 6-10 mediciones en una jornada de trabajo de 8 horas efectivas, en función de las circunstancias logísticas y de la localización de emplazamientos con las condiciones adecuadas de pendiente topográfica y superficie uniforme y plana. No obstante, se constata la existencia de otras metodologías que también permiten obtener modelos de Vs en profundidad de una forma no invasiva y en base a medidas superficiales, con similares ventajas a las que ofrece ReMi. En este sentido, se citan los métodos SASW (Spectral Analysis of Surface Waves) Nazarian y Stokoe II (1984) y MASW (Multichannel Analysis of Surface Waves) (Park et al., 1999).

En esta tesis doctoral, la toma de datos ReMi está orientada a la obtención de modelos de Vs en los primeros $30 \mathrm{~m}$ profundidad en emplazamientos seleccionados en la isla de El Hierro, que permitan calcular, en cada uno, una curva de amplificación sísmica en función de la frecuencia. Por medio de esta curva de amplificación espectral, se pretende estimar la amplificación sísmica del material geológico de las capas más superficiales, con una exactitud mayor que la proporcionada únicamente por la cartografía de amplificación sísmica de las Islas Canarias, a obtener de acuerdo a la metodología de Borcherdt (1994) (sección 2.1).

Simultáneamiente, se pretende utilizar la curva de amplificación sísmica espectral para llevar a cabo una segunda aproximación a la determinación de valores sintéticos realistas de la aceleración y la intensidad sísmicas en la región donde se sitúan los emplazamientos (El Hierro), por medio de algoritmos de simulación estocástica de acelerogramas sintéticos (capítulo 10). Para poder emplear estos algoritmos, es preciso calibrarlos previamente, a partir de los registros de aceleración disponibles en la región (capítulo 8). Por este motivo, se busca que los emplazamientos de medida ReMi se sitúen en las proximidades de estaciones acelerométricas, con el fin de disponer de un número suficiente de registros de aceleración que haga posible la calibración del algoritmo en la región. 
Además, es de interés que los emplazamientos de medida ReMi presenten una capacidad de amplificación variable, en base a la primera cartografía de amplificación sísmica (2.1). En este sentido, se persigue la comparación de los nuevos valores de amplificación sísmica obtenidos en los emplazamientos a partir de las medidas ReMi con respecto a los valores proporcionados por el mapa de amplificación sísmica.

\subsubsection{Antecedentes del método ReMi: SASW, MASW}

Como antecedentes del método ReMi pueden relacionarse diversas metodologías no invasivas (surface geophysical methods), que permiten caracterizar la distribución en profundidad de la velocidad Vs a partir de medidas tomadas en superficie. Estas metodologías representan un avance significativo en el estudio de este parámetro, en contraposición a las metodologías tradicionales de muestreo directo, como los sondeos o el análisis de muestras de suelo y roca en laboratorio.

De acuerdo con Louie (2001), como antecedentes del método ReMi pueden citarse los métodos de prospección SASW y MASW, que también proporcionan, a partir de mediciones de campo, la estructura de Vs en los primeros $30 \mathrm{~m}$ de profundidad. El método SASW (Spectral Analysis of Surface Waves) utiliza una fuente sísmica artificial para calcular velocidades de fase de ondas superficiales Rayleigh, a partir de las cuales se interpretan los perfiles de Vs (Nazarian y Stokoe II, 1984). En cambio, el método MASW (Multichannel Analysis of Surface Waves) incorpora medidas de ruido sísmico ambiental a las mediciones realizadas mediante la fuente artificial, con el fin de mejorar los resultados obtenidos por el SASW (Park et al., 1999).

Existen también otras técnicas que tratan de utilizar medidas de onda P y onda S para estimar la estructura de Vs, como explica el trabajo de Williams et al. (1994) en Louie (2001). Sin embargo, estas técnicas no han conseguido un éxito similar al de los métodos basados en las estimaciones de las velocidades de ondas superficiales en cuanto a la contrastación de sus resultados con datos de sondeos (borehole) o de movimientos del terreno (Louie, 2001).

Descrito por Nazarian y Stokoe II (1984) por primera vez, el método SASW utiliza una fuente artificial de energía sísmica, la cual es registrada varias veces por dos sismómetros de frecuencia $1 \mathrm{~Hz}$ que se encuentran situados a distancias de 1 a 500 m (Nazarian y Desai, 1993). De acuerdo con Louie (2001), a partir de los registros de estos sismómetros, las velocidades de fase de las ondas Rayleigh son interpretadas por medio de la comparación de los espectros de amplitud y fase diferencial que han sido obtenidos para cada activación de la fuente. Puesto que la interpretación se realiza en el dominio de la frecuencia, el método SASW supone que las llegadas más energéticas corresponden a ondas Rayleigh. Por este motivo, este método no permite diferenciar adecuadamente las velocidades de las ondas Rayleigh de las debidas a otros tipos de ondas en determinados emplazamientos, en los que la amplitud del ruido supera a la amplitud de la señal procedente de la fuente artificial (ciudades), o bien en los emplazamientos donde las fases de las ondas de cuerpo son más energéticas que las ondas Rayleigh.

El método MASW ha sido desarrollado a partir del trabajo de Park et al. (1999), ante las limitaciones del SASW en presencia de ruido. Como indica Louie (2001), el registro simultáneo de 12 o más sensores situados en distancias de 1-2 m a 50-100 
$m$ con respecto a una fuente impulsiva o vibratoria da redundancia estadística a las medidas de velocidades de fase. La representación de los datos de todos los canales con respecto a la frecuencia, variable con el tiempo, también permite la identificación y desestimación de las ondas Rayleigh de los modos no fundamentales y de los ruidos coherentes.

De acuerdo con Louie (2001), el método ReMi combina la simplicidad operacional del método SASW con la resolución para profundidades someras que ofrece el MASW. Al igual que el método MASW, ReMi separa las ondas Rayleigh de las ondas de cuerpo, ondas aéreas y otros ruidos coherentes a partir de la conservación de los sismogramas originales y de la aplicación de un análisis de velocidad en el dominio del tiempo.

A nivel de utilización de los métodos presentados, Stephenson et al. (2005) obtienen modelos de Vs por medio de los métodos ReMi y MASW en California, que son comparados con los modelos obtenidos a partir de sondeos de hasta $260 \mathrm{~m}$ de profundidad. Los autores generan modelos con las dos técnicas no invasivas, para las profundidades 30,50 y $100 \mathrm{~m}$, constatando su coincidencia con los resultados de los sondeos al menos hasta el $15 \%$ de estas profundidades, y concluyen que ReMi y MASW pueden ser complementarios para llevar a cabo la microzonación sísmica de entornos urbanos.

\subsubsection{Fundamentos teóricos del método ReMi}

El método Refraction Microtremor (ReMi) permite obtener la estructura de Vs hasta $100 \mathrm{~m}$ de profundidad a partir del análisis espectral de las ondas superficiales Rayleigh, contenidas en el ruido sísmico ambiental producido por el tráfico y otras actividades comunes en medios urbanos (Louie, 2001).

El conocimiento de la estructura de Vs en profundidad por medio del análisis espectral de las ondas Rayleigh se fundamenta en el hecho de que la velocidad de propagación de estas ondas, concretamente la velocidad de fase $\left(C_{R}\right)$, es función de la distribución de Vs en profundidad. Debido a que las ondas Rayleigh son dispersivas, $C_{R}$ cambia con la longitud de onda $(\lambda)$, o con la frecuencia $(f)$, cuando existen cambios verticales en la distribución de Vs en profundidad (Figura 2.9).

La profundidad afectada por las ondas Rayleigh es proporcional a $\lambda$ e inversamente proporcional a $f$. En consecuencia, cuanto mayor es $\lambda$ la profundidad alcanzada también es mayor, mientras que ondas Rayleigh con baja $\lambda$ sólo se transmiten en estratos más superficiales.

La representación de la dependencia de la velocidad de fase $C_{R}$ con la frecuencia $f$ constituye la curva de dispersión de la onda Rayleigh (Figura 2.10). La determinación de esta curva, de forma experimental e in situ, así como su posterior modelización por medio de un modelo inicial de velocidad Vs para el emplazamiento, constituye el fundamento principal del método ReMi. Este modelo inicial es modificado sucesivamente con el fin de obtener una curva de dispersión sintética que modelice razonablemente bien la curva experimental, obteniéndose así el modelo final de Vs. Debido a la dispersión de las ondas Rayleigh, el modelo final de Vs obtenido es el modelo de Vs del emplazamiento, el cual puede contener capas de baja velocidad relativa. 


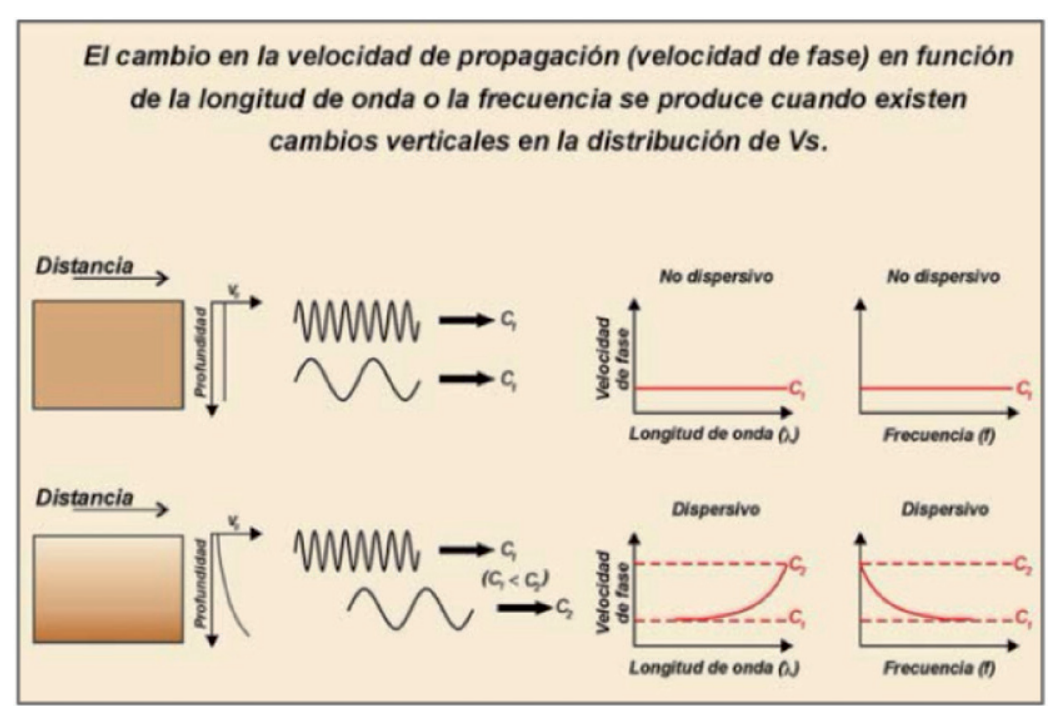

Figura 2.9: Relación entre la velocidad de fase $C_{R}$ de las ondas Rayleigh y los cambios verticales en la distribución de Vs en profundidad. Tomado de Granda et al. (2005).
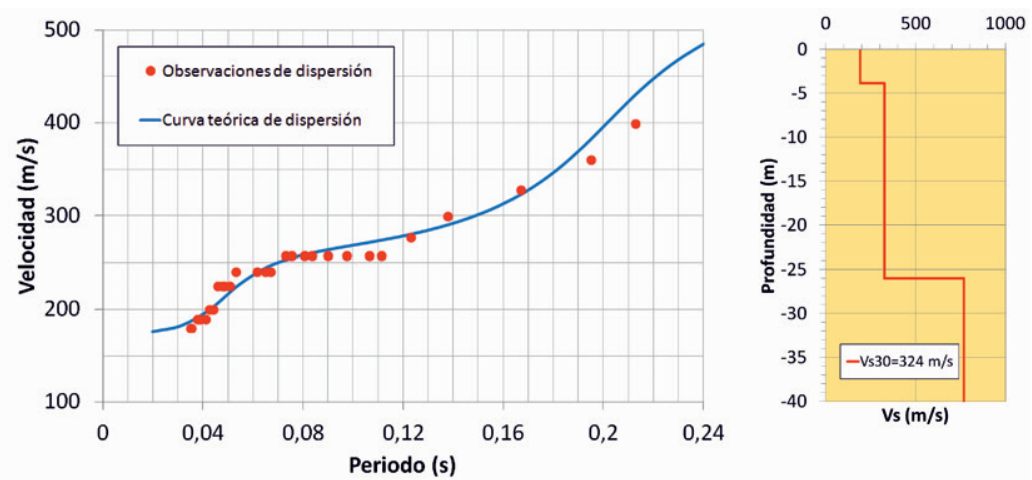

Figura 2.10: Curva de dispersión experimental de la onda Rayleigh y curva teórica (izquierda), la cual ha sido generada a partir del modelo de Vs local (derecha). 


\subsubsection{Adquisición de medidas ReMi}

La adquisición de medidas de ruido sísmico ambiental, por medio del método ReMi, puede realizarse con un equipo de sísmica de refracción, empleando un sismógrafo multicanal con conversor analógico-digital de alta resolución y geófonos de alta frecuencia. Respecto a la configuración del dispositivo de medida, la más utilizada es una configuración lineal con sensores equiespaciados, la cual permite realizar las medidas adecuadamente en ambientes urbanos. No obstante, Granda et al. (2005) indica que los geófonos pueden disponerse también según configuraciones en L, triangulares o circulares. Como demuestran estudios recientes como los de Hayashi (2005), las diferencias entre los resultados conseguidos con las distintas configuraciones no son relevantes.

Aunque el método ReMi prevee únicamente el registro de ruido sísmico ambiental, resulta conveniente también generar artificialmente ondas Rayleigh en un extremo del dispositivo (Rueda y Mezcua, 2013), mediante el golpeo fuerte del suelo con una maza que impacte sobre una placa metálica, o mediante la circulación de un vehículo en las proximidades del dispositivo.

La profundidad de investigación depende tanto de la longitud del dispositivo de medida como del rango de frecuencias registrado. A este respecto, se pueden muestrear profundidades de hasta $100 \mathrm{~m}$ con líneas de geófonos de $8 \mathrm{~Hz}$ y con una longitud de $200 \mathrm{~m}$ Louie (2001). Granda et al. (2005) añaden que la separación entre los geófonos y su frecuencia natural condicionan la capacidad de resolución del método para muestrear adecuadamente los niveles superficiales.

Para asegurar el registro de todas las frecuencias contenidas en las ondas de corte, el intervalo de muestreo debe ser al menos de $2 \mathrm{~ms}$ en ventanas de $30 \mathrm{~s}$ (Rueda y Mezcua, 2013). Este muestreo recomienda la aplicación de un filtro paso-alta de al menos $250 \mathrm{~Hz}$ para eliminar el efecto aliasing, el cual puede conllevar confusiones en la identificación de la curva de dispersión de las ondas Rayleigh, y, en su caso, de un filtro paso-baja siempre menor que $4 \mathrm{~Hz}$.

De acuerdo con Rueda y Mezcua (2013), una configuración de dispositivo adecuada para alcanzar con seguridad profundidades que permitan calcular la Vs30 (40 m) consiste en varias líneas de 24 geófonos de 4,5 Hz, que se emplazan en el terreno con una separación constante de $3 \mathrm{~m}$, resultando en una longitud del dispositivo de $69 \mathrm{~m}$. Granda et al. (2005) señalan el problema de conseguir un buen acoplamiento de los geófonos sobre el terreno, indicando que, en zonas pavimentadas, resulta práctico instalar los geófonos sobre placas metálicas que puedan ser arrastradas fácilmente sobre el suelo. Rueda y Mezcua (2013) coinciden también en esta solución, recordando la instalación sobre pica metálica si el terreno permite la hinca del geófono.

\subsubsection{Análisis espectral para la construcción del diagrama de la transformada lentitud (p)-frecuencia (f)}

Los datos adquiridos en campo se deben procesar en tres fases bien diferenciadas (Figura 2.11): 


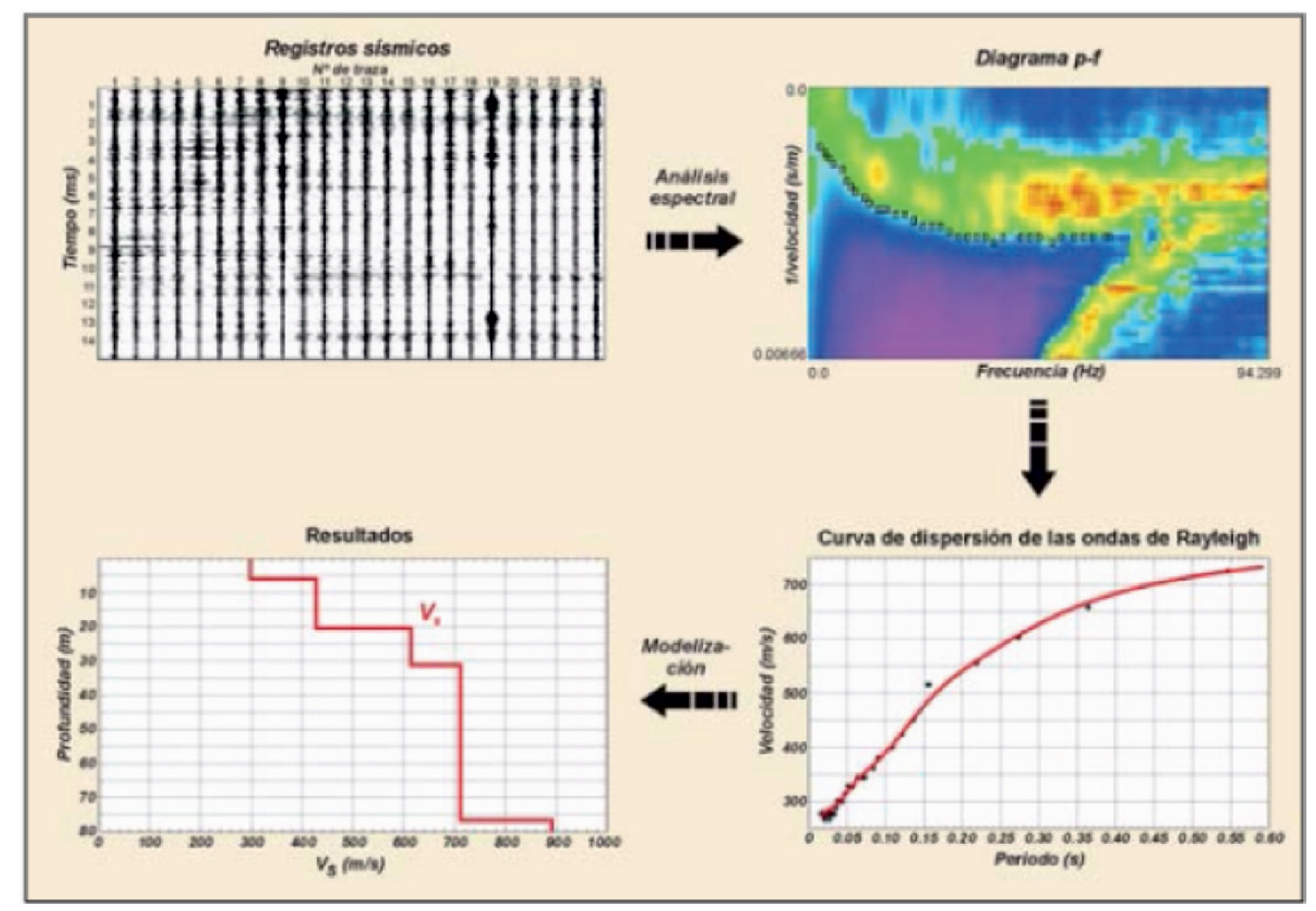

Figura 2.11: Procesado completo de las medidas ReMi: Diagrama de la transformada lentitud-frecuencia $(p-f)$, curva de dispersión experimental de las ondas Rayleigh y modelización por medio un modelo de velocidad Vs. Tomado de Granda et al. (2005).

- Obtención del diagrama de la transformada $p-f$ de la inversa de la velocidad de fase o lentitud $(p)$ en función de la frecuencia $(f)$ en cada registro

- Suma de diagramas de la transformada $p-f$ de los registros y obtención de la curva de dispersión de las ondas Rayleigh para el emplazamiento

- Modelización de la curva de dispersión de las ondas Rayleigh y obtención del modelo final de Vs para el emplazamiento

Primeramente, se realiza un análisis espectral de cada ventana de registro, para obtener el diagrama de la transformada $p-f$ de la inversa de la velocidad de fase o lentitud o parámetro del rayo $(p)$ en función de la frecuencia $f$, que permite separar las ondas Rayleigh de otras llegadas sísmicas. Este análisis requiere la aplicación de la transformada lentitud-tiempo de interceptación $(p-\tau)$, o slant-stack, sobre el registro (Thorson y Claerbout, 1985). Esta transformada considera una sección de registro $\mathrm{A}(\mathrm{x}, \mathrm{t})$ de múltiples sismogramas y los convierte a amplitudes relativas del parámetro del rayo y del tiempo de interceptación. De acuerdo con Louie (2001), la transformación es:

$$
A(p, \tau)=\int A(x, t) d x=\int A(x, \tau+p x) d x
$$

Siguiendo a Rueda y Mezcua (2013), la variable $x=j d x$ se discretiza en $n x$ intervalos, siendo $j$ entero y $d x$ la distancia entre geófonos. El tiempo $t=i d t$ se 
discretiza de forma similar, siendo $d t$ el intervalo de muestreo. Entonces, se obtiene la transformada $p-\tau$ en su forma discreta, con $p=p_{0}+l d p$, y $\tau=k d t$ :

$$
\begin{aligned}
A(p, \tau)= & \sum A(x, t)=\sum A\left(p_{0}+l d p, k d t\right) \\
& =\sum_{j=0}^{n x-1} A(j d x, i d t) \\
& =\sum_{j=0}^{n x-1} A(j d x, \tau+p x)
\end{aligned}
$$

Para pasar al dominio lentitud-frecuencia $(p-f)$, se aplica la transformada de Fourier en el eje $\tau$ :

$$
F_{A}(p, f)=\int A(p, \tau) e^{-i 2 \pi f \tau} d \tau
$$

De acuerdo con Louie (2001), el análisis espectral de la lentitud se hace utilizando la función de densidad espectral cruzada o espectro de potencia cruzada $S_{A}(p, f)$, que es igual a la magnitud al cuadrado de la transformada compleja de Fourier:

$$
S_{A}(p, f)=F_{A}(p, f) \cdot \overline{F_{A}(p, f)}
$$

siendo $\overline{F_{A}(p, f)}$ la conjugada compleja.

La suma de las dos transformadas $(p, f)$ de cada traza de registro, en dos direcciones hacia delante y hacia atrás, permite representar el registro con un solo eje de lentitud:

$$
S_{A}(|p|, f)=\left[S_{A}(p, f)\right]_{p \geq 0}+\left[S_{A}(-p, f)\right]_{p<0}
$$

\subsubsection{Construcción de la curva de dispersión de las ondas Rayleigh}

Para construir la curva de dispersión correspondiente a cada emplazamiento de medida, debe realizarse primeramente la suma, pixel a pixel, de los diagramas de las transformadas $p-f$, para todas las ventanas de registro en las cuales se han llevado a cabo las mediciones de ruido sísmico ambiental, con el fin de generar el diagrama $p-f$ correspondiente al emplazamiento. Teniendo en cuenta que las ondas dispersivas corresponden a los máximos de energía, la curva de dispersión correspondiente a las ondas Rayleigh vendrá representada por la envolvente de menor velocidad aparente de fase (Louie, 2001) ("Diagrama $p$ - f" en Figura 2.11). No es recomendable seleccionar la velocidad de fase de mayor energía, pues en ese caso se podrían obtener velocidades aparentes mayores que la real, pues las ondas que llegan de forma oblicua a los geófonos tienen una velocidad aparente mayor que las que se propagan a lo largo de la misma. 
Por tanto, la construcción de la curva de dispersión de las ondas Rayleigh se realizará seleccionando manualmente las menores velocidades de fase que marquen la tendencia del máximo de energía en función de la frecuencia ("Curva de dispersión de las ondas de Rayleigh" en Figura 2.11).

\subsubsection{Modelización de la curva de dispersión y obtención del modelo de Vs en los primeros $30 \mathrm{~m}$ de profundidad}

Por último, se procede a modelizar la curva de dispersión de las ondas Rayleigh obtenida experimentalmente, partiendo de un modelo inicial de velocidad Vs que abarque, como mínimo, los primeros $30 \mathrm{~m}$ de profundidad y que se establece en base a la información geológica y sismológica de la zona. Por medio de iteraciones sucesivas, se calcula la velocidad de fase para cada frecuencia (Louie, 2001), a fin de compararla con la velocidad de fase observada. De esta forma, en cada iteración se obtiene una curva de dispersión teórica, que depende del modelo de Vs empleado, que se compara con la curva de dispersión experimental. Se llevan a cabo varias iteraciones de este proceso hasta que la curva teórica ajusta la curva experimental con un error cuadrático medio (root-mean-square, RMS) aceptable. El modelo final de Vs que permite obtener este error RMS se considera como la estructura de Vs del emplazamiento ("Resultados" en Figura 2.11), y puede emplearse para calcular el valor de Vs30 correspondiente.

Es posible introducir inversiones de velocidad en el modelo de Vs, consistentes en capas de menor velocidad que las capas suprayacentes. Estas capas de baja velocidad pueden estar relacionadas con rupturas en la pendiente de la curva de dispersión experimental, y que se corresponden con cambios en la tendencia de la envolvente del máximo de energía de la transformada $p-f$ del emplazamiento. En todo caso, la introducción de una capa de baja velocidad debe estar avalada por las observaciones de la secuencia estratigráfica local, tomadas en campo, y además producir una reducción significativa del error RMS del ajuste de la curva teórica a los puntos de dispersión experimentales.

\subsection{Determinación de la amplificación sísmica en función de la frecuencia a partir del modelo de Vs en los primeros $30 \mathrm{~m}$ de profundidad}

En este trabajo se ha utilizado la estructura de Vs en los primeros $30 \mathrm{~m}$ de profundidad en un emplazamiento, obtenida experimentalmente a partir de medidas de ruido sísmico ambiental y por medio del método ReMi, con el fin de estimar en el citado emplazamiento la curva de amplificación sísmica en función de la frecuencia de la señal. Algunos autores han cuestionado que una distribución o estructura de Vs en profundidad sea suficiente para cuantificar la amplificación sísmica en un emplazamiento (Wald y Mori, 2000; Mucciarelli y Gallipolli, 2006; Castellaro et al., 2008). No obstante, existe una gran cantidad de trabajos que, partiendo del modelo local de Vs en profundidad, calculan la amplificación sísmica, como los de Stephenson et al. (2005), Odum et al. (2010) y Odum et al. (2013), entre otros. 


\subsubsection{Cálculo de la amplificación teórica de una onda SH que se propaga en una estructura de capas con velocidad constante}

Considerando la propagación de una onda SH (onda plana) en una estructura de capas de velocidad constante, Joyner et al. (1981) utilizan la raíz cuadrada de la impedancia sísmica efectiva para calcular la amplificación teórica de la onda plana en función de la frecuencia, conforme atraviesa un estrato de velocidad Vs conocida. Esta aproximación es conocida como aproximación del cuarto de longitud de onda (quarter-wavelength approximation). Como se ha referido en la subsección 2.2.1, Stephenson et al. (2005) obtienen resultados satisfactorios con esta aproximación, al utilizarla para comparar los resultados derivados de modelos de velocidad Vs de un mismo emplazamiento, proporcionados por los métodos ReMi y MASW, con los obtenidos a partir de medidas directas de velocidad en sondeos. Adicionalmente, Odum et al. $(2010,2013)$ utilizan la aproximación del cuarto de longitud de onda para obtener las amplificaciones espectrales en distintos emplazamientos a partir de los modelos de Vs obtenidos previamente por ReMi.

La amplificación teórica obtenida por Joyner et al. (1981) constituye una primera aproximación a la amplificación basada en la solución completa de la propagación de la onda plana en la estructura de capas de velocidad constante. Esta solución es obtenida por el método de la matriz de propagación (propagator matrix method), desarrollado por Haskell (1960).

Con respecto a otras aproximaciones a la amplificación sísmica, puede citarse la utilización de la solución continua de la ecuación de ondas (Kanai, 1951; Matthiesen et al., 1964; Roesset y Whitman, 1969; Lysmer et al., 1971) para calcular la amplificación sísmica suponiendo un conjunto de capas con módulo de cizalla constante (Idriss y Sun, 1992). Si se considera el movimiento del terreno como la suma de ondas sinusoidales de diferentes frecuencias, la amplificación del terreno se puede modelizar por medio de una función de transferencia (EduPro Civil Systems, 2012).

\subsubsection{Implementación de la solución completa en algoritmos de cálculo de la amplificación sísmica en función de la frecuencia: NRATTLE}

La determinación de la amplificación sísmica espectral en los emplazamientos con un modelo de Vs en profundidad, obtenido con el método ReMi, se realizará por medio del método de la matriz de propagación de Haskell (1960), con el fin de calcular la solución completa de la propagación de la onda plana $(\mathrm{SH})$ en la estructura de Vs. A este respecto, es necesario que esta estructura presente capas con velocidad constante. El cálculo de esta solución completa se lleva a cabo por medio de su implementación en el algoritmo de cálculo de la amplificación sísmica en función de la frecuencia NRATTLE. Este código, escrito originalmente por C. Mueller, del Servicio Geológico de Estados Unidos (United States Geological Survey, USGS), y modificado posteriormente por R. Herrmann, de la Universidad de San Luis (Saint Louis University), se incluye en el paquete de simulación SMSIM (Boore, 2005). Utilizando este algoritmo, se procede a generar la curva de amplificación sísmica 
espectral en cada emplazamiento, partiendo de la correspondiente estructura de Vs en los primeros $30 \mathrm{~m}$ de profundidad.

\subsubsection{Cálculo del periodo fundamental del suelo a partir de la curva de amplificación sísmica}

A partir de la curva de amplificación sísmica espectral de cada emplazamiento, se calculará el periodo fundamental del suelo, tomando el valor de la frecuencia en la cual la curva de amplificación alcanza su primer máximo, que corresponde con el modo fundamental de vibración, y considerando un rango de frecuencias menores que $10 \mathrm{~Hz}$. Sin embargo, este valor teórico del periodo fundamental necesita ser contrastado con un valor experimental, a fin de comprobar su validez. Por este motivo, en la siguiente sección 2.4 se plantea la determinación experimental del periodo fundamental, mediante la razón espectral del registro de las componentes horizontal y vertical de registros de ruido ambiente, siguiendo el método $\mathrm{H} / \mathrm{V}$.

\subsection{Determinación experimental del periodo fun- damental del suelo mediante el método $\mathrm{H} / \mathrm{V}$}

La determinación experimental del periodo fundamental del suelo por medio del método $\mathrm{H} / \mathrm{V}$ en emplazamientos determinados se ha agrupado en las metodologías de microzonación sísmica cuyo fin es la estimación de la amplificación sísmica a partir de medidas directas e in situ de las características mecánicas de los materiales geológicos (capítulo 1). La clasificación de este método en este grupo de metodologías se ha realizado en contraposición a las metodologías de microzonación que obtienen mapas de amplificación en base a criterios geológicos o topográficos. No obstante, el método $\mathrm{H} / \mathrm{V}$ realmente no busca determinar un parámetro mecánico del material geológico, como es Vs30, aunque sí parte de mediciones de ruido ambiental, realizadas en el propio emplazamiento, con el fin de estimar la frecuencia fundamental de resonancia del suelo.

En esta sección se describe el fundamento del método $\mathrm{H} / \mathrm{V}$, y su empleo en el procesado de muestras de ruido sísmico de estaciones de registro de velocidad cercanas a algunos de los emplazamientos de medida ReMi en El Hierro. Como resultado del procesado, se determinarán valores experimentales del periodo fundamental del suelo que pueden ser contrastados con los valores teóricos procedentes de las curvas de amplificación espectral de los citados emplazamientos.

\subsubsection{Fundamentos del método $\mathrm{H} / \mathrm{V}$}

El ratio espectral $\mathrm{H} / \mathrm{V}$ es el cociente entre los espectros de amplitud de Fourier de las componentes horizontal y vertical de un microtremor, entendiendo por microtremor el ruido ambiental de alta frecuencia (mayor que $1 \mathrm{~Hz}$ ), originado predominantemente por actividades antrópicas (tráfico, industria, etc.) y constituido tanto por ondas de cuerpo como por ondas superficiales. Nogoshi e Igarashi (1971), y posteriormente Nakamura (1989), han descrito la correlación existente entre la 
frecuencia correspondiente al máximo ratio $\mathrm{H} / \mathrm{V}$ y la frecuencia fundamental de resonancia del suelo en el emplazamiento donde se realiza la medida. Sin embargo, Lermo y Chávez-García (1994) han cuestionado la fiabilidad del método H/V para estimar la amplificación de las ondas sísmicas, indicando que este método sólo permite obtener valores de la amplificación en el caso de que la geología local sea relativamente simple, y también que estos valores carecen de precisión. No obstante, los autores no cuestionan la validez del método para la obtención del periodo fundamental.

\subsubsection{Obtención y análisis de la curva de la relación $\mathrm{H} / \mathrm{V}$}

Siguiendo las recomendaciones del proyecto SESAME para la implementación del método $\mathrm{H} / \mathrm{V}$, en cuanto a la toma de medidas, su procesado y su interpretación (SESAME European research project, 2004), la toma de medidas de ruido sísmico ambiental debe realizarse preferentemente con sensores de corto periodo (periodo natural alrededor de $1 \mathrm{~s}$ ). Se debe evitar la utilización de acelerómetros con este fin, pues no son lo suficientemente sensibles a los niveles de vibración ambiental. No se recomienda, asimismo, adquirir medidas con sismómetros de banda ancha (con periodo natural superior a $20 \mathrm{~s}$ ), pues requieren un tiempo de estabilización prolongado. En cuanto a las condiciones experimentales, (SESAME European research project, 2004) recomienda tomar una frecuencia de muestreo de $50 \mathrm{~Hz}$, y un tiempo de registro que cumpla la siguiente condición: $f_{0}>10 / l_{w}$, siendo $f_{0}$ la menor frecuencia de interés en el experimento (en este caso $1 \mathrm{~Hz}$, al medirse microtremores), y $l_{w}$ la duración temporal de la ventana de registro. Para frecuencias $f_{0}$ mayores que $1 \mathrm{~Hz}$, SESAME European research project (2004) recomienda registrar 10 ventanas de ruido de al menos $10 \mathrm{~s}$ de duración.

A partir de las mediciones de ruido ambiental obtenidas en campo, se realiza el procesado de los datos adquiridos con el fin de obtener la curva de la relación $\mathrm{H} / \mathrm{V}$ en el emplazamiento de medida. De acuerdo con las directrices del proyecto SESAME, el procesado de los datos se lleva a cabo por medio de un procedimiento de selección de ventanas sobre el registro de cada componente (SESAME European research project, 2003), de forma que se conserven las partes más estacionarias de las vibraciones ambientales y se eviten los transitorios, frecuentemente asociados con fuentes específicas (pasos y tráfico cercano). Simultáneamente, es necesario descartar vibraciones ambientales con amplitudes anormalmente bajas, introduciendo un límite mínimo bajo el cual no debe caer la señal dentro de cualquier ventana que se seleccione. Esta metodología calcula, en cada ventana, el ratio espectral $\mathrm{H} / \mathrm{V}$, siendo posible realizar filtrados y suavizados, y combinar las componentes horizontales (NS y EW) (SESAME European research project, 2003). A continuación, se obtiene la curva promedio de los ratios espectrales $\mathrm{H} / \mathrm{V}$ que se hayan calculado para las ventanas. La frecuencia fundamental o de pico se calcula como la media de las frecuencias de pico de las ventanas, con una determinada desviación estándar.

La metodología de esta tesis doctoral propone, en algunos de los emplazamientos seleccionados para la adquisición de medidas de ReMi en El Hierro (sección 2.2), la producción de la curva de la relación $\mathrm{H} / \mathrm{V}$ en función de la frecuencia según Nakamura (1989). Con este fin, se utilizarán muestras de ruido sísmico del registro continuo de las estaciones de registro de velocidad del Instituto Geográfico Nacional 
(IGN), con una duración temporal determinada. Por este motivo, la determinación experimental del periodo fundamental a partir de la curva de la relación $\mathrm{H} / \mathrm{V}$ sólo será posible en los emplazamientos situados en las proximidades de estaciones de registro de velocidad.

Las ventanas temporales de ruido, de duración constante, se procesarán utilizando el software GEOPSY, escrito por Marc Wathelet de ISTerre (Francia). Esta herramienta permite obtener, para cada ventana, la curva de amplificación espectral $\mathrm{H} / \mathrm{V}$, que representa la relación $\mathrm{H} / \mathrm{V}$ en función de la frecuencia. Adicionalmente, el código genera un gráfico con la variación del ratio $\mathrm{H} / \mathrm{V}$ con la frecuencia y el acimut. A partir de estos resultados, se selecciona la ventana temporal que presente un máximo del ratio espectral $\mathrm{H} / \mathrm{V}$ contenido en el intervalo de frecuencias $(0,10) \mathrm{Hz}$. De esta forma, se utiliza el mismo rango de frecuencias que el empleado en el análisis de la curva de amplificación espectral obtenida a partir del modelo de Vs (sección 2.3), con el fin de determinar la frecuencia fundamental del suelo. Además, la ventana seleccionada debe asegurar, en la medida de lo posible, que el máximo ratio $\mathrm{H} / \mathrm{V}$ ocurre en todos los acimutes comprendidos entre $0^{\circ}$ a $180^{\circ}$, para poder garantizar la bondad del resultado obtenido, y también demostrar que la fuente de ruido no está focalizada y que no tiene un origen antrópico (fundamentalmente industrial).

\subsubsection{Determinación del periodo fundamental}

En la ventana seleccionada, se utiliza la curva del ratio $\mathrm{H} / \mathrm{V}$ en función de la frecuencia, considerando que el máximo ratio no se encuentra focalizado, para determinar de forma experimental el periodo fundamental del suelo en el emplazamiento. Con este fin, se toma la frecuencia correspondiente al máximo ratio $\mathrm{H} / \mathrm{V}$, comprendido entre 0 y $10 \mathrm{~Hz}$ de frecuencia. Este resultado se compara con el periodo fundamental del suelo calculado a partir de la curva de amplificación espectral del emplazamiento, que se obtiene a partir del modelo de Vs proporcionado por el método ReMi (sección 2.3). La coincidencia entre ambas determinaciones del periodo fundamental del suelo permitirá certificar adecuadamente la validez del periodo procedente de la curva de amplificación espectral del emplazamiento, que tiene un carácter teórico.

\subsection{Cálculo de la aceleración máxima y de la inten- sidad sintéticas considerando una fuente sísmi- ca puntual}

En esta sección se aborda el cálculo en primera aproximación de los valores sintéticos realistas de la aceleración máxima del terreno (Peak Ground Acceleration, PGA) y de la intensidad sísmica en cualquier punto de la Península Ibérica, las Islas Baleares y las Islas Canarias, ante la ocurrencia de un terremoto. La determinación de estos valores sintéticos constituye la simulación de un escenario sísmico en dicho emplazamiento, tanto en términos de aceleración como en términos de intensidad. En este contexto, se considera que la fuente sísmica es puntual, y por tanto se calcula la PGA con una relación o ley de atenuación que introduce como 
variables fundamentales la magnitud del terremoto y la distancia al foco sísmico. Esta primera aproximación también permite tomar como valor válido de la capacidad de amplificación del material geológico aflorante los factores de amplificación de Borcherdt (1994), que son proporcionados por el mapa de amplificación sísmica (sección 2.1), que depende en última instancia de las características geotécnicas de las unidades geológicas. Las intensidades se calculan a partir de las PGA sintéticas, por medio de una correlación previamente seleccionada entre la aceleración máxima y la intensidad sísmica.

El objetivo de esta parte de la metodología consiste en seleccionar las relaciones de atenuación aplicables en la Península Ibérica y las Islas Baleares, y paralelamente en las Islas Canarias, que puedan considerarse de óptima utilización en un determinado intervalo de magnitudes y de distancias, con el fin de preparar su implementación en la herramienta de desarrollo del Sistema de Información Geográfica QGIS, que se explica en la sección 2.8. Esta selección se va a realizar por medio de un proceso de calibración, que implica calcular las aceleraciones sintéticas en distancias epicentrales o hipocentrales comparables con las de los acelerómetros que han registrado acelerogramas para un conjunto de terremotos de calibración, previamente seleccionado. Se comparan a continuación las aceleraciones calculadas con las observadas en este rango de distancias, para poder valorar cualitativamente el ajuste de cada relación de atenuación a las observaciones. Finalmente, se escogen los modelos de atenuación que presenten un mejor ajuste, que serán utilizados en la simulación de los escenarios sísmicos para terremotos de diseño, o con alta probabilidad de ocurrencia en el futuro. Un objetivo complementario consiste en seleccionar determinadas relaciones de atenuación recomendadas para su utilización en un contexto mundial, con el fin de implementarlas también en la herramienta de desarrollo del Sistema QGIS.

\subsubsection{Leyes de atenuación obtenidas a partir del ajuste em- pírico de registros de aceleración}

Las relaciones empíricas de atenuación en términos de aceleración máxima del terreno, PGA, se obtienen por medio del ajuste de un determinado modelo de atenuación de la aceleración con la distancia a las aceleraciones máximas del terreno, o aceleraciones pico, que se han determinado a partir de los acelerogramas de determinados terremotos, normalmente localizados en la misma región. Este modelo de atenuación describe la variación que experimenta la aceleración máxima del terreno en función de la distancia, para determinados valores de la magnitud, aunque también pueden incorporar otras variables predictivas, como el efecto de sitio o el mecanismo focal de la fuente. De esta forma, se obtienen ecuaciones que pueden ser utilizadas, a priori, para llevar a cabo la simulación del escenario sísmico en la misma región.

Respecto a la variable predictiva del efecto de sitio, cada modelo de atenuación expresa la PGA con respecto a un determinado tipo de material geológico, que normalmente coincide con el material en el cual se encuentran instaladas las estaciones que han registrado los datos utilizados en la determinación del modelo. Este material geológico se entiende como un terreno de referencia. De acuerdo con la sección2.1, este material puede asociarse a una determinada clase de emplazamiento 
de la clasificación de síntesis a realizar, y en función de esta clase, pueden conocerse los factores de amplificación de Borcherdt (1994) que describen el terreno de referencia. Esta consideración es fundamental para poder amplificar adecuadamente las aceleraciones calculadas con el modelo de atenuación. Concretamente, al calcular la PGA en un punto que pertenezca a la misma clase de emplazamiento que el terreno de referencia, esta PGA no debe ser modificada por el factor de amplificación local, a fin de no introducir una redundancia en la consideración del efecto de sitio. En cambio, la utilización del modelo de atenuación requiere reescalar los factores de amplificación que corresponden a las distintas clases de la clasificación de síntesis, de forma que el factor de amplificación correspondiente al terreno de referencia sea igual a 1 .

El análisis de las leyes de atenuación potencialmente aplicables en la región en estudio requiere en primer lugar de la realización de una investigación bibliográfica de las leyes de atenuación obtenidas a partir de registros de aceleración en la misma región. En el caso de la Península Ibérica y las Islas Baleares, y también en el de las Islas Canarias, no se dispone de suficientes registros de aceleración para poder obtener leyes de atenuación en cada tipología de terreno. Esta circunstancia hace necesaria una revisión de las leyes de atenuación correspondientes a otras partes del mundo y que puedan ser de aplicación en cada territorio.

La aplicabilidad de la relación de atenuación se valora primeramente en función de que haya sido obtenida o no en un ambiente tectónico parecido. En segundo lugar, se revisan los rangos de aplicación de la relación de atenuación en magnitud y en distancia, con el fin de comprobar si son compatibles con los intervalos de las magnitudes de los terremotos de la región de estudio que han producido acelerograma, y con los rangos de distancias de las estaciones acelerométricas con respecto al foco sísmico. Esta comprobación se realizará en tres pasos:

1. Comprobación de que el rango de distancias epicentrales o hipocentrales de los datos del modelo de atenuación contiene a los rangos de distancias epicentrales o hipocentrales de los acelerómetros

2. Si el rango de profundidades de los datos del modelo es restringido, comprobación de que contiene al rango de profundidades de los terremotos registrados

3. Comprobación de que los rangos de magnitudes de los datos del modelo contienen a algunas de las magnitudes de los terremotos registrados

En tercer lugar, aquellas relaciones de atenuación que resultan aplicables en el territorio pasan al proceso de calibración que se explica en la siguiente subsección 2.5.2.

En la Península Ibérica y las Islas Baleares, la selección de las leyes de atenuación a utilizar se ha realizado a partir del análisis de ocho relaciones empíricas de atenuación de movimientos fuertes que pueden ser de aplicación en el área de la Península Ibérica. En particular, se trata de cuantificar la incertidumbre asociada a cada relación de atenuación por medio de la metodología del árbol lógico (Coppersmith y Youngs, 1986), de forma que esta incertidumbre pueda ser empleada en un análisis de peligrosidad sísmica determinista. De esta forma, se asigna un peso normalizado a cada ley de atenuación, el cual depende del cumplimiento de algunos de los diez criterios de Bommer et al. (2010) y del ajuste de las predicciones de la 
ley a las intensidades observadas para terremotos seleccionados, en lo que constituye la calibración de la ley de atenuación. En este sentido, se utiliza la conversión de las PGA y las PGV calculadas a intensidades.

En esta tesis doctoral, se han elegido 5 relaciones de atenuación en términos de PGA, de entre las relaciones analizadas y calibradas. Como criterios de selección, se han considerado la fecha de publicación más reciente, y en segundo lugar la bondad del ajuste de las intensidades calculadas a las intensidades observadas y el cumplimiento de los criterios de Bommer et al. (2010).

En las Islas Canarias, se ha realizado el proceso completo de selección de las leyes de atenuación, de acuerdo con la presente metodología. Por tanto, se ha comenzado con un análisis riguroso de las relaciones de atenuación obtenidas en distintas regiones del mundo, susceptibles de aplicación en el archipiélago por corresponder a ambientes tectónicos parecidos al de las islas, en particular por haber sido obtenidas en ambientes volcánicos. A continuación, se han analizado sus rangos de aplicación en magnitudes y distancias, en comparación con los rangos de magnitudes y distancias para un conjunto de 20 terremotos de $\mathrm{Mw}>4,0$ localizados en El Hierro durante la crisis sísmica de julio-octubre de 2011, ligada a la reactivación volcánica de 2011-2012, la crisis sísmica de junio de 2012 y la crisis de marzo de 2013, que han producido 36 acelerogramas. Los datos acelerométricos de estos terremotos han sido proporcionados por el IGN a través de un catálogo de acelerogramas que cubre los periodos citados, siendo el intervalo total de magnitudes Mw 2,4-4,9.

En cuanto a las magnitudes, se han comparado las magnitudes de aplicación de cada modelo de atenuación con el intervalo de magnitudes Mw 4,1-4,9 para los terremotos de El Hierro. Con respecto a las distancias, se ha comparado el rango de aplicación de la ley de atenuación en distancias epicentrales o hipocentrales con las distancias epicentrales o hipocentrales de los acelerómetros de El Hierro que han registrado acelerograma para estos terremotos. En este sentido, se tiene un rango de distancias epicentrales de 3,4-37 km. En ambos casos, se han seleccionado las relaciones de atenuación con rangos de magnitudes y distancias coherentes con los datos acelerométricos de El Hierro, de acuerdo a los criterios expresados.

Para comparar los rangos de magnitudes, se han convertido a magnitud momento (Mw) las magnitudes utilizadas por los modelos de atenuación y que se encuentran expresadas en otras escalas. Para la conversión de magnitud local $M_{L}$ a $\mathrm{Mw}$, se ha utilizado la correlación de Ristau et al. (2005), $\mathrm{Mw}=M_{L}+0,6$, obtenida para terremotos con $\mathrm{Mw}$ 3,4-7,5 localizados en zona de subducción en el W de Canadá. La correlación de Ristau et al. (2005) es válida para $M \geq 3,6$. Siguiendo a Wells y Coppersmith (1994), se ha empleado la conversión de magnitud de las ondas superficiales $M_{S}$ a $\mathrm{Mw}, \mathrm{Mw}=M_{S}$, válida para $5,7<M<8$. Con respecto a la distancia al foco sísmico, se han considerado las distancias epicentral e hipocentral. Para analizar las relaciones de atenuación con rangos de distancias aplicables expresados en distancia Joyner y Boore $\left(R_{j b}\right)$ (Joyner y Boore, 1981), se ha considerado que esta distancia es equiparable a la distancia epicentral para magnitudes $M w<5,5$.

Con respecto a las relaciones de atenuación aplicables en un contexto mundial, se han estudiado las leyes de atenuación recomendadas por el proyecto Global Earthquake Model (Stewart et al., 2013), en función de los principales ambientes tectónicos: 
- Regiones continentales estables

- Zonas de subducción

- Regiones corticales activas

Stewart et al. (2013) recomiendan utilizar relaciones de atenuación asociadas a terrenos de referencia en roca, y de esta forma amplificar las aceleraciones calculadas con un término no lineal de efecto de sitio. Con estas consideraciones, en esta tesis doctoral se ha elegido 1 relación de atenuación en cada ambiente tectónico, que haya sido obtenida en un terreno de referencia en roca, de forma que no sea necesaria la amplificación de las PGA calculadas con el término no lineal de efecto de sitio.

\subsubsection{Calibración de relaciones de atenuación a partir de la comparación entre las aceleraciones máximas sintéticas y las aceleraciones máximas registradas}

En los siguientes apartados de esta sección, se particulariza la metodología al problema de selección de relaciones de atenuación óptimas en las Islas Canarias, en función de la calibración de las mismas con los datos acelerométricos proporcionados por el IGN y correspondientes a El Hierro. La calibración de las relaciones de atenuación aplicables en Canarias se ha realizado en base al mismo conjunto de datos acelerométricos que han servido para valorar los rangos de aplicación en magnitudes y distancias (subsección 2.5.1). Previamente a la calibración, es preciso preparar los datos acelerométricos observados. A partir de cada acelerograma, debe calcularse la PGA observada en función de la definición que proporcione el modelo de atenuación. En este sentido la definición de PGA se aproxima en función de los valores pico de cada componente de acelerograma.

Con cada relación de atenuación, se ha calculado la aceleración máxima, PGA, sintética producida por un terremoto de magnitud intermedia con respecto al rango de magnitudes de los terremotos reales que intervienen en la calibración, y en distancias epicentrales comparables a las distancias epicentrales de los acelerómetros que han registrado cada terremoto. Se obtiene de esta forma una curva de PGA sintética, que debe ajustar las observaciones discretas de PGA. En el caso de El Hierro, las magnitudes de los terremotos de calibración se sitúan entre 4,1 y 4,9 Mw, por lo que la magnitud considerada equidistante se ha tomado como 4,5 $\mathrm{Mw}$. Las distancias epicentrales de los acelerómetros se sitúan entre 3,4 y $37 \mathrm{~km}$, por lo que la curva de atenuación sintética deberá tomar valores, al menos, en estas distancias.

Los valores de PGA calculados con cada relación de atenuación se entienden expresados en su condición del terreno de referencia (subsección 2.5.1). Es claro que, para poder realizar la calibración, las PGA observadas deben corregirse a este nivel de referencia. Esta corrección se realiza en términos de los factores de amplificación del terreno (Borcherdt, 1994) que proporciona el mapa de amplificación sísmica de las Islas Canarias (sección 2.1). En este punto, debe justificarse el factor de amplificación a utilizar. El factor de amplificación para altas frecuencias de Borcherdt (1994), $F_{a}$ se utiliza para modificar las aceleraciones en distancias epicentrales cortas, donde las altas frecuencias de las ondas sísmicas no han sido aún atenuadas. El factor 
para bajas frecuencias, $F_{v}$, modifica normalmente las velocidades a distancias más grandes, en las cuales sólo permanecen las bajas frecuencias. En el caso de los datos acelerométricos de El Hierro, la localización de los eventos de alta magnitud y de las estaciones acelerométricas del IGN en la isla permiten considerar que las distancias epicentrales son cortas y que, por tanto, se puede utilizar el factor $F_{a}$.

La corrección de las PGA observadas se realiza teniendo en cuenta el factor de amplificación $F_{a}$ que proporciona el mapa de amplificación para el emplazamiento donde se sitúa el acelerómetro, y el factor de amplificación $F_{a} *$ del material geológico de referencia del modelo de atenuación. Bajo la premisa de que, en acelerómetros instalados sobre la misma clase de emplazamiento que la clase que corresponde al terreno de referencia (sección 2.1), la PGA observada no experimenta ninguna corrección, se reescalan todos los factores de amplificación $F_{a}$ de los acelerómetros en función del factor $F_{a} *$ del terreno de referencia, de manera que el factor de amplificación modificado para el mismo sea igual a 1. Este reescalamiento se realiza dividiendo los factores de amplificación entre el factor $F_{a} *$ del terreno de referencia. Por tanto, las PGA observadas se corrigen a la condición del terreno de referencia dividiendo su valor por el factor $F_{a}$ ya reescalado.

La comparación de la curva de PGA sintética con las PGA observadas se lleva a cabo de forma cualtitativa, teniendo en cuenta si los valores observados se ajustan a la curva, o bien quedan comprendidos entre los límites del error de los valores calculados. En este contexto, un buen ajuste se consigue si las observaciones se constriñen, en todo caso, al área comprendida entre las curvas de PGA sintética que incorporan el error de $2 \sigma$, siendo $\sigma$ la desviación estándar del modelo de atenuación. Las diferencias entre PGA observadas y sintéticas pueden considerarse entonces aceptables, para el rango de distancias de los datos observados y el rango de magnitudes de los terremotos de calibración. La calibración, por tanto, permite valorar la bondad del ajuste de las predicciones de la ley de atenuación a los datos observados.

\subsubsection{Selección de leyes de atenuación óptimas para distintas escalas de trabajo y en función de la magnitud del terremoto}

En función de los resultados de la calibración, se eligen las relaciones de atenuación que proporcionan los valores de PGA sintética más próximos a las PGA observadas, con un mejor ajuste. Estas relaciones se consideran óptimas, en función de la magnitud del terremoto y de la escala de trabajo. Es decir, las leyes de atenuación así seleccionadas se podrán utilizar para simular escenarios sísmicos, en términos de aceleración, para terremotos de magnitudes comprendidas en el intervalo de magnitudes de los terremotos utilizados en la calibración. Las relaciones de atenuación óptimas también se podrán utilizar a distancias epicentrales que también estén contenidas en el rango de distancias epicentrales que corresponda a las aceleraciones observadas.

En las Islas Canarias, se han seleccionado 3 modelos de atenuación en dos fases distintas. En la primera fase, se ha examinado la bondad del ajuste de las leyes de atenuación a las PGA registradas en El Hierro y corregidas a la condición del terreno de referencia correspondiente. Este ajuste se ha entendido en dos vertientes, 
por un lado de una forma general y cualitativa, de acuerdo con las directrices de la subsección 6.2.3, y por otra parte contabilizando el número de PGA registradas que coinciden con el valor de la PGA sintética al alinearse a la curva de atenuación de la PGA. Las relaciones de atenuación con buen ajuste han pasado a la segunda fase, en la cual se han valorado de nuevo los rangos de distancias epicentrales o hipocentrales y de magnitudes de los datos acelerométricos empleados en la determinación de cada modelo. Se seleccionan los modelos de atenuación con un rango de distancias y de magnitudes lo más amplio posible, pues éstos marcan la escala de trabajo y el rango de magnitudes para los cuales los modelos pueden simular aceleraciones sintéticas realistas.

\subsubsection{Correlaciones entre los parámetros del movimiento fuer- te y la intensidad sísmica}

Con objeto de convertir en intensidades sintéticas las PGA sintéticas obtenidas por medio de las relaciones de atenuación seleccionadas como óptimas en la Península Ibérica, las Islas Baleares y las Islas Canarias, se debe elegir una correlación adecuada entre la PGA y la intensidad sísmica, en la escala EMS-98. Esta correlación, que también se aplica para convertir las PGA calculadas con las 3 relaciones de alcance mundial, se programa en la herramienta de desarrollo del Sistema QGIS.

Con este fin, se realiza una investigación bibliográfica de las distintas correlaciones publicadas en la literatura sismológica. En este sentido, se revisan las correlaciones de la PGA (Peak Ground Acceleration) y de la PGV (Peak Ground Velocity) con respecto a la Intensidad Modificada de Mercalli, por su equivalencia con la escala EMS-98 (Wald et al., 1999a; Atkinson y Kaka, 2007; Caprio et al., 2015).

\subsection{Estimación de la atenuación anelástica de la litosfera}

En esta parte de la metodología se pretende caracterizar la atenuación anelástica de la litosfera en las Islas Canarias. Como se ha explicado en la sección 2.2, la generación de curvas de amplificación espectral en emplazamientos de medida ReMi en El Hierro se ha orientado a su incorporación como variable necesaria a un algoritmo de simulación estocástica de acelerogramas sintéticos en la isla (sección 2.7). Este algoritmo requiere también de la implementación de la atenuación de la litosfera regional, por lo cual resulta necesario llevar a cabo un estudio de la atenuación anelástica en el archipiélago, que permita regionalizar adecuadamente los parámetros que la definen.

\subsubsection{Metodologías de estimación del factor de calidad Q a partir del análisis de las ondas de coda}

La atenuación del medio atravesado por una onda sísmica en su camino hacia la superficie se define como el decaimiento de la amplitud de la onda conforme se 
propaga. La atenuación es producida por dos causas, una de carácter geométrico y otra definida como la atenuación anelástica. La atenuación anelástica es causada por dos mecanismos principales, la absorción intrínseca en el medio anelástico y la dispersión en las heterogeneidades del medio. La absorción intrínseca convierte la energía de las ondas sísmicas en calor y deformaciones plásticas, conforme las ondas se propagan a través del medio anelástico, mientras que la dispersión introduce un cambio en la dirección de propagación de las ondas sísmicas.

La atenuación anelástica total se suele representar por el factor de calidad Q, que se define en términos de la fracción de energía perdida durante el ciclo de una onda. El factor de calidad Q depende de la frecuencia (f), y de acuerdo con Mitchell (1981) aumenta con la misma según la siguiente expresión:

$$
Q(f)=Q_{0}\left[\frac{f}{f_{0}}\right]^{\nu}
$$

donde $Q_{0}$ es el factor de calidad a la frecuencia de referencia $f_{0}$ y $\nu$ expresa el grado de heterogeneidad de la corteza Aki (1981).

El factor Q puede ser expresado como la combinación de los dos mecanismos que causan la atenuación anelástica, la absorción intrínseca $\left(Q_{i}\right)$ y la dispersión $\left(Q_{s}\right)$, escribiéndose Q como:

$$
\frac{1}{Q}=\frac{1}{Q_{i}}+\frac{1}{Q_{s}}
$$

A partir del desarrollo de la teoría de las ondas de coda (Aki, 1969), los registros de ondas de coda han sido frecuentemente utilizados para estimar la atenuación anelástica. Los diferentes modelos de onda de coda dependen del tipo de proceso dispersivo considerado. La hipótesis de dispersión simple supone que la onda experimenta sólo una interacción o cambio en su dirección conforme se propaga desde el foco sísmico hasta la superficie. En cambio, la hipótesis de difusión considera un medio fuertemente dispersivo. La mayor parte de los métodos utilizados para calcular Q a partir de las ondas de coda se basan en la distribución aleatoria de las heterogeneidades que introducen la dispersión en el medio de propagación. Aki y Chouet (1975) han interpretado las ondas de coda de terremotos locales de baja magnitud como ondas retrodispersivas que proceden de numerosas heterogeneidades uniformemente distribuidas en la corteza terrestre. Estos autores proponen un modelo de dispersión simple, que es utilizado posteriormente por Havskov et al. (1989) para desarrollar su metodología de estimación de Q para un valor concreto de frecuencia f, por medio del ajuste de una envolvente que decae con el tiempo a la señal filtrada de la onda de coda.

Partiendo de los resultados de Aki (1969) relativos al modelo de dispersión simple, Herrmann (1980) propone un método para estimar Q a partir de la frecuencia predominante $f_{p}$ observada en la onda de coda, que se considera como una función del tiempo t. Con este fin, asume que la frecuencia $f_{p}$ depende del espectro de la fuente sísmica, de la respuesta intrumental y del filtro Q de la Tierra. Pujades et al. (1990) modifican el método de Herrmann (1980) para considerar también la dependencia frecuencial de Q. En este sentido, asumen que, para frecuencias cercanas a 1 $\mathrm{Hz}, \mathrm{Q}$ depende de $f_{p}$ de acuerdo con la siguiente expresión: 


$$
Q\left(f_{p}\right)=Q_{0}\left[\frac{f_{p}{ }^{\nu}}{f_{0}}{ }^{\nu}\right.
$$

Por tanto, Pujades et al. (1990) estiman $Q_{0}$ y $\nu$ a partir de lecturas de la frecuencia $f_{p}$ y del tiempo t realizadas en los sismogramas.

\subsubsection{Metodología de Havskov et al. (1989) para la determi- nación de $\mathrm{Q}$ en función de la frecuencia de filtrado de la señal}

En base al método de retrodispersión simple publicado por Aki y Chouet (1975), estos autores muestran que el decaimiento de la amplitud $\mathrm{A}(\mathrm{f}, \mathrm{t})$ de la onda de coda en una determinada frecuencia $f$ y un determinado lapso de tiempo $t$, medido desde el tiempo origen del terremoto, es una función de Q, de acuerdo con la expresión siguiente:

$$
\ln (A(f, t))+\beta \ln (t)=\ln (S(f))-\pi f t / Q
$$

donde $\mathrm{S}(\mathrm{f})$ es el factor de la fuente y $\beta$ el parámetro de atenuación geométrica ( 1 para ondas de cuerpo y 0,5 para ondas superficiales). Havskov et al. (1989) utilizan este resultado para calcular el factor Q para una determinada frecuencia, por medio de la modelización de la envolvente de la amplitud de la onda de coda como una función del tiempo t. Considerando la ecuación 2.12, los autores llevan a cabo una regresión lineal de la función $\ln (A(f, t))+\beta \ln (t)$ respecto de la variable t para una frecuencia $\mathrm{f}$ constante. Para determinar A(f,t), siguen el trabajo de Rautian y Khalturin (1978) y estiman la amplitud de la onda de coda por medio de la aplicación de un filtro paso-banda sobre la señal, con frecuencia central $f$ y ancho de banda pequeño, y del ajuste de una envolvente decreciente con el tiempo a la señal así filtrada. De la ecuación 2.12, el factor $\mathrm{Q}$ para una frecuencia f constante es calculado por medio del término que expresa la dependencia temporal de la envolvente, $-\pi f t / Q$.

En esta tesis doctoral, se determinará la atenuación anelástica de la litosfera regional en Canarias, en términos del factor de calidad Q, a partir del análisis de las ondas de coda registradas en estaciones de registro de velocidad. Siguiendo la metodología de Havskov et al. (1989), se propone obtener el factor Q que corresponde a cada trayectoria estación-epicentro, en función de 5 bandas frecuenciales de filtrado de la señal, con frecuencia central y ancho de banda conocidos. Con este fin, y atendiendo a la metodología, es preciso filtrar previamente el registro de la onda de coda con un filtro paso-banda, construido con las 5 frecuencias centrales y anchos de banda escogidos, y ajustar la envolvente a la amplitud de la señal filtrada. La regresión lineal de los valores de la envolvente en función del tiempo permitirá obtener $\mathrm{Q}$ en función de la frecuencia de filtrado.

\subsubsection{Regionalización de los parámetros $Q_{0} \mathbf{y} \nu$}

Para realizar la regionalización espacial de los parámetros que expresan la dependencia frecuencial del factor de calidad $\mathrm{Q}, Q_{0}$ y $\nu$, se siguen los trabajos de Canas 
et al. (1995) y de González Drigo et al. (2003), entre otros. Se propone asignar cada observación Q al punto medio de la correspondiente trayectoria estación-epicentro. Las observaciones $\mathrm{Q}$ así distribuidas se interpolan sobre una malla de ancho fijo que abarque la distribución de los puntos medios de las trayectorias, de forma que, en cada punto de esta malla, se tiene una colección de valores $\mathrm{Q}$, correspondientes a las diferentes frecuencias de filtrado. A partir de estos valores, en cada punto de la malla se determinan los parámetros $Q_{0}$ y $\nu$, para lo cual se ajustan los valores $\mathrm{Q}$ de cada punto de la malla a la ecuación 2.9, tomando $f_{0}=1 \mathrm{~Hz}$. Por medio de los valores $Q_{0}$ y $\nu$ de cada punto de la malla se obtienen los mapas de contornos para cada parámetro, que permiten llevar a cabo la regionalización de la atenuación anelástica en Canarias.

Para completar el estudio de la atenuación anelástica en la litosfera regional, resulta conveniente analizar la variación de los parámetros $Q_{0}$ y $\nu$ en función del tiempo. El conocimiento de la evolución temporal de ambos parámetros permite su correlación con la evolución de la sismicidad y de la deformación, entre otros parámetros geofísicos y geodésicos, la cual es de gran interés ante el desarrollo de una erupción volcánica. En este contexto, la evolución temporal de los parámetros de la atenuación puede discretizarse en su determinación por días, y en una misma estación de registro de velocidad, que proporcionará los registros de onda de coda necesarios. En Canarias, se ha estudiado la evolución temporal de $Q_{0}$ y $\nu$ durante la crisis sísmica de julio-octubre de 2011 y la erupción volcánica posterior (2011-2012), tratando de trazar correlaciones del comportamiento de estos parámetros con el de los parámetros geofísicos y geodésicos monitorizados por el IGN en el mismo periodo de tiempo (López et al., 2012).

Siguiendo la metodología de Havskov et al. (1989), será preciso obtener en una estación de registro de velocidad fija, una colección de valores medios de Q para cada frecuencia de estudio, considerando todas las trayectorias estación-epicentro generadas por los terremotos de un mismo día. Estos valores medios de Q se obtendrán a partir de las observaciones de Q correspondientes que cumplan el criterio de Chauvenet. Se seleccionarán a continuación los valores medios de Q calculados con un mínimo de 2 observaciones de Q y, por tanto, con desviaciones estándar finitas. A partir de esta selección, es necesario disponer de valores medios de Q para 3 frecuencias distintas, para aplicar la ecuación 2.9 con $f_{0}=1 H z$ y obtener los parámetros $Q_{0}$ y $\nu$ con desviaciones estándar aceptables.

\subsection{Simulación estocástica de acelerogramas sinté- ticos considerando una fuente sísmica extensa}

La simulación de escenarios bajo la consideración de fuente puntual se basa en la predicción del movimiento sísmico fuerte a partir de una expresión que permita su obtención conociendo, además de la magnitud y la distancia, la amplificación sísmica del terreno (efecto de sitio) en el punto de cálculo. Si se quiere predecir el movimiento sísmico fuerte con mayor precisión, se ha de tener en cuenta que la energía liberada por el sismo no se libera de forma esférica (foco puntual), sino que las dimensiones de la falla responsable alteran esta radiación. Es decir, existirán diferentes emisiones 
de energía según las distintas direcciones, e incluso se pueden presentar fenómenos de directividad que también modificarán la distribución acimutal de la energía.

En esta parte de la metodología se aborda la simulación de movimientos sísmicos fuertes utilizando una modelización para cada punto basada en la aproximación estocástica que, de forma inicial, propusieron Hanks (1979) y McGuire y Hanks (1980) basándose en el modelo de Brune (1970) para el espectro en la fuente, y que Boore (1983) generalizó para otros modelos más complejos, extendiéndola a la simulación de registros temporales.

Existen dos aproximaciones dentro de esta modelización estocástica: una basada en la consideración de la fuente como puntual y otra más complicada que considera un modelo de fuente extensa. Ambas aproximaciones son válidas y su consideración depende mucho del rango de distancias a considerar, de la magnitud del terremoto y también de las precisiones que se quieran alcanzar. En cualquier caso, el modelo de fuente extensa está basado en el modelo de fuente puntual aplicable a cada uno de los elementos en que la falla pueda dividirse. Por esta razón se describirá la esencia del método puntual, el cual será aplicado a continuación al modelo de fuente extensa que se estima como el más apropiado a la simulación de movimientos sísmicos fuertes en El Hierro.

\subsubsection{Modelo estocástico de fuente puntual}

La consideración del espectro en la fuente constituye la parte fundamental de la aproximación estocástica de la generación de un acelerograma en un punto. Este espectro dependerá del modelo de fuente considerado. Por ejemplo, el modelo de falla circular propuesto por Brune (1970) es el modelo más sencillo, pues considera una sola frecuencia de esquina cuyo valor está relacionado con las dimensiones del área de ruptura y de la caída de esfuerzos que se genera al producirse el sismo. Este espectro en la fuente $\mathrm{F}\left(\mathrm{f}\right.$ ) es función del momento sísmico $M_{0}$ (en la parte plana del espectro). Por tanto, se puede afirmar que el movimiento sísmico fuerte generado $\mathrm{A}\left(M_{0}, \mathrm{D}, \mathrm{f}\right)$ es:

$$
A\left(M_{0}, D, f\right)=F\left(M_{0}, f\right) T(D, f, \text { duracion }) S(f) I(f)
$$

donde:

- D es la distancia de observación desde el foco

- f es la frecuencia

- $T(D, f, d u r a c i o n)$ es el efecto de la trayectoria desde el foco al punto de observación

- S(f) es el efecto de sitio de observación que podrá amplificar o disminuir la señal que llega, y que se corresponde con los últimos metros de la trayectoria. Generalmente, se consideran los últimos $30 \mathrm{~m}$ 
- I(f) es el efecto del instrumento que modifica la señal de entrada, ya sea amplificándola, filtrándola o modificando sus características, obteniéndose aceleración, velocidad o desplazamiento del movimiento del suelo

\section{Función fuente}

Este es el elemento más decisivo en la simulación. Debe considerarse cuál de los diferentes modelos de fuente es el que más se aproxima a la fuente que se está considerando. Como ya se ha avanzado, el modelo más comúnmente utilizado por su sencillez y los buenos resultados que se obtienen es el modelo de Brune (1970), que relaciona la frecuencia de esquina $f_{0}$ con las demás variables que definen el modelo:

$$
f_{0}=4,9 \cdot 10^{6} V_{s}\left(\frac{\triangle \sigma}{M_{0}}\right)^{(1 / 3)}
$$

siendo $V_{s}$ la velocidad de las ondas $\mathrm{S}$ y $\triangle \sigma$ la caída de esfuerzos.

En esta expresión, la velocidad $V_{s}$ se expresa en $\mathrm{km} / \mathrm{s}, M_{0}$ en $d y n \cdot \mathrm{cm}$ y la caída de esfuerzos en bares.

Este modelo es también denominado $\omega^{2}$, dado que la pendiente de la frecuencia angular es cuadrática, para frecuencias superiores a la frecuencia de esquina.

Dado que existen otros modelos de fuente que se han desarrollado, como los de Atkinson y Boore (1995), Haddon (1996) y Atkinson y Silva (2000) entre otros, se puede generalizar para expresarlos todos en una simple expresión:

$$
F\left(M_{0}, f\right)=C M_{0} E\left(M_{0}, f\right)
$$

donde $E\left(M_{0}, f\right)$ es el espectro de desplazamiento en la fuente, que en el caso general puede tener más de una frecuencia de esquina y que por tanto se representa como:

$$
E\left(M_{0}, f\right)=E_{a}\left(M_{0}, f\right) \cdot E_{b}\left(M_{0}, f\right)
$$

donde a y b representan genéricamente las frecuencias de esquina de cada modelo. Así, se tiene que el modelo de Brune (1970) se representa según esta expresión:

$$
\begin{aligned}
& E_{a}=\frac{1}{1+\left(f / f_{a}\right)^{2}} \\
& E_{b}=1
\end{aligned}
$$

La constante $\mathrm{C}$ representa los aspectos del cuadro de radiación del modelo que depende de los ángulos de acimut e incidencia, $R(\theta \phi)$. Es decir:

$$
C=\frac{(R(\theta \phi) V F)}{4 \pi \rho_{S} V_{S}^{3} D_{0}}
$$

V representa la partición de la energía total de la onda de cizalla en sus componentes horizontales y $\mathrm{F}$ el efecto de interacción en la superficie libre. $\rho_{S}$ es la 
densidad del material en la fuente, $V_{S}$ la velocidad de las ondas de cizalla en la fuente y $D_{0}$ la distancia de referencia a partir de la que se considera el proceso lineal. Generalmente, $D_{0}$ se considera $1 \mathrm{~km}$, en los modelos más comunes.

\section{Trayectoria}

Elegido el espectro en la fuente, debe ser trasladado a través de la trayectoria de las ondas hasta el punto más próximo al punto de observación. Aunque un estudio preciso necesitaría de un conocimiento detallado de la estructura de velocidad correspondiente al camino recorrido, en general el estudio se limita al conocimiento de la atenuación de la energía transmitida en forma de ondas sísmicas tanto por ensanchamiento del frente de ondas (atenuación geométrica) como por efecto de falta del medio a ser asimilado como perfectamente elástico, que se representa por $Q^{-1}$ (atenuación anelástica). Estos dos elementos que intervienen en la atenuación se suelen presentar juntos ya que ambos dependen de la distancia recorrida:

$$
T(D, f)=G(D) e^{-\frac{\pi f D}{Q(f) C}}
$$

siendo la atenuación geométrica:

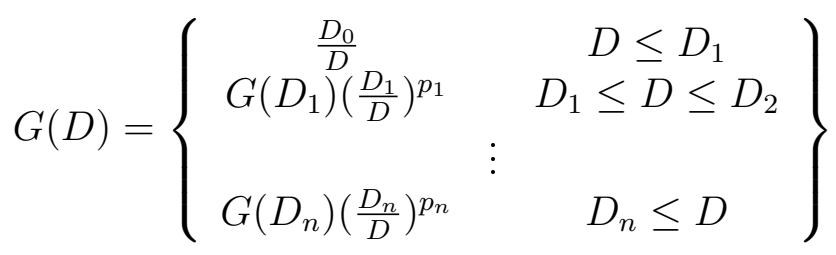

Los valores más frecuentemente observados de distancias en las que existe uniformidad en la atenuación son $D_{0}=1 \mathrm{~km}, D_{1}=60-70 \mathrm{~km}$ y los coeficientes correspondientes son $p_{1}=-1,0$ y $p_{2}=-0,5$.

Generalmente, estos valores se obtienen por observación de la atenuación de las ondas con la distancia para observaciones en la zona de estudio.

En cuanto a la atenuación anelástica, es necesario un estudio del valor de $Q$ en la zona. Dado que el caso de El Hierro esta variable puede ser muy diferente de los modelos estándar utilizados, se ha estudiado su valor de acuerdo con los criterios de la sección 2.6.

\section{Efecto de sitio}

La modificación que se produce en la señal sísmica por efecto de la composición y propiedades elásticas del suelo en los últimos metros (generalmente se consideran entre 30-100 m) puede ser muy importante. Por esta razón, la determinación de este efecto merece un estudio separado en esta tesis. Por una parte, y de forma general, se plantea la determinación de los factores de amplificación para altas $F_{a}$ y bajas $F_{v}$ frecuencias (Borcherdt, 1994) de todo el territorio canario, de acuerdo con la sección 2.1. Sin embargo, dada la importancia que tiene este efecto, se propone también una estimación en segunda aproximación, por medio de la determinación de los valores Vs30 de los puntos en los que se disponen instrumentos, así como otras 
determinaciones de las características del suelo, de acuerdo con las secciones $2.2 \mathrm{y}$ 2.3 .

Sin embargo, en el efecto de sitio se pueden individualizar dos efectos, un primer efecto que depende de la trayectoria y un segundo efecto que es independiente de la misma. Cuando se mide el efecto de sitio en un emplazamiento, ambos factores están combinados y es imposible su distinción. No obstante, en un proceso de simulación en el que se pretende alterar una señal por el efecto de sitio intervendrán ambos factores separadamente. Un primer efecto, debido a la geología local (Vs30), que es dependiente de la frecuencia y que generalmente supone una amplificación de la señal; y un segundo efecto de atenuación, que es independiente de la atenuación de la trayectoria ya estudiada. Este segundo efecto de atenuación dependiente de la frecuencia se puede expresar como:

$$
A T T(f)=e^{-\pi \chi_{0} f}
$$

donde $\chi_{0}$ es el coeficiente responsable de la atenuación de la señal dependiente de la frecuencia.

De esta forma, el efecto sitio se introduce en la simulación estocástica mediante una curva de amplificación del sitio, generada a partir del valor de Vs30 y por el efecto de atenuación dependiente de la frecuencia $\chi_{0}$.

\section{Efecto de corteza}

Este efecto se refiere al que introduce la composición de la corteza hasta los últimos metros cercanos a la superficie, donde interviene el efecto sitio. Es pues la modificación que sufre la señal emitida desde el foco en su paso a través del medio. En la literatura existe un gran número de estimaciones de este efecto de corteza basados en el conocimiento detallado de la estructura de velocidad con la profundidad desde la base de la corteza hasta la parte más superficial. Los más representativos son: Frankel y Clayton (1986), Boore y Joyner (1997), Kamai et al. (2013), y el más reciente de Campbell y Boore (2016). En todos ellos se tratan estructuras correspondientes a regiones activas continentales.

\section{Duración}

La duración del evento simulado en un punto dependerá de la trayectoria y de la duración del proceso de ruptura. Estas duraciones se determinan de forma empírica para diferentes modelos de trayectoria. La duración total puede descomponerse en la duración del proceso en la fuente y en la duración dependiente de la trayectoria. La primera componente está ligada a la frecuencia de esquina del modelo de fuente seleccionado, mientras la segunda es más difícil de evaluar, aunque evidentemente tiene relación con la magnitud. Kempton y Stewart (2006) y Ghofrani y Atkinson (2014) representan la duración como una función suma de la fuente, el camino recorrido y los parámetros del sitio, mientras otros como Bommer et al. (2009) o Bora et al. (2014), como una función más complicada. En general, en los últimos estudios se observa que la dependencia de la trayectoria como $0,005 R$, utilizada en gran cantidad de trabajos, no se corresponde con la realidad. 
En esta tesis doctoral se ha adoptado, a la vista de los datos existentes, una expresión de la duración que proporcione valores grandes como los que se observan. Se ha considerado la relación de Boore y Thompson (2015), suponiendo que la variable de distancia utilizada por los autores es semejante a la distancia hipocentral.

\section{Obtención del movimiento del suelo correspondiente al modelo}

Una vez obtenido el modelo de fuente y los efectos de la trayectoria es sencillo obtener la simulación en el dominio del tiempo. En la Figura 2.12 se muestra el proceso de obtención del registro en aceleración correspondiente al modelo presentado, de acuerdo con Boore (2003). Al ruido blanco generado aleatoriamente, de duración igual a la prevista en el modelo, se le aplica una ventana que simule la forma normal de un sismo con decrecimiento exponencial de su amplitud con el tiempo. El registro así obtenido se pasa al dominio de la frecuencia, normalizándose su espectro por la raíz cuadrada de la amplitud media cuadrática del espectro de amplitudes. Este espectro normalizado se multiplica por el espectro del modelo seleccionado en la fuente para, posteriormente y mediante la inversa de la transformada de Fourier, obtener el registro temporal correspondiente.

\subsubsection{Modelo estocástico de fuente extensa}

La consideración de una fuente extensa surge cuando se tratan terremotos muy grandes o cuando se pretende realizar la modelización a distancias en las que un modelo puntual no puede aplicarse. En general, la inclusión de efectos como la geometría de la superficie de falla, la distribución de deslizamiento en la falla o el efecto de directividad son propiedades de la fuente que deben ser necesariamente incluidas en la modelización estocástica. Hartzell (1978) propuso dividir la falla correspondiente en $\mathrm{N}$ subfallas a las que se podía aplicar el modelo de fuente puntual, de tal forma que la suma de todas permitiera obtener el efecto de la falla total. Así, cada uno de los modelos puntuales, con el consiguiente retraso, se suman para obtener el registro de aceleración del evento suma.

$$
a(t)_{i, j}=\sum_{i=1}^{n l} \sum_{j=1}^{n w} a_{i, j}\left(t+\triangle_{i, j}\right)
$$

donde $n l$ y $n w$ son el número de subfallas a lo largo de la longitud y anchura respectivamente de la falla, y $\triangle_{i, j}$ es el retraso para cada radiación emitida por la sufalla correspondiente.

Existen modelizaciones de fuente extensa para simulación estocástica como las de Joyner y Boore (1986) o Beresnev y Atkinson (1998) en las que el resultado final es dependiente del número de subfallas utilizadas, lo que hace que la utilización del método no sea conveniente. Sin embargo, el trabajo de Motazedian y Atkinson (2005) soslaya esta dependencia basándose en la frecuencia de esquina dinámica. Es decir, la frecuencia de esquina del modelo es dependiente del tiempo y así la ruptura controla el contenido frecuencial de cada una de las subfuentes: 

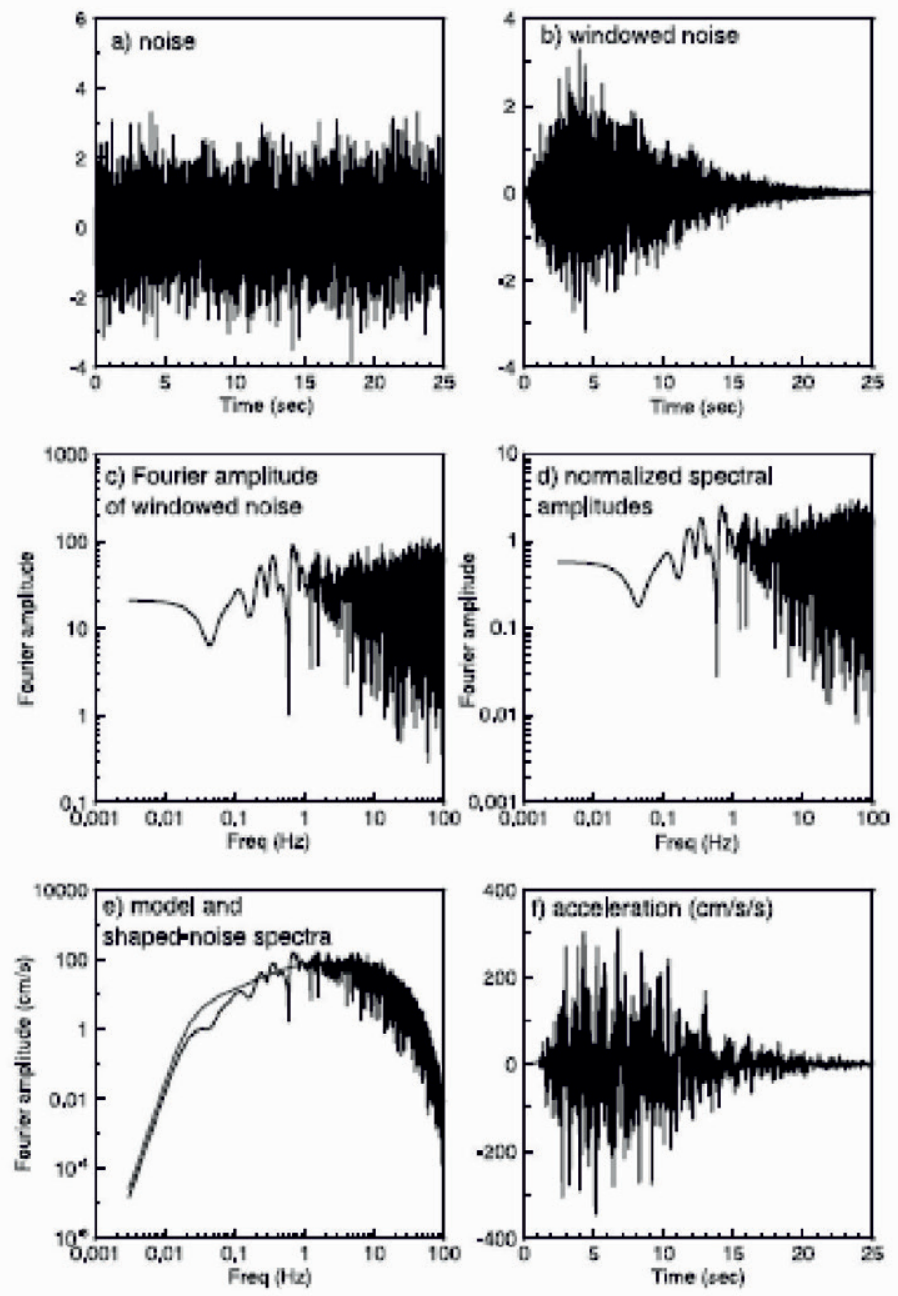

Figura 2.12: Proceso de simulación de movimientos sísmicos fuertes en el dominio del tiempo, por medio del método estocástico, con obtención del registro en aceleración. Tomado de Boore (2003). 


$$
f_{0 i j}(t)=N_{R}(t)^{-1 / 3} 4,9 E+6 V_{S}\left(\frac{\triangle \sigma}{M_{0 p r o}}\right)^{1 / 3}
$$

donde $M_{0 \text { pro }}$ es el valor medio del momento sobre las N subfallas, $N_{R}(t)$ es el número acumulado de subfallas que han roto en el tiempo t, E es la energía radiada total a altas frecuencias desde la falla completa, $V_{s}$ es la velocidad de las ondas S y $\triangle \sigma$ es la caída de esfuerzos.

Este modelo tiene además otras ventajas sobre los modelos anteriormente propuestos, y es que la energía radiada a altas frecuencias es independiente del número de subfallas utilizado. Además, es posible utilizar el modelo para sismos grandes, obteniéndose un control en la proporción de energía radiada a altas y bajas frecuencias.

La versión propuesta por Motazedian y Atkinson (2005) se ha materializado en el programa de cálculo EXSIM, que es el utilizado en esta tesis.

\subsubsection{Calibración del algoritmo de simulación}

Como se ha explicado, se ha seleccionado el programa EXSIM para realizar la simulación estocástica de los acelerogramas de un terremoto con suficientes registros acelerométricos ocurrido en El Hierro, con el fin de calibrar el algoritmo por medio de la comparación de estos registros con las aceleraciones calculadas en los puntos donde se sitúan las estaciones acelerométricas. En este contexto, el terremoto de calibración debe tener magnitud alta $(>4,5)$.

Para proceder a la calibración del algoritmo, en primer lugar se tratan los registros de aceleración del terremoto de calibración. Se calculan los espectros de respuesta en términos de pseudoaceleración para cada una de las componentes del acelerograma (Vertical, Este y Norte), para un amortiguamiento del \%5. También se obtiene la transformada de Fourier del registro de aceleración. A continuación, se simulan los acelerogramas sintéticos de los terremotos en los puntos donde se localizan las estaciones acelerométricas, y a partir de los acelerogramas sintéticos se obtienen los espectros de respuesta y la transformada de Fourier correspondientes.

El proceso de calibración comienza con la comparación de los acelerogramas registrados con los correspondientes acelerogramas sintéticos. A continuación, se realiza el análisis del ajuste de las transformadas de Fourier del registro de aceleración y del acelerograma calculado en cada estación, simultáneamente al análisis del ajuste del espectro de respuesta registrado al espectro de respuesta simulado. De esta forma, se estudian las posibles coincidencias en función de la frecuencia. Las diferencias observadas llevan a realizar un análisis de sensibilidad de los parámetros de entrada en el algoritmo, para que, a través de la modificación de los mismos, se reduzcan las discrepancias detectadas. 


\subsection{Generación de mapas de aceleraciones máxi- mas sintéticas y de intensidades sintéticas}

En este apartado de la metodología se explican los fundamentos del diseño de una herramienta o plugin de desarrollo del Sistema de Información Geográfica (SIG) QGIS, producto del proyecto oficial del Open Source Geospatial Foundation (OSGeo). Esta herramienta permite implementar en el SIG algunos de los resultados obtenidos en la tesis doctoral, con el fin de producir de forma automática mapas de aceleraciones máximas sintéticas (mapas de sacudidas) y de intensidades sintéticas ante la ocurrencia de un terremoto. La generación de estos mapas es la expresión de la simulación del escenario sísmico, que se realiza en base al primer nivel de aproximación a los valores sintéticos realistas de la aceleración sísmica, haciendo uso de las leyes de atenuación óptimas ya establecidas, y del mapa de amplificación sísmica. A partir de las aceleraciones máximas sintéticas que permiten obtener el mapa de sacudidas, la herramienta determinará las intensidades sintéticas.

\subsubsection{Antecedentes}

La metodología de Wald et al. (1999b) para la simulación de escenarios sísmicos se basa en la producción automática de mapas de aceleraciones máximas (PGA) y de velocidades máximas (PGV) del movimiento del terreno ante la ocurrencia de un terremoto en el sur de California, llamados ShakeMaps. La metodología seguida consiste en cuatro pasos bien diferenciados, donde cabe destacar la toma en consideración del efecto de sitio, a partir de la geología local. En primer lugar, se requiere calcular la aceleración o velocidad sintética en términos de roca, en una malla de puntos equiespaciados que cubra la región en estudio. Bajo una aproximación a una fuente puntual, se plantea la utilización de una relación de atenuación que permita obtener las aceleraciones o velocidades en roca.

Como segundo paso, se requiere conocer los valores de las aceleraciones y velocidades observadas en las estaciones de registro distribuidas en la región en estudio. Estos datos deben corregirse a condiciones de roca, para lo cual Wald et al. (1999b) utilizan los factores de amplificación sísmica en función de la frecuencia de Borcherdt (1994). Siguiendo la metodología de Borcherdt (1994), los autores han clasificado previamente las unidades geológicas locales en tres clases de emplazamiento, y han obtenido para cada estación de registro el factor de amplificación que le corresponde en función de la geología. A continuación, las aceleraciones y velocidades observadas, junto con las aceleraciones y velocidades sintéticas, se interpolan sobre una malla de puntos más densa que la inicial, obteniendo valores interpolados en roca.

Por último, los valores interpolados son amplificados por medio de los factores de amplificación que les corresponden en función de las características geológicas del emplazamiento de cada punto, de forma que se recuperan las aceleraciones y velocidades registradas inicialmente. Ajustando una función suave a la distribución de los valores interpolados, se obtiene un mapa de contornos de las aceleraciones máximas (mapa de sacudidas) y de las velocidades máximas. Wald et al. (1999b) también proponen la conversión de las PGA y las PGV simuladas en intensidades sísmicas en la Escala Modificada de Mercalli, haciendo uso de las correlaciones publicadas por Wald et al. (1999a). 
La metodología de Wald et al. (1999b) es utilizada por Geological Survey of Japan (2014) para la generación rápida de mapas de velocidades máximas y de intensidades sintéticas en Japón, los QuakeMaps. Estos mapas tienen en cuenta los registros de estaciones sísmicas y la capacidad de amplificación sísmica del terreno para todo el territorio a partir de un mapa de amplificación. Este mapa expresa la amplificación en términos de Vs30, y se ha obtenido a partir de datos sobre geología superficial y condiciones geomorfológicas.

Distintos códigos que permiten la simulación de escenarios sísmicos se encuentran actualmente a disposición tanto de los investigadores en la materia como de las autoridades responsables en la toma de decisiones sobre protección civil. Por este motivo, estos códigos se encuentran orientados a la simulación del riesgo sísmico en una determinada región ante la ocurrencia de un terremoto. En España, el código SES 2002 (Dirección General de Protección Civil y Dirección General del Instituto Geográfico Nacional, 2002) fue desarrollado con objeto de simular escenarios sísmicos en términos de intensidad, como paso previo a la estimación de los daños a personas y construcciones que produciría un terremoto de determinada magnitud en el ámbito de la Península Ibérica. Dada la magnitud mb del terremoto, el programa calcula la intensidad epicentral y, a continuación, selecciona una ley de atenuación de entre las publicadas por Martín Martín (1983) en función de la zona sismogenética donde se localiza el epicentro. Por medio de la relación de atenuación seleccionada, el programa calcula un valor de intensidad sintética en cualquier municipio a partir de la intensidad epicentral. Sin embargo, no se tiene en cuenta ninguna estimación de la amplificación sísmica del terreno para corregir las intensidades sintéticas calculadas.

Más recientemente, Fazendeiro Sá et al. (2016) han desarrollado una nueva herramienta de simulación de riesgo sísmico (Simulador de Risco Sísmico, SIRCO) para Portugal, en base a una metodología que podría ser aplicable al conjunto de la Península Ibérica. Al comparar los resultados de las simulaciones de terremotos históricos de Mw estimada superior a 6, los autores obtienen intensidades sintéticas equiparables a las isosistas previamente obtenidas, y también coherentes con otros programas de estimación del riesgo, como ERSTA (Fazendeiro Sá et al., 2016). La herramienta obtiene las intensidades sintéticas directamente, utilizando una serie de relaciones de atenuación en intensidad, y también combinaciones entre las mismas (particularmente los modelos de Esteva y Rosenblueth (1964) y de Oliveira y Sousa (1996)). El código tiene en cuenta la amplificación sísmica, a partir del contraste de impedancias (producto de la densidad y la Vs) entre los sedimentos y la roca.

\subsubsection{Cálculo de aceleraciones máximas sintéticas en la con- dición del terreno de referencia}

La herramienta plugin de cálculo de escenarios sísmicos en términos de aceleración incorpora los siguientes resultados a obtener en la tesis:

- Mapa de amplificación sísmica de la Península Ibérica y las Islas Baleares, y mapa de amplificación sísmica de las Islas Canarias. Ambos mapas se deben obtener en formato shapefile (.shp), de forma que sean fácilmente descargables y operativos en un entorno SIG. 
- Leyes de atenuación seleccionadas como óptimas en la Península Ibérica y en las Islas Baleares

- Leyes de atenuación seleccionadas como óptimas en las Islas Canarias

- Correlación entre la aceleración sísmica y la intensidad sísmica

Adicionalmente, también se implementan las 3 leyes de atenuación de alcance mundial referidas en la subsección 2.5.1, con el fin de posibilitar la aplicación del plugin en áreas geográficas más amplias que las de la Península Ibérica, las Islas Baleares y las Islas Canarias.

De acuerdo a la metodología de Wald et al. (1999b), el plugin se diseña para realizar los cálculos de aceleración máxima sintética por medio de las relaciones de atenuación. Se plantea un primer caso, en el que no se cuente con valores de aceleración observada en las estaciones de registro. En este supuesto, las variables de entrada del plugin comprenden, por una parte, los parámetros de localización del terremoto y la magnitud, y por otra las coordenadas de los puntos donde se van a calcular las aceleraciones:

- Coordenadas geográficas del epicentro

- Profundidad del hipocentro

- Magnitud del terremoto

- Puntos de cálculo, distribuidos en una malla de paso constante

Las coordenadas y la profundidad del foco sísmico serán empleadas para obtener la distancia epicentral o hipocentral entre el foco sísmico y los puntos de la malla de cálculo, de acuerdo con las variables predictivas de la relación de atenuación que se utilice. A partir de la distancia y de la magnitud, el plugin obtiene la aceleración máxima sintética en cada punto de la malla de cálculo por medio de las relaciones de atenuación.

La capacidad de amplificación sísmica del terreno es considerada en base a los mapas de amplificación de la Península Ibérica y de las Islas Baleares, y de las Islas Canarias, obtenidos de acuerdo con la sección 2.1. Entendiendo que, principalmente, el plugin trabajará en un intervalo de distancias epicentrales cortas, en cada punto de cálculo la herramienta obtiene factor de amplificación $\left(F_{a}\right)$ para altas frecuencias (2-10 Hz) o periodos cortos (0,1-0,5 s) (Borcherdt, 1994).

De acuerdo con la subsección 2.5.1, el valor de aceleración máxima sintética calculado en cada punto de la malla de cálculo $\left(a_{r e f}\right)$ está referido o corregido al material geológico de referencia de la ley de atenuación empleada. Este terreno de referencia coincide con el material geológico en el cual se han instalado los acelerómetros que han proporcionado los datos de aceleración que se han utilizado a su vez para obtener la ley de atenuación. En consecuencia, en cada modelo de atenuación es necesario conocer la condición del terreno de referencia, y también la clase de emplazamiento que le corresponde, de acuerdo con la clasificación de síntesis que sirve de soporte al mapa de amplificación sísmica (sección 2.1). El plugin debe ser 
programado de forma que incorpore el factor de amplificación $F_{a} *$ que le corresponde a cada ley de atenuación, en función de su terreno de referencia.

$\mathrm{Al}$ calcular la aceleración máxima sintética en cada punto de la malla de cálculo $\left(a_{r e f}\right)$, la herramienta la modifica con un factor de amplificación $F_{a}$ de la clase de emplazamiento donde se sitúa este punto, el cual ha sido previamente reescalado en función del factor de la clase de emplazamiento que le corresponde a cada ley de atenuación $\left(F_{a} *\right)$. Esta consideración implica que, en cada punto de cálculo el valor de $a_{r e f}$ es multiplicado por el cociente entre el factor de amplificación del punto $F_{a}$ y el factor de amplificación que se ha asociado a la ley de atenuación $\left(F_{a} *\right)$ :

$$
a_{\text {suelo }}=a_{r e f} \cdot \frac{F_{a}}{F_{a} *}
$$

donde $a_{\text {suelo }}$ el valor de aceleración máxima sintética final, resultado de la consideración de la amplificación sísmica.

No obstante, en el caso de que la relación de atenuación incluya un término de efecto de sitio, el plugin se programará para calcular la aceleración máxima tomando el término referido a roca. En este sentido, se tomará como $F_{a} *$ de la condición del terreno de referencia de la ley de atenuación el factor de amplificación de la clase de emplazamiento de la clasificación de síntesis que mejor se ajuste a la definición de roca dada por los autores del modelo de atenuación. Es decir, se elegirá la clase de emplazamiento con un rango de Vs30 coherente con el intervalo de Vs30 para el terreno definido como roca.

\subsubsection{Corrección de aceleraciones máximas registradas a la condición del terreno de referencia}

De acuerdo a la metodología utilizada por Wald et al. (1999b), el plugin debe considerar el caso de disponer de los valores de aceleración observada en las estaciones de registro. En este caso, se plantean como variables de entrada adicionales los valores de la aceleración máxima horizontal que han sido registrados en las estaciones acelerométricas en tiempo real. La metodología prevee la interpolación conjunta de las aceleraciones sintéticas que se han calculado con la relación de atenuación $\left(a_{r e f}\right)$ y las aceleraciones registradas, por lo cual es preciso incluir también una densificación de la malla de cálculo inicial. Por tanto, en este caso el plugin incorpora dos variables adicionales:

- Aceleración máxima horizontal registrada

- Malla de interpolación de paso constante

Wald et al. (1999b) proponen la corrección de las aceleraciones observadas a roca, pues las aceleraciones que calculan por medio de las relaciones de atenuación ya se encuentran expresadas en términos de roca. Sin embargo, en el diseño del plugin cada relación de atenuación que se utiliza tiene asignado un factor $F_{a} *$ para su terreno referencia, que es el que marca el nivel al cual se deben reducir o corregir las aceleraciones observadas. Como se ha explicado, las aceleraciones sintéticas $a_{r e f}$ que se han 
calculado con cada relación ya se expresan en términos de este terreno de referencia. Por tanto, es preciso corregir a este mismo nivel las aceleraciones registradas. Siendo $a_{o b s}$ un valor de aceleración registrado en un acelerómetro, esta corrección implica que se divide su valor por el cociente entre el factor $F_{a}$ del acelerómetro, de acuerdo con el mapa de amplificación, y el factor $F_{a} *$ del terreno de referencia de la relación de atenuación que se ha utilizado para calcular las aceleraciones sintéticas:

$$
a_{o b s-r e f}=\frac{a_{o b s}}{\frac{F_{a}}{F_{a}}}
$$

donde $a_{\text {obs-ref }}$ es el valor de aceleración observada, una vez corregida al terreno de referencia de la relación de atenuación (comúnmente roca).

$\mathrm{Al}$ incorporar las aceleraciones observadas en las estaciones acelerométricas, Wald et al. (1999b) consideran que los valores de aceleración sintética calculados en puntos de la malla de cálculo relativamente cercanos $(<30 \mathrm{~km}$, que es el paso de malla empleado por los autores) a las estaciones introducen una información redundante. Por este motivo, se programa el plugin para que elimine del proceso de cálculo las aceleraciones calculadas en puntos cuya distancia a cualquier estación sea igual o inferior al paso de la malla de cálculo.

\subsubsection{Interpolación de aceleraciones máximas sintéticas y re- gistradas y amplificación a suelo}

Las aceleraciones $a_{r e f}$ restantes y las aceleraciones observadas y corregidas al terreno de referencia $\left(a_{o b s-r e f}\right)$ son interpoladas sobre los puntos de la malla de interpolación, obteniendo en cada punto de esta malla un valor $a_{\text {int }}$. Las aceleraciones interpoladas serán llevadas de nuevo al nivel de amplificación original (condiciones de suelo), multiplicando por el cociente entre el factor $F_{a}$ de cada punto de la malla de interpolación, de acuerdo con el mapa de amplificación, y el factor $F_{a} *$ del terreno de referencia de la relación de atenuación, obteniéndose de este modo los valores finales de la aceleración máxima sintética:

$$
a_{\text {int-suelo }}=a_{\text {int }} \frac{F_{a}}{F_{a} *}
$$

donde $a_{\text {int-suelo }}$ es el valor de la aceleración máxima sintética final, resultado de la interpolación con las aceleraciones observadas y de la consideración de la amplificación sísmica.

\subsubsection{Producción de mapas de sacudidas y de intensidades sintéticas}

Las aceleraciones máximas sintéticas finales se obtienen en dos casos específicos:

1. Consideración del mapa de amplificación

2. Consideración del mapa de amplificación e incorporación de aceleraciones máximas horizontales observadas 
En el caso 1, el plugin realiza una interpolación bivariante a partir de los puntos de la malla de cálculo, con los valores de $a_{\text {suelo, }}$, de forma que crea un mapa de contornos de esta variable. Este mapa constituye el mapa de sacudidas final.

En el caso 2, el plugin realiza la misma interpolación a partir de los puntos de la malla de interpolación, con los valores de $a_{\text {int-suelo, }}$ para obtener el mapa de contornos que constituye el mapa de sacudidas final. En este caso, el plugin también calcula los valores de intensidad en cada punto de la malla de interpolación. Las aceleraciones $a_{\text {int-suelo }}$ son convertidas a intensidades sintéticas en escala EMS98, haciendo uso de una determinada correlación entre la aceleración sísmica y la intensidad sísmica a obtener de acuerdo con la subsección 2.5.4. 


\section{Capítulo 3}

\section{MAPA DE AMPLIFICACIÓN SÍSMICA DE LA PENÍNSULA IBÉRICA, LAS ISLAS BALEARES Y LAS ISLAS CANARIAS}

En una primera aproximación, se pretende cuantificar la capacidad de amplificación sísmica de los materiales aflorantes en la Península Ibérica, las Islas Baleares y las Islas Canarias por medio de un mapa de amplificación sísmica para el territorio. Para generar el mapa, se han utilizado dos bases de partida bien diferenciadas: cuatro clasificaciones de suelos y rocas en función de su capacidad de amplificación sísmica, publicadas en la literatura sismológica, y las unidades geológicas descritas por la cartografía geológica oficial de España. De acuerdo con la metodología de Borcherdt (1994), las unidades geológicas han sido clasificadas de acuerdo a las clases de emplazamiento de una clasificación de síntesis, la cual ha sido previamente diseñada teniendo en cuenta los criterios empleados por las clasificaciones de partida. Las clases de emplazamiento de la clasificación de síntesis se caracterizan por un intervalo de valores de Vs30, entre otras propiedades geotécnicas. Como resultado del proceso de clasificación de las unidades geológicas, se ha obtenido un primer mapa de clases de emplazamiento que informa sobre el rango de Vs30 en cualquier punto del mismo. De acuerdo con la metodología citada, se han calculado los factores de amplificación para periodos cortos $\left(F_{a}\right)$ y periodos medios $\left(F_{v}\right)$ del movimiento del terreno que corresponden a cada clase de emplazamiento, con el fin de asignar estos factores a las unidades geológicas en función de la clase a la cual pertenecen. La representación de la distribución espacial de las unidades, una vez que se les han asignado sus correspondientes valores de $F_{a} F_{v}$, constituye el mapa de amplificación sísmica del territorio. 


\subsection{Base de partida 1: Clasificaciones de suelos y rocas según su capacidad de amplificación sís- mica}

Por medio de una investigación bibliográfica preliminar, se han estudiado cuatro clasificaciones de suelos y rocas en función de su capacidad de amplificación sísmica. A este respecto, se ha analizado la clasificación de Borcherdt (1994), la clasificación incluida en el Uniform Building Code (UBC) de 1997 (International Council of Building Officials, 1997) y las dos clasificaciones previstas por las normativas de construcción sismorresistente del NEHRP de 1997 y 2003 (Building Seismic Safety Council, 1997, 2004).

Borcherdt (1994) (Tabla 2.1) utiliza como criterios de clasificación la descripción geotécnica, el intervalo de valores de Vs30 y el espesor mínimo del material que se pretende analizar. Respecto al intervalo de valores de Vs30, se indican el valor mínimo, máximo y promedio. Asimismo, la clasificación que propone distingue cuatro clases de emplazamiento principales (SC-I, SC-II, SC-III y SC-IV). No obstante, y dado que las clases SC-I y SC-IV se subdividen a su vez en dos subclases (SC-Ia, SC-Ib, SC-IVa, SC-IVb), esta clasificación permite establecer seis clases de emplazamiento diferentes. Puede destacarse que el reconocimiento de la subclase SC-IVb (suelos blandos de estudio específico) exige llevar a cabo investigaciones geotécnicas in situ.

De acuerdo con International Council of Building Officials (1997), el UBC de 1997 diferencia seis clases de emplazamiento, llamadas tipos de perfiles de suelo $\left(S_{A}, S_{B}\right.$, $\left.S_{C}, S_{D}, S_{E}, S_{F}\right)$, según el promedio de tres propiedades geotécnicas en los primeros 100 pies $(30,480 \mathrm{~m})$ de profundidad: velocidad de onda de corte, resistencia según el ensayo SPT y resistencia a cortante y sin drenar (Tabla 3.1). Además, establece la descripción genérica de cada perfil expresando su compacidad y consistencia. Por ejemplo, el perfil $S_{C}$ se describe como suelo muy denso y roca blanda, deduciéndose que este tipo de perfil corresponde a un suelo muy compacto o bien a una roca de poca consistencia. Por tanto, se puede afirmar que los criterios empleados por esta clasificación son equiparables a la Vs30, a la resistencia según el ensayo SPT y la resistencia a cortante y sin drenar en los primeros $30 \mathrm{~m}$ de profundidad, y a la descripción geotécnica en términos de compacidad y consistencia.

Según Bachmann y Bonneville (2000), el tipo de perfil de suelo $S_{E}$ también engloba a cualquier perfil de suelo con una potencia de más de 10 pies $(3,048 \mathrm{~m})$ de arcilla blanda. En este sentido, se define la arcilla blanda como un suelo con Índice de Plasticidad $P I>20$, contenido en humedad $w_{m c} \geq 40 \%$ y resistencia a cortante y $\sin$ drenar $s_{u}<25 \mathrm{kPa}$.

La normativa de construcción sismorresistente del NEHRP de 1997 (Building Seismic Safety Council, 1997) presenta una clasificación de emplazamientos en función de su respuesta sísmica que prevee 6 clases (A-F). Asimismo, recurre a los criterios geotécnicos siguientes: descripción geotécnica, velocidad Vs30, resistencia media a cortante y sin drenar en los primeros $30 \mathrm{~m}$ de profundidad y la resistencia media según el ensayo SPT en los primeros $30 \mathrm{~m}$ de profundidad (Tabla 3.2).

De acuerdo a Building Seismic Safety Council (1997), la normativa del NEHRP recomienda utilizar la clase D cuando las propiedades del suelo no sean conocidas con 
suficiente detalle para poder asignar una determinada clase. No es necesario asumir la clase $\mathrm{E}$ o $\mathrm{F}$ a menos que la autoridad competente determine que estas clases podrían estar presentes en el emplazamiento, o bien la clase E o F sea establecida por datos geotécnicos.

La clasificación de la normativa sismorresistente del NEHRP de 2003 (Building Seismic Safety Council, 2004) es prácticamente idéntica a la clasificación incluida en la normativa de 1997 (Building Seismic Safety Council, 1997). Nuevamente, se presentan 6 clases de emplazamiento (A-F), que se diferencian en base a su descripción geotécnica, a su valor de la velocidad media de onda de corte ante esfuerzos cortantes pequeños en los primeros $30 \mathrm{~m}$ de profundidad, y a sus valores de la resistencia media a cortante y sin drenar y de la resistencia media según el ensayo SPT en los primeros $30 \mathrm{~m}$ de profundidad (Tabla 3.3).

Se entiende que la velocidad media de onda de corte ante esfuerzos cortantes pequeños en los primeros $30 \mathrm{~m}$ de profundidad es equiparable a la Vs30 y que, por tanto, esta clasificación utiliza los mismas propiedades geotécnicas que Building Seismic Safety Council (1997). No obstante, se ha observado una diferencia respecto a la clasificación de 1997, pues la descripción geotécnica de uno de los suelos característicos de la clase $\mathrm{F}$ (arcillas de consistencia blanda a medio dura de potencia mayor que $36 \mathrm{~m}$ ) es ampliada, incluyéndose el valor de resistencia media a cortante y sin drenar (Tabla 3.3).

La normativa del NEHRP de 2003 (Building Seismic Safety Council, 2004) permite asignar la clase D en aquellos emplazamientos donde las propiedades del suelo no sean conocidas con suficiente detalle para poder determinar una clase de acuerdo a la Tabla 3.3, a menos que la autoridad competente determine que las clases $\mathrm{E}$ o $\mathrm{F}$ podrían ser aplicables en el emplazamiento, o bien la clase E o F sea establecida a partir de datos geotécnicos.

\subsubsection{Clasificación de síntesis}

Las cuatro clasificaciones que se han analizado se basan en dos criterios geotécnicos comunes, la descripción geotécnica en términos de compacidad y consistencia y el intervalo de Vs30. De acuerdo a estos dos criterios, las cuatro clasificaciones establecen seis clases de emplazamiento, desde rocas duras hasta suelo blandos que requieren de investigaciones geotécnicas in situ para su valoración. Se ha constatado que cada una de las clases de emplazamiento de una clasificación equivale a una y sólo una clase de cada una de las restantes clasificaciones, ya que la descripción geotécnica proporcionada por cada clasificación es razonablemente parecida a la descripción geotécnica procedente de las restantes clasificaciones. Además, los rangos de Vs30 que caracterizan a una determinada clase son coincidentes en todas las clasificaciones. Adicionalmente, los intervalos de resistencia media según el ensayo SPT y de resistencia media a cortante y sin drenar en los primeros $30 \mathrm{~m}$ de profundidad, que se pueden consultar en las clasificaciones del UBC y NEHRP (International Council of Building Officials, 1997; Building Seismic Safety Council, 1997, 2004), tampoco varían.

Por ejemplo, la clase SC-Ia de la clasificación de Borcherdt (1994) equivale a la clase $S_{A}$ de la clasificación del UBC de 1997 (International Council of Building 
Officials, 1997) y a la clase A de las clasificaciones del NEHRP de 1997 y 2003 (Building Seismic Safety Council, 1997, 2004). Esta afirmación se basa en la igualdad de las descripciónes geotécnicas proporcionadas por las clasificaciones (Roca dura en Tabla 2.1, Tabla 3.1, Tabla 3.2 y Tabla 3.3), así como en la similitud en los intervalos de Vs30. A este respecto, Borcherdt (1994) indica que Vs30>1400 m/s (Tabla 2.1), mientras que el resto de las clasificaciones presentan una Vs30 >1500 $\mathrm{m} / \mathrm{s}$ (Tabla 3.1, Tabla 3.2 y Tabla 3.3).

Dado que cada clasificación contiene una clase de emplazamiento que tiene su equivalente en cada una de las restantes clasificaciones, ha sido posible construir una clasificación de síntesis (Tabla 3.4). Esta nueva clasificación presenta las seis clases de emplazamiento equivalentes que se encuentran las clasificaciones de partida, e incorpora los criterios geotécnicos de estas clasificaciones. La clasificación de síntesis, por tanto, se articula según las siguientes cinco propiedades geotécnicas:

- Descripción geotécnica (en términos de compacidad y consistencia)

- $\operatorname{Vs} 30(\mathrm{~m} / \mathrm{s})$

- Resistencia media según el ensayo SPT en los primeros $30 \mathrm{~m}$ de profundidad (golpes/pie)

- Resistencia media a cortante y sin drenar en los primeros $30 \mathrm{~m}$ de profundidad $(\mathrm{kPa})$

- Espesor mínimo (m)

Cada clase de emplazamiento de la clasificación de síntesis presenta las propiedades geotécnicas de las clases equivalentes de cada clasificación de partida. Asimismo, presenta una respuesta sísmica determinada o capacidad de amplificación sísmica. 
Tabla 3.1: Tipos de perfil de suelo según el Uniform Building Code de 1997 (International Council of Building Officials, 1997).

\begin{tabular}{|c|c|c|c|c|}
\hline \multirow{3}{*}{$\begin{array}{l}\text { Tipo de perfil de } \\
\text { suelo }\end{array}$} & \multicolumn{4}{|c|}{ Propiedades para los primeros 100 pies } \\
\hline & \multicolumn{4}{|c|}{ Promedio de valores } \\
\hline & $\begin{array}{l}\text { Denominación / } \\
\text { Descripción ge- } \\
\text { nérica }\end{array}$ & $\begin{array}{l}\text { Velocidad de on- } \\
\text { da de corte, } \bar{v}_{s} \\
(\mathrm{~m} / \mathrm{s})\end{array}$ & $\begin{array}{l}\text { Ensayo SPT, } N \\
\text { (o } \bar{N}_{C H} \text { para } \\
\text { capas de sue- } \\
\text { lo no cohesivo) } \\
\text { (golpes/pie) }\end{array}$ & $\begin{array}{l}\text { Resistencia } \\
\text { a cortante } y \\
\text { sin drenar, } \bar{S}_{u} \\
(\mathrm{kPa})\end{array}$ \\
\hline$S_{A}$ & Roca dura & $>1500$ & & \\
\hline$S_{B}$ & Roca & 760 a 1500 & & \\
\hline$S_{C}$ & $\begin{array}{l}\text { Suelo muy den- } \\
\text { so y roca blanda }\end{array}$ & 360 a 760 & $>50$ & $>100$ \\
\hline$S_{D}$ & $\begin{array}{lll}\begin{array}{l}\text { Perfil de } \\
\text { duro }\end{array} & \\
\end{array}$ & 180 a 360 & 15 a 50 & 50 a 100 \\
\hline$S_{E}$ & $\begin{array}{l}\text { Perfil de suelo } \\
\text { blando }\end{array}$ & $<180$ & $<15$ & $<50$ \\
\hline$S_{F}$ & \multicolumn{4}{|c|}{ Suelo que requiere evaluación específica in situ } \\
\hline
\end{tabular}


Tabla 3.2: Definiciones de clases de emplazamiento según la normativa de construcción sismorresistente del NEHRP de 1997 (Building Seismic Safety Council, 1997). PI es el Índice de Plasticidad, $w$ es el contenido en humedad, $s u$ es la resistencia a cortante y sin drenar y $H$ la potencia del suelo.

\begin{tabular}{|c|c|c|c|c|}
\hline $\begin{array}{l}\text { Clase de empla- } \\
\text { zamiento }\end{array}$ & Descripción geotécnica & $\operatorname{Vs} 30(\mathrm{~m} / \mathrm{s})$ & $\begin{array}{l}\text { Resistencia } \\
\text { media según } \\
\text { el ensayo } \\
\text { SPT en los } \\
\text { primeros } \\
30 \text { m de } \\
\text { profundidad, } \\
\bar{N} \text { (golpes) }\end{array}$ & $\begin{array}{l}\text { Resistencia } \\
\text { media a } \\
\text { cortante y } \\
\text { sin drenar en } \\
\text { los primeros } \\
30 \text { m de } \\
\text { profundidad, } \\
\bar{s}_{u}(\mathrm{kPa})\end{array}$ \\
\hline A & Roca dura & $V s 30>1500$ & & \\
\hline B & Roca & $760<V s 30 \leq 1500$ & & \\
\hline $\mathrm{C}$ & $\begin{array}{l}\text { Suelo muy denso y roca blan- } \\
\text { da }\end{array}$ & $360<V s 30 \leq 760$ & $\bar{N}>50$ & $\bar{s}_{u}>100$ \\
\hline $\mathrm{D}$ & Suelo duro & $180 \leq V s 30 \leq 360$ & $15 \leq \bar{N} \leq 50$ & $\begin{array}{l}50 \leq \bar{s}_{u} \leq \\
100\end{array}$ \\
\hline \multirow{2}{*}{$\mathrm{E}$} & Perfil de suelo & $V s 30<180$ & $\bar{N}<15$ & $\bar{s}_{u}<50$ \\
\hline & $\begin{array}{l}\text { Perfil con una potencia de } \\
\text { más de } 3 \mathrm{~m} \text { de arcilla blanda, } \\
\text { definida como un suelo con } \\
P I>20, w \geq 40 \% \text { y } s_{u}<25 \\
\mathrm{kPa}\end{array}$ & & & \\
\hline $\mathrm{F}$ & $\begin{array}{l}\text { Suelos que requieren evalua- } \\
\text { ciones específicas in situ: } \\
\text { 1. Suelos susceptibles de ro- } \\
\text { tura o colapso bajo carga sís- } \\
\text { mica tales como suelos li- } \\
\text { cuefactibles, arcillas deforma- } \\
\text { bles (quick and highly sensi- } \\
\text { tive clays }) \text {, suelos colapsables } \\
\text { débilmente cementados } \\
\text { 2. Turbas y/o arcillas alta- } \\
\text { mente orgánicas ( } H>3 \text { m } \\
\text { de turba y/o arcilla altamen- } \\
\text { te orgánica) } \\
\text { 3. Arcillas de muy alta plasti- } \\
\text { cidad ( } H>8 \text { m con } P I>75) \\
\text { 4. Arcillas de consistencia } \\
\text { blanda a medio dura muy po- } \\
\text { tentes }(H>36 \text { m) }\end{array}$ & & & \\
\hline
\end{tabular}


Tabla 3.3: Definiciones de clases de emplazamiento según la normativa de construcción sismorresistente del NEHRP de 2003 (Building Seismic Safety Council, 2004). PI es el Índice de Plasticidad, $w$ es el contenido en humedad, $s_{u}$ es la resistencia a cortante y sin drenar y $H$ la potencia del suelo.

\begin{tabular}{|c|c|c|c|c|}
\hline $\begin{array}{l}\text { Clase de empla- } \\
\text { zamiento }\end{array}$ & Descripción geotécnica & $\operatorname{Vs} 30(\mathrm{~m} / \mathrm{s})$ & $\begin{array}{l}\text { Resistencia } \\
\text { media según } \\
\text { el ensayo } \\
\text { SPT en los } \\
\text { primeros } \\
30 \text { m de } \\
\text { profundidad, } \\
\bar{N} \text { (golpes) }\end{array}$ & $\begin{array}{l}\text { Resistencia } \\
\text { media a } \\
\text { cortante y } \\
\text { sin drenar en } \\
\text { los primeros } \\
30 \mathrm{~m} \text { de } \\
\text { profundidad, } \\
\bar{s}_{u}(\mathrm{kPa})\end{array}$ \\
\hline $\mathrm{A}$ & Roca dura & $V s 30>1500$ & & \\
\hline $\mathrm{B}$ & Roca & $760<V s 30 \leq 1500$ & & \\
\hline $\mathrm{C}$ & $\begin{array}{l}\text { Suelo muy denso y roca blan- } \\
\text { da }\end{array}$ & $360<V s 30 \leq 760$ & $\bar{N}>50$ & $\bar{s}_{u}>100$ \\
\hline $\mathrm{D}$ & Suelo duro & $180 \leq V s 30 \leq 360$ & $15 \leq \bar{N} \leq 50$ & $\begin{array}{l}50 \leq \bar{s}_{u} \leq \\
100\end{array}$ \\
\hline \multirow{2}{*}{$\mathrm{E}$} & Perfil de suelo & $V s 30<180$ & $N<15$ & $\bar{s}_{u}<50$ \\
\hline & $\begin{array}{l}\text { Perfil de suelo con una poten- } \\
\text { cia de más de } 3 \mathrm{~m} \text { de arcilla } \\
\text { blanda, definida como un sue- } \\
\text { lo con } P I>20, w \geq 40 \% \text { y } \\
s_{u}<25 \mathrm{kPa}\end{array}$ & & & \\
\hline $\mathrm{F}$ & $\begin{array}{l}\text { Suelos que requieren evalua- } \\
\text { ciones específicas in situ: } \\
\text { 1. Suelos susceptibles de ro- } \\
\text { tura o colapso bajo carga sís- } \\
\text { mica tales como suelos li- } \\
\text { cuefactibles, arcillas deforma- } \\
\text { bles (quick and highly sensi- } \\
\text { tive clays), suelos colapsables } \\
\text { débilmente cementados } \\
2 \text {. Turba y/o arcillas altamen- } \\
\text { te orgánicas ( } H>3 \text { m de tur- } \\
\text { ba y/o arcilla altamente orgá- } \\
\text { nica) } \\
3 \text {. Arcillas de muy alta plasti- } \\
\text { cidad ( } H>8 \text { m con } P I>75) \\
4 . \text { Arcillas de consistencia } \\
\text { blanda a medio dura muy po- } \\
\text { tentes }\left(H>36 \text { m) con } s_{u}\right. \\
<50 \mathrm{kPa}\end{array}$ & & & \\
\hline
\end{tabular}




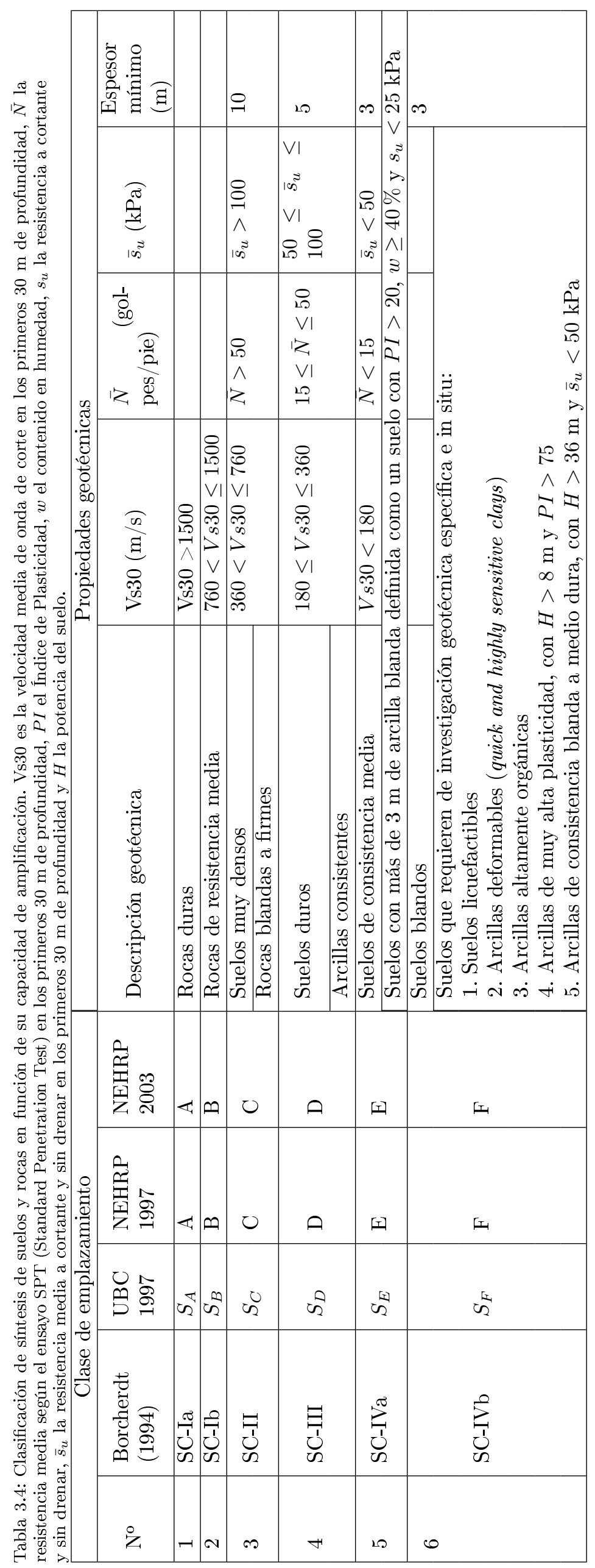




\subsection{Base de partida 2: Cartografía geológica 1:1.000.000 de la Península Ibérica y las Islas Baleares y cartografía geológica 1:50.000 de las Islas Ca- narias}

Las unidades geológicas aflorantes en el territorio de la Península Ibérica, las Islas Baleares y las Islas Canarias han sido analizadas de acuerdo con la información proporcionada por la cartografía geológica oficial de España. Debido a la gran diferencia existente entre las características geológicas de la Península Ibérica y las Islas Baleares y las mismas características de las Islas Canarias, el estudio se ha realizado en dos partes bien diferenciadas. En primer lugar, se ha revisado el Mapa Geológico de España a escala 1:1.000.000 (Instituto Geológico y Minero de España, 1994), con el fin de analizar las unidades geológicas aflorantes en la Península Ibérica e Islas Baleares. En segundo lugar, las unidades correspondientes al archipiélago canario se han estudiado según la cartografía geológica continua GEODE de las Islas Canarias, a escala 1:50.000 (GEODE, 2014).

Según el Mapa Geológico de España a escala 1:1.000.000 (Instituto Geológico y Minero de España, 1994), en la Península Ibérica y las Islas Baleares se diferencian 134 unidades geológicas (Figura 3.1). Estas unidades son descritas por medio de distintas características: dominio geológico, litología genérica, descripción litológica y edad cronoestratigráfica (detallada hasta el nivel de piso). Adicionalmente, algunas unidades son descritas también de acuerdo a su facies distintiva. Todas las unidades se identifican por un número de referencia o sigla, que aparece en la leyenda del Mapa Geológico.

En esta cartografía, las unidades son caracterizadas por medio de 102 descripciones litológicas (Figura 3.1). En función de la descripción litológica, las unidades se encuadran en diferentes grupos con una litología genérica determinada, los cuales a su vez pueden corresponder o no a un dominio geológico. En la Tabla 3.5 se muestran las litologías genéricas correspondientes a cada una de las 134 unidades geológicas presentes en la Península Ibérica y las Islas Baleares. 

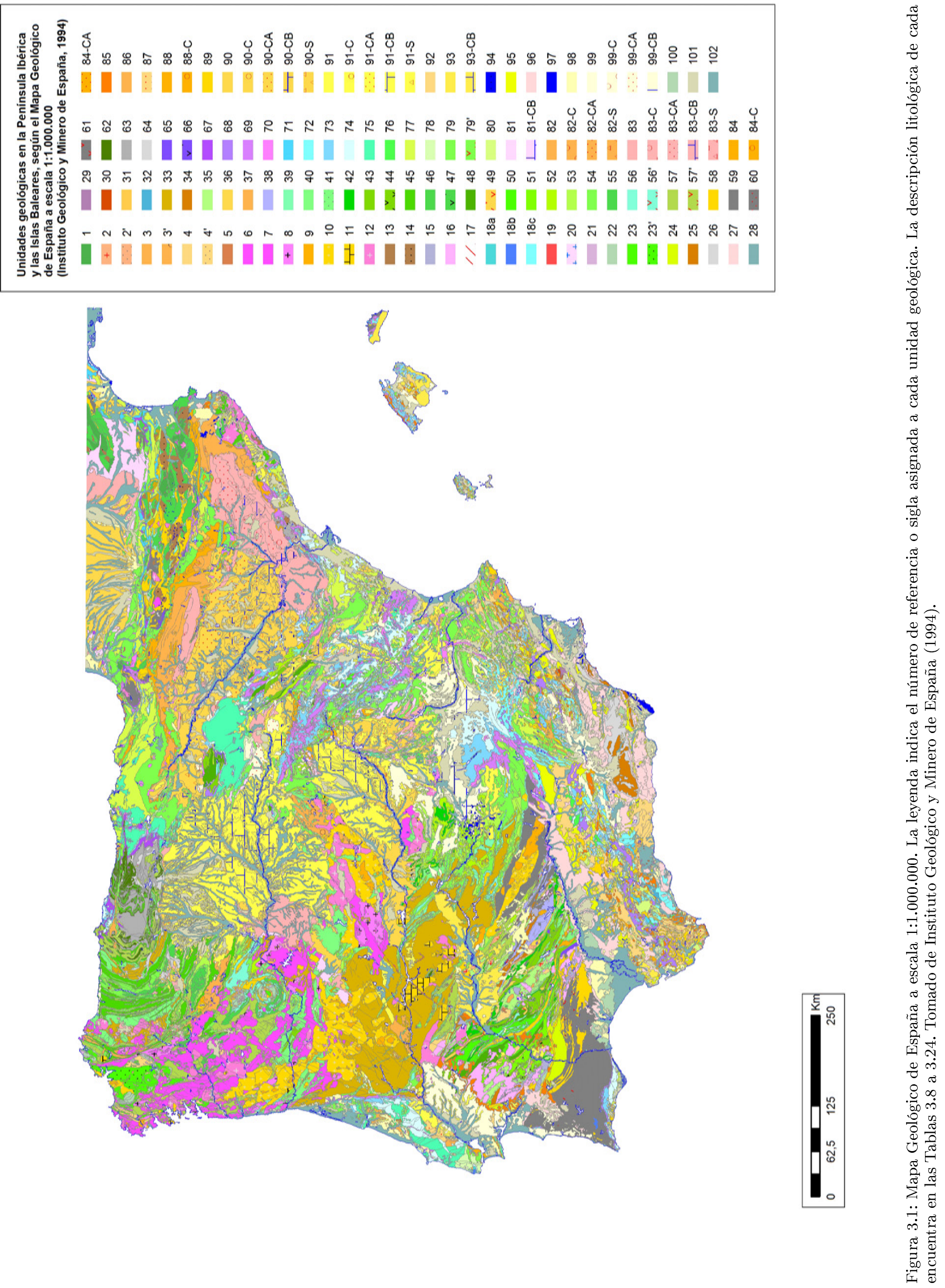
Tabla 3.5: Litologías genéricas correspondientes a las unidades geológicas de la Península Ibérica y las Islas Baleares, de acuerdo con el Mapa Geológico de España a escala 1:1.000.000 (Instituto Geológico y Minero de España, 1994). $\mathrm{C}$ indica litofacies de conglomerados, CA litofacies de conglomerados y areniscas, CB litofacies carbonatada y $\mathrm{S}$ litofacies sulfatada.

\begin{tabular}{|c|c|}
\hline Unidad geológica & Litología genérica \\
\hline $1,2,2 ', 3,3,4,4,5,6,7,8$ & Granitoides de emplazamiento meso-catazonal \\
\hline 9,10 & Granitoides de emplazamiento epi-mesozonal \\
\hline 11,12 & $\begin{array}{l}\text { Granitoides postcinematicos de emplazamien- } \\
\text { to epizonal }\end{array}$ \\
\hline 13,14 & Plutonismo orogénico colisional peralumínico \\
\hline 15,16 & Plutonismo orogénico calcoalcalino toleítico \\
\hline 17 & Granitoides alcalinos postcinemáticos \\
\hline $18 \mathrm{a}, 18 \mathrm{~b}, 18 \mathrm{c}, 19,20$ & Rocas plutónicas alpinas \\
\hline 21 & Rocas básicas y ultrabásicas \\
\hline $22,23,23,24,25,26,27,28$ & Rocas metamórficas \\
\hline $29,30,31,32$ & Rocas sedimentarias (Béticas; zonas internas) \\
\hline $33,34,35,36,37,38,39$ & Gneises; esquistos; mármoles y vulcanitas \\
\hline $\begin{array}{l}40,41,42,43,44,45,46,47,48,49,50,51, \\
52,53,54,55\end{array}$ & $\begin{array}{l}\text { Cuarcitas; pizarras; areniscas; calizas y vulca- } \\
\text { nitas }\end{array}$ \\
\hline $56,56^{\prime}, 57,57^{\prime}, 58$ & Areniscas; pizarras y calizas \\
\hline $59,60,61,62,63,64$ & $\begin{array}{l}\text { Conglomerados; areniscas; pizarras; calizas y } \\
\text { vulcanitas; Carbón }\end{array}$ \\
\hline 65,66 & Conglomerados; areniscas y lutitas; Vulcanitas \\
\hline $67,68,69,70$ & $\begin{array}{l}\text { Conglomerados; areniscas; calizas; yesos y ar- } \\
\text { cillas versicolores }\end{array}$ \\
\hline $71,72,73,74,75$ & $\begin{array}{l}\text { Calizas; dolomías y margas; Conglomerados y } \\
\text { areniscas }\end{array}$ \\
\hline $76,77,78,79,79^{\prime}$ & Calizas; dolomías y margas; Areniscas \\
\hline $\begin{array}{l}\text { 80, 81, 81-CB, 82, 82-C, 82-CA, 82-S, 83, 83-C, } \\
\text { 83-CA, 83-CB, 83-S }\end{array}$ & $\begin{array}{l}\text { Conglomerados; areniscas; arcillas y calizas; } \\
\text { Evaporitas }\end{array}$ \\
\hline $84,84-\mathrm{C}, 84-\mathrm{CA}, 85,86$ & Turbiditas calcáreas \\
\hline $\begin{array}{l}87,88,88-\mathrm{C}, 89,90,90-\mathrm{C}, 90-\mathrm{CA}, 90-\mathrm{CB}, 90-\mathrm{S} \\
\text { 91, 91-C, 91-CA, 91-CB, 91-S, 92, 93, 93-CB, } \\
94,95,96,97,98,99,99-\mathrm{C}, 99-\mathrm{CA}, 99-\mathrm{CB}, 100\end{array}$ & $\begin{array}{l}\text { Conglomerados; areniscas; arcillas; calizas y } \\
\text { evaporitas; Vulcanitas básicas }\end{array}$ \\
\hline 101,102 & Conglomerados; gravas; arenas y limos \\
\hline
\end{tabular}

La cartografía geológica continua GEODE de las Islas Canarias a escala 1:50.000 permite diferenciar 804 unidades geológicas en las Islas Canarias. Está cartografía está constituida por diferentes regiones GEODE, cada una de las cuales abarca el territorio de una isla en concreto (GEODE, 2014). En la Tabla 3.6 se muestra la correspondencia entre cada isla y la región GEODE que coincide con su territorio.

Cada región GEODE se compone a su vez por un número determinado de unidades. Estas unidades son agrupadas en sistemas, dominios, edificios, ciclos volcánicos o macizos plutónicos, de acuerdo a la leyenda cronoestratigráfica del mapa de la región en cuestión. En cuanto a los dominios y ciclos volcánicos, éstos pueden subdividirse en subdominios y subdivisiones, respectivamente. Adicionalmente, pueden especificarse formaciones y miembros como entidades agrupadoras de las unidades.

Cada unidad es caracterizada con referencia a su litología y edad cronoestratigráfica a nivel de serie. También es definida por un código alfanumérico, que aparece como rótulo en la leyenda cronoestratigráfica del mapa de la región GEODE correspondiente.

Con respecto a la litología, la información proporcionada por la cartografía GEODE consta de: 
- Descripción de la unidad geológica

- Descripción extensa de la unidad geológica. Consiste en información complementaria sobre las características de la unidad en cuanto a aspectos morfológicos, petrofísicos y litológicos, entre otros.

Aunque la cartografía GEODE proporciona información sobre 804 unidades diferentes en el archipiélago canario, sólo aparecen representadas 759 unidades en el conjunto de los mapas de todas las regiones GEODE. Esta circunstancia se debe a la estructura de los datos alfanuméricos que da soporte a la cartografía, que consiste en tablas de datos interrelacionadas entre sí a partir de campos o columnas comunes (GEODE, 2014). En este contexto, la tabla DESC_LITOLOGIA presenta la descripción litológica, la descripción litológica extensa y el código alfanumérico de cada unidad, por medio de los campos LIT_NOMBRE, LIT_LEYENDA y LIT_COD_LEYENDA, respectivamente (Tabla 3.7). La tabla LIT_DESC contiene los identificadores de los polígonos que representan los afloramientos de cada unidad, contenidos en el campo LIT_ID. Ambas tablas tienen en común un campo LIT_CODIGO, que es único para cada unidad, como se ilustra en la Tabla 3.7. Existen 804 valores distintos del campo LIT_CODIGO en la tabla DESC_LITOLOGIA, pero este número se reduce a 784 en la tabla LIT_DESC. Es decir, existen 20 valores de LIT_CODIGO sin polígono asignado, lo cual implica que existen 20 unidades sin afloramiento cartografiado. Adicionalmente, 25 unidades con polígono asignado no aparecen representadas en los mapas de las regiones GEODE que les corresponden. Por tanto, se tiene un total de 45 unidades geológicas cuyos afloramientos no tienen representación espacial en la cartografía. De este hecho se deriva que sólo 759 unidades geológicas están realmente cartografiadas, aunque la cartografía GEODE (GEODE, 2014) incluya la descripción de 804 unidades. En este punto, cabe especificar que una de estas unidades (LIT_CODIGO 0) está reservada para masas de agua o recintos sin codificar. Por tanto, la cartografía GEODE representa los afloramientos de 758 unidades geológicas susceptibles de ser analizadas y clasificadas de acuerdo a la clasificación de síntesis (Tabla 3.4).

En las Figuras 3.2, 3.3, 3.4, 3.5, 3.6, 3.7 y 3.8 se presentan los mapas geológicos de las regiones GEODE correspondientes a las islas de El Hierro, Fuerteventura, Gran Canaria, La Gomera, La Palma, Lanzarote y Tenerife, respectivamente. Asimismo, la descripción de las características litológicas de las unidades geológicas de estas islas se encuentra en las Tablas A.1, A.2, A.3, A.4, A.5, A.6 y A.7, de acuerdo con el orden en el que se han citado.

Tabla 3.6: Regiones GEODE que corresponden a las Islas Canarias, según la cartografía geológica continua GEODE a escala 1:50.000 (GEODE, 2014).

\begin{tabular}{|l|l|}
\hline Isla & Región GEODE \\
\hline El Hierro & 2916 \\
\hline Fuerteventura & 2911 \\
\hline Gran Canaria & 2912 \\
\hline La Gomera & 2914 \\
\hline La Palma & 2915 \\
\hline Lanzarote & 2910 \\
\hline Tenerife & 2913 \\
\hline
\end{tabular}


Tabla 3.7: Tablas DESC_LITOLOGIA y LIT_DESC, y atributos correspondientes, de acuerdo a la base de datos de la cartografía geológica continua GEODE a escala 1:50.000 (GEODE, 2014).

\begin{tabular}{|l|l|}
\hline Tabla & Atributo \\
\hline \multirow{4}{*}{ DESC_LITOLOGIA } & LIT_COD_LEYENDA \\
\cline { 2 - 2 } & LIT_CODIGO \\
\cline { 2 - 2 } & LIT_LEYENDA \\
\cline { 2 - 2 } & LIT_NOMBRE \\
\hline \multirow{2}{*}{ LIT_DESC } & LIT_CODIGO \\
\cline { 2 - 2 } & LIT_ID \\
\hline
\end{tabular}

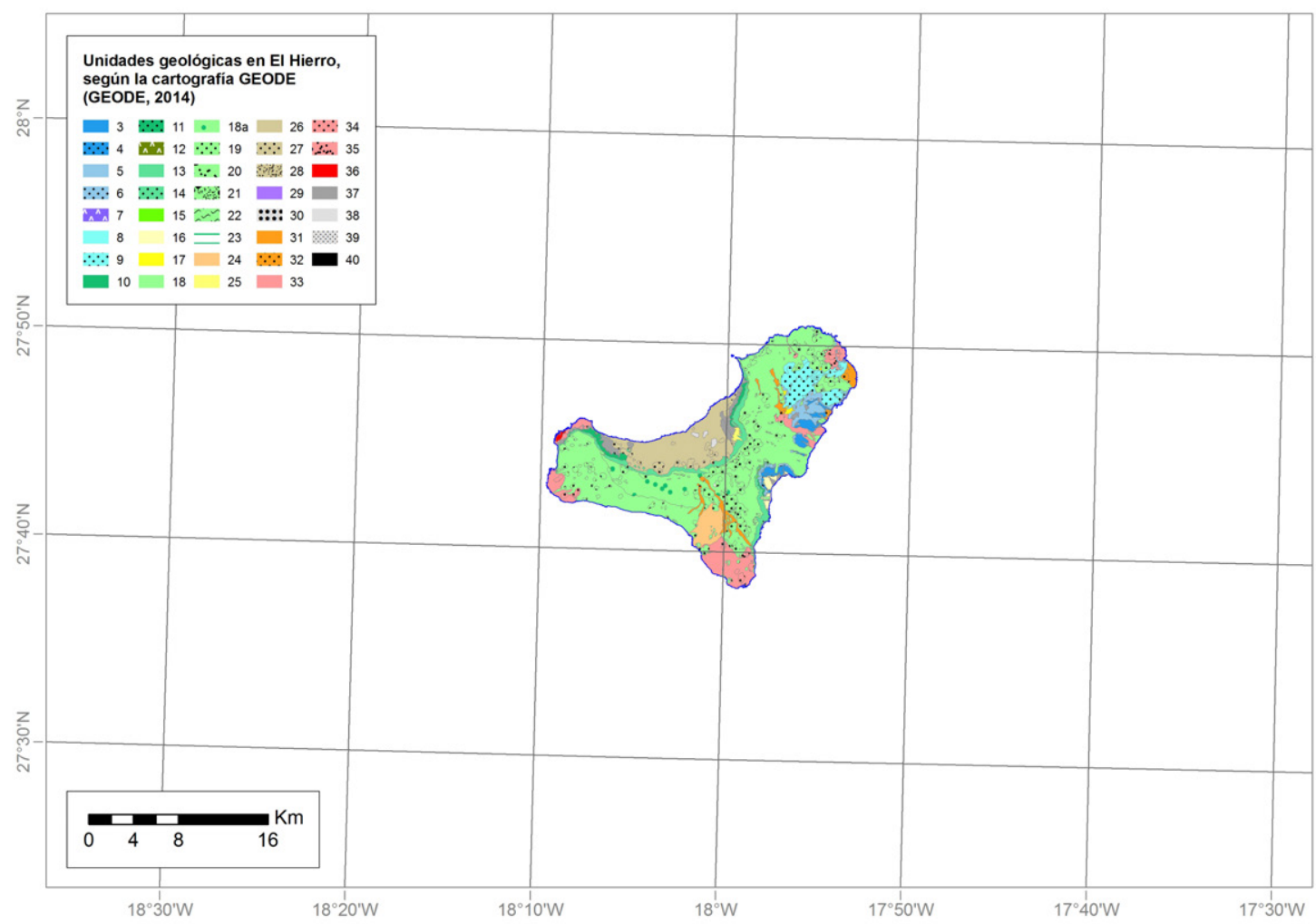

Figura 3.2: Mapa geológico continuo GEODE a escala 1:50.000 de El Hierro (Zona 2916). La leyenda indica el código alfanumérico asignado a cada unidad geológica. La descripción de cada unidad en cuanto a su litología se encuentra en la Tabla A.1. Tomado de GEODE (2014). 


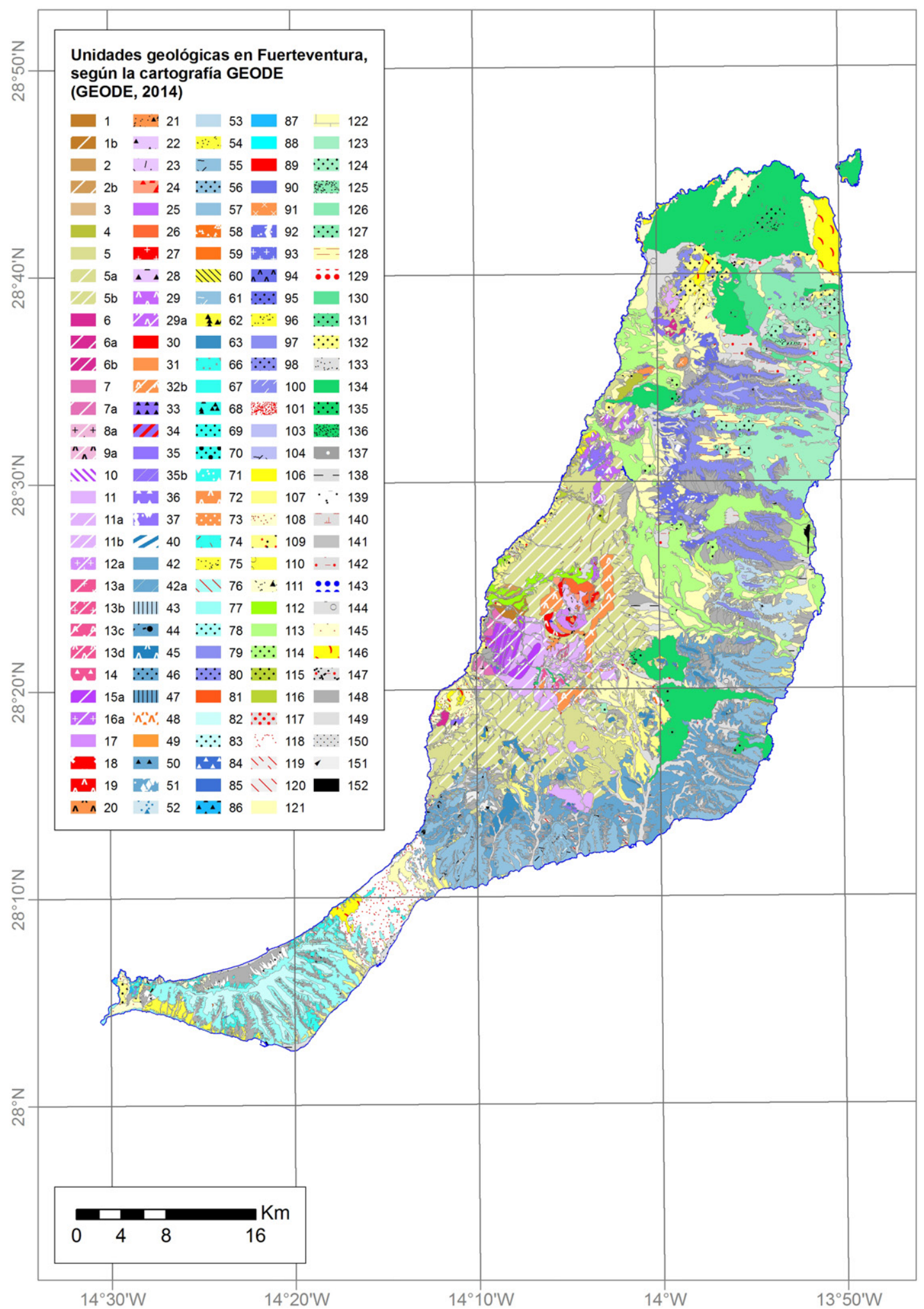

Figura 3.3: Mapa geológico continuo GEODE a escala 1:50.000 de Fuerteventura (Zona 2911). La leyenda indica el código alfanumérico asignado a cada unidad geológica. La descripción de cada unidad en cuanto a su litología se encuentra en la Tabla A.2. Tomado de GEODE (2014). 


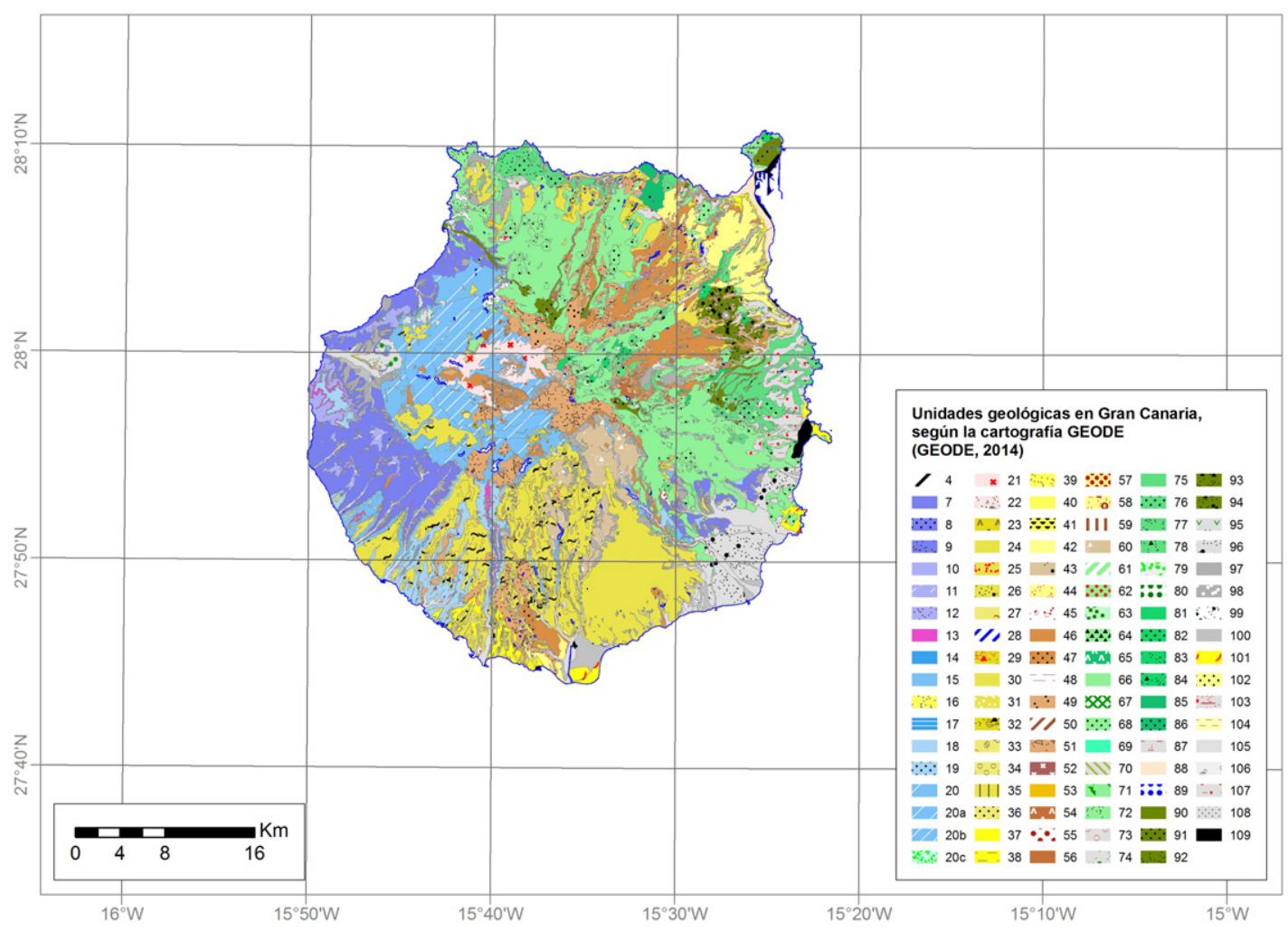

Figura 3.4: Mapa geológico continuo GEODE a escala 1:50.000 de Gran Canaria (Zona 2912). La leyenda indica el código alfanumérico asignado a cada unidad geológica. La descripción de cada unidad en cuanto a su litología se encuentra en la Tabla A.3. Tomado de GEODE (2014). 


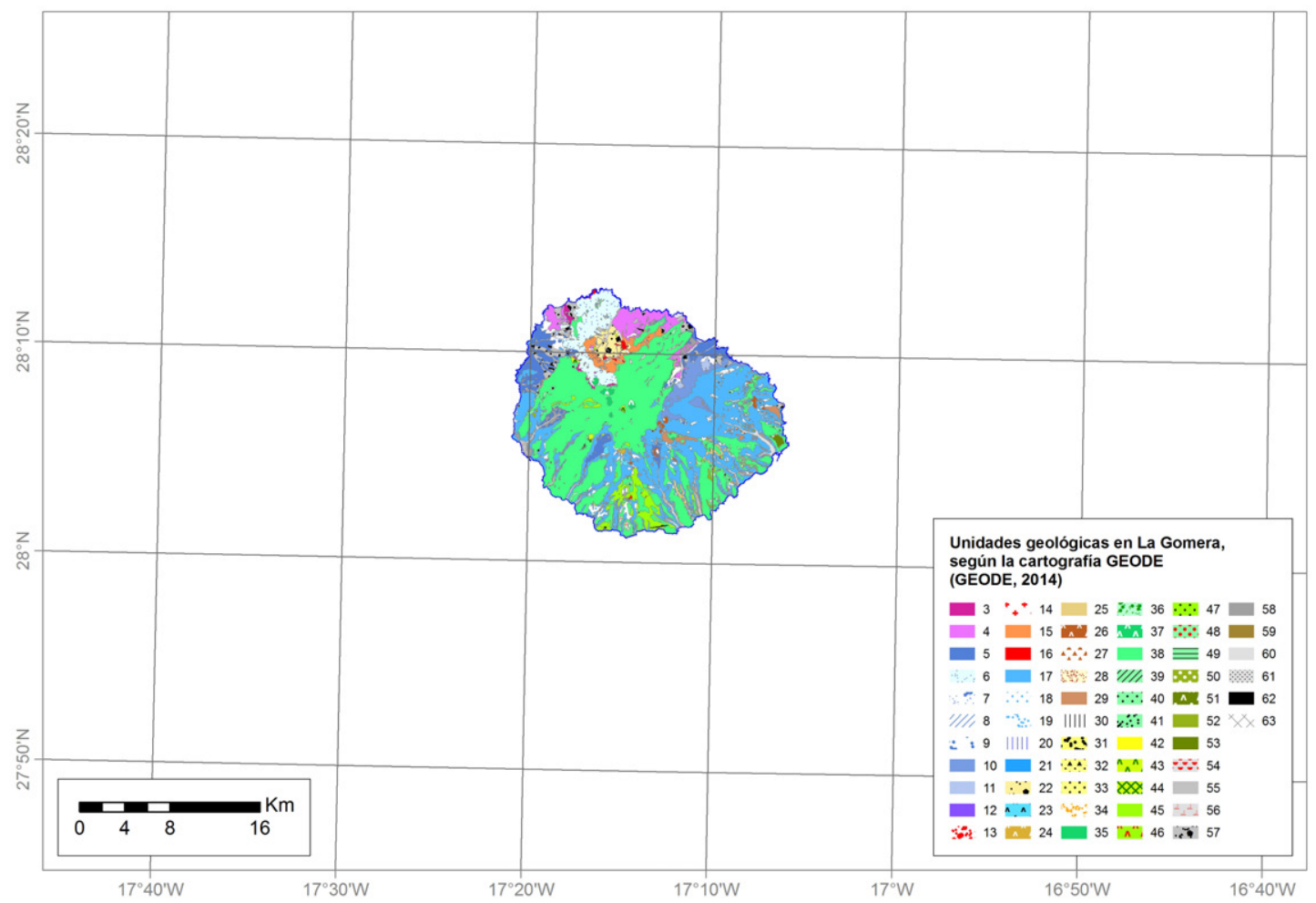

Figura 3.5: Mapa geológico continuo GEODE a escala 1:50.000 de La Gomera (Zona 2914). La leyenda indica el código alfanumérico asignado a cada unidad geológica. La descripción de cada unidad en cuanto a su litología se encuentra en la Tabla A.4. Tomado de GEODE (2014). 


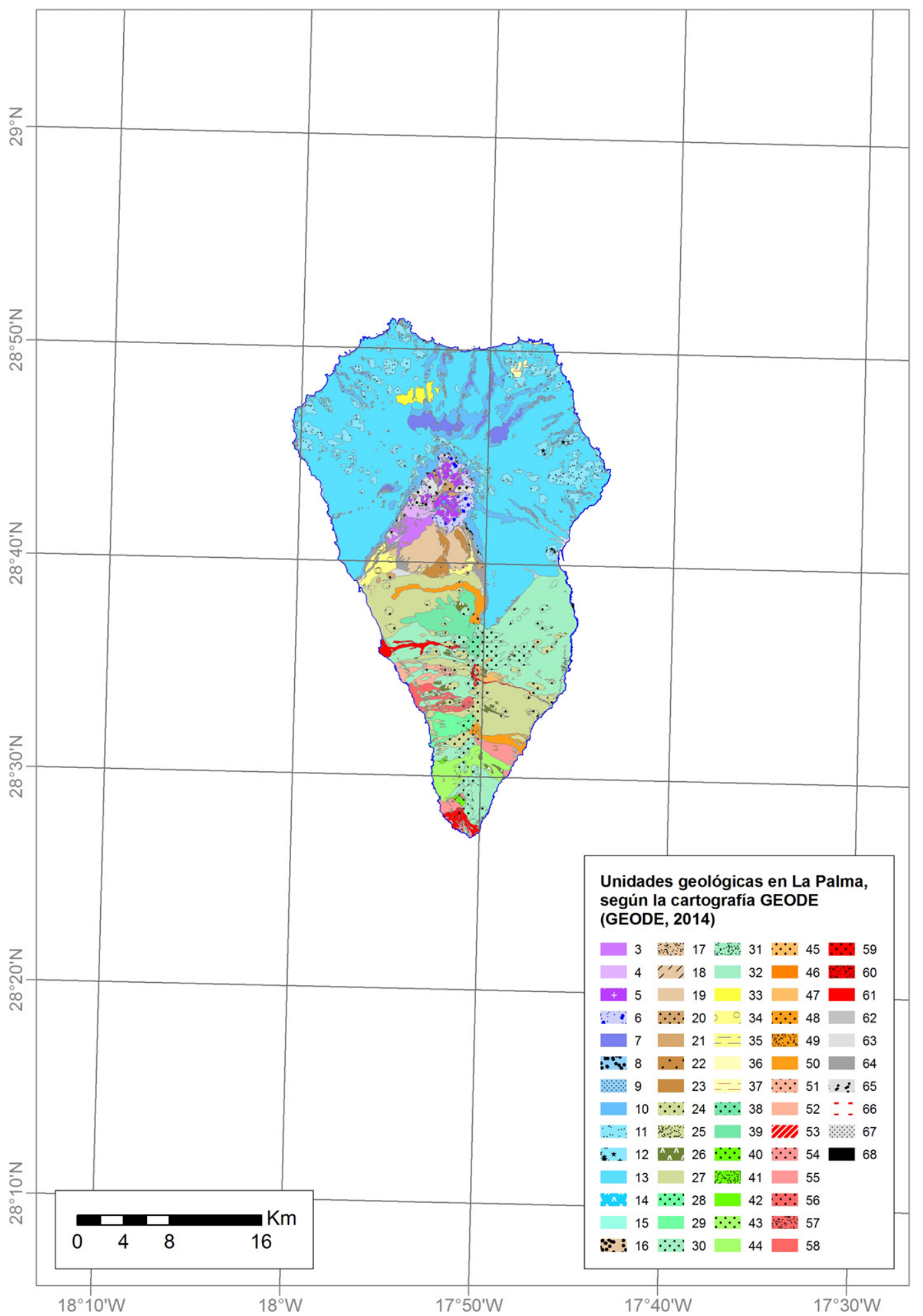

Figura 3.6: Mapa geológico continuo GEODE a escala 1:50.000 de La Palma (Zona 2915). La leyenda indica el código alfanumérico asignado a cada unidad geológica. La descripción de cada unidad en cuanto a su litología se encuentra en la Tabla A.5. Tomado de GEODE (2014). 


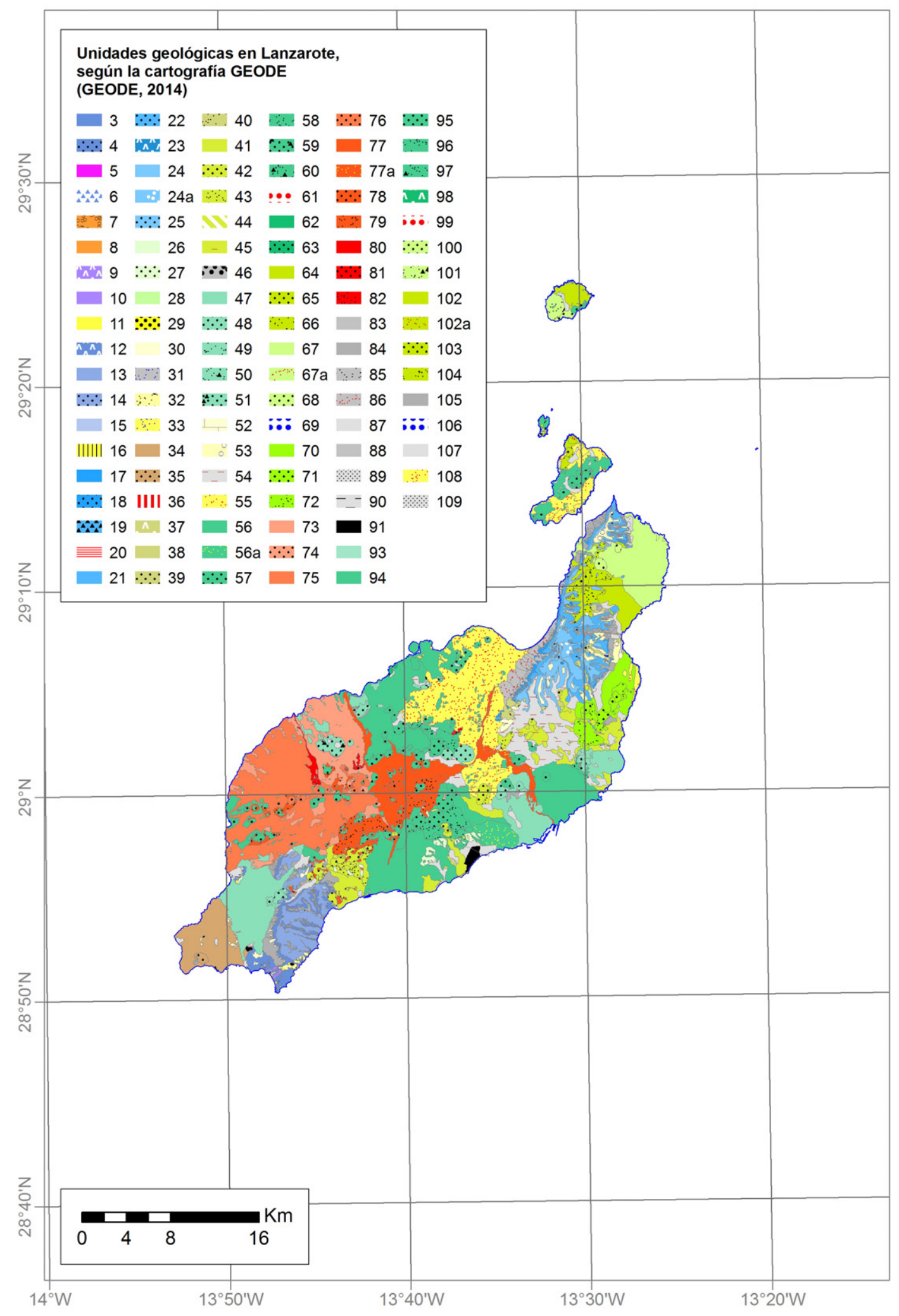

Figura 3.7: Mapa geológico continuo GEODE a escala 1:50.000 de Lanzarote (Zona 2910). La leyenda indica el código alfanumérico asignado a cada unidad geológica. La descripción de cada unidad en cuanto a su litología se encuentra en la Tabla A.6. Tomado de GEODE (2014). 


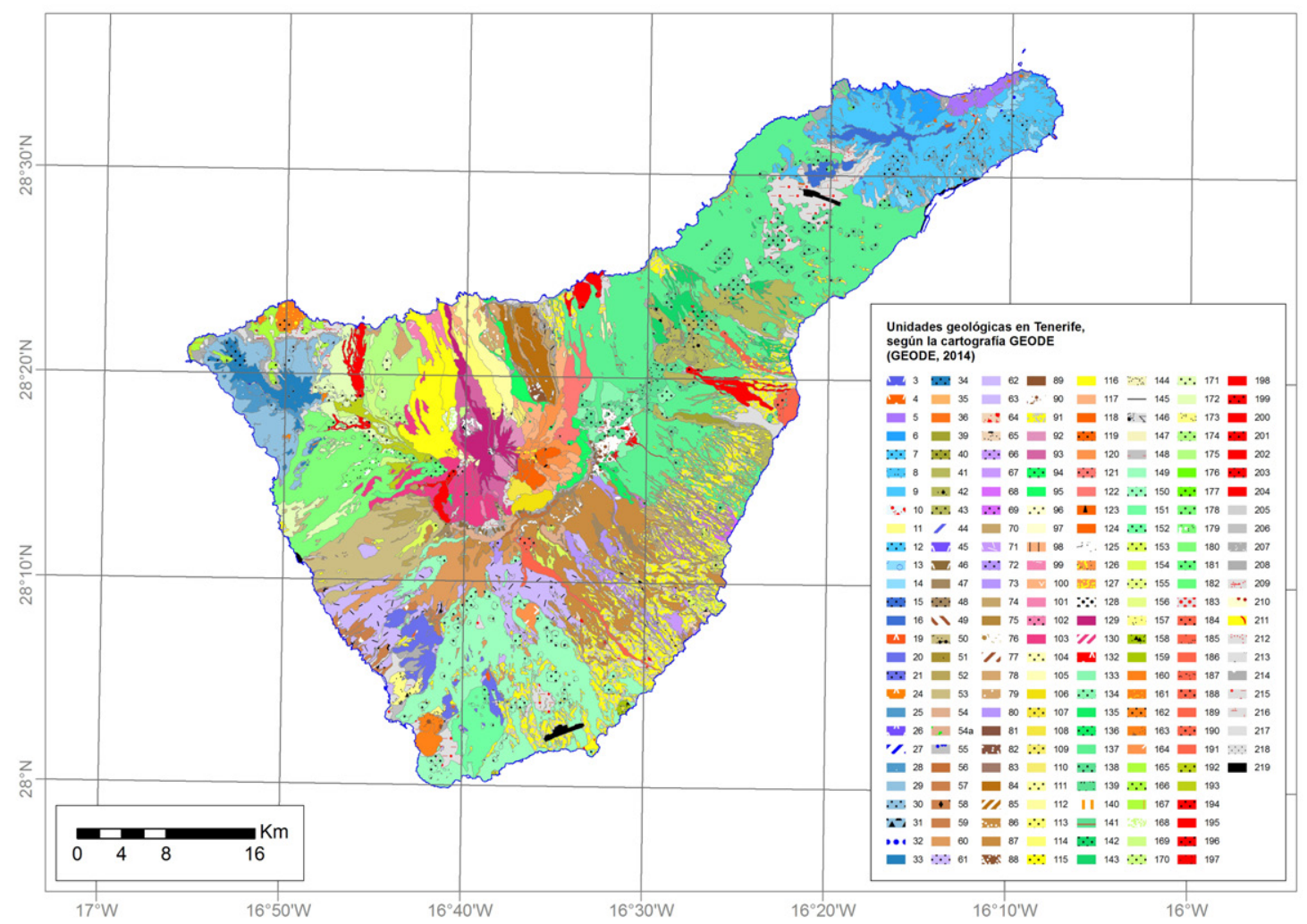

Figura 3.8: Mapa geológico continuo GEODE a escala 1:50.000 de Tenerife (Zona 2912). La leyenda indica el código alfanumérico asignado a cada unidad geológica. La descripción de cada unidad en cuanto a su litología se encuentra en la Tabla A.7. Tomado de GEODE (2014). 


\subsection{Clasificación de las unidades geológicas según la clasificación de síntesis}

Las unidades geológicas descritas en la Península Ibérica, las Islas Baleares y las Islas Canarias han sido agrupadas de acuerdo con las clases de emplazamiento de la clasificación de síntesis (Tabla 3.4), con el fin de asignar a cada unidad el valor de Vs30 de la clase correspondiente.

Siguiendo la metodología de Borcherdt (1994), el proceso de clasificación ha comenzado con la estimación de las propiedades geotécnicas cualitativas de cada unidad geológica: resistencia, compacidad y consistencia (Figura 2.6). En el caso de las unidades de la Península Ibérica e Islas Baleares, estas propiedades han sido inferidas considerando la descripción litológica y la edad cronoestratigráfica de cada unidad (Instituto Geológico y Minero de España, 1994). En el caso de las unidades geológicas de las Islas Canarias, se han analizado la edad cronoestratigráfica y las descripciones relativas a la litología (GEODE, 2014).

Una vez caracterizada cada unidad en función de su resistencia, compacidad y consistencia, se ha realizado la comparación de estas propiedades con la descripción geotécnica de cada una de las clases de emplazamiento de la clasificación de síntesis. Como se ha indicado, la descripción geotécnica de cada clase refleja el grado de compacidad y consistencia de la misma (Tabla 3.4), y a la vez permite estimar de una forma cualitativa el grado de resistencia. Finalmente, se ha asignado a la unidad la clase de emplazamiento cuya descripción geotécnica es coherente con las propiedades de la unidad en estudio.

Debe tenerse en cuenta que la clasificación de las unidades geológicas se ha realizado considerando exclusivamente características y descripciones de materiales aflorantes en superficie, sin tener en cuenta su posible variación en profundidad. Esta limitación viene dada por la información disponible en la cartografía geológica oficial para la totalidad del territorio, y que se limita a unidades compuestas por materiales superficiales. En el caso de varias unidades, tanto su descripción litológica como su edad cronoestratigráfica han planteado la disyuntiva de su clasificación en dos o más clases de emplazamiento. Valorando conjuntamente ambos parámetros, algunas de estas unidades se han asociado con la clase de emplazamiento de menor resistencia, optando por tanto por un criterio conservador para resolver la ambigüedad en relación a la asignación de la clase de emplazamiento.

En cuanto a la terminología empleada en el proceso de clasificación que se describe a continuación, se utiliza el término "roca volcánica" para hacer referencia a las rocas formadas por enfriamiento de lava volcánica. Este término se distingue del término "piroclasto", que es utilizado para describir los fragmentos de magma o de roca encajante proyectados al aire por una explosión volcánica.

\subsubsection{Unidades geológicas de la Península Ibérica y las Islas Baleares}

Para explicar el proceso de clasificación de las unidades geológicas de la Península Ibérica y las Islas Baleares, se presentan los dominios geológicos descriptivos de 
las unidades y las litologías genéricas que contiene cada uno, de acuerdo a Instituto Geológico y Minero de España (1994). En el caso de cada litología genérica, se detallan las unidades que engloba, y para cada unidad se indican la sigla que la identifica en la leyenda del Mapa Geológico a escala 1:1.000.000, la descripción litológica y la edad cronoestratigráfica (eonotema, eratema, sistema o serie). En este punto, se justifica qué clase de emplazamiento se asigna a la unidad en cuestión, de acuerdo con la metodología de Borcherdt (1994). Las unidades geológicas que no corresponden a ningún dominio geológico se introducen mediante su litología genérica, procediendo de forma análoga a la explicada para el caso de las unidades con dominio geológico.

\section{Dominio geológico: Rocas plutónicas prehercínicas}

Bajo el dominio geológico "Rocas plutónicas prehercínicas" se encuadra la litología genérica "Granitoides de emplazamiento meso-catazonal". Esta litología genérica comprende seis unidades geológicas que corresponden a cuatro descripciones litológicas diferentes, y que se presentan en la Tabla 3.8.

Los granitoides de emplazamiento meso-catazonal son rocas plutónicas graníticas, localizadas originalmente en un ambiente de metamorfismo de grado alto a medio. Las unidades 2, 3, 3', 4 y 4' corresponden a granitoides de diversas composiciones: alcalinos, calcoalcalinos y peralumínicos. Por su parte, la unidad 5 se asocia a conjuntos de rocas alcalinas. La unidad 1 consiste en serpentinitas, que son rocas metamórficas bien cristalizadas y compuestas principalmente por serpentina, producto de la alteración del olivino.

Desde un punto de vista geotécnico, las seis unidades pueden describirse como rocas duras, ya que presentan rocas plutónicas, salvo la unidad 1 (rocas metamórficas). Por tanto, se clasifican en la clase de emplazamiento 1 de la clasificación de síntesis (Tabla 3.4), cuya descripción geotécnica consiste en rocas duras.

\section{Dominio geológico: Rocas plutónicas hercínicas}

Las unidades pertenecientes al dominio geológico de "Rocas plutónicas hercínicas" presentan seis litologías genéricas diferentes (Tabla 3.9):

- Granitoides de emplazamiento meso-catazonal

- Granitoides de emplazamiento epi-mesozonal

Tabla 3.8: Unidades del dominio geológico "Rocas plutónicas prehercínicas". Tomado de Instituto Geológico y Minero de España (1994), elaboración propia.

\begin{tabular}{|l|l|l|l|l|}
\hline $\begin{array}{l}\text { Litología genéri- } \\
\text { ca }\end{array}$ & Siglas & Descripción litológica & $\begin{array}{l}\text { Eonotema/ } \\
\text { Sistema }\end{array}$ & Clase de emplazamiento \\
\hline \multirow{3}{*}{$\begin{array}{l}\text { Granitoides de } \\
\text { emplazamiento } \\
\text { meso-catazonal }\end{array}$} & 1 & $\begin{array}{l}\text { Rocas ultrabásicas (ser- } \\
\text { pentinitas). Margen activo }\end{array}$ & Proterozoico & 1 \\
\cline { 2 - 5 } & 2,2, & Granitoides calcoalcalinos & Proterozoico & 1 \\
\cline { 2 - 5 } & 3,3, & $\begin{array}{l}\text { Granitoides peralumínicos } \\
\text { (marco colisional) }\end{array}$ & Proterozoico & 1 \\
\cline { 2 - 5 } & 5,4 & $\begin{array}{l}\text { Granitoides alcalinos (mar- } \\
\text { co extensional) }\end{array}$ & $\begin{array}{l}\text { Cámbrico- } \\
\text { Ordovícico }\end{array}$ & 1 \\
\cline { 2 - 5 } & 5 & $\begin{array}{l}\text { Complejos alcalinos ácidos- } \\
\text { básicos }\end{array}$ & $\begin{array}{l}\text { Cámbrico- } \\
\text { Ordovícico }\end{array}$ & 1 \\
\hline
\end{tabular}


- Granitoides postcinemáticos de emplazamiento epizonal

- Plutonismo orogénico colisional peralumínico

- Plutonismo orogénico calcoalcalino toleítico

- Granitoides alcalinos postcinemáticos

La litología genérica "Granitoides de emplazamiento meso-catazonal" comprende las unidades 6,7 y 8, en las cuales aparecen rocas graníticas de diversa composición que corresponden a un ambiente de metamorfismo de grado alto a medio. En este ambiente, algunas rocas se han formado a partir de la fusión de otras preexistentes, como los complejos migmatíticos-anatécticos de la unidad 6.

Las litologías genéricas "Granitoides de emplazamiento epi-mesozonal" (unidades 9 y 10) y "Granitoides postcinemáticos de emplazamiento epizonal" (unidades 11 y 12) corresponden a rocas graníticas de diversa composición y emplazadas en un ambiente con grado de metamorfismo medio a bajo. Las unidades 13 y 14 se describen como rocas plutónicas fundamentalmente básicas que se enmarcan en un plutonismo orogénico colisional peralumínico, mientras que las unidades 15 y 16 son rocas plutónicas que derivan de un plutonismo orogénico calcoalcalino toleítico. Por último, la unidad 17 corresponde a granitoides alcalinos postcinemáticos.

Todas las unidades de este dominio contienen rocas plutónicas. Por tanto, pueden ser descritas como rocas duras y clasificarse en la clase de emplazamiento 1 de la clasificación de síntesis.

\section{Dominio geológico: Rocas plutónicas alpinas}

Este dominio geológico agrupa cinco unidades geológicas que tienen la misma litología genérica, "Rocas plutónicas alpinas", como se observa en la Tabla 3.10. Las unidades 18a, 18b y 18c presentan idéntica descripción litológica como metagranitos, que son rocas de metamorfismo incipiente que proceden del granito. La unidad 19 se describe como diques doleríticos, los cuales tienen una composición pobre en cuarzo pero rica en plagioclasas, y la unidad 20 consta de peridotitas, de composición básica a ultrabásica.

Las unidades 19 y 20 corresponden a rocas plutónicas de diferente composición, mientras que las unidades 18a, 18b y 18c presentan rocas metamórficas. Tanto las rocas plutónicas como las rocas metamórficas pueden describirse geotécnicamente como rocas duras, por lo cual las unidades se clasifican en la clase de emplazamiento 1 .

\section{Dominio geológico: Unidades alóctonas del Macizo Hespérico}

Bajo el dominio geológico"Unidades alóctonas del Macizo Hespérico" se engloban dos litologías genéricas (Tabla 3.11):

- Rocas básicas y ultrabásicas

- Rocas metamórficas 
Tabla 3.9: Unidades del dominio geológico "Rocas plutónicas hercínicas". La abreviatura s.l. (sensu lato) indica que la referencia a la que acompaña se entiende en un sentido amplio. Tomado de Instituto Geológico y Minero de España (1994), elaboración propia.

\begin{tabular}{|c|c|c|c|c|}
\hline $\begin{array}{l}\text { Litología genéri- } \\
\text { ca }\end{array}$ & Sigla & Descripción litológica & Sistema & Clase de emplazamiento \\
\hline \multirow{3}{*}{$\begin{array}{l}\text { Granitoides de } \\
\text { emplazamiento } \\
\text { meso-catazonal }\end{array}$} & 6 & $\begin{array}{l}\text { Complejos migmatíticos- } \\
\text { anatécticos }\end{array}$ & $\begin{array}{l}\text { Devónico- } \\
\text { Carbonífero- } \\
\text { Pérmico } \\
\end{array}$ & 1 \\
\hline & 7 & Granitoides de dos micas & $\begin{array}{l}\text { Devónico- } \\
\text { Carbonífero- } \\
\text { Pérmico } \\
\end{array}$ & 1 \\
\hline & 8 & Granitoides biotíticos & $\begin{array}{l}\text { Devónico- } \\
\text { Carbonífero- } \\
\text { Pérmico }\end{array}$ & 1 \\
\hline \multirow{2}{*}{$\begin{array}{l}\text { Granitoides de } \\
\text { emplazamiento } \\
\text { epi-mesozonal }\end{array}$} & 9 & Granitoides de dos micas & $\begin{array}{l}\text { Devónico- } \\
\text { Carbonífero- } \\
\text { Pérmico }\end{array}$ & 1 \\
\hline & 10 & Granitoides biotíticos & $\begin{array}{l}\text { Devónico- } \\
\text { Carbonífero- } \\
\text { Pérmico } \\
\end{array}$ & 1 \\
\hline \multirow{2}{*}{$\begin{array}{l}\text { Granitoides } \\
\text { postcinemáticos de } \\
\text { emplazamiento } \\
\text { epizonal }\end{array}$} & 11 & Granitoides de dos micas & $\begin{array}{l}\text { Devónico- } \\
\text { Carbonífero- } \\
\text { Pérmico } \\
\end{array}$ & 1 \\
\hline & 12 & Granitoides biotíticos & $\begin{array}{l}\text { Devónico- } \\
\text { Carbonífero- } \\
\text { Pérmico }\end{array}$ & 1 \\
\hline \multirow{2}{*}{$\begin{array}{l}\text { Plutonismo } \\
\text { orogénico } \\
\text { colisional } \\
\text { peralumínico }\end{array}$} & 13 & $\begin{array}{l}\text { Rocas intermedias y bási- } \\
\text { cas }\end{array}$ & $\begin{array}{l}\text { Devónico- } \\
\text { Carbonífero- } \\
\text { Pérmico }\end{array}$ & 1 \\
\hline & 14 & $\begin{array}{l}\text { Complejos ácidos y básicos } \\
\text { indiferenciados }\end{array}$ & $\begin{array}{l}\text { Devónico- } \\
\text { Carbonífero- } \\
\text { Pérmico } \\
\end{array}$ & 1 \\
\hline \multirow{2}{*}{$\begin{array}{l}\text { Plutonismo } \\
\text { orogénico } \\
\text { calcoalcalino } \\
\text { toleítico }\end{array}$} & 15 & $\begin{array}{l}\text { Rocas básicas y ultrabási- } \\
\text { cas }\end{array}$ & $\begin{array}{l}\text { Devónico- } \\
\text { Carbonífero- } \\
\text { Pérmico } \\
\end{array}$ & 1 \\
\hline & 16 & $\begin{array}{l}\text { Granitoides s.l. indiferen- } \\
\text { ciado }\end{array}$ & $\begin{array}{l}\text { Devónico- } \\
\text { Carbonífero- } \\
\text { Pérmico } \\
\end{array}$ & 1 \\
\hline $\begin{array}{l}\text { Granitoides alcali- } \\
\text { nos postcinemáti- } \\
\text { cos }\end{array}$ & 17 & $\begin{array}{l}\text { Granitoides de tendencia } \\
\text { alcalina postcinemáticos }\end{array}$ & $\begin{array}{l}\text { Devónico- } \\
\text { Carbonífero- } \\
\text { Pérmico } \\
\end{array}$ & 1 \\
\hline
\end{tabular}

Tabla 3.10: Unidades del dominio geológico "Rocas plutónicas alpinas". Tomado de Instituto Geológico y Minero de España (1994), elaboración propia.

\begin{tabular}{|l|l|l|l|l|}
\hline $\begin{array}{l}\text { Litología genéri- } \\
\text { ca }\end{array}$ & Siglas & Descripción litológica & Eratema & Clase de emplazamiento \\
\hline \multirow{2}{*}{$\begin{array}{l}\text { Rocas plutónicas } \\
\text { alpinas }\end{array}$} & $\begin{array}{l}18 \mathrm{a}, 18 \mathrm{~b}, \\
18 \mathrm{c}\end{array}$ & Metagranitos & $\begin{array}{l}\text { Mesozoico- } \\
\text { Cenozoico }\end{array}$ & 1 \\
\cline { 2 - 5 } & 19 & Diques doleríticos & $\begin{array}{l}\text { Mesozoico- } \\
\text { Cenozoico }\end{array}$ & 1 \\
\cline { 2 - 5 } & 20 & Peridotitas & Cenozoico & 1 \\
\hline
\end{tabular}

Tabla 3.11: Unidades del dominio geológico "Unidades alóctonas del Macizo Hespérico". Tomado de Instituto Geológico y Minero de España (1994), elaboración propia.

\begin{tabular}{|l|l|l|l|l|}
\hline $\begin{array}{l}\text { Litología genéri- } \\
\text { ca }\end{array}$ & Siglas & Descripción litológica & $\begin{array}{l}\text { Eonotema/ } \\
\text { Eratema }\end{array}$ & Clase de emplazamiento \\
\hline $\begin{array}{l}\text { Rocas básicas y ul- } \\
\text { trabásicas }\end{array}$ & 21 & $\begin{array}{l}\text { Rocas básicas y ultrabási- } \\
\text { cas }\end{array}$ & $\begin{array}{l}\text { Proterozoico- } \\
\text { Paleozoico }\end{array}$ & 1 \\
\hline $\begin{array}{l}\text { Rocas } \\
\text { metamórficas }\end{array}$ & 22 & $\begin{array}{l}\text { Esquistos gneiseofélsicos y } \\
\text { metabasitas }\end{array}$ & $\begin{array}{l}\text { Proterozoico- } \\
\text { Paleozoico }\end{array}$ & 1 \\
\cline { 2 - 5 } & 23, & $\begin{array}{l}\text { Serpentinitas, metaba- } \\
\text { sitas y metavulcanitas } \\
\text { ácidas (Metasedimentos } \\
\text { septentrionales) }\end{array}$ & $\begin{array}{l}\text { Proterozoico- } \\
\text { Paleozoico }\end{array}$ & 1 \\
\cline { 2 - 5 } & 24 & $\begin{array}{l}\text { Esquistos y cuarcitas (Me- } \\
\text { tasedimentos meridionales) }\end{array}$ & $\begin{array}{l}\text { Proterozoico- } \\
\text { Paleozoico }\end{array}$ & 1 \\
\hline
\end{tabular}


La litología genérica "Rocas básicas y ultrabásicas" incluye la unidad 21, cuya descripción litológica presenta rocas ígneas de composición básica y ultrabásica. La litología genérica "Rocas metamórficas" agrupa a cuatro unidades (22, 23, 23' y 24), que tienen litologías distintas entre sí pero consistentes en rocas metamórficas. La unidad 22 se describe como esquistos gneiseofélsicos, con alto contenido en cuarzo y feldespatos, y metabasitas. Las unidades 23 y 23' presentan la misma descripción litológica, consistiendo en serpentinitas, metabasitas y metavulcanitas ácidas, mientras que la unidad 24 consta de esquistos y cuarcitas.

Las cinco unidades presentan rocas ígneas y metamórficas datadas en el tránsito del Proterozoico al Paleozoico. Esta edad cronoestratigráfica y la alta resistencia de este tipo de rocas permite considerar las unidades como rocas duras, y por tanto clasificarlas en la clase de emplazamiento 1.

\section{Dominio geológico: Bloque de Alborán}

El dominio geológico "Bloque de Alborán" comprende dos litologías genéricas (Tabla 3.12):

- Rocas metamórficas

- Rocas sedimentarias (Béticas; zonas internas)

Dentro de la litología genérica "Rocas metamórficas" se encuentran cuatro unidades $(25,26,27,28)$ que se describen como rocas metamórficas de diversa composición y con edad comprendida entre el comienzo del Paleozoico y el Triásico. En las unidades 27 y 28 aparecen, además, rocas sedimentarias (calizas, dolomías, pelitas, areniscas, grauvacas y conglomerados).

Las unidades 25 y 26 se componen íntegramente de rocas metamórficas (micaesquistos, anfibolitas, serpentinitas y mármoles), por lo cual pueden considerarse como rocas duras y, por tanto, encuadrarse en la clase de emplazamiento 1.

Las unidades 27 y 28 presentan rocas metamórficas acompañadas de rocas sedimentarias. Así, la unidad 27 comprende gneises, migmatitas, micaesquistos, esquistos, filitas y mármoles (rocas metamórficas) y calizas y dolomías del Triásico (rocas sedimentarias) (Tabla 3.12). Las calizas y dolomías de edad mesozoica (TriásicoJurásico), o edad más antigua, pueden considerarse como rocas duras. Por tanto, y dado que la unidad 27 también presenta rocas metamórficas, es razonable afirmar que esta unidad tiene la resistencia de una roca dura, y, por tanto, que pertenece a la clase 1.

En cuanto a la unidad 28, su descripción litológica comprende cinco tipos de rocas sedimentarias (calizas, pelitas, areniscas, grauvacas y conglomerados) por un solo tipo de roca metamórfica (filita). La presencia de las rocas sedimentarias detríticas (como la arenisca) indica que la resistencia de esta unidad es menor que la de una roca dura, aunque también aparezcan las filitas y las calizas paleozoicas. La razón consiste en el hecho de que, en general, las rocas detríticas tienen una resistencia inferior a la resistencia que alcanzan rocas duras como las ígneas y metamórficas, o bien las calizas y dolomías del Triásico-Jurásico. Por tanto, puede considerarse una resistencia media para la unidad 28, y clasificar esta unidad en la clase de 
emplazamiento 2, en coherencia con la descripción geotécnica de esta clase como rocas de resistencia media (Tabla 3.4).

Bajo la litología genérica "Rocas sedimentarias (Béticas; zonas internas)" se agrupan las unidades 29, 30, 31 y 32 , que se componen de rocas sedimentarias, en su mayoría de edad mesozoica (Tabla 3.12). En las unidades 29 y 30, aparecen dolomías y calizas del Mesozoico (Triásico y Jurásico-Cretácico) junto a rocas sedimentarias detríticas: margas, areniscas y conglomerados en el caso de la unidad 29, y margocalizas y margas arenosas en el caso de la unidad 30. Estas rocas detríticas presentan menor resistencia que las dolomías y calizas mesozoicas (rocas duras), y por tanto hacen conveniente la clasificación de las unidades 29 y 30 en la clase de emplazamiento 2. Por medio del mismo razonamiento, las unidades 31 y 32 son clasificadas en la clase 2, ya que se describen como calizas y dolomías, pero acompañadas de margas y areniscas (Tabla 3.12).

\section{Litología genérica: Gneises; esquistos; mármoles y vulcanitas}

Como muestra la Tabla 3.13, la litología genérica "Gneises; esquistos; mármoles y vulcanitas" encuadra siete unidades geológicas que se componen de rocas metamórficas y sedimentarias, exceptuando la unidad 36, la cual consiste básicamente en rocas volcánicas.

Las unidades 34 y 37 presentan exclusivamente rocas metamórficas, que, como se ha indicado, son descritas geotécnicamente como rocas duras. Por tanto, estas dos unidades son clasificadas en la clase de emplazamiento 1. La unidad 38 también se encuadra en la clase 1 pues, aunque presenta grauvacas (roca sedimentaria detrítica), consta además de cuatro tipos de rocas metamórficas (como la cuarcita). La unidad 36 presenta rocas volcánicas y/o vulcanoclásticas, las cuales tienen alta resistencia y por tanto permiten asignar la clase de emplazamiento 1 a esta unidad.

En relación a las unidades 33, 35 y 39, la Tabla 3.13 indica que estas unidades presentan rocas metamórficas de grado de metamorfismo bajo (pizarras) junto a rocas sedimentarias, mayoritariamente detríticas. Estas rocas tienen una edad bastante antigua (tránsito del Proterozoico al Paleozoico) y, por tanto, las unidades podrían encuadrarse en la clase de emplazamiento 1. Sin embargo, la presencia de las rocas detríticas induce a cuestionar que la resistencia del conjunto sea la de una roca dura, y aconseja adoptar el criterio conservador y agrupar las unidades 33, 35 y 39 en la clase 2 (Tabla 3.4).

\section{Litología genérica: Cuarcitas; pizarras; areniscas; calizas y vulcanitas}

La litología genérica "Cuarcitas; pizarras; areniscas; calizas y vulcanitas" agrupa dieciséis unidades de edad paleozoica (Vendiense-Ordovícico Superior) (Tabla 3.14). Estas unidades pueden estar compuestas exclusivamente por rocas metamórficas (46, 47, 48, 49, 50, 51), por rocas volcánicas $(41,44)$, por rocas sedimentarias y metamórficas $(40,42,45,52,53,54,55)$ o por rocas sedimentarias (43). Por tanto, se analizará cada uno de estos grupos de unidades de forma separada, con el fin de asignar a cada unidad la clase de emplazamiento que le corresponde.

Las unidades compuestas sólo por rocas metamórficas $(46,47,48,49,50,51)$ presentan la resistencia propia de las rocas duras y se encuadran en la clase de 
Tabla 3.12: Unidades del dominio geológico "Bloque de Alborán". Tomado de Instituto Geológico y Minero de España (1994), elaboración propia.

\begin{tabular}{|c|c|c|c|c|}
\hline $\begin{array}{l}\text { Litología genéri- } \\
\text { ca }\end{array}$ & Sigla & Descripción litológica & $\begin{array}{l}\text { Eonotema/ } \\
\text { Eratema/ } \\
\text { Sistema }\end{array}$ & Clase de emplazamiento \\
\hline \multirow{4}{*}{$\begin{array}{l}\text { Rocas } \\
\text { metamórficas }\end{array}$} & 25 & $\begin{array}{l}\text { Micaesquistos grafitosos } \\
\text { con granate (M. del Veleta) }\end{array}$ & $\begin{array}{l}\text { Proterozoico- } \\
\text { Paleozoico }\end{array}$ & 1 \\
\hline & 26 & $\begin{array}{l}\text { Anfibolitas, serpentinitas, } \\
\text { micaesquistos, y mármoles } \\
\text { (M. del Mulhacén) }\end{array}$ & Triásico & 1 \\
\hline & 27 & $\begin{array}{l}\text { Gneises, migmatitas, mi- } \\
\text { caesquistos, esquistos, fili- } \\
\text { tas, mármoles, calizas, y } \\
\text { dolomías (M. Alpujárride) }\end{array}$ & Triásico & 1 \\
\hline & 28 & $\begin{array}{l}\text { Filitas, pelitas, areniscas, } \\
\text { grauvacas, calizas y conglo- } \\
\text { merados }\end{array}$ & $\begin{array}{l}\text { Proterozoico- } \\
\text { Paleozoico }\end{array}$ & 2 \\
\hline \multirow{4}{*}{$\begin{array}{l}\text { Rocas } \\
\text { sedimentarias } \\
\text { (Béticas; zonas } \\
\text { internas) }\end{array}$} & 29 & $\begin{array}{l}\text { Dolomías, areniscas, con- } \\
\text { glomerados, arcillas, y } \\
\text { margas }\end{array}$ & Triásico & 2 \\
\hline & 30 & $\begin{array}{l}\text { Dolomías, calizas, margo- } \\
\text { calizas, y margas arenosas }\end{array}$ & $\begin{array}{l}\text { Jurásico- } \\
\text { Cretácico }\end{array}$ & 2 \\
\hline & 31 & $\begin{array}{l}\text { Filitas, areniscas, calizas, } \\
\text { dolomías, margas }\end{array}$ & $\begin{array}{l}\text { Paleozoico- } \\
\text { Mesozoico }\end{array}$ & 2 \\
\hline & 32 & $\begin{array}{l}\text { Dolomías, calizas, margas, } \\
\text { areniscas y arcillas (M. } \\
\text { Dorsal) }\end{array}$ & Mesozoico & 2 \\
\hline
\end{tabular}

Tabla 3.13: Unidades de la litología genérica "Gneises; esquistos; mármoles y vulcanitas". Tomado de Instituto Geológico y Minero de España (1994), elaboración propia.

\begin{tabular}{|l|l|l|l|}
\hline Sigla & Descripción litológica & Eonotema/Sistema & $\begin{array}{l}\text { Clase de emplaza- } \\
\text { miento }\end{array}$ \\
\hline 33 & $\begin{array}{l}\text { Pizarras, grauvacas, conglomerados o } \\
\text { porfiroides }\end{array}$ & Proterozoico & 2 \\
\hline 34 & $\begin{array}{l}\text { Gneises y anfibolitas } \\
\text { Esquistos o pizarras, grauvacas y lidi- } \\
\text { tas }\end{array}$ & Proterozoico & 1 \\
\hline 36 & $\begin{array}{l}\text { Vulcanitas y/o rocas vulcanoclásticas } \\
\text { y metasedimentos }\end{array}$ & Vendiense & 2 \\
\hline 37 & $\begin{array}{l}\text { Gneises, migmatitas, cuarcitas y már- } \\
\text { moles }\end{array}$ & Rifeense-Vendiense-Cámbrico & 1 \\
\hline 38 & $\begin{array}{l}\text { Cuarcitas, gneises, esquistos, pizarras } \\
\text { grauvacas }\end{array}$ & Rifeense-Vendiense-Cámbrico & 1 \\
\hline 39 & $\begin{array}{l}\text { Pizarras, areniscas, conglomerados y } \\
\text { calizas }\end{array}$ & Vendiense & 2 \\
\hline
\end{tabular}


emplazamiento 1, con independencia de su composición. El mismo criterio se aplica a las unidades compuestas exclusivamente por rocas volcánicas $(41,44)$, de manera que también se les asigna la clase 1 (Tabla 3.4).

Las unidades consistentes en rocas sedimentarias y metamórficas pertenecen a la clase de emplazamiento 2 (rocas de resistencia media). La razón reside en la presencia de la arenisca, que, como ya se ha indicado, es una roca de menor resistencia que las rocas duras, como las rocas metamórficas, ígneas o las calizas del Triásico-Jurásico. Algunas de estas unidades presentan junto a las areniscas rocas metamórficas como la cuarcita (unidades 45, 52, 54), o bien calizas paleozoicas (unidades 40, 42, 45, $54)$. No obstante, se adopta el criterio conservador para asignar la clase 2 a todas las unidades $(40,42,45,52,53,54,55)$.

La unidad 43 (Tabla 3.14) se compone de calizas y dolomías del Paleozoico (Cámbrico Inferior). Esta descripción litológica y edad cronoestratigráfica permiten, por tanto, estimar que la unidad tiene la resistencia de una roca dura, y que puede ser clasificada dentro de la clase de emplazamiento 1.

\section{Litología genérica: Areniscas; pizarras y calizas}

Las cinco unidades agrupadas bajo la litología genérica "Areniscas; pizarras y calizas" responden a tres descripciones litológicas concretas, como se puede ver en la Tabla 3.15. Cada unidad consiste en rocas sedimentarias de resistencia media (liditas o areniscas) y rocas metamórficas (cuarcitas, ampelitas, pizarras o esquistos), que aparecen en algunos casos junto a rocas sedimentarias duras (calizas) y rocas vulcanoclásticas. La presencia de las rocas sedimentarias de resistencia media desaconseja asignar a estas unidades la clase de emplazamiento 1, revelando en cambio la conveniencia de adoptar el criterio conservador y encuadrarlas en la clase 2 (Tabla 3.4).

\section{Litología genérica: Conglomerados; areniscas; pizarras; calizas y vulcani- tas; carbón}

Esta litología genérica engloba cinco unidades (Tabla 3.16) que presentan rocas volcánicas (unidades 60,61 ), rocas sedimentarias $(62,64)$ o una mezcla de rocas metamórficas y sedimentarias $(59,63)$. Siguiendo el razonamiento de los apartados anteriores, las unidades 60 y 61 pertenecen a la clase de emplazamiento 1 (rocas duras), pues están compuestas de rocas volcánicas. Las unidades 59 y 63 presentan pizarras junto a areniscas y calizas. Dado que el bajo grado de metamorfismo de la pizarra no invita a clasificar esta roca como roca dura, y teniendo en cuenta la resistencia media de la arenisca, se han clasificado estas dos unidades en la clase 2 (rocas de resistencia media).

La unidad 62 se compone de calizas y dolomías del Carbonífero, que pueden considerarse como rocas duras, por lo cual esta unidad pertenece a la clase de emplazamiento 1. Finalmente, la unidad 64 presenta conglomerados, areniscas, lutitas y carbón, que son rocas sedimentarias con menor resistencia que la exigida por la clase 2. Esta descripción litológica, en cambio, es compatible con la descripción geotécnica de la clase de emplazamiento 3 como rocas blandas a firmes, por lo que se ha clasificado la unidad 64 en la citada clase 3. 
Tabla 3.14: Unidades de la litología genérica "Cuarcitas; pizarras; areniscas; calizas y vulcanitas". Tomado de Instituto Geológico y Minero de España (1994), elaboración propia.

\begin{tabular}{|l|l|l|l|}
\hline Sigla & Descripción litológica & Sistema/Serie & $\begin{array}{l}\text { Clase de emplaza- } \\
\text { miento }\end{array}$ \\
\hline 40 & $\begin{array}{l}\text { Pizarras, grauvacas o arcosas, conglo- } \\
\text { merados y calizas }\end{array}$ & Vendiense-Cámbrico \\
$\begin{array}{l}\text { Vulcanitas ácidas y rocas vulcanoclás- } \\
\text { ticas }\end{array}$ & Vendiense & 2 \\
\hline 41 & Areniscas, pizarras y calizas & Cámbrico Inferior & 2 \\
\hline 43 & Calizas y dolomías & Cámbrico Inferior & 1 \\
\hline 44 & Rocas vulcanoclásticas & Cámbrico Inferior & 1 \\
\hline 45 & $\begin{array}{l}\text { Areniscas, pizarras, cuarcitas o calizas } \\
\text { y conglomerados }\end{array}$ & $\begin{array}{l}\text { Cámbrico Inferior-Cámbrico } \\
\text { Medio-Cámbrico Superior- } \\
\text { Ordovícico Inferior }\end{array}$ & 2 \\
\hline 46 & Esquistos y paragneises & Cámbrico-Ordovícico & 1 \\
\hline 47 & Metavulcanitas & Ordovícico Medio & 1 \\
\hline 48 & Pizarras y/o esquistos arenosos & $\begin{array}{l}\text { Cámbrico Medio-Cámbrico Su- } \\
\text { perior }\end{array}$ & 1 \\
\hline 49 & Metavulcanitas básicas & Cámbrico Medio & 1 \\
\hline 50 & Pizarras y/o esquistos; metavulcanitas & Ordovícico & 1 \\
\hline 51 & Pizarras y/o esquistos y cuarcitas & Ordovícico-Silúrico & 2 \\
\hline 52 & $\begin{array}{l}\text { Conglomerados, areniscas, cuarcitas y } \\
\text { pizarras }\end{array}$ & Ordovícico Inferior & 2 \\
\hline 53 & Ortocuarcitas, areniscas y pizarras & Ordovícico Inferior & 2 \\
\hline 54 & $\begin{array}{l}\text { Pizarras, areniscas, cuarcitas y calizas } \\
\text { o rocas vulcanoclásticas }\end{array}$ & $\begin{array}{l}\text { Ordovícico Medio-Ordovícico } \\
\text { Superior }\end{array}$ & 2 \\
\hline 55 & Pizarras y areniscas & Ordovícico Superior & 2 \\
\hline
\end{tabular}

Tabla 3.15: Unidades de la litología genérica "Areniscas; pizarras y calizas". Tomado de Instituto Geológico y Minero de España (1994), elaboración propia.

\begin{tabular}{|l|l|l|l|}
\hline Siglas & Descripción litológica & Sistema/Serie & $\begin{array}{l}\text { Clase de emplaza- } \\
\text { miento }\end{array}$ \\
\hline 56,56, & $\begin{array}{l}\text { Ampelitas, cuarcitas, liditas y rocas } \\
\text { vulcanoclásticas }\end{array}$ & Silúrico & 2 \\
\hline 57,57, & $\begin{array}{l}\text { Pizarras, esquistos, areniscas, calizas, } \\
\text { ampelitas y liditas }\end{array}$ & Silúrico-Devónico & 2 \\
\hline 58 & $\begin{array}{l}\text { Areniscas, pizarras, calizas, cuarcitas y } \\
\text { rocas vulcanoclásticas }\end{array}$ & $\begin{array}{l}\text { Devónico Inferior-Devónico } \\
\text { Medio-Devónico Superior- } \\
\text { Carbonífero Inferior }\end{array}$ & 2 \\
\hline
\end{tabular}

Tabla 3.16: Unidades de la litología genérica "Conglomerados; areniscas; pizarras; calizas y vulcanitas; carbón". Tomado de Instituto Geológico y Minero de España (1994), elaboración propia.

\begin{tabular}{|l|l|l|l|}
\hline Sigla & Descripción litológica & Sistema/Serie & $\begin{array}{l}\text { Clase de emplaza- } \\
\text { miento }\end{array}$ \\
\hline 59 & $\begin{array}{l}\text { Pizarras y grauvacas; conglomerados y } \\
\text { calizas }\end{array}$ & Devónico-Carbonífero & 2 \\
\hline 60 & Vulcanitas bimodales & Devónico & 1 \\
\hline 61 & Vulcanitas y rocas vulcanoclásticas & Carbonífero Inferior & 1 \\
\hline 62 & Calizas y dolomías & Carbonífero & 1 \\
\hline 63 & $\begin{array}{l}\text { Pizarras, areniscas, conglomerados, } \\
\text { carbón y calizas }\end{array}$ & Carbonífero & 2 \\
\hline 64 & $\begin{array}{l}\text { Conglomerados, areniscas, lutitas y } \\
\text { carbón }\end{array}$ & Carbonífero Superior & 3 \\
\hline
\end{tabular}




\section{Litología genérica: Conglomerados; areniscas y lutitas; vulcanitas}

Bajo esta litología genérica se clasifican dos unidades $(65,66)$, cuya descripción puede consultarse en la Tabla 3.17. La unidad 65 se compone de rocas sedimentarias de diversa resistencia y de rocas volcánicas. Debido a la presencia de vulcanitas y calizas, que se consideran rocas duras, junto con areniscas, a las cuales se atribuye una resistencia media, se ha preferido clasificar esta unidad en la clase 2 (rocas de resistencia media), de acuerdo a la Tabla 3.4. La presencia de lutitas, para las que se estima una resistencia pequeña, junto con las citadas rocas puede constituir un argumento para clasificar la unidad en una clase con resistencia inferior a la de la clase 2. No obstante, el hecho de que la edad de todas las rocas sea bastante antigua (Carbonífero-Pérmico) invita a estimar una resistencia media para la unidad. En cuanto a las unidad 66, ésta se encuadra en la clase de emplazamiento 1 (rocas duras), pues consta de rocas volcánicas.

\section{Litología genérica: Conglomerados; areniscas; calizas; yesos y arcillas ver- sicolores}

Bajo esta litología genérica se agrupan cuatro unidades, compuestas íntegramente por rocas sedimentarias detríticas, carbonatadas o evaporíticas, cuyas descripciones litológicas pueden consultarse en la Tabla 3.18.

Las unidades 67 y 68 tienen una descripción litológica idéntica, constando de conglomerados, areniscas, arcillas, dolomías, calizas y yesos. Aparecen calizas y dolomías del Triásico (rocas duras) junto a arcillas y yesos, que podrían constituir una razón para clasificar ambas unidades en la clase de emplazamiento 3 (rocas blandas a firmes). Sin embargo, la presencia de las calizas y dolomías se considera suficiente para clasificar las unidades 67 y 68 en la clase 2 (rocas de resistencia media). Análogamente se ha procedido en el caso de la unidad 69, que presenta una descripción litológica que difiere de la anterior en la presencia de margas en lugar de yesos.

En cuanto a la unidad 70, ésta se compone de arcillas versicolores (de varios colores), y de yesos de facies Keuper (Triásico Medio-Superior). La presencia de estas arcillas se considera suficiente para asignar a esta unidad la clase de emplazamiento 3 , a tenor de la coherencia entre la resistencia estimada para este tipo de material triásico y la descripción geotécnica de la clase 3 como suelos muy densos, como se puede comprobar en la Tabla 3.4.

\section{Litología genérica: Calizas; dolomías y margas; conglomerados y areniscas}

Como se observa en la Tabla 3.19, esta litología genérica agrupa a cinco unidades, de las cuales tres se componen fundamentalmente de rocas sedimentarias carbonata-

Tabla 3.17: Unidades de la litología genérica "Conglomerados; areniscas y lutitas; vulcanitas". Tomado de Instituto Geológico y Minero de España (1994), elaboración propia.

\begin{tabular}{|l|l|l|l|}
\hline Sigla & Descripción litológica & Sistema & $\begin{array}{l}\text { Clase de emplaza- } \\
\text { miento }\end{array}$ \\
\hline 65 & $\begin{array}{l}\text { Lutitas, areniscas, conglomerados y } \\
\text { vulcanitas o calizas }\end{array}$ & Carbonífero-Pérmico & 2 \\
\hline 66 & Vulcanitas básicas & Pérmico & 1 \\
\hline
\end{tabular}


das (unidades 71,72 y 73). La unidad 74 presenta rocas carbonatadas junto a rocas volcánicas, mientras que la unidad 75 se compone de rocas detríticas y margas.

Las unidades 72 y 73 se componen de dolomías y calizas del Jurásico, que son rocas duras y permiten clasificar estas unidades en la clase de emplazamiento 1. En cambio, las unidades 71 y 74 consisten en calizas de la misma edad cronoestratigráfica, que aparecen junto a margas. La presencia de las margas recomienda clasificar estas unidades en la clase de emplazamiento 2, pues la resistencia de las mismas es menor que en el caso de que sólo se presentasen calizas. En cuanto a la unidad 75, ésta podría pertenecer a la clase de emplazamiento 4 (suelos duros), al contener arenas y margas (Tabla 3.19), pero la presencia de las areniscas y la edad de la unidad (Jurásico-Cretácico) inducen a considerar que la resistencia de la unidad será mayor, y por tanto la unidad ha sido clasificada en la clase de emplazamiento 3 (suelos muy densos/rocas blandas a firmes).

\section{Litología genérica: Calizas; dolomías y margas; areniscas}

La litología genérica "Calizas; dolomías y margas; areniscas" engloba a cinco unidades compuestas principalmente de rocas y sedimentos detríticos y rocas carbonatadas de edad cretácica, como se ve en la Tabla 3.20. Estas cinco unidades se encuadran en la clase de emplazamiento 3, pues son consideradas como suelos muy densos o, alternativamente, como rocas blandas a firmes.

En el caso de las unidades 76 y 77, se encuentran rocas blandas (margas turbidíticas), rocas duras (calizas) y sedimentos detríticos (arenas). A pesar de la presencia de arenas, se considera que estas unidades se componen básicamente de rocas blandas a firmes, y por tanto se clasifican en la clase de emplazamiento 3 (Tabla 3.4).

La unidad 78 se compone de sedimentos detríticos (gravas, arenas, arcillas), que aparecen junto a areniscas y carbón. La presencia de gravas y areniscas indica que esta unidad puede describirse geotécnicamente como suelos muy densos, a pesar de la presencia de arenas y arcillas, y por tanto es razonable asignarle la clase de emplazamiento 3 .

Las unidades 79 y 79’ tienen la misma descripción litológica. Básicamente presentan margas, calizas margosas y arcillas con niveles turbidíticos. Por tanto, podría plantearse la disyuntiva entre clasificar estas unidades en la clase 3 (rocas blandas a firmes) y clasificarlas en la clase 4 (suelos duros), puesto que la presencia de las arcillas, de edad cretácica (Tabla 3.20), podría ser considerada como suficiente para optar por esta última clase. Sin embargo, puede afirmarse que las calizas margosas

Tabla 3.18: Unidades de la litología genérica "Conglomerados; areniscas; calizas; yesos y arcillas versicolores". Tomado de Instituto Geológico y Minero de España (1994), elaboración propia.

\begin{tabular}{|l|l|l|l|}
\hline Sigla & Descripción litológica & Sistema/Facies & $\begin{array}{l}\text { Clase de emplaza- } \\
\text { miento }\end{array}$ \\
\hline 67 & $\begin{array}{l}\text { Conglomerados, areniscas, arcillas, ca- } \\
\text { lizas, dolomías y yesos }\end{array}$ & Pérmico-Triásico & 2 \\
\hline 68 & $\begin{array}{l}\text { Areniscas, conglomerados, dolomías, } \\
\text { calizas, arcillas y yesos }\end{array}$ & Triásico \\
\hline 69 & $\begin{array}{l}\text { Conglomerados, areniscas, arcillas, do- } \\
\text { lomías, calizas y margas }\end{array}$ & Buntsandstein-Muschelkalk & 2 \\
\hline 70 & Arcillas versicolores y yesos & Keuper & 3 \\
\hline
\end{tabular}


proporcionan a estas unidades una resistencia mayor que la de los suelos duros. Por tanto, las unidades 79 y $79^{\prime}$ también se han clasificado en la clase 3 .

\section{Litología genérica: Conglomerados; areniscas; arcillas y calizas; evaporitas}

Hasta 12 unidades se agrupan bajo esta litología genérica, aunque presentan solamente cuatro descripciones litológicas diferentes, como se ve en la Tabla 3.21. En primer lugar, la unidad 80 se compone de dolomías, calizas y margas del tránsito del Cretácico al Paleógeno, que están acompañadas de areniscas y arcillas. La presencia de areniscas, calizas y dolomías, en contraste con la de las arcillas, permite considerar esta unidad como consistente en rocas blandas a firmes y, por tanto, clasificarla en la clase de emplazamiento 3 (Tabla 3.4). En segundo lugar, las unidades 81 y 81 -CB comparten la misma descripción litológica aunque difieren en la facies en la que se manifiestan. Esta descripción incluye fundamentalmente conglomerados, areniscas, margas, calizas y yesos. Aunque aparecen calizas, la presencia de margas y areniscas recomienda clasificar esta unidad en la clase 3 como rocas blandas a firmes.

Las unidades 82, 82-S, 82-CA y 82-C comparten la misma descripción litológica, aunque con distintas facies, presentando areniscas silíceas y arcillas del Paleógeno. La presencia de estas areniscas permite describir estas cuatro unidades como rocas blandas a firmes y clasificarlas en la clase 3 .

Por último, las unidades 83, 83-CB, 83-S, 83-CA y 83-C tienen la misma descripción litológica, constando de turbiditas, calizas, areniscas y margas arenosas. Si bien aparecen turbiditas (sedimentos detríticos) y margas, estas unidades no pueden ser consideradas como suelos duros (clase de emplazamiento 4), pues también presentan calizas y areniscas. Por esta razón, estas cinco unidades pueden ser clasificadas como rocas blandas a firmes (clase 3$)$.

\section{Litología genérica: Turbiditas calcáreas}

Las cinco unidades que se agrupan bajo la litología genérica "Turbiditas calcáreas" presentan tres descripciones litológicas que, si bien son diferentes, presentan muchas similitudes, como se muestra en la Tabla 3.22. En primer lugar, las unidades 84, 84-CA y 84-C (que tienen la misma descripción litológica), así como la unidad 85, constan básicamente de turbiditas calcáreas, margas, areniscas y calizas del Paleógeno. Estas rocas paleógenas pueden considerarse como rocas blandas a firmes, las cuales aparecen acompañadas de las turbiditas, que se equiparan a sedimentos, en este caso de origen calcáreo. Por este motivo, podría presentarse la disyuntiva

Tabla 3.19: Unidades de la litología genérica "Calizas; dolomías y margas; conglomerados y areniscas". Tomado de Instituto Geológico y Minero de España (1994), elaboración propia.

\begin{tabular}{|c|c|c|c|}
\hline Sigla & Descripción litológica & Sistema/Serie & $\begin{array}{l}\text { Clase de emplaza- } \\
\text { miento }\end{array}$ \\
\hline 71 & Dolomías, margas y calizas nodulosas & Triásico-Jurásico & 2 \\
\hline 72 & $\begin{array}{l}\text { Dolomías, calizas, calizas oolíticas y } \\
\text { nodulosas }\end{array}$ & Jurásico-Cretácico & 1 \\
\hline 73 & Dolomías, calizas y calizas nodulosas & Lías (Jurásico Inferior) & 1 \\
\hline 74 & $\begin{array}{l}\text { Calizas, margas, calizas nodulosas y } \\
\text { radiolaritas. Rocas volcánicas }\end{array}$ & Jurásico-Cretácico & 2 \\
\hline 75 & $\begin{array}{l}\text { Conglomerados, areniscas, arenas y } \\
\text { margas }\end{array}$ & Jurásico-Cretácico & 3 \\
\hline
\end{tabular}


Tabla 3.20: Unidades de la litología genérica "Calizas; dolomías y margas; areniscas". Tomado de Instituto Geológico y Minero de España (1994), elaboración propia.

\begin{tabular}{|l|l|l|l|}
\hline Siglas & Descripción litológica & Sistema/Serie & $\begin{array}{l}\text { Clase de emplaza- } \\
\text { miento }\end{array}$ \\
\hline 76 & $\begin{array}{l}\text { Turbiditas silíceas, margas con turbi- } \\
\text { ditas y margocalizas. Calizas bioclásti- } \\
\text { cas, calcarenitas, arenas, margas, dolo- } \\
\text { mías y calizas }\end{array}$ & Cretácico & 3 \\
\hline 77 & $\begin{array}{l}\text { Margas y margocalizas. Margas arcillo- } \\
\text { sas turbidíticas. Calizas arenosas, are- } \\
\text { niscas, arenas y margas }\end{array}$ & Cretácico Inferior & 3 \\
\hline 78 & $\begin{array}{l}\text { Gravas, arenas, areniscas y arcillas. } \\
\text { Carbón }\end{array}$ & Cretácico & 3 \\
\hline 79,79, & $\begin{array}{l}\text { Margas y arcillas con niveles turbidí- } \\
\text { ticos. Margocalizas y calizas margosas } \\
\text { (Capas rojas })\end{array}$ & Cretácico Superior & 3 \\
\hline
\end{tabular}

Tabla 3.21: Unidades de la litología genérica "Conglomerados; areniscas; arcillas y calizas; evaporitas". Tomado de Instituto Geológico y Minero de España (1994), elaboración propia.

\begin{tabular}{|c|c|c|c|}
\hline Siglas & Descripción litológica & Serie & $\begin{array}{l}\text { Clase de emplaza- } \\
\text { miento }\end{array}$ \\
\hline 80 & $\begin{array}{l}\text { Dolomías, calizas y margas. } \\
\text { Margocalizas, calizas areno- } \\
\text { sas, areniscas y arcillas }\end{array}$ & $\begin{array}{l}\text { Cretácico Superior-Paleoceno- } \\
\text { Eoceno }\end{array}$ & 3 \\
\hline $81,81-\mathrm{CB}$ & $\begin{array}{l}\text { Conglomerados, } \\
\text { calizas, margas, arcillas, } \\
\text { yesos y/o sales sodico- } \\
\text { potásicas }\end{array}$ & $\begin{array}{l}\text { Cretácico Superior-Paleoceno- } \\
\text { Eoceno }\end{array}$ & 3 \\
\hline $\begin{array}{l}82,82-\mathrm{S}, 82-\mathrm{CA}, \\
82-\mathrm{C}\end{array}$ & Areniscas silíceas y arcillas & Eoceno & 3 \\
\hline $\begin{array}{l}\text { 83, 83-CB, 83-S, } \\
83-\mathrm{C}\end{array}$ & $\begin{array}{l}\text { Turbiditas calcáreas. Calizas, } \\
\text { calizas arenosas, areniscas y } \\
\text { margas arenosas }\end{array}$ & Eoceno-Oligoceno-Mioceno & 3 \\
\hline 83-CA & $\begin{array}{l}\text { Turbiditas calcáreas. Calizas, } \\
\text { calizas arenosas, areniscas y } \\
\text { margas arenosas }\end{array}$ & Paleoceno-Eoceno & 3 \\
\hline
\end{tabular}


entre clasificar estas cuatro unidades en la clase de emplazamiento 3 y encuadrarlas en la clase 4 (Tabla 3.4). La presencia de las calizas y areniscas puede permitir estimar una resistencia acorde a la descripción geotécnica de la clase 3(rocas blandas a firmes) y por tanto clasificar las unidades en esta clase.

La unidad 86 consta de conglomerados del Paleógeno-Neógeno, así como de arenas, arcillas, margas y areniscas. A partir de esta descripción litológica es posible inferir una resistencia coherente con la descripción geotécnica de la clase de emplazamiento 4 (suelos duros), por lo que se asigna a esta unidad 86 la clase 4 .

\section{Litología genérica: Conglomerados; areniscas; arcillas; calizas y evapori- tas; vulcanitas básicas}

Bajo esta litología genérica se agrupan veintisiete unidades que responden a catorce descripciones litológicas distintas, como se observa en la Tabla 3.23. A excepción de las unidades 94 y 97, compuestas por rocas volcánicas, las unidades constan de sedimentos y rocas sedimentarias detríticas, carbonatadas o evaporíticas.

Con el fin de asignar a cada unidad su respectiva clase de emplazamiento de la clasificación de síntesis (Tabla 3.4), se han agrupado las unidades con la misma descripción litológica. En primer lugar, la unidad 87 se describe como conglomerados, areniscas, arcillas y calizas y/o yesos del Paleógeno-Neógeno. Teniendo en cuenta la edad de las areniscas y calizas presentes, se ha optado por el criterio conservador de manera que se ha desestimado encuadrar esta unidad en la clase 3 (rocas blandas a firmes). En consecuencia, se ha englobado esta unidad en la clase 4, a partir de la existencia de las arcillas y conglomerados terciarios. Con respecto a las unidades 88 y 88-C, éstas constan de areniscas silíceas turbidíticas y calizas y margas arenosas, que conforman un conjunto de rocas equiparable a rocas blandas a firmes. Por tanto, estas unidades se encuadran en la clase 3.

La unidad 89 se compone básicamente de calizas, calcarenitas y diversas clases de margas. La predominancia de las margas permite estimar una resistencia más acorde con la de los suelos duros que con la de las rocas blandas a firmes, por lo que esta unidad se encuadra en la clase de emplazamiento 4. En cuanto a las unidades 90, 90-CB, 90-S, 90-CA y 90-C, su descripción litológica presenta calizas arrecifales, calcarenitas, conglomerados y arcillas con olistolitos. Las calizas y calcarenitas pueden tratarse conjuntamente como rocas blandas a firmes (clase 3), mientras que los conglomerados y las arcillas son más coherentes con la descripción geotécnica de la

Tabla 3.22: Unidades de la litología genérica "Turbiditas calcáreas". Tomado de Instituto Geológico y Minero de España (1994), elaboración propia.

\begin{tabular}{|l|l|l|l|}
\hline Siglas & Descripción litológica & Serie & $\begin{array}{l}\text { Clase de emplaza- } \\
\text { miento }\end{array}$ \\
\hline 84 & $\begin{array}{l}\text { Turbiditas calcáreas, calizas, margas, } \\
\text { conglomerados, areniscas y arcillas. } \\
\text { Calizas lacustres }\end{array}$ & Paleoceno-Eoceno & 3 \\
\hline $\begin{array}{l}\text { 8ur-CA, } \\
84-\mathrm{C}\end{array}$ & $\begin{array}{l}\text { Turbiditas calcáreas, calizas, margas, } \\
\text { Conglomerados, areniscas y arcillas. } \\
\text { Calizas lacustres }\end{array}$ & Paleoceno-Eoceno & 3 \\
\hline 85 & $\begin{array}{l}\text { Turbiditas calcáreas, calizas y margas. } \\
\text { Pudingas, areniscas y margas arenosas. } \\
\text { Areniscas y calizas lacustres }\end{array}$ & Eoceno-Oligoceno-Mioceno & 3 \\
\hline 86 & $\begin{array}{l}\text { Conglomerados, areniscas, arenas, ar- } \\
\text { cillas, margas y yesos }\end{array}$ & Paleoceno-Oligoceno-Mioceno & 4 \\
\hline
\end{tabular}


clase 4 (Tabla 3.4) como suelos duros y arcillas consistentes. Adoptando de nuevo el criterio conservador, se ha asignado a estas cinco unidades la clase 4.

Las unidades 91, 91-CB, 91-S, 91-CA y 91-C se describen como conglomerados, areniscas, calizas, yesos, arenas y arcillas de edad miocena. Recurriendo al criterio conservador, se ha considerado que estas cinco unidades son equiparables a suelos duros y por tanto se les ha asignado la clase de emplazamiento 4. La razón se basa en que se ha otorgado una mayor relevancia a los conglomerados, arenas y arcillas en la estimación de la resistencia de las unidades, con respecto a la importancia que pudieran tener las areniscas y las calizas. Por su parte, la unidad 92 se compone básicamente de conglomerados y margas miocenas, que pueden considerarse también como suelos duros de clase 4. Las unidades 93 y $93-\mathrm{CB}$ se describen como conglomerados, areniscas, calizas y arcillas. Aplicando de nuevo el criterio conservador, la asignación de la clase de emplazamiento 4 a estas dos unidades se ha basado en la mayor importancia que pueden tener los conglomerados y arcillas en la estimación de la resistencia de las unidades, frente a las areniscas y calizas.

En las unidades 94 y 97 aparecen rocas volcánicas de composición alcalina, por tanto se les asigna la clase de emplazamiento 1 (rocas duras) (Tabla 3.4). La unidad 95 presenta conglomerados y margas con niveles turbidíticos, que permiten asignarle la clase 4 de una forma conservadora, aunque también aparezcan otras rocas, como areniscas y calizas arrecifales. La unidad 96 también se encuadra en la clase 4, pues presenta conglomerados, arenas y limos amarillos, acompañados de calizas lacustres (Tabla 3.23).

La descripción litológica de la unidad 98 como calcarenitas, arenas y limos amarillos del Plioceno induce a considerar esta unidad como suelos de consistencia media y a clasificarla en la clase 5. Las arenas y calcarenitas pudieran ser suficientes para clasificar la unidad en la clase 4, pero la presencia de los limos invita a estimar una resistencia algo menor y más coherente con la de la clase 5. Por último, las unidades 99, 99-CB, 99-CA, 99-C y 100 se han clasificado en la clase 4, pues constan fundamentalmente de conglomerados y arcillas pliocuaternarias, conteniendo además areniscas, y calizas (unidades 99, 99-CB, 99-CA y 99-C). Como en el caso de otras descripciones litológicas, se sigue el criterio conservador para dar mayor peso a los conglomerados y las arcillas en la asignación de la clase de emplazamiento de la clasificación de síntesis (Tabla 3.4).

\section{Litología genérica: Conglomerados; gravas; arenas y limos}

Tanto la unidad 101 como la 102 se componen de sedimentos de origen detrítico y edad cuaternaria, como se observa en la Tabla 3.24. Según su descripción litológica, estos sedimentos aparecen en ambientes deposicionales fluviales y litorales. A partir de la clasificación de síntesis (Tabla 3.4), se ha asignado a estas dos unidades la clase de emplazamiento 6, pues la descripción litológica, edad y ambiente deposicional correspondientes permiten inferir una resistencia global, en cada caso, que es razonablemente coherente con la descripción geotécnica de esta clase de emplazamiento como suelos blandos. 
Tabla 3.23: Unidades de la litología genérica "Conglomerados; areniscas; arcillas; calizas y evaporitas; vulcanitas básicas". Tomado de Instituto Geológico y Minero de España (1994), elaboración propia.

\begin{tabular}{|c|c|c|c|}
\hline Siglas & Descripción litológica & Serie & $\begin{array}{l}\text { Clase de emplaza- } \\
\text { miento }\end{array}$ \\
\hline 87 & $\begin{array}{l}\text { Conglomerados, areniscas y arcillas. } \\
\text { Calizas y/o yesos }\end{array}$ & Oligoceno-Mioceno & 4 \\
\hline $88,88-\mathrm{C}$ & $\begin{array}{l}\text { Areniscas silíceas turbidíticas. Cali- } \\
\text { zas y margas arenosas }\end{array}$ & Mioceno & 3 \\
\hline 89 & $\begin{array}{l}\text { Calizas, biocalcarenitas y margas. } \\
\text { Margas y margocalizas blancas con } \\
\text { radiolarios (moronitas o albarizas) }\end{array}$ & Mioceno & 4 \\
\hline $\begin{array}{l}\text { 90, } 90-\mathrm{CB}, \quad 90-\mathrm{S} \\
\text { 90-CA, 90-C }\end{array}$ & $\begin{array}{l}\text { Calizas arrecifales, calcarenitas y } \\
\text { conglomerados. Arcillas con olistoli- } \\
\text { tos }\end{array}$ & Mioceno & 4 \\
\hline $\begin{array}{l}\text { 91, 91-CB, 91-S, } \\
\text { 91-CA, 91-C }\end{array}$ & $\begin{array}{l}\text { Conglomerados, areniscas, arenas ar- } \\
\text { cósicas, arcillas, calizas y yesos }\end{array}$ & Mioceno & 4 \\
\hline 92 & $\begin{array}{l}\text { Conglomerados, calizas y margas. } \\
\text { Margas con olistostromas de origen } \\
\text { diverso }\end{array}$ & Mioceno & 4 \\
\hline $93,93-\mathrm{CB}$ & $\begin{array}{l}\text { Conglomerados, areniscas, arcillas, } \\
\text { calizas y yesos }\end{array}$ & Mioceno & 4 \\
\hline 94 & $\begin{array}{l}\text { Rocas volcánicas calcoalcalinas (an- } \\
\text { desitas, dacitas, riolitas, shoshonitas, } \\
\text { lamproítas) }\end{array}$ & Mioceno & 1 \\
\hline 95 & $\begin{array}{l}\text { Conglomerados, calcarenitas, calizas } \\
\text { arrecifales, areniscas y margas con } \\
\text { niveles turbidíticos }\end{array}$ & Mioceno & 4 \\
\hline 96 & $\begin{array}{l}\text { Conglomerados, arenas, arrecifes, li- } \\
\text { mos amarillos, yesos y sales haloi- } \\
\text { deas. Conglomerados, arenas y cali- } \\
\text { zas lacustres }\end{array}$ & Mioceno & 4 \\
\hline 97 & Basaltos alcalinos & $\begin{array}{l}\text { Plioceno- } \\
\text { Pleistoceno- } \\
\text { Holoceno } \\
\end{array}$ & 1 \\
\hline 98 & $\begin{array}{l}\text { Calcarenitas, arenas y limos amari- } \\
\text { llos }\end{array}$ & Plioceno & 5 \\
\hline $\begin{array}{l}\text { 99, 99-CB, 99-CA, } \\
99-\mathrm{C}\end{array}$ & $\begin{array}{l}\text { Conglomerados, areniscas, arcillas, } \\
\text { calizas y/o yesos }\end{array}$ & $\begin{array}{l}\text { Plioceno-Pleistoceno } \\
\text { Inferior }\end{array}$ & 4 \\
\hline 100 & Conglomerados, areniscas y arcillas & $\begin{array}{l}\text { Plioceno-Pleistoceno } \\
\text { Inferior }\end{array}$ & 4 \\
\hline
\end{tabular}

Tabla 3.24: Unidades de la litología genérica "Conglomerados; gravas; arenas y limos". Tomado de Instituto Geológico y Minero de España (1994), elaboración propia.

\begin{tabular}{|l|l|l|l|}
\hline Sigla & Descripción litológica & Serie & $\begin{array}{l}\text { Clase de emplaza- } \\
\text { miento }\end{array}$ \\
\hline 101 & $\begin{array}{l}\text { Conglomerados, gravas, arenas, arenis- } \\
\text { cas, arenas, limos y arcillas. Terrazas } \\
\text { fluviales y marinas }\end{array}$ & Pleistoceno & 6 \\
\hline 102 & $\begin{array}{l}\text { Gravas, arenas, arcillas y limos. Alu- } \\
\text { vial, playas, flechas litorales }\end{array}$ & Holoceno & 6 \\
\hline
\end{tabular}




\subsubsection{Unidades geológicas de las Islas Canarias}

La clasificación de las unidades geológicas de las Islas Canarias se ha llevado a cabo por medio de la asignación de una clase de emplazamiento a cada una de las unidades que constituyen las regiones GEODE en las que se estructura la cartografía GEODE en el archipiélago canario (GEODE, 2014). En este sentido, sólo se han clasificado las 758 unidades cartografiadas y con información relativa a su litología. A estos efectos, se han tomado en consideración las dos descripciones litológicas de cada unidad y su edad cronoestratigráfica, hasta el nivel de serie. El proceso de clasificación ha seguido los mismos criterios que los seguidos en la Península Ibérica y las Islas Baleares, de acuerdo a la metodología de Borcherdt (1994).

A continuación se detalla el citado proceso para las unidades geológicas de cada una de las regiones GEODE. Las unidades son introducidas de acuerdo al sistema, dominio, edificio, ciclo volcánico, macizo plutónico, formación o miembro al cual pertenecen, según la leyenda cronoestratigráfica del mapa de la región GEODE. Para cada unidad, se indica el código alfanumérico que la identifica en la leyenda cronoestratigráfica, así como su descripción litológica breve y su edad cronoestratigráfica.

\section{El Hierro (Región 2916)}

Según GEODE (2014), la cartografía GEODE en El Hierro distingue 41 unidades geólogicas, cuyas edades cronoestratigráficas comienzan en el Pleistoceno InferiorMedio. Las correspondientes descripciones litológicas y edades cronoestratigráficas se muestran en la Tabla A.1. De acuerdo con la leyenda cronoestratigráfica de la región 2916 de la cartografía GEODE, que corresponde a esta isla (GEODE, 2014), las unidades se distribuyen en 12 grupos, que pueden ser consultados en la Tabla 3.25. De las 41 unidades geológicas mencionadas, 2 no aparecen cartografiadas y por tanto no se han clasificado: unidades 1 y 2 .

Tabla 3.25: División de las unidades del mapa geológico continuo GEODE a escala 1:50.000 de El Hierro (Zona 2916), de acuerdo a la leyenda cronoestratigráfica. La abreviatura s.l. (sensu lato) indica que la referencia a la que acompaña se entiende en un sentido amplio. La abreviatura s.s. (sensu stricto) indica que la referencia se entiende en un sentido estricto. Tomado de GEODE (2014).

\begin{tabular}{|l|l|l|}
\hline $\begin{array}{l}\text { Código de la } \\
\text { unidad geológica }\end{array}$ & $\begin{array}{l}\text { Sistema/Dominio/Edificio/Ciclo volcánico/Macizo } \\
\text { plutónico/Formación/Miembro }\end{array}$ \\
\cline { 1 - 2 } 1,2 & Rocas filonianas & \multirow{2}{*}{ Edificio Tiñor } \\
\hline 3,4 & Tramo inferior & \\
\hline $5,6,7$ & Tramo tabular & \multirow{2}{*}{ Edificio El Golfo-Las Playas } \\
\hline 8,9 & Conjunto volcánico Ventejis-Picos-Moles & \multirow{2}{*}{ Volcanismo de las dorsales } \\
\hline $10,11,12$ & Tramo inferior & \multirow{2}{*}{ Tramo medio-superior } \\
\hline $13,14,15$ & Volcanismo de las dorsales (s.s.) & \\
\hline $21,22,23,24$ & Volcanismo de relleno de El Golfo & \\
\hline $25,26,27,28$ & Emisiones subrecientes & \\
\hline $29,30,31,32$ & Emisiones recientes & Posible emisión histórica (1793?) \\
\hline $33,34,35$ & Formaciones sedimentarias recientes & \\
\hline $36,38,39,40$ & &
\end{tabular}


Edificio Tiñor (Tramo inferior) La unidad 3 se describe como coladas basálticas, basaníticas y tefríticas, como puede observarse en la Tabla A.1. La descripción litológica extensa de esta unidad (GEODE, 2014) consiste en basaltos de diferentes composiciones. Dado que los basaltos son rocas volcánicas, permiten inferir que la resistencia de esta unidad es equivalente a la de las rocas duras. La descripción geotécnica de la clase de emplazamiento 1 (Tabla 3.4) consiste también en rocas duras, por tanto es razonable suponer que la resistencia de la unidad 3 es equiparable a la resistencia de esta clase de emplazamiento. En consecuencia, se clasifica la unidad 3 en la clase 1. En cuanto a la unidad 4, ésta se compone de conos de tefra basálticos, como muestra la Tabla A.1. Según la descripción litológica extensa de esta unidad (GEODE, 2014), estos conos presentan piroclastos heterométricos (escorias, bombas, lapilli y cenizas) de basaltos de distintas composiciones. Estos piroclastos conforman un suelo con una compacidad o consistencia que puede considerarse alta, lo que les confiere una resistencia algo menor que la de una roca blanda. Trasladando estas consideraciones a la clasificación de síntesis de la Tabla 3.4, es razonable asignar la clase de emplazamiento 4 a la unidad 4, puesto que la descricpión geotécnica de la clase 4 consiste en suelos duros, que tienen una consistencia alta. Adicionalmente, la descripción geotécnica de la clase 3 como rocas firmes a blandas/suelos muy densos (Tabla 3.4) permite constatar que la resistencia de esta clase es superior a la inferida para la unidad 4. Por este motivo, la selección de la clase de emplazamiento 4 resulta de nuevo adecuada, pues ésta presenta la compacidad/consistencia, y por tanto la resistencia, inmediatamente inferiores a las propiedades geotécnicas propias de la clase 3 .

Edificio Tiñor (Tramo tabular) La unidad 5 consiste en coladas basálticas, basaníticas y tefríticas (Tabla A.1). Debido a la presencia de rocas volcánicas (basaltos, basanitas y tefritas), se aplica el criterio adoptado para clasificar la unidad 3, también compuesta por rocas volcánicas, en la clase de emplazamiento 1 (Tabla 3.4). La unidad 6 se compone de conos de tefra basálticos. Se procede, por tanto, de forma similar a la seguida en el caso de la unidad 4, considerando que los depósitos piroclásticos presentes en la unidad 6 constituyen suelos de alta compacidad/consistencia y que, por tanto, deben ser clasificados como emplazamientos de clase 4 (Tabla 3.4). Con respecto a la unidad 7 , su descripción litológica consta de cuerpos intrusivos básicos (Tabla A.1), que están compuestos por basaltos piroxénicos porfídicos y basaltos afaníticos (GEODE, 2014). De nuevo, se adopta el criterio de clasificación de las unidades compuestas por rocas volcánicas, como los basaltos, para encuadrar la unidad 7 en la clase de emplazamiento 1.

Edificio Tiñor (Conjunto volcánico Ventejis-Picos-Moles) De acuerdo a la Tabla A.1, la unidad 8 se describe como coladas basálticas y tefríticas. Esta unidad presenta rocas volcánicas (basaltos y tefritas), por lo cual debe aplicarse el criterio ya explicado de clasificación de las unidades con rocas volcánicas en la clase de emplazamiento 1 (Tabla 3.4). La unidad 9 está compuesta por conos de tefra basálticos con intercalaciones hidromagmáticas. Como se ha mencionado al analizar las unidades 4 ó 6 , los conos de tefra se presentan como depósitos piroclásticos, asimilables a suelos de alta compacidad o consistencia, y por tanto clasificables en la clase de emplazamiento 4 . Por tanto, la unidad 9 es encuadrada en esta clase. 
Edificio El Golfo-Las Playas (Tramo inferior) La unidad 10 se compone de coladas basálticas, traquibasálticas y tefríticas, como se puede observar en la Tabla A.1. La descripción litológica extensa de GEODE (2014) indica que estas coladas presentan basaltos de distintas composiciones, los cuales, al ser rocas volcánicas, permiten clasificar la unidad 10 en la clase de emplazamiento 1 (Tabla 3.4). La unidad 11 se describe como conos de tefra basálticos e hidromagmáticos. Por tanto, puede adoptarse el criterio de clasificación de los depósitos piroclásticos como suelos de alta compacidad/consistencia y pertenecientes a la clase de emplazamiento 4. La unidad 12, por su parte, consiste en cuerpos intrusivos de basaltos y traquibasaltos (GEODE, 2014). En función de la presencia de estas rocas volcánicas, se clasifica la unidad 12 en la clase de emplazamiento 1.

Edificio El Golfo-Las Playas (Tramo medio-superior) Según la Tabla A.1, la unidad 13 se describe como coladas basálticas, traquibasálticas, basaníticas y tefríticas. Como se ha explicado, debido a que la descripción litológica de esta unidad se fundamenta en rocas volcánicas, como los basaltos y los traquibasaltos, se procede a clasificar la unidad en la clase de emplazamiento 1 (Tabla 3.4). En cuanto a la unidad 14, ésta se compone de conos de tefra basálticos, traquibasálticos, basaníticos y tefríticos (Tabla A.1). En función de esta descripción, se adopta el criterio de clasificar las unidades con depósitos piroclásticos en la clase de emplazamiento 4. La unidad 15 es descrita como coladas de traquitas y traquitas máficas. Las traquitas son rocas volcánicas, por lo cual permiten utilizar el criterio de clasificación de las unidades con este tipo de rocas en la clase de emplazamiento 1.

Volcanismo de las dorsales (s.l.) (Volcanismo de las dorsales (s.s.)) En este apartado se justifica la clasificación de las unidades correspondientes al volcanismo de las dorsales en un sentido estricto (s.s., sensu stricto): unidades 16, 17, 18, 18a, 19, 20, 21, 22, 23 y 24. Estas unidades se enmarcan a su vez en un grupo más genérico de volcanismo de dorsales, ya entendido en un sentido amplio (s.l., sensu lato), el cual comprende también las unidades 25, 26, 27, 28, 29, 30, 31, 32, 33, 34, 35 y 36 .

Según la Tabla A.1, la unidad 16 se presenta en depósitos sedimentarios detríticos, que consisten en bloques, arenas con cantos y brechas caóticas (GEODE, 2014). Los sedimentos de tamaño arena y superior (en este caso las arenas y los bloques) son indicativos de una compacidad o consistencia alta, como se ha inferido en el caso de los depósitos piroclásticos. En función de la descripción geotécnica de la clase de emplazamiento 4 como suelos cohesivos duros (Tabla 3.4), y por tanto con alta consistencia, se ha considerado que la resistencia de esta clase es equiparable a la resistencia de la unidad 16, pues el grado de compacidad/consistencia es parecido. Por tanto, se clasifica la unidad 16 en la clase 4 . La unidad 17 se compone de sedimentos arenosos-arcillosos (Tabla A.1), cuya descripción litológica extensa se concreta en limos con cantos y tobas de distintas clases (GEODE, 2014). Se considera que, al contener granulometrías de tamaño limo, estos sedimentos conforman suelos de baja consistencia, y por tanto blandos. A tenor de la descripción geotécnica de la clase de emplazamiento 6 como suelos blandos (Tabla 3.4), se estima que la resistencia de la unidad 17 es coherente con la resistencia de la clase 6. La unidad 18 consiste en coladas basálticas, traquibasálticas, basaníticas y tefríticas. Se adopta por tanto 
el criterio de clasificación de las unidades con rocas volcánicas (en este caso basaltos, traquibasaltos, basanitas y tefritas) en la clase de emplazamiento 1 (Tabla 3.4). La unidad 18a se describe como mantos de lapilli sobre coladas (Tabla A.1). Estos mantos configuran depósitos de lapillis, que son piroclastos con una granulometría fina. Este bajo tamaño de grano recomienda suponer una consistencia menor que la consistencia alta que se ha considerado para el caso de los depósitos piroclásticos. Por tanto, se infiere una consistencia media para la unidad 18a, coherente con la de la clase de emplazamiento 5 (Tabla 3.4 ), pues esta clase se describe geotécnicamente como suelos de consistencia media. La unidad 19, por su parte, se compone de conos de tefra basálticos, traquibasálticos, basaníticos y tefríticos. Dado que la unidad configura un depósito piroclástico, se aplica el criterio de clasificarla en la clase de emplazamiento 4. En cuanto a la unidad 20, la descripción litológica extensa de GEODE (2014) presenta lapillis y cenizas. En función de la baja granulometría de estos piroclastos, se adopta el mismo criterio conservador que el aplicado para clasificar la unidad 18a, en el sentido de considerar que la unidad tiene una consistencia media, y no alta. Por tanto, la unidad 20 se encuadra en la clase de emplazamiento 5 (Tabla 3.4).

La unidad 21 consiste en depósitos piroclásticos freatomagmáticos (Tabla A.1), cuya descripción litológica extensa se concreta en depósitos de cenizas con cantos (GEODE, 2014). Debido a que estos depósitos están formados fundamentalmente por cenizas, y a su baja granulometría, se considera que la consistencia de la unidad 21 es baja. Por tanto, la resistencia de esta unidad es equiparable a la de la clase de emplazamiento 6, pues ésta se describe geotécnicamente como suelos blandos y, por tanto, con baja consistencia. La unidad 22 se describe como depósitos piroclásticos compuestos de cineritas grises, arenas con pómez y lapilli (GEODE, 2014). Se procede como en el caso de la unidad 20, en el sentido de considerar los depósitos de cenizas y lapilli como suelos de consistencia media, y por tanto clasificables en la clase de emplazamiento 5 (Tabla 3.4). Las unidades 23 y 24 se describen como coladas traquíticas y basálticas, respectivamente (Tabla A.1). Se aplica por tanto el criterio de clasificación de las unidades con rocas volcánicas (en esta unidad, traquitas y basaltos) en la clase de emplazamiento 1.

Volcanismo de las dorsales (s.l.) (Volcanismo de relleno de El Golfo) La unidad 25 tiene una descripción litológica extensa que consiste en depósitos detríticos muy heterométricos, brechas, tobas epiclásticas y depósitos coluviales (GEODE, 2014). La fuerte heterometría presente, y el desconocimiento de una granulometría predominante, recomiendan aplicar un criterio conservador e inferir una consistencia media para esta unidad. Considerando la descripción geotécnica de la clase de emplazamiento 5 (Tabla 3.4) como suelos de consistencia media, es razonable suponer que su resistencia es similar a la de la unidad 25. Por tanto, se clasifica esta unidad en la clase 5. La unidad 26 presenta coladas basálticas, basaníticas y tefríticas (Tabla A.1). En este caso, se adopta el criterio de clasificación de las unidades con rocas volcánicas (basaltos, basanitas y tefritas) en la clase de emplazamiento 1. Con respecto a la unidad 27, ésta consta de conos de tefra basálticos, basaníticos y tefríticos. Por tanto, se aplica el criterio de clasificar las unidades con depósitos piroclásticos en la clase de emplazamiento 4. La unidad 28 es descrita por GEODE (2014) como lapillis y cenizas. Debido a la baja granulometría de los piroclastos presentes, se pro- 
cede análogamente al caso de la unidad 20, considerando que la consistencia global de la unidad es media. Por tanto, se asigna la clase de emplazamiento 5 (Tabla 3.4) a la unidad 28.

Volcanismo de las dorsales (s.l.) (Emisiones subrecientes) La unidad 29 configura niveles de playa fósiles (Tabla A.1), que son descritos más ampliamente por GEODE (2014) como compuestos por gravas. Debido a que esta unidad constituye depósitos sedimentarios detríticos con granulometría de tamaño superior al de las arenas (en este caso, tamaño grava), se procede como en el caso de la unidad 16, considerando que la compacidad/consistencia de la unidad 29 es alta. Por tanto, esta propiedad permite equiparar la resistencia de la unidad 29 a la de la clase de emplazamiento 4 (Tabla 3.4), pues ésta se describe geotécnicamente como suelos duros, es decir, con alta consistencia. La unidad 30 se describe como coluviones antiguos, que se disponen junto con brechas compactadas (GEODE, 2014). Los coluviones son depósitos constituidos por la acumulación de materiales en las laderas, debido a la acción de la gravedad. Se entiende que la compacidad/consistencia global de este tipo de depósitos es media-alta. Considerando la descripción geotécnica de la clase de emplazamiento 4 (Tabla 3.4) como suelos duros, es decir, de alta consistencia, se considera que la resistencia de la unidad 30 es equivalente a la resistencia de esta clase. Además, la presencia de las brechas compactadas también contribuye a este nivel de resistencia. Consecuentemente, se encuadra esta unidad en la clase 4. La unidad 31 consiste fundamentalmente en coladas de basaltos olivínicos y basaltos olivínico-piroxénicos (GEODE, 2014). Al presentarse rocas volcánicas, la unidad 31 es clasificable como emplazamiento de clase 1 (Tabla 3.4). La unidad 32 se describe como conos de tefra basálticos, basaníticos y foidíticos (Tabla A.1). Se aplica, por tanto, el criterio de clasificación de las unidades con depósitos piroclásticos en la clase de emplazamiento 4 , y se clasifica la unidad 32 en esta clase.

Volcanismo de las dorsales (s.l.) (Emisiones recientes) La unidad 33 se compone de coladas basálticas, basaníticas y foidíticas (Tabla A.1). En función de la presencia de basaltos, basanitas y foiditas, se aplica el criterio de clasificación de las unidades con rocas volcánicas en la clase de emplazamiento 1 (Tabla 3.4). La unidad 34 consiste en conos de tefra basálticos, basaníticos y foidíticos. Estas litologías configuran depósitos piroclásticos, por lo cual la unidad 34 se clasifica en la clase de emplazamiento 4. En cuanto a la unidad 35, ésta consta de piroclastos de dispersión basálticos, basaníticos y foidíticos (Tabla A.1), los cuales son descritos como cenizas y lapillis por GEODE (2014). Se considera nuevamente que los depósitos de cenizas y lapillis tienen consistencia media, y se clasifica, por tanto, la unidad 35 en la clase de emplazamiento 5 .

Volcanismo de las dorsales (s.l.) (Posible emisión histórica (1793?)) La unidad 36 es descrita como coladas basálticas (Tabla A.1). Como los basaltos son rocas volcánicas, se consideran rocas duras, y por tanto se clasifica la unidad 36 en la clase de emplazamiento 1 (Tabla 3.4).

Formaciones sedimentarias recientes La unidad 37 consiste en coluviones y depósitos de ladera (Tabla A.1). La descripción litológica extensa de GEODE (2014) 
aporta que estos depósitos contienen brechas, bloques, cantos, arenas y limos. La unidad forma depósitos sedimentarios con clastos gruesos (bloques, cantos, arenas), y también con brechas, por lo que la resistencia global es equiparable a la de los suelos duros que describen la clase de emplazamiento 4 (Tabla 3.4). La unidad 38 configura depósitos aluviales con arenas, gravas y bloques. Considerando estas litologías, se aplica el criterio de clasificación seguido para las unidades 16 y 29, en el sentido de estimar que la compacidad/consistencia de los depósitos detríticos con tamaño de grano igual o superior al de las arenas es alta. Por tanto, se clasifica la unidad 38 en la clase de emplazamiento 4. Idéntico criterio se sigue para clasificar la unidad 39 en la clase de emplazamiento 4, pues su descripción extensa consta de depósitos de arenas y gravas (GEODE, 2014). Finalmente, la unidad 40 se describe como depósitos antrópicos (Tabla A.1). En este caso, se adopta un criterio conservador y se considera que la consistencia/compacidad de estos materiales es muy baja. Por tanto, se consideran pertenecientes a la clase 6 , a tenor de la descripción geotécnica de esta clase como suelos blandos, y por tanto con baja consistencia.

En la Tabla 3.26 se resumen las clases de emplazamiento que corresponden a las unidades de El Hierro, de acuerdo al procedimiento de clasificación explicado.

\section{Fuerteventura (Región 2911)}

De acuerdo con la cartografía de la región GEODE 2911, que corresponde a Fuerteventura (GEODE, 2014), en la isla afloran 185 unidades geológicas, datadas desde el Cretácico hasta el Holoceno, cuyas descripciones litológicas y edades cronoestratigráficas se detallan en la Tabla A.2. Según la leyenda cronoestratigráfica de esta cartografía, las unidades pertenecen a 20 grupos distintos, que se presentan en la Tabla 3.27. Del conjunto de las 185 unidades geológicas referidas, 26 no aparecen cartografiadas y por tanto no se han clasificado: unidades 1a, 2a, 7b, 8, 8b, 9, 9b, 12, 12b, 15, 15b, 16, 16b, 29b, 32, 32a, 35a, 38, 39, 41, 64, 65, 77a, 99, 102 y 105.

Sedimentos de fondo oceánico La unidades 1 y 1 b se describen como ritmitas, lutitas, areniscas y calizas, pertenencientes al Complejo Basal de la isla, con $<20 \%$ y $>75 \%$ de diques, respectivamente. Estas litologías, de edad cretácica, pueden equipararse a las turbiditas, que son series alternantes de rocas detríticas (arenisca, lutita), a veces carbonatadas. La resistencia de esta unidad puede por tanto considerarse equivalente a la resistencia de la clase de emplazamiento 3 de la clasificación de síntesis (Tabla 3.4), descrita como rocas blandas a firmes. Como conclusión, las unidades 1 y 1 b son englobadas en la clase 3. Siguiendo el mismo razonamiento, las unidades 2 y $2 \mathrm{~b}$ también se clasifican en la clase 3 , pues se componen de lutitas y areniscas del Cretácico (Tabla A.2). En cuanto a la unidad 3, consistente en lutitas, margas y calizas del Cretácico, la presencia de las calizas cretácicas podría aumentar la resistencia de la unidad y plantear la posibilidad de clasificarla en la clase 2. No obstante, las margas presentes, cuya descripción geotécnica es acorde a la de la clase 3 de la clasificación de síntesis (rocas blandas a firmes), hacen preferible la clasificación de esta unidad en la clase de emplazamiento 3.

Lavas y piroclastos en parte submarinos La unidad 4 se describe como rocas carbonatadas y silicificadas del Cretácico Superior-Oligoceno. Dada la edad de las 
Tabla 3.26: Resumen de la clasificación de las unidades del mapa geológico continuo GEODE a escala 1:50.000 de El Hierro (Zona 2916), de acuerdo a la clasificación de síntesis. La abreviatura s.l. ( sensu lato) indica que la referencia a la que acompaña se entiende en un sentido amplio. La abreviatura s.s. (sensu stricto) indica que la referencia se entiende en un sentido estricto. Tomado de GEODE (2014), elaboración propia.

\begin{tabular}{|c|c|c|c|}
\hline \multicolumn{2}{|c|}{$\begin{array}{l}\text { Sistema/Dominio/Edificio/Ciclo } \\
\text { volcánico/Macizo } \\
\text { plutónico/Formación/Miembro }\end{array}$} & $\begin{array}{l}\text { Código de la unidad } \\
\text { geológica }\end{array}$ & $\begin{array}{l}\text { Clase de } \\
\text { emplazamiento }\end{array}$ \\
\hline \multirow{7}{*}{ Edificio Tiñor } & \multirow{2}{*}{ Tramo inferior } & 3 & 1 \\
\hline & & 4 & 4 \\
\hline & \multirow{3}{*}{ Tramo tabular } & 5 & 1 \\
\hline & & 6 & 4 \\
\hline & & 7 & 1 \\
\hline & \multirow{2}{*}{$\begin{array}{l}\text { Conjunto volcánico } \\
\text { Ventejis-Picos-Moles }\end{array}$} & 8 & 1 \\
\hline & & 9 & 4 \\
\hline \multirow{6}{*}{$\begin{array}{l}\text { Edificio El } \\
\text { Golfo-Las Playas }\end{array}$} & \multirow{3}{*}{ Tramo inferior } & 10 & 1 \\
\hline & & 11 & 4 \\
\hline & & 12 & 1 \\
\hline & \multirow{3}{*}{ Tramo medio-superior } & 13 & 1 \\
\hline & & 14 & 4 \\
\hline & & 15 & 1 \\
\hline \multirow{22}{*}{$\begin{array}{l}\text { Volcanismo de las } \\
\text { dorsales (s.l.) }\end{array}$} & \multirow{10}{*}{$\begin{array}{l}\text { Volcanismo de las dorsales } \\
\text { (s.s.) }\end{array}$} & 16 & 4 \\
\hline & & 17 & 6 \\
\hline & & 18 & 1 \\
\hline & & $18 \mathrm{a}$ & 5 \\
\hline & & 19 & 4 \\
\hline & & 20 & 5 \\
\hline & & 21 & 6 \\
\hline & & 22 & 5 \\
\hline & & 23 & 1 \\
\hline & & 24 & 1 \\
\hline & \multirow{4}{*}{$\begin{array}{l}\text { Volcanismo de relleno de } \\
\text { El Golfo }\end{array}$} & 25 & 5 \\
\hline & & 26 & 1 \\
\hline & & 27 & 4 \\
\hline & & 28 & 5 \\
\hline & \multirow{4}{*}{ Emisiones subrecientes } & 29 & 4 \\
\hline & & 30 & 4 \\
\hline & & 31 & 1 \\
\hline & & 32 & 4 \\
\hline & \multirow{3}{*}{ Emisiones recientes } & 33 & 1 \\
\hline & & 34 & 4 \\
\hline & & 35 & 5 \\
\hline & $\begin{array}{l}\text { Posible emisión histórica } \\
(1793 ?)\end{array}$ & 36 & 1 \\
\hline \multirow{4}{*}{\multicolumn{2}{|c|}{ Formaciones sedimentarias recientes }} & 37 & 5 \\
\hline & & 38 & 4 \\
\hline & & 39 & 4 \\
\hline & & 40 & 6 \\
\hline
\end{tabular}

rocas carbonatadas presentes, se estima que su resistencia es equiparable a la de la clase de emplazamiento 2 (Rocas de resistencia media), y por tanto se asigna esta clase a la unidad 4. Respecto a las unidades 5, 5a y 5b, éstas se describen como lavas, tobas y brechas del Complejo Basal, con diferentes porcentajes de diques y edades comprendidas entre el Cretácico Superior y el Oligoceno. Estas unidades se componen por tanto de materiales volcánicos diversos, algunos brechificados e intercalados con sedimentos cretácicos. Por esta razón, puede considerarse una consistencia global firme, acorde con la descripción de la clase de emplazamiento 3 (Tabla 3.4).

Serie intrusiva ultraalcalina Las unidades $6,6 \mathrm{a}$ y $6 \mathrm{~b}$ consisten en piroxenitas melteigitas, ijolitas, sienitas y carbonatitas del Complejo Basal, con diferentes porcentajes de diques (Tabla A.2). Puesto que estas litologías corresponden a rocas plutónicas, que son consideradas como rocas duras, las tres unidades se clasifican en la clase de emplazamiento 1 (Tabla 3.4). Por su parte, las unidades 7 y 7 a se 
Tabla 3.27: División de las unidades del mapa geológico continuo GEODE a escala 1:50.000 de Fuerteventura (Zona 2911), de acuerdo a la leyenda cronoestratigráfica. Tomado de GEODE (2014).

\begin{tabular}{|c|c|}
\hline Código de la unidad geológica & $\begin{array}{l}\text { Sistema/Dominio/Edificio/Ciclo vol- } \\
\text { cánico/Macizo plutónico/Formación/ } \\
\text { Miembro }\end{array}$ \\
\hline $1,1 \mathrm{a}, 1 \mathrm{~b}, 2,2 \mathrm{a}, 2 \mathrm{~b}, 3$ & Sedimentos de fondo oceánico \\
\hline $4,5,5 \mathrm{a}, 5 \mathrm{~b}$ & Lavas y piroclastos en parte submarinos \\
\hline $6,6 \mathrm{a}, 6 \mathrm{~b}, 7,7 \mathrm{a}, 7 \mathrm{~b}, 8,8 \mathrm{a}, 8 \mathrm{~b}, 9,9 \mathrm{a}, 9 \mathrm{~b}$ & Serie intrusiva ultraalcalina \\
\hline $\begin{array}{l}10,11,11 \mathrm{a}, 11 \mathrm{~b}, 12,12 \mathrm{a}, 12 \mathrm{~b}, 13 \mathrm{a}, 13 \mathrm{~b} \\
13 \mathrm{c}, 13 \mathrm{~d}, 14\end{array}$ & Series plutónicas indiferenciadas \\
\hline $15,15 \mathrm{a}, 15 \mathrm{~b}, 16,16 \mathrm{a}, 16 \mathrm{~b}$ & Macizo máfico-ultramáfico de Mezquez \\
\hline $17,18,19,20,21$ & Complejo circular Vega Río Palmas \\
\hline $22,23,24,25,26,27,31,32,32 \mathrm{a}, 32 \mathrm{~b}$ & Edificio Betancuria \\
\hline $28,29,29 \mathrm{a}, 29 \mathrm{~b}, 30$ & $\begin{array}{l}\text { Dominio del Complejo Basal. Edificio Morro } \\
\text { Negro-Morro del Sol }\end{array}$ \\
\hline $33,34,35,35 \mathrm{a}, 35 \mathrm{~b}, 36,37$ & Formaciones de transición \\
\hline $38,39,40,41$ & Rocas filonianas del Edificio Gran Tarajal \\
\hline $\begin{array}{l}42,42 \mathrm{a}, 43,44,45,46,47,48,49,50, \\
51,52,53,54,55,56,57,58,59,60,61, \\
62,63\end{array}$ & Edificio Gran Tarajal \\
\hline 64,65 & Rocas filonianas del Edificio Jandía \\
\hline $\begin{array}{l}66,67,68,69,70,71,72,73,74,75,76, \\
77,77 \mathrm{a}, 78,79,80,81,82,83,84,85, \\
86,87\end{array}$ & Edificio Jandía \\
\hline 88,89 & Rocas filonianas del Edificio Tetir \\
\hline $\begin{array}{l}90,91,92,93,94,95,96,97,98,99,100, \\
101,102,103,104\end{array}$ & Edificio Tetir \\
\hline 105 & Rocas filonianas pliocenas \\
\hline $\begin{array}{l}106,107,108,109,110,111,112,113, \\
114,115,116,117\end{array}$ & Fase pliocena \\
\hline $118,119,120,121,122,123,124$ & Formaciones sedimentarias plio-pleistocenas \\
\hline $\begin{array}{l}125,126,127,128,129,130,131,132, \\
133,134,135\end{array}$ & $\begin{array}{l}\text { Fase pleistocena media-holocena (episodios re- } \\
\text { cientes) }\end{array}$ \\
\hline $\begin{array}{l}136,137,138,139,140,141,142,143, \\
144,145,146,147,148,149,150,151, \\
152\end{array}$ & Formaciones sedimentarias recientes \\
\hline
\end{tabular}

componen de traquitas, fonolitas y sienitas del Complejo Basal, con porcentajes de diques menores que el $20 \%$, y comprendidos entre el 20 y el $75 \%$, respectivamente. Las traquitas y fonolitas son rocas volcánicas, y las sienitas rocas plutónicas, por lo cual ambas unidades se consideran compuestas de rocas duras y, por tanto, se encuadran en la clase de emplazamiento 1. En cuanto a la unidad 8a, ésta forma parte del macizo sienítico de El Recogedero, perteneciente al Complejo Basal (Tabla A.2). De acuerdo con la descripción litológica extensa proporcionada por GEODE (2014), esta unidad presenta sienitas alcalinas, con un porcentaje de diques comprendido entre el 20 y el $75 \%$. Dado que las sienitas alcalinas son rocas plutónicas, y por tanto rocas duras, se asigna a esta unidad la clase de emplazamiento 1. La unidad 9a consiste en un intrusivo fonolítico con diques entre el 20 y el $75 \%$. Puesto que las fonolitas son rocas volcánicas (rocas duras), la unidad 9a también es englobada en la clase de emplazamiento 1.

Series plutónicas indiferenciadas La unidad 10 se compone de brechas de intrusión, de acuerdo a la Tabla A.2. Este tipo de brecha está formado por cantos de 
naturaleza plutónica y volcánica, muy compactados. Es por esta razón que la unidad puede considerarse equiparable a una roca blanda a firme, y por tanto clasificarse en la clase de emplazamiento 3 de la clasificación de síntesis (Tabla 3.4). Las unidades 11, 11a y 11b consisten en intrusiones de gabros del Complejo Basal, con diferentes porcentajes de diques (Tabla A.2). Dado que los gabros son rocas plutónicas, y por tanto rocas duras, estas unidades se encuadran en la clase de emplazamiento 1. En cuanto a la unidad 12a, ésta se compone de werhlitas y piroxenitas del Complejo Basal, con porcentaje de diques comprendido entre el 20 y el $75 \%$. Ambas litologías corresponden a rocas plutónicas ultrabásicas, consideradas rocas duras y con resistencia alta. Por tanto, a la unidad 12a se le asigna la clase 1 . Con respecto a las unidades 13a, 13b, 13c y 13d, su descripción litológica consiste en intrusivos sálicos traquíticos y sieníticos del Complejo Basal, con porcentajes de diques $>20 \%$. Las descripciones litológicas extensas de estas unidades coinciden en describirlas como brechas formadas por fragmentos de diques traquíticos y de sienitas, y por algunos fragmentos de gabros (GEODE, 2014). Debido a la naturaleza brechoide de estas litologías, se considera que su resistencia puede ser coherente con la de la clase de emplazamiento 3, pues ésta consiste en rocas blandas a firmes (Tabla 3.4). Por esta razón, se engloban las unidades 13a, 13b, 13c y 13d en la clase 3. Utilizando el mismo razonamiento, la unidad 14 se clasifica también en la clase 3 , pues se describe como brechas sálicas compactas.

Macizo máfico-ultramáfico de Mezquez La unidad 15a se describe como werhlitas y piroxenitas del Complejo Basal con porcentaje de diques comprendido entre el 20 y el $75 \%$. Puesto que ambas litologías pertenecen al grupo de las rocas plutónicas ultrabásicas, y presentan alta resistencia, se encuadran en la clase de emplazamiento 1 (Rocas duras), de acuerdo a la Tabla 3.4. En cuanto a la unidad 16a, ésta se compone de gabros del Complejo Basal, con porcentaje de diques comprendido entre el 20 y el $75 \%$. Debido a que los gabros son rocas plutónicas, y por tanto rocas duras, se asigna a la unidad 16a la clase de emplazamiento 1.

Complejo circular Vega Río Palmas La unidad 17 consiste en leucogabros bandeados de grano medio (Tabla A.2). Siguiendo el razonamiento expuesto, el hecho de que los gabros sean rocas plutónicas confiere a esta litología la resistencia propia de las rocas duras (clase de emplazamiento 1 en la Tabla 3.4). Por tanto, se asigna la clase 1 a esta unidad. La unidad 18 se compone de sienitas nefelínicas que, al ser también rocas plutónicas, permiten encuadrar la unidad en la clase 1. Por su parte, la unidad 19 se describe como sienitas nefelínicas con fracturas (diaclasas). Esta fracturación produce una disminución en la resistencia inferida para esta unidad. Es por esta razón que la unidad 19 se clasifica en la clase de emplazamiento 2 (Rocas de resistencia media), de acuerdo a la Tabla 3.4. En cuanto a la unidad 20, su descripción litológica consiste en tobas e intrusivos traquíticos indiferenciados. De acuerdo con la descripción litológica extensa proporcionada por GEODE (2014), estos materiales se presentan en un dique heterogéneo con traquitas (rocas volcánicas) brechoides, a veces con textura tobácea (piroclástica). Se entiende por tanto que esta descripción es equiparable a la descripción geotécnica de la clase de emplazamiento 3 (Tabla 3.4), como rocas blandas a firmes. Como resultado, se asigna la clase 3 a la unidad 20. La unidad 21 está constituida por depósitos de tobas y 
brechas sálicas de carácter volcánico. Siguiendo nuevamente la descripción extensa para esta unidad (GEODE, 2014), estos depósitos están compuestos por fragmentos de traquitas y sienitas empastados por una matriz tobácea muy compactada. Esta alta compacidad permite considerar esta unidad como un suelo muy denso, en coherencia con la descripción geotécnica de la clase de emplazamiento 3. Por tanto, se encuadra la unidad 21 en la clase 3.

Edificio Betancuria La unidad 22 se describe como brechas basálticas muy compactadas. Considerando que esta descripción es equiparable a la descripción geotécnica de la clase de emplazamiento 3 como rocas blandas a firmes (Tabla 3.4), se asigna esta clase a la unidad 22. La unidad 23 consiste en lavas e intrusivos con basaltos (Tabla A.2). Atendiendo al hecho de que el basalto es una roca volcánica, se considera equiparable a una roca dura, en coherencia con la descripción geotécnica de la clase de emplazamiento 1 . Se encuadra por tanto la unidad 23 en la clase 1. La unidad 24 se compone de tobas, brechas traquíticas y traquibasaltos, formando depósitos piroclásticos muy compactados (GEODE, 2014). Esta alta compacidad permite considerar esta unidad como un suelo muy denso, y por tanto clasificarla en la clase de emplazamiento 3 (Tabla 3.4). En cuanto a la unidad 25, ésta consiste en gabros, los cuales, al ser rocas plutónicas, permiten ser considerados como roca duras. Por tanto, de acuerdo con la Tabla 3.4, se asigna la clase de emplazamiento 1 a esta unidad. La unidad 26 se describe como intrusivos compuestos por rocas masivas volcánicas (traquitas), de acuerdo a GEODE (2014) . La presencia de rocas volcánicas permite considerar que la unidad 26 se compone de rocas duras, y por tanto clasificar esta unidad en la clase 1 . Por su parte, la unidad 27 consiste en sienitas, las cuales, al ser rocas plutónicas, se consideran rocas duras. Por tanto, se asigna la clase 1 a la unidad 27. La unidad 31 está constituida por cuerpos intrusivos, compuestos a su vez por rocas de naturaleza volcánica (traquitas). Nuevamente, la presencia de rocas volcánicas permite clasificar esta unidad en la clase 1. La unidad $32 \mathrm{~b}$ consiste en una intrusión masiva de diques traquíticos y traquibasálticos del Complejo Basal, con porcentajes de diques $>75 \%$. Debido a la presencia de rocas volcánicas (traquitas, traquibasaltos), se clasifica esta unidad en la clase 1.

Dominio del Complejo Basal. Edificio Morro Negro-Morro del Sol La unidad 28 se describe como brechas líticas de extrusión, de acuerdo con la Tabla A.2. Para asignarle una clase de emplazamiento, se sigue el razonamiento ya expuesto para las unidades compuestas por brechas, según el cual se infiere una resistencia para este tipo de rocas que es equiparable a la de la clase de emplazamiento 3, pues ésta se describe como rocas firmes a blandas (Tabla 3.4). Es por esta razón por la que se asigna la clase 3 a la unidad 28. Las unidades 29 y 29a se componen de leucogabros y melanogabros del Complejo Basal, con porcentajes de diques menores que el $20 \%$, y comprendidos entre el 20 y el $75 \%$, respectivamente. Nuevamente, se sigue el criterio de considerar las rocas plutónicas como rocas duras. Por tanto, estas dos unidades se clasifican en la clase de emplazamiento 1 (Tabla 3.4). Por este razonamiento, la unidad 30 también se encuadra en la clase 1, pues se describe como sienitas. 
Formaciones de transición Como se puede comprobar en la Tabla A.2, la unidad 33 tiene la misma descripción litológica que la unidad 28 (brechas líticas de extrusión). Por tanto, también se clasifica en la clase de emplazamiento 3. La unidad 34 se compone de tobas rojizas. Las tobas son depósitos piroclásticos, para los cuales se infiere una resistencia propia de suelos de alta compacidad o consistencia, pero en todo caso menor que la resistencia de las rocas blandas a firmes características de la clase de emplazamiento 3 (Tabla 3.4). De acuerdo a la descripción geotécnica de la clase de emplazamiento 4 como suelos cohesivos duros, resulta razonable equiparar la resistencia de la unidad 34 a la resistencia propia de la clase 4, pues esta unidad se podría considerar como un suelo cohesivo duro, o granular denso, en función de su alta compacidad/consistencia. Por tanto, se asigna la clase 4 a la unidad 34. Por su parte, las unidades 35 y 35b consisten en coladas basálticas del Complejo Basal, atravesadas por diques en porcentajes menores que el $20 \%$, y mayores que el $75 \%$, respectivamente. Siguiendo el razonamiento expuesto para la clasificación de rocas volcánicas, el basalto se considera equiparable a una roca dura, y por tanto la unidades 35 y 35b se clasifican en la clase de emplazamiento 1. La unidad 36 se describe como una brecha tectónica con escasa matriz (GEODE, 2014). Esta característica de la matriz hace aconsejable adoptar un criterio más conservador que el seguido para clasificar las unidades de naturaleza brechoide, infiriendo una compacidad menor. Por tanto, la unidad 36 se clasifica en la clase de emplazamiento 4 (Tabla 3.4). En cambio, para clasificar la unidad 37, descrita como brechas de salinas (Tabla A.2), se recupera el criterio general de clasificar las unidades con brechas en la clase de emplazamiento 3.

Rocas filonianas del Edificio Gran Tarajal Según la Tabla A.2, la unidad 40 consiste en intrusiones basálticas (sills). Siguiendo el razonamiento expuesto para el caso de rocas volcánicas, como los basaltos, la unidad 40 se clasifica en la clase de emplazamiento 1 (Tabla 3.4).

Edificio Gran Tarajal La unidad 42 se describe como coladas basálticas olivínicopiroxénicas e indiferenciadas, con alteración y fracturación (disyunción columnar), de acuerdo a la descripción litológica extensa proporcionada por GEODE (2014). Por su parte, la unidad 42a se presenta en la Tabla A.2 como coladas basálticas de la misma composición que la de la unidad 42, y con intensa fracturación. Ambas unidades se componen de rocas volcánicas con distinto grado de fracturación, por lo cual su resistencia no puede ser equiparable a la de las rocas duras propias de la clase de emplazamiento 1 (Tabla 3.4). Por tanto, procede adoptar un criterio conservador y considerar una resistencia menor, más acorde con la de la clase de emplazamiento 2 (Rocas de resistencia media). La unidad 43 consiste en pegmatitoides, que, al ser rocas plutónicas, se consideran rocas duras. Por tanto, la unidad 43 se clasifica en la clase de emplazamiento 1 . En cuanto a la unidad 44, ésta consiste en brechas y coladas brechificadas. Siguiendo el criterio adoptado para las unidades compuestas por brechas, se asigna la clase de emplazamiento 3 (Tabla 3.4) a esta unidad. Según GEODE (2014), la unidad 45 se describe como intrusiones de naturaleza volcánica (basáltica), con fuerte fracturación. En este caso, se procede con el mismo criterio conservador que en el caso de las unidades 42 y 42a, en el sentido de considerar la resistencia de las rocas volcánicas con fracturación como equiparable a la resistencia 
de la clase de emplazamiento 2. Por tanto, la unidad 45 se encuadra en la clase 2.

Con respecto a la unidad 46, ésta se describe como conos de tefra basálticos y niveles de lapilli. De acuerdo a GEODE (2014), se trata de depósitos piroclásticos marcadamente heterométricos. En este caso, se sigue el criterio adoptado para clasificar la unidad 34, en el sentido de que la resistencia de los depósitos piroclásticos puede considerarse equiparable a la de los suelos cohesivos duros, que caracterizan la clase de emplazamiento 4 (Tabla 3.4). Por tanto, se clasifica la unidad 46 en la clase 4. La unidad 47 se compone de depósitos piroclásticos mixtos (Tabla A.2). Según la descripción litológica extensa de GEODE (2014), estos depósitos consisten en materiales brechoides alternados con niveles de cineríticos (ceniza) y escorias de tamaño pequeño. En función de su carácter piroclástico y heterométrico, se vuelve a seguir el criterio de considerar la resistencia de estos depósitos como equivalente a la de la clase de emplazamiento 4 . Por tanto, se clasifica la unidad 47 en esta clase. Por su parte, la unidad 48 consiste en intrusiones de naturaleza volcánica (traquitas) con fracturación (lajeado) (GEODE, 2014). En este caso, se adopta el criterio de considerar la resistencia de las rocas volcánicas con fracturación como equiparable a la resistencia de la clase de emplazamiento 2, con el fin de clasificar la unidad 48 en esta clase. En relación a la unidad 49, ésta se clasifica en la clase de emplazamiento 1, pues se describe como coladas sálicas que se componen de traquitas (GEODE, 2014). Por tanto, la presencia de estas rocas volcánicas permite considerar que la resistencia de la unidad 49 es coherente con la resistencia de las rocas duras, características de la clase 1 . La unidad 50 tiene una descripción litológica extensa consistente en brechas con muy escasa matriz (GEODE, 2014). La poca importancia de la matriz en el conjunto de esta litología recomiendan adoptar un criterio más conservador que el seguido para clasificar las unidades de naturaleza brechoide, y por tanto encuadrar la unidad 50 en la clase de emplazamiento 4 .

La unidades 51 y 52 consisten en brechas de diferentes tipos (Tabla A.2). Siguiendo el razonamiento ya expuesto para clasificar las unidades compuestas por brechas, estas unidades se clasifican en la clase de emplazamiento 3 (Tabla 3.4). La unidad 53 se describe como coladas basálticas de diversa composición, por lo cual se sigue el criterio adoptado para clasificar las unidades compuestas por rocas volcánicas, y se engloba esta unidad en la clase de emplazamiento 1. En cuanto a la unidad 54, ésta consiste en depósitos de arenas y conglomerados en ambiente aluvial. En función de la presencia de sedimentos detríticos de tamaño igual o superior al de las arenas, se infiere una resistencia acorde a la de los suelos de alta compacidad o consistencia, al igual que en el caso de los depósitos piroclásticos. Según la descripción geotécnica de la clase de emplazamiento 4 como suelos cohesivos duros (Tabla 3.4), puede considerarse que la resistencia de la unidad 54 es equiparable, por tanto, a la de esta clase de emplazamiento. En consecuencia, se clasifica la unidad 54 en la clase 4. La unidad 55 se describe como coladas basálticas con fracturación (disyunción columnar) (GEODE, 2014). Siguiendo el criterio adoptado para clasificar las rocas volcánicas con fracturación, se encuadra la unidad 55 en la clase de emplazamiento 2 (Tabla 3.4).

La unidad 56 se compone de depósitos piroclásticos heterométricos (bloques, bombas, escorias y lapilli), con capas de lapilli (GEODE, 2014). En función de esta litología, se vuelve a considerar su resistencia como equivalente a la de la clase de emplazamiento 4 (Tabla 3.4). Por tanto, se clasifica la unidad 56 en esta clase. 
Las unidades 57 y 59 son descritas por GEODE (2014) como coladas basálticas y traquíticas, respectivamente. Ambas unidades presentan fracturación (diaclasado y disyunción columnar). A tenor de la presencia, en los dos casos, de rocas volcánicas con fracturación, las dos unidades se clasifican en la clase de emplazamiento 2. En relación a la unidad 58, ésta consiste en brechas traquíticas (Tabla A.2). En función de la naturaleza brechoide de esta unidad, se sigue el criterio de clasificarla en la clase de emplazamiento 3. La unidad 60 se describe como depósitos de conglomerados aluviales en matriz arenosa (GEODE, 2014). En este caso, se sigue el mismo criterio que el adoptado para clasificar la unidad 54, considerando que los depósitos detríticos de naturaleza aluvial, y que contienen sedimentos con tamaño de grano igual o superior al tamaño arena, presentan una resistencia equiparable a la de la clase de emplazamiento 4 (Suelos duros).

Las unidades 61 y 63 se describen como coladas basálticas de distinta composición, con diaclasado y disyunción columnar, respectivamente, de acuerdo a la descripción extensa proporcionada por GEODE (2014). La presencia de esta fracturación permite adoptar de nuevo el criterio de clasificar las rocas volcánicas fracturadas en la clase de emplazamiento 2. Por tanto, ambas unidades, 61 y 63 , se encuadran en esta clase. En cuanto a la unidad 62, ésta consiste en sedimentos aluviales localmente con intercalaciones de brechas (Tabla A.2). La descripción litológica extensa añade que estos depósitos tienen naturaleza brechoide, con tamaños decimétricos y matriz arenosa, y hacia techo pasan a depósitos sedimentarios más seleccionados y con menor tamaño de grano (GEODE, 2014). Esta heterometría recomienda desestimar el criterio general seguido para clasificar las unidades con brechas en la clase de emplazamiento 3, e inferir una resistencia más acorde a la de los suelos cohesivos duros, característicos de la clase de emplazamiento 4 (Tabla 3.4). Por tanto, se clasifica la unidad 62 en la clase 4.

Edificio Jandía De acuerdo a la Tabla A.2, la unidad 66 se describe como coladas basálticas olivínico-piroxénicas, brechas y depósitos piroclásticos. La presencia de rocas volcánicas junto con brechas y depósitos piroclásticos recomienda inferir una resistencia menor que la de las clases de emplazamiento 1 y 2 , que son las que agrupan las rocas volcánicas, en función de su grado de fracturación. Por tanto, se considera que la resistencia de esta unidad es acorde con la resistencia de las rocas blandas a firmes que caracterizan la descripción geotécnica de la clase de emplazamiento 3 (Tabla 3.4). En consecuencia, se clasifica las unidad 66 en la clase 3. La unidad 67 consiste en coladas basálticas muy alteradas, de acuerdo con la descripción litológica extensa de GEODE (2014). Este elevado grado de alteración recomienda considerar una resistencia menor que la resistencia atribuida a las rocas volcánicas, la cual es equiparable a la de la clase de emplazamiento 1. Por tanto, para la unidad 67 se infiere una resistencia equivalente a la de la clase de emplazamiento 2 , y por tanto esta unidad se clasifica en la clase 2. La unidad 68 se compone de brechas, coladas basálticas, intrusivos y diques (Tabla A.2). Para clasificar esta unidad, se sigue el mismo criterio que el adoptado en la clasificación de la unidad 66, considerando que la presencia de las brechas es un factor determinante para encuadrar la unidad en la clase de emplazamiento 3. Por su parte, las unidades 69 y 70 consisten en piroclastos basálticos y conos enterrados, y en conos piroclásticos hidromagmáticos y mixtos, respectivamente (Tabla A.2). Como en el caso de otras unidades consistentes en 
depósitos piroclásticos, se sigue el criterio de clasificar estas unidades en la clase de emplazamiento 4.

La unidad 71 se describe como brechas del tipo "Debris-avalanche" y del tipo ampuyenta (Tabla A.2). Siguiendo el criterio expuesto para clasificar las unidades con brechas, la unidad 71 se encuadra en la clase de emplazamiento 3 (Tabla 3.4). En cuanto a la unidad 72, su descripción como intrusivos traquíticos lleva a clasificar la unidad en la clase de emplazamiento 1, pues se sigue el criterio ya adoptado para clasificar las rocas volcánicas, como son las traquitas. En cuanto a la unidad 73, ésta consiste en tobas sálicas, que configuran depósitos de naturaleza piroclástica que se clasifican en la clase de emplazamiento 4, siguiendo el criterio utilizado en el caso de otras unidades consistentes en este tipo de depósitos. En cuanto a la unidad 74, en la Tabla A.2 se indica que su descripción litológica está integrada por coladas basálticas olivínico-piroxénicas. Dado que la unidad está compuesta por rocas volcánicas (basaltos), la unidad se clasifica en la clase de emplazamiento 1. La unidad 75 se compone de sedimentos detríticos y depósitos epiclásticos, sin una descripción más detallada (Tabla A.2). Debido a que se desconoce la consistencia de esta unidad, se entiende que tiene una consistencia media. Por tanto, en coherencia con la descripción geotécnica de la clase de emplazamiento 5 como suelos de consistencia media, se clasifica la unidad 75 en esta clase de emplazamiento.

Las unidades 76 y 79 se describen como coladas de basaltos (Tabla A.2), y por tanto se componen de rocas volcánicas, por lo cual se clasifican en la clase de emplazamiento 1. La unidad 77 consiste en coladas basálticas con fracturación (disyunción columnar), de acuerdo con la descripción litológica extensa de GEODE (2014). En función de esta fracturación, se adopta el criterio de clasificar la unidad en la clase de emplazamiento 2 (Tabla 3.4). Con respecto a las unidades 78 y 80 , éstas están compuestas por piroclastos basálticos, por lo que se recurre de nuevo al criterio de clasificar las unidades consistentes en depósitos piroclásticos en la clase de emplazamiento 4 .

La unidad 81 consiste en una intrusión sálica, compuesta por rocas plutónicas, a tenor de la descripción litológica extensa de GEODE (2014). Se considera que la presencia de rocas plutónicas confiere a esta unidad la misma resistencia que la de la clase de emplazamiento 1 (Tabla 3.4). La unidad 82 se describe como coladas basálticas con fracturación (disyunción prismática y partición en bloques) (GEODE, 2014). De acuerdo con el criterio seguido para clasificar las unidades con rocas volcánicas con fracturación, se encuadra esta unidad en la clase de emplazamiento 2. La unidad 83 consiste en piroclastos basálticos, por tanto se sigue el criterio ya expuesto para clasificar esta unidad en la clase de emplazamiento 4. Las unidades 84 y 86 coinciden en ser descritas como brechas (Tabla A.2). En función de esta descripción, se sigue el criterio de clasificar las unidades de naturaleza brechoide en la clase de emplazamiento 3 . Con respecto a la unidad 85, ésta consiste en una intrusión de composición básica, con potente fracturación (disyunción columnar), a tenor de la descripción litológica extensa de GEODE (2014). Al presentarse rocas plutónicas con fracturación, se infiere una resistencia equiparable a la de la clase de emplazamiento 2 (Tabla 3.4). Por tanto, se sigue el criterio adoptado en la clasificación de las unidades con rocas volcánicas fracturadas, y se encuadra la unidad 85 en la clase 2. Este mismo criterio se utiliza para clasificar la unidad 87 en la clase 2, puesto que su descripción litológica extensa (GEODE, 2014) consiste en coladas 
de naturaleza volcánica (basaltos, basanitas y nefelinitas) con fuerte fracturación (disyunción columnar).

Rocas filonianas del Edificio Tetir De acuerdo con la Tabla A.2, las unidades 88 y 89 se describen como diques y sills de composición básica y sálica, respectivamente. En función de la presencia de rocas plutónicas, se aplica el criterio de clasificar las unidades con este tipo de rocas en la clase de emplazamiento 1 (Tabla 3.4).

Edificio Tetir La unidad 90 se describe como coladas basálticas que presentan acusada alteración, de acuerdo con la descripción litológica extensa de GEODE (2014). En este caso, se aplica el criterio adoptado para clasificar la unidad 67, considerando la alteración de las rocas volcánicas (basaltos) como indicador de una resistencia menor que la correspondiente a la clase de emplazamiento 1 (Tabla 3.4). Por este motivo, se clasifica la unidad 90 en la clase de emplazamiento 2. La unidad 91 es descrita como rocas volcánicas (cuarzotraquitas) con fracturación (disyunción o lajeado) (GEODE, 2014). Se adopta el criterio de clasificar las unidades con rocas volcánicas fracturadas en la clase de emplazamiento 2, para encuadrar por tanto la unidad 91 en esta clase. Por su parte, la unidad 92 consiste en brechas líticas del tipo ampuyenta (Tabla A.2). Teniendo en cuenta la correspondencia ya establecida entre las litologías con brechas y la clase de emplazamiento 3, se clasifica la unidad 92 en esta clase. En cuanto a las unidades 93 y 94, ambas se clasifican en la clase de emplazamiento 1. La unidad 93 se compone de gabros y pegmatitoides (Tabla A.2), que al ser rocas plutónicas y por tanto rocas duras, corresponden claramente a esta clase. La unidad 94 consiste en una intrusión básica. Por tanto, la presencia de rocas plutónicas permite inferir que la unidad tiene una resistencia equiparable a la de las rocas duras y, por tanto, a la de la clase 1. La unidad 95 se describe como depósitos piroclásticos (Tabla A.2), para los cuales se adopta el criterio de considerar su resistencia como equiparable a la de los suelos cohesivos duros, procediendo por tanto a clasificar la unidad en la clase de emplazamiento 4 (Tabla 3.4).

La unidad 96 consiste en sedimentos y depósitos epiclásticos (Tabla A.2). Se configuran en depósitos detríticos que, según la descripción litológica extensa de GEODE (2014), tienen una matriz arenosa fina y granoselección positiva (el tamaño de los cantos disminuye a techo). Podría adoptarse el mismo criterio de clasificación que el utilizado para la unidad 75 y considerar una consistencia media. Sin embargo, la presencia de la matriz fina permite suponer una consistencia mayor, con lo cual la resistencia de la unidad 96 puede entenderse como más acorde a la de un suelo duro, que es característico de la clase de emplazamiento 4 (Tabla 3.4). Por tanto, se clasifica la unidad 96 en esta clase. Siguiendo a GEODE (2014), la unidad 97 se compone de coladas basálticas con fracturación (disyunción columnar). Por tanto, se utiliza el criterio ya expuesto de clasificación de las rocas volcánicas fracturadas, para englobar la unidad 97 en la clase de emplazamiento 2. En cuanto a la unidad 98, ésta se describe como conos de tefra, que GEODE (2014) describe más extensamente como compuestos de bombas, escorias y lapillis. Estos depósitos consisten por tanto en piroclastos heterométricos, lo que permite clasificar la unidad en la clase de emplazamiento 4. La unidad 100 presenta coladas de basaltos plagioclásicos (Tabla A.2). Se sigue en este caso el criterio ya expuesto de clasificar las rocas volcánicas (basaltos) en la clase de emplazamiento 1. 
La unidad 101 se describe como almagres y niveles edáficos rubefactados (Tabla A.2). Considerando que los almagres son sedimentos arcillosos rubefactados por coladas suprayacentes, su consistencia puede estimarse como alta. Por tanto, la unidad puede tener una resistencia acorde a la de los suelos duros que describen geotécnicamente la clase de emplazamiento 4, y encuadrarse en esta clase. En cuanto a las unidades 103 y 104, la Tabla A.2 las describe como coladas basálticas de distinta composición, por lo que se adopta el criterio de clasificar las unidades con rocas volcánicas en la clase de emplazamiento 1.

Fase pliocena La unidad 106 se compone de arenas y conglomerados (Tabla A.2). La sola presencia de areniscas podría ser indicativa de una resistencia propia de rocas blandas a firmes, y por tanto de la clasificación de la unidad en la clase de emplazamiento 3 (Tabla 3.4). Sin embargo, y dado que la descripción litológica de la unidad también incluye conglomerados, parece conveniente inferir una resistencia menor, y clasificar la unidad en la clase de emplazamiento 4. La unidad 107 consta de arenas y conglomerados en depósitos aluviales. En función de la presencia de las arenas, se adopta el criterio de clasificar los depósitos detríticos con arenas y sedimentos de tamaños superiores en la clase de emplazamiento 4. La unidad 108 consiste en arenas eólicas pliocenas, que se encuentran fuertemente cementadas según la descripción litológica extensa de GEODE (2014). Este alto grado de cementación permite inferir una consistencia alta, y por tanto una resistencia acorde a la de los suelos duros que caracterizan la clase de emplazamiento 4. La unidad 109 se compone de arenas eólicas con intercalaciones de derrubios de ladera. De acuerdo a la descripción litológica extensa de GEODE (2014), estos sedimentos constituyen depósitos aluviales detríticos y heterométricos (contienen arenas y cantos), intercalados con arenas eólicas de granulometría fina. A partir de la presencia de las arenas en estos depósitos detríticos, podría suponerse una consistencia alta y clasificar la unidad en la clase de emplazamiento 4 (Suelos duros) (Tabla 3.4). Sin embargo, las intercalaciones de arenas eólicas hacen recomendable adoptar un criterio conservador y rebajar la consistencia global de la unidad, pasando a considerarla como media. Teniendo en cuenta la descripción geotécnica de la clase de emplazamiento 5 como suelos de consistencia media, se clasifica, por tanto, la unidad 109 en esta clase de emplazamiento. De acuerdo con la Tabla A.2, la unidad 110 consiste en sedimentos conglomeráticos y depósitos caóticos. En este caso, se adopta el criterio de clasificar las unidades con sedimentos detríticos de tamaño arena o superior en la clase de emplazamiento 4.

La unidad 111 (Tabla A.2) se compone de depósitos de deslizamiento con intercalaciones eólicas. Según la descripción litológica extensa de GEODE (2014), estos depósitos son de naturaleza detrítica y están constituidos por arenas y conglomerados heterométricos, con intercalaciones de areniscas con cantos. En esta caso, se considera que la resistencia de estos depósitos detríticos no es disminuida por las intercalaciones eólicas, pues éstas presentan areniscas y cantos, que son sedimentos de tamaño de grano superior al de las arenas y conglomerados. Por tanto, la resistencia global de la unidad 111 se considera equiparable a la de la clase de emplazamiento 4 (Tabla 3.4). Por su parte, la unidad 117 es descrita como depósitos aluviales con arenas y cantos, y con escasa matriz (GEODE, 2014). En este caso, se vuelve a aplicar el criterio de clasificación de los depósitos detríticos con arenas y 
sedimentos de tamaños superiores en la clase 4. La unidad 112 tiene una descripción litológica extensa (GEODE, 2014) consistente en coladas basálticas con fracturación (disyunción columnar), similar a la de la unidad 116 (coladas basaníticas con disyunción columnar). Por tanto, se aplica el criterio de clasificar las unidades con rocas volcánicas fracturadas en la clase de emplazamiento 2, para encuadrar las unidades 112 y 116 en esta clase. En cuanto a la unidad 113, ésta se describe como coladas basálticas olivínicas (Tabla A.2), por lo que se clasifica en la clase de emplazamiento 1, adoptando el criterio de encuadrar en esta clase las unidades con rocas volcánicas. Las unidades 114 y 115 se describen como centros de emisión y conos de cinder (Tabla A.2). Dado que ambas unidades consisten en depósitos piroclásticos, se sigue el criterio de clasificar las unidades con este tipo de depósitos en la clase de emplazamiento 4.

Formaciones sedimentarias plio-pleistocenas De acuerdo con la Tabla A.2, la unidad 118 se describe como arenas eólicas plio-pleistocenas. A partir de esta descripción litológica, se infiere una consistencia alta, que es equiparable a la de la clase de emplazamiento 4 (Tabla 3.4), en virtud de la descripción geotécnica de esta clase como suelos duros. Las unidades 119 y 120 tienen descripciones litológicas coincidentes entre sí, que consisten en glacis (Tabla A.2). Las respectivas descripciones litológicas extensas, proporcionadas por GEODE (2014), indican que ambas unidades se componen de depósitos de arenas y gravas con una matriz areno-arcillosa. La presencia de la matriz, de tamaño de grano más fino que los sedimentos heterométricos que soporta, permite reforzar la consideración de una consistencia global acorde a la de los suelos duros, y por tanto clasificar ambas unidades en la clase de emplazamiento 4.

Las unidades 121 y 122 configuran depósitos de carácter evaporítico (caliche). A partir de esta descripción, se infiere una consistencia media, más baja en todo caso que la de los depósitos sedimentarios de carácter detrítico. Esta consistencia es coherente con la descripción geotécnica de la clase de emplazamiento 5 (Suelos de consistencia media), por lo que ambas unidades se clasifican en esta clase. La unidad 123 consiste en coladas basálticas con fracturación (disyunción columnar), de acuerdo con GEODE (2014). Adoptando el criterio de clasificar las rocas volcánicas (basaltos) fracturadas en la clase de emplazamiento 2 (Tabla 3.4), se encuadra la unidad 123 en esta clase. En cuanto a la unidad 124, ésta se describe como conos de tefra (Tabla A.2). Dado que esta litología implica la presencia de depósitos piroclásticos, se aplica el criterio de clasificación de las unidades con este tipo de depósitos en la clase de emplazamiento 4.

Fase pleistocena media-holocena (episodios recientes) La unidad 125 se describe litológicamente como piroclastos de dispersión (Tabla A.2), con una granulometría fina (lapilli) (GEODE, 2014). En función del bajo tamaño de grano que presentan estos piroclastos, se infiere una consistencia menor que la que se ha supuesto para los depósitos piroclásticos (alta consistencia). En este caso, por tanto, se infiere una consistencia media, equiparable a la de la clase de emplazamiento 5 (Tabla 3.4), pues esta clase se describe geotécnicamente como suelos de consistencia media. Como consecuencia, se clasifica la unidad 125 en la clase de emplazamiento 5. De acuerdo a GEODE (2014), la unidad 126 tiene una descripción litológica extensa 
que presenta coladas basálticas con fracturación (disyunción columnar). En este caso, se aplica el criterio de clasificación de las rocas volcánicas fracturadas (basaltos) en la clase de emplazamiento 2. Por su parte, la unidad 127 se compone de conos de tefra, que al ser depósitos piroclásticos permiten la clasificación de esta unidad en la clase de emplazamiento 4 (Tabla 3.4). La unidad 128 presenta arenas y arcillas rojas (Tabla A.2). La presencia de las arcillas disminuye la alta consistencia que tienen los sedimentos arenosos, que es equiparable a la de los suelos duros característicos de la clase de emplazamiento 4. Por tanto, la unidad 128 presenta una consistencia menor, que es por tanto coherente con la descripción geotécnica de la clase de emplazamiento 5 como suelos de consistencia media (Tabla 3.4). En cambio, la unidad 129 consiste en depósitos detríticos de arenas y conglomerados. Consecuentemente, se puede aplicar el criterio de clasificación de las unidades con depósitos detríticos compuestos por arenas y sedimentos de tamaños superiores, con el fin de encuadrar la unidad 129 en la clase de emplazamiento 4. La unidad 130 tiene una descripción litológica extensa consistente en coladas basálticas compuestas por bloques y cascotes (GEODE, 2014). Dado que se puede suponer una cierta heterometría en los materiales que componen la colada, se debe aplicar un criterio conservador, similar al adoptado para considerar como media la resistencia de las coladas volcánicas con fracturación, y en todo caso menor que la resistencia de las rocas duras propias de la clase de emplazamiento 1 (Tabla 3.4). Por tanto, se infiere una resistencia para la unidad 130 coherente con la de la clase de emplazamiento 2 (Rocas de resistencia media).

La unidades 131 y 135 consisten en conos de tefra (Tabla A.2). Dado que esta litología implica la presencia de depósitos piroclásticos, se adopta el criterio de clasificar las unidades con este tipo de depósitos en la clase de emplazamiento 4. La unidad 132 se compone de arenas eólicas consolidadas del Pleistoceno. En función del alto grado de consolidación descrito y de la granulometría del sedimento (arena), se infiere que la consistencia de la unidad es alta, y por tanto que su resistencia es equiparable a la de la clase de emplazamiento 4, descrita como suelos duros (Tabla 3.4). Por su parte, la unidad 133 presenta una descripción litológica extensa (GEODE, 2014) que consiste en sedimentos de rambla con conglomerados y arenas. Se aplica en este caso el criterio de clasificación de este tipo de depósitos detríticos en la clase de emplazamiento 4. La unidad 134 consiste en coladas basálticas, que al presentar rocas volcánicas permiten la clasificación de la unidad en la clase de emplazamiento 1 (Rocas duras).

Formaciones sedimentarias recientes La unidad 136 consiste en depósitos de piroclastos de dispersión, cuyo fino tamaño (lapilli) es explicitado en la descripción litológica extensa de GEODE (2014). Como en el caso de la unidad 125, se considera que la unidad 136 tiene una consistencia media, debido al bajo tamaño de grano que presentan los piroclastos, por lo cual esta unidad es clasificable en la clase de emplazamiento 5 (Tabla 3.4). La unidad 137 consiste en coluviones antiguos (Tabla A.2), que son descritos por GEODE (2014) como consistentes en arenas y gravas muy heterométricas. Por tanto, se aplica el criterio de clasificar los depósitos detríticos con arenas y sedimentos de tamaños superiores en la clase de emplazamiento 4 . Este mismo criterio se utiliza para clasificar la unidad 138 en la clase 4, pues esta unidad se describe como depósitos de arenas y cantos muy heterométricos (GEODE, 2014). 
La unidad 139 también se clasifica en la clase de emplazamiento 4, ya que GEODE (2014) la describe como depósitos de deslizamientos gravitacionales que presentan cantos y bloques. En este caso, la presencia de sedimentos de tamaño superior al de la arena refuerza la consideración de la consistencia global de la unidad como equiparable a la de la clase 4 . La unidad 140 se configura como depósitos lacustres de arenas, arcillas y limos. La presencia de las arcillas y los limos disminuyen la consistencia de este depósito detrítico, pasando de una consistencia alta (que proporcionarían las arenas) a una media. En coherencia con la descripción geotécnica de la clase de emplazamiento 5 como suelos de consistencia media, se encuadra la unidad 140 en esta clase.

La unidad 141 tiene una descripción litológica extensa consistente en depósitos de terrazas aluviales con arenas y conglomerados, con baja consolidación (GEODE, 2014). En función del bajo grado de consolidación de los sedimentos presentes, se infiere una consistencia y resistencia menores que las que se han considerado para los depósitos detríticos con arenas y sedimentos de tamaños superiores. Por tanto, considerando una consistencia media, se encuadra la unidad 141 en la clase de emplazamiento 5 (Tabla 3.4). A partir de la descripción de la unidad 142 como depósitos areno-arcillosos, suelos y cuarzo (Tabla A.2), se vuelve a considerar que la arcilla es indicativa de una consistencia media. Por tanto, en coherencia con la descripción geotécnica de la clase de emplazamiento 5 (Tabla 3.4), se clasifica la unidad 142 en esta clase. La unidad 143 consiste en depósitos marinos que presentan areniscas y conglomerados (GEODE, 2014). La presencia de los conglomerados permite inferir una resistencia menor que la que proporcionan las areniscas, consideradas como rocas blandas a firmes, y con resistencia equiparable a la resistencia de la clase de emplazamiento 3 (Tabla 3.4). Por tanto, se considera una resistencia global para la unidad 143 que es coherente con la resistencia de la clase de emplazamiento 4 . Con respecto a la unidad 144, la descripción litológica extensa de GEODE (2014) indica que consiste en depósitos detríticos mal seleccionados y muy heterométricos. Se aplica el criterio de clasificar los depósitos detríticos con sedimentos de tamaño arena o superior en la clase de emplazamiento 4. Las unidades 145 y 146 consisten en arenas eólicas sueltas, a tenor de su descripción litológica extensa (GEODE, 2014). Se puede afirmar objetivamente, pues, que la compacidad de estos sedimentos es muy baja. En este contexto, la resistencia de los mismos puede equipararse a la de la clase de emplazamiento 6, que se describe geotécnicamente como suelos blandos, es decir, suelos cohesivos con consistencia mínima. La razón de esta correlación estriba en la razonable similitud que se supone entre la resistencia de los suelos poco compactos y la resistencia de los suelos de baja consistencia que caracterizan la clase 6 .

La unidad 147 se describe como suelos con recubrimiento de lapilli (Tabla A.2). En función de la presencia de lapillis junto a los sedimentos detríticos de esta unidad (GEODE, 2014), se considera que el fino tamaño de grano de estos piroclastos es indicativo de una consistencia global media, como en el caso de las unidades 125 y 136 . Por tanto, se clasifica la unidad 147 en la clase de emplazamiento 5 (Tabla 3.4). La unidad 148 presenta una descripción litológica extensa (GEODE, 2014) que consiste en depósitos detríticos (arenas, cantos y bloques), con alta heterometría y escasa selección. Se aplica por tanto el criterio de clasificar las unidades con sedimentos arenosos o de tamaño superior en la clase de emplazamiento 4. De acuerdo a la (Tabla A.2), la unidad 149 consiste en depósitos de barranco y/o aluviales. Su des- 
cripción litológica extensa los describe como altamente heterométricos, conteniendo gravas, arenas y limos (GEODE, 2014). Como ya se ha razonado, la presencia de los limos es suficiente para considerar que la consistencia de esta unidad pasa de alta a media, y por tanto para clasificar la misma en la clase de emplazamiento 5 .

La unidad 150 se presenta en playas de arena y cantos (Tabla A.2). Configura depósitos de playa, donde los sedimentos se encuentran normalmente sueltos. Por tanto, las granulometrías de tamaño arena y superior (canto) que se observan no son suficientes para una clasificación en la clase de emplazamiento 4, sino que se opta por considerar que la compacidad/consistencia global es baja. Por tanto, la unidad se clasifica en la clase de emplazamiento 6 como suelos blandos (Tabla 3.4). La unidad 151 se describe como depósitos de deslizamiento poco consolidados que presentan bloques (GEODE, 2014). Dado el bajo grado de consolidación de los bloques, se aplica el criterio ya adoptado en el caso de la unidad 141, para inferir una consistencia y resistencia equiparables a la de los suelos de consistencia media, característicos de la clase de emplazamiento 5 (Tabla 3.4). Por último, la unidad 152 presenta depósitos antrópicos de bloques, organizados caóticamente (GEODE, 2014). En este caso, se adopta un criterio conservador para considerar una consistencia/ compacidad muy baja para estos materiales, por lo cual se consideran suelos blandos y se clasifican en la clase de emplazamiento 6 (Tabla 3.4).

En la Tabla 3.28 se presenta un resumen de la clasificación de las unidades de Fuerteventura, de acuerdo al procedimiento descrito.

Tabla 3.28: Resumen de la clasificación de las unidades del mapa geológico continuo GEODE a escala 1:50.000 de Fuerteventura (Zona 2911), de acuerdo a la clasificación de síntesis. Tomado de GEODE (2014), elaboración propia.

\begin{tabular}{|c|c|c|}
\hline $\begin{array}{l}\text { Sistema/Dominio/Edificio/Ciclo vol- } \\
\text { cánico/Macizo plutónico/Formación/ } \\
\text { Miembro }\end{array}$ & $\begin{array}{l}\text { Código de la unidad } \\
\text { geológica }\end{array}$ & $\begin{array}{l}\text { Clase de emplazamien- } \\
\text { to }\end{array}$ \\
\hline \multirow{5}{*}{ Sedimentos de fondo oceánico } & 1 & 3 \\
\hline & $1 \mathrm{~b}$ & 3 \\
\hline & 2 & 3 \\
\hline & $2 \mathrm{~b}$ & 3 \\
\hline & 3 & 3 \\
\hline \multirow{4}{*}{ Lavas y piroclastos en parte submarinos } & 4 & 2 \\
\hline & 5 & 3 \\
\hline & $5 \mathrm{a}$ & 3 \\
\hline & $5 \mathrm{~b}$ & 3 \\
\hline \multirow{7}{*}{ Serie intrusiva ultraalcalina } & 6 & 1 \\
\hline & $6 \mathrm{a}$ & 1 \\
\hline & $6 \mathrm{~b}$ & 1 \\
\hline & 7 & 1 \\
\hline & $7 \mathrm{a}$ & 1 \\
\hline & $8 \mathrm{a}$ & 1 \\
\hline & $9 \mathrm{a}$ & 1 \\
\hline \multirow{10}{*}{ Series plutónicas indiferenciadas } & 10 & 3 \\
\hline & 11 & 1 \\
\hline & $11 \mathrm{a}$ & 1 \\
\hline & $11 \mathrm{~b}$ & 1 \\
\hline & $12 \mathrm{a}$ & 1 \\
\hline & $13 a$ & 3 \\
\hline & $13 \mathrm{~b}$ & 3 \\
\hline & $13 c$ & 3 \\
\hline & $13 \mathrm{~d}$ & 3 \\
\hline & 14 & 3 \\
\hline \multirow{2}{*}{ Macizo máfico-ultramáfico de Mezquez } & $15 \mathrm{a}$ & 1 \\
\hline & $16 \mathrm{a}$ & 1 \\
\hline
\end{tabular}


(continuación)

\begin{tabular}{|c|c|c|}
\hline $\begin{array}{l}\text { Sistema/Dominio/Edificio/Ciclo vol- } \\
\text { cánico/Macizo plutónico/Formación/ } \\
\text { Miembro }\end{array}$ & $\begin{array}{l}\text { Código de la unidad } \\
\text { geológica }\end{array}$ & $\begin{array}{l}\text { Clase de emplazamien- } \\
\text { to }\end{array}$ \\
\hline \multirow{5}{*}{ Complejo circular Vega Río Palmas } & 17 & 1 \\
\hline & 18 & 1 \\
\hline & 19 & 2 \\
\hline & 20 & 3 \\
\hline & 21 & 3 \\
\hline \multirow{8}{*}{ Edificio Betancuria } & 22 & 3 \\
\hline & 23 & 1 \\
\hline & 24 & 3 \\
\hline & 25 & 1 \\
\hline & 26 & 1 \\
\hline & 27 & 1 \\
\hline & 31 & 1 \\
\hline & $32 \mathrm{~b}$ & 1 \\
\hline \multirow{4}{*}{$\begin{array}{l}\text { Dominio del Complejo Basal. Edificio Morro } \\
\text { Negro-Morro del Sol }\end{array}$} & 28 & 3 \\
\hline & 29 & 1 \\
\hline & $29 \mathrm{a}$ & 1 \\
\hline & 30 & 1 \\
\hline \multirow{6}{*}{ Formaciones de transición } & 33 & 3 \\
\hline & 34 & 4 \\
\hline & 35 & 1 \\
\hline & $35 \mathrm{~b}$ & 1 \\
\hline & 36 & 4 \\
\hline & 37 & 3 \\
\hline Rocas filonianas del Edificio Gran Tarajal & 40 & 1 \\
\hline \multirow{23}{*}{ Edificio Gran Tarajal } & 42 & 2 \\
\hline & $42 \mathrm{a}$ & 2 \\
\hline & 43 & 1 \\
\hline & 44 & 3 \\
\hline & 45 & 2 \\
\hline & 46 & 4 \\
\hline & 47 & 4 \\
\hline & 48 & 2 \\
\hline & 49 & 1 \\
\hline & 50 & 4 \\
\hline & 51 & 3 \\
\hline & 52 & 3 \\
\hline & 53 & 1 \\
\hline & 54 & 4 \\
\hline & 55 & 2 \\
\hline & 56 & 4 \\
\hline & 57 & 2 \\
\hline & 58 & 3 \\
\hline & 59 & 2 \\
\hline & 60 & 4 \\
\hline & 61 & 2 \\
\hline & 62 & 4 \\
\hline & 63 & 2 \\
\hline
\end{tabular}

(continúa) 
(continuación)

\begin{tabular}{|c|c|c|}
\hline $\begin{array}{l}\text { Sistema/Dominio/Edificio/Ciclo vol- } \\
\text { cánico/Macizo plutónico/Formación/ } \\
\text { Miembro }\end{array}$ & $\begin{array}{l}\text { Código de la unidad } \\
\text { geológica }\end{array}$ & $\begin{array}{l}\text { Clase de emplazamien- } \\
\text { to }\end{array}$ \\
\hline \multirow{22}{*}{ Edificio Jandía } & 66 & 3 \\
\hline & 67 & 2 \\
\hline & 68 & 3 \\
\hline & 69 & 4 \\
\hline & 70 & 4 \\
\hline & 71 & 3 \\
\hline & 72 & 1 \\
\hline & 73 & 4 \\
\hline & 74 & 1 \\
\hline & 75 & 5 \\
\hline & 76 & 1 \\
\hline & 77 & 2 \\
\hline & 78 & 4 \\
\hline & 79 & 1 \\
\hline & 80 & 4 \\
\hline & 81 & 1 \\
\hline & 82 & 2 \\
\hline & 83 & 4 \\
\hline & 84 & 3 \\
\hline & 85 & 2 \\
\hline & 86 & 3 \\
\hline & 87 & 2 \\
\hline \multirow{2}{*}{ Rocas filonianas del Edificio Tetir } & 88 & 1 \\
\hline & 89 & 1 \\
\hline \multirow{13}{*}{ Edificio Tetir } & 90 & 2 \\
\hline & 91 & 2 \\
\hline & 92 & 3 \\
\hline & 93 & 1 \\
\hline & 94 & 1 \\
\hline & 95 & 4 \\
\hline & 96 & 4 \\
\hline & 97 & 2 \\
\hline & 98 & 4 \\
\hline & 100 & 1 \\
\hline & 101 & 4 \\
\hline & 103 & 1 \\
\hline & 104 & 1 \\
\hline \multirow{12}{*}{ Fase pliocena } & 106 & 4 \\
\hline & 107 & 4 \\
\hline & 108 & 4 \\
\hline & 109 & 5 \\
\hline & 110 & 4 \\
\hline & 111 & 4 \\
\hline & 112 & 2 \\
\hline & 113 & 1 \\
\hline & 114 & 4 \\
\hline & 115 & 4 \\
\hline & 116 & 2 \\
\hline & 117 & 4 \\
\hline \multirow{7}{*}{ Formaciones sedimentarias plio-pleistocenas } & 118 & 4 \\
\hline & 119 & 4 \\
\hline & 120 & 4 \\
\hline & 121 & 5 \\
\hline & 122 & 5 \\
\hline & 123 & 2 \\
\hline & 124 & 4 \\
\hline \multirow{11}{*}{$\begin{array}{l}\text { Fase pleistocena media-holocena (episodios } \\
\text { recientes) }\end{array}$} & 125 & 5 \\
\hline & 126 & 2 \\
\hline & 127 & 4 \\
\hline & 128 & 5 \\
\hline & 129 & 4 \\
\hline & 130 & 2 \\
\hline & 131 & 4 \\
\hline & 132 & 5 \\
\hline & 133 & 4 \\
\hline & 134 & 1 \\
\hline & 135 & 4 \\
\hline
\end{tabular}


(continuación)

\begin{tabular}{|l|l|l|}
\hline $\begin{array}{l}\text { Sistema/Dominio/Edificio/Ciclo vol- } \\
\text { cánico/Macizo plutónico/Formación/ } \\
\text { Miembro }\end{array}$ & $\begin{array}{l}\text { Código de la unidad } \\
\text { geológica }\end{array}$ & $\begin{array}{l}\text { Clase de emplazamien- } \\
\text { to }\end{array}$ \\
\hline \multirow{5}{*}{ Formaciones sedimentarias recientes } & 136 & 5 \\
\cline { 2 - 3 } & 137 & 4 \\
\cline { 2 - 3 } & 138 & 4 \\
\cline { 2 - 3 } & 139 & 4 \\
\cline { 2 - 3 } & 140 & 5 \\
\cline { 2 - 3 } & 141 & 5 \\
\cline { 2 - 3 } & 143 & 4 \\
\cline { 2 - 3 } & 144 & 6 \\
\cline { 2 - 3 } & 145 & 6 \\
\cline { 2 - 3 } & 146 & 5 \\
\cline { 2 - 3 } & 148 & 5 \\
\hline & 149 & 5 \\
\hline
\end{tabular}

\section{Gran Canaria (Región 2912)}

La región GEODE que corresponde a la isla de Gran Canaria es la región 2912 (GEODE, 2014). La cartografía de esta región GEODE distingue 112 unidades geológicas en la isla, con edades cronoestratigráficas comprendidas entre el Mioceno Medio y el Holoceno. En la Tabla A.3 se muestran las descripciones litológicas y edades cronoestratigráficas correspondientes a estas unidades, las cuales son agrupadas por la leyenda cronoestratigráfica de la cartografía en 14 grupos (Tabla 3.29). Del conjunto de 112 unidades geológicas descritas para la región 2912 (GEODE, 2014), 5 no aparecen cartografiadas y por tanto no se han clasificado: unidades 1,2 , 3,5 y 6 .

Rocas filonianas La unidad 4 se describe como diques de basanitas, tefritas, basaltos y fonolitas, y diques básicos en general, como se muestra en la Tabla A.3. Esta unidad se compone, por tanto, de rocas volcánicas (basaltos, basanitas, tefritas y fonolitas), cuya resistencia es equivalente a la de una roca dura. Considerando la descripción geotécnica de la clase de emplazamiento 1 como rocas duras (Tabla 3.4), se asigna esta clase a la unidad 4.

Ciclo I (Formación basáltica) De acuerdo con la Tabla A.3, la unidad 7 consiste en lavas basáltico-olivínicas, olivínico-piroxénicas y plagioclásicas, con traquibasaltos subordinados, y en hawaiitas, benmoreitas y mugearitas. En función de esta descripción, la unidad 7 presenta rocas volcánicas, como los basaltos y traquibasaltos, las benmoreitas y mugearitas (que son traquiandesitas), y las hawaiitas (que es un traquibasalto). Se aplica, por tanto, el criterio de clasificación de las unidades con rocas volcánicas en la clase de emplazamiento 1 (Tabla 3.4), al igual que en el caso de la unidad 4. En cuanto a la unidad 8, su descripción litológica consta de conos de tefra y piroclastos basálticos (lapillis, escorias y bombas), según la Tabla A.3. Esta unidad constituye claramente un depósito piroclástico, que se considera equivalente a un suelo con alta compacidad/consistencia. Consiguientemente, la resistencia del depósito es coherente con la resistencia de la clase de emplazamiento 4 (Tabla 3.4), a 
Tabla 3.29: División de las unidades del mapa geológico continuo GEODE a escala 1:50.000 de Gran Canaria (Zona 2912), de acuerdo con la leyenda cronoestratigráfica. Tomado de GEODE (2014).

\begin{tabular}{|c|c|c|c|}
\hline $\begin{array}{l}\text { Código de la } \\
\text { unidad geológica }\end{array}$ & \multicolumn{3}{|c|}{$\begin{array}{l}\text { Sistema/Dominio/Edificio/Ciclo volcánico/Macizo } \\
\text { plutónico/Formación/Miembro }\end{array}$} \\
\hline $1,2,3,4,5,6$ & \multicolumn{3}{|c|}{ Rocas filonianas } \\
\hline $7,8,9,10,11,12$ & \multicolumn{2}{|l|}{ Formación basáltica } & \multirow{4}{*}{ Ciclo I } \\
\hline $\begin{array}{l}13,14,15,16,17,18 \\
19\end{array}$ & Dominio extracaldera & Formación & \\
\hline $\begin{array}{l}20,20 \mathrm{a}, 20 \mathrm{~b}, 20 \mathrm{c}, 21, \\
22\end{array}$ & Dominio intracaldera & $\begin{array}{l}\text { traquitico- } \\
\text { fonolítica }\end{array}$ & \\
\hline $\begin{array}{l}23,24,25,26,27,28, \\
29,30,31,32,33,34, \\
35,36\end{array}$ & \multicolumn{2}{|l|}{ Formación fonolítica } & \\
\hline 37,38 & \multicolumn{2}{|l|}{ Miembro inferior } & \multirow{3}{*}{$\begin{array}{l}\text { Formación detrítica } \\
\text { de Las Palmas }\end{array}$} \\
\hline $39,40,41$ & \multicolumn{2}{|l|}{ Miembro medio } & \\
\hline 42 & \multicolumn{2}{|l|}{ Miembro superior } & \\
\hline $\begin{array}{l}43,44,45,46,47,48, \\
49,50,51,52,53,54, \\
55,56,57,58,59,60\end{array}$ & \multicolumn{3}{|l|}{ Ciclo Roque Nublo } \\
\hline $\begin{array}{l}61,62,63,64,65,66, \\
67,68,69,70,71,72, \\
73,74\end{array}$ & \multicolumn{2}{|l|}{ Medio e inferior } & \multirow[t]{2}{*}{$\begin{array}{l}\text { Ciclo Post-Roque } \\
\text { Nublo }\end{array}$} \\
\hline $75,76,77,78$ & \multicolumn{2}{|l|}{ Superior } & \\
\hline $\begin{array}{l}79,80,81,82,83,84 \\
85,86,87\end{array}$ & \multicolumn{2}{|l|}{ Inferior } & \multirow[t]{2}{*}{ Ciclo Reciente } \\
\hline $\begin{array}{l}88,89,90,91,92,93 \\
94,95\end{array}$ & \multicolumn{2}{|l|}{ Superior } & \\
\hline $\begin{array}{l}96,97,98,99,100,101, \\
102,103,104,105,106, \\
107,108,109\end{array}$ & \multicolumn{3}{|c|}{ Depósitos sedimentarios recientes } \\
\hline
\end{tabular}

tenor de la descripción geotécnica de esta clase como suelos cohesivos duros, es decir, de alta consistencia. Por esta razón, se asigna la clase 4 a la unidad 8. En cambio, la unidad 9 se compone de lapillis de dispersión, que son piroclastos con granulometría fina. A partir del pequeño tamaño de grano de estos piroclastos, se considera que la consistencia del depósito que integran es menor que la consistencia alta que se ha supuesto para los depósitos piroclásticos con granulometrías mayores (escorias, bombas). Por tanto, la unidad 9 tiene una consistencia media, que es equiparable a la de la clase de emplazamiento 5 (Tabla 3.4), en función de su descripción geotécnica como suelos de consistencia media. Consecuentemente, se clasifica la unidad 9 en la clase 5 .

La unidad 10 se describe como lavas basálticas y traquibasaltos, con hawaiitas y mugearitas (Tabla A.3). Como se ha indicado para la unidad 7, esta descripción consiste básicamente en rocas volcánicas (basaltos, traquibasaltos y traquiandesitas), que permiten clasificar la unidad 10 en la clase de emplazamiento 1 (Tabla 3.4). Siguiendo este mismo criterio, la unidad 11 también se clasifica en la clase 1, pues se compone de coladas basálticas y traquibasálticas indiferenciadas. La unidad 12, por su parte, consiste en piroclastos de tipo lapilli y escoria (Tabla A.3). Aplicando el criterio seguido en la clasificación de la unidad 8, se encuadra la unidad 12 en la clase de emplazamiento 4, pues configura un depósito piroclástico con alta consistencia. 
Ciclo I (Formación traquítico-fonolítica, dominio extracaldera) De acuerdo con la Tabla A.3, la unidad 13 presenta, de muro a techo, tobas riolítico-traquíticas, traquibasaltos e intercalaciones ignimbríticas, y tobas traquibasálticas. Las tobas son depósitos piroclásticos, que, como se ha indicado, equivalen a suelos de alta consistencia. Estas tobas se encuentran intercaladas con traquibasaltos (rocas duras) e ignimbritas, que son rocas piroclásticas con matriz y que, por esta razón, se consideran rocas blandas a firmes. La predominancia de las tobas en esta unidad recomienda considerar que su resistencia global es la propia de un suelo duro, a pesar de la presencia de rocas de diversas consistencias. Por tanto, la unidad 13 se clasifica en la clase de emplazamiento 4 (Tabla 3.4). La unidad 14, por su parte, se compone de lavas basálticas y traquibasálticas. Debido a la presencia de rocas volcánicas (basaltos y traquibasaltos), se clasifica la unidad en la clase de emplazamiento 1. La unidad 15 es descrita como coladas riolítico-traquíticas (Tabla A.3). Según la descripción litológica extensa de GEODE (2014), las rocas volcánicas presentes (traquitas y riolitas) presentan fracturación (lajeado). Por este motivo, la resistencia inferida para esta unidad disminuye con respecto a la resistencia considerada para las rocas volcánicas (rocas duras y clasificables en la clase de emplazamiento 1). De acuerdo con la Tabla 3.4, la resistencia de la unidad 15 se considera más acorde con la descripción geotécnica de la clase de emplazamiento 2 como rocas de resistencia media. Por tanto, se clasifica la unidad 15 en la clase 2. Atendiendo a la descripción litológica extensa de GEODE (2014) para la unidad 16, se observa una alternancia de niveles arenosos gruesos y conglomeráticos con capas limoso-arcillosas. En función de la presencia de las capas finas, se considera que la consistencia global es media, y por tanto se clasifica la unidad en la clase de emplazamiento 5 (Tabla 3.4).

La unidad 17 presenta lavas de basaltos plagioclásicos (Tabla A.3), que al ser rocas volcánicas permiten clasificar esta unidad en la clase de emplazamiento 1. La unidad 18 se describe como ignimbritas, coladas piroclásticas y coladas riolíticotraquíticas. Las ignimbritas, al ser consideradas como rocas blandas a firmes, tienen una resistencia equivalente a la de la clase de emplazamiento 3 (Tabla 3.4), en función de la descripción geotécnica de esta clase. Sin embargo, la presencia de las coladas de piroclastos recomienda disminuir la resistencia global de la unidad, correlacionando la misma con la resistencia de los suelos duros que caracterizan la clase de emplazamiento 4 (Tabla 3.4). Por tanto, se encuadra la unidad 18 en la clase 4. En cuanto a la unidad 19, ésta se compone de piroclástos basálticos (Tabla A.3), incluyendo bombas, escorias y lapillis (GEODE, 2014). A partir de la heterometría de los piroclastos que componen esta unidad, se entiende que ésta configura un depósito piroclástico genérico, por lo que se adopta el criterio ya utilizado en el caso de la unidad 8, y se clasifica la unidad 19 en la clase de emplazamiento 4.

Ciclo I (Formación traquítico-fonolítica, dominio intracaldera) La unidad 20 se describe como tobas ignimbríticas y coladas riolítico-traquíticas (Tabla A.3). La presencia de piroclastos (tobas ignimbríticas) junto con rocas volcánicas (riolitas y traquitas) recomienda inferir una resistencia global inferior a la que presentarían estas rocas volcánicas de forma aislada. Por esta razón, la resistencia no sería equiparable ni a la resistencia de las rocas duras que caracterizan la clase de emplazamiento 1 (Tabla 3.4), ni tampoco a la de las rocas de resistencia media, propias de la clase de emplazamiento 2. Se prefiere, por tanto, suponer que la resistencia de la uni- 
dad 20 es coherente con la resistencia de las rocas blandas a firmes que describen geotécnicamente la clase de emplazamiento 3 (Tabla 3.4). Las unidades 20a y 20b son también descritas como tobas ignimbríticas y coladas riolítico-traquíticas (Tabla A.3), aunque presentan diferentes porcentajes de diques. No obstante, se adopta el mismo criterio que el escogido para clasificar la unidad 20, en función de la presencia de las tobas junto con las riolitas y traquitas, para clasificar las unidades 20a y $20 \mathrm{~b}$ en la clase de emplazamiento 3 . Con este mismo criterio, también se encuadra la unidad 20c en la clase 3 (Tabla 3.4), pues su descripción litológica consta de niveles de alteración hidrotermal en las tobas ignimbríticas y coladas riolítico-traquíticas (Tabla A.3).

La unidad 21 se compone de sienitas alcalinas y peralcalinas (Tabla A.3). Las sienitas son rocas plutónicas, para las cuales se infiere la misma resistencia que la inferida para las rocas volcánicas. Por tanto, la resistencia de la unidad 21 es coherente con la de las rocas duras que caracterizan la clase de emplazamiento 1 (Tabla 3.4). En cuanto a la unidad 22, ésta se describe como una brecha volcánica (Tabla A.3). Esta roca está formada fundamentalmente por fragmentos traquíticos y traquibasálticos y una matriz escasa (GEODE, 2014). A partir de esta descripción, es razonable inferir una resistencia equiparable a la de las rocas blandas a firmes, que caracterizan la clase de emplazamiento 3 (Tabla 3.4). Por tanto, se clasifica la unidad 22 en la clase 3.

Ciclo I (Formación fonolítica) De acuerdo con la Tabla A.3, la unidad 23 consta de intrusiones de fonolitas, las cuales presentan fracturación (disyunción columnar), según la descripción litológica extensa de GEODE (2014). Se adopta por tanto el mismo criterio que el seguido en el caso de la unidad 15, en el sentido de considerar las rocas volcánicas (fonolitas en la unidad 23) con fracturación como rocas de resistencia media y clasificables en la clase de emplazamiento 2 (Tabla 3.4). Consecuentemente, se encuadra la unidad 23 en la clase 2. La unidad 24 se compone de ignimbritas y lavas fonolítico-traquíticas peralcalinas (Tabla A.3). Debido a la presencia de las ignimbritas, que son consideradas como rocas blandas a firmes, se infiere una resistencia global para la unidad 24 equiparable a la de la clase de emplazamiento 3 (Tabla 3.4), a pesar de la presencia de rocas volcánicas (traquitas y fonolitas). Se adopta por tanto un criterio conservador, desestimando que la resistencia global de la unidad 24 sea equiparable a la resistencia de la clase de emplazamiento 1 , a la que se asignarían las rocas volcánicas. Por tanto, se clasifica la unidad 24 en la clase 3.

Las unidades 25 y 26 consisten en ignimbritas fonolíticas (Tabla A.3). Como se ha indicado en el caso de las unidades 13 y 24, las ignimbritas son rocas piroclásticas con matriz que, por esta razón, son consideradas como rocas blandas a firmes. Por tanto, y de acuerdo con la descripción litológica de la clase de emplazamiento 3 (Tabla 3.4), las unidades 25 y 26 se clasifican en la clase 3 . La unidad 27 consiste en ignimbritas fonolíticas y coladas piroclásticas (Tabla A.3). Debido a la presencia de los piroclastos junto con las ignimbritas, se considera que la unidad es equiparable a un suelo de alta consistencia, y no a una roca blanda a firme. De acuerdo con la descripción litológica de la clase de emplazamiento 4 (Tabla 3.4) como suelos duros, se clasifica por tanto la unidad 27 en esta clase. La unidad 28 consta de lavas basálticas que, de acuerdo con la descripción litológica extensa de GEODE (2014), 
presentan fracturación (disyunción columnar). Al presentar rocas volcánicas fracturadas, se adopta el criterio de clasificar la unidad 28 en la clase de emplazamiento 2 (Tabla 3.4).

De acuerdo con la Tabla A.3, la unidad 29 se compone de brechas fonolíticas,ignimbritas fonolíticas y sedimentos epiclásticos. En función de esta descripción litológica, se estima que la resistencia global de la unidad 29 es equiparable a la de una roca blanda a firme, pues se encuentran brechas e ignimbritas, para las cuales ya se ha razonado su equiparación a este tipo de roca (véase el criterio adoptado para las unidades $22,25,26)$. Por tanto, la unidad 29 se encuadra en la clase de emplazamiento 3 (Tabla 3.4). La unidad 30 consiste en lavas fonolíticas, fonolítico-nefelínicas y traquíticas (Tabla A.3). Según la descripción litológica extensa de GEODE (2014), las rocas volcánicas presentes (fonolitas y traquitas) presentan fracturación (lajeado). En consecuencia, se adopta el criterio de clasificación de las unidades con rocas volcánicas fracturadas en la clase de emplazamiento 2 (Tabla 3.4). La unidad 31 se describe como coladas piroclásticas de diversos tipos (Tabla A.3). Esta unidad configura por tanto un depósito de tipo piroclástico, el cual es clasificable en la clase de emplazamiento 4 (Tabla 3.4), al ser su resistencia equiparable a la de un suelo cohesivo duro. En cuanto a la unidad 32, su descripción litológica como ignimbritas y coladas piroclásticas (Tabla A.3) es complementada por la ampliación de GEODE (2014), siendo posible concluir que estas ignimbritas presentan una matriz cinerítica, es decir, con tamaño de grano igual al de las cenizas. A partir de este bajo tamaño de grano para la matriz, es razonable suponer que la resistencia de las ignimbritas es acorde con la de las rocas blandas a firmes, pues esta matriz fina refuerza la consistencia media de este tipo de rocas. Por otra parte, la Tabla A.3 también describe la presencia de la brecha ignimbrítica de Arucas, para la cual GEODE (2014) detalla igualmente una matriz cinerítica. Por tanto, la resistencia de esta brecha también es compatible con la de las roca blandas a firmes. Consecuentemente, la resistencia de la unidad 32 en su conjunto puede entenderse equiparable a la de las rocas blandas a firmes y, a partir de la descripción litológica de la clase de emplazamiento 3 (Tabla 3.4), la unidad 32 puede ser clasificada en esta clase.

La unidad 33 consiste en una brecha volcánica de tipo "block and ash" (Tabla A.3). En función de la presencia de esta brecha, se sigue el criterio adoptado para la unidad 22, en el sentido de considerar que la resistencia de las litologías brechoides es compatible con la resistencia de las rocas blandas a firmes, características de la clase de emplazamiento 3 (Tabla 3.4). Por tanto, se clasifica la unidad 33 en la clase 3. La unidad 34 se compone de tobas e ignimbritas (Tabla A.3). La presencia de las tobas, que son depósitos piroclásticos, recomienda inferir una resistencia global acorde con la de los suelos duros (clase de emplazamiento 4 en la Tabla 3.4), a pesar de que las ignimbritas podrían abrir la posibilidad de equiparar la resistencia global de la unidad a la resistencia de las rocas blandas a firmes (clase de emplazamiento 3). Por tanto, se encuadra la unidad 34 en la clase de emplazamiento 4. Por su parte, la unidad 35 se describe como ignimbritas soldadas, por lo que se clasifica directamente en la clase de emplazamiento 3. En cuanto a la unidad 36, ésta se compone de piroclastos de tamaño igual al de los lapillis (GEODE, 2014). Siguiendo el criterio adoptado en el caso de la unidad 9, se infiere que la consistencia global de la unidad 36 es media, y por tanto equivalente a la consistencia de la clase de emplazamiento 5 (Tabla 3.4). 
Formación detrítica de Las Palmas (Miembro inferior) La unidad 37 se compone de conglomerados y arenas (Tabla A.3). A partir de la presencia de sedimentos detríticos de tamaño igual o superior al de las arenas, se considera que la resistencia global de esta unidad es coherente con la resistencia de los suelos de alta compacidad/consistencia. La descripción geotécnica de la clase de emplazamiento 4 como suelos cohesivos duros (Tabla 3.4) hace posible, por tanto, considerar la resistencia de la unidad 37 como equiparable a la resistencia de esta clase. En consecuencia, se clasifica la unidad 37 en la clase 4 . En cuanto a la unidad 38, ésta consta de conglomerados y arenas continentales con intercalaciones de niveles detríticos marinos. Aplicando el mismo criterio que el utilizado para clasificar la unidad 37, se encuadra la unidad 38 en la clase de emplazamiento 4.

Formación detrítica de Las Palmas (Miembro medio) De acuerdo con la Tabla A.3, la unidad 39 se compone de sedimentos aluviales con intercalaciones piroclásticas indiferenciadas. La descripción litológica extensa de GEODE (2014) indica que los sedimentos corresponden a conglomerados, arcillas y limos. Si bien la unidad conforma un depósito sedimentario detrítico para el cual podría inferirse una consistencia alta, la presencia de limos recomienda disminuir la consistencia global de la unidad de alta a media. De acuerdo con la descripción geotécnica de la clase de emplazamiento 5 como suelos de consistencia media, la unidad 39 en se clasifica en esta clase. La unidad 40 es descrita como facies de conglomerados y arenas marinas (Tabla A.3). Por tanto, se adopta el mismo criterio que el utilizado para clasificar la unidad 37, en el sentido de clasificar las unidades con sedimentos detríticos de tamaño igual o superior al de las arenas en la clase de emplazamiento 4 (Tabla 3.4). Con respecto a la unidad 41, ésta se compone de arenas eólicas (Tabla A.3). A partir de esta descripción, se infiere que la compacidad/consistencia de estas arenas es muy baja. Su resistencia puede equipararse a la de la clase de emplazamiento 6 (Tabla 3.4), pues ésta se describe geotécnicamente como suelos blandos, es decir, suelos cohesivos con consistencia mínima. Por tanto, se clasifica la unidad 41 en la clase 6 .

Formación detrítica de Las Palmas (Miembro superior) La unidad 42 se describe como facies detríticas de arenas y conglomerados poco clasificados, de acuerdo con la Tabla A.3. En función de la presencia de las arenas y los conglomerados, se adopta el criterio de clasificar la unidad en la clase de emplazamiento 4 (Tabla 3.4).

Ciclo Roque Nublo La unidad 43 se compone de sedimentos, depósitos epiclásticos y brechas volcánicas (Tabla A.3). La unidad configura depósitos constituidos por sedimentos de origen volcánico, que se encuentran acompañados por materiales brechoides compuestos por fragmentos del mismo origen. En función de la presencia de estos sedimentos, se considera que la resistencia de esta unidad es menor que la resistencia de los materiales brechoides, que es equivalente a la de las rocas blandas a firmes propias de la clase de emplazamiento 3 (Tabla 3.4). Por tanto, se otorga a la unidad 43 una resistencia equiparable a la de los suelos de alta consistencia que caracterizan la clase de emplazamiento 4. La unidad 44 consiste en sedimentos aluviales de origen basáltico y depósitos caóticos fluvio-torrenciales (Tabla A.3). De acuerdo con la descripción litológica extensa de GEODE (2014), estos depósitos 
contienen conglomerados que se alternan con arenas, arcillas y limos. La presencia de los limos recomienda considerar una consistencia media para la unidad 44, como en el caso de la unidad 39, y clasificarla en la clase de emplazamiento 5. En cuanto a la unidad 45, ésta se describe como coladas piroclásticas y depósitos epiclásticos. Debido a la naturaleza piroclástica de estos depósitos, se adopta el criterio de clasificarlos en la clase de emplazamiento 4 (Tabla 3.4). La unidad 46 consta de lavas basálticas, basaníticas y tefríticas, y de hawaiitas y benmoreitas. De acuerdo con la descripción litológica extensa de GEODE (2014), las rocas volcánicas presentes (basaltos, basanitas, tefritas, traquibasaltos y traquiandesitas) presentan fracturación (disyunción columnar y lajeado). Por este motivo, se consideran rocas de resistencia media y se clasifican en la clase de emplazamiento 2 (Tabla 3.4).

La unidad 47 presenta conos de tefra y conos piroclásticos con lapillis, escorias y bombas (Tabla A.3). Como la unidad constituye un depósito piroclástico, se clasifica en la clase de emplazamiento 4 (Tabla 3.4). De acuerdo con GEODE (2014), la unidad 48 consta de depósitos epiclásticos de arenas y conglomerados. En este caso, se aplica el criterio de clasificación de las unidades con sedimentos detríticos de tamaño igual o superior al de las arenas en la clase de emplazamiento 4 . La unidad 49 es descrita por GEODE (2014) como una alternancia de brechas y coladas volcánicas (traquitas, fonolitas y tefritas). En función de la presencia de las brechas, se adopta el criterio de considerar la resistencia global de la unidad como equivalente a la de una roca blanda a firme, a pesar de la presencia de las rocas volcánicas. Con este criterio conservador, se clasifica la unidad 49 en la clase de emplazamiento 3 (Tabla 3.4). Según la Tabla A.3, la unidad 50 se describe como depósitos hidromagmáticos con niveles de arenas marinas intercalados. La descripción litológica extensa de GEODE (2014) detalla que estos depósitos hidromagmáticos presentan fragmentos centimétricos de pómez o pumita y fragmentos milimétricos básico-sálicos . Se infiere una resistencia global equivalente a la de los suelos de alta compacidad/consistencia, debido a la presencia de las arenas marinas y de los fragmentos volcánicos de tamaño grueso que constituyen los depósitos hidromagmáticos. Por tanto, la unidad 50 se clasifica en la clase de emplazamiento 4 (Tabla 3.4).

La unidad 51 es descrita como la brecha volcánica Roque Nublo (Tabla A.3), que comprende un conjunto de materiales brechoides muy consolidados y con matriz cinerítica y vitroclástica (GEODE, 2014). En función de estas propiedades, se considera que la unidad 51 se compone de rocas blandas a firmes y, por tanto, ésta se clasifica en la clase de emplazamiento 3 (Tabla 3.4). La unidad 52 consta de gabros alcalinos de grano medio y facies pegmatoides (Tabla A.3). Por tanto, se adopta el criterio de clasificación de las unidades con rocas plutónicas (gabros) en la clase de emplazamiento 1 (Tabla 3.4). La unidad 53 consiste en lavas traquíticas, por lo que se sigue el criterio de clasificación de las unidades con rocas volcánicas (traquitas) en la clase de emplazamiento 1 . La unidad 54 consta de intrusiones de fonolitas y foiditas. De acuerdo con GEODE (2014), estas rocas volcánicas presentan fracturación (disyunción columnar), por lo que se consideran como rocas de resistencia media, y por tanto la unidad 54 se clasifica en la clase de emplazamiento 2 (Tabla 3.4).

De acuerdo con GEODE (2014), la unidad 55 presenta alternancias de depósitos sedimentarios con piroclastos de caída e hiladas de cineritas y tobas finas. Debido a la presencia de las tobas finas, que son depósitos piroclásticos de granulometría fina, y de las cineritas, que se componen de cenizas, se considera que la consistencia de esta 
unidad es media. A partir de la descripción geotécnica de la clase de emplazamiento 5 como suelos de consistencia media (Tabla 3.4), se clasifica la unidad 55 en esta clase. La unidad 56 se compone de lavas fonolíticas, tefríticas y benmoreíticas, por lo cual se adopta el criterio de clasificación de las unidades con rocas volcánicas (fonolitas, tefritas y traquiandesitas) en la clase de emplazamiento 1. La unidad 57 se describe como depósitos conglomeráticos aluviales y torrenciales, que presentan también arenas y arcillas (Tabla A.3). Teniendo en cuenta la litología predominante (conglomerado), se aplica el criterio de clasificación de los depósitos detríticos con sedimentos de tamaño igual o superior al de las arenas en la clase de emplazamiento 4 (Tabla 3.4).

La unidad 58 es descrita como facies laháricas y "mud flows" de las unidades del Roque Nublo (Tabla A.3). De acuerdo con GEODE (2014), esta unidad presenta niveles de arenas que se alternan con niveles de conglomerados. Adoptando el criterio de clasificación de los depósitos detríticos con arenas y sedimentos de tamaño superior en la clase de emplazamiento 4 (Tabla 3.4), se encuadra la unidad 58 en esta clase. Este mismo criterio se utiliza igualmente para clasificar la unidad 59 en la citada clase 4, puesto que esta unidad se describe como conglomerados y depósitos epiclásticos (Tabla A.3). En cuanto a la unidad 60, ésta presenta depósitos de deslizamientos gravitacionales que, según GEODE (2014), constan de materiales brechoides de diversos espesores, dispuestos caóticamente. A partir de la inestabilidad que se presume para este tipo de depósito, se entiende que su resistencia es menor que la inferida para los materiales brechoides, que corresponden a la clase de emplazamiento 3. Por tanto, la unidad 60 se encuadra en la clase de emplazamiento 4.

Ciclo Post-Roque Nublo (Medio e inferior) De acuerdo con la Tabla A.3, la unidad 61 se describe como depósitos epiclásticos. Según GEODE (2014), estos depósitos contienen materiales detríticos gruesos, fragmentos básicos de tamaño mili-decimétrico y lapillis. La granulometría predominante es gruesa y, por tanto, es razonable considerar que la compacidad/consistencia de la unidad es equiparable a la de los depósitos detríticos con arenas o sedimentos de tamaño superior. Por tanto, se infiere que la resistencia de la unidad 61 es equivalente a la de los suelos cohesivos duros que caracterizan la clase de emplazamiento 4 (Tabla 3.4). La unidad 62 consiste en depósitos volcanoclásticos y tobas (Tabla A.3), por lo cual configura un depósito piroclástico. En este sentido, también se clasifica en la clase de emplazamiento 4. La unidad 63 es descrita como lapillis, depósitos epiclásticos y freatomagmáticos (Tabla A.3). La descripción litológica extensa de GEODE (2014) detalla que la unidad consta fundamentalmente de tobas, con lapillis y arenas finas interestratificadas. Considerando que la unidad corresponde a un depósito piroclástico, ésta se clasifica en la clase de emplazamiento 4. La unidad 64 consiste en brechas líticas, por lo cual se aplica el criterio de clasificación de los materiales brechoides en la clase de emplazamiento 3 (Tabla 3.4).

La unidad 65 es descrita como diques-domo basanítico-nefeliníticos (Tabla A.3). A partir de esta composición, se adopta el criterio de clasificar las unidades con rocas volcánicas (basanitas y nefelinitas) en la clase de emplazamiento 1 (Tabla 3.4). La unidad 66 consiste en lavas basanítico-nefeliníticas, basálticas y basáltico olivínicopiroxénicas (Tabla A.3). De acuerdo con GEODE (2014), estas lavas presentan frac- 
turación (disyunción columnar). Por tanto, se considera que la fracturación de las rocas volcánicas presentes (basanitas y basaltos) disminuye su resistencia, de forma que se clasifican en la clase de emplazamiento 2 como rocas de resistencia media (Tabla 3.4). La unidad 67 consta de tobas piroclásticas (Tabla A.3), por lo cual configura un depósito piroclástico, claramente clasificable en la clase de emplazamiento 4 (Tabla 3.4). Aplicando este mismo criterio, la unidad 68 se encuadra en la clase 4, pues se describe como conos de tefra y piroclásticos, con bombas, escorias y lapillis (Tabla A.3).

La unidad 69 se compone de niveles fluvio-marinos de gravas (Tabla A.3). Como la granulometría del sedimento detrítico (grava) que constituye esta unidad es superior al tamaño de las arenas, la unidad se clasifica en la clase de emplazamiento 4 (Tabla 3.4). La unidad 70 es descrita por GEODE (2014) como depósitos aluviales de conglomerados con niveles de arenas. En este caso, se vuelve a aplicar el criterio de clasificación de las unidades con depósitos detríticos de arenas o de sedimentos de tamaño superior al de las arenas en la clase de emplazamiento 4. La unidad 71 consiste en brechas de tipo "block and ash" (Tabla A.3), las cuales presentan una matriz piroclástica de tipo lapilli muy fina (GEODE, 2014). Esta unidad presenta materiales brechoides, que se consideran como rocas blandas a firmes. Esta inferencia también se apoya en la presencia de la matriz muy fina, que incrementa la consistencia de la roca. Por tanto, la unidad 71 se clasifica en la clase de emplazamiento 3 (Tabla 3.4).

De acuerdo con la Tabla A.3, la unidad 72 se describe como piroclastos de dispersión, los cuales corresponden a lapillis (GEODE, 2014). Considerando la fina granulometría del piroclasto, se adopta el mismo criterio que el seguido en el caso de la unidad 9, infiriendo una consistencia media para la unidad 72. Por esta razón, esta unidad se encuadra en la clase de emplazamiento 5 (Tabla 3.4). La unidad 73 es descrita como depósitos aluviales de arenas y gravas (Tabla A.3). Al presentarse sedimentos de tamaño arena y grava, se sigue el criterio ya establecido de clasificación de las unidades con sedimentos detríticos de tamaño igual o superior al de las arenas en la clase de emplazamiento 4 (Tabla 3.4). La unidad 74 se compone de depósitos de deslizamientos gravitacionales, con bloques empastados en material arcillo-arenoso (GEODE, 2014). En función de la gruesa granulometría del sedimento (bloques), se vuelve a aplicar el mismo criterio para encuadrar esta unidad en la clase de emplazamiento 4.

Ciclo Post-Roque Nublo (Superior) La unidad 75 consiste en lavas basaníticonefeliníticas, tefrítico-fonolíticas, basaníticas y basálticas (Tabla A.3). La descripción litológica extensa de GEODE (2014) indica que las lavas presentan fracturación (disyunción columnar). Por tanto, las rocas volcánicas presentes (basanitas, tefritas y basaltos) se consideran como rocas de resistencia media, y la unidad 75 se clasifica en la clase de emplazamiento 2 (Tabla 3.4). La unidad 76 consta de conos de tefra y piroclásticos, con bombas, escorias y lapillis (Tabla A.3). Se aplica, por tanto, el criterio de clasificación de las unidades con depósitos piroclásticos en la clase de emplazamiento 4 (Tabla 3.4). La unidad 77 se describe como piroclastos de dispersión de clase lapilli (GEODE, 2014). Por tanto, se adopta el criterio de considerar las unidades que constituyen depósitos de lapilli como suelos de consistencia media y, por tanto, clasificables en la clase de emplazamiento 5 (Tabla 3.4). En cuanto a 
la unidad 78, ésta consiste en depósitos freatomagmáticos, que, de acuerdo con la descripción litológica extensa de GEODE (2014), presentan fragmentos volcánicos centimétricos con niveles de lapilli intercalados. En función de la granulometría gruesa que se encuentra en estos depósitos, se sigue el criterio general de clasificación de los depósitos piroclásticos como suelos cohesivos duros, y se encuadra la unidad 78 en la clase de emplazamiento 4.

Ciclo Reciente (Inferior) La unidad 79 es descrita por GEODE (2014) como sedimentos aluviales de arenas, cantos y bloques. En función de las granulometrías que presentan estos sedimentos, se aplica el criterio de clasificación de las unidades con sedimentos detríticos de tamaños de grano iguales o superiores al de las arenas en la clase de emplazamiento 4 (Tabla 3.4). La unidad 80 consiste en arenas de playa fosilíferas, que constituyen una rasa marina situada a alturas de $30 \mathrm{~m}$ o de $35 \mathrm{~m}$ sobre el nivel del mar (Tabla A.3). Se aplica por tanto el mismo criterio que el utilizado para clasificar la unidad 79, encuadrando también la unidad 80 en la clase de emplazamiento 4. Según la descripción litológica extensa de GEODE (2014), la unidad 81 se compone de lavas de composición basanítica y con disyunción columnar grosera. Por tanto, se sigue el criterio de clasificación de las unidades con rocas volcánicas (basanitas) con fracturación (disyunción columnar) en la clase de emplazamiento 2 (Tabla 3.4). La unidad 82 consiste en conos piroclásticos y conos de tefra, con bombas, escorias y lapillis (Tabla A.3). Dado que esta unidad constituye un depósito piroclástico, se clasifica en la clase de emplazamiento 4. En cambio, la unidad 83 presenta depósitos de lapilli (GEODE, 2014), por lo cual se sigue el criterio de clasificación de las unidades con este tipo de depósitos en la clase de emplazamiento 5 (Tabla 3.4).

La descripción litológica extensa de la unidad 84 (GEODE, 2014) señala que la unidad configura depósitos freatomagmáticos, que contienen materiales compactos de naturaleza areno-arcillosa, que se encuentran intercalados por niveles de lapilli. Debido a que la granulometría del sedimento es próxima al tamaño de las arenas, y también a la descripción de la compacidad de los depósitos, se entiende que la compacidad global de la unidad 84 puede suponerse alta. En este sentido, la resistencia de la unidad es equiparable a la de los suelos cohesivos duros que caracterizan la clase de emplazamiento 4 (Tabla 3.4). Por tanto, la unidad 84 se clasifica en la clase 4. La unidad 85 consiste en lavas tefríticas y fonolíticas (Tabla A.3). En consecuencia, puede aplicarse el criterio de clasificación de las unidades con rocas volcánicas (tefritas y fonolitas) en la clase de emplazamiento 1 (Tabla 3.4). En cuanto a la unidad 86, ésta es descrita como conos de tefra (Tabla A.3), por tanto se adopta el criterio de clasificación de las unidades con depósitos piroclásticos en la clase de emplazamiento 4. La unidad 87 consta de depósitos lacustres limo-arcillosos. En función de la baja granulometría de los sedimentos existentes, es razonable considerar que la consistencia de la unidad 87 es baja. Debido a esta baja consistencia, la resistencia de la unidad es equiparable a la resistencia de la clase de emplazamiento 6 (Tabla 3.4), a tenor de su descripción geotécnica como suelos blandos.

Ciclo Reciente (Superior) La unidad 88 se compone de arenas de playa con cantos y fauna de Strombus (Tabla A.3). La descripción litológica extensa de GEODE (2014) indica que el depósito está constituido por areniscas, que suelen presentar 
en su parte alta conglomerados de cantos básicos . La resistencia de la arenisca puede ser considerada como equivalente a la de una roca blanda a firme. De acuerdo con la descripción geotécnica de la clase de emplazamiento 3 como rocas blandas a firmes (Tabla 3.4), se procede a clasificar la unidad 88 en esta clase. La unidad 89 se describe como una rasa holocena que está constituida por arenas finas (GEODE, 2014). Se aplica por tanto el criterio de clasificación de las unidades con sedimentos detríticos de tamaños de grano iguales o superiores al tamaño de las arenas en la clase de emplazamiento 4 (Tabla 3.4). Según GEODE (2014), la unidad 90 consiste en lavas basaníticas con fracturación (disyunción columnar o esferoidal). Debido a la presencia de rocas volcánicas fracturadas, se consideran rocas de resistencia media y se clasifica la unidad 90 en la clase de emplazamiento 2 (Tabla 3.4). La unidad 91 presenta conos de tefra y piroclásticos, con bombas, escorias y lapillis (Tabla A.3). Como esta unidad constituye depósitos piroclásticos, se clasifica en la clase de emplazamiento 4.

Las unidades 92, 94 y 95 son descritas por GEODE (2014) como depósitos de lapilli. En este sentido,se adopta el criterio de clasificación de las unidades con depósitos de lapilli en la clase de emplazamiento 5 (Tabla 3.4). Con respecto a la unidad 93, GEODE (2014) la describe como depósitos freatomagmáticos con alternancia de niveles de lapillis y de materiales arenosos finos. En función del tamaño de grano de las arenas encontradas en esta unidad, se considera que la compacidad global es alta. Por tanto, la resistencia global es equiparable a la resistencia de la clase de emplazamiento 4, de acuerdo con su descripción geotécnica como suelos duros (Tabla 3.4).

Depósitos sedimentarios recientes La unidad 96 consiste en sedimentos conglomeráticos y arenas fluviales (Tabla A.3). La descripción litológica extensa de GEODE (2014) precisa que la unidad configura depósitos aluviales con gravas, arenas y limos. La presencia de limos recomienda adoptar un criterio conservador, disminuyendo la consistencia global de la unidad de alta a media. Por tanto, la resistencia de la unidad 96 es equivalente a la de la clase de emplazamiento 5 (Tabla 3.4). La unidad 97 se describe como depósitos de ladera y coluviones, a veces con arenas fosilíferas, conos de deyección con intercalaciones eólicas, y glacis-conos (Tabla A.3). La unidad constituye depósitos de ladera que presentan arenas, por lo cual resulta razonable aplicar el criterio de clasificación de las unidades con sedimentos detríticos de granulometrías iguales o superiores a la de las arenas en la clase de emplazamiento 4. La unidad 98 se describe como depósitos caóticos y cuñas de deslizamientos de ladera gravitacionales (Tabla A.3). De acuerdo con la descripción litológica extensa de GEODE (2014), estos depósitos están constituidos por bloques y cantos en matriz detrítica más fina. De acuerdo con el criterio de clasificación de los depósitos de arenas o sedimentos de tamaño superior en la clase de emplazamiento 4 (Tabla 3.4), es razonable encuadrar esta unidad en la citada clase, pues contiene sedimentos gruesos (bloques, cantos), y además éstos están soportados por una matriz más fina, que favorece su compacidad. La disposición caótica de los depósitos podría plantear el dilema de disminuir la compacidad global de la unidad y clasificar la misma en la clase de emplazamiento 5 , si bien se considera que los argumentos anteriores (granulometría gruesa con matriz fina) son suficientes para clasificar la unidad 98 en la clase de emplazamiento 4. Respecto a la unidad 99, ésta 
corresponde a depósitos epiclásticos que, de acuerdo con GEODE (2014), contienen fragmentos de origen volcánico y sedimentario englobados en matriz piroclástica. En base a la naturaleza piroclástica de la matriz, resulta razonable clasificar la unidad 99 en la clase de emplazamiento 4.

La unidad 100 consiste en depósitos y sedimentos aluviales antiguos, y de terrazas fluviales (Tabla A.3). Dado que GEODE (2014) explicita la composición de estos depósitos en arenas y gravas, se aplica el criterio de clasificación de los depósitos detríticos con sedimentos de tamaño igual o superior al de las arenas en la clase de emplazamiento 4 (Tabla 3.4). La unidad 101 se describe como arenas eólicas y dunas (Tabla A.3). A partir de la baja compacidad/consistencia que se presupone a este tipo de arenas, que forman dunas, se clasifica la unidad 101 en la clase de emplazamiento 6, pues ésta se describe como suelos blandos (Tabla 3.4), es decir, de baja consistencia. La unidad 102 consta de arenas eólicas fósiles (Tabla A.3). En este caso, la descripción de estas arenas como fósiles y la edad de la unidad (Pleistoceno Superior-Holoceno) permiten considerar que las arenas se comportan como un suelo de alta compacidad/consistencia. Por tanto, la unidad 102 se clasifica en la clase de emplazamiento 4, cuya descripción geotécnica consiste en suelos duros (Tabla 3.4). La unidad 103 consiste en depósitos de arenas y arcillas indiferenciados (Tabla A.3). En función de la presencia de las arenas, se aplica el criterio de clasificación de los depósitos detríticos con sedimentos de esta granulometría en la clase de emplazamiento 4 .

La unidad 104 se describe como arenas marrones fosilíferas sobre dunas fósiles (Tabla A.3). Se adopta por tanto el criterio de clasificación de las unidades con sedimentos de tamaño arena o superior en la clase de emplazamiento 4 (Tabla 3.4). La unidad 105 es descrita como depósitos aluviales y de fondo de barranco (Tabla A.3). La descripción litológica extensa de GEODE (2014) añade que los depósitos aluviales contienen gravas y arenas. Aplicando de nuevo el mismo criterio que el utilizado en el caso de la unidad 104, se encuadra la unidad 105 en la clase de emplazamiento 4. En cuanto a la unidad 106, ésta se compone de materiales granulosos, de 1-2 cm de tamaño de grano, con intercalaciones de cantos más gruesos y material piroclástico fino (GEODE, 2014), conformando depósitos eluvio-coluviales (Tabla A.3). En función del tamaño de grano (grava) de los materiales granulosos, también se clasifica la unidad 106 en la clase de emplazamiento 4.

La unidad 107 consiste en depósitos de suelos y depósitos de recubrimiento, otros depósitos indiferenciados y a veces rellenos de fondo de valle (Tabla A.3). La descripción litológica extensa de GEODE (2014) precisa que estos depósitos están constituidos por limos arcillosos y material piroclástico retrabajado. La fina granulometría presente (limo, arcilla) indica que la consistencia global de la unidad es media, por lo que ésta puede encuadrarse en la clase de emplazamiento 5 (Tabla 3.4), en base a su descripción geotécnica como suelos de consistencia media. La unidad 108 configura depósitos de playas con gravas y arenas (GEODE, 2014). En función de la baja compacidad/consistencia que se presupone para este tipo de depósitos, la unidad 108 se clasifica en la clase de emplazamiento 6 (Tabla 3.4) como suelos blandos, es decir, de baja consistencia. Por último, la unidad 109 configura depósitos antrópicos (rellenos), de acuerdo con la Tabla A.3 y la descripción litológica extensa de GEODE (2014). Siguiendo un criterio conservador, se considera la compacidad/consistencia de este tipo de depósitos como baja. Por tanto, la unidad 109 
se encuadra en la clase 6 .

En la Tabla 3.30 se presenta un resumen de la clasificación de las unidades de Gran Canaria, de acuerdo con el procedimiento descrito.

Tabla 3.30: Resumen de la clasificación de las unidades del mapa geológico continuo GEODE a escala 1:50.000 de Gran Canaria (Zona 2912), de acuerdo a la clasificación de síntesis. Tomado de GEODE (2014), elaboración propia.

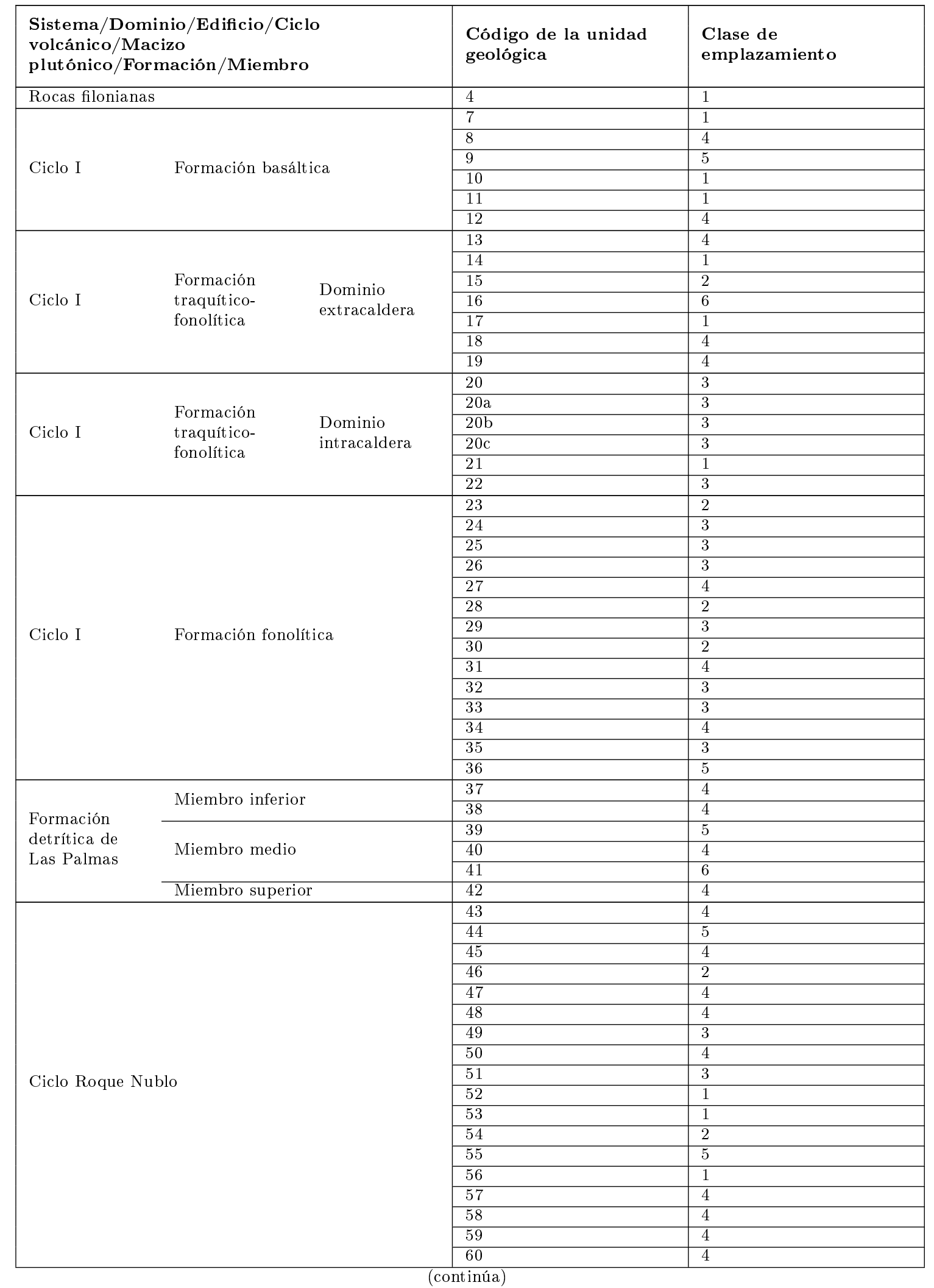


(continuación)

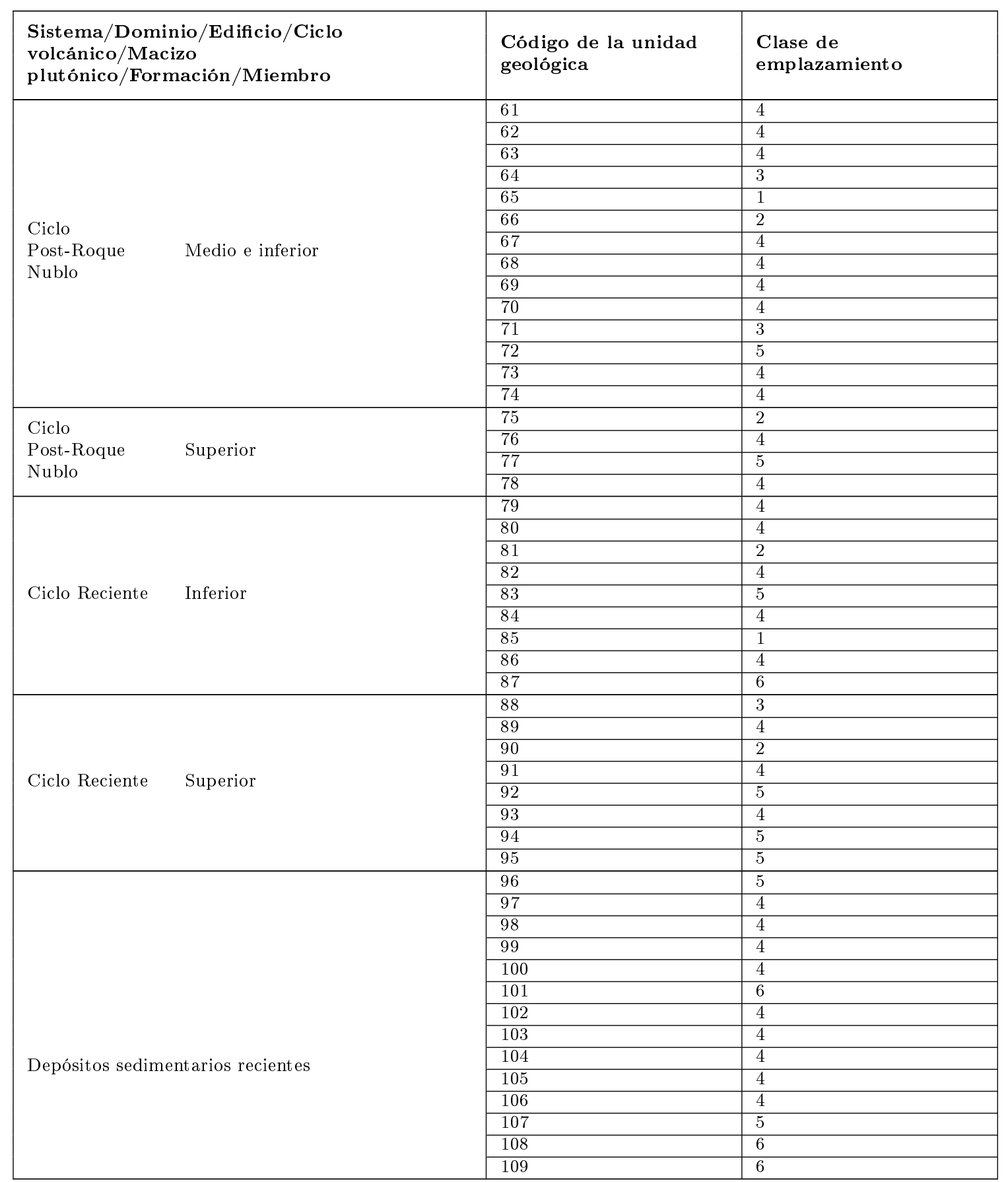

\section{La Gomera (Región 2914)}

La región GEODE que corresponde a La Gomera es la región 2914. De acuerdo a la cartografía de la misma (GEODE, 2014), en La Gomera afloran 63 unidades geológicas, datadas desde el Mioceno Medio-Superior hasta el Cuaternario. Las descripciones litológicas y edades cronoestratigráficas de estas unidades se detallan en la Tabla A.4. Según la leyenda cronoestratigráfica de la cartografía (GEODE, 2014), las unidades pertenecen a 8 grupos distintos, que se presentan en la Tabla 3.31. De las 63 unidades geológicas descritas en la región 2914, 2 no aparecen cartografiadas 
y por tanto no se han clasificado: unidades 1 y 2.

Tabla 3.31: División de las unidades del mapa geológico continuo GEODE a escala 1:50.000 de La Gomera (Zona 2914), de acuerdo a la leyenda cronoestratigráfica. Tomado de GEODE (2014).

\begin{tabular}{|l|l|l|}
\hline $\begin{array}{l}\text { Código de la } \\
\text { unidad geológica }\end{array}$ & $\begin{array}{l}\text { Sistema/Dominio/Edificio/Ciclo volcánico/Macizo } \\
\text { plutónico/Formación/Miembro }\end{array}$ \\
\hline 1,2 & Rocas filonianas & \multirow{2}{*}{ Complejo traquítico-fonolítico } \\
\hline 3,4 & Complejo Basal & \\
\hline $5,6,7$ & Tramo inferior & \\
\hline $8,9,10,11,12$ & Tramo medio & \\
\hline $13,14,15,16$ & \\
\hline $\begin{array}{l}17,18,19,20,21,22, \\
23,24,25,26,27,28,\end{array}$ & Primer ciclo volcánico \\
29 & \\
\hline $30,31,32,33,34,35$, & \\
$36,37,38,39,40,41$, & Segundo ciclo volcánico \\
$42,43,44,45,46,47$, & \\
$48,49,50,51,52,53$ & \\
\hline $54,55,56,57,58,59$, & Formaciones sedimentarias cuaternarias \\
$60,61,62,63$ & \\
\hline
\end{tabular}

Complejo Basal La unidad 3 se describe como lavas, tobas y sedimentos detríticos y carbonatados, como se observa en la Tabla A.4. La presencia de lavas permite considerar que la unidad contiene rocas volcánicas, cuya resistencia es equivalente a la de las rocas duras que caracterizan a la clase de emplazamiento 1, a tenor de la descripción geotécnica de ésta última (Tabla 3.4). En cambio, las tobas son depósitos piroclásticos, cuya compacidad/consistencia se supone alta. En este sentido, la resistencia de las tobas es equiparable a la de la clase de emplazamiento 4 (Tabla 3.4), en función de su descripción geotécnica como suelos cohesivos duros, es decir, de alta consistencia. La aparición de rocas volcánicas junto con estos depósitos piroclásticos, y también junto con otros sedimentos de tipo detrítico y carbonatado hacen recomendable la adopción de un criterio conservador. En este sentido, se prefiere considerar la unidad 3 en su conjunto como un suelo de alta consistencia, y por tanto clasificarla en la clase de emplazamiento 4. La unidad 4 es descrita como rocas plutónicas básicas y ultrabásicas (Tabla A.4). Para este tipo de rocas también se supone una resistencia equivalente a la de las rocas duras. Sin embargo, la descripción litológica extensa, proporcionada por GEODE (2014), precisa que estas rocas presentan fracturación, por lo cual su resistencia disminuye con respecto a la resistencia de las rocas duras, de alta a media. Teniendo en cuenta la descripción geotécnica de la clase de emplazamiento 2 como rocas de resistencia media (Tabla 3.4), resulta razonable, por tanto, clasificar la unidad 4 en la clase 2.

Complejo traquítico-fonolítico (Tramo inferior) La unidad 5 se compone de coladas basálticas con niveles de brechas subordinadas (Tabla A.4). De acuerdo con la descripción litológica extensa de GEODE (2014), se observa alteración y brechificación en las coladas, que contienen rocas volcánicas (basaltos). Estas circunstancias permiten suponer que la resistencia global de la unidad 5 no es la misma que la resistencia que tendrían las rocas volcánicas (basaltos) de forma aislada. Por tanto, se infiere que la resistencia global de la unidad pasa de alta a media, y que es equiparable a la de la clase de emplazamiento 2 (Tabla 3.4), en vez de ser equivalente a 
la resistencia de la clase de emplazamiento 1 . Por tanto, se clasifica la unidad 5 en la clase 2. La unidad 6 consiste en conos de tefra (Tabla A.4). De acuerdo con la descripción litológica extensa de GEODE (2014), estos conos configuran depósitos piroclásticos, con piroclastos de clase escoria y lapilli. La compacidad/consistencia de estos depósitos puede considerarse alta. Por tanto, la resistencia de la unidad 6 es equiparable a la de la clase de emplazamiento 4 (Tabla 3.4), atendiendo a su descripción geotécnica como suelos duros (suelos de alta consistencia). Según la descripción litológica extensa de la unidad 7 (GEODE, 2014), ésta consiste en brechas relativamente deleznables (sueltas), con fragmentos heterométricos de origen basáltico. Aunque las brechas son rocas sedimentarias detríticas, normalmente compuestas por clastos de tamaño grueso (grava), en esta unidad presentan una compacidad escasa, que recomienda inferir una resistencia más acorde a la de un suelo. Adoptando un criterio conservador, se prefiere equiparar esta resistencia a la de un suelo de consistencia media. En función de la descripción geotécnica de la clase de emplazamiento 5 como suelos de consistencia media (Tabla 3.4), se encuadra la unidad 7 en la clase 5 .

Complejo traquítico-fonolítico (Tramo medio) La unidad 8 se compone de depósitos hidromagmáticos (Tabla A.4) que, según la descripción litológica extensa de GEODE (2014), están constituidos por lapillis y cenizas. Por tanto, esta unidad configura depósitos de piroclastos de granulometría fina. La compacidad/ consistencia de este tipo de depósitos piroclásticos no puede considerarse alta sino media, por razón de este menor tamaño de grano. Por tanto, la unidad 8 se clasifica en la clase de emplazamiento 5, en función de su descripción geotécnica como suelos de consistencia media (Tabla 3.4). La unidad 9 es descrita como brechas líticas polimícticas, o heterogéneas (Tabla A.4). Dado que las brechas son rocas sedimentarias de tamaño de grano grueso, se infiere que su resistencia es equiparable a la de las rocas blandas a firmes, con consistencia media-baja. Por tanto, y a partir de la descripción geotécnica de la clase de emplazamiento 3 como rocas blandas a firmes, se encuadra la unidad 9 en esta clase. La unidad 10, por su parte, presenta una alternancia de coladas basálticas y brechas. Como se ha razonado al argumentar la clasificación de la unidad 5 en la clase de emplazamiento 2 (Tabla 3.4), la presencia de las brechas junto con las rocas volcánicas (basaltos) recomienda considerar que la resistencia global de la unidad 10 es coherente con la de las rocas de resistencia media que caracterizan esta clase de emplazamiento. Por tanto, se encuadra la unidad 10 en la clase 2.

La unidad 11 es descrita como coladas basálticas (Tabla A.4). Como se considera que la resistencia de las rocas volcánicas (basaltos) es equivalente a la resistencia de las rocas duras, la unidad 11 se clasifica en la clase de emplazamiento 1 (Tabla 3.4), atendiendo a la descripción geotécnica de esta clase de emplazamiento como rocas duras. Este mismo criterio se utiliza para encuadrar la unidad 12 también en la clase de emplazamiento 1, pues esta unidad se describe como intrusivos basálticos y traquibasálticos, es decir, compuestos por las rocas volcánicas basalto y traquibasalto.

Complejo traquítico-fonolítico La unidad 13 consiste en depósitos piroclásticos sálicos con tobas, brechas, aglutinados e ignimbritas (Tabla A.4). Las ignimbritas son rocas piroclásticas con matriz que, al igual que las brechas, se consideran rocas 
blandas a firmes. En cuanto a los aglutinados, son rocas formadas por piroclastos que, depositados a alta temperatura, han quedado deformados plásticamente. Al ser también rocas piroclásticas, se consideran como rocas blandas a firmes. En este contexto, se entiende que los piroclastos de las tobas no serían suficientes para disminuir la resistencia propia de las rocas blandas a firmes. Por tanto, esta resistencia puede considerarse como la resistencia global de la unidad 13, y así se pasa a clasificar la misma en la clase de emplazamiento 3 (Tabla 3.4), en función de su descripción geotécnica como rocas blandas a firmes. En cuanto a la unidad 14, ésta consta de sienitas (Tabla A.4). Dado que las sienitas son rocas plutónicas, se considera que su resistencia es equivalente a la de las rocas duras que caracterizan la clase de emplazamiento 1 (Tabla 3.4). Por tanto, se encuadra la unidad 14 en esta clase.

La unidad 15 se describe como una intrusión masiva de diques sálicos y lavas subordinadas (Tabla A.4). La descripción litológica extensa de GEODE (2014) añade que las rocas volcánicas presentes (traquitas) tienen fracturación intensa. Por tanto, se aplica el mismo criterio que el utilizado para clasificar la unidad 4, en el sentido de considerar las rocas volcánicas fracturadas como rocas de resistencia media. Por tanto, se clasifica la unidad 15 en la clase de emplazamiento 2 (Tabla 3.4). La unidad 16 es descrita como intrusivos sálicos, preferentemente fonolíticos (Tabla A.4). A tenor de la presencia de rocas volcánicas (fonolitas), se adopta el criterio de clasificación de las unidades con rocas volcánicas en la clase de emplazamiento 1 (Tabla 3.4).

Primer ciclo volcánico La unidad 17 se describe como coladas basálticas (Tabla A.4). De acuerdo con la descripción litológica extensa de GEODE (2014), las rocas volcánicas de esta unidad (basaltos) presentan fracturación (disyunción columnar), por lo que se consideran rocas de resistencia media, siguiendo el criterio utilizado en la clasificación de la unidad 4. Por tanto, la unidad 17 se clasifica en la clase de emplazamiento 2 (Tabla 3.4). La unidad 18 consiste en conos de tefra que, al ser depósitos piroclásticos, permiten considerar que la unidad conforma un suelo de alta consistencia, siguiendo el criterio adoptado para clasificar la unidad 6. Por tanto, la unidad 18 se clasifica en la clase de emplazamiento 4 (Tabla 3.4). Como se observa en la Tabla A.4, la unidad 19 se compone de piroclastos basálticos de dispersión que, de acuerdo con GEODE (2014), corresponden a la clase lapilli. En función de la fina granulometría de los piroclastos, se considera que la resistencia de la unidad 19 es equiparable a la de los suelos de consistencia media y, por tanto, se procede a clasificar la unidad en la clase de emplazamiento 5 (Tabla 3.4). La unidad 20 consta de brechas de basaltos plagioclásicos (Tabla A.4). Dado que se presentan materiales brechoides, se consideran como rocas blandas a firmes, siguiendo el criterio ya utilizado en el caso de la unidad 9. Por tanto, la unidad 20 se encuadra en la clase de emplazamiento 3. En cuanto a la unidad 21, ésta se compone de coladas traquibasálticas, por lo que se adopta el criterio de clasificación de las unidades con rocas volcánicas (traquibasaltos) en la clase de emplazamiento 1 (Tabla 3.4).

La unidad 22 configura el "debris-avalanche" de Vallehermoso (Tabla A.4), que presenta materiales brechoides y tobáceos (GEODE, 2014). En función de la presencia de los materiales brechoides, se considera que la resistencia de la unidad es equiparable a la de las rocas blandas a firmes, por lo cual la unidad se clasifica en la clase de emplazamiento 3 (Tabla 3.4). La unidad 23 se describe como intrusivos basálticos y traquibasálticos, en los que se observa una fuerte fracturación (disyun- 
ción columnar), de acuerdo con GEODE (2014). Se aplica por tanto el criterio de clasificación de las unidades con rocas volcánicas (basaltos y traquibasaltos) fracturadas en la clase de emplazamiento 2 (Tabla 3.4). Las unidades 24 y 26 consisten fundamentalmente en intrusivos de traquitas (Tabla A.4). Como estas unidades presentan rocas volcánicas (traquitas), se encuadran en la clase de emplazamiento 1 (Tabla 3.4). Con este mismo criterio, también se clasifica la unidad 25 en la clase 1 , pues esta unidad se compone de coladas de traquitas máficas (Tabla A.4).

La unidad 27 se describe como brechas de intrusión (Tabla A.4). Por tanto, se adopta el criterio de clasificación de las unidades con materiales brechoides en la clase de emplazamiento 3 (Tabla 3.4). La unidad 28 presenta tobas piroclásticas sálicas y/o traquíticas (Tabla A.4), por lo cual puede considerarse que la resistencia de estos depósitos piroclásticos es equiparable a la de los suelos de consistencia alta. Como consecuencia, la unidad 28 se encuadra en la clase de emplazamiento 4 (Tabla 3.4). Con respecto a la unidad 29, ésta es descrita como coladas traquíticas y traquibasálticas. Por tanto, se adopta el criterio de clasificación de las unidades con rocas volcánicas (traquitas y traquibasaltos) en la clase de emplazamiento 1 (Tabla 3.4).

Segundo ciclo volcánico La unidad 30 se describe como brechas básicas (facies deslizadas), de acuerdo con la Tabla A.4. La descripción litológica extensa de GEODE (2014) precisa, por su parte, que estas brechas presentan una matriz arenosa, que contribuye a la compacidad general del material. Teniendo en cuenta este aspecto y el criterio seguido en cuanto a la equiparación de los materiales brechoides como rocas blandas a firmes, se clasifica la unidad 30 en la clase de emplazamiento 3 (Tabla 3.4). La unidad 31 es descrita como brechas tipo "Debris-avalanche", de acuerdo con la Tabla A.4. La descripción litológica extensa de GEODE (2014) indica también la presencia de matriz arenosa. Por tanto, es claro que puede aplicarse el mismo criterio que el adoptado en el caso de la unidad 30, por lo cual la unidad 31 se encuadra también en la clase de emplazamiento 3. En cuanto a la unidad 32, ésta consiste en brechas y sedimentos indiferenciados (Tabla A.4). En este caso, la descripción litológica extensa de GEODE (2014) aclara que la unidad se compone de un nivel de cantos y bloques en matriz arenosa, niveles de piroclastos y brechas, y un nivel de origen aluvial con cantos en matriz arenosa. Aparecen, por tanto, sedimentos detríticos gruesos (bloques, cantos), soportados por matriz arenosa, que permiten suponer que la compacidad de la unidad es alta, y que su resistencia puede equipararse a la de un suelo de alta compacidad o consistencia. Por otra parte, los niveles de piroclastos tienen una resistencia equivalente a la resistencia de este tipo de suelo. En consecuencia, es razonable clasificar la unidad 32 en la clase de emplazamiento 4, pues su descripción geotécnica consiste en suelos duros, es decir, de alta consistencia (Tabla 3.4). Con respecto a la unidad 33, ésta se describe como sedimentos detríticos aluviales (Tabla A.4), los cuales son descritos por GEODE (2014) como arenas muy finas, bloques y cantos. A partir de la granulometría predominantemente gruesa (bloques, cantos), y también de la presencia de las arenas, se entiende que la resistencia global de la unidad es equiparable a la de un suelo de alta compacidad/consistencia. Por tanto, la unidad 33 es clasificada en la clase de emplazamiento 4, a tenor de su descripción geotécnica (Tabla 3.4).

La unidad 34 se compone de tobas y brechas sálicas y básicas (Tabla A.4). Dado 
que se presentan materiales brechoides, se infiere que la resistencia de esta unidad es equivalente a la de las rocas blandas a firmes. Por tanto, la unidad 34 se encuadra en la clase de emplazamiento 3 (Tabla 3.4). La unidad 35 consiste en una alternancia de coladas y piroclastos basálticos. Las rocas volcánicas presentes son basaltos y traquibasaltos (GEODE, 2014). Estas rocas, al estar acompañadas de piroclastos de clase escoria (GEODE, 2014), tienen una resistencia menor que la de las rocas duras que caracterizan la clase de emplazamiento 1 (Tabla 3.4). Por tanto, se infiere una resistencia menor, y se clasifica la unidad 35 en la clase de emplazamiento 2, en función de su descripción geotécnica como rocas de resistencia media (Tabla 3.4). La unidad 36 configura niveles piroclásticos mixtos (Tabla A.4). Considerando que la resistencia de los depósitos de naturaleza piroclástica es equiparable a la de los suelos de alta consistencia, la unidad 36 se clasifica en la clase de emplazamiento 4 (Tabla 3.4). La unidad 37 presenta intrusivos basálticos y traquibasálticos, los cuales han experimentado fuerte fracturación (disyunción columnar), de acuerdo con GEODE (2014). Por tanto, se adopta el criterio ya utilizado en el caso de las unidades 4, 17 y 23, en el sentido de equiparar la resistencia de las rocas volcánicas (basaltos, traquibasaltos) fracturadas a la de las rocas de resistencia media, para clasificar las unidades correspondientes en la clase de emplazamiento 2 (Tabla 3.4). Por tanto, la unidad 37 se encuadra en la clase 2. En cuanto a la unidad 38, ésta consta de coladas basálticas y traquibasálticas (Tabla A.4). Por tanto, se adopta el criterio de clasificación de las unidades con rocas volcánicas (basaltos, traquibasaltos) en la clase de emplazamiento 1 (Tabla 3.4).

La unidad 39 se describe como centros de emisión freatomagmáticos (Tabla A.4). La descripción litológica extensa de GEODE (2014) indica que la unidad consiste en depósitos piroclásticos que contienen lapillis o, alternativamente, escorias y lapillis. Debido a la predominancia de piroclastos finos (lapillis), se adopta el mismo criterio que el seguido en el caso de la unidad 19, al considerar que la compacidad/consistencia global de los depósitos de lapilli es media. Por tanto, la unidad 39 se clasifica en la clase de emplazamiento 5, a tenor de su descripción geotécnica como suelos de consistencia media (Tabla 3.4). La unidad 40 se compone de conos de tefra basálticos y traquibasálticos (Tabla A.4). Dado que los depósitos de tefra son piroclásticos, se adopta el criterio de clasificación de las unidades con este tipo de depósitos en la clase de emplazamiento 4 (Tabla 3.4). La unidad 41 es descrita como depósitos piroclásticos basálticos de dispersión (Tabla A.4). Entendiendo que los depósitos de piroclastos de dispersión presentan fracciones finas, se puede aplicar el criterio de clasificación empleado en el caso de las unidad 19, considerando que la resistencia de estos depósitos es equiparable a la resistencia de los suelos de consistencia media. A tenor de la descripción geotécnica de la clase de emplazamiento 5 como suelos de consistencia media (Tabla 3.4), la unidad 41 se encuadra en esta clase. En cuanto a la unidad 42, ésta se describe como conglomerado aluvial (Tabla A.4). De acuerdo con la descripción litológica extensa de GEODE (2014), la unidad conforma un depósito detrítico constituido por fragmentos métricos cementados por una matriz arenosa. Dado que los clastos son gruesos, de tamaño grava, y que se encuentran soportados por la matriz, se equipara la resistencia de la unidad a la resistencia de los suelos de alta compacidad/consistencia. Por tanto, la unidad 42 se encuadra en la clase de emplazamiento 4 (Tabla 3.4), en función de su descripción geotécnica como suelos duros.

La unidad 43 consiste en intrusivos sálicos, traquíticos y fonolíticos (Tabla A.4). 
En función de la presencia de rocas volcánicas (traquitas, fonolitas), esta unidad se clasifica en la clase de emplazamiento 1 (Tabla 3.4). La unidad 44 se describe como tobas y brechas de borde de intrusión (Tabla A.4). En función de la presencia de las brechas, se infiere que la resistencia de la unidad es equivalente a la resistencia de las rocas blandas a firmes, por lo cual se encuadra en la clase de emplazamiento 3 (Tabla 3.4). En cuanto a las unidades 45 y 46, éstas constan de coladas de traquitas máficas y de intrusivos de traquitas máficas, respectivamente. De acuerdo con la descripción litológica extensa proporcionada por GEODE (2014) para ambas unidades, las rocas volcánicas presentes (traquitas) tienen fracturación (disyunción columnar y lajeado). Por tanto, se adopta el criterio de clasificación de las unidades con rocas volcánicas fracturadas en la clase de emplazamiento 2 (Tabla 3.4), con el fin de encuadrar las unidades 45 y 46 en esta clase.

La unidad 47 se describe como conos de tefra y piroclastos de dispersión (Tabla A.4). La descripción litológica extensa de GEODE (2014) precisa que la unidad conforma depósitos piroclásticos con bloques, bombas y escorias, por tanto su resistencia es equiparable a la de los suelos de alta consistencia. En función de la descripción geotécnica de la clase de emplazamiento 4 (Tabla 3.4), se encuadra la unidad 47 en esta clase. La unidad 48 es descrita como depósitos piroclásticos sálicos (Tabla A.4). Por tanto, se aplica el mismo criterio que el empleado en el caso de la unidad 47, con el fin de clasificar la unidad 48 en la clase de emplazamiento 4. En cuanto a la unidad 49, ésta consiste en depósitos hidromagmáticos (Tabla A.4), que son descritos más extensamente por GEODE (2014) como compuestos por piroclastos de tamaños variables centimétricos, con presencia de niveles de tipo cinerítico. Dado que la unidad constituye un depósito piroclástico, se sigue el criterio de clasificación de las unidades con este tipo de depósitos en la clase de emplazamiento 4 (Tabla 3.4). Con respecto a la unidad 50, ésta consiste en depósitos piroclásticos con lapillis y escorias (GEODE, 2014). A partir de la presencia de los lapillis, se entiende que la consistencia global de la unidad 50 es media, y, a tenor de la descripción geotécnica de la clase de emplazamiento 5 (Tabla 3.4), se encuadra la unidad en esta clase.

La unidad 51 se describe como intrusivos basálticos (Tabla A.4), que, de acuerdo con GEODE (2014), contienen rocas volcánicas con fracturación (disyunción columnar). Por tanto, se considera que la resistencia global de la unidad es equiparable a la de las rocas de resistencia media, y en consecuencia, la unidad es clasificada en la clase de emplazamiento 2 (Tabla 3.4). La unidad 52 se compone de coladas basálticas y traquibasálticas (Tabla A.4). Según GEODE (2014), las rocas volcánicas (basaltos y traquibasaltos) de esta unidad presentan fracturación (disyunción columnar). Por tanto, se vuelve a aplicar el criterio seguido en el caso de la unidad 51, y se encuadra la unidad 52 en la clase de emplazamiento 2. En cuanto a la unidad 53, ésta se describe como coladas basálticas y traquibasálticas con recubrimientos superficiales. La descripción litológica extensa de esta unidad (GEODE, 2014) indica que los basaltos y traquibasaltos presentan lajeado, por lo cual se consideran fracturados, y, como consecuencia, la unidad 53 se clasifica también en la clase de emplazamiento 2.

Formaciones sedimentarias cuaternarias De acuerdo con la Tabla A.4, la unidad 54 configura depósitos de arenas eólicas. En base a la escasa compacidad/ consistencia que se infiere para este tipo de material, presumiblemente suelto, se 
equipara su resistencia a la de un suelo blando, por tanto con baja consistencia. En función de la descripción geotécnica de la clase de emplazamiento 6 como suelos blandos (Tabla 3.4), se clasifica la unidad 54 en esta clase. La unidad 55 consiste en depósitos aluviales antiguos y terrazas, los cuales presentan gravas y arenas, a tenor de la descripción litológica extensa de GEODE (2014). En función de las granulometrías de estos sedimentos, iguales o superiores al tamaño de las arenas, se infiere que la compacidad/consistencia del depósito aluvial es alta. Considerando la descripción geotécnica de la clase de emplazamiento 4 (Tabla 3.4) como suelos duros, es decir, de alta consistencia, se clasifica la unidad 55 en esta clase. La unidad 56 se describe como suelos y depósitos areno-arcillosos y aluviales indiferenciados. Según GEODE (2014), estos depósitos detríticos pueden ser poco consolidados, presentar cantos en matriz arenosa o bien presentar arcillas. En base a esta descripción, se entiende que la escasa consolidación de algunos depósitos, junto con la importante presencia de arcillas, recomiendan adoptar un criterio conservador, y suponer que la consistencia global de la unidad es media, a pesar de observar granulometrías superiores al tamaño arcilla (arena, canto). Por tanto, la unidad 56 se clasifica en la clase de emplazamiento 5, pues la Tabla 3.4 indica que su descripción geotécnica consiste en suelos de consistencia media. Con respecto a la unidad 57, ésta se compone de depósitos de deslizamiento, coladas de derrubios y desplomes costeros (Tabla A.4). La descripción litológica extensa de GEODE (2014) aporta que los depósitos de deslizamiento contienen sedimentos de clase bloque, canto y arena en matriz arcillosa. Como se presentan granulometrías de tamaño igual o superior al de las arenas (bloque, canto, arena), y además los sedimentos correspondientes están soportados por una matriz fina (arcilla), se puede considerar que la compacidad/ consistencia global de la unidad es alta. Por tanto, se equipara su resistencia a la de los suelos cohesivos duros que caracterizan la clase de emplazamiento 4 (Tabla 3.4), y se clasifica la unidad 57 en esta clase.

La unidad 58 se describe como coluviones y depósitos de ladera (Tabla A.4) que, de acuerdo con GEODE (2014), presentan gravas y arenas sin ordenación interna. En función del tamaño de grano observado, igual y superior al tamaño de las arenas, se clasifica la unidad en la clase de emplazamiento 4 (Tabla 3.4). La unidad 59 es descrita como una rasa marina erbanense (Tabla A.4), que configura un nivel conglomerático (GEODE, 2014). Dado que los conglomerados están formados por sedimentos gruesos, de tamaño grava, se puede equiparar su resistencia a la de un suelo de alta compacidad/consistencia. En función de la descripción geotécnica de la clase de emplazamiento 4 como suelos duros (Tabla 3.4), se clasifica la unidad 59 en esta clase. La unidad 60 consiste en depósitos aluviales y fondos de barranco (Tabla A.4). De acuerdo con la descripción litológica extensa de esta unidad (GEODE, 2014), los depósitos aluviales contienen bloques, cantos y arenas. Por tanto, se adopta el criterio de clasificación de los depósitos detríticos con tamaños de grano iguales o superiores al de las arenas en la clase de emplazamiento 4 (Tabla 3.4).

La unidad 61 configura playas de arenas y cantos (Tabla A.4). Se infiere que la compacidad/consistencia de los depósitos de playas es baja. A tenor de la descripción geotécnica de la clase de emplazamiento 6 (Tabla 3.4) como suelos blandos (de baja consistencia), se clasifica la unidad 61 en esta clase. La unidad 62 se compone de rellenos antrópicos (Tabla A.4). En este caso, se adopta un criterio conservador para considerar también la compacidad/consistencia de este tipo de depósitos como baja, y clasificar la unidad 62 en la clase de emplazamiento 6. Por último, la unidad 63 
se describe como una zona de fracturación y brechificación (Tabla A.4). Debido a que esta unidad presenta materiales fracturados y brechificados, puede adoptarse el criterio de clasificación de las unidades con materiales brechoides en la clase de emplazamiento 3, en función de su descripción geotécnica como rocas blandas a firmes (Tabla 3.4).

En la Tabla 3.32 se resumen las clases de emplazamiento que corresponden a las unidades de La Gomera, de acuerdo con el procedimiento de clasificación que se ha explicado.

\section{La Palma (Región 2915)}

De acuerdo con la cartografía de la región GEODE 2915, que abarca la isla de La Palma (GEODE, 2014), afloran 68 unidades geológicas, con edades cronoestratigráficas comprendidas entre el Plioceno y el Holoceno. Las descripciones litológicas y edades cronostratigráficas de estas unidades se detallan en la Tabla A.5. Según la leyenda cronoestratigráfica de esta cartografía, las unidades pertenecen a 22 grupos distintos, que se presentan en la Tabla 3.33.

Rocas filonianas La unidad 1 se describe como diques basálticos aflorando en superficie, como se observa en la Tabla A.5. En función de las rocas volcánicas que presenta esta unidad (basaltos), se infiere que la resistencia global de la misma es equivalente a la resistencia de las rocas duras. Dado que la descripción geotécnica de la clase de emplazamiento 1 consiste en rocas duras (Tabla 3.4), se clasifica la unidad 1 en esta clase de emplazamiento. La unidad 2 consiste en diques basálticos cortados en galerías (Tabla A.5). Considerando que el impacto inherente a las galerías ha introducido fracturación en las rocas volcánicas de esta unidad (basaltos), se entiende que su resistencia es menor que la de las rocas duras. En este contexto, y recurriendo a la clasificación de síntesis (Tabla 3.4), es razonable suponer que la resistencia de la clase de emplazamiento 2, descrita geotécnicamente como rocas de resistencia media, es razonablemente equiparable a la resistencia de la unidad 2. Por esta razón, la unidad 2 se clasifica en esta clase de emplazamiento.

Edificio submarino (seamount) La unidad 3 se describe como lavas almohadilladas, brechas, aglomerados y hialoclastitas, metasomatizados a rocas verdes (Tabla A.5). De acuerdo con la descripción litológica extensa de GEODE (2014), las brechas que aparecen en esta unidad son rocas constituidas por almohadillas y por fragmentos de almohadillas, en referencia a los materiales lávicos igualmente encontrados. Del mismo modo, las brechas también pueden estar compuestas de almohadillas, bombas y lapillis escoriáceos, en referencia a los piroclastos producidos por la erupción que originó las lavas mencionadas. En cuanto a las hialoclastitas, este tipo de rocas consiste en una brecha rica en vidrio volcánico y que presenta partículas de lapillis o cenizas. De acuerdo con la Tabla A.5, también se encuentran aglomerados, que consisten en material piroclástico, consolidado o no, compuesto fundamentalmente por piroclastos redondeados de tamaño milimétrico. Atendiendo a la predominancia observada de rocas de naturaleza brechoide, con fragmentos de 
Tabla 3.32: Resumen de la clasificación de las unidades del mapa geológico continuo GEODE a escala 1:50.000 de La Gomera (Zona 2914), de acuerdo a la clasificación de síntesis. Tomado de GEODE (2014), elaboración propia.

\begin{tabular}{|c|c|c|}
\hline $\begin{array}{l}\text { Sistema/Dominio/Edificio/Ciclo } \\
\text { volcánico/Macizo } \\
\text { plutónico/Formación/Miembro }\end{array}$ & $\begin{array}{l}\text { Código de la unidad } \\
\text { geológica }\end{array}$ & $\begin{array}{l}\text { Clase de } \\
\text { emplazamiento }\end{array}$ \\
\hline \multirow{2}{*}{ Complejo Basal } & 3 & 4 \\
\hline & 4 & 2 \\
\hline \multirow{12}{*}{$\begin{array}{l}\text { Complejo } \\
\text { traquítico- } \\
\text { fonolítico }\end{array}$} & 5 & 2 \\
\hline & 6 & 4 \\
\hline & 7 & 5 \\
\hline & 8 & 5 \\
\hline & 9 & 3 \\
\hline & 10 & 2 \\
\hline & 11 & 1 \\
\hline & 12 & 1 \\
\hline & 13 & 3 \\
\hline & 14 & 1 \\
\hline & 15 & 2 \\
\hline & 16 & 1 \\
\hline \multirow{13}{*}{ Primer ciclo volcánico } & 17 & 2 \\
\hline & 18 & 4 \\
\hline & 19 & 5 \\
\hline & 20 & 3 \\
\hline & 21 & 1 \\
\hline & 22 & 3 \\
\hline & 23 & 2 \\
\hline & 24 & 1 \\
\hline & 25 & 1 \\
\hline & 26 & 1 \\
\hline & 27 & 3 \\
\hline & 28 & 4 \\
\hline & 29 & 1 \\
\hline \multirow{24}{*}{ Segundo ciclo volcánico } & 30 & 3 \\
\hline & 31 & 3 \\
\hline & 32 & 4 \\
\hline & 33 & 4 \\
\hline & 34 & 3 \\
\hline & 35 & 2 \\
\hline & 36 & 4 \\
\hline & 37 & 2 \\
\hline & 38 & 1 \\
\hline & 39 & 5 \\
\hline & 40 & 4 \\
\hline & 41 & 5 \\
\hline & 42 & 4 \\
\hline & 43 & 1 \\
\hline & 44 & 3 \\
\hline & 45 & 2 \\
\hline & 46 & 2 \\
\hline & 47 & 4 \\
\hline & 48 & 4 \\
\hline & 49 & 4 \\
\hline & 50 & 5 \\
\hline & 51 & 2 \\
\hline & 52 & 2 \\
\hline & 53 & 2 \\
\hline \multirow{10}{*}{ Formaciones sedimentarias cuaternarias } & 54 & 6 \\
\hline & 55 & 4 \\
\hline & 56 & 5 \\
\hline & 57 & 4 \\
\hline & 58 & 4 \\
\hline & 59 & 4 \\
\hline & 60 & 4 \\
\hline & 61 & 6 \\
\hline & 62 & 6 \\
\hline & 63 & 3 \\
\hline
\end{tabular}


Tabla 3.33: División de las unidades del mapa geológico continuo GEODE a escala 1:50.000 de La Palma (Zona 2915), de acuerdo a la leyenda cronoestratigráfica. Tomado de GEODE (2014).

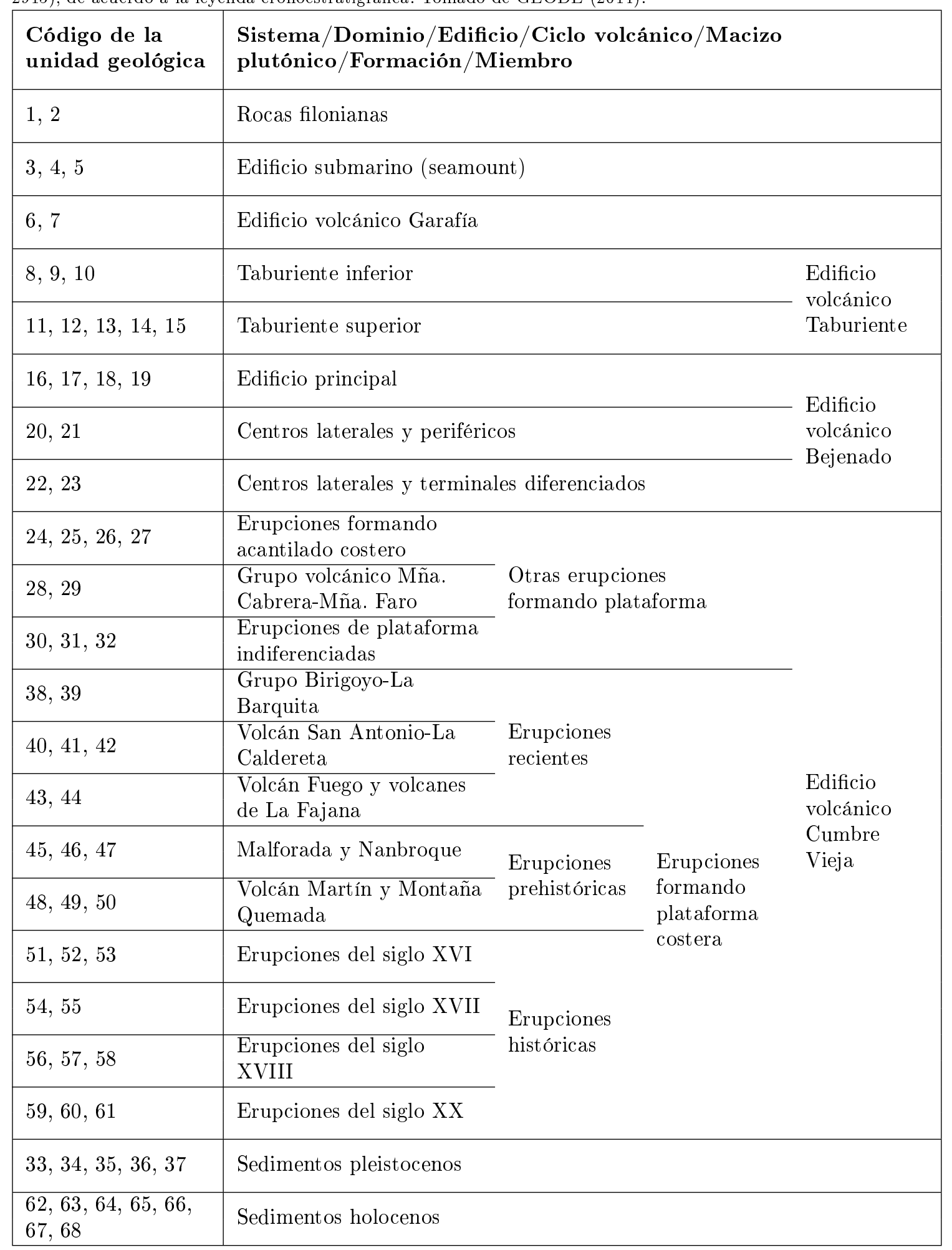


distinto tamaño, se entiende que la resistencia global de la unidad puede ser equiparable a la de las rocas blandas a firmes que describen geotécnicamente la clase de emplazamiento 3 (Tabla 3.4). En consecuencia, se clasifica la unidad 3 en la clase 3.

La unidad 4 consiste en domos o domos colada traquíticos o fonolíticos metasomatizados (Tabla A.5). De acuerdo con la descripción litológica extensa de GEODE (2014), esta unidad contiene rocas volcánicas (fonolitas y traquitas) en facies brechoides y masivas. Las facies brechoides observadas pueden introducir una disminución en la resistencia alta que se ha inferido para las rocas volcánicas, de acuerdo con el criterio de clasificación de la unidad 1 en la clase de emplazamiento 1 . Por este motivo, es razonable suponer que la resistencia de la unidad 4 es menor que la correspondiente a la clase 1. En función de la descripción geotécnica de la clase de emplazamiento 2 como rocas de resistencia media (Tabla 3.4), se encuadra la unidad 4 en esta clase. Según la Tabla A.5, la unidad 5 consiste en gabros, los cuales pertenecen al grupo de las rocas plutónicas. Considerando que la resistencia de las rocas plutónicas es equiparable a la resistencia de las rocas duras, y que la clase de emplazamiento 1 se describe geotécnicamente como rocas duras (Tabla 3.4), se clasifica la unidad 5 en la clase de emplazamiento 1.

Edificio volcánico Garafía La unidad 6 se compone de sedimentos, aglomerados y brechas, según la descripción litológica de la Tabla A.5. De acuerdo con GEODE (2014), los sedimentos son de tipo detrítico, con cantos redondeados en matriz muy alterada, y se encuentran acompañados de tobas, que son depósitos piroclásticos . La importancia que este material piroclástico y los sedimentos referidos tienen en el conjunto de la unidad permiten inferir que la misma conforma un suelo con compacidad/consistencia alta. Por tanto, se considera que su resistencia es equivalente a la resistencia de la clase de emplazamiento 4, pues, según la Tabla 3.4, esta clase tiene una descripción geotécnica en términos de suelos cohesivos duros, por tanto, de alta consistencia. Por tanto, la unidad 6 se clasifica en la clase 4 . La unidad 7 es descrita como lavas y piroclastos basálticos con gran densidad de diques basálticos. Dado que las rocas volcánicas (basaltos) de lavas y diques afloran junto con piroclastos, se infiere una resistencia global menor que la correspondiente a las rocas duras que caracterizan la clase de emplazamiento 1 (Tabla 3.4). Por tanto, se clasifica la unidad 7 en la clase de emplazamiento 2 como rocas de resistencia media.

Edificio volcánico Taburiente (Taburiente inferior) De acuerdo con la Tabla A.5, la unidad 8 consiste en aglomerados. Como estos materiales son de tipo piroclástico, y presentan principalmente escorias y bombas (GEODE, 2014), su resistencia es equiparable a resistencia de un suelo de alta compacidad/consistencia. A tenor de la descripción geotécnica de la clase de emplazamiento 4 como suelos duros (Tabla 3.4), la unidad 8 se clasifica en esta clase de emplazamiento. En cuanto a la unidad 9, se vuelve a aplicar este criterio de clasificación, pues la descripción litológica de la unidad presenta piroclastos basálticos (Tabla A.5). Por tanto, la unidad 9 también se clasifica en la clase de emplazamiento 4. La unidad 10 consta de coladas basálticas, por lo que se adopta el criterio de clasificación ya empleado en el caso de la unidad 1, en el sentido de agrupar las unidades con rocas volcánicas (basaltos en la unidad 10) en la clase de emplazamiento 1 (Tabla 3.4). 
Edificio volcánico Taburiente (Taburiente superior) La unidad 11 se describe como piroclastos basálticos (Tabla A.5). En función de la naturaleza piroclástica de esta unidad, se aplica el criterio de clasificación de las unidades con depósitos piroclásticos en la clase de emplazamiento 4 (Tabla 3.4). La unidad 12 es descrita como depósitos freatomagmáticos por la Tabla A.5. GEODE (2014) ofrece una descripción litológica extensa para esta unidad, en la cual refiere la presencia de tobas coronadas por ignimbritas fuertemente cementadas. Las ignimbritas son rocas piroclásticas con matriz, las cuales son equiparables a rocas blandas a firmes, atendiendo también a la fuerte cementación que presentan en esta unidad. No obstante, la presencia de las tobas a muro de estas ignimbritas, que constituyen materiales piroclásticos, plantea la conveniencia de aplicar un criterio conservador y considerar que el conjunto de la unidad 12 tiene una resistencia menor y equivalente a la de un suelo duro. De acuerdo con la descripción geotécnica de la clase de emplazamiento 4 (Tabla 3.4), la unidad 12 se encuadra, por tanto, en esta clase. La unidad 13, por su parte, consiste en coladas basálticas (Tabla A.5), por lo cual se adopta el criterio de clasificación de las unidades con rocas volcánicas en la clase de emplazamiento 1 (Tabla 3.4).

Como se observa en la Tabla A.5, la unidad 14 se compone de gabros, gabros olivínicos y gabros alcalinos. Dado que los gabros son rocas plutónicas, se sigue el criterio utilizado en el caso de la unidad 5, considerando que las unidades con rocas plutónicas son clasificables en la clase de emplazamiento 1 (Tabla 3.4). La unidad 15 se describe como lavas terminales diferenciadas (tefritas y fonolitas). A partir de la presencia de estas rocas volcánicas, se encuadra también la unidad 15 en la clase de emplazamiento 1.

Edificio volcánico Bejenado (Edificio principal) La unidad 16 consiste en aglomerados y depósitos de deslizamiento (Tabla A.5). Dado que los aglomerados consisten en material piroclástico, consolidado o no, se considera que la resistencia global de la unidad es equivalente al de un suelo duro, por lo cual la unidad se clasifica en la clase de emplazamiento 4 (Tabla 3.4). La unidad 17 es descrita como piroclastos basálticos, por lo cual se adopta el criterio de clasificación de las unidades con depósitos piroclásticos en la clase de emplazamiento 4 (Tabla 3.4). Con respecto a la unidad 18, ésta configura depósitos freatomagmáticos (Tabla A.5). Puesto que esta descripción litológica es idéntica a la de la unidad 12, se aplica el mismo criterio de clasificación en la clase de emplazamiento 4, considerando que la resistencia de este tipo de depósitos es equivalente a la de un suelo duro. La unidad 19 se compone de coladas basálticas, en las cuales las rocas volcánicas de clase basalto se encuentran fuertemente fracturadas (GEODE, 2014). A partir de esta descripción, se considera que la resistencia de la unidad es media, y por tanto se clasifica en la clase de emplazamiento 2 (Tabla 3.4).

Edificio volcánico Bejenado (Centros laterales y periféricos) La unidad 20 se describe como piroclastos basálticos con intercalaciones de lavas, aglomerados y sedimentos (Tabla A.5). La unidad conforma un depósito piroclástico que, al contener también los aglomerados y sedimentos, puede equipararse a un suelo de alta consistencia. Por tanto, se clasifica en la clase de emplazamiento 4 (Tabla 3.4). La 
unidad 21 consta de coladas basálticas, por lo cual se adopta el criterio de clasificación de las unidades con rocas volcánicas en la clase de emplazamiento 1.

Edificio volcánico Bejenado (Centros laterales y terminales diferenciados) La unidad 22 es descrita como conos de piroclastos basálticos, basaníticos y tefríticos (Tabla A.5). Debido a que esta unidad constituye depósitos piroclásticos, se clasifica en la clase de emplazamiento 4 (Tabla 3.4) como suelos duros. La unidad 23 consiste en coladas basálticas, tefríticas y fonolíticas. Por tanto, se aplica el criterio de clasificación de la unidades con rocas volcánicas (basaltos, tefritas y fonolitas) en la clase de emplazamiento 1.

Edificio volcánico Cumbre Vieja (Otras erupciones formando plataforma, erupciones formando acantilado costero) La unidad 24 se compone de conos de piroclastos basálticos (Tabla A.5), por lo cual se adopta el criterio de clasificación de las unidades con depósitos piroclásticos en la clase de emplazamiento 4 (Tabla 3.4). La unidad 25 consta de conos y depósitos freatomagmáticos, los cuales están formados por materiales hialoclastíticos (GEODE, 2014). En función del fino tamaño de grano de las hialoclastitas (lapilli, ceniza) se considera que su resistencia es equivalente a la de la clase de emplazamiento 4 (suelos duros), en vez de a la resistencia de la clase de emplazamiento 3 (rocas blandas a firmes), como los materiales brechoides. Por tanto, la unidad 25 se clasifica en la clase de emplazamiento 4. La unidad 26 consiste en domos y coladas de tefritas y fonolitas, por tanto se sigue el criterio de clasificar las unidades con rocas volcánicas (tefritas, fonolitas) en la clase de emplazamiento 1 (Tabla 3.4). Este mismo criterio se utiliza para clasificar la unidad 27 en la clase 1, pues ésta se describe como coladas basálticas (Tabla A.5).

Edificio volcánico Cumbre Vieja (Otras erupciones formando plataforma, grupo volcánico Mña. Cabrera-Mña. Faro) Según la Tabla A.5, la unidad 28 consta de conos de piroclastos basálticos. Puesto que la unidad constituye un depósito piroclástico, se adopta el criterio de clasificación de este tipo de depósitos en la clase de emplazamiento 4 (Tabla 3.4). La unidad 29 se compone de coladas de basanitas, tefritas y fonolitas, por tanto se considera que las rocas volcánicas presentes (basanitas, tefritas y fonolitas) tienen la misma resistencia que las rocas duras y se clasifican en la clase de emplazamiento 1 (Tabla 3.4).

Edificio volcánico Cumbre Vieja (Otras erupciones formando plataforma, erupciones de plataforma indiferenciadas) La unidad 30 es descrita por la Tabla A.5 como conos de piroclastos basálticos. En consecuencia, esta unidad se clasifica en la clase de emplazamiento 4 (Tabla 3.4). La unidad 31 se describe como conos y depósitos freatomagmáticos. Procediendo de la misma forma que en el caso de la unidad 18, se considera que la presencia de depósitos freatomagmáticos es indicativa de una resistencia equiparable a la de un suelo duro. Por tanto, la unidad 31 es encuadrada en la clase de emplazamiento 4. Con respecto a la unidad 32, ésta consta de coladas basálticas, por lo cual se utiliza el criterio de clasificación de las unidades con rocas volcánicas en la clase de emplazamiento 1 (Tabla 3.4). 
Edificio volcánico Cumbre Vieja (Erupciones formando plataforma costera, erupciones recientes-grupo Birigoyo-La Barquita) La unidad 38 presenta conos de piroclastos basálticos (Tabla A.5). En consecuencia, se adopta el criterio de clasificación de las unidades con depósitos piroclásticos en la clase de emplazamiento 4 (Tabla 3.4). La unidad 39 consta de coladas basálticas, para las cuales GEODE (2014) aporta una descripción litológica en términos de tefritas y tefritas fonolíticas. Debido a la presencia de estas rocas volcánicas, la unidad 39 se clasifica en la clase de emplazamiento 1 (Tabla 3.4).

Edificio volcánico Cumbre Vieja (Erupciones formando plataforma costera, erupciones recientes-volcán San Antonio-La Caldereta) La unidad 40 es descrita como conos de piroclastos basálticos (Tabla A.5). Por tanto, se utiliza el criterio de clasificación de las unidades con depósitos piroclásticos en la clase de emplazamiento 4 (Tabla 3.4). La unidad 41 configura depósitos freatomagmáticos (Tabla A.5). En este caso, se considera que la resistencia de este tipo de depósitos es equiparable a la de un suelo duro, procediendo con el mismo criterio que el observado para clasificar las unidades 18 y 31. Consecuentemente, la unidad 41 es agrupada dentro de la clase de emplazamiento 4. En relación a la unidad 42, ésta consiste en coladas basálticas (Tabla A.5). Por tanto, se adopta el criterio de clasificación de las unidades con rocas volcánicas (basaltos en la unidad 42) en la clase de emplazamiento 1 (Tabla 3.4).

Edificio volcánico Cumbre Vieja (Erupciones formando plataforma costera, erupciones recientes-volcán Fuego y volcanes de La Fajana) La unidad 43 se describe como conos de piroclastos basálticos, basaníticos y tefríticos (Tabla A.5). En función del carácter piroclástico de la unidad, ésta es clasificada en la clase de emplazamiento 4 (Tabla 3.4). La unidad 44 constituye coladas basálticas, por tanto se aplica el criterio de clasificación de las unidades con rocas volcánicas (basaltos en esta unidad) en la clase de emplazamiento 1 (Tabla 3.4).

Edificio volcánico Cumbre Vieja (Erupciones formando plataforma costera, erupciones prehistóricas-Malforada y Nanbroque) La unidad 45 consiste en conos de piroclastos basálticos, basaníticos y tefríticos (Tabla A.5). Por tanto, se utiliza el criterio de clasificación de las unidades con depósitos piroclásticos en la clase de emplazamiento 4 (Tabla 3.4). La unidad 46 es descrita como intrusiones de fonolitas juveniles, por lo cual se considera que las rocas volcánicas presentes (fonolitas) presentan una resistencia equiparable a la de las rocas duras, procediendo a clasificar esta unidad en la clase de emplazamiento 1. Con este mismo criterio, se encuadra la unidad 47 en la clase 1, pues GEODE (2014) indica que esta unidad presenta lavas que varían de tefritas fonolíticas a fonolitas.

Edificio volcánico Cumbre Vieja (Erupciones formando plataforma costera, erupciones prehistóricas-Volcán Martín y Montaña Quemada) La unidad 48 se describe como conos de piroclastos basálticos (Tabla A.5). Dado que la unidad constituye un depósito piroclástico, se adopta el criterio de clasificación de las unidades con este tipo de depósitos en la clase de emplazamiento 4 (Tabla 3.4). 
La unidad 49 consta de depósitos freatomagmáticos (Tabla A.5), por lo cual se sigue el criterio empleado en la clasificación de las unidades 18, 31 y 41, en el sentido de encuadrar este tipo de depósitos en la clase de emplazamiento 4. La unidad 50 presenta coladas basálticas, por tanto se clasifica en la clase de emplazamiento 1 (Tabla 3.4), al ser los basaltos rocas volcánicas.

Edificio volcánico Cumbre Vieja (Erupciones formando plataforma costera, erupciones históricas-Erupciones del siglo XVI) La descripción litológica de la unidad 51 consiste en conos de piroclastos basálticos (Tabla A.5), por lo cual se clasifica en la clase de emplazamiento 4 (Tabla 3.4), siguiendo el criterio ya establecido. La unidad 52 se describe como coladas basálticas, por tanto se utiliza el criterio de clasificación de las unidades con rocas volcánicas (basaltos) en la clase de emplazamiento 1 (Tabla 3.4). Siguiendo este criterio, la unidad 53 también se clasifica en la clase 1, pues se compone de fonolitas juveniles intrusivas, de acuerdo con la Tabla A.5, siendo también las fonolitas rocas volcánicas.

Edificio volcánico Cumbre Vieja (Erupciones formando plataforma costera, erupciones históricas-Erupciones del siglo XVII) De acuerdo con la Tabla A.5, la unidad 54 se describe como conos de piroclastos basálticos, por tanto se clasifica en la clase de emplazamiento 4 (Tabla 3.4). La unidad 55 es descrita como coladas basálticas, por lo que se aplica el criterio de clasificación de las unidades con rocas volcánicas (basaltos) en la clase de emplazamiento 1.

Edificio volcánico Cumbre Vieja (Erupciones formando plataforma costera, erupciones históricas-Erupciones del siglo XVIII) La unidad 56 está formada por conos de piroclastos basálticos (Tabla A.5). Siguiendo el criterio establecido, esta unidad se clasifica en la clase de emplazamiento 4 (Tabla 3.4). La unidad 57 se describe como depósitos freatomagmáticos, para los cuales se aplica el criterio ya empleado, de clasificación como suelos duros en la clase de emplazamiento 4 . La unidad 58 consiste en coladas basálticas que, al presentar rocas volcánicas (basaltos), permiten la clasificación de la unidad en la clase de emplazamiento 1 (Tabla 3.4).

Edificio volcánico Cumbre Vieja (Erupciones formando plataforma costera, erupciones históricas-Erupciones del siglo XX) La unidad 59 es descrita como conos de piroclastos basálticos (Tabla A.5), por lo tanto se clasifica en la clase de emplazamiento 4 (Tabla 3.4). La unidad 60 configura depósitos freatomagmáticos, por lo que se adopta el criterio de clasificación de este tipo de depósitos en la clase de emplazamiento 4 . Con respecto a la unidad 61, ésta presenta coladas basálticas (Tabla A.5). En función de la presencia de rocas volcánicas (basaltos), esta unidad se clasifica en la clase de emplazamiento 1 (Tabla 3.4).

Sedimentos pleistocenos La unidad 33 constituye la formación sedimentaria de La Mata, con depósitos de ladera y lahares (Tabla A.5). Esta unidad, de acuerdo con GEODE (2014), presenta clastos de tamaño bloque en matriz arcillosa, siendo de origen aluvial o bien asimilable a un depósito de "debris-flow" (colada clástica). A 
partir del tamaño particularmente alto de los clastos (bloque), puede inferirse que la compacidad del depósito es también alta, y por tanto su resistencia es equivalente a la de un suelo de alta compacidad/consistencia. En base a la descripción geotécnica de la clase de emplazamiento 4 como suelos duros (Tabla 3.4), es decir, de alta consistencia, se considera que la resistencia de la unidad 33 es equiparable a la resistencia de esta clase de emplazamiento. Por tanto, la unidad 33 se clasifica en la clase de emplazamiento 4. De acuerdo con la Tabla A.5, la unidad 34 está compuesta por materiales epiclásticos de "fan-delta" marino. La descripción litológica extensa proporcionada por GEODE (2014) para esta unidad indica que la misma presenta conglomerados y gravas. El alto tamaño de grano (grava) de los clastos permite considerar que la resistencia global de la unidad también es equivalente a la de un suelo de alta compacidad/consistencia. Por tanto, y en función de la descripción geotécnica de la clase de emplazamiento 4 (Tabla 3.4), la unidad 34 se clasifica en esta clase. En el caso de la unidad 35, puede aplicarse el mismo criterio de clasificación que el utilizado para las unidades 33 y 34 . Esta unidad es descrita como materiales epiclásticos de "fan-delta" lacustre (Tabla A.5), que presentan tamaños gruesos y arcillas (GEODE, 2014). El tamaño de grano grueso de los sedimentos es razón, de nuevo, para inferir una resistencia acorde con la de los suelos duros, y por tanto para clasificar la unidad 35 en la clase de emplazamiento 4.

La unidad 36 conforma coluviones y depósitos de ladera (Tabla A.5). Según GEODE (2014), los coluviones de esta unidad están constituidos por los restos de una plataforma costera que ha quedado a resguardo de la erosión marina por depósitos de piedemonte más recientes. Entre estos coluviones, los más antiguos se encuentran muy compactados. Bajo estas premisas, se considera que la compacidad/consistencia global de la unidad es alta. A partir de la descripción geotécnica de la clase de emplazamiento 4 (Tabla 3.4) como suelos duros (de alta consistencia), se infiere que la resistencia de la unidad 36 es equivalente a la de esta clase. Por tanto, la unidad 36 se encuadra en la clase de emplazamiento 4. La unidad 37 contiene suelos de alteración de lapilli (Tabla A.5). En función del fino tamaño de grano del piroclasto presente (lapilli), es razonable inferir que la compacidad/consistencia global de la unidad es menor que la que se ha atribuido a los depósitos piroclásticos en sentido general. Por tanto, se prefiere suponer una compacidad/consistencia media a una alta, y por tanto se considera conveniente clasificar la unidad 37 en la clase de emplazamiento 5, en base a su descripción geotécnica como suelos de consistencia media (Tabla 3.4).

Sedimentos holocenos La unidad 62 se describe como terrazas aluviales recientes (Tabla A.5). De acuerdo con la descripción litológica extensa de GEODE (2014), los depósitos aluviales referidos presentan arenas gruesas y bloques. En función de la granulometría gruesa observada (arenas gruesas, bloques) en los sedimentos existentes, se infiere que la resistencia de la unidad es equiparable a la de un suelo de alta compacidad/consistencia. Por tanto, la unidad 62 se clasifica en la clase de emplazamiento 4 (Tabla 3.4), pues ésta se describe geotécnicamente como suelos duros, es decir, de alta consistencia. La unidad 63 corresponde a depósitos aluviales de relleno de barrancos que, de acuerdo con GEODE (2014), también contienen arenas gruesas y bloques. Por tanto, se aplica el criterio empleado para clasificar la unidad 62 , considerando que el grueso tamaño de grano observado es indicativo de la perte- 
nencia de la unidad 63 a la clase de emplazamiento 4. La unidad 64 es descrita como coluviones y depósitos de ladera (Tabla A.5), que presentan la típica estructura en capas de diferente granulometría (GEODE, 2014). A partir de esta descripción, se considera que la compacidad/consistencia global de la unidad es media-alta, por lo cual se infiere que su resistencia es equivalente a la de los suelos duros que caracterizan la clase de emplazamiento 4 (Tabla 3.4). Por tanto, la unidad 64 se clasifica en la clase 4. La unidad 65 se presenta como avalanchas y desplomes ("rock falls"), de acuerdo con la Tabla A.5. La descripción litológica extensa de GEODE (2014) precisa que los fragmentos que constituyen los depósitos de avalanchas y desplomes de esta unidad se disponen caóticamente. Se infiere una compacidad/consistencia media-alta para el conjunto de estos depósitos, equiparándose por tanto la resistencia de la unidad 65 a la de la clase de emplazamiento 4, por razón de su descripción geotécnica (Tabla 3.4).

La unidad 66 consiste en depósitos de playa cementados ("beach rocks"), como se indica en la Tabla A.5. A partir de la cementación presente en estos depósitos de playa, se entiende que los sedimentos pueden tener una consistencia media. Por otra parte, no se dispone de una descripción litológica extensa que permita conocer la granulometría del sedimento. Por estas dos razones, es razonable suponer que la consistencia global de la unidad es media. En función de la descripción geotécnica de la clase de emplazamiento 5 como suelos de consistencia media (Tabla 3.4), se equiparan la resistencia de la unidad 66 y la de la clase de emplazamiento 5, para agrupar la citada unidad en esta clase. En cuanto a la unidad 67, ésta forma playas de arenas y cantos. En este sentido, se infiere que la compacidad/consistencia de los depósitos de playas es baja, independientemente de la granulometría del sedimento. A tenor de la descripción geotécnica de la clase de emplazamiento 6 (Tabla 3.4) como suelos blandos (de baja consistencia), la unidad 67 es clasificada en esta clase. Por último, la unidad 68 se compone de rellenos antrópicos (Tabla A.5). En este caso, se considera también la compacidad/consistencia de este tipo de depósitos como baja, y se clasifica la unidad 68 en la clase de emplazamiento 6.

En la Tabla 3.34 se presenta un resumen de la clasificación de las unidades geológicas de La Palma, de acuerdo con el procedimiento que se ha explicado.

Tabla 3.34: Resumen de la clasificación de las unidades del mapa geológico continuo GEODE a escala 1:50.000 de La Palma (Zona 2915), de acuerdo a la clasificación de síntesis. Tomado de GEODE (2014), elaboración propia.

\begin{tabular}{|c|c|c|}
\hline $\begin{array}{l}\text { Sistema/Dominio/Edificio/Ciclo volcánico/Macizo } \\
\text { plutónico/Formación/Miembro }\end{array}$ & $\begin{array}{l}\text { Código de la } \\
\text { unidad } \\
\text { geológica }\end{array}$ & $\begin{array}{l}\text { Clase de em- } \\
\text { plazamiento }\end{array}$ \\
\hline \multirow{2}{*}{ Rocas filonianas } & 1 & 1 \\
\hline & 2 & 2 \\
\hline \multirow{3}{*}{ Edificio submarino (seamount) } & 3 & 3 \\
\hline & 4 & 2 \\
\hline & 5 & 1 \\
\hline \multirow[b]{2}{*}{ Edificio volcánico Garafía } & 6 & 4 \\
\hline & 7 & 1 \\
\hline \multirow{8}{*}{$\begin{array}{l}\text { Edificio } \\
\text { volcánico } \\
\text { Taburiente }\end{array}$} & 8 & 4 \\
\hline & 9 & 4 \\
\hline & 10 & 1 \\
\hline & 11 & 4 \\
\hline & 12 & 4 \\
\hline & 13 & 1 \\
\hline & 14 & 1 \\
\hline & 15 & 1 \\
\hline
\end{tabular}




\begin{tabular}{|c|c|c|c|c|c|}
\hline \multicolumn{4}{|c|}{$\begin{array}{l}\text { Sistema/Dominio/Edificio/Ciclo volcánico/Macizo } \\
\text { plutónico/Formación/Miembro }\end{array}$} & \multirow{2}{*}{$\begin{array}{l}\text { Código de la } \\
\text { unidad } \\
\text { geológica }\end{array}$} & \multirow{2}{*}{$\begin{array}{l}\text { Clase de em- } \\
\text { plazamiento }\end{array}$} \\
\hline \multirow{8}{*}{$\begin{array}{l}\text { Edificio } \\
\text { volcánico } \\
\text { Bejenado }\end{array}$} & \multirow{4}{*}{\multicolumn{3}{|c|}{ Edificio principal }} & & \\
\hline & & & & 17 & 4 \\
\hline & & & & 18 & 4 \\
\hline & & & & 19 & 2 \\
\hline & \multirow{2}{*}{\multicolumn{3}{|c|}{ Centros laterales y periféricos }} & 20 & 4 \\
\hline & & & & 21 & 1 \\
\hline & \multirow{2}{*}{\multicolumn{3}{|c|}{ Centros laterales y terminales diferenciados }} & 22 & 4 \\
\hline & & & & 23 & 1 \\
\hline \multirow{33}{*}{$\begin{array}{l}\text { Edificio } \\
\text { volcánico } \\
\text { Cumbre Vi }\end{array}$} & \multirow{9}{*}{$\begin{array}{l}\text { Otras } \\
\text { erupciones } \\
\text { formando } \\
\text { plataforma }\end{array}$} & \multirow{4}{*}{\multicolumn{2}{|c|}{$\begin{array}{l}\text { Erupciones formando acantilado } \\
\text { costero }\end{array}$}} & 24 & 4 \\
\hline & & & & 25 & 4 \\
\hline & & & & 26 & 1 \\
\hline & & & & 27 & 1 \\
\hline & & \multirow{2}{*}{\multicolumn{2}{|c|}{$\begin{array}{l}\text { Grupo volcánico Mña. Cabrera-Mña. } \\
\text { Faro }\end{array}$}} & 28 & 4 \\
\hline & & & & 29 & 1 \\
\hline & & \multirow{3}{*}{\multicolumn{2}{|c|}{$\begin{array}{l}\text { Erupciones de plataforma } \\
\text { indiferenciadas }\end{array}$}} & 30 & 4 \\
\hline & & & & 31 & 4 \\
\hline & & & & 32 & 1 \\
\hline & \multirow{24}{*}{$\begin{array}{l}\text { Erupciones } \\
\text { formando } \\
\text { plataforma } \\
\text { costera }\end{array}$} & \multirow{7}{*}{$\begin{array}{l}\text { Erupciones } \\
\text { recientes }\end{array}$} & \multirow{2}{*}{$\begin{array}{l}\text { Grupo Birigoyo-La } \\
\text { Barquita }\end{array}$} & 38 & 4 \\
\hline & & & & 39 & 1 \\
\hline & & & \multirow{3}{*}{$\begin{array}{l}\text { Volcán San } \\
\text { Antonio-La Caldereta }\end{array}$} & 40 & 4 \\
\hline & & & & 41 & 4 \\
\hline & & & & 42 & 1 \\
\hline & & & \multirow{2}{*}{$\begin{array}{l}\text { Volcán Fuego y } \\
\text { volcanes de La Fajana }\end{array}$} & 43 & 4 \\
\hline & & & & 44 & 1 \\
\hline & & & \multirow{3}{*}{$\begin{array}{l}\text { Malforada y } \\
\text { Nanbroque }\end{array}$} & 45 & 4 \\
\hline & & & & 46 & 1 \\
\hline & & Erupciones & & 47 & 1 \\
\hline & & prehistóricas & \multirow{3}{*}{$\begin{array}{l}\text { Volcán Martín y } \\
\text { Montaña Quemada }\end{array}$} & 48 & 4 \\
\hline & & & & 49 & 4 \\
\hline & & & & 50 & 1 \\
\hline & & & & 51 & 4 \\
\hline & & & $\begin{array}{l}\text { Erupciones del siglo } \\
\text { XVI }\end{array}$ & 52 & 1 \\
\hline & & & & 53 & 1 \\
\hline & & & Erupciones del siglo & 54 & 4 \\
\hline & & & XVII & 55 & 1 \\
\hline & & Erupciones & Frunciones del siolo & 56 & 4 \\
\hline & & & $\begin{array}{l}\text { Erupciones del siglo } \\
\text { XVII }\end{array}$ & 57 & 4 \\
\hline & & & & 58 & 1 \\
\hline & & & & 59 & 4 \\
\hline & & & $\begin{array}{l}\text { Erupciones del siglo } \\
\text { XX }\end{array}$ & 60 & 4 \\
\hline & & & & 61 & 1 \\
\hline & & & & 33 & 4 \\
\hline & & & & 34 & 4 \\
\hline Sedimento & ocenos & & & 35 & 4 \\
\hline & & & & 36 & 4 \\
\hline & & & & 37 & 5 \\
\hline & & & & 62 & 4 \\
\hline & & & & 63 & 4 \\
\hline & & & & 64 & 4 \\
\hline Sedimentc & enos & & & 65 & 4 \\
\hline & & & & 66 & 5 \\
\hline & & & & 67 & 6 \\
\hline & & & & 68 & 6 \\
\hline
\end{tabular}

\section{Lanzarote (Región 2910)}

La región GEODE que corresponde a la isla de Lanzarote es la región 2910 (GEODE, 2014). La cartografía de esta región GEODE distingue 114 unidades geológicas en la isla, cuyas edades cronoestratigráficas están comprendidas entre el Mioceno Inferior y el Holoceno. En la Tabla A.6 se muestran las descripciones litológicas y edades cronoestratigráficas correspondientes a estas unidades. Las 114 unidades de 
esta región GEODE se agrupan en 22 grupos, de acuerdo con la leyenda cronoestratigráfica de la cartografía (Tabla 3.35). De este conjunto de unidades geológicas, 3 no aparecen cartografiadas y por tanto no se han clasificado: unidades 1, 2 y 92.

Tabla 3.35: División de las unidades del mapa geológico continuo GEODE a escala 1:50.000 de Lanzarote (Zona 2910), de acuerdo a la leyenda cronoestratigráfica. Tomado de GEODE (2014).

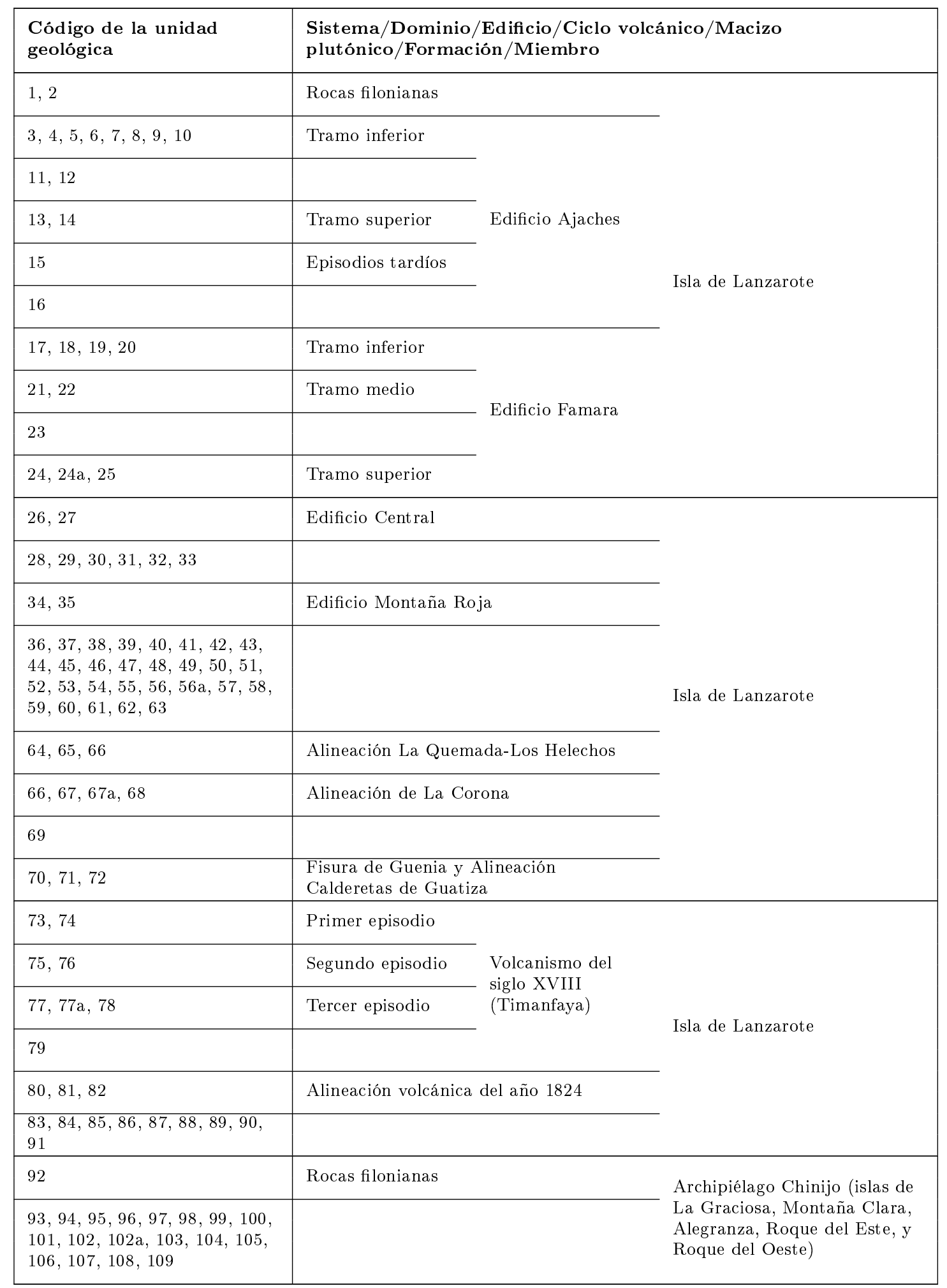


Isla de Lanzarote (Edificio Ajaches, tramo inferior) En la isla de Lanzarote, el Edificio Ajaches contiene las unidades comprendidas entre la unidad 3 y la unidad 16 (Tabla A.6). Las unidades contenidas en el tramo inferior del edificio, de acuerdo con la leyenda cronoestratigráfica de la cartografía GEODE, son las que tienen código 3, 4, 5, 6, 7, 8, 9 y 10 (Tabla 3.35).

En este contexto, la unidad 3 es descrita como coladas basálticas (Tabla A.6), si bien GEODE (2014) proporciona una descripción litológica extensa que precisa que los basaltos presentan fracturación (disyunción columnar). La resistencia de las rocas volcánicas se considera equiparable a la resistencia de las rocas duras, pero al presentarse fracturación, el criterio conservador recomienda disminuir esta resistencia. De acuerdo con la clasificación de síntesis (Tabla 3.4), la resistencia de la unidad 3 no podría corresponder a la resistencia de la clase de emplazamiento 1, descrita geotécnicamente como rocas duras, aunque sí sería razonable plantear esta correspondencia con la resistencia de la clase de emplazamiento 2, pues su descripción geotécnica consiste en rocas de resistencia media (Tabla 3.4). En consecuencia, la unidad 3 se clasifica en la clase 2 . La unidad 4 se compone de conos de tefra y piroclásticos basálticos (Tabla A.6). Esta unidad conforma, por tanto, depósitos piroclásticos, cuya compacidad/consistencia puede considerarse alta. La resistencia de la unidad se equipara a la resistencia de la clase de emplazamiento 4 (Tabla 3.4), pues su descripción geotécnica consiste en suelos duros y, por tanto, de alta consistencia. Según la Tabla A.6, la descripción litológica de la unidad 5 consta de gabros, que son rocas plutónicas, y por tanto con una resistencia equiparable a la de las rocas duras. Sin embargo, y de acuerdo con la descripción litológica extensa de GEODE (2014), estos gabros presentan fracturación (disyunción columnar), por lo cual su resistencia se considera menor que la de las rocas duras. En función de la descripción geotécnica de la clase de emplazamiento 2 como rocas de resistencia media (Tabla 3.4), se encuadra la unidad 5 en la clase 2. La unidad 6 se compone de brechas (Tabla A.6), que son rocas sedimentarias compuestas por clastos de tamaño equiparable al de las gravas. Al ser una roca sedimentaria, la resistencia de las brechas se considera menor que la resistencia de la clase de emplazamiento 2, y más acorde por tanto con la resistencia de la clase de emplazamiento 3, descrita geotécnicamente como rocas blandas a firmes (Tabla 3.4). Por tanto, la unidad 6 se agrupa en la clase 3 .

La unidad 7 consiste en tobas sálicas (Tabla A.6). Puesto que las tobas son depósitos piroclásticos, se considera que tienen una consistencia alta, y por tanto una resistencia equivalente a la resistencia de los suelos duros que describen la clase de emplazamiento 4 (Tabla 3.4). Por tanto, se adopta el criterio de clasificación empleado en el caso de la unidad 4, y se encuadra la unidad 7 en la clase de emplazamiento 4. La unidad 8 es descrita como intrusivos traquíticos (Tabla A.6). Al contener rocas volcánicas (traquitas), puede considerarse que la resistencia de esta unidad es equivalente a la de la clase de emplazamiento 1, a partir de su descripción geotécnica como rocas duras (Tabla 3.4). La unidad 9 consta de intrusiones mugearíticas-benmoreíticas, por lo cual presenta rocas volcánicas de composición traquítica (GEODE, 2014). En este caso, se adopta el mismo criterio que el utilizado en el caso de la unidad 8, en el sentido de clasificar las unidades con rocas volcánicas en la clase de emplazamiento 1 . Con respecto a la unidad 10, ésta consta de coladas mugearíticas-benmoreíticas, presentando las mismas rocas volcánicas de composición traquítica que la unidad 9. Por tanto, la unidad 10 se clasifica también 
en la clase de emplazamiento 1.

Isla de Lanzarote (Edificio Ajaches, tramo superior) La unidad 13 se describe como coladas basálticas (Tabla A.6), las cuales presentan fracturación (disyunción columnar) de acuerdo con la descripción litológica extensa de GEODE (2014). En función de la presencia de roca volcánica fracturada, se aplica el mismo criterio de clasificación que el utilizado para la unidad 3, en el sentido de considerar la resistencia de esta litología como media y equiparable a la resistencia de la clase de emplazamiento 2, a tenor de su descripción geotécnica (Tabla 3.4). Por tanto, la unidad 13 se encuadra en la clase 2. La unidad 14, por su parte, consiste en conos de tefra y piroclastos basálticos y de dispersión (Tabla A.6). En este caso, se adopta el criterio de clasificación de los depósitos piroclásticos en la clase de emplazamiento 4, en función de su descripción geotécnica como suelos de consistencia alta o duros (Tabla 3.4).

Isla de Lanzarote (Edificio Ajaches, episodios tardíos) La unidad 15 consiste en coladas basálticas (Tabla A.6). La descripción litológica extensa de GEODE (2014) indica que los basaltos de esta unidad tienen disyunción columnar y por tanto fracturación. Por este motivo, se vuelve a aplicar el criterio ya empleado en el caso de las unidades 3 y 13, y se clasifica la unidad 15 como rocas de resistencia media y pertenecientes a la clase de emplazamiento 2 (Tabla 3.4).

Isla de Lanzarote (Edificio Ajaches) En este apartado se describe el proceso de clasificación de las unidades restantes del Edificio Ajaches, para las cuales la leyenda cronoestratigráfica de la cartografía GEODE no establece su pertenencia a ningún subgrupo del mencionado edificio (Tabla 3.35). Los códigos de estas unidades son 11,12 y 16 .

La unidad 11 contiene arenas y conglomerados, de acuerdo con la Tabla A.6. En función de las granulometrías presentes, con tamaño igual o superior al de las arenas, se infiere que la resistencia de la unidad es equiparable a la de un suelo de alta compacidad/consistencia. Por tanto, la unidad 11 se clasifica en la clase de emplazamiento 4 (Tabla 3.4), pues ésta se describe geotécnicamente como suelos duros, es decir, de alta consistencia. La unidad 16 tiene una descripción litológica similar a la de la unidad 11 (Tabla A.6), por lo cual puede aplicarse el mismo criterio, y considerar que las unidades con depósitos detríticos con sedimentos de tamaño arena o superior pertenecen a la clase de emplazamiento 4 . Con respecto a la unidad 12, ésta se describe como intrusivos basálticos y básicos en general, si bien la descripción litológica extensa de GEODE (2014) precisa que las rocas volcánicas presentes tienen fracturación (disyunción columnar). Por tanto, se adopta el criterio de clasificación de las unidades con rocas volcánicas fracturadas en la clase de emplazamiento 2 (Tabla 3.4).

Isla de Lanzarote (Edificio Famara, tramo inferior) En el Edificio Famara afloran las unidades comprendidas entre la unidad 17 y la unidad 25 (Tabla A.6). Las unidades contenidas en el tramo inferior del edificio, de acuerdo con la leyenda 
cronoestratigráfica de la cartografía GEODE, son las que tienen código 17, 18, 19 y 20 (Tabla 3.35).

La unidad 17 se compone de coladas basálticas y basálticas olivínicas (Tabla A.6). De acuerdo con la descripción litológica extensa de GEODE (2014), los basaltos de esta unidad se presentan con fracturación (disyunción columnar y lajeado). De nuevo, se emplea el criterio de clasificación de las rocas volcánicas fracturadas como rocas de resistencia media y clasificables en la clase de emplazamiento 2 (Tabla 3.4). La unidad 18 consta de piroclastos basálticos y conos de tefra enterrados. Se adopta, por tanto, el criterio de clasificación de las unidades con depósitos piroclásticos en la clase de emplazamiento 4 (Tabla 3.4). La unidad 19 se describe como brechas líticas basálticas (Tabla A.6), presentando una matriz fina y muy consolidada (GEODE, 2014). En función de esta descripción, se equipara la resistencia de la unidad 19 a la resistencia propia de una roca blanda a firme, como en el caso de la unidad 6. Esta correspondencia es coherente también con la existencia de la matriz fina y consolidada, que contribuye a la compacidad general de las brechas, y por tanto a su resistencia. A partir de la descripción geotécnica de la clase de emplazamiento 3 como rocas blandas a firmes (Tabla 3.4), la unidad 19 es clasificada en esta clase. Con respecto a la unidad 20, ésta se describe como arenas eólicas que, según GEODE (2014), se encuentran cementadas. La granulometría del sedimento (arenas) y la existencia de cemento permiten suponer que la compacidad/consistencia general de la unidad es alta, y que por tanto su resistencia puede equipararse a la de los suelos duros característicos de la clase de emplazamiento 4 (Tabla 3.4).

Isla de Lanzarote (Edificio Famara, tramo medio) La unidad 21 se describe como coladas basálticas y basálticas olivínicas (Tabla A.6). De acuerdo con GEODE (2014), las rocas volcánicas de esta unidad (basaltos) presentan fracturación (disyunción columnar). En consecuencia, la unidad 21 se clasifica en la clase de emplazamiento 2 (Tabla 3.4). La unidad 22 es descrita como piroclastos basálticos, piroclastos basálticos de dispersión y conos de tefra enterrados (Tabla A.6). Teniendo en cuenta esta litología, se aplica el criterio de clasificación de las unidades con depósitos piroclásticos en la clase de emplazamiento 4 (Tabla 3.4).

Isla de Lanzarote (Edificio Famara, tramo superior) De acuerdo con la Tabla A.6, la unidad 24 está constituida por coladas de tipo basáltico, que presentan fracturación (disyunción columnar) (GEODE, 2014). Por tanto, se aplica el criterio de clasificación de las unidades con rocas volcánicas fracturadas en la clase de emplazamiento 2 (Tabla 3.4). La unidad 24a conforma recubrimientos de lapillis sobre coladas basálticas (Tabla A.6). En función de la granulometría fina del piroclasto (lapilli), la compacidad/consistencia de esta unidad no se infiere como alta sino como media. Consecuentemente, su resistencia se equipara con la resistencia de la clase de emplazamiento 5, pues su descripción geotécnica consiste en suelos de consistencia media (Tabla 3.4). Por tanto, la unidad 24a se clasifica en la clase 5. En cuanto a la unidad 25, ésta se compone de piroclastos basálticos y conos de tefra, con piroclastos de clase bomba, escoria y lapilli (GEODE, 2014), por lo cual se aplica el criterio general de clasificación de las unidades con depósitos piroclásticos en la clase de emplazamiento 4 (Tabla 3.4). 
Isla de Lanzarote (Edificio Famara) En este apartado se describe el proceso de clasificación de la unidad 23, la cual no pertenece a ningún subgrupo del Edificio Famara (Tabla 3.35). Esta unidad consiste en intrusivos basálticos y básicos, en general (Tabla A.6). Las rocas volcánicas de clase basalto afloran con fracturación (disyunción columnar), según la descripción litológica extensa de GEODE (2014). Por este motivo, se sigue el criterio de clasificación de las unidades con rocas volcánicas fracturadas en la clase de emplazamiento 2 (Tabla 3.4), y se encuadra la unidad 23 en esta clase.

Isla de Lanzarote (Edificio Central) En el Edificio Central afloran las unidades 26 y 27 (Tabla A.6). La unidad 26 conforma coladas basálticas, por tanto se considera que la resistencia de las rocas volcánicas presentes (basaltos) es equivalente a la de las rocas duras. En consecuencia, esta unidad se clasifica en la clase de emplazamiento 1, a tenor de su descripción geotécnica (Tabla 3.4). La unidad 27 consiste en conos de tefra, que al constituir depósitos piroclásticos permiten clasificar esta unidad en la clase de emplazamiento 4 (Tabla 3.4) .

Isla de Lanzarote (Edificio Montaña Roja) En el Edificio Montaña Roja, GEODE (2014) ha descrito las unidades 34 y 35, con las precisiones expresadas en la Tabla A.6. La unidad 34 se compone de coladas basálticas y basálticas olivínicas, por lo cual se adopta el criterio de clasificación de las rocas volcánicas (basaltos en la unidad 34) como rocas duras, y por tanto pertenecientes a la clase de emplazamiento 1 (Tabla 3.4). La unidad 35 consiste en conos de tefra, siendo posible adoptar el criterio de clasificación de las unidades con depósitos piroclásticos en la clase de emplazamiento 4 (Tabla 3.4).

Isla de Lanzarote (Alineación La Quemada-Los Helechos) La Alineación La Quemada-Los Helechos comprende las unidades 64, 65 y 66, cuyas descripciones litológicas pueden consultarse en la Tabla A.6. La unidad 64 es descrita como coladas de tipo basáltico con fracturación (disyunción columnar). Consecuentemente, se adopta el criterio de clasificación de las unidades con rocas volcánicas fracturadas en la clase de emplazamiento 2 (Tabla 3.4). La unidad 65 consta de conos de tefra y centros de emisión con lapillis, escorias y bombas. Debido a que la unidad constituye depósitos de naturaleza piroclástica, se clasifica en la clase de emplazamiento 4 (Tabla 3.4). En cuanto a la unidad 66, ésta presenta piroclastos de dispersión, a los cuales GEODE (2014) adjudica la clase lapilli. Por tanto, se recupera el criterio de clasificación aplicado en el caso de la unidad 24a, en el sentido de considerar los depósitos de lapilli como suelos de consistencia media. En consecuencia, y a tenor de la descripción geotécnica de la clase de emplazamiento 5 (Tabla 3.4), la unidad 66 es agrupada en esta clase.

Isla de Lanzarote (Alineación de La Corona) De acuerdo con la leyenda cronoestratigráfica de la cartografía de la región 2910 (Tabla 3.35), la Alineación de La Corona agrupa las unidades 66, 67 y 67 a y 68, cuyas descripciones litológicas pueden consultarse en la Tabla A.6. La clasificación de la unidad 66 ya ha sido justificada en el apartado dedicado a la Alineación La Quemada-Los Helechos, por lo que puede 
ser consultada en el mismo. Con respecto a la unidad 67, su descripción litológica consiste en coladas basálticas y basálticas olivínicas. Dado que la descripción litológica extensa de GEODE (2014) añade que los basaltos de esta unidad presentan fracturación (disyunción columnar), se adopta el criterio de clasificación de las unidades con rocas volcánicas fracturadas (basaltos en la unidad 67) en la clase de emplazamiento 2 (Tabla 3.4). La unidad 67a configura un recubrimiento de arenas sobre coladas (Tabla A.6). En función de la granulometría de las arenas, se entiende que el recubrimiento descrito forma un suelo de alta compacidad/consistencia, clasificable en la clase de emplazamiento 4 como suelos duros. Con respecto a la unidad 68, ésta es descrita como conos de tefra compuestos por lapillis (GEODE, 2014). En función de la fina granulometría del piroclasto, se aplica el criterio de clasificación de las unidades con depósitos de lapilli en la clase de emplazamiento 5 (Tabla 3.4).

\section{Isla de Lanzarote (Fisura de Guenia y Alineación Calderetas de Guatiza)}

La Fisura de Guenia y Alineación Calderetas de Guatiza agrupa las unidades 70, 71 y 72. De acuerdo con la Tabla A.6, la unidad 70 configura coladas basálticas y basálticas olivínicas, por lo cual se adopta el criterio de clasificación de las rocas volcánicas (basaltos en la unidad 70) en la clase de emplazamiento 1 (Tabla 3.4). La unidad 71 se describe como conos de tefra, y por tanto puede analizarse en función de estos depósitos piroclásticos. De acuerdo al criterio ya establecido para este tipo de depósitos, la resistencia global de la unidad 71 es coherente con la de la clase de emplazamiento 4 (Tabla 3.4). La unidad 72 es descrita como piroclastos de dispersión con lapillis (GEODE, 2014). Por este motivo, se clasifica como suelos de consistencia media en la clase de emplazamiento 5 (Tabla 3.4).

Isla de Lanzarote (Volcanismo del siglo XVIII (Timanfaya), primer episodio) El volcanismo del siglo XVIII engloba las unidades comprendidas entre la unidad 73 y la unidad 79 (Tabla A.6). Las unidades correspondientes al primer episodio de este volcanismo, de acuerdo con la leyenda cronoestratigráfica de la cartografía GEODE, son las que tienen código 73 y 74 .

La unidad 73 se describe como coladas basálticas y basálticas olivínicas, de acuerdo con la (Tabla A.6). Dado que la unidad está formada por rocas volcánicas (basaltos), se clasifica en la clase de emplazamiento 1 (Tabla 3.4). La unidad 74 es descrita como conos de tefra, por lo cual se aplica el criterio de clasificación de las unidades con depósitos piroclásticos en la clase de emplazamiento 4 (Tabla 3.4).

Isla de Lanzarote (Volcanismo del siglo XVIII (Timanfaya), segundo episodio) La unidad 75 se describe como coladas de tipo basáltico (Tabla A.6), por lo cual se adopta el criterio de clasificación de las unidades con rocas volcánicas (basaltos en la unidad 75) en la clase de emplazamiento 1 (Tabla 3.4). La unidad 76 configura conos de tefra (Tabla A.6). Puesto esta descripción litológica corresponde a la de un depósito piroclástico, la unidad 76 se clasifica en la clase de emplazamiento 4 (Tabla 3.4).

Isla de Lanzarote (Volcanismo del siglo XVIII (Timanfaya), tercer episodio) La unidad 77 consiste en coladas basálticas y basálticas olivínicas (Tabla A.6). 
Por tanto, se adopta el criterio de clasificación de las unidades con rocas volcánicas (basaltos) en la clase de emplazamiento 1 (Tabla 3.4). La unidad 77a corresponde a un recubrimiento de arenas sobre coladas. Atendiendo a la granulometría de las arenas, el recubrimiento descrito forma un suelo de alta compacidad/consistencia, y se clasifica en la clase de emplazamiento 4 como suelos duros. La unidad 78 constituye conos de tefra (Tabla A.6), por lo cual se aplica el criterio de clasificación de las unidades con depósitos piroclásticos en la clase de emplazamiento 4 (Tabla 3.4).

Isla de Lanzarote (Volcanismo del siglo XVIII (Timanfaya) En este apartado se incluye la justificación de la clasificación de la unidad 79, pues ésta no pertenece a ningún episodio del volcanismo del siglo XVIII (Tabla 3.35). De acuerdo con la Tabla A.6, esta unidad agrupa los piroclastos de dispersión de los tres episodios ya estudiados. Dado que la descripción litológica extensa que proporciona GEODE (2014) indica que los piroclastos son de clase lapilli, se adopta el criterio de clasificación de las unidades con depósitos de lapilli en la clase de emplazamiento 5 (Tabla 3.4).

Isla de Lanzarote (Alineación volcánica del año 1824) La Alineación volcánica del año 1824 agrupa las unidades 80, 81 y 82. Según la Tabla A.6, la unidad 80 se describe como coladas basálticas. Al estar compuesta por rocas volcánicas (basaltos), esta unidad se clasifica en la clase de emplazamiento 1 (Tabla 3.4). La unidad 81 conforma conos de tefra (Tabla A.6), que contienen lapillis y escasas bombas (GEODE, 2014). En función de la predominancia de los piroclastos finos (lapillis), se infiere que la resistencia global de la unidad 81 equivale a la de los suelos de consistencia media, y por tanto a la resistencia de la clase de emplazamiento 5 (Tabla 3.4). Con este mismo criterio, la unidad 82 también se engloba en la clase 5, pues se describe como piroclastos de dispersión con lapillis (GEODE, 2014).

Isla de Lanzarote En este apartado se describe el proceso de clasificación de las unidades restantes de la isla de Lanzarote, para las cuales la leyenda cronoestratigráfica de la cartografía GEODE no establece su pertenencia a ningún grupo (Tabla 3.35). Los códigos de estas unidades son 28, 29, 30, 31, 32, 33, 36, 37, 38, 39, 40, 41, 42, 43, 44, 45, 46, 47, 48, 49, 50, 51, 52, 53, 54, 55, 56, 56a, 57, 58, 59, 60, $61,62,63,69,83,84,85,86,87,88,89,90$ у 91 .

La unidad 28 se describe como coladas basálticas (Tabla A.6). Al presentar rocas volcánicas (basaltos), esta unidad se clasifica en la clase de emplazamiento 1 (Tabla 3.4). La unidad 29 presenta una rasa marina pliocena con areniscas y conglomerados (GEODE, 2014). Siendo la arenisca una roca sedimentaria detrítica con clastos de tamaño arena, su resistencia es equivalente a la de una roca blanda a firme. Por este motivo, es razonable equiparar la resistencia de la unidad 29 a la resistencia de la clase de emplazamiento 3, pues ésta se describe geotécnicamente como rocas blandas a firmes (Tabla 3.4). La unidad 30 constituye depósitos de glacis y glacis-cono con arenas, cantos y conglomerados. La granulometría de los sedimentos presentes es fundamentalmente gruesa, con un tamaño mínimo de clasto equivalente al de las arenas. En este contexto, se adopta el mismo criterio que el utilizado para clasificar las unidades 11 y 16, al inferir que la resistencia de un depósito detrítico 
con sedimentos de tamaño arena o superior es equiparable a la resistencia de un suelo de alta compacidad/consistencia. En función de la descripción geotécnica de la clase de emplazamiento 4 como suelos duros, o de alta consistencia (Tabla 3.4), la unidad 30 se clasifica en esta clase.

De acuerdo con la descripción litológica extensa de la unidad 31 (GEODE, 2014), ésta consiste en depósitos de laderas con bloques y gravas en matriz areno-arcillosa. A partir de la gruesa granulometría del sedimento presente (grava, bloque), superior a la de las arenas, es razonable inferir que la resistencia global de la unidad 31 es equivalente a la de un suelo duro. Esta inferencia también es coherente con la presencia de la matriz más fina, que contribuye a la consistencia de esta unidad. Consiguientemente, la unidad 31 se clasifica en la clase de emplazamiento 4 (Tabla 3.4). La unidad 32 es descrita como conglomerados y arenas (Tabla A.6), por tanto se considera que la presencia de las arenas permite equiparar la resistencia de la unidad a la de la clase de emplazamiento 4. Con respecto a la unidad 33, ésta consta de arenas eólicas pliocenas y plio-pleistocenas. En función de esta descripción, se aplica el mismo criterio que el empleado en el caso de la unidad 32, y también se clasifica la unidad 33 en la clase de emplazamiento 4.

La descripción litológica extensa de GEODE (2014) indica que la unidad 36 está compuesta por areniscas cementadas con conglomerados. En función de esta descripción, se aplica el criterio de clasificación de la unidad 29, considerando que las areniscas cementadas permiten inferir una resistencia global equivalente a la de las rocas blandas a firmes que describen geotécnicamente la clase de emplazamiento 3 (Tabla 3.4). Por tanto, la unidad 36 se clasifica en la clase 3. La unidad 37 corresponde a un intrusivo basáltico con fracturación (disyunción columnar), de acuerdo con GEODE (2014). En función de esta descripción, se adopta el criterio de clasificación de las unidades con rocas volcánicas fracturadas (basaltos) en la clase de emplazamiento 2 (Tabla 3.4). La unidad 38 se describe fundamentalmente como coladas basálticas (Tabla A.6), por tanto se clasifican en la clase de emplazamiento 1 (Tabla 3.4), siguiendo el criterio ya explicado para las unidades con rocas volcánicas. En cuanto a la unidad 39, ésta se describe como conos de tefra y piroclastos basálticos (lapillis, escorias y bombas). Consecuentemente, se aplica el criterio de clasificación de las unidades con depósitos piroclásticos en la clase de emplazamiento 4 (Tabla 3.4).

La unidad 40 se describe como piroclastos de dispersión de diversas alineaciones volcánicas (Tabla A.6). De acuerdo con la descripción litológica extensa de GEODE (2014), estos piroclastos son de clase lapilli. Por tanto, la resistencia de la unidad se considera equivalente a la resistencia de los suelos de consistencia media que caracterizan la clase de emplazamiento 5 (Tabla 3.4), y la unidad 40 es clasificada en esta clase. La unidad 41 es descrita como coladas basálticas y basálticas olivínicas (Tabla A.6). En función de la presencia de rocas volcánicas (basaltos), la unidad se clasifica en la clase de emplazamiento 1 (Tabla 3.4). Con respecto a la unidad 42, ésta configura conos de tefra de diversas alineaciones volcánicas. Dado que los depósitos de tefra son piroclásticos, se aplica el criterio de clasificación de las unidades con depósitos piroclásticos en la clase de emplazamiento 4 (Tabla 3.4). La unidad 43 presenta piroclastos de dispersión, consistentes en lapillis (GEODE, 2014), por lo cual se aplica el criterio de clasificación de las unidades con depósitos de lapilli en la clase de emplazamiento 5 (Tabla 3.4). 
La unidad 44 consiste en edificios hidromagmáticos y piroclastos freatomagmáticos (Tabla A.6). De acuerdo con la descripción litológica extensa de GEODE (2014), en esta unidad afloran tobas con fragmentos líticos centimétricos. En función de la naturaleza piroclástica de las tobas, se adopta el criterio de clasificación de las unidades con depósitos piroclásticos en la clase de emplazamiento 4 (Tabla 3.4). A partir de la descripción litológica extensa de la unidad 45 como materiales arcillosos (GEODE, 2014), y de la fina granulometría del sedimento (arcilla), se considera que la consistencia global de la unidad es media. Como la clase de emplazamiento 5 se describe geotécnicamente en términos de suelos de consistencia media (Tabla 3.4), la unidad 45 es clasificada en esta clase. La unidad 46 es descrita como depósitos aluviales con conglomerados y arenas. La granulometría más pequeña del sedimento corresponde al tamaño de las arenas, por lo que se considera que la resistencia de esta unidad equivale a la de los suelos de alta compacidad/consistencia. Por tanto, se clasifica la unidad 46 en la clase de emplazamiento 4 . La unidad 47 presenta coladas basálticas y basálticas olivínicas (Tabla A.6), por lo cual se sigue el criterio de clasificación de las unidades con rocas volcánicas (basaltos) en la clase de emplazamiento 1 (Tabla 3.4).

La unidad 48 consta de conos de tefra y centros de emisión con lapillis, escorias y bombas (Tabla A.6). A partir de la presencia de estos depósitos piroclásticos, la unidad 48 se clasifica en la clase de emplazamiento 4 (Tabla 3.4). La unidad 49 consiste en piroclastos y lapillis de dispersión, considerando pues que la predominancia de los lapillis, con granulometría fina, es indicativa de la pertenencia de esta unidad a la clase de emplazamiento 5 (Tabla 3.4). La unidad 50 se compone de piroclastos freatomagmáticos y conos y edificios hidromagmáticos, si bien la descripción litológica extensa de GEODE (2014) indica que la unidad presenta cineritas y tobas. La presencia de las cineritas, compuestas de cenizas y por tanto con una granulometría fina, recomienda considerar la consistencia global de la unidad como media. A partir de la descripción geotécnica de la clase de emplazamiento 5 como suelos de consistencia media (Tabla 3.4), se clasifica la unidad 50 en la clase 5. La unidad 51 se describe como conos piroclásticos mixtos (estrombolianos-hidromagmáticos), con tobas, brechas y lapillis (GEODE, 2014). La litología de esta unidad es fundamentalmente piroclástica (tobas, lapillis), por tanto se adopta el criterio de clasificación de las unidades con depósitos piroclásticos en la clase de emplazamiento 4 .

La unidad 52 se describe como depósitos de caliches y zonas de incipiente encalichamiento (Tabla A.6). Los depósitos de caliches consisten en materiales carbonatados, con un tamaño de grano pequeño. Puede inferirse, con esta información, que la consistencia del depósito es media. En coherencia con la descripción geotécnica de la clase de emplazamiento 5 como suelos de consistencia media (Tabla 3.4), la unidad 52 se clasifica en esta clase. La unidad 53 presenta depósitos piroclásticos y arenosos alterados. Aunque esta descripción litológica podría implicar la clasificación de la unidad en la clase de emplazamiento 4, se prefiere la clasificación en la clase de emplazamiento 5, ya que la descripción litológica extensa de la unidad (GEODE, 2014) precisa que los materiales presentes son areno-arcillosos. Al presentarse una granulometría de tamaño arcilla, y por tanto fina, se adopta un criterio conservador para considerar que la consistencia de la unidad no es alta (clase 4) sino media (clase 5). La unidad 54 conforma depósitos aluvio-coluviales y areno-arcillosos (Tabla A.6), si bien la descripción extensa de GEODE (2014) puntualiza que los materiales son realmente areno-arcillo-limosos. La unidad contiene sedimentos con una elevada fracción 
de finos, como las arcillas y los limos. Adoptando de nuevo un criterio conservador, esta predominancia de finos hace recomendable rebajar la consistencia de la unidad hasta un nivel bajo. Como la descripción geotécnica de la clase de emplazamiento 6 consiste en suelos blandos, de baja consistencia (Tabla 3.4), resulta razonable equiparar la resistencia de la unidad 54 con la de la clase 6 . La unidad 55 se compone de arenas eólicas y arenas sobre sustrato, que se encuentran sueltas y por tanto con una compacidad mínima (GEODE, 2014). Es por este motivo que la compacidad de la unidad se considera coherente con la descripción geotécnica de la clase de emplazamiento 6 (suelos blandos).

La unidad 56 se describe como coladas basálticas y basálticas olivínicas (Tabla A.6). Como la unidad contiene rocas volcánicas (basaltos), se clasifica en la clase de emplazamiento 1 (Tabla 3.4). La unidad 56a consiste en recubrimientos de arenas sobre coladas (Tabla A.6). En función de la granulometría de las arenas, se entiende que el recubrimiento descrito forma un suelo de alta compacidad/ consistencia, y por tanto se clasifica en la clase de emplazamiento 4. La unidad 57 consta de conos de tefra y centros de emisión con lapilli, escorias y bombas. En función de la presencia de estos depósitos piroclásticos, la unidad 57 se clasifica en la clase de emplazamiento 4 (Tabla 3.4). La unidad 58 es descrita como piroclastos de dispersión, que presentan lapillis de acuerdo con la descripción extensa de GEODE (2014). Por tanto, se aplica el criterio de clasificación de los depósitos de lapilli en la clase de emplazamiento 5 (Tabla 3.4). La unidad 59 configura depósitos piroclásticos mixtos (estrombolianos-hidromagmáticos), de acuerdo con la Tabla A.6. Estos depósitos piroclásticos, que presentan bombas, escorias y lapillis (GEODE, 2014), permiten la clasificación de la unidad 59 en la clase de emplazamiento 4.

La unidad 60 consiste en depósitos piroclásticos hidromagmáticos y conos de tobas (Tabla A.6). En función de la presencia de piroclastos finos de clase lapilli y cineritas, compuestas por cenizas (GEODE, 2014), la unidad se clasifica en la clase de emplazamiento 5 como suelos de consistencia media (Tabla 3.4). La unidad 61 contiene areniscas con conglomerados, según la descripción litológica extensa de GEODE (2014). Dado que las areniscas se consideran como rocas blandas a firmes, se considera que la resistencia global de la unidad es equiparable a la de la clase de emplazamiento 3, por razón de su descripción geotécnica como rocas blandas a firmes (Tabla 3.4). La unidad 62 corresponde a coladas basálticas, por lo cual se adopta el criterio de clasificación de las unidades con rocas volcánicas (basaltos en la unidad 62) en la clase de emplazamiento 1 (Tabla 3.4). La unidad 63 es descrita como conos de tefra (Tabla A.6). Debido a que los conos de tefra son depósitos piroclásticos, se encuadra la unidad 63 en la clase de emplazamiento 4. La unidad 69 presenta areniscas con conglomerados, de acuerdo con la descripción litológica extensa de GEODE (2014). En función de la presencia de las areniscas, se infiere que la resistencia global de la unidad es equivalente a la de la clase de emplazamiento 3 (Tabla 3.4).

La unidad 83 se describe como depósitos de deslizamientos gravitacionales y deslizamientos de ladera (Tabla A.6). Según la descripción litológica extensa de GEODE (2014), estos depósitos son heterométricos, están constituidos por fragmentos o megabloques y tienen escasa matriz terrosa. A partir del elevado tamaño de los fragmentos observados, mayor que el tamaño de las arenas, puede considerarse que la compacidad global de los depósitos es alta. Por tanto, la resistencia de la unidad 83 
se corresponde con la resistencia de la clase de emplazamiento 4, a tenor de su descripción geotécnica como suelos duros (Tabla 3.4). La unidad 84 conforma depósitos de ladera y coluviones con arenas y gravas. De acuerdo con la descripción extensa de GEODE (2014), estos depósitos presentan cantos y bloques de tamaño centidecimétrico. El tamaño de grano mayoritario es el de las gravas, pues comprende los tamaños de los clastos de clase canto y bloque. Al ser este tamaño superior al de las arenas, es indicativo de que la unidad 84 es clasificable en la clase de emplazamiento 4. La unidad 85 es descrita como depósitos de deslizamientos gravitacionales y de ladera (Tabla A.6), los cuales presentan bloques y cantos (GEODE, 2014). Por tanto, se vuelve a aplicar el criterio establecido para los depósitos de tamaño de grano superior al de las arenas, y se clasifica la unidad 85 en la clase de emplazamiento 4. La unidad 86 se describe como depósitos de ladera con bloques y cantos en matriz arenosa (GEODE, 2014). Consecuentemente, y a partir de la granulometría de los sedimentos (bloque y canto), la unidad se clasifica en la clase de emplazamiento 4 . La unidad 87 configura depósitos aluviales, de barrancos y de fondos de valle, con conglomerados, gravas, arenas y arcillas (Tabla A.6). De acuerdo con la descripción litológica extensa de GEODE (2014), afloran bloques y cantos en matriz arenosa, y además materiales areno-arcillosos. Se considera que los bloques y cantos, soportados además por la matriz arenosa, permiten considerar que la compacidad de la unidad es alta y que, por tanto, ésta es clasificable en la clase de emplazamiento 4.

La unidad 88 conforma depósitos de terrazas con gravas y arenas (GEODE, 2014). A partir de la granulometría de los sedimentos constitutivos de estos depósitos (grava, arena), se equipara la resistencia global de la unidad a la de la clase de emplazamiento 4 (Tabla 3.4). La unidad 89 se describe como playas de arenas y de cantos (Tabla A.6). Como la unidad consiste en depósitos de playa, se infiere que su compacidad/consistencia global es baja, a pesar de que los tamaños de grano presentes son al menos equivalentes al tamaño arena. A tenor de la descripción geotécnica de la clase de emplazamiento 6 (Tabla 3.4) como suelos blandos, y por tanto de baja consistencia, la unidad 89 se clasifica en la clase 6. La unidad 90 es descrita por GEODE (2014) como depósitos cuaternarios indiferenciados, generalmente antrópicos. Adoptando un criterio conservador, se supone que la compacidad/consistencia de este tipo de depósitos es baja, y, consecuentemente, se clasifica la unidad 90 en la clase de emplazamiento 6 . La unidad 91 consiste en depósitos antrópicos con materiales limo-arcillosos, arenas y gravas. Aplicando el criterio seguido en el caso de la unidad 90, y también atendiendo a la presencia de los materiales limo-arcillosos, la unidad 91 se encuadra igualmente en la clase de emplazamiento 6.

\section{Archipiélago Chinijo (islas de La Graciosa, Montaña Clara, Alegranza,} Roque del Este, y Roque del Oeste) En este apartado se describe el proceso de clasificación de las unidades que afloran en el Archipiélago Chinijo, que comprende las islas de La Graciosa, Montaña Clara, Alegranza, el Roque del Este y el Roque del Oeste. Según la leyenda cronoestratigráfica de la cartografía GEODE (Tabla 3.35), los códigos de estas unidades son 93, 94, 95, 96, 97, 98, 99, 100, 101, 102, 102a, 103, 104, 105, 106, 107, 108 y 109.

La unidad 93 comprende las coladas basálticas olivínicas que forman el sustrato de los primeros episodios en la isla de La Graciosa (Tabla A.6). A partir de la presencia de los basaltos, rocas volcánicas, la unidad se clasifica como rocas duras en 
la clase de emplazamiento 1 (Tabla 3.4). Idéntico criterio se utiliza para clasificar la unidad 94 en la clase 1, porque su descripción litológica consta de coladas basálticas olivínicas (Tabla A.6). La unidad 95 se describe como conos de tefra estrombolianos. Al ser depósitos de naturaleza piroclástica, como se ha indicado, los conos de tefra son indicativos de la pertenencia de la unidad 95 a la clase de emplazamiento 4 (suelos duros, Tabla 3.4). La unidad 96 consiste en piroclastos basálticos de dispersión, de clase lapilli (GEODE, 2014). Como la unidad corresponde a depósitos de lapillis, se equipara su resistencia a la de los suelos de consistencia media que describen geotécnicamente la clase de emplazamiento 5 (Tabla 3.4). Con este mismo criterio, se clasifica la unidad 97 en la clase 5, pues la descripción litológica extensa de GEODE (2014) establece que la unidad forma depósitos piroclásticos basálticos hidromagmáticos, con piroclastos de clase lapilli.

La unidad 98 consiste en un intrusivo basáltico, que al presentar rocas volcánicas (basaltos), implica la clasificación de la unidad en la clase de emplazamiento 1 (Tabla 3.4). La unidad 99 es descrita por GEODE (2014) como areniscas con conglomerados. Puesto que las areniscas se consideran como rocas blandas a firmes, la descripción geotécnica de la clase de emplazamiento 3 (Tabla 3.4) permite clasificar la unidad 99 en esta clase. La unidad 100 se describe como piroclastos basálticos hidromagmáticos y estrombolianos (Tabla A.6), que corresponden a bombas, escorias y lapillis (GEODE, 2014). Por tanto, se adopta el criterio de clasificación de las unidades con depósitos piroclásticos en la clase de emplazamiento 4 (Tabla 3.4). La unidad 101 forma depósitos piroclásticos basálticos hidromagmáticos (Tabla A.6), que según la descripción extensa de GEODE (2014) contienen lapillis. Considerando la fina granulometría del piroclasto (lapilli), se equipara la resistencia de la unidad a la resistencia de los suelos de consistencia media que describen geotécnicamente la clase de emplazamiento 5 (Tabla 3.4).

La unidad 102 es descrita como coladas basálticas olivínicas (Tabla A.6). Dado que la unidad presenta rocas volcánicas (basaltos), se aplica el criterio de clasificación establecido para encuadrar la misma en la clase de emplazamiento 1 (Tabla 3.4). La unidad 102a consiste en recubrimientos de arenas sobre coladas (Tabla A.6). En función de la granulometría de las arenas, se entiende que el recubrimiento descrito forma un suelo de alta compacidad/consistencia, clasificable en la clase de emplazamiento 4. La unidad 103 presenta conos de tefra, por lo cual se sigue el criterio de clasificación de las unidades con depósitos piroclásticos en la clase de emplazamiento 4 (Tabla 3.4). La unidad 104 se compone de piroclastos basálticos, de dispersión y estrombolianos (Tabla A.6). Consecuentemente, se aplica el mismo criterio para clasificar la unidad 104 en la clase de emplazamiento 4. La unidad 105 se describe como depósitos de ladera, de carácter detrítico y con granulometrías gruesas (GEODE, 2014). En función de estas granulometrías, se considera que la compacidad/consistencia global de la unidad es alta, y se clasifica también en la clase de emplazamiento 4 .

La descripción litológica extensa de la unidad 106 consiste en areniscas con conglomerados (GEODE, 2014). A partir de la correspondencia establecida entre la resistencia de las areniscas y la de las rocas blandas a firmes que constituyen la descripción geotécnica de la clase de emplazamiento 3 (Tabla 3.4), la unidad 106 es clasificada en esta clase. La unidad 107 se describe como depósitos aluviales arcilloareno-limosos (GEODE, 2014). El bajo tamaño de grano de los sedimentos de tipo 
arcilla y limo se consideran indicativos de una compacidad/consistencia baja. De acuerdo con la descripción geotécnica de la clase de emplazamiento 6 (Tabla 3.4) como suelos blandos, la unidad 107 se clasifica en esta clase. La unidad 108 consta de arenas eólicas y recubrimientos de arenas eólicas (Tabla A.6). Aplicando el criterio seguido en la clasificación de la unidad 55, se entiende que la compacidad de las arenas eólicas es mínima, al encontrarse sueltas. Por tanto, la resistencia de la unidad 108 es equiparable a la de un suelo blando o de baja consistencia, y a partir de la descripción geotécnica de la clase de emplazamiento 6 (Tabla 3.4), es razonable clasificar la unidad en esta clase. Por último, la unidad 109 aflora como playas de arenas y playas en general (Tabla A.6). Considerando que los depósitos de playa tienen una compacidad o consistencia baja, la unidad 109 se clasifica también en la clase de emplazamiento 6 .

En la Tabla 3.36 se presenta un resumen de la clasificación de las unidades geológicas de la Región 2910 que corresponden a la Isla de Lanzarote, de acuerdo con el procedimiento indicado. El resumen de la clasificación de las unidades geológicas de la Región 2910 y pertenecientes al Archipiélago Chinijo (islas de La Graciosa, Montaña Clara, Alegranza, Roque del Este, y Roque del Oeste) se presenta en la Tabla 3.37

Tabla 3.36: Resumen de la clasificación de las unidades del mapa geológico continuo GEODE a escala 1:50.000 de la Región 2910 (Isla de Lanzarote), de acuerdo a la clasificación de síntesis. Tomado de GEODE (2014), elaboración propia.

\begin{tabular}{|c|c|c|c|}
\hline \multicolumn{2}{|c|}{$\begin{array}{l}\text { Sistema/Dominio/Edificio/Ciclo } \\
\text { volcánico/Macizo } \\
\text { plutónico/Formación/Miembro }\end{array}$} & $\begin{array}{l}\text { Código de la } \\
\text { unidad geológica }\end{array}$ & $\begin{array}{l}\text { Clase de } \\
\text { emplazamiento }\end{array}$ \\
\hline \multirow{14}{*}{ Edificio Ajaches } & \multirow{8}{*}{ Tramo inferior } & 3 & 2 \\
\hline & & 4 & 4 \\
\hline & & 5 & 2 \\
\hline & & 6 & 3 \\
\hline & & 7 & 4 \\
\hline & & 8 & 1 \\
\hline & & 9 & 1 \\
\hline & & 10 & 1 \\
\hline & \multirow{4}{*}{ Tramo superior } & 11 & 4 \\
\hline & & 12 & 2 \\
\hline & & 13 & 2 \\
\hline & & 14 & 4 \\
\hline & \multirow[t]{2}{*}{ Episodios tardíos } & 15 & 2 \\
\hline & & 16 & 4 \\
\hline \multirow{10}{*}{ Edificio Famara } & \multirow{4}{*}{ Tramo inferior } & 17 & 2 \\
\hline & & 18 & 4 \\
\hline & & 19 & 3 \\
\hline & & 20 & 4 \\
\hline & \multirow{3}{*}{ Tramo medio } & 21 & 2 \\
\hline & & 22 & 4 \\
\hline & & 23 & 2 \\
\hline & \multirow{3}{*}{ Tramo superior } & 24 & 2 \\
\hline & & $24 \mathrm{a}$ & 5 \\
\hline & & 25 & 4 \\
\hline \multirow{2}{*}{\multicolumn{2}{|c|}{ Edificio Central }} & 26 & 1 \\
\hline & & 27 & 4 \\
\hline & & 28 & 1 \\
\hline & & 29 & 3 \\
\hline & & 30 & 4 \\
\hline & & 31 & 4 \\
\hline & & 32 & 4 \\
\hline & & 33 & 4 \\
\hline \multirow{2}{*}{\multicolumn{2}{|c|}{ Edificio Montaña Roja }} & 34 & 1 \\
\hline & & 35 & 4 \\
\hline
\end{tabular}


(continuación)

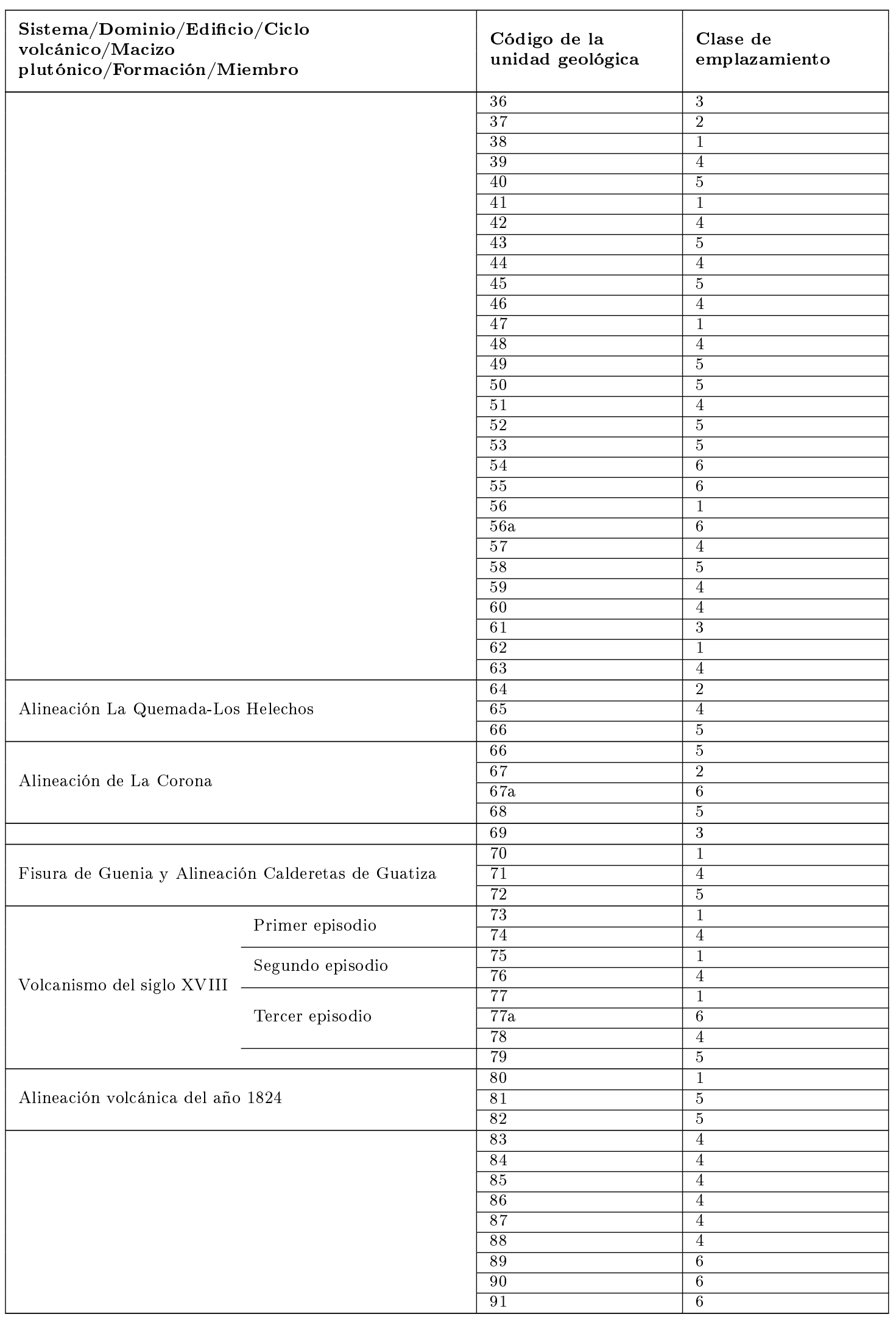


Tabla 3.37: Resumen de la clasificación de las unidades del mapa geológico continuo GEODE a escala 1:50.000 de la Región 2910 (Archipiélago Chinijo (islas de La Graciosa, Montaña Clara, Alegranza, Roque del Este, y Roque del Oeste)), de acuerdo a la clasificación de síntesis. Tomado de GEODE (2014), elaboración propia.

\begin{tabular}{|l|l|}
\hline Código de la unidad geológica & Clase de emplazamiento \\
\hline 93 & 1 \\
\hline 94 & 1 \\
\hline 95 & 4 \\
\hline 96 & 5 \\
\hline 97 & 5 \\
\hline 98 & 1 \\
\hline 99 & 3 \\
\hline 100 & 4 \\
\hline 101 & 5 \\
\hline 102 & 1 \\
\hline $102 \mathrm{a}$ & 6 \\
\hline 103 & 4 \\
\hline 104 & 4 \\
\hline 105 & 4 \\
\hline 106 & 3 \\
\hline 107 & 6 \\
\hline 108 & 6 \\
\hline 109 & 6 \\
\hline
\end{tabular}

\section{Tenerife (Región 2913)}

De acuerdo con la cartografía de la región GEODE 2913, que abarca la isla de Tenerife (GEODE, 2014), afloran 220 unidades geológicas, con edades cronoestratigráficas comprendidas entre el Mioceno Medio y el Holoceno. Las descripciones litológicas y edades cronostratigráficas de estas unidades se detallan en la Tabla A.7. Según la leyenda cronoestratigráfica de esta cartografía, las unidades pertenecen a 52 grupos distintos, que se presentan en la Tabla 3.38. Del conjunto de 220 unidades descritas para la región 2913,9 no aparecen cartografiadas y por tanto no se han clasificado: unidades 1, 2, 17, 18, 22, 23, 37, 38 y 131.

Tabla 3.38: División de las unidades del mapa geológico continuo GEODE a escala 1:50.000 de Tenerife (Zona 2913), de acuerdo con la leyenda cronoestratigráfica. Tomado de GEODE (2014).

\begin{tabular}{|c|c|c|}
\hline $\begin{array}{l}\text { Código de la unidad } \\
\text { geológica }\end{array}$ & \multicolumn{2}{|c|}{$\begin{array}{l}\text { Sistema/Dominio/Edificio/Ciclo volcánico/Macizo } \\
\text { plutónico/Formación/Miembro }\end{array}$} \\
\hline $1,2,3,4$ & $\begin{array}{l}\text { Rocas filonianas y subvol- } 1^{\circ} \text { Ciclo } \\
\text { cánicas }\end{array}$ & \multirow{5}{*}{ Macizo de Anaga } \\
\hline 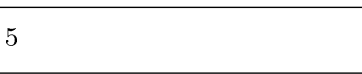 & $\begin{array}{ll}\text { Complejo del Arco de Ta- } & \text { (Molcanico } \\
\text { ganana } & \text { Plio- } \\
\end{array}$ & \\
\hline $6,7,8,9,10,11,12$ & & \\
\hline 13,14 & Formación Chamorga & \\
\hline 15,16 & $\begin{array}{lll}\text { Formación } & \text { Chinamada- } & \text { volcánico } \\
\text { Batanes } & & \text { (Plioceno) }\end{array}$ & \\
\hline $17,18,19$ & Rocas filonianas y subvolcánicas & \multirow{2}{*}{ Macizo del Roque del Conde } \\
\hline 20,21 & & \\
\hline $22,23,24$ & Rocas filonianas y subvolcánicas & \multirow{4}{*}{ Macizo de Teno } \\
\hline 25,26 & Edificio inferior & \\
\hline $27,28,29,30,31$ & Edificio Carrizales & \\
\hline $32,33,34,35,36$ & Formación tabular & \\
\hline 37,38 & Rocas filonianas y subvolcánicas & \multirow{3}{*}{ Edificio Dorsal } \\
\hline 39,40 & Tramo inferior & \\
\hline $41,42,43$ & Tramo superior & \\
\hline $44,45,46$ & Rocas filonianas y subvolcánicas & \multirow{3}{*}{ Primeros Edificios Cañadas } \\
\hline $47,48,49,50$ & Edificio Cañadas I, y su destrucción & \\
\hline $51,52,53,54,54 \mathrm{a}$ & Edificios El Cedro, y Ucanca inferior & \\
\hline $\begin{array}{l}55,56,57,58,59,60,61, \\
62,63,64,65,66,67,68, \\
69,70,71,72,73\end{array}$ & Dominio de las laderas de Cañadas & \multirow[t]{2}{*}{ Series Cañadas Intermedias } \\
\hline $74,75,76,77$ & Dominio de Tigaiga & \\
\hline
\end{tabular}


(continuación)

\begin{tabular}{|c|c|c|c|}
\hline $\begin{array}{l}\text { Código de la unidad } \\
\text { geológica }\end{array}$ & \multicolumn{3}{|c|}{$\begin{array}{l}\text { Sistema/Dominio/Edificio/Ciclo volcánico/Macizo } \\
\text { plutónico/Formación/Miembro }\end{array}$} \\
\hline $78,79,80,81,82,83,84,85$ & \multicolumn{2}{|l|}{ Edificio Las Pilas } & \multirow{2}{*}{$\begin{array}{l}\text { Grupo Guajara y Edificio Las } \\
\text { Pilas }\end{array}$} \\
\hline 86,87 & \multicolumn{2}{|l|}{ Grupo Guajara } & \\
\hline $88,89,90$ & \multicolumn{2}{|l|}{ Formación Diego Hernández } & \multirow{2}{*}{ Edificios y formaciones Cañadas } \\
\hline 91 & & & \\
\hline 92,93 & \multicolumn{2}{|l|}{ Edificio Teide } & \multirow{9}{*}{ Erupciones postcaldera } \\
\hline 94,95 & \multicolumn{2}{|l|}{ Emisiones básicas antiguas } & \\
\hline 96,97 & \multicolumn{2}{|c|}{ Pequeñas emisiones sálicas antiguas } & \\
\hline $98,99,100$ & \multicolumn{2}{|c|}{ Sin asignación de edificio } & \\
\hline $101,102,103$ & \multicolumn{2}{|c|}{ Edificio Pico Viejo } & \\
\hline 104,105 & \multicolumn{2}{|c|}{ Pequeñas emisiones sálicas periféricas } & \\
\hline $\begin{array}{l}106,107,108,109,110 \\
111,112,113,114,115,116\end{array}$ & \multicolumn{2}{|c|}{ Edificios domáticos sálicos periféricos } & \\
\hline $\begin{array}{l}117,118,119,120,121 \\
122,123,124\end{array}$ & \multicolumn{2}{|l|}{ Dominio de Montaña Blanca } & \\
\hline $125,126,127,128,129,130$ & & & \\
\hline 131,132 & \multicolumn{2}{|c|}{ Rocas filonianas y subvolcánicas } & \multirow{4}{*}{ Eje NE o de La Dorsal } \\
\hline 133,134 & \multirow{2}{*}{\multicolumn{2}{|c|}{$\begin{array}{l}\text { Erupciones Inferiores } \\
\text { Fisura de Colmenar-Llanos de Los Infantes }\end{array}$}} & \\
\hline 135,136 & & & \\
\hline $\begin{array}{l}137, \quad 138,139, \quad 140,141 \\
142,143,144,145,146,147\end{array}$ & \multicolumn{2}{|c|}{ Erupciones Superiores } & \\
\hline $148,149,150$ & \multicolumn{2}{|l|}{ Erupciones Inferiores } & \multirow{2}{*}{ Eje SE-NO } \\
\hline 151,152 & \multicolumn{2}{|c|}{ Erupciones Superiores } & \\
\hline $153,154,155,156$ & \multicolumn{3}{|c|}{ Erupciones fuera de los ejes principales } \\
\hline $157,158,159$ & \multirow{2}{*}{\multicolumn{2}{|c|}{ Erupciones hidromagmáticas }} & \multirow{2}{*}{$\begin{array}{l}\text { Erupciones costeras y periféricas } \\
\text { (mayoritariamente sálicas) }\end{array}$} \\
\hline $160,161,162,163,164$ & & & \\
\hline 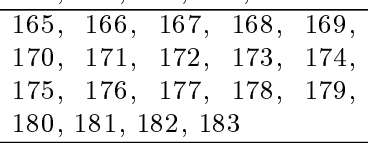 & \multicolumn{3}{|l|}{ Eje NO } \\
\hline $184,185,186$ & \multicolumn{2}{|l|}{ Volcán de Güimar } & \multirow{4}{*}{ Erupciones subhistóricas } \\
\hline $187,188,189$ & Volcán de las Arenas & & \\
\hline 190,191 & \multicolumn{2}{|l|}{ Volcán de Arafo } & \\
\hline 192,193 & \multicolumn{2}{|c|}{ Volcán de Montaña Reventada } & \\
\hline 194,195 & Erupción fisural del Taoro & \begin{tabular}{lr}
\multicolumn{2}{l}{ Erupciones } \\
del & siglo \\
XIV &
\end{tabular} & \\
\hline $196,197,198$ & $\begin{array}{l}\text { Fisura eruptiva de Sie- } \\
\text { te Fuentes-Fasnia Güimar } \\
(1704-05)\end{array}$ & $\begin{array}{l}\text { Erupciones } \\
\text { del siglo }\end{array}$ & Erupciones históricas \\
\hline 199,200 & $\begin{array}{l}\text { Erupción de Montaña Ne- } \\
\text { gra o Garachico (1706) }\end{array}$ & XVIII & \\
\hline 201,202 & $\begin{array}{l}\text { Erupción del Chahorra o } \\
\text { Narices del Teide (1798) }\end{array}$ & & \\
\hline 203,204 & $\begin{array}{l}\text { Erupción del Chinyero } \\
(1909)\end{array}$ & \begin{tabular}{l}
\multicolumn{2}{l}{ Erupciones } \\
del \\
XX
\end{tabular} & \\
\hline $\begin{array}{l}205,206,207,208,209 \\
210,211,212,213,214 \\
215,216,217,218,219\end{array}$ & Depósitos sedimentarios cua & ternarios & \\
\hline
\end{tabular}

Macizo de Anaga ( $1^{\circ}$ Ciclo volcánico (Mio-Plioceno), rocas filonianas y subvolcánicas) De acuerdo con la leyenda cronoestratigráfica de la cartografía de la región GEODE 2913, el Macizo de Anaga contiene las unidades comprendidas entre la unidad 3 y la unidad 16 (Tabla A.7). El macizo se subdivide a su vez en un primer ciclo volcánico (Mio-Plioceno) y en un segundo ciclo (Plioceno). Dentro del primer ciclo volcánico, las unidades agrupadas como rocas filonianas y subvolcánicas, de acuerdo con la leyenda cronoestratigráfica, son las que tienen código 3 y 4 (Tabla 3.38).

La unidad 3 se describe como intrusivos básicos (Tabla A.7). Se entiende que, al 
consistir en rocas plutónicas, su resistencia se considera equiparable a la resistencia de las rocas duras. Dado que la descripción geotécnica de la clase de emplazamiento 1 (Tabla 3.4) consta de rocas duras, es razonable asumir que la resistencia de la unidad 3 es equiparable también a la de la clase 1. Por tanto, la unidad 3 se clasifica en la clase 1. La unidad 4 es descrita como roques fonolíticos. De acuerdo con la descripción litológica extensa de GEODE (2014), estos roques constituyen intrusiones compuestas por roca volcánica (fonolita) que se encuentra fracturada (disyunción columnar). La resistencia de las rocas volcánicas también se considera equivalente a la de las rocas duras, pero en esta unidad, las fonolitas existentes se encuentran fracturadas, por lo que se asume que su resistencia es menor que la propia de las rocas volcánicas. De acuerdo con la Tabla 3.4, la unidad 4 se encuadra en la clase de emplazamiento 2, en función de su descripción geotécnica como rocas de resistencia media, y por tanto menor que la resistencia de la clase 1 (rocas duras).

Macizo de Anaga ( $1^{\circ}$ Ciclo volcánico (Mio-Plioceno), complejo del Arco de Taganana) La unidad 5 es descrita como coladas basálticas y brechas polimícticas atravesadas por intensa malla de diques (Tabla A.7). La presencia de brechas junto a las rocas volcánicas (basaltos) recomienda considerar una resistencia menor que la resistencia de las rocas duras. La razón estriba en el carácter sedimentario de las brechas, que se componen de clastos de tamaño grava, y para las cuales se supone una resistencia equiparable a la de las rocas de consistencia media, o rocas blandas a firmes. En consecuencia, se infiere que la resistencia global de la unidad 5 es media, y por este motivo se clasifica en la clase de emplazamiento 2 (Tabla 3.4).

Macizo de Anaga ( $1^{\circ}$ Ciclo volcánico (Mio-Plioceno) En este apartado se describe el proceso de clasificación de las unidades restantes del primer ciclo volcánico del Macizo de Anaga, para las cuales la leyenda cronoestratigráfica de la cartografía GEODE no establece su pertenencia a ningún subgrupo de este ciclo (Tabla 3.38). Los códigos de estas unidades son 6, 7, 8, 9, 10, 11 y 12.

La unidad 6 conforma coladas básicas alteradas (Tabla A.7). Las rocas volcánicas de estas coladas (basaltos) presentan un fuerte grado de alteración (GEODE, 2014), que implica que la resistencia de la unidad es equiparable a la de las rocas de resistencia media (clase de emplazamiento 2, Tabla 3.4). La unidad 7 aflora en centros de emisión estrombolianos, que corresponden a depósitos piroclásticos. La compacidad/consistencia de este tipo de depósitos se considera alta, por lo cual su resistencia es equiparable a la de la clase de emplazamiento 4 (Tabla 3.4), que se describe geotécnicamente como suelos duros o de alta consistencia. Por tanto, la unidad 7 se clasifica en la clase 4 . La unidad 8 consta de piroclastos de dispersión, que, según la descripción litológica extensa de GEODE (2014), presentan una granulometría fina (clase lapilli). En función del bajo tamaño de grano del piroclasto, la compacidad/consistencia global de la unidad se infiere como media, y no alta, como en el caso de un depósito piroclástico de carácter general. De acuerdo con la descripción geotécnica de la clase de emplazamiento 5 (suelos de consistencia media, Tabla 3.4), la resistencia de la unidad 8 se equipara con la resistencia de la clase 5. La Tabla A.7 describe la unidad 9 en términos de coladas basálticas con niveles piroclásticos subordinados, si bien la descripción extensa de GEODE (2014) indica 
que la unidad sólo presenta coladas de basaltos (rocas volcánicas). Por tanto, la unidad 9 se clasifica en la clase de emplazamiento 1.

La unidad 10 configura un "debris-avalanche" con niveles sedimentarios (Tabla A.7). De acuerdo con la descripción litológica extensa de GEODE (2014), la unidad contiene niveles de brechas caóticas de deslizamientos y niveles de naturaleza epiclástica. En función de la presencia de estos niveles, para los cuales se supone una compacidad baja, la resistencia de la unidad es menor que la de las brechas (clasificables en la clase de emplazamiento 3), y por tanto es equiparable a la de un suelo duro (clase de emplazamiento 4, Tabla 3.4). La unidad 11 es descrita como depósitos epiclásticos y sedimentos "intramontanos" (Tabla A.7). De acuerdo con la descripción litológica extensa de GEODE (2014), estos depósitos contienen arenas y cantos. A partir de la granulometría observada, con un tamaño mínimo igual al de las arenas, se supone que la compacidad/consistencia de los depósitos es alta. Por esta razón, la resistencia global de la unidad 11 es equivalente a la resistencia de la clase de emplazamiento 4 (Tabla 3.4), en función de su descripción geotécnica como suelos duros (de alta consistencia). Consecuentemente, la unidad 11 se clasifica en la clase 4. La unidad 12 se compone de piroclastos de caída con niveles hidromagmáticos. De acuerdo con la descripción litológica extensa de GEODE (2014), contiene niveles de potencia media de lapillis estrombolianos de color amarillento y niveles piroclásticos finos de aspecto hidromagmático. Al presentarse piroclastos finos, la resistencia de la unidad es equiparable a la de un suelo de consistencia media (clase de emplazamiento 5).

Macizo de Anaga (2 ${ }^{\circ}$ Ciclo volcánico (Plioceno), formación Chamorga) Dentro del segundo ciclo volcánico (Plioceno), las unidades agrupadas en la formación Chamorga, según la leyenda cronoestratigráfica, son las que tienen código 13 y 14 (Tabla 3.38). La unidad 13 es descrita como brechas, domos, depósitos volcanosedimentarios y coladas tefríticas y fonolíticas (Tabla A.7). La presencia de las brechas junto con las rocas volcánicas (tefritas, fonolitas) recomienda inferir una resistencia menor que la resistencia adjudicable a las rocas volcánicas (clase de emplazamiento 1). De acuerdo con la clasificación de síntesis (Tabla 3.4), la resistencia de la unidad 13 es equivalente, por tanto, a la resistencia de la clase de emplazamiento 2, en función de su descripción geotécnica como rocas de resistencia media. La unidad 14, por su parte, consta de coladas intermedias y fonolitas máficas. En función de la presencia de las rocas volcánicas (fonolitas), la unidad es clasificada en la clase de emplazamiento 1.

Macizo de Anaga ( $2^{\circ}$ Ciclo volcánico (Plioceno), formación ChinamadaBatanes) La unidad 15 contiene centros de emisión estrombolianos (Tabla A.7). Análogamente al caso de la unidad 7, se considera que la unidad 15 es clasificable en la clase de emplazamiento 4 (Tabla 3.4), al consistir en depósitos piroclásticos cuya resistencia es equiparable a la resistencia de los suelos duros o de alta consistencia. La unidad 16 consiste en coladas basálticas y basaníticas subhorizontales. Dado que la unidad presenta rocas volcánicas, de composición básica, se aplica el criterio de clasificación, ya establecido, en la clase de emplazamiento 1 (Tabla 3.4). 
Macizo del Roque del Conde (Rocas filonianas y subvolcánicas) De acuerdo con la leyenda cronoestratigráfica de la cartografía de la región GEODE 2913, el Macizo del Roque del Conde engloba las unidades comprendidas entre la unidad 19 y la unidad 21 (Tabla A.7). La única unidad cartografiada y encuadrada dentro del subgrupo de rocas filonianas y subvolcánicas, de acuerdo con la leyenda cronoestratigráfica, es la unidad con código 19 (Tabla 3.38). Esta unidad aflora como domos sálicos (Tabla A.7), y, de acuerdo con la descripción litológica extensa de GEODE (2014), presenta rocas volcánicas (fonolitas, traquitas) y plutónicas (theralitas, que pertenecen al grupo de los gabros). Tanto las rocas volcánicas como las plutónicas se consideran como rocas duras, por lo cual la unidad 19 se clasifica en la clase de emplazamiento 1 (Tabla 3.4).

Macizo del Roque del Conde En este apartado se describe el proceso de clasificación de las unidades restantes del Macizo del Roque del Conde, para las cuales la leyenda cronoestratigráfica de la cartografía GEODE no establece su pertenencia a ningún subgrupo (Tabla 3.38). Los códigos de estas unidades son 20 y 21.

La unidad 20 se describe como coladas basálticas (Tabla A.7). Por tanto se aplica el criterio de clasificación de las unidades con rocas volcánicas (basaltos en la unidad 20) en la clase de emplazamiento 1 (Tabla 3.4). La unidad 21 consta de piroclastos basálticos, por lo cual se considera que su resistencia global es equiparable a la de los suelos de alta consistencia. Según la descripción geotécnica de la clase de emplazamiento 4 (Tabla 3.4), la unidad 21 se clasifica en esta clase.

Macizo de Teno (Rocas filonianas y subvolcánicas) La unidad 24 se describe como roques sálicos (Tabla A.7). De acuerdo con la descripción litológica extensa de GEODE (2014), estos roques están representados por afloramientos de fonolitas. $\mathrm{Al}$ ser rocas volcánicas, las fonolitas son indicativas de la clasificación de la unidad 24 en la clase de emplazamiento 1 (Tabla 3.4) como rocas duras.

Macizo de Teno (Edificio inferior) La unidad 25 constituye coladas de basaltos plagioclásicos, piroxénicos, ankaramitas, escorias y brechas (Tabla A.7). Como se observa, en esta descripción litológica predominan las rocas volcánicas: basaltos y ankaramitas, éstas últimas pertenecientes al grupo de las basanitas. Por este motivo, se infiere que la resistencia de la unidad es equiparable a la de las rocas duras que describen geotécnicamente la clase de emplazamiento 1 (Tabla 3.4). Por tanto, la unidad 25 pertenece a esta clase. De acuerdo con la descripción litológica extensa de la unidad 26 (GEODE, 2014), ésta consiste en una intrusión básica con rocas plutónicas (doleritas, microgabros). Al contener rocas plutónicas, la unidad 26 es agrupada en la clase de emplazamiento 1.

Macizo de Teno (Edificio Carrizales) La unidad 27 se describe como brechas de basaltos plagioclásicos (Tabla A.7). De acuerdo con la descripción litológica extensa de GEODE (2014), la unidad presenta una brecha heterométrica, con matriz arcilloso-arenosa. Como se ha indicado al justificar la clasificación de la unidad 5, la resistencia de las brechas se considera equivalente a la resistencia de las rocas blandas a firmes, o de consistencia media, pues las primeras tienen carácter sedimentario 
y se componen de clastos de tamaño equiparable al de las gravas. Además, la brecha de la unidad 27 presenta una matriz de grano más fino (arcilla-arena), que contribuye a la consistencia general de la roca. A partir de la descripción geotécnica de la clase de emplazamiento 3 como rocas blandas a firmes (Tabla 3.4), se considera, consecuentemente, que la resistencia de la unidad 27 es coherente con la de esta clase de emplazamiento. Por tanto, la unidad 27 es agrupada en la clase 3. La unidad 28 es descrita como coladas básicas, escorias y brechas, si bien la descripción litológica extensa de GEODE (2014) puntualiza que esta unidad presenta brechas y lapillis con intercalaciones delgadas de coladas basálticas y, en su parte alta, intercalaciones de depósitos de escorias. El carácter fundamentalmente piroclástico de la unidad 28 (escorias, lapillis) recomienda adoptar un criterio conservador e inferir una resistencia global acorde con la resistencia de la clase de emplazamiento 4 (Tabla 3.4), considerando su descripción geotécnica como suelos duros o de alta consistencia. La unidad 29 consiste en coladas basálticas y traquibasálticas con intercalaciones de escorias (Tabla A.7). A partir de la presencia de rocas volcánicas (basaltos), la unidad se clasifica en la clase de emplazamiento 1 (Tabla 3.4) como rocas duras.

La unidad 30 se compone de piroclastos basálticos (Tabla A.7). Por tanto, se sigue el criterio de clasificación de las unidades con depósitos piroclásticos, con el fin de encuadrar la unidad 30 en la clase de emplazamiento 4 (Tabla 3.4). La unidad 31 consta de brechas basálticas laháricas, que se encuentran compactadas (GEODE, 2014). De acuerdo con esta descripción litológica, se aplica el mismo criterio que el considerado en el caso de la unidad 27, en el sentido de clasificar la unidad 31 en la clase de emplazamiento 3 (Tabla 3.4) como rocas blandas a firmes.

Macizo de Teno (Formación tabular) El afloramiento de la unidad 32 consiste en una brecha basáltica polimíctica (Tabla A.7), que presenta una matriz arcillosoarenosa (GEODE, 2014). Por tanto, se vuelve a aplicar el criterio ya considerado en el caso de las unidades 27 y 31, en el sentido de encuadrar las unidades con brechas en la clase de emplazamiento 3 (Tabla 3.4). Considerando también que la presencia de la matriz fina es una contribución a la consistencia y resistencia global de la unidad 32, la misma es clasificada en la clase 3. La unidad 33 se describe como coladas basálticas tabulares (Tabla A.7). De acuerdo con la descripción litológica extensa de GEODE (2014), esta unidad contiene rocas volcánicas (basaltos) con fracturación (disyunción columnar grosera). En base a la clasificación de la unidad 4 , se considera que la resistencia de las rocas volcánicas fracturadas es acorde con la de la clase de emplazamiento 2 (Tabla 3.4), por razón de su descripción geotécnica como rocas de resistencia media. Por tanto, la unidad 33 es agrupada en la clase 2. La unidad 34 consta de piroclastos basálticos, con bombas, escorias, lapillis y cenizas (GEODE, 2014). En este caso, se adopta el criterio de clasificación de las unidades con depósitos piroclásticos en la clase de emplazamiento 4 (Tabla 3.4).

Las unidades 35 y 36 consisten en traquitas y traquibasaltos-fonolitas máficas, respectivamente (Tabla A.7). A partir de estas descripciones litológicas en términos de rocas volcánicas (traquitas, traquibasaltos, fonolitas), ambas unidades, 35 y 36 , se clasifican en la clase de emplazamiento 1 (Tabla 3.4), de acuerdo con el criterio establecido para las unidades con descripciones basadas en rocas volcánicas. 
Edificio Dorsal (Tramo inferior) La unidad 39 se describe como coladas basálticas (Tabla A.7). Dado que la unidad contiene rocas volcánicas (basaltos), se aplica el criterio de clasificación de las unidades con rocas volcánicas en la clase de emplazamiento 1 (Tabla 3.4). La unidad 40 se compone de piroclastos basálticos, por lo cual se clasifica en la clase de emplazamiento 4 (Tabla 3.4) como suelos duros.

Edificio Dorsal (Tramo superior) La unidad 41 es descrita como coladas basálticas (Tabla A.7). Se procede de forma análoga a la seguida en la clasificación de las unidades con rocas volcánicas, y se clasifica la unidad 41 en la clase de emplazamiento 1 (Tabla 3.4), pues esta unidad presenta basaltos en su descripción litológica. La unidad 42 se compone de aglomerados con intercalaciones de coladas básicas (Tabla A.7). De acuerdo con la descripción litológica extensa de GEODE (2014), los aglomerados de esta unidad consisten en cantos centimétricos subangulosos y subredondeados de composición basáltica, que están cementados por una matriz vítrea. En función de la cementación de los clastos gruesos que se observan (tamaño grava), se entiende que la compacidad/consistencia global de la unidad 42 es equivalente a la de una roca blanda a firme, y superior a la de un suelo de alta compacidad/consistencia. Por tanto, y en coherencia con la descripción geotécnica de la clase de emplazamiento 3 como rocas blandas a firmes (Tabla 3.4), la unidad 42 se encuadra en la clase 3 , en lugar de en la clase 4 . Con respecto a la unidad 43, ésta consta de piroclastos basálticos (Tabla A.7), cuya resistencia sí se considera equivalente a la de los suelos duros o de alta consistencia que caracterizan la clase de emplazamiento 4 (Tabla 3.4).

Primeros Edificios Cañadas (Rocas filonianas y subvolcánicas) La unidad 44 se describe como diques básicos, de acuerdo con la Tabla A.7. Según la descripción litológica extensa de GEODE (2014), la unidad presenta las rocas volcánicas basalto y traquibasalto, por tanto se clasifica en la clase de emplazamiento 1 (Tabla 3.4). La unidad 45 consta de intrusivos básicos, que al presentar rocas plutónicas también son indicativos de la pertenencia de esta unidad a la clase de emplazamiento 1. En cuanto a la unidad 46, ésta consiste en intrusivos y diques sálicos (Tabla A.7), compuestos por fonolitas (GEODE, 2014). Dado que las fonolitas son rocas volcánicas, la unidad se clasifica también en la clase 1.

Primeros Edificios Cañadas (Edificio Cañadas I, y su destrucción) Según la Tabla A.7, la unidad 47 se describe como basaltos plagioclásicos con traquitas y fonolitas intercaladas. Debido a la presencia de rocas volcánicas (basaltos, traquitas, fonolitas), la unidad se clasifica en la clase de emplazamiento 1 (Tabla 3.4). La unidad 48 se compone de piroclastos basálticos, por tanto se adopta el criterio de clasificación de las unidades con depósitos piroclásticos en la clase de emplazamiento 4 (Tabla 3.4).

La unidad 49 es descrita como brecha poligénica y aglomerados, según la Tabla A.7. En función del afloramiento de brechas con matriz muy compactada, como indica GEODE (2014), se considera que la resistencia global de la unidad es equiparable a la de las rocas blandas a firmes que describen geotécnicamente la clase de emplazamiento 3 (Tabla 3.4). Se procede, en consecuencia, a agrupar la unidad 49 
en la clase 3. La unidad 50 consiste en brechas polimícticas atravesadas por diques, por lo cual se aplica el criterio general de clasificación de las unidades compuestas por brechas en la clase de emplazamiento 3, análogamente al caso de las unidades 27,31 y 32 .

Primeros Edificios Cañadas (Edificios El Cedro, y Ucanca inferior) La unidad 51 consiste en brechas y coladas traquibasálticas, como se observa en la Tabla A.7. A pesar de la presencia de rocas volcánicas (traquibasaltos), la presencia de las brechas recomienda aplicar un criterio conservador, y considerar que la resistencia global de la unidad es equivalente a la de una roca blanda a firme. Por tanto, y de acuerdo con la descripción geotécnica de la clase de emplazamiento 3 (Tabla 3.4), la unidad 51 es clasificada en esta clase. La unidad 52 se describe en primer lugar como fonolitas (Tabla A.7), si bien (GEODE, 2014) amplía esta descripción a las traquitas. Por tanto, la unidad se compone de rocas volcánicas, y se clasifica en la clase de emplazamiento 1 (Tabla 3.4). La unidad 53 consta de traquibasaltos (Tabla A.7), por lo cual se vuelve a aplicar el criterio de clasificación de las unidades con rocas volcánicas en la clase de emplazamiento 1.

Las unidades 54 y 54 a se describen como tobas, pumitas y depósitos volcanoclásticos hidrotermalizados y atravesados por diques sálicos (Tabla A.7). Las tobas son depósitos piroclásticos, por lo que se considera que ambas unidades constituyen depósitos de esta clase, y que es posible equiparar la correspondiente resistencia global a la resistencia de la clase de emplazamiento 4 (Tabla 3.4). En consecuencia, las unidades 54 y 54a se encuadran en la clase 4 como suelos duros.

Series Cañadas Intermedias (Dominio de las laderas de Cañadas) La unidad 55 se describe como derrubios de ladera (Tabla A.7). De acuerdo con la descripción litológica extensa de GEODE (2014), esta unidad constituye un depósito con bloques y cantos heterométricos y mal seleccionados. Atendiendo a la gruesa granulometría de los clastos (bloques, cantos), superior al tamaño de las arenas, se infiere que la resistencia global de la unidad 55 es equiparable a la de los suelos de duros o de alta consistencia que describen geotécnicamente la clase de emplazamiento 4 (Tabla 3.4). La unidad 56 consiste en traquibasaltos inferiores con intercalaciones de traquitas y fonolitas máficas. Como esta unidad contiene rocas volcánicas (traquibasaltos, traquitas, fonolitas), se clasifica en la clase de emplazamiento 1 (Tabla 3.4) como rocas duras. Con este mismo criterio, la unidad 57 se clasifica también en la clase 1, pues su descripción litológica presenta coladas de fonolitas y traquitas. La unidad 58 constituye un aglomerado traquítico, el cual, de acuerdo con la descripción litológica extensa de GEODE (2014), se compone de cantos de basalto soportados por una matriz pumítica. Se considera que la resistencia de la unidad 58 es equivalente a la de una roca blanda a firme a partir de la presencia de la matriz, que contribuye a la compacidad y consolidación global de la unidad. A partir de la descripción geotécnica de la clase de emplazamiento 3 como rocas blandas a firmes (Tabla 3.4), la unidad 58 es clasificada en esta clase.

La unidad 59 consiste en ignimbritas (Tabla A.7). Al ser rocas piroclásticas con matriz, la resistencia de las ignimbritas puede considerarse equivalente a la de las rocas blandas a firmes. Por tanto, y atendiendo a la descripción geotécnica de la clase de emplazamiento 3 (Tabla 3.4), la unidad 59 se clasifica en esta clase. La unidad 60 
se compone de fonolitas con fracturación (lajeado), según la descripción litológica extensa de GEODE (2014). En este caso, se adopta el criterio de clasificación de las unidades con rocas volcánicas fracturadas en la clase de emplazamiento 2 (Tabla 3.4) como rocas de resistencia media. La unidad 61 consta de piroclastos basálticos (Tabla A.7), por tanto su resistencia se infiere equiparable a la de un suelo duro o de alta consistencia, y se clasifica en la clase de emplazamiento 4 (Tabla 3.4). La unidad 62 se compone de basaltos (Tabla A.7), que al ser rocas volcánicas permiten la clasificación de la unidad en la clase de emplazamiento 1 (Tabla 3.4).

La unidad 63 es descrita como traquibasaltos con piroclastos intercalados (Tabla A.7). Dado que las rocas volcánicas presentes (basalto y traquibasalto) están medianamente degradadas (GEODE, 2014), se adopta un criterio conservador para clasificar la unidad en la clase de emplazamiento 2 como rocas de resistencia media (Tabla 3.4). La unidad 64 configura depósitos laháricos sálicos, los cuales presentan cantos heterométricos y cementados, de acuerdo a la descripción litológica extensa de GEODE (2014). Se aplica por tanto el criterio ya utilizado para clasificar la unidad 42, en el sentido de considerar que la cementación de los cantos implica una resistencia acorde con la de las rocas blandas a firmes que caracterizan la clase de emplazamiento 3 (Tabla 3.4). Consecuentemente, la unidad 64 se clasifica en la clase 3. La unidad 65 se compone de ignimbritas asociadas con tobas, según la descripción litológica extensa de GEODE (2014). Aunque la resistencia de las ignimbritas es equivalente a la de una roca blanda a firme, la presencia de las tobas, como depósitos piroclásticos, recomienda disminuir de forma conservadora la resistencia global de la unidad. Por tanto, esta resistencia se considera equiparable a la de un suelo de alta consistencia, y la unidad 65 se clasifica en la clase de emplazamiento 4 (Tabla 3.4). La unidad 66 consta de piroclastos basálticos (Tabla A.7), por lo cual también se clasifica en la clase de emplazamiento 4 .

La unidad 67 se describe como basaltos y tefritas (Tabla A.7), que al ser rocas volcánicas permiten la clasificación de la unidad en la clase de emplazamiento 1 (Tabla 3.4) como rocas duras. Utilizando el mismo criterio, la unidad 68 también se clasifica en la clase 1, pues se describe como coladas basálticas (Tabla A.7). La unidad 69 se compone de piroclastos basálticos, siendo posible adoptar el criterio de clasificación de las unidades con depósitos piroclásticos en la clase de emplazamiento 4 (Tabla 3.4). La unidad 70, por su parte, presenta traquibasaltos y fonolitas máficas (Tabla A.7). Dado que los traquibasaltos y las fonolitas son rocas volcánicas, la unidad 70 se clasifica en la clase de emplazamiento 1.

La unidad 71 aflora como traquibasaltos (Tabla A.7), si bien GEODE (2014) describe también fonolitas. En función de la presencia de estas rocas volcánicas, la unidad 71 se clasifica en la clase de emplazamiento 1 (Tabla 3.4) como rocas duras. La unidad 72 consta de piroclastos basálticos, por lo cual se aplica el criterio de clasificación de las unidades con depósitos piroclásticos en la clase de emplazamiento 4 (Tabla 3.4). La unidad 73 se describe como coladas basálticas (Tabla A.7), por tanto se agrupa en la clase de emplazamiento 1 de acuerdo con las rocas volcánicas que contiene (basaltos).

Series Cañadas Intermedias (Dominio de Tigaiga) La unidad 74 está constituida por coladas basálticas y traquibasálticas con intercalaciones fonolíticas, según 
la Tabla A.7. En base al afloramiento de rocas volcánicas (basaltos y traquibasaltos), la unidad se clasifica en la clase de emplazamiento 1 (Tabla 3.4). La unidad 75 presenta coladas de traquibasaltos y fonolitas con intercalaciones de tobas sálicas. La presencia de las tobas junto con las rocas volcánicas (basaltos, traquibasaltos y fonolitas, de acuerdo con la descripción extensa de GEODE (2014)) recomienda disminuir la resistencia global de la unidad, desde la resistencia propia de las rocas duras (clase de emplazamiento 1) hasta una resistencia media, acorde con la resistencia de las rocas que describen la clase de emplazamiento 2 (Tabla 3.4). Por tanto, la unidad 75 se encuadra en la clase de emplazamiento 2. Según la Tabla A.7, la unidad 76 consiste en ignimbritas y tobas sálicas. Como se ha razonado en el caso de la unidad 65, la asociación de las ignimbritas con las tobas recomienda considerar la resistencia global de la unidad como equiparable a la resistencia de los suelos de alta consistencia que caracterizan la clase de emplazamiento 4 (Tabla 3.4). Por tanto, la unidad 76 se clasifica en la clase 4. La unidad 77 es descrita como coladas fonolíticas y, al presentar rocas volcánicas (fonolitas), es clasificable en la clase de emplazamiento 1.

Grupo Guajara y Edificio Las Pilas (Edificio Las Pilas) La unidad 78 consiste en coladas traquibasálticas con intercalaciones de basaltos y fonolitas subordinadas y de piroclastos (Tabla A.7). A partir de la presencia de rocas volcánicas (basaltos, traquibasaltos y fonolitas) junto con depósitos piroclásticos, se adopta el criterio empleado en la clasificación de la unidad 75, en el sentido de considerar que los piroclastos diminuyen la resistencia global de la unidad, y de equiparar ésta a la resistencia de la clase de emplazamiento 2 (Tabla 3.4). Por tanto, la unidad 78 se clasifica en la clase 2. La descripción litológica de la unidad 79 consta de coladas fonolíticas con depósitos laháricos y tobas pumíticas asociadas. En este caso, se vuelve a aplicar el criterio de clasificación de las unidades 75 y 78 , considerando que las rocas volcánicas de la unidad 79 (fonolitas) y los depósitos piroclásticos que las acompañan (incluyendo las tobas) son suficientes para clasificar esta unidad en la clase 2. La unidad 80 constituye coladas basálticas, que al contener rocas volcánicas (basaltos), permiten clasificar la unidad en la clase de emplazamiento 1 (Tabla 3.4). La unidad 81 presenta coladas de fonolitas y fonolitas máficas (Tabla A.7). Siendo las fonolitas rocas volcánicas, la unidad 81 también se clasifica en la clase de emplazamiento 1.

La unidad 82 se describe como tobas pumíticas intercaladas (Tabla A.7). Aplicando el criterio general establecido para las unidades con depósitos piroclásticos (tobas), la unidad 82 se clasifica en la clase de emplazamiento 4 (Tabla 3.4). La unidad 83 consiste en coladas fonolíticas, en las que las rocas volcánicas presentes (fonolitas) presentan fracturación (lajeado), de acuerdo con la descripción litológica extensa de GEODE (2014). Por tanto, se emplea el criterio de clasificación de las unidades con rocas volcánicas fracturadas en la clase de emplazamiento 2 (Tabla 3.4). La unidad 84 es descrita como coladas basálticas y traquibasálticas con traquitas subordinadas. A partir de la presencia de las rocas volcánicas basalto y traquibasalto, la unidad 84 es agrupada en la clase de emplazamiento 1 (Tabla 3.4). Análogamente, la unidad 85 también se clasifica en la clase de emplazamiento 1, siendo su descripción litológica coladas fonolíticas (Tabla A.7). 
Grupo Guajara y Edificio Las Pilas (Grupo Guajara) La unidad 86 se describe como tobas sálicas (coladas piroclásticas y piroclastos pumíticos), de acuerdo con la Tabla A.7. Como la unidad constituye depósitos piroclásticos (tobas), se aplica el criterio de clasificación de este tipo de depósitos en la clase de emplazamiento 4 (Tabla 3.4). La unidad 87 es descrita como coladas fonolíticas de grandes escarpes, por lo que se clasifica en la clase de emplazamiento 1 en función de la presencia de las fonolitas como rocas volcánicas.

Edificios y formaciones Cañadas (Formación Diego Hernández) De acuerdo con la leyenda cronoestratigráfica de la cartografía de la región GEODE 2913, los edificios y formaciones Cañadas agrupan las unidades comprendidas entre la unidad 88 y la unidad 91 (Tabla A.7). En este contexto, las unidades pertenecientes a la Formación Diego Hernández, de acuerdo con la leyenda cronoestratigráfica, son las unidades con código 88, 89 y 90 (Tabla 3.38).

Según la Tabla A.7, la unidad 88 consiste en tobas sálicas estrombolianas e hidromagmáticas, con niveles sedimentarios y coladas básicas intercaladas. Considerando que las tobas, como depósitos piroclásticos, y los niveles sedimentarios son razón para inferir que la compacidad/consistencia de la unidad es alta, la resistencia de la misma es equivalente a la de los suelos duros que caracterizan la clase de emplazamiento 4 (Tabla 3.4). La unidad 89 corresponde a una colada fonolítica masiva (Tabla A.7). De acuerdo con la descripción litológica extensa de GEODE (2014), las fonolitas de esta unidad se presentan con fracturación (disyunción columnar), por tanto se sigue el criterio de clasificación de las unidades con rocas volcánicas (fonolitas) fracturadas en la clase de emplazamiento 2 (Tabla 3.4). La unidad 90 forma un depósito brechoide polimíctico sin consolidar, con cantos subangulosos de sienitas. La falta de consolidación de los clastos también es descrita por GEODE (2014), indicando directamente que los depósitos se encuentran sueltos. Por tanto, se entiende que la compacidad global de la unidad es mínima, y que, por tanto, su resistencia es equivalente a la de un suelo suelto. En base a la descripción geotécnica de la clase de emplazamiento 6 (Tabla 3.4) como suelos blandos, es decir, de baja consistencia, se encuadra la unidad 90 en esta clase.

Edificios y formaciones Cañadas En este apartado se describe el proceso de clasificación de la unidad 91, la cual, aunque forma parte de los edificios y formaciones Cañadas, no pertenece a ningún subgrupo, según la leyenda cronoestratigráfica de la cartografía GEODE (Tabla 3.38). Esta unidad se describe como piroclastos sálicos indiferenciados (Tabla A.7), que constituyen depósitos con distintos grados de compactación (GEODE, 2014). En este caso, se adopta el criterio de clasificación de las unidades con depósitos piroclásticos en la clase de emplazamiento 4 (Tabla 3.4), y se agrupa la unidad 91 en esta clase.

Erupciones postcaldera (Edificio Teide) De acuerdo con la leyenda cronoestratigráfica de la cartografía de la región GEODE 2913, las erupciones postcaldera engloban las unidades comprendidas entre la unidad 92 y la unidad 130 (Tabla A.7). Las unidades pertenecientes al subgrupo Edificio Teide, de acuerdo con la leyenda cronoestratigráfica, son las unidades con código 92 y 93 (Tabla 3.38). 
La unidad 92 comprende las coladas antiguas del Teide (Tabla A.7). En la descripción litológica extensa de GEODE (2014) se indica que estas coladas son de tipo "aa" y que se encuentran bastante degradadas. Esta degradación de la roca volcánica aflorante recomienda la clasificación en la clase de emplazamiento 2 (Tabla 3.4) como rocas de resistencia media. La unidad 93 se describe como coladas intermedias del Teide (Tabla A.7). Entendiendo que están constituidas por rocas volcánicas, la resistencia de estas coladas es equivalente a la de las rocas duras que describen geotécnicamente la clase de emplazamiento 1.

Erupciones postcaldera (Emisiones básicas antiguas) La unidad 94 configura conos basálticos periféricos (Tabla A.7), compuestos por bombas, lapillis y cenizas (GEODE, 2014). Dado que la unidad constituye un depósito piroclástico, se clasifica en la clase de emplazamiento 4 (Tabla 3.4). La unidad 95 consiste en coladas basálticas-traquibasálticas, con rocas volcánicas (basaltos y traquibasaltos) que permiten su clasificación en la clase de emplazamiento 1.

Erupciones postcaldera (Pequeñas emisiones sálicas antiguas) La unidad 96 se describe como conos sálicos periféricos, según la Tabla A.7. En consecuencia, constituye depósitos piroclásticos y se clasifica en la clase de emplazamiento 4 (Tabla 3.4). La unidad 97 es descrita como coladas sálicas indiferenciadas. Entendiendo que las mismas están compuestas por rocas volcánicas sálicas, con $<40 \%$ de minerales ferromagnesianos, la unidad se agrupa en la clase de emplazamiento 1.

Erupciones postcaldera (Sin asignación de edificio) Las unidades 98, 99 y 100 se clasifican en la clase de emplazamiento 1 (Tabla 3.4) pues se describen como rocas volcánicas de distintas composiciones. Como indica la Tabla A.7, la unidad 98 presenta traquitas y fonolitas, la unidad 99 contiene basaltos y la unidad 100 corresponde a traquitas.

Erupciones postcaldera (Edificio Pico Viejo) La unidad 101 consta de lavas basálticas "pahoehoe" (Tabla A.7). Se aplica, por tanto, el criterio de clasificación de las unidades con rocas volcánicas en la clase de emplazamiento 1 (Tabla 3.4). Respecto a la unidad 102, su descripción litológica extensa (GEODE, 2014) indica que consiste en depósitos piroclásticos formados por bombas, escorias y lapillis. En este caso, se considera que su resistencia es equiparable a la de los suelos duros que describen geotécnicamente la clase de emplazamiento 4 (Tabla 3.4). La unidad 103 es descrita como coladas basálticas y traquibasálticas "aa", por lo cual se vuelve a adoptar el criterio de clasificación de las unidades con rocas volcánicas, para agrupar la unidad 103 en la clase de emplazamiento 1.

Erupciones postcaldera (Pequeñas emisiones sálicas periféricas) La unidad 104 configura centros de emisión fonolíticos (Tabla A.7). Se sigue, por tanto, el criterio de clasificación de las unidades con depósitos piroclásticos en la clase de emplazamiento 4 (Tabla 3.4). La unidad 105 es descrita como coladas fonolíticas de pequeños centros de emisión. Al contener rocas volcánicas (fonolitas), la unidad se clasifica en la clase de emplazamiento 1. 
Erupciones postcaldera (Edificios domáticos sálicos periféricos) Las unidades 106, 108 y 110 se describen como coladas fonolíticas, según la Tabla A.7. Debido a la presencia de rocas volcánicas (fonolitas), las tres unidades se clasifican en la clase de emplazamiento 1 (Tabla 3.4). Las unidades 107 y 109 conforman conos piroclásticos ( Tabla A.7), por lo que se clasifican en la clase de emplazamiento 4 (Tabla 3.4) siguiendo el criterio establecido para los depósitos piroclásticos.

Las unidades 111, 113 y 115 son descritas como domos fonolíticos, de acuerdo con la Tabla A.7. Presentan por tanto fonolitas, que al ser rocas volcánicas son indicativas de la pertenencia de las tres unidades a la clase de emplazamiento 1 (Tabla 3.4). Utilizando el mismo criterio, las unidades 112, 114 y 116 también se clasifican en la clase 1, pues sus descripciones litológicas consisten en coladas fonolíticas.

Erupciones postcaldera (Dominio de Montaña Blanca) Las unidades 117 y 120 coinciden en su descripción litológica como coladas fonolíticas (Tabla A.7). Como ambas unidades presentan rocas volcánicas (fonolitas), se clasifican en la clase de emplazamiento 1 (Tabla 3.4). La unidad 118 configura la fisura eruptiva fonolítica de Montaña Blanca (Tabla A.7), en la cual GEODE (2014) observa el afloramiento de coladas fonolíticas. Consecuentemente, se adopta en este caso el criterio general de clasificación de las unidades con rocas volcánicas en la clase de emplazamiento 1. En el caso de la unidad 119, su descripción litológica extensa (GEODE, 2014) corresponde a pequeños centros de emisión fonolíticos de tipo domático. Dado que la unidad forma depósitos piroclásticos, debe ser agrupada en la clase de emplazamiento 4 (Tabla 3.4).

La unidad 121 se compone de piroclastos traquibasálticos (Tabla A.7), que corresponden a la clase escoria (GEODE, 2014). Por tanto, se adopta el criterio de clasificación de las unidades con depósitos piroclásticos, y se encuadra la unidad 121 en la clase de emplazamiento 4 (Tabla 3.4). La unidad 122 se describe como coladas traquibasálticas (Tabla A.7). Siguiendo el criterio de clasificación de las unidades con rocas volcánicas, esta unidad se encuadra en la clase de emplazamiento 1, puesto que presenta traquibasaltos. La unidad 123 corresponde al domo de Montaña Rajada (Tabla A.7). Como un domo es una intrusión, se entiende que está constituido por rocas plutónicas. Dado que estas rocas se consideran indicativas de una resistencia acorde con la de las rocas duras, la unidad 123 se clasifica también en la clase 1. La unidad 124 aflora como coladas fonolíticas, con rocas volcánicas (fonolitas) que presentan fracturación (grietas), según la descripción extensa de GEODE (2014). Se considera que las rocas volcánicas fracturadas tienen una resistencia media, por lo que la unidad 124 es encuadrada en la clase de emplazamiento 2 (Tabla 3.4) como rocas de resistencia media.

Erupciones postcaldera En este apartado se describe el proceso de clasificación de las unidades restantes de las erupciones postcaldera, para las cuales la leyenda cronoestratigráfica de la cartografía GEODE no establece su pertenencia a ningún subgrupo (Tabla 3.38). Los códigos de estas unidades son 125, 126, 127, 128, 129 y 130 .

La unidad 125 se describe como derrubios y zona de alteración en la ladera $\mathrm{N}$ del Teide (Tabla A.7). Los derrubios proceden de la alteración de piroclastos y afloran 
sueltos, como indica la descripción litológica extensa de GEODE (2014). Por tanto, constituyen un suelo de baja compacidad, y la resistencia de la unidad 125 se infiere como equivalente a la de un suelo blando o de baja consistencia, clasificable en la clase de emplazamiento 6 (Tabla 3.4). La unidad 126 es descrita como centro de emisión fonolítico (Tabla A.7), por lo que constituye un depósito piroclástico clasificable en la clase de emplazamiento 4. La unidad 127 se compone de pumitas de dispersión (Tabla A.7), que forman depósitos piroclásticos. Por tanto, la unidad 127 también se clasifica en la clase 4 . La unidad 128 corresponde al cono superior del Teide (Tabla A.7) y también forma un depósito piroclástico, por lo que se clasifica igualmente en la clase 4.

La unidad 129 es descrita como coladas traquifonolíticas (Tabla A.7). Al incluir rocas volcánicas, de composición traquifonolítica, la unidad se clasifica en la clase de emplazamiento 1 (Tabla 3.4). La unidad 130 se describe como depósitos hidromagmáticos, que son depósitos de naturaleza piroclástica y, generalmente, de compacidad media al contener piroclastos de tamaño pequeño (lapillis). Por tanto, se adopta un criterio conservador y se infiere que la consistencia de la unidad 130 es media, y que su resistencia es equiparable a la de la clase de emplazamiento 5, de acuerdo con su descripción geotécnica como suelos de consistencia media (Tabla 3.4). Por tanto, la unidad 130 se agrupa en la clase 5.

Eje NE o de La Dorsal (Rocas filonianas y subvolcánicas) La unidad 132 se describe como domos y diques traquíticos (Tabla A.7). Esta intrusiones contienen rocas de composición traquítica que les confieren una resistencia alta, que es equivalente a la de las rocas duras que caracterizan la clase de emplazamiento 1 (Tabla 3.4).

Eje NE o de La Dorsal (Erupciones Inferiores) La unidad 133 es descrita como coladas basálticas con piroclastos intercalados (Tabla A.7). Dado que se presentan rocas volcánicas (basaltos) junto con piroclastos, se infiere una resistencia global para la unidad menor que la resistencia de las rocas duras (clase de emplazamiento 1). Por este motivo, la unidad 133 se clasifica en la clase de emplazamiento 2 como rocas de resistencia media (Tabla 3.4). La unidad 134 se compone de piroclastos basálticos, por lo cual se aplica el criterio de clasificación de las unidades con depósitos piroclásticos en la clase de emplazamiento 4, como suelos duros.

Eje NE o de La Dorsal (Fisura de Colmenar-Llanos de Los Infantes) La unidad 135 consiste en coladas basálticas, según la Tabla A.7. A partir de las rocas volcánicas presentes (basaltos), la unidad se clasifica en la clase de emplazamiento 1 (Tabla 3.4). La unidad 136 consta de piroclastos basálticos (bombas y lapillis), según la descripción litológica extensa de GEODE (2014), por lo cual se considera su resistencia equiparable a la de un suelo duro, y se clasifica en la clase de emplazamiento 4 .

Eje NE o de La Dorsal (Erupciones Superiores) La unidad 137 se describe como coladas basálticas (Tabla A.7). Como la unidad presenta rocas volcánicas (basaltos), se clasifica en la clase de emplazamiento 1 (Tabla 3.4). La unidad 138 se 
compone de piroclastos basálticos, por tanto se aplica el criterio de clasificación de las unidades con depósitos piroclásticos en la clase de emplazamiento 4 (Tabla 3.4). La unidad 139 consta de piroclastos basálticos finos de dispersión (Tabla A.7). De acuerdo con la descripción litológica extensa de GEODE (2014), la unidad forma depósitos piroclásticos de lapillis y cenizas. La presencia de estas granulometrías finas recomienda considerar una compacidad/consistencia media para la unidad 139, por lo cual su resistencia es equiparable a la de los suelos de consistencia media que caracterizan la clase de emplazamiento 5 (Tabla 3.4). En este sentido, la unidad 139 es clasificada en la clase 5. La unidad 140 aflora como coladas de traquitasfonolitas máficas. En función de la presencia de rocas volcánicas (traquitas-fonolitas), la unidad se clasifica en la clase de emplazamiento 1 como rocas duras.

La unidad 141 se describe como coladas de fonolitas máficas (Tabla A.7). Por tanto, se adopta el criterio de clasificación de las unidades con rocas volcánicas (fonolitas) en la clase de emplazamiento 1 (Tabla 3.4). La unidad 142 consta de piroclastos de traquibasaltos y fonolitas máficas, por tanto, la unidad se clasifica en la clase de emplazamiento 4 (Tabla 3.4) como suelos duros. La unidad 143 es descrita como coladas de traquibasaltos y fonolitas máficas, por lo cual se clasifica en la clase de emplazamiento 1 al presentar las rocas volcánicas traquibasaltos y fonolitas. La unidad 144 consiste en depósitos de limos y arenas finas, que alternan con arenas gruesas, según la descripción litológica extensa de GEODE (2014). En función del bajo tamaño de grano de los sedimentos (limos, arenas finas), la compacidad/consistencia de la unidad se infiere como media. Por tanto, la unidad se clasifica en la clase de emplazamiento 5 (Tabla 3.4).

La unidad 145 forma depósitos de barranco intercalados entre coladas (Tabla A.7), que GEODE (2014) describe compuestos por gravas y arenas, con matriz arenoso-arcillosa. Como el tamaño mínimo de grano de los sedimentos es igual al de las arenas, se infiere que la compacidad de los depósitos es alta, y por tanto que la resistencia global de la unidad 145 es equivalente a la resistencia de la clase de emplazamiento 4 (Tabla 3.4). Esta conclusión se apoya también en la contribución de la matriz arenoso-arcilloso a la compacidad general de los depósitos. La unidad 146 se describe como depósitos de avalanchas "debris flow". Como indica la descripción litológica extensa de GEODE (2014), estos depósitos contienen materiales fragmentarios fuertemente heterométricos, con tamaños que alcanzan al tamaño de los bloques. Se entiende, en este caso, que la compacidad/consistencia global de la unidad es alta, y ésta se clasifica en la clase de emplazamiento 4. La unidad 147 aflora como arenas con estratificación cruzada (Tabla A.7). Al constituir depósitos con sedimentos de tamaño arena, la unidad se clasifica en la clase de emplazamiento 4 como suelos duros.

Eje SE-NO (Erupciones Inferiores) La unidad 148 se describe como suelos antiguos del Pleistoceno Superior, de acuerdo con la Tabla A.7. Según la descripción litológica extensa de GEODE (2014), están formados por alteración de materiales sálicos. Se infiere que su resistencia es equivalente a la de un suelo con compacidad muy alta. Por tanto, a partir de la descripción geotécnica de la clase de emplazamiento 3 como suelos muy densos (Tabla 3.4), la unidad 148 se clasifica en esta clase. La unidad 149 consiste en coladas basálticas, que al presentar rocas volcánicas (basaltos), permite la clasificación de la unidad en la clase de emplazamiento 1 
(Tabla 3.4). La unidad 150 consta de piroclastos basálticos (Tabla A.7), por lo que se sigue el criterio de clasificación ya establecido para los depósitos piroclásticos, y se clasifica la unidad 150 en la clase de emplazamiento 4 (Tabla 3.4).

Eje SE-NO (Erupciones Superiores) La unidad 151 es descrita como coladas basálticas y traquibasálticas (Tabla A.7). Debido al afloramiento de rocas volcánicas (basaltos y traquibasaltos), la unidad se clasifica en la clase de emplazamiento 1 (Tabla 3.4). La unidad 152 se compone de piroclastos basálticos, por lo cual se clasifica en la clase de emplazamiento 4 (Tabla 3.4) como suelos duros.

Erupciones fuera de los ejes principales Las unidades 153 y 155 constan de piroclastos basálticos y traquibasálticos, respectivamente (Tabla A.7). Por tanto, se aplica el criterio de clasificación de las unidades con depósitos piroclásticos, para encuadrar ambas unidades en la clase de emplazamiento 4 (Tabla 3.4). Por su parte, las unidades 154 y 156 se describen como coladas basálticas y traquibasálticas-tefríticas, respectivamente (Tabla A.7). Como ambas unidades presentan rocas volcánicas (basaltos en el caso de la unidad 154 y rocas volcánicas de composición traquibasálticatefrítica en la unidad 156), se clasifican en la clase de emplazamiento 1 (Tabla 3.4).

Erupciones costeras y periféricas (mayoritariamente sálicas) (Erupciones hidromagmáticas) De acuerdo con la leyenda cronoestratigráfica de la cartografía de la región GEODE 2913, las erupciones costeras y periféricas (mayoritariamente sálicas) agrupan las unidades comprendidas entre la unidad 157 y la unidad 164 (Tabla A.7). Las unidades pertenecientes al subgrupo de erupciones hidromagmáticas, de acuerdo con la leyenda cronoestratigráfica, son las unidades con código 157, 158 y 159 (Tabla 3.38).

La unidad 157 consiste en tobas traquíticas y fonolíticas (Tabla A.7). Como las tobas son depósitos piroclásticos, la unidad se clasifica en la clase de emplazamiento 4 (Tabla 3.4). Empleando el mismo criterio de clasificación, la unidad 158 también se encuadra en la clase 4, pues su descripción litológica se expresa en términos de piroclastos basálticos (Tabla A.7). La unidad 159 se describe como coladas basálticas, por tanto, al contener rocas volcánicas (basaltos), se clasifica en la clase de emplazamiento 1 (Tabla 3.4).

Erupciones costeras y periféricas (mayoritariamente sálicas) En este apartado se describe el proceso de clasificación de las unidades agrupadas en las erupciones costeras y periféricas (mayoritariamente sálicas) y que no pertenecen a ningún subgrupo, según la leyenda cronoestratigráfica de la cartografía GEODE (Tabla 3.38). Los códigos de estas unidades son 160, 161, 162, 163 y 164.

La unidad 160 se describe como coladas sálicas (Tabla A.7). De acuerdo con la descripción litológica extensa de GEODE (2014), estas coladas presentan rocas volcánicas (fonolitas, traquitas) con fracturación (disyunción columnar). Por tanto, se adopta el criterio de clasificación de las unidades con rocas volcánicas fracturadas en la clase de emplazamiento 2 (Tabla 3.4), como rocas de resistencia media. La unidad 161 consiste en coladas sálicas con recubrimiento de pumitas (Tabla A.7). De acuerdo con GEODE (2014), las coladas contienen fonolitas (rocas volcánicas) junto 
con pumitas (depósitos piroclásticos), los cuales disminuyen la resistencia global de la unidad, desde la propia de las rocas duras de la clase de emplazamiento 1 hasta la de las rocas de resistencia media de la clase de emplazamiento 2. La unidad 162 es descrita como piroclastos sálicos, por tanto forman depósitos piroclásticos que, de acuerdo al criterio establecido al efecto, se clasifican en la clase de emplazamiento 4 (Tabla 3.4) como suelos duros.

La unidad 163 se compone de tobas y brechas sálicas (Tabla A.7). A pesar de la presencia de las brechas, se aplica un criterio conservador para considerar que las tobas, como depósitos piroclásticos, determinan que la resistencia de la unidad 163 es equiparable a la resistencia de los suelos duros que caracterizan la clase de emplazamiento 4 (Tabla 3.4). Por tanto, esta unidad se clasifica en la clase 4. La unidad 164 forma el domo de Lomo de Simón (Tabla A.7). Dado que la descripción litológica extensa de GEODE (2014) indica que afloran rocas volcánicas (fonolitas y traquitas), la unidad se clasifica en la clase de emplazamiento 1 (Tabla 3.4).

Eje NO La unidad 165 se describe como coladas basálticas y traquibasálticas (Tabla A.7). En función de la presencia de rocas volcánicas (basaltos y traquibasaltos), la unidad se clasifica en la clase de emplazamiento 1 (Tabla 3.4). La unidades 166 y 168 se componen de piroclastos basálticos y traquibasálticos, y de piroclastos sálicos, respectivamente (Tabla A.7). Ambas unidades configuran depósitos piroclásticos clasificables en la clase de emplazamiento 4 (Tabla 3.4), de acuerdo al criterio establecido para este tipo de depósitos. La unidad 167 aflora en coladas y pumitas encalichadas (Tabla A.7). Entendiendo que la unidad está compuesta por coladas de roca volcánica, al encontrarse éstas junto con piroclastos (pumitas), se considera que la resistencia global de la unidad disminuye, y por tanto que la unidad pasa de clasificarse en la clase de emplazamiento 1 como roca dura a agruparse en la clase de emplazamiento 2 como roca de resistencia media.

La unidades 169 y 172 son descritas como coladas de traquitas-fonolitas máficas, y como coladas basálticas y traquibasálticas, respectivamente (Tabla A.7). En ambas unidades, las coladas presentan rocas volcánicas: traquitas-fonolitas en la unidad 169 y basaltos y traquibasaltos en la unidad 172. Por este motivo, las dos unidades se clasifican en la clase de emplazamiento 1 (Tabla 3.4). Las unidades 170 y 171 constan de piroclastos de traquitas-fonolitas máficas, y de piroclastos basálticos y traquibasálticos, respectivamente. En consecuencia, se adopta el criterio de clasificación de las unidades con depósitos piroclásticos, y ambas unidades se agrupan en la clase de emplazamiento 4 (Tabla 3.4).

La unidad 173 se compone de sedimentos indiferenciados, según la Tabla A.7. De acuerdo con la descripción litológica extensa de GEODE (2014), en esta unidad se observan depósitos sedimentarios de lapillis, arenas y cantos. A partir de la granulometría del sedimento (arena, canto), con tamaño de grano igual o superior al de las arenas, se infiere que la compacidad/consistencia de los depósitos es alta, y por tanto que la resistencia global de la unidad es equivalente a la resistencia de los suelos duros que describen geotécnicamente la clase de emplazamiento 4 (Tabla 3.4). Por tanto, la unidad 173 se encuadra en la clase 4. La unidad 174 consiste en piroclastos traquibasálticos, por tanto se clasifica en la clase de emplazamiento 4. La unidad 175 es descrita como coladas de traquibasaltos y traquitas-fonolitas máficas, 
con basaltos subordinados (Tabla A.7). Dado que la unidad presenta rocas volcánicas (traquibasaltos, traquitas-fonolitas), se clasifica en la clase de emplazamiento 1 (Tabla 3.4). La unidad 176 forma coladas de pequeños edificios al E de Montaña Reventada (Tabla A.7). Considerando que la unidad está constituida por coladas de roca volcánica, se clasifica en la clase de emplazamiento 1.

Las unidades 177 y 178 corresponden a piroclastos de distintas composiciones (Tabla A.7). Al formar depósitos piroclásticos, ambas unidades se clasifican de acuerdo con el criterio de clasificación empleado para este tipo de depósitos, y se agrupan consecuentemente en la clase de emplazamiento 4 (Tabla 3.4). La unidad 179 se describe como piroclastos sobre coladas. De acuerdo con la descripción litológica extensa de GEODE (2014), muchos de los piroclastos han sido arrastrados, pero disminuyen la resistencia la roca volcánica aflorante en las coladas. Por tanto, la resistencia global de la unidad corresponde a la de las rocas de resistencia media que caracterizan la clase de emplazamiento 2. La unidad 180 consiste en coladas basálticas y traquibasálticas (Tabla A.7). En función la presencia de rocas volcánicas (basaltos y traquibasaltos), esta unidad se clasifica en la clase de emplazamiento 1 (Tabla 3.4).

La unidad 181 consta de piroclastos basálticos (Tabla A.7). En este caso, se adopta el criterio de clasificación de las unidades con depósitos piroclásticos en la clase de emplazamiento 4 (Tabla 3.4). La unidad 182 es descrita como coladas basálticas y presenta rocas volcánicas (basaltos), por tanto se clasifica en la clase de emplazamiento 1 (Tabla 3.4). La unidad 183 se describe como playa fósil, con edad Pleistoceno Superior (Tabla A.7). Al conformar depósitos de arenas, se considera que la compacidad/consistencia de la unidad es alta, y por tanto que su resistencia global es equivalente a la resistencia de la clase de emplazamiento 4. Como resultado, la unidad 183 se clasifica en la clase 4 .

Erupciones subhistóricas (Volcán de Güimar) La unidad 184 se compone de piroclastos basálticos, según la Tabla A.7. Al configurar depósitos piroclásticos, la unidad se clasifica en la clase de emplazamiento 4 (Tabla 3.4) como suelos duros, de acuerdo al criterio establecido. La unidad 185 consiste en piroclastos basálticos finos de dispersión (Tabla A.7). La descripción litológica extensa de GEODE (2014) precisa que estos piroclastos son de clase lapilli, considerándose que esta fina granulometría implica que la compacidad/consistencia de la unidad es media. Por tanto, y en función de la descripción geotécnica de la clase de emplazamiento 5 como suelos de consistencia media (Tabla 3.4), se equipara la resistencia de la unidad 185 a la resistencia de esta clase. La unidad 186 se describe como coladas basálticas (Tabla A.7), y al presentarse rocas volcánicas (basaltos) se clasifica la unidad en la clase de emplazamiento 1 (Tabla 3.4).

Erupciones subhistóricas (Volcán de las Arenas) La unidad 187 consta de piroclastos de dispersión con granulometría fina, de acuerdo con la descripción litológica extensa de GEODE (2014). Aplicando el mismo criterio que el utilizado en la clasificación de la unidad 185, la resistencia de la unidad 187 se infiere como equivalente a la de los suelos de consistencia media que describen geotécnicamente la clase de emplazamiento 5 (Tabla 3.4). La unidad 188 consiste en piroclastos basálticos (Tabla A.7), por lo cual se adopta el criterio general de clasificación de las unidades 
con depósitos piroclásticos, y se encuadra la unidad en la clase de emplazamiento 4 (Tabla 3.4). La unidad 189 se describe como coladas basálticas-traquibasálticas, y al contener rocas volcánicas (basaltos y traquibasaltos), se clasifica en la clase de emplazamiento 1 (Tabla 3.4).

Erupciones subhistóricas (Volcán de Arafo) La unidad 190 se describe como piroclastos basálticos (Tabla A.7). Como la unidad forma depósitos piroclásticos, se agrupa en la clase de emplazamiento 4 (Tabla 3.4). La unidad 191 corresponde a coladas basálticas, por lo que se clasifica en la clase de emplazamiento 1, en función de la presencia de rocas volcánicas (basaltos).

Erupciones subhistóricas (Volcán de Montaña Reventada) La unidad 192 se compone de piroclastos traquíticos (Tabla A.7), por tanto se adopta el criterio de clasificación de las unidades con depósitos piroclásticos en la clase de emplazamiento 4 (Tabla 3.4). La unidad 193 consiste en coladas de traquitas máficas. En función del afloramiento de rocas volcánicas (traquitas), la unidad se clasifica en la clase de emplazamiento 1.

Erupciones históricas (Erupciones del siglo XIV, Erupción fisural del Taoro) La unidad 194 consta de piroclastos (Tabla A.7), siendo posible su clasificación como depósitos piroclásticos en la clase de emplazamiento 4 (Tabla 3.4). La unidad 195 se describe como coladas traquibasálticas que, al presentar rocas volcánicas (traquibasaltos), permiten la clasificación de la unidad en la clase de emplazamiento 1 .

Erupciones históricas (Erupciones del siglo XVIII, Fisura eruptiva de Siete Fuentes-Fasnia Güimar (1704-05)) La unidad 196 se describe como piroclastos basálticos (Tabla A.7), por lo que forma depósitos piroclásticos clasificables en la clase de emplazamiento 4 (Tabla 3.4) como suelos duros. La unidad 197 es descrita como piroclastos finos. En este caso, se adopta el criterio de clasificación ya establecido para las unidades con piroclastos de granulometría fina (clase lapilli o ceniza), en el sentido de considerar que la consistencia de la unidad es media, y que su resistencia es equivalente a la de la clase de emplazamiento 5, de acuerdo con su descripción geotécnica como suelos de consistencia media(Tabla 3.4). La unidad 198 consiste en coladas basálticas (Tabla A.7), por tanto se aplica el criterio de clasificación de las unidades con rocas volcánicas (basaltos en la unidad 198) en la clase de emplazamiento 1 (Tabla 3.4).

Erupciones históricas (Erupciones del siglo XVIII, Erupción de Montaña Negra o Garachico (1706)) La unidad 199 se compone de piroclastos basálticos (Tabla A.7). Se adopta, por tanto, el criterio de clasificación de las unidades con depósitos piroclásticos en la clase de emplazamiento 4 (Tabla 3.4). La unidad 200 consiste en coladas basálticas. Dada la presencia de rocas volcánicas (basaltos), la unidad se clasifica en la clase de emplazamiento 1 (Tabla 3.4). 
Erupciones históricas (Erupciones del siglo XVIII, Erupción del Chahorra o Narices del Teide (1798)) La unidad 201 es descrita como piroclastos basálticos (Tabla A.7), por lo que se clasifica en la clase de emplazamiento 4 (Tabla 3.4). La unidad 202 consiste en coladas basálticas (Tabla A.7), y en función de las rocas volcánicas existentes (basaltos), se clasifica en la clase de emplazamiento 1.

Erupciones históricas (Erupciones del siglo XX, Erupción del Chinyero (1909)) La unidad 203 se describe como piroclastos basálticos (Tabla A.7), por tanto se clasifica en la clase de emplazamiento 4 (Tabla 3.4). La unidad 204 es descrita como coladas basálticas. Dado que presenta rocas volcánicas (basaltos), se clasifica en la clase de emplazamiento 1.

Depósitos sedimentarios cuaternarios La unidad 205 se describe como conos de deyección antiguos (Tabla A.7). A partir de esta descripción litológica, se entiende que la compacidad/consistencia global de la unidad es alta, siendo equiparable su resistencia a la de la clase de emplazamiento 4, pues ésta se describe geotécnicamente como suelos duros o de alta consistencia (Tabla 3.4). La unidad 206 es descrita como aluviales antiguos y terrazas. Se considera que estos depósitos tienen una compacidad/consistencia alta, y que su resistencia es equiparable a la de la clase de emplazamiento 4. La unidad 207 corresponde a depósitos de deslizamiento, con bloques y arenas, de acuerdo a la descripción litológica extensa de GEODE (2014). A partir de la gruesa granulometría de los depósitos (bloque, arena), se infiere que la compacidad/consistencia de la unidad es alta, y que, por tanto, su resistencia es equiparable a la resistencia de la clase de emplazamiento 4. Consecuentemente, se clasifica la unidad 207 en la clase 4. La unidad 208 se describe como depósitos de ladera y de conos de deyección (Tabla A.7). De acuerdo con la descripción litológica extensa de GEODE (2014), los depósitos de ladera presentan bloques y cantos entremezclados con matriz fina de arenas y arcillas. Ambos tipos de depósitos presentan una compacidad alta, y por tanto una resistencia equiparable a la de la clase de emplazamiento 4 .

Según la descripción litológica extensa de GEODE (2014), la unidad 209 forma depósitos sedimentarios indiferenciados, con bloques, gravas, arenas y arcillas. En base a la presencia de granulometrías iguales o superiores al tamaño arena (grava, arena), se infiere que la compacidad/consistencia global de la unidad es alta, y que por tanto su resistencia es acorde con la de los suelos duros que caracterizan la clase de emplazamiento 4 (Tabla 3.4). La unidad 210 se describe como depósitos pumíticos sedimentarios (Tabla A.7). Considerando que los depósitos de pumita son depósitos piroclásticos, se aplica el criterio establecido y se clasifica la unidad en la clase de emplazamiento 4. La unidad 211 configura dunas y médanos (Tabla A.7), los cuales son descritos por GEODE (2014) como depósitos de arenas eólicas cementadas. En función del tamaño de grano de las arenas y de su cementación, se considera que la compacidad/consistencia global de estos depósitos es alta. En consecuencia, la unidad 211 se clasifica en la clase de emplazamiento 4 (Tabla 3.4). La unidad 212 se describe como suelos rojos de alteración piroclástica, que se consideran consiguientemente como depósitos piroclásticos. Por este motivo se adopta el criterio de 
clasificación establecido para este tipo de depósitos, y se clasifica la unidad 212 en la clase de emplazamiento 4 .

La unidad 213 se describe como depósitos aluviales y de fondo de valle (Tabla A.7). De acuerdo con la descripción litológica extensa de GEODE (2014), estos depósitos están constituidos por gravas, arenas, arcillas y limos. La presencia de sedimentos finos de clase limo y arcilla implica una reducción de la resistencia que aportan los sedimentos gruesos (grava, arena), de forma que la resistencia global se equipara a la de los suelos de consistencia media de la clase de emplazamiento 5 (Tabla 3.4). La unidad 214 consiste en depósitos de ladera indiferenciados, que contienen bloques, gravas, arenas y arcillas (GEODE, 2014). A partir de la presencia de granulometrías iguales o superiores al tamaño arena (grava, arena), se considera que la compacidad/consistencia global de la unidad es alta, y que su resistencia es equiparable a la de la clase de emplazamiento 4 (Tabla 3.4). La unidad 215 se describe como suelos (Tabla A.7), aunque GEODE (2014) precisa que la unidad forma depósitos arenoso-arcillosos. A partir de la presencia de los sedimentos de arena, la compacidad/consistencia global de la unidad se considera alta, y su resistencia equiparable a la resistencia de la clase de emplazamiento 4. La unidad 216 consiste en sedimentos lacustres de limos arcillosos. En base a la fina granulometría de estos sedimentos (limo), la consistencia de la unidad es media, y por tanto ésta se clasifica en la clase de emplazamiento 5 (Tabla 3.4).

De acuerdo con la Tabla A.7, la unidad 217 configura depósitos de barranco/ rambla. La descripción litológica extensa de GEODE (2014) indica que estos depósitos corresponden a conos de deyección con bloques y cantos. En función de la gruesa granulometría de los clastos (bloque, canto), superior al tamaño de las arenas, se infiere que la resistencia de la unidad es equiparable a la resistencia de la clase de emplazamiento 4 (Tabla 3.4). La unidad 218 aflora como playas de arenas y cantos (Tabla A.7). Dado que la unidad forma depósitos de playa, se considera que su compacidad/consistencia global es baja, a pesar de que los tamaños de grano presentes son al menos equivalentes al tamaño arena. En función de la descripción geotécnica de la clase de emplazamiento 6 (Tabla 3.4) como suelos blandos, o de baja consistencia, la unidad 218 se clasifica en esta clase. Finalmente, la unidad 219, descrita como depósitos antrópicos, también se clasifica en la clase 6, pues, adoptando un criterio conservador, se considera que la compacidad/consistencia de este tipo de depósitos es baja.

En la Tabla 3.39 se resumen las clases de emplazamiento que corresponden a las unidades de Tenerife, de acuerdo con el procedimiento de clasificación que se ha explicado. 
Tabla 3.39: Resumen de la clasificación de las unidades del mapa geológico continuo GEODE a escala 1:50.000 de Tenerife (Zona 2913), de acuerdo a la clasificación de síntesis. Tomado de GEODE (2014), elaboración propia.

\begin{tabular}{|c|c|c|c|}
\hline \multicolumn{2}{|c|}{$\begin{array}{l}\text { Sistema/Dominio/Edificio/Ciclo volcánico/Macizo } \\
\text { plutónico/Formación/Miembro }\end{array}$} & $\begin{array}{l}\text { Código de la } \\
\text { unidad geológica }\end{array}$ & $\begin{array}{l}\text { Clase de } \\
\text { emplazamiento }\end{array}$ \\
\hline \multirow{14}{*}{$\begin{array}{l}\text { Macizo de } \\
\text { Anaga }\end{array}$} & \multirow{10}{*}{$\begin{array}{l}\text { Rocas filonianas y } \\
\text { subvolcánicas } \\
\text { Complejo del Arco de Tagana- } \\
\text { na }\end{array}$} & 3 & 1 \\
\hline & & 4 & 2 \\
\hline & & 5 & 2 \\
\hline & & 6 & 1 \\
\hline & & 7 & 4 \\
\hline & & 8 & 5 \\
\hline & & 9 & 2 \\
\hline & & 10 & 6 \\
\hline & & 11 & 4 \\
\hline & & 12 & 4 \\
\hline & \multirow{2}{*}{ Formación Chamorga } & 13 & 2 \\
\hline & & 14 & 1 \\
\hline & \multirow{2}{*}{$\begin{array}{l}\text { Formación } \\
\text { Chinamada-Batanes }\end{array}$} & 15 & 4 \\
\hline & & 16 & 1 \\
\hline \multirow{3}{*}{$\begin{array}{l}\text { Macizo del } \\
\text { Roque del } \\
\text { Conde }\end{array}$} & \multirow[t]{3}{*}{ Rocas filonianas y subvolcánicas } & 19 & 1 \\
\hline & & 20 & 1 \\
\hline & & 21 & 4 \\
\hline \multirow{13}{*}{$\begin{array}{l}\text { Macizo de } \\
\text { Teno }\end{array}$} & Rocas filonianas y subvolcánicas & 24 & 1 \\
\hline & \multirow{2}{*}{ Edificio inferior } & 25 & 1 \\
\hline & & 26 & 1 \\
\hline & \multirow{5}{*}{ Edificio Carrizales } & 27 & 3 \\
\hline & & 28 & 4 \\
\hline & & 29 & 1 \\
\hline & & 30 & 4 \\
\hline & & 31 & 3 \\
\hline & \multirow{5}{*}{ Formación tabular } & 32 & 3 \\
\hline & & 33 & 2 \\
\hline & & 34 & 4 \\
\hline & & 35 & 1 \\
\hline & & 36 & 1 \\
\hline \multirow{5}{*}{ Edificio Dorsal } & \multirow{2}{*}{ Tramo inferior } & 39 & 1 \\
\hline & & 40 & 4 \\
\hline & \multirow{3}{*}{ Tramo superior } & 41 & 1 \\
\hline & & 42 & 3 \\
\hline & & 43 & 4 \\
\hline \multirow{12}{*}{$\begin{array}{l}\text { Primeros } \\
\text { Edificios } \\
\text { Cañadas }\end{array}$} & & 44 & 1 \\
\hline & Rocas filonianas y subvolcánicas & 45 & 1 \\
\hline & & 46 & 1 \\
\hline & & 47 & 1 \\
\hline & & 48 & 4 \\
\hline & Edificio Cañadas 1, y su destrucción & 49 & 3 \\
\hline & & 50 & 3 \\
\hline & & 51 & 3 \\
\hline & Fdificios $\mathrm{Fl}$ Codro Ucanco inforior & 52 & 1 \\
\hline & Edificios El Cedro, y Ucanca inferior & 53 & 1 \\
\hline & & 54 & 4 \\
\hline & & $54 \mathrm{a}$ & 4 \\
\hline & & 55 & 4 \\
\hline & & 56 & 1 \\
\hline Sorioc $C a \tilde{n} a d a$ & & 57 & 1 \\
\hline Series Canadas & Dominio de las laderas de Cañadas & 58 & 3 \\
\hline & & 59 & 3 \\
\hline & & 60 & 2 \\
\hline & & 61 & 4 \\
\hline & & 62 & 1 \\
\hline & & 63 & 1 \\
\hline Series Cañadas & Dominis do loc lodoros do $\mathrm{Con}$ doc & 64 & 3 \\
\hline Intermedias & Dominio de las laderas de Canadas & 65 & 4 \\
\hline & & 66 & 4 \\
\hline & & 67 & 1 \\
\hline
\end{tabular}


(continuación)

\begin{tabular}{|c|c|c|c|}
\hline \multicolumn{2}{|c|}{$\begin{array}{l}\text { Sistema/Dominio/Edificio/Ciclo volcánico/Macizo } \\
\text { plutónico/Formación/Miembro }\end{array}$} & \multirow{2}{*}{$\begin{array}{l}\text { Código de la } \\
\text { unidad geológica }\end{array}$} & \multirow{2}{*}{$\begin{array}{l}\text { Clase de } \\
\text { emplazamiento }\end{array}$} \\
\hline \multirow{6}{*}{$\begin{array}{l}\text { Series Cañadas } \\
\text { Intermedias }\end{array}$} & \multirow{6}{*}{ Dominio de las laderas de Cañadas } & & \\
\hline & & 69 & 4 \\
\hline & & 70 & 1 \\
\hline & & 71 & 1 \\
\hline & & 72 & 4 \\
\hline & & 73 & 1 \\
\hline \multirow{4}{*}{$\begin{array}{l}\text { Series Cañadas } \\
\text { Intermedias }\end{array}$} & \multirow{4}{*}{ Dominio de Tigaiga } & 74 & 1 \\
\hline & & 75 & 2 \\
\hline & & 76 & 4 \\
\hline & & 77 & 1 \\
\hline \multirow{10}{*}{$\begin{array}{l}\text { Grupo } \\
\text { Guajara y } \\
\text { Edificio Las } \\
\text { Pilas }\end{array}$} & \multirow{8}{*}{ Edificio Las Pilas } & 78 & 2 \\
\hline & & 79 & 2 \\
\hline & & 80 & 1 \\
\hline & & 81 & 1 \\
\hline & & 82 & 4 \\
\hline & & 83 & 2 \\
\hline & & 84 & 1 \\
\hline & & 85 & 1 \\
\hline & \multirow{2}{*}{ Grupo Guajara } & 86 & 4 \\
\hline & & 87 & 1 \\
\hline \multirow{4}{*}{$\begin{array}{l}\text { Edificios y } \\
\text { formaciones } \\
\text { Cañadas }\end{array}$} & \multirow{4}{*}{ Formación Diego Hernández } & 88 & 4 \\
\hline & & 89 & 2 \\
\hline & & 90 & 6 \\
\hline & & 91 & 4 \\
\hline \multirow{14}{*}{$\begin{array}{l}\text { Erupciones } \\
\text { postcaldera }\end{array}$} & \multirow{2}{*}{ Edificio Teide } & 92 & 4 \\
\hline & & 93 & 1 \\
\hline & \multirow{2}{*}{ Emisiones básicas antiguas } & 94 & 4 \\
\hline & & 95 & 1 \\
\hline & & 96 & 4 \\
\hline & Pequeñas emisiones sálicas antiguas & 97 & 1 \\
\hline & & 98 & 1 \\
\hline & Sin asignación de edificio & 99 & 1 \\
\hline & & 100 & 1 \\
\hline & & 101 & 1 \\
\hline & Edificio Pico Viejo & 102 & 4 \\
\hline & & 103 & 1 \\
\hline & & 104 & 4 \\
\hline & Pequenas emisiones sálicas perifericas & 105 & 1 \\
\hline & & 106 & 1 \\
\hline & & 107 & 4 \\
\hline & & 108 & 1 \\
\hline & & 109 & 4 \\
\hline & & 110 & 1 \\
\hline Erupciones & Edificios domáticos sálicos periféricos & 111 & 1 \\
\hline & & 112 & 1 \\
\hline & & 113 & 1 \\
\hline & & 114 & 1 \\
\hline & & 115 & 1 \\
\hline & & 116 & 1 \\
\hline & & 117 & 1 \\
\hline & & 118 & 1 \\
\hline & & 119 & 1 \\
\hline Erupciones & Dominis de Montaña Blanca & 120 & 1 \\
\hline postcaldera & Dominıo de Montana Blanca & 121 & 4 \\
\hline & & 122 & 1 \\
\hline & & 123 & 1 \\
\hline & & 124 & 2 \\
\hline & & 125 & 4 \\
\hline & & 126 & 4 \\
\hline Erupciones & & 127 & 4 \\
\hline postcaldera & & 128 & 4 \\
\hline & & 129 & 1 \\
\hline & & 130 & 5 \\
\hline
\end{tabular}


(continuación)

\begin{tabular}{|c|c|c|c|}
\hline \multicolumn{2}{|c|}{$\begin{array}{l}\text { Sistema/Dominio/Edificio/Ciclo volcánico/Macizo } \\
\text { plutónico/Formación/Miembro }\end{array}$} & \multirow{2}{*}{$\begin{array}{l}\text { Código de la } \\
\text { unidad geológica }\end{array}$} & \multirow{2}{*}{$\begin{array}{l}\begin{array}{l}\text { Clase de } \\
\text { emplazamiento }\end{array} \\
1\end{array}$} \\
\hline \multirow{16}{*}{$\begin{array}{l}\text { Eje NE o de } \\
\text { La Dorsal }\end{array}$} & Rocas filonianas y subvolcánicas & & \\
\hline & \multirow{2}{*}{ Erupciones Inferiores } & 133 & 2 \\
\hline & & 134 & 4 \\
\hline & \multirow{2}{*}{ Fisura de Colmenar-Llanos de Los Infantes } & 135 & 1 \\
\hline & & 136 & 4 \\
\hline & \multirow{11}{*}{ Erupciones Superiores } & 137 & 1 \\
\hline & & 138 & 4 \\
\hline & & 139 & 5 \\
\hline & & 140 & 1 \\
\hline & & 141 & 1 \\
\hline & & 142 & 4 \\
\hline & & 143 & 1 \\
\hline & & 144 & 5 \\
\hline & & 145 & 4 \\
\hline & & 146 & 4 \\
\hline & & 147 & 4 \\
\hline \multirow{5}{*}{ Eje SE-NO } & \multirow{3}{*}{ Erupciones Inferiores } & 148 & 3 \\
\hline & & 149 & 1 \\
\hline & & 150 & 4 \\
\hline & \multirow{2}{*}{ Erupciones Superiores } & 151 & 1 \\
\hline & & 152 & 4 \\
\hline \multirow{4}{*}{\multicolumn{2}{|c|}{ Erupciones fuera de los ejes principales }} & 153 & 4 \\
\hline & & 154 & 1 \\
\hline & & 155 & 4 \\
\hline & & 156 & 1 \\
\hline \multirow{8}{*}{$\begin{array}{l}\text { Erupciones } \\
\text { costeras y } \\
\text { periféricas } \\
\text { (mayoritaria- } \\
\text { mente } \\
\text { sálicas) }\end{array}$} & \multirow{8}{*}{ Erupciones hidromagmáticas } & 157 & 4 \\
\hline & & 158 & 4 \\
\hline & & 159 & 1 \\
\hline & & 160 & 2 \\
\hline & & 161 & 1 \\
\hline & & 162 & 4 \\
\hline & & 163 & 4 \\
\hline & & 164 & 1 \\
\hline \multirow{19}{*}{ Eje NO } & & 165 & 1 \\
\hline & & 166 & 4 \\
\hline & & 167 & 1 \\
\hline & & 168 & 4 \\
\hline & & 169 & 1 \\
\hline & & 170 & 4 \\
\hline & & 171 & 4 \\
\hline & & 172 & 1 \\
\hline & & 173 & 4 \\
\hline & & 174 & 4 \\
\hline & & 175 & 1 \\
\hline & & 176 & 1 \\
\hline & & 177 & 4 \\
\hline & & 178 & 4 \\
\hline & & 179 & 4 \\
\hline & & 180 & 1 \\
\hline & & 181 & 4 \\
\hline & & 182 & 1 \\
\hline & & 183 & 4 \\
\hline \multirow{10}{*}{$\begin{array}{l}\text { Erupciones } \\
\text { subhistóricas }\end{array}$} & \multirow{3}{*}{ Volcán de Güimar } & 184 & 4 \\
\hline & & 185 & 5 \\
\hline & & 186 & 1 \\
\hline & & 187 & 5 \\
\hline & Volcán de las Arenas & 188 & 4 \\
\hline & & 189 & 1 \\
\hline & Volcán de Arafo & 190 & 4 \\
\hline & volcan de Arafo & 191 & 1 \\
\hline & Volcán de Montaña Beyentada & 192 & 4 \\
\hline & volcan de Montana Keventada & 193 & 1 \\
\hline
\end{tabular}




\begin{tabular}{|c|c|c|c|c|}
\hline \multicolumn{3}{|c|}{$\begin{array}{l}\text { Sistema/Dominio/Edificio/Ciclo volcánico/Macizo } \\
\text { plutónico/Formación/Miembro }\end{array}$} & $\begin{array}{l}\text { Código de la } \\
\text { unidad geológica }\end{array}$ & $\begin{array}{l}\text { Clase de } \\
\text { emplazamiento }\end{array}$ \\
\hline \multirow{11}{*}{$\begin{array}{l}\text { Erupciones } \\
\text { históricas }\end{array}$} & \multirow{2}{*}{$\begin{array}{l}\text { Erupciones del } \\
\text { siglo XIV }\end{array}$} & \multirow{2}{*}{ Erupción fisural del Taoro } & 194 & 4 \\
\hline & & & 195 & 1 \\
\hline & \multirow{7}{*}{$\begin{array}{l}\text { Erupciones del } \\
\text { siglo XVIII }\end{array}$} & \multirow{3}{*}{$\begin{array}{l}\text { Fisura eruptiva de Siete } \\
\text { Fuentes-Fasnia Güimar } \\
(1704-05)\end{array}$} & 196 & 4 \\
\hline & & & 197 & 5 \\
\hline & & & 198 & 1 \\
\hline & & \multirow{4}{*}{$\begin{array}{l}\text { Erupción de Montaña Negra o } \\
\text { Garachico (1706) } \\
\text { Erupción del Chahorra o } \\
\text { Narices del Teide (1798) }\end{array}$} & 199 & 4 \\
\hline & & & 200 & 1 \\
\hline & & & 201 & 4 \\
\hline & & & 202 & 1 \\
\hline & \multirow{2}{*}{$\begin{array}{l}\text { Erupciones del } \\
\text { siglo XX }\end{array}$} & \multirow{2}{*}{ Erupción del Chinyero (1909) } & 203 & 4 \\
\hline & & & 204 & 1 \\
\hline \multirow{15}{*}{\multicolumn{3}{|c|}{ Depósitos sedimentarios cuaternarios }} & 205 & 4 \\
\hline & & & 206 & 4 \\
\hline & & & 207 & 4 \\
\hline & & & 208 & 4 \\
\hline & & & 209 & 4 \\
\hline & & & 210 & 4 \\
\hline & & & 211 & 4 \\
\hline & & & 212 & 4 \\
\hline & & & 213 & 6 \\
\hline & & & 214 & 4 \\
\hline & & & 215 & 4 \\
\hline & & & 216 & 5 \\
\hline & & & 217 & 4 \\
\hline & & & 218 & 6 \\
\hline & & & 219 & 6 \\
\hline
\end{tabular}

\subsection{Obtención del mapa de clases de emplazamien- to y Vs30}

Una vez que se ha concluido el proceso de clasificación de las unidades geológicas de la Península Ibérica y las Islas Baleares, y de las unidades geológicas de las Islas Canarias, se ha asignado a cada unidad el intervalo de valores de Vs30 correspondiente a la clase de emplazamiento a la cual pertenece la unidad, de acuerdo con la clasificación de síntesis (Tabla 3.4). En este sentido, debe puntualizarse que la clase de emplazamiento 6 no corresponde a ningún rango de valores de Vs30, pues su descripción geotécnica prevee que los suelos a agrupar en esta clase requieren de investigación geotécnica específica e in situ.

En el caso de la Península y de las Islas Baleares, la asignación de valores de Vs30 a las unidades geológicas del Mapa Geológico de España a escala 1:1.000.000, representadas en la Figura 3.1, ha permitido obtener el mapa de clases de emplazamiento y Vs30 de este territorio (Figura 3.9). Análogamente, la asignación de valores de Vs30 a las unidades correspondientes a la cartografía geológica continua GEODE de las Islas Canarias a escala 1:50.000, representadas en las Figuras 3.2, 3.3, 3.4, 3.5, 3.6, 3.7 y 3.8, ha permitido obtener el mapa de clases de emplazamiento y Vs30 del archipiélago (Figura 3.10). 

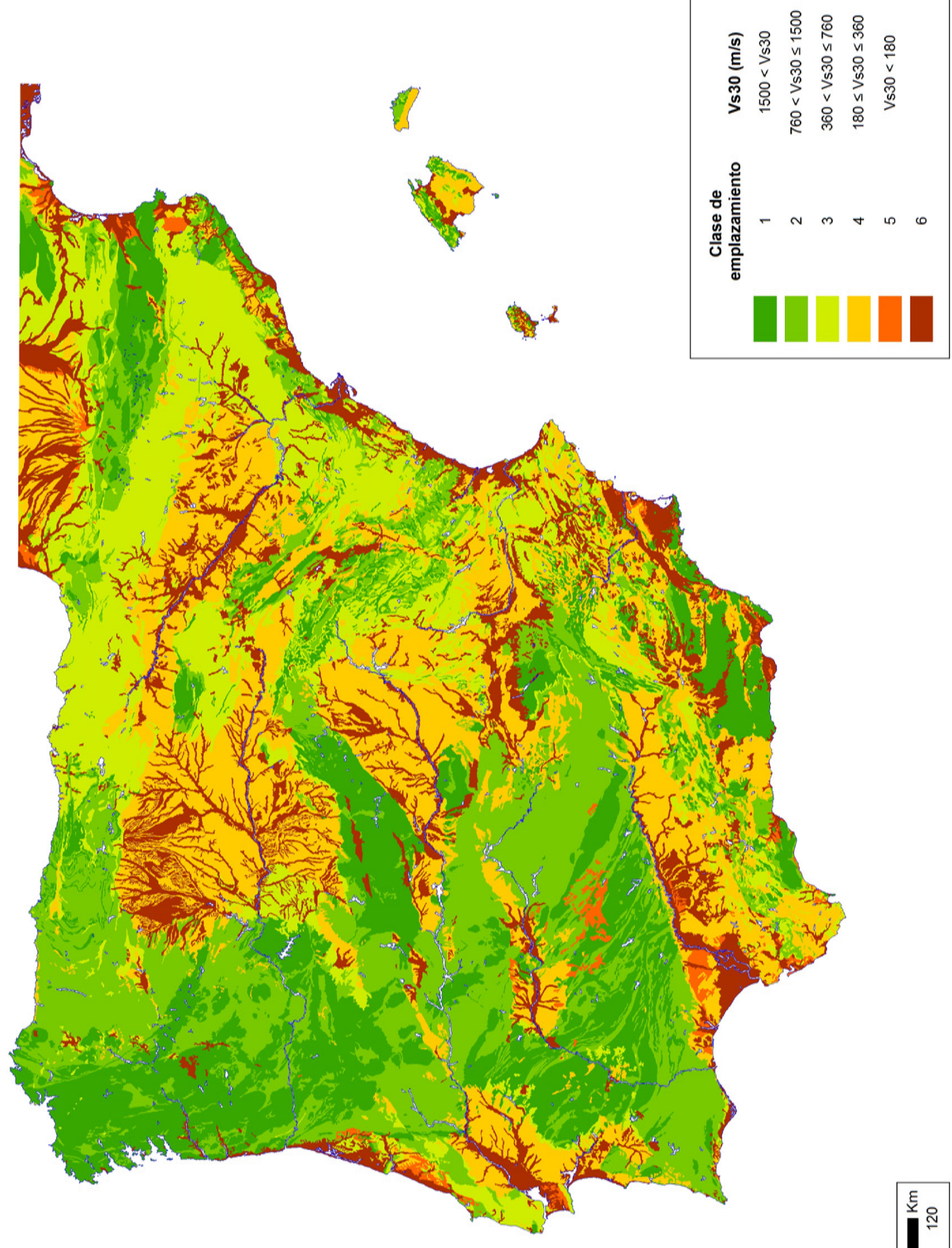

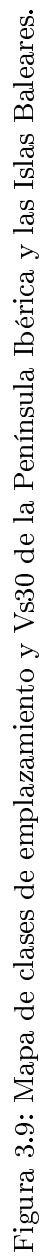




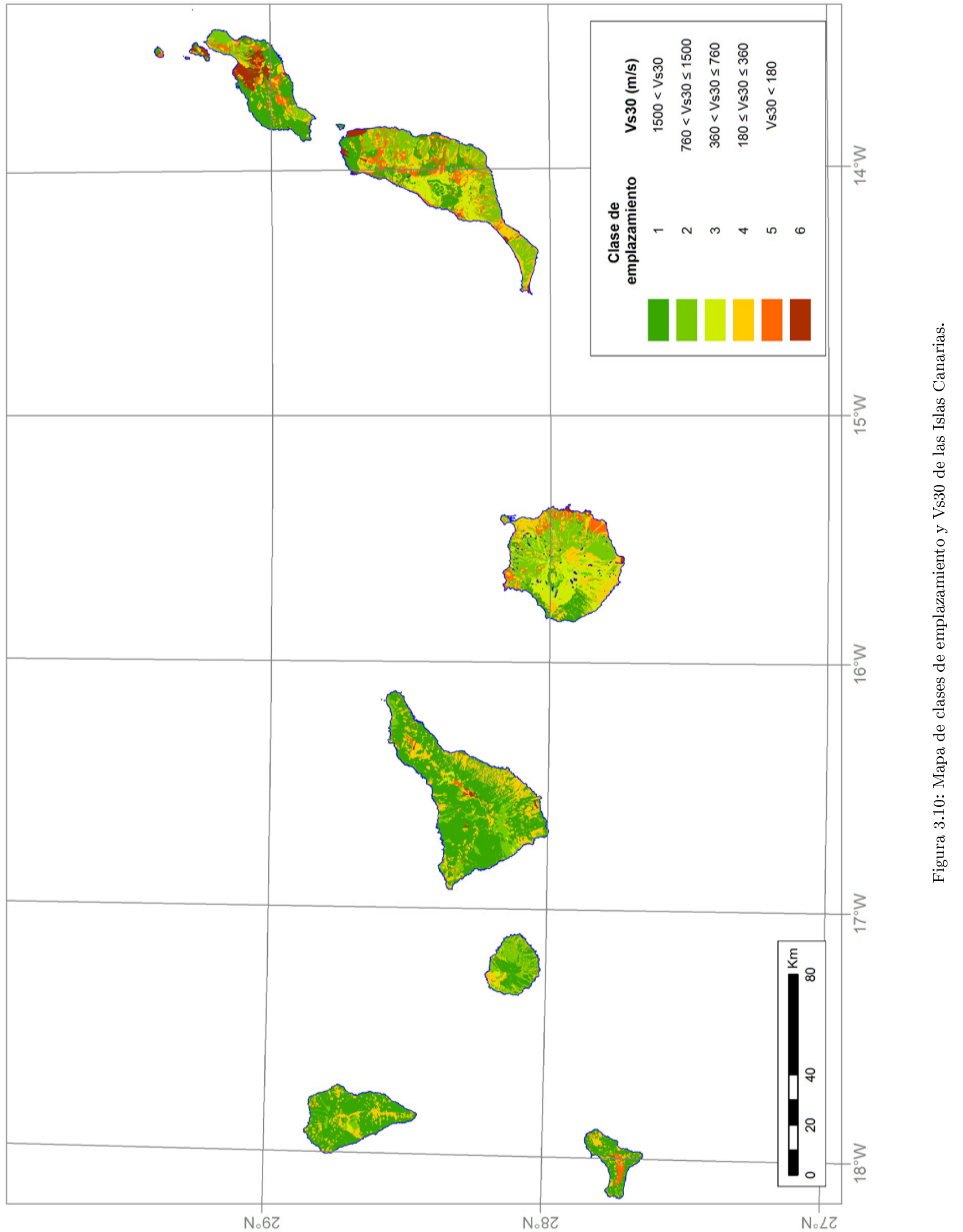




\subsection{Cálculo de factores de amplificación en fun- ción de la frecuencia del movimiento del te- rreno para cada clase de emplazamiento (Bor- cherdt, 1994)}

A partir de los mapas de clases de emplazamiento y Vs30 de la Península Ibérica y las Islas Baleares, y de las Islas Canarias, la producción de la cartografía de amplificación sísmica de cada territorio precisa de la cuantificación de la amplificación de cada una de las clases de emplazamiento de la clasificación de síntesis (Tabla 3.4). Siguiendo la metodología de Borcherdt (1994), esta cuantificación se ha realizado por medio de la estimación del factor de amplificación $\left(F_{a}\right)$ para altas frecuencias $(2-10 \mathrm{~Hz})$ o periodos cortos $(0,1-0,5 \mathrm{~s})$ y del factor de amplificación $\left(F_{v}\right)$ para bajas frecuencias $(0,5-2,5 \mathrm{~Hz})$ o periodos medios $(0,4-2,0 \mathrm{~s})$. De acuerdo con los requerimientos de esta metodología para el cálculo de los dos factores de amplificación, se ha escogido como condición del terreno de referencia la clase de emplazamiento SC-Ib de la clasificación de Borcherdt (1994) (Tabla 2.1), con un valor de Vs30 $\left(v_{0}\right)$ igual a $1050 \mathrm{~m} / \mathrm{s}$. También se ha seleccionado el nivel espectral inicial del movimiento del terreno $(I)$, que depende de la aceleración máxima del terreno que, ocurrido un terremoto, puede experimentar el emplazamiento donde se quiere estimar la amplificación. En este sentido, la elección de $I$ se ha realizado de acuerdo con el mapa de peligrosidad sísmica de España en términos de aceleración sísmica básica, presentado por la Norma de Construcción Sismorresistente NCSE-02 (Ministerio de Fomento, 2003). La aceleración sísmica básica es un valor característico de aceleración horizontal del terreno, y en el presente trabajo se ha considerado que es un valor válido de la aceleración máxima del terreno. De acuerdo con el mapa de peligrosidad sísmica, los valores de aceleración sísmica básica están comprendidos entre $0,04 \mathrm{y} 0,16 \mathrm{~g}$, siendo $\mathrm{g}=9,8 \mathrm{~m} / \mathrm{s}^{2}$. Por esta razón, se ha estimado $0,1 \mathrm{~g}$ como un valor representativo para la Península Ibérica, las Islas Baleares y las Islas Canarias. En consecuencia, se ha seleccionado un valor $I$ de $0,1 \mathrm{~g}$.

Los factores de amplificación $F_{a}$ y $F_{v}$ de cada clase de emplazamiento se han calculado mediante las ecuaciones $7 \mathrm{a}$ y $7 \mathrm{~b}$ de Borcherdt (1994) (ecuaciones $2.1 \mathrm{y}$ 2.2). Para utilizar estas ecuaciones, se requiere conocer, además del valor de Vs30 de la condición del terreno de referencia $\left(v_{0}=1050 \mathrm{~m} / \mathrm{s}\right)$, el valor medio del intervalo de valores de Vs30 de cada clase de emplazamiento $(v)$ y los parámetros $m_{a}$ y $m_{v}$.

Los parámetros $m_{a}$ y $m_{v}$ dependen a su vez de los factores de amplificación para periodos cortos y periodos medios correspondientes a la clase de emplazamiento SCIV de la clasificación de Borcherdt (1994) (Tabla 2.1), y se expresan de acuerdo a las ecuaciones 2c y $2 \mathrm{~b}$ de Borcherdt (1994) (ecuaciones 2.3 y 2.4). No obstante, siguiendo la metodología de Borcherdt (1994), los valores de $m_{a}$ y $m_{v}$ se han seleccionado fácilmente de entre los valores tabulados de la Tabla 2.2, que dependen del valor de $I$ y de la condición del terreno de referencia. Por tanto, con el valor de $I$ ya seleccionado $(0,1 \mathrm{~g})$ y la condición del terreno de referencia escogida (SC-Ib), se han obtenido como valores de $m_{a}$ y $m_{v} 0,35$ y 0,65 , respectivamente.

El valor medio del intervalo de valores de Vs30 de cada clase de emplazamiento $(v)$ se ha obtenido a partir del correspondiente intervalo que figura en la Tabla 3.4. En este sentido, el valor de $v$ se ha obtenido como la media aritmética de los valores 
de Vs30 de los extremos del intervalo, para las clases 2, 3 y 4. Sin embargo, en las clases 1,5 y 6 no consta un intervalo delimitado por dos extremos, motivo por el cual se han estimado valores representativos del rango de valores que toma Vs30 las citadas clases, que se asumen como valores medios de los citados rangos. De esta forma, en la Tabla 3.40 se pueden consultar los 6 valores de $v$ para las 6 clases de la Tabla 3.4.

Finalmente, por medio de las ecuaciones 2.1 y 2.2 , y de los valores de $v_{0}, v, m_{a}$ y $m_{v}$, se han calculado los factores de amplificación $F_{a}$ y $F_{v}$ de cada clase de emplazamiento de la clasificación de síntesis. En la Tabla 3.40 se presentan los resultados de $F_{a}$ y $F_{v}$ de cada clase, observándose que ambos factores de amplificación aumentan desde la clase 1 (rocas duras, Tabla 3.4) hasta la clase 6 (suelos blandos, Tabla 3.4).

Tabla 3.40: Valores medios del intervalo de valores de Vs $30(v)$, fac-
tor de amplificación para periodos cortos $\left(F_{a}\right)$ y factor de amplifi-
cación para periodos medios $\left(F_{v}\right)$ de cada clase de emplazamiento
de la clasificación de síntesis (Tabla 3.4$)$.
\begin{tabular}{|c|r|r|r|}
\hline Clase de emplazamiento & $v \mathbf{( m / s )}$ & $F_{a}$ & $F_{v}$ \\
\hline 1 & 1600 & 0,86 & 0,76 \\
\hline 2 & 1130 & 0,97 & 0,95 \\
\hline 3 & 560 & 1,25 & 1,50 \\
\hline 4 & 270 & 1,61 & 2,42 \\
\hline 5 & 160 & 1,93 & 3,40 \\
\hline 6 & 130 & 2,08 & 3,89 \\
\hline
\end{tabular}

\subsection{Obtención del mapa de amplificación sísmica}

El mapa de amplificación sísmica de cada uno de los territorios en estudio (Península Ibérica e Islas Baleares, e Islas Canarias) se obtiene por medio de la asignación a cada unidad geológica de los factores de amplificación $F_{a}$ y $F_{v}$ de la clase de emplazamiento a la que pertenece. De esta forma, el mapa permite cuantificar, en cualquier punto, la amplificación sísmica por medio de ambos factores.

Los mapas de clases de emplazamiento y Vs30 de la Península Ibérica y las Islas Baleares (Figura 3.9) y de las Islas Canarias (Figura 3.10) ya representan la clasificación de las unidades geológicas, y por tanto permiten obtener directamente la distribución espacial de los dos factores de amplificación, por medio de la correspondencia entre cada clase y los valores de los factores de amplificación asociados a la misma (Tabla 3.40). En consecuencia, el mapa de amplificación sísmica de la Península Ibérica y las Islas Baleares (Figura 3.11) se ha generado a partir del mapa de clases de emplazamiento del territorio. Como se observa en la Figura 3.11, en una primera aproximación los factores de amplificación son coherentes con la resistencia de las rocas o suelos de cada punto del mapa. Por ejemplo, los suelos blandos de las cuencas fluviales son representados como zonas de alta amplificación, mientras que las rocas duras de las cordilleras montañosas son representadas como zonas de baja amplificación. Aplicando el mismo proceso, el mapa de amplificación sísmica de las Islas Canarias (Figura 3.12) ha sido producido a partir del mapa de clases de emplazamiento del archipiélago. 


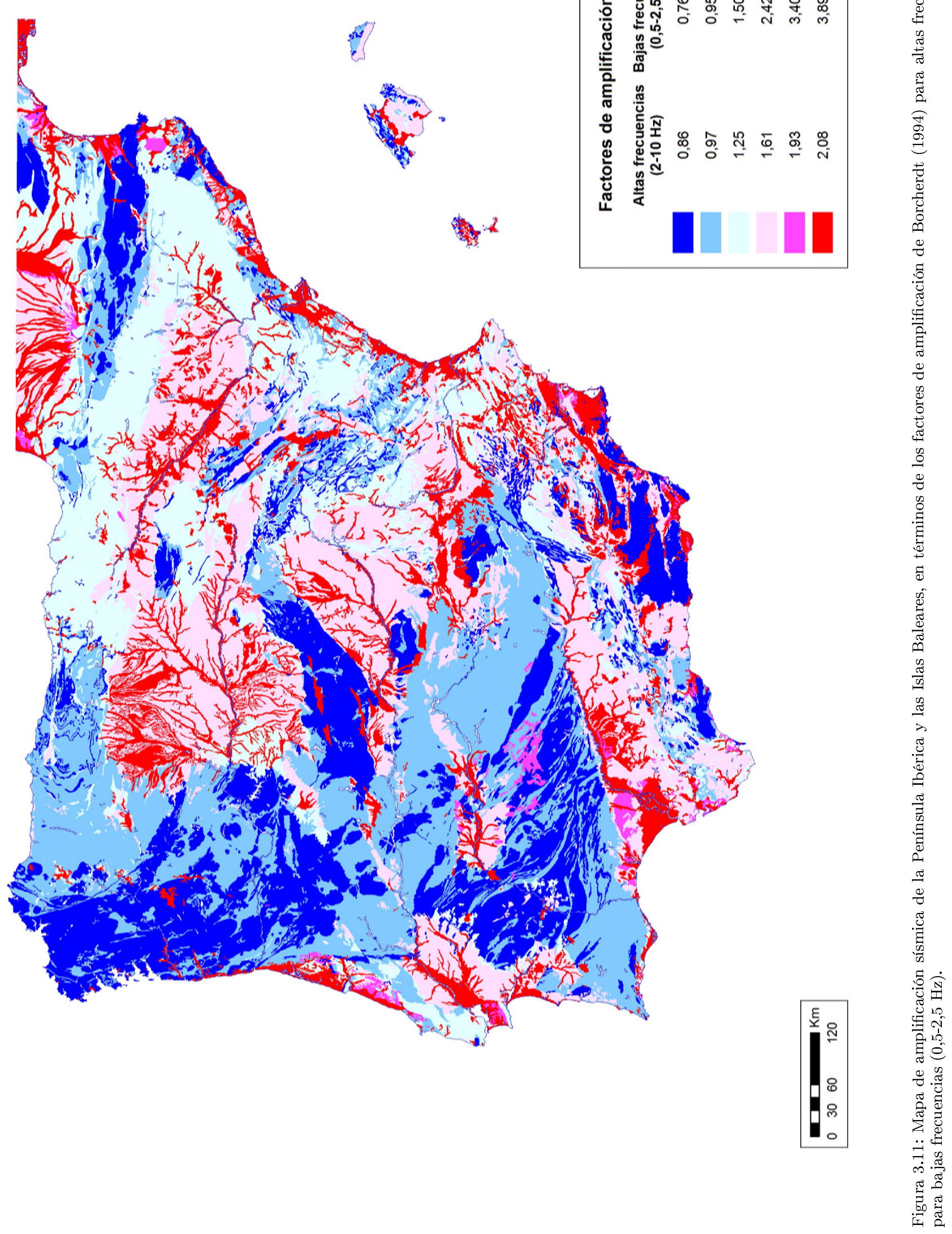




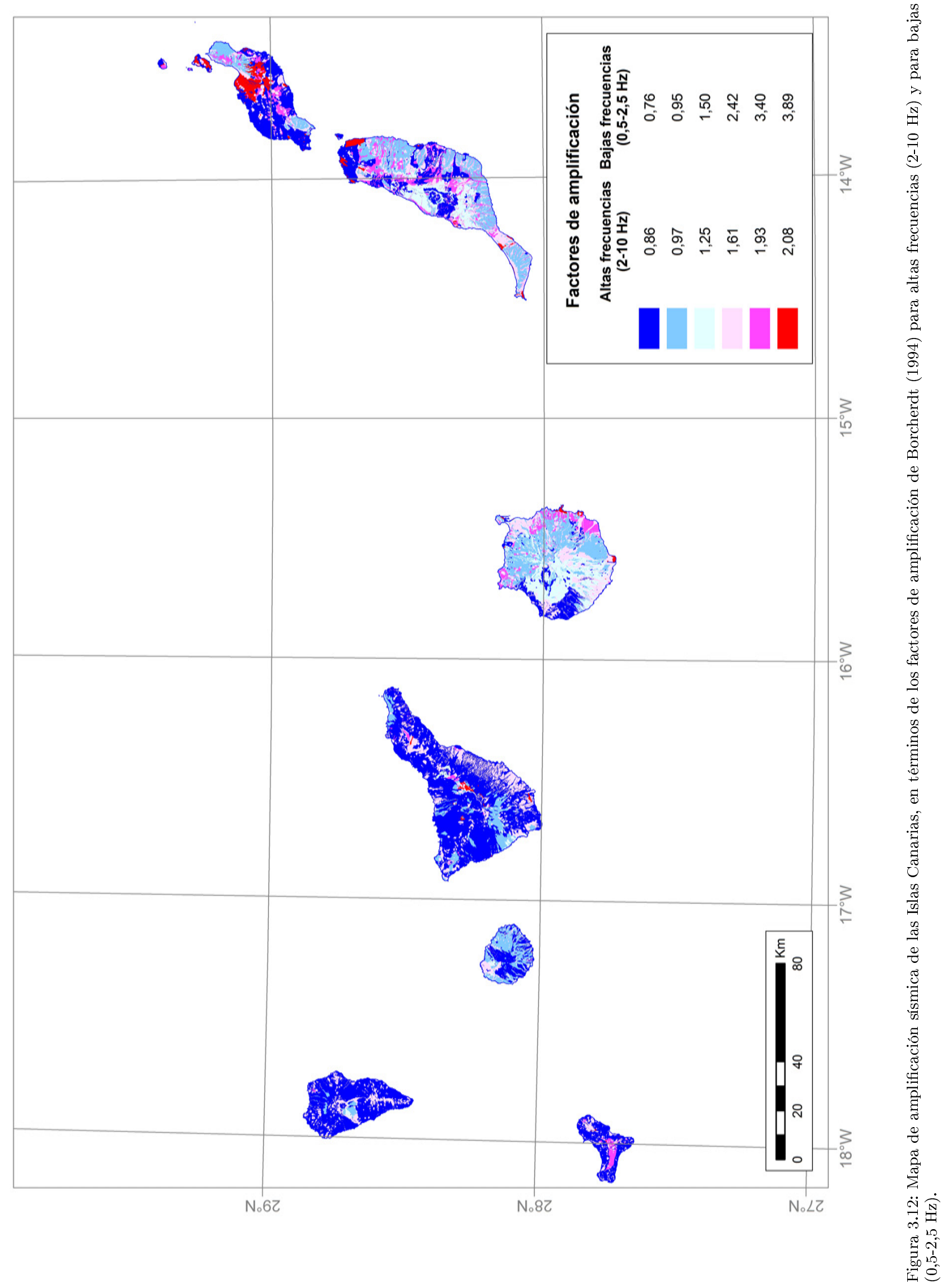




\section{Capítulo 4}

\section{DETERMINACIÓN DE LA AMPLIFICACIÓN SÍSMICA EN FUNCIÓN DE LA FRECUENCIA EN EL HIERRO (ISLAS CANARIAS) MEDIANTE LA DISTRIBUCIÓN DE LA VELOCIDAD DE LA ONDA DE CORTE EN LOS PRIMEROS $30 \mathrm{M}$ DE PROFUNDIDAD}

En el contexto de una segunda aproximación a la capacidad de amplificación sísmica del territorio de la Península Ibérica, las Islas Baleares y las Islas Canarias, se ha planteado la realización de una microzonación sísmica en una región determinada, por medio de la estimación directa de la estructura de velocidades de onda de corte $(\mathrm{Vs})$ en los primeros $30 \mathrm{~m}$ de profundidad en determinados emplazamientos. Estos modelos de Vs son utilizados posteriormente como variables para la generación de la curva de amplificación sísmica en función de la frecuencia en los emplazamientos correspondientes.

La curva de amplificación sísmica espectral constituye una variable necesaria para poder simular escenarios sísmicos realistas en términos de aceleración y de intensidad en una región, por medio de la simulación estocástica de acelerogramas sintéticos. Con el fin de poder llevar a cabo esta simulación en la región donde se localizan los emplazamientos (capítulo 10), es preciso calibrar previamente el algoritmo de simulación en la región (capítulo 8), para lo cual es preciso contar con suficientes registros de aceleración. Por este motivo, se pretende que los emplazamientos de medida se sitúen en las proximidades de estaciones acelerométricas. Al mismo tiempo, se buscan emplazamientos que presenten distintos factores de amplificación, de acuerdo con los mapas de amplificación sísmica que se han obtenido en 
el capítulo 3 (Figuras 3.11 y 3.12).

El reciente proceso de reactivación volcánica ocurrido en la isla de El Hierro (Islas Canarias) dio lugar a una erupción volcánica submarina, que comenzó en octubre de 2011 y fue dada por finalizada en marzo de 2012. De acuerdo con López et al. (2012), la erupción fue precedida por casi 10000 terremotos que, con una magnitud momento $(\mathrm{Mw})$ máxima de 4,0, fueron localizados en la isla entre julio y octubre de 2011. Esta crisis sísmica, unida a las crisis sucedidas en junio de 2012 y marzo de 2013, y que presentaron eventos con Mw hasta 5,0, ha permitido el registro de 202 acelerogramas en las estaciones acelerométricas dispuestas por el Instituto Geográfico Nacional (IGN) en la isla, de acuerdo con el catálogo de acelerogramas correspondiente a las citadas crisis y proporcionado por el IGN. Por tanto, se ha considerado que El Hierro constituye una región adecuada para la determinación de la amplificación sísmica espectral a partir del modelo de Vs en profundidad, debido a la ingente cantidad de registros acelerométricos disponibles, y también por la presencia de hasta cuatro clases de emplazamiento en la isla, de acuerdo con la Figura 3.12. En este punto, es preciso puntualizar, sin embargo, que El Hierro es la única isla del archipiélago canario donde no afloran unidades geológicas de cada una de las 6 clases de emplazamiento de la clasificación de síntesis (Tabla 3.4 y Figura 3.12).

\subsection{Campaña de adquisición de medidas ReMi}

Entre el 11 y el 15 de noviembre de 2013 se llevó a cabo una campaña de medidas de ruido sísmico ambiental por medio del método ReMi (Refraction Microtremor) en 25 emplazamientos distribuidos en la isla de El Hierro. Estos emplazamientos se escogieron de acuerdo a criterios de cercanía a las distintas estaciones sísmicas y geodésicas desplegadas por el IGN en la isla, a los afloramientos de máxima amplificación y a los núcleos urbanos. En función del parámetro medido, se ha estudiado la situación de las estaciones acelerométricas, las estaciones de registro de velocidad, las estaciones centrales de los arrays sísmicos y las estaciones GPS instaladas por el IGN. Los datos relativos a las estaciones de registro de velocidad y a los acelerómetros se obtuvieron a partir de Instituto Geográfico Nacional (2015a) e Instituto Geográfico Nacional (2017b), respectivamente, mientras que los relativos a los arrays sísmicos y a las estaciones de GPS fueron proporcionados directamente por el Instituto Geográfico Nacional tras cursar la oportuna petición.

En la Figura 4.1 se presenta la distribución espacial de los emplazamientos de medida ReMi en El Hierro, junto con la instrumentación del IGN, así como también se incluyen las clases de emplazamiento y de Vs30, en coherencia con el mapa de la Figura 3.10. En la Tabla B.1 se refieren las coordenadas geográficas y la altitud de los emplazamientos de medida. Adicionalmente, se indica el nombre y tipo de la estación del IGN que se localiza en las proximidades del emplazamiento, en su caso: Estación de registro de velocidad, estación central de array sísmico, acelerómetro, o estación GPS. Para los emplazamientos situados en núcleos urbanos, se indica el nombre de la localidad o entidad de población. Por último, y en el caso de los puntos de máxima amplificación, se precisa la clase de emplazamiento correspondiente, según la Tabla 3.4). Respecto a los acelerómetros relacionados en la Tabla B.1, se señala 


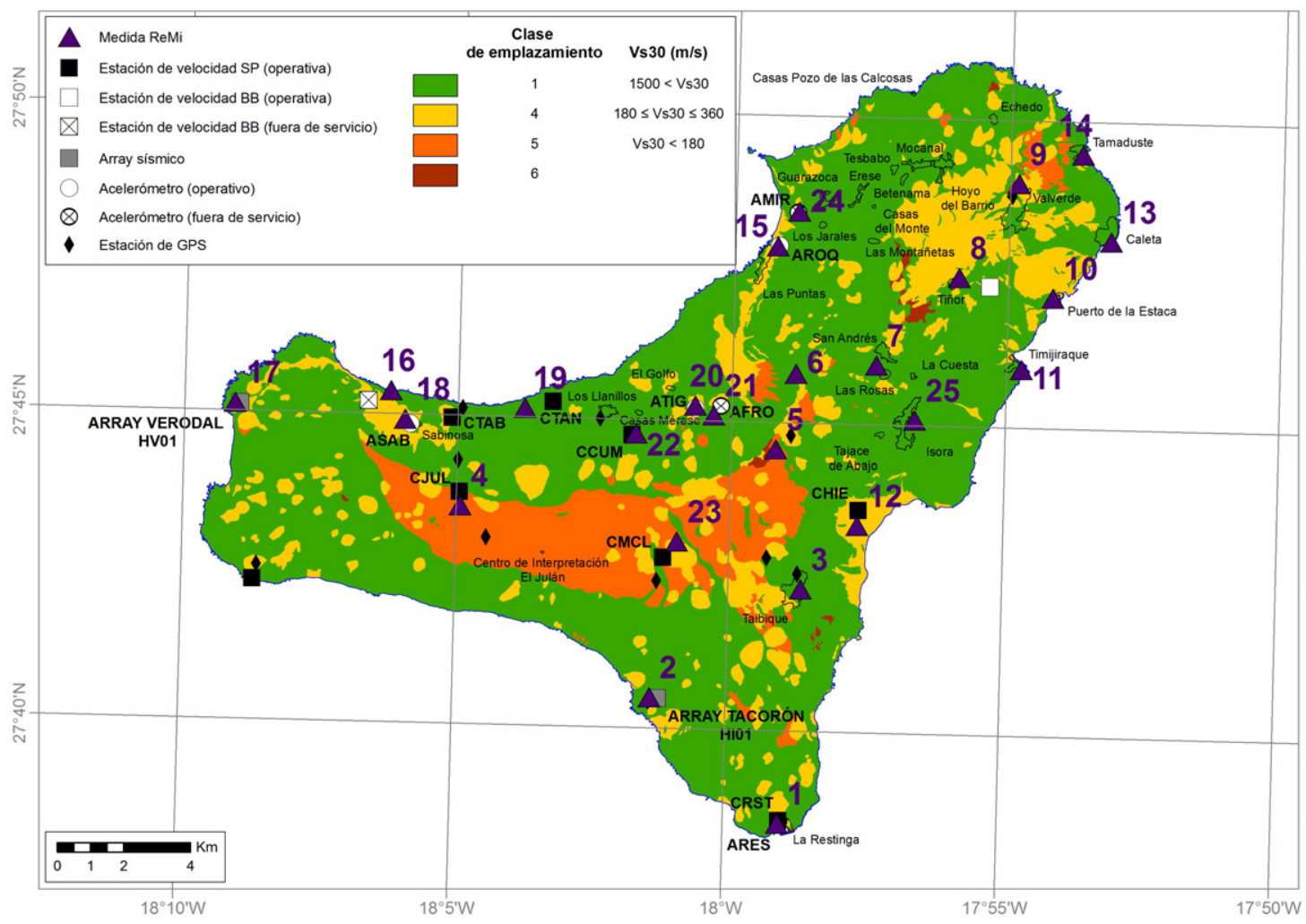

Figura 4.1: Emplazamientos correspondientes a la campaña de adquisición de medidas ReMi en El Hierro en noviembre de 2013. La instrumentación sísmica y geodésica dispuesta por el IGN en la isla aparece también representada, incluyendo las estaciones de registro de velocidad de corto periodo (SP) y de banda ancha (BB), en función de su operatividad (Instituto Geográfico Nacional, 2015a); las estaciones centrales de arrays sísmicos, los acelerómetros en función de su operatividad (Instituto Geográfico Nacional, 2017b), y las estaciones de GPS. Se presenta asimismo el mapa de clases de emplazamiento y de Vs30 de El Hierro, de acuerdo con el mapa general para las Islas Canarias de la Figura 3.10. La información relativa a los arrays sísmicos y a las estaciones de GPS ha sido proporcionada directamente por el Instituto Geográfico Nacional, previa petición motivada.

que, a fecha de marzo de 2017, sólo las estaciones acelerométricas AROQ, ASAB y ATIG se encuentran operativas, mientras que AFRO, AMIR y ARES se encuentran fuera de servicio, de acuerdo con el listado de acelerógrafos publicado por Instituto Geográfico Nacional (2017b).

En base a las configuraciones y dispositivos de adquisición de medidas utilizados por Rueda et al. (2015) en su trabajo de microzonificación sísmica de las localidades de Torreperogil y Sabiote (Jaén), en cada emplazamiento de medida en El Hierro se han dispuesto dos líneas de 12 geófonos de $4,5 \mathrm{~Hz}$ y componente vertical, con el fin de alcanzar profundidades de al menos $40 \mathrm{~m}$. Los geófonos se han emplazado sobre el terreno siguiendo un perfil lineal y con una separación constante de $3 \mathrm{~m}$, resultando en una longitud del dispositivo de $69 \mathrm{~m}$. Cada línea constituye un único cable sísmico, al cual se conectan los geófonos correspondientes, y que a su vez se conecta a un equipo de sísmica de refracción DAQlink III de Seismic Source, propiedad del IGN, con software de adquisición de datos Vibrascope V2.4.59. Este equipo consiste en un digitalizador de 24 canales con 24 bits de resolución, 144 dB de rango dinámico y una frecuencia de muestreo de hasta $48000 \mathrm{~Hz}$. Los canales están sincronizados mediante un reloj GPS. El digitalizador se encuentra conectado a un ordenador portátil vía Ethernet, en el cual se trabaja con el software de adquisición y se almacenan los datos en formato SEG-2 (ficheros .sgy). 


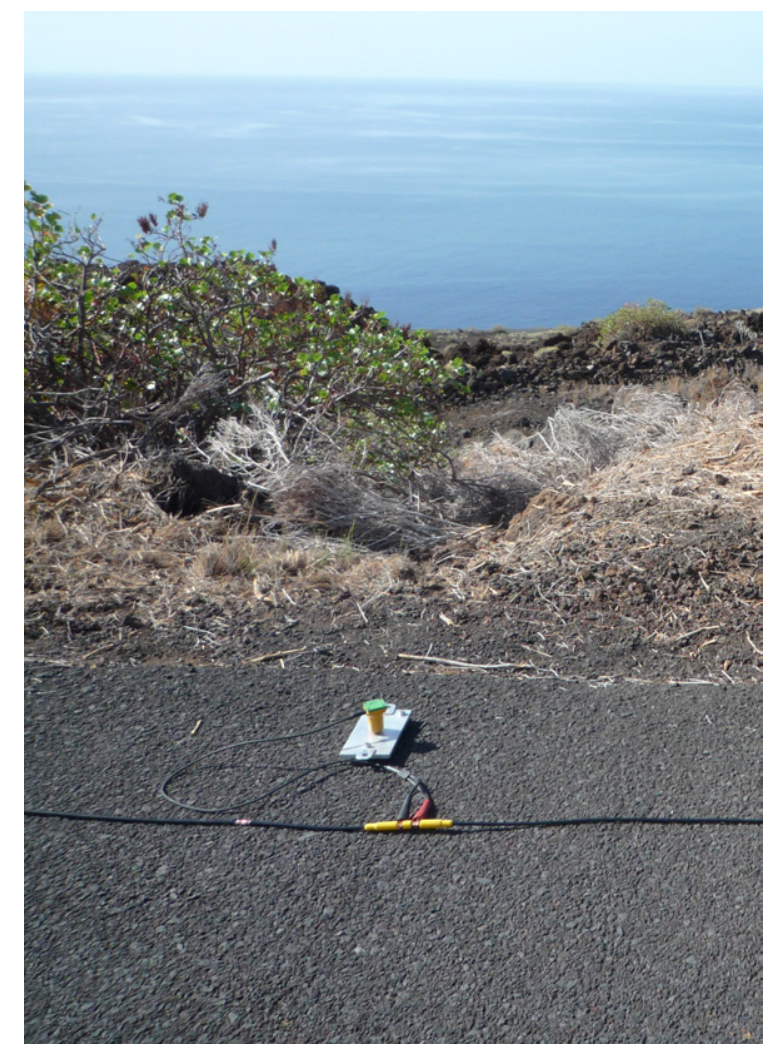

Figura 4.2: Geófono perfectamente instalado sobre el terreno por medio de su acoplamiento previo a una placa de duraluminio, durante la toma de medidas de ruido sísmico ambiental por medio del método ReMi, en el emplazamiento $n^{\circ} 2$ (Array sísmico de Tacorón, en el municipio de El Pinar).

Para la instalación de los geófonos sobre el terreno duro, se han utilizado 24 placas de duraluminio con tres puntos de apoyo, provistas de un orificio al cual se han enroscado los sensores. De esta forma, se ha conseguido que los geófonos se apoyen perfectamente sobre el suelo, cuando no era posible utilizar las picas para hincarlos (Figura 4.2).

Para aumentar el nivel de ruido sísmico ambiental durante el proceso de medición, se han generado también ondas superficiales de forma artificial en un extremo del dispositivo, con el fin de aumentar la calidad del registro. Con este fin, un ayudante ha golpeado fuertemente con una maza de $6 \mathrm{~kg}$ una placa metálica dispuesta sobre el terreno, a $4 \mathrm{~m}$ del extremo, realizando el golpeo a intervalos de $2 \mathrm{~s}$ aproximadamente (Figura 4.3). De esta forma, se han tomado 10 ventanas de registro, de $30 \mathrm{~s}$ de duración cada una. En 2 ventanas se ha registrado sólo el ruido ambiente, y en las 8 restantes el ruido y las ondas superficiales generadas.

Paralelamente a la toma de datos en cada emplazamiento, se ha realizado una reseña geológica sobre el mismo, para poder inferir un modelo de velocidad Vs en profundidad del citado emplazamiento. Concretamente, se ha tratado de describir la columna estratigráfica de la zona, sobre todo la secuencia de capas que se sitúa bajo la línea de geófonos. De esta forma, se pretende recabar la información geológica necesaria para poder construir el citado modelo de Vs y así iniciar el proceso de modelización de la curva de dispersión experimental, a obtener a partir de las medidas. En la Tabla C.1 se puede consultar la información geológica de cada emplazamiento. 


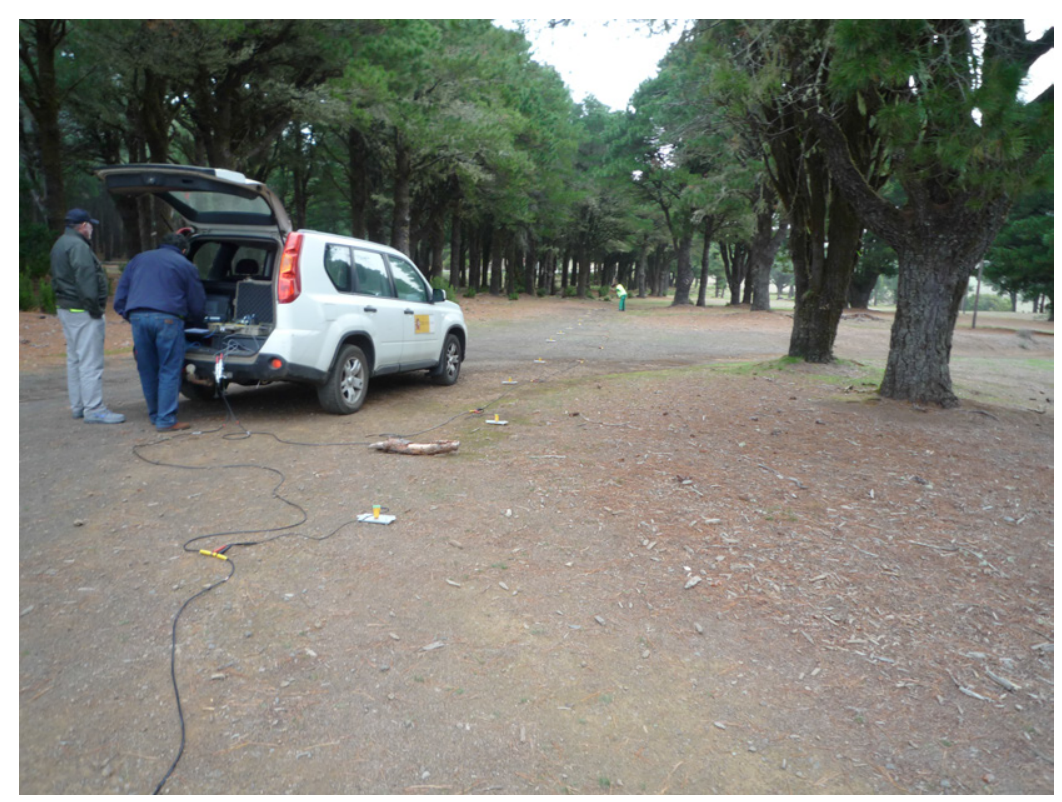

Figura 4.3: Líneas sísmicas con 24 geófonos equiespaciados $3 \mathrm{~m}$, desplegadas en el emplazamiento $n^{\circ} 5$ (municipio de Valverde) y preparadas para la toma de medidas con el método ReMi. Se observa, en primer plano, la conexión de las líneas al equipo digitalizador. Al fondo, el ayudante golpea la placa metálica dispuesta en el extremo del dispositivo con la maza de $6 \mathrm{~kg}$.

\subsection{Procesado de datos}

El tratamiento de los registros obtenidos en cada emplazamiento de medida se ha realizado con el software SeisOpt@ ReMiVspect Version 4.0. Previamente, ha sido preciso importar estos datos a partir de un fichero con formato SEG-Y, que contiene las 24 trazas de cada una de las 10 ventanas de registro o planos de registro.

A cada uno de los planos de registro se le realiza un preprocesado, que consiste en eliminar el desplazamiento temporal y realizar una ecualización de todas las trazas. Esta ecualización normaliza las 24 trazas a la mayor amplitud. A continuación, se elimina la información de la geometría en la cabecera del fichero, pues la simplicidad de la geometría lineal del dispositivo de medida que se ha empleado permite su sencilla introducción en la siguiente fase del procesado, consistente en el análisis espectral de las ventanas de registro.

El análisis espectral de cada ventana de registro tiene el fin de obtener el diagrama de la transformada lentitud-frecuencia $(p-f)$. Implica calcular la transformada lentitud-tiempo de interceptación $(p-\tau)$, la cual se convierte en la imagen en el dominio $p-f$ mediante una transformada de Fourier. Para calcular la transformada $p-\tau$ se introduce el intervalo de muestreo empleado, 0,002 s (que implica 500 muestras por segundo); la separación entre geófonos, $3 \mathrm{~m}$; la frecuencia máxima a analizar, $50 \mathrm{~Hz}$; así como la velocidad mínima, de $200 \mathrm{~m} / \mathrm{s}$. En este punto, se destaca que tanto la frecuencia máxima como la velocidad mínima permiten escalar el diagrama $p-f$. De esta forma, se busca apreciar las velocidades que permiten identificar el máximo de energía o amplitud espectral de la transformada y que corresponde al modo fundamental de las ondas Rayleigh. Este máximo de amplitud se distingue por tener una pendiente que disminuye hacia las altas frecuencias.

Si en el diagrama $p-f$ correspondiente a un plano de registro se observa una 


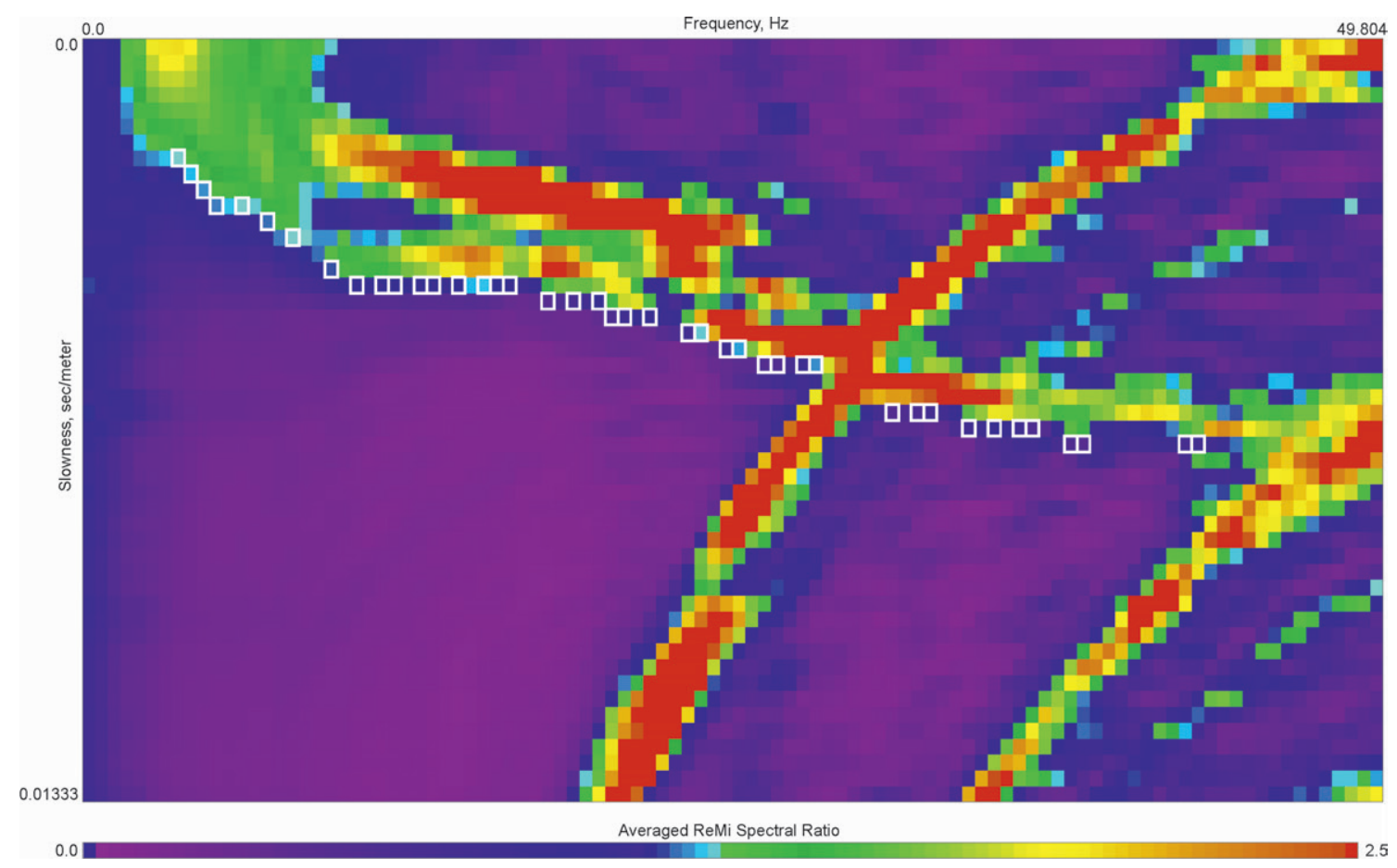

Figura 4.4: Diagrama de la transformada lentitud-frecuencia $(p-f)$ definitiva para el emplazamiento $n^{\circ} 23$, resultado de la suma de las diez transformadas individuales obtenidas, una por cada ventana de registro. Los puntos cuadrados blancos son los puntos de dispersión experimentales que se han seleccionado en este emplazamiento.

interrupción clara, del orden de $5 \mathrm{~Hz}$, del máximo de energía, es preciso descartar la transformada $p-f$ asociada. Se seleccionan, en definitiva, los planos de registro que presentan un máximo de energía claramente identificable, coherente y continuo, a lo largo de un intervalo de frecuencias lo suficientemente ancho. Por tanto, la visualización de los diagramas lentitud-frecuencia de los 10 planos de registro tiene el fin de seleccionar aquellas transformadas $p-f$ que pueden combinarse para generar la transformada definitiva del emplazamiento. En este contexto, se obtiene una imagen $p-f$ para el emplazamiento a partir de la suma, píxel a píxel, de los diagramas $p-f$ de los planos de registro cuyas transformadas se han seleccionado. Se está así en disposición de acometer la siguiente fase del procesado, consistente en la construcción de la curva de dispersión experimental de las ondas Rayleigh en el emplazamiento en cuestión.

En la Figura 4.4 se observa, para el emplazamiento $n^{\circ} 23$ de la Figura 4.1, el diagrama resultado de la combinación o suma de las transformadas $p-f$ para las 10 ventanas de registro.

De acuerdo con Louie (2001), la curva de dispersión correspondiente a las ondas Rayleigh está representada por la envolvente del máximo de energía de la transformada $p-f$ que, a su vez, presenta la menor velocidad aparente de fase. Para construir esta curva, se han seleccionado manualmente las menores velocidades de fase que marcan la tendencia de este máximo de energía en función de la frecuencia.

En la Figura 4.4 se observan, para el emplazamiento $n^{\circ} 23$, los picados experimentales de la envolvente de menor velocidad aparente de fase que corresponde al máximo de energía de la transformada definitiva $p-f$. Estos picados se señalan como puntos cuadrados blancos, y constituyen la curva de dispersión experimental para 


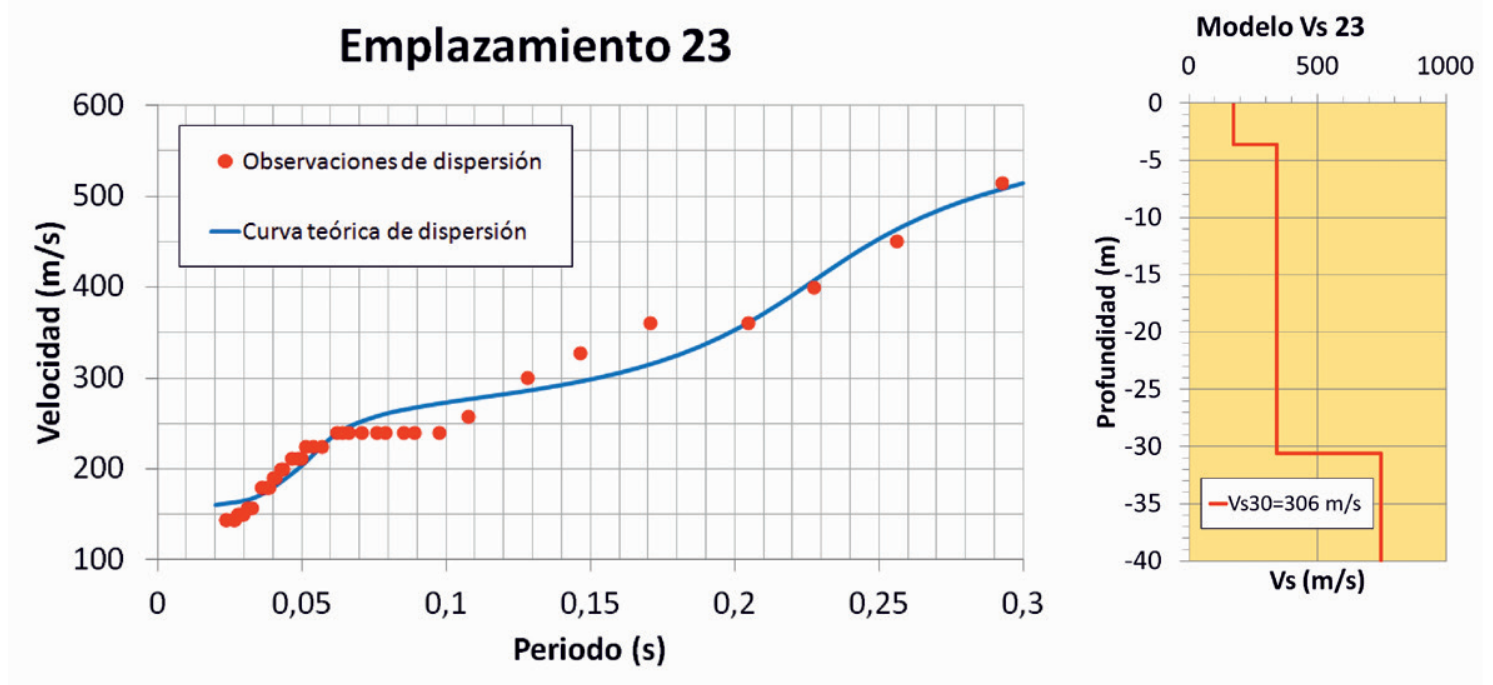

Figura 4.5: Observaciones que constituyen la curva de dispersión experimental del emplazamiento $n^{\circ} 23$, a las que se ajusta la curva de dispersión teórica que se ha obtenido a partir del modelo de velocidad Vs. El error cuadrático medio (error RMS) del ajuste es de $16 \mathrm{~m} / \mathrm{s}$, y el valor de Vs30 que corresponde al modelo Vs es de $306 \mathrm{~m} / \mathrm{s}$.

este emplazamiento. En el Apéndice D se presenta el diagrama de la transformada lentitud-frecuencia $p-f$ que se ha obtenido en cada uno de los 25 emplazamientos de medida ReMi en El Hierro, incluyendo los puntos de dispersión experimentales como cuadrados blancos.

Los picados experimentales se definen por su frecuencia y lentitud, y se transforman al espacio velocidad-periodo con el fin de representar convenientemente la curva de dispersión experimental del emplazamiento. En la Figura 4.5 (izquierda) se puede observar la curva de dispersión experimental para el emplazamiento $n^{\circ} 23$.

La última fase del procesado de datos consiste en la modelización de la curva de dispersión experimental, la cual se ha llevado a cabo con el software SeisOpt $₫$ ReMiDispert Version 4.0. A partir de un modelo inicial de Vs en profundidad, se ha generado una curva de dispersión teórica con el fin de ajustarla a los puntos de dispersión observados o experimentales. Por medio de la variación de los parámetros de velocidad y espesor del modelo, se ha tratado de mejorar este ajuste, en un proceso iterativo de prueba y error. Es decir, con cada cambio en los parámetros del modelo, se ha generado una nueva curva de dispersión téorica que debe ajustarse a los puntos observados. Obtenido un error cuadrático medio (root-mean-square, RMS) aceptable para el ajuste, se detiene el proceso y selecciona el modelo de Vs correspondiente como la estructura definitiva de Vs del emplazamiento.

En todos los emplazamientos, se ha considerado inicialmente un modelo de Vs coherente con las observaciones de campo que constituyen la reseña geológica de cada emplazamiento. Además, los respectivos modelos tienen una profundidad de hasta $40 \mathrm{~m}$, con el fin de asegurar la posterior determinación de Vs30. En función del emplazamiento, los modelos constan de 3 ó 4 capas, a las cuales se les asigna una velocidad constante Vs.

En la Figura 4.5 (izquierda) se puede observar la curva de dispersión teórica del emplazamiento $n^{\circ} 23$, junto con el modelo de Vs (Figura 4.5, derecha), a partir del cual se ha obtenido. También se presenta el ajuste de la curva teórica a los puntos de 

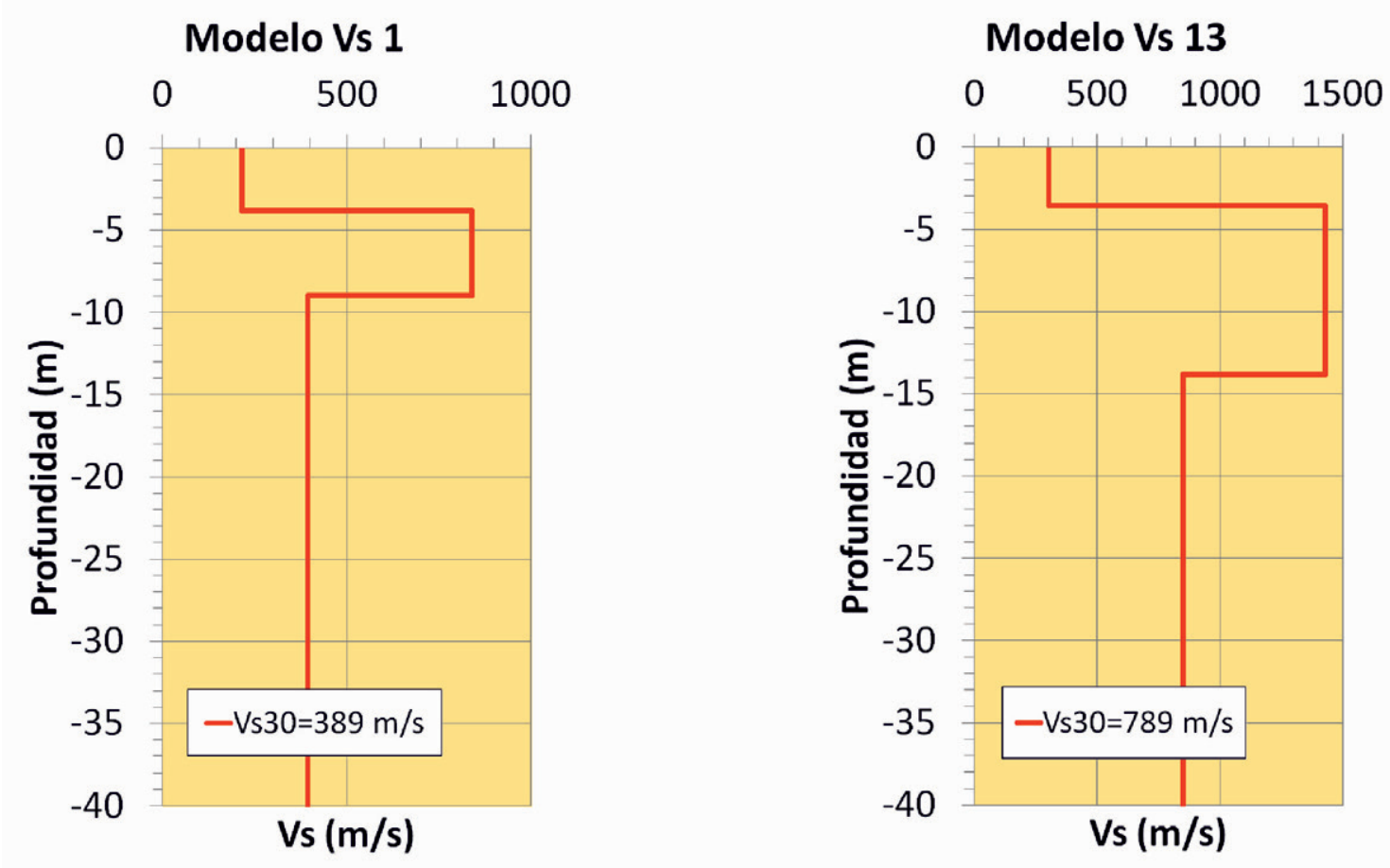

Figura 4.6: Modelos de Vs de los emplazamientos $n^{\circ} 1$ y 13, en los que se ha introducido una capa de baja velocidad de acuerdo con las consideraciones realizadas en el texto. Se muestra, asimismo, el valor de Vs30 que corresponde a cada modelo de Vs.

dispersión experimentales, que corresponde a un error cuadrático medio de $16 \mathrm{~m} / \mathrm{s}$, el cual se considera aceptable de acuerdo con la velocidad máxima del modelo (749 $\mathrm{m} / \mathrm{s}$ ). Este procesado de datos se ha realizado en los 25 emplazamientos distribuidos en la isla de El Hierro. En el Apéndice E se puede consultar el ajuste de la curva de dispersión teórica a los puntos de dispersión observados en cada emplazamiento y los modelos de velocidad Vs obtenidos.

En dos de los 25 emplazamientos de medida (números 1 y 13), se optó por un modelo de Vs con capa de baja velocidad. En el caso del emplazamiento $n^{\circ} 1$ (Figura 4.6, izquierda), la capa más superficial tiene $3,8 \mathrm{~m}$ de espesor, con velocidad $216 \mathrm{~m} / \mathrm{s}$, que se corresponde con los detríticos finos observados al nivel de la carretera sobre la que apoya la línea de geófonos (Tabla C.1). Bajo la misma, se sitúa una capa que alcanza los $9,0 \mathrm{~m}$ de profundidad, con $838 \mathrm{~m} / \mathrm{s}$ y que puede corresponder a la capa de basalto que se ha observado por encima de la línea de geófonos. Esta capa se habría desmontado para acoger la carretera, y por tanto se situaría también bajo el dispositivo de medida. La capa subyacente es de $394 \mathrm{~m} / \mathrm{s}$ y por tanto introduce una inversión de velocidad. Esta capa se corresponde con la capa métrica de lapillis en explotación que se ha observado bajo el dispositivo de medida, en campo.

Con respecto al emplazamiento 13 (Figura 4.6, derecha), la primera capa tiene un espesor de $3,55 \mathrm{~m}$ y velocidad de $301 \mathrm{~m} / \mathrm{s}$, y se corresponde con el relleno sobre el que se ha dispuesto la línea de geófonos (Tabla C.1). Por debajo, aparece una capa con velocidad de $1429 \mathrm{~m} / \mathrm{s}$, que se asocia a los basaltos observados en campo, llegando a los 13,79 $\mathrm{m}$ de profundidad. Bajo esta capa, se ha introducido otra de menor velocidad $(848 \mathrm{~m} / \mathrm{s})$, que no se puede correlacionar con ninguna capa observada en campo. Sin embargo, la introducción de esta capa se ha considerado como solución 
imprescindible para poder obtener un error aceptable $(R M S=16 \mathrm{~m} / \mathrm{s})$ en el ajuste de la curva de dispersión modelizada a los puntos de dispersión experimentales (Figura E.13 en el Apéndice E). Esta inversión de velocidad con la profundidad puede explicarse a partir del diagrama $p-f$ de este emplazamiento (Figura D.13 en el Apéndice D), ya que muestra una ruptura de la pendiente de la envolvente de mínima velocidad del máximo de energía. Esta ruptura se sitúa a $30 \mathrm{~Hz}$, que pueden considerarse frecuencias relativamente altas. Por ello, la ruptura puede explicar esta inversión a una profundidad relativamente baja, como son 13,79 m sobre el total de los $40 \mathrm{~m}$ en estudio.

\subsection{Valores de Vs30 en los emplazamientos de me- dida}

A partir de los modelos de Vs que se han obtenido como resultado del procesado de los datos registrados en los 25 emplazamientos, en cada uno se ha obtenido el valor correspondiente de Vs30. En la Tabla 4.1 se presentan los valores de Vs30 de cada emplazamiento, junto con el error RMS del ajuste de la curva de dispersión teórica a los puntos de dispersión observados que ha permitido obtener cada valor de Vs30. También se incluye el intervalo de valores de Vs30 de la clase en la cual se sitúa cada emplazamiento, de acuerdo con el mapa de clases de emplazamiento y Vs30 de las Islas Canarias (Figura 3.10) y con la Figura 4.1. En la Figura 4.7 se muestra la distribución espacial de los valores de Vs30 obtenidos en El Hierro con ReMi, sobre el mapa de clases de emplazamiento y de Vs30 correspondiente a la isla.

\subsubsection{Comparación de los valores de Vs30 en los emplaza- mientos de medida con los valores de Vs30 del mapa}

Como se observa en la Tabla 4.1, en la mayoría de los emplazamientos, los valores de Vs30 calculados a partir de las medidas de ReMi no están contenidos por los intervalos de Vs30 de las clases de emplazamiento del mapa a las que corresponden estos emplazamientos. Por ejemplo, los valores de Vs30 calculados para todos los emplazamientos pertenecientes a la clase de emplazamiento 1 (Vs30>1500 m/s, Figura 4.1) son menores que $1500 \mathrm{~m} / \mathrm{s}$ (Tabla 4.1). Esta situación se pone especialmente de manifiesto en los emplazamientos 2, 6, 19, 21, 22 y 24, para los cuales la Vs30 calculada es menor que $400 \mathrm{~m} / \mathrm{s}$. Además, sólo en tres emplazamientos (15, 18 y 23) el mapa proporciona intervalos de Vs30 que contienen a los correspondientes valores de Vs30 calculados a partir de las medidas ReMi (Tabla 4.1). Estos emplazamientos, 15, 18 y 23 pertenecen a la clase $4(180 \leq V s 30 \leq 360)$, y presentan una Vs30 calculada a partir de las medidas ReMi igual a 340, 308 y $306 \mathrm{~m} / \mathrm{s}$, respectivamente.

Las diferencias observadas entre los valores de Vs30 que proporciona ReMi y los intervalos de Vs30 del mapa se deben, fundamentalmente, al hecho de que el mapa de Vs30 se ha generado teniendo en cuenta la geología superficial, mientras que el método ReMi muestrea los primeros $30 \mathrm{~m}$ de profundidad con el fin de obtener el mismo parámetro Vs30. Las discrepancias encontradas en los emplazamientos de clase 1 pueden deberse también a la alteración y fracturación del material geológico 
Tabla 4.1: Valores obtenidos para Vs30 y para el error RMS del ajuste (m/s) en los 25 emplazamientos de medida ReMi en El Hierro, e intervalos de valores de Vs30 proporcionados por el mapa de clases de emplazamiento y Vs30 de las Islas Canarias (Figuras 3.10) y 4.1).

\begin{tabular}{|c|c|c|c|}
\hline $\begin{array}{l}\text { Número de } \\
\text { emplazamiento }\end{array}$ & Vs30 ReMi (m/s) & $\operatorname{RMS}(\mathbf{m} / \mathbf{s})$ & Vs30 Mapa $(\mathrm{m} / \mathrm{s})$ \\
\hline 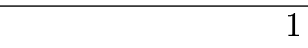 & 389 & 13 & $180 \leq V_{s} 30 \leq 360$ \\
\hline 2 & 326 & 11 & $\mathrm{Vs} 30>1500$ \\
\hline 3 & 411 & 9 & $\mathrm{Vs} 30>1500$ \\
\hline 4 & 300 & 11 & Vs30 < 180 \\
\hline 5 & 350 & 7 & Vs30 $<180$ \\
\hline 6 & 315 & 11 & $\mathrm{Vs} 30>1500$ \\
\hline 7 & 508 & 28 & $\mathrm{Vs} 30>1500$ \\
\hline 8 & 408 & 14 & $\mathrm{Vs} 30>1500$ \\
\hline 9 & 564 & 10 & $180 \leq \mathrm{Vs} 30 \leq 360$ \\
\hline 10 & 417 & 15 & $\mathrm{Vs} 30>1500$ \\
\hline 11 & 764 & 18 & $\mathrm{Vs} 30>1500$ \\
\hline 12 & 431 & 16 & $180 \leq \mathrm{Vs} 30 \leq 360$ \\
\hline 13 & 789 & 16 & $\mathrm{Vs} 30>1500$ \\
\hline 14 & 431 & 15 & $\mathrm{Vs} 30>1500$ \\
\hline 15 & 340 & 10 & $180 \leq V_{s} 30 \leq 360$ \\
\hline 16 & 449 & 10 & Vs30 > 1500 \\
\hline 17 & 507 & 12 & Vs30 > 1500 \\
\hline 18 & 308 & 10 & $180 \leq \mathrm{Vs} 30 \leq 360$ \\
\hline 19 & 350 & 16 & $\mathrm{Vs} 30>1500$ \\
\hline 20 & 473 & 10 & $180 \leq \mathrm{Vs} 30 \leq 360$ \\
\hline 21 & 367 & 15 & $\mathrm{Vs} 30>1500$ \\
\hline 22 & 321 & 15 & $\mathrm{Vs} 30>1500$ \\
\hline 23 & 306 & 16 & $180 \leq \mathrm{Vs} 30 \leq 360$ \\
\hline 24 & 324 & 11 & $\mathrm{Vs} 30>1500$ \\
\hline 25 & 412 & 14 & $\mathrm{Vs} 30>1500$ \\
\hline
\end{tabular}




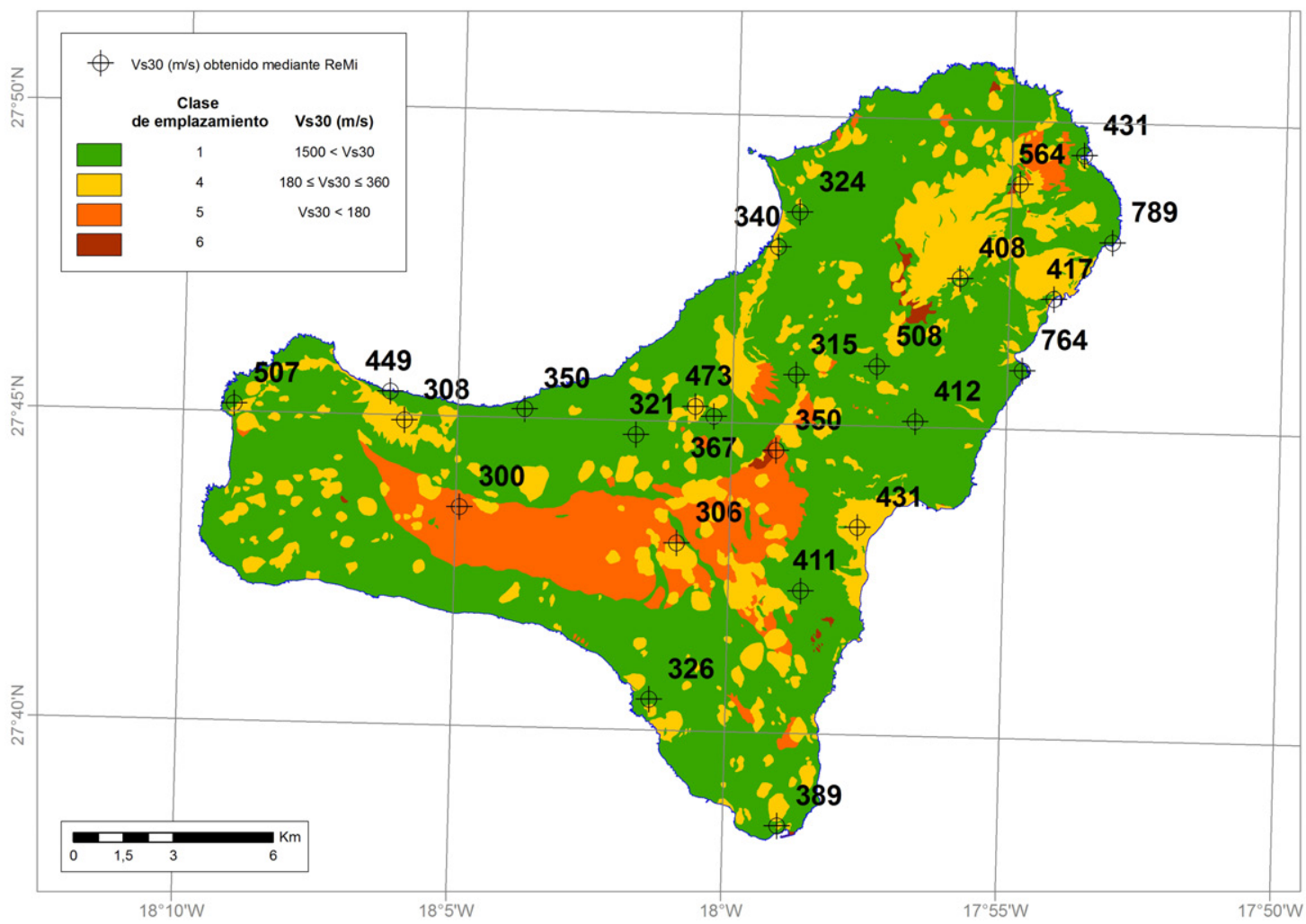

Figura 4.7: Distribución espacial de los valores de Vs30 obtenidos en El Hierro por medio del método ReMi. Se presenta igualmente el mapa de clases de emplazamiento y de Vs30 de la isla, de acuerdo con el mapa general para las Islas Canarias de la Figura 3.10. 
aflorante (rocas duras). Este material presenta, por tanto, una resistencia real, conocida a partir del valor Vs30 de ReMi, que es menor que la resistencia inferida para la clase 1 del mapa a partir de la descripción geotécnica de esta clase. En cambio, es razonable que el mapa de Vs30 resuelva mejor el valor de este parámetro en los emplazamientos de clase 4 (suelos de compacidad/consistencia alta), pues la resistencia de este tipo de material no es susceptible a un cambio tan sensible ante la acción de los agentes erosivos.

\subsection{Curvas de amplificación sísmica espectral en los emplazamientos de medida}

En cada emplazamiento de medida ReMi, se ha generado una curva de amplificación sísmica espectral, utilizando únicamente la estructura de Vs en los primeros $30 \mathrm{~m}$ de profundidad, siguiendo de esta forma el método utilizado por Rueda et al. (2015) para determinar la amplificación espectral las localidades de Torreperogil y Sabiote (Jaén). Con este fin, y dado que cada modelo de Vs tiene capas de velocidad constante, se ha empleado el algoritmo de cálculo de la amplificación sísmica en función de la frecuencia NRATTLE (Boore, 2005), que implementa la solución completa de la propagación de una onda plana $(\mathrm{SH})$ en una estructura de capas con velocidad constante, y que se obtiene de acuerdo con el método de la matriz de propagación de Haskell (1960).

El procesado de los modelos de Vs de cada emplazamiento con NRATTLE ha requerido la especificación de un valor del factor de calidad de la atenuación anelástica, Q, y un valor de densidad para cada capa. Como densidad, se ha escogido un valor genérico de $2,0 \mathrm{~g} / \mathrm{cm}^{3}$, que se asume válido para el rango de profundidades del modelo $(0-40 \mathrm{~m})$. En cuanto a Q, se toma un valor del factor de calidad para la frecuencia de $1 \mathrm{~Hz}, Q_{0}$ general para la isla de El Hierro. A este respecto, en primera instancia se ha considerado el factor $Q_{0}=113$ que aporta Canas et al. (1995) para la isla, en su trabajo de regionalización de la atenuación anelástica en Canarias, el cual es coherente con los valores obtenidos en esta tesis doctoral y que se encuentran en el capítulo 7.

También se han especificado, a los efectos del procesado con NRATTLE, la velocidad Vs y la densidad del semiespacio, entendido como la región donde se sitúa la fuente sísmica, y que en todo caso está situada a profundidades superiores a las que comprende el modelo de Vs. En este sentido, se han estimado una Vs de 1,80 km/s y una densidad de $2,72 \mathrm{~g} / \mathrm{cm}^{3}$ para el semiespacio. Por último, se ha introducido el ángulo de incidencia de las ondas que llegan a la estructura de capas, tomando el valor de $5^{\circ}$.

A partir de la curva de amplificación espectral de un emplazamiento, se puede obtener el valor del periodo fundamental del suelo, considerando la frecuencia a la cual se produce el primer máximo de la curva de amplificación, en el intervalo de frecuencias en $\mathrm{Hz}(0,10)$. Este valor pico de la curva se toma como la máxima amplificación en el emplazamiento. Bajo estas consideraciones, se analiza la Figura 4.8, en la cual se presentan, a modo de ejemplo, las curvas de amplificación espectral para los emplazamientos número 4, 17 y 22. En el emplazamiento $n^{\circ} 4$, la máxima 
amplificación corresponde a $2,1 \mathrm{~Hz}$ de frecuencia, que equivale a un periodo fundamental de $0,5 \mathrm{~s}$. En el emplazamiento $n^{\circ} 17$, la amplificación máxima se observa a $4,6 \mathrm{~Hz}$, o bien a $0,2 \mathrm{~s}$ de periodo fundamental. En el emplazamiento $n^{\circ} 22$, la amplificación máxima es alcanzada a la frecuencia de 2,9 Hz, que corresponde a un periodo fundamental de $0,3 \mathrm{~s}$. En la Tabla 4.2 se recogen los valores de máxima amplificación y periodo fundamental para cada uno de los 25 emplazamientos de El Hierro. Igualmente, se expresa el factor de amplificación para altas frecuencias $\left(F_{a}\right)$ de la clase de emplazamiento a la cual pertenece cada punto, de acuerdo con el mapa de amplificación sísmica de las Islas Canarias (Figura 3.12).

En el Apéndice E se puede consultar la curva de amplificación espectral obtenida para cada uno de los 25 emplazamientos de medida ReMi, de acuerdo con el correspondiente modelo de Vs.

\footnotetext{
Tabla 4.2: Valores de la máxima amplificacíon y del periodo fundamental (s) en los 25 emplazamientos de medida ReMi en El Hierro, obtenidos a partir de las curvas de amplificación espectral calculadas con los respectivos modelos de velocidad Vs. También se presentan los factores de amplificación $F_{a}$ para altas frecuencias $(2-10 \mathrm{~Hz})$ que corresponden a los emplazamientos, de acuerdo con el mapa de amplificación sísmica de las Islas Canarias (Figura 3.12).
}

\begin{tabular}{|r|r|r|r|}
\hline $\begin{array}{l}\text { Número de } \\
\text { emplazamiento }\end{array}$ & $\begin{array}{l}\text { Máxima } \\
\text { amplificación }\end{array}$ & $\begin{array}{l}\text { Periodo } \\
\text { fundamental (s) }\end{array}$ & $F_{a}$ Mapa \\
\hline 1 & 5,8 & 0,4 & 1,6 \\
\hline 2 & 6,7 & 0,4 & 0,9 \\
\hline 3 & 5,9 & 0,3 & 0,9 \\
\hline 4 & 7,0 & 0,5 & 1,9 \\
\hline 5 & 5,9 & 0,4 & 1,9 \\
\hline 6 & 7,2 & 0,4 & 0,9 \\
\hline 7 & 4,5 & 0,3 & 0,9 \\
\hline 8 & 5,2 & 0,3 & 0,9 \\
\hline 9 & 4,0 & 0,2 & 1,6 \\
\hline 10 & 5,4 & 0,3 & 0,9 \\
\hline 11 & 2,9 & 0,2 & 0,9 \\
\hline 12 & 5,1 & 0,3 & 1,6 \\
\hline 13 & 2,8 & 0,2 & 0,9 \\
\hline 14 & 5,5 & 0,2 & 0,9 \\
\hline 15 & 6,3 & 0,4 & 1,6 \\
\hline 16 & 4,7 & 0,3 & 0,9 \\
\hline 17 & 4,7 & 0,2 & 0,9 \\
\hline 18 & 6,7 & 0,4 & 1,6 \\
\hline 19 & 6,1 & 0,3 & 0,9 \\
\hline 20 & 4,8 & 0,2 & 1,6 \\
\hline 21 & 5,8 & 0,3 & 0,9 \\
\hline 22 & 6,8 & 0,3 & 0,9 \\
\hline 23 & 7,0 & 0,4 & 1,6 \\
\hline 24 & 7,1 & 0,3 & 0,9 \\
\hline 25 & 5,8 & 0,3 & 0,9 \\
\hline & & & \\
\hline & & & 0,3 \\
\hline
\end{tabular}

Odum et al. (2010, 2013) han obtenido la respuesta espectral en diferentes emplazamientos por medio del cálculo teórico de la propagación de la onda plana SH a través de modelos de Vs obtenidos por ReMi (aproximación del cuarto de longitud de onda, 2.3.1). En particular, las medidas ReMi fueron realizadas en cinco emplazamientos del ANSS (Advanced National Seismic System) en Illinois e Indiana (Odum et al., 2010), y en 27 emplazamientos en Puerto Rico (Odum et al., 2013). En ambos trabajos, la mayoría de las amplificaciones espectrales se encuentran comprendidas entre 2 y 8 . Como se observa en la Tabla 4.2, las máximas amplificaciones espectrales 

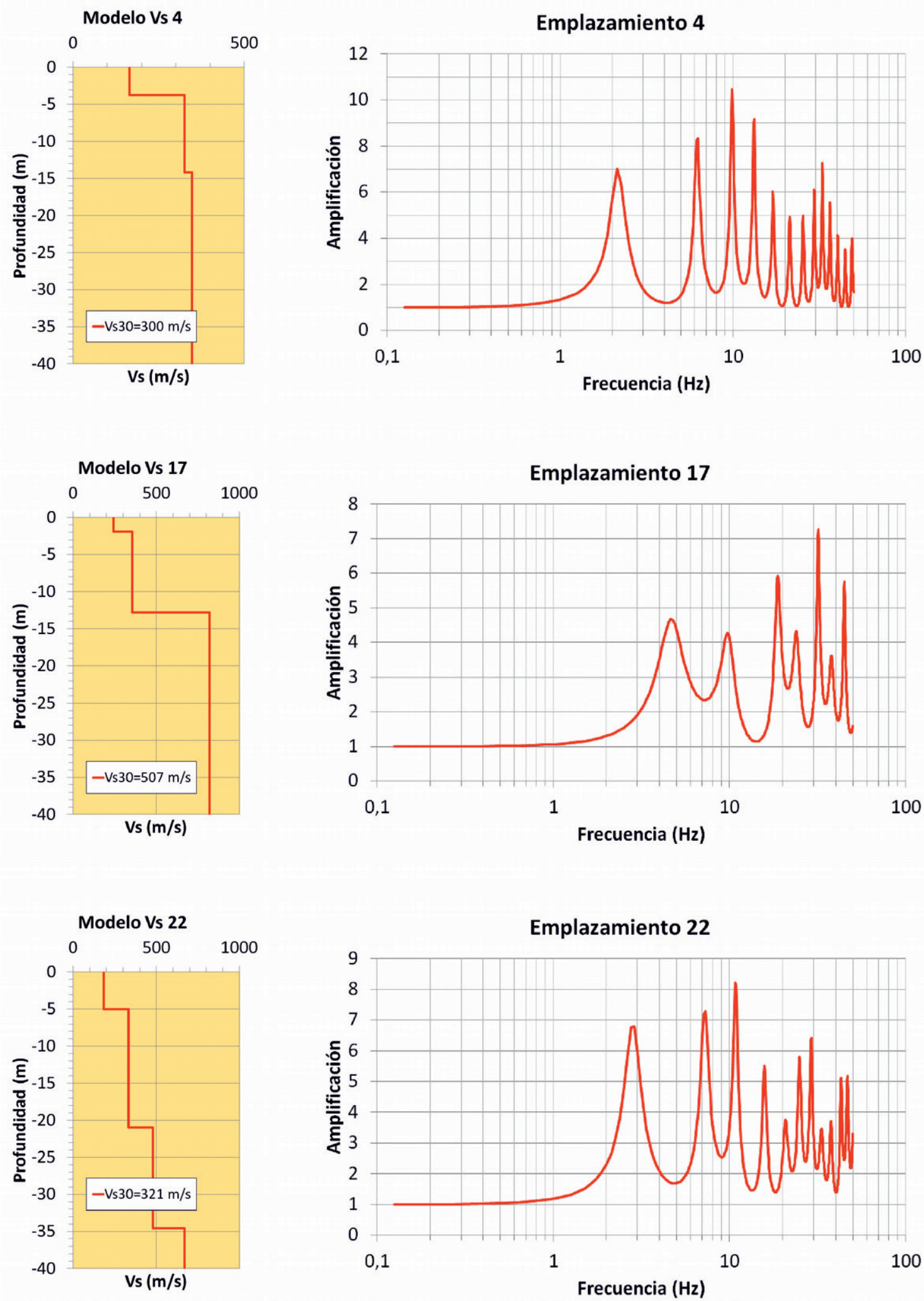

Figura 4.8: Curvas de amplificación espectral obtenidas a partir de los modelos de velocidad Vs en los emplazamientos $n^{\circ} 4,17$ y 22 . 
calculadas en El Hierro están incluidas en el rango entre 2,8 y 7,2. Por tanto, los niveles de amplificación calculados en la isla pueden considerarse como comparables con los publicados por Odum et al. (2010, 2013), siendo interesante esta coherencia en el caso de los resultados de Puerto Rico, al ser también una isla con carácter volcánico.

\subsubsection{Comparación de los valores máximos de amplificación en los emplazamientos de medida con los factores de amplificación del mapa}

Obtenido el valor de la máxima amplificación de cada emplazamiento, a partir de la correspondiente curva de amplificación espectral (Tabla 4.2, se ha procedido a comparar la máxima amplificación con el factor de amplificación para altas frecuencias $\left(F_{a}\right)$ que corresponde al emplazamiento, según el mapa de amplificación sísmica de las Islas Canarias (Figura 3.12), y que también se puede consultar en la Tabla 4.2. De esta forma, se pretende comprobar la validez del mapa de amplificación sísmica en El Hierro. El motivo para emplear el factor $F_{a}$, y no el factor de amplificación para bajas frecuencias $F_{v}$, reside en la similitud entre el rango de frecuencias de $F_{a}$ $(2-10 \mathrm{~Hz})$ y el intervalo de frecuencias en el cual se localiza la frecuencia asociada al periodo fundamental del suelo y a la máxima amplificación del emplazamiento, y que abarca frecuencias menores que $10 \mathrm{~Hz}$. No es posible utilizar $F_{v}$, pues el intervalo de frecuencias en el cual es aplicable abarca desde 0,5 hasta 2,5 Hz Borcherdt (1994), insuficiente para poder comparar este factor con las amplificaciones espectrales para todas las frecuencias inferiores a $10 \mathrm{~Hz}$.

Como se observa en la Tabla 4.2, el valor de la máxima amplificación para todos los emplazamientos es mayor que el correspondiente valor de $F_{a}$, siendo la máxima diferencia entre ambos parámetros 6,3 (emplazamiento $n^{\circ} 6$ ). En emplazamientos con el mismo valor de $F_{a}, F_{a}=0,9$, la máxima amplificación oscila entre 2,8 (emplazamiento $\left.n^{\circ} 13\right)$ y 7,2 (emplazamiento $\left.n^{\circ} 6\right)$. Esta variabilidad refleja claramente la divergencia importante que existe entre la primera aproximación a la amplificación sísmica del terreno que representa el factor $F_{a}$ y la segunda aproximación que implica la curva de amplificación espectral, al introducir el modelo local de velocidades que se ha obtenido experimentalmente a partir de medidas ReMi.

En los restantes emplazamientos, con $F_{a}=1,6$ y $F_{a}=1,9$, también se observan diferencias importantes entre el factor de amplificación del mapa de amplificación sísmica y la máxima amplificación que se obtiene a partir de la curva de amplificación espectral. En particular, en los 7 emplazamientos con $F_{a}=1,6$, esta diferencia alcanza 5,4 (emplazamiento 23), mientras que en los 2 emplazamientos con $F_{a}=1,9$, la diferencia es al menos de 4 , tomando valores de 4 y 5,1 en los emplazamientos 5 y 4 , respectivamente (Tabla 4.2).

Las diferencias encontradas entre el factor de amplificación $F_{a}$ que proporciona el mapa de amplificación y la máxima amplificación procedente de la curva de amplificación espectral se deben seguramente a la consideración del modelo local de Vs obtenido con el método ReMi en el proceso de generación de la curva de amplificación, y al cálculo de $F_{a}$ a partir del valor de Vs30 proporcionado por el mapa de clases de emplazamiento y Vs30. Como se ha explicado en la subsección 4.3.1, los 
valores de Vs30 obtenidos experimentalmente ya presentan diferencias importantes con respecto a los valores Vs30 de este mapa. Por tanto, es razonable pensar que las diferencias encontradas en cuanto a la velocidad Vs entre la cartografía y los resultados del método ReMi se reflejen finalmente en las divergencias constatadas en la amplificación. 


\section{Capítulo 5}

\section{DETERMINACIÓN EXPERIMENTAL DEL PERIODO FUNDAMENTAL DEL SUELO EN EL HIERRO MEDIANTE EL MÉTODO H/V}

Obtenidas las curvas de amplificación espectral en cada uno de los 25 emplazamientos de medida de ruido sísmico ambiental por medio del método ReMi (Apéndice E, Tabla 4.2), en determinados emplazamientos se ha obtenido, de forma experimental, el periodo fundamental del suelo por medio de la aplicación del método $\mathrm{H} / \mathrm{V}$ (sección 2.4). El objetivo de esta nueva microzonación sísmica en El Hierro ha consistido, del mismo modo que la campaña de medidas con ReMi, en la estimación de la amplificación sísmica local con mayor exactitud que la proporcionada por el mapa de amplificación sísmica (Figura 3.12). Con los resultados obtenidos para el periodo fundamental, se ha llevado a cabo una comparación de los mismos con los valores de periodo fundamental derivados de las curvas de amplificación espectral, sólo en aquellos emplazamientos donde se ha utilizado el método $\mathrm{H} / \mathrm{V}$.

\subsection{Adquisición de muestras de ruido sísmico am- biental a partir del registro continuo de las es- taciones de registro de velocidad del Instituto Geográfico Nacional (IGN) en El Hierro}

De acuerdo con la Figura 4.1 y con la Tabla B.1, 6 de los 25 emplazamientos de medidas ReMi se sitúan en las cercanías de estaciones de registro de velocidad del IGN: emplazamientos $n^{\circ} 1$ (estación CRST), 4 (CJUL), 12 (CHIE), 19 (CTAB y CTAN), 22 (CCUM) y 23 (CMCL). Además, de los 19 emplazamientos restantes, 2 se encuentran en las proximidades de las estaciones centrales de los dos arrays sísmicos desplegados por el IGN en la isla: emplazamientos $n^{\circ} 2$ (estación HI01 del array de 
Tacorón) y $n^{\circ} 17$ (estación HV01 del array de El Verodal). Siguiendo a Nakamura (1989), en estos 8 emplazamientos se ha tratado de estimar el periodo fundamental por medio del ratio espectral de las componentes horizontal $(\mathrm{H})$ y vertical $(\mathrm{V})$ del ruido ambiental. En particular, se busca la frecuencia correspondiente al máximo ratio $\mathrm{H} / \mathrm{V}$, que es la que se encuentra correlacionada con la frecuencia fundamental de resonancia del suelo.

Para determinar el ratio $\mathrm{H} / \mathrm{V}$ en cada emplazamiento, se han empleado muestras de ruido sísmico del registro continuo de las citadas estaciones, proporcionadas en formato SAC por el IGN, en ventanas temporales de 30 minutos de duración. En este contexto, no se han podido emplear los registros de las estaciones CHIE y CMCL (cercanas a los emplazamientos $n^{\circ} 12$ y 23, respectivamente) pues éstas consisten en sismómetros de corto periodo de componente vertical. Esta circunstancia implica la ausencia de registro para las componentes horizontales y, por tanto, no permite la aplicación del método $\mathrm{H} / \mathrm{V}$ en estos emplazamientos. Por tanto, sólo ha sido posible determinar experimentalmente el periodo fundamental en los 6 emplazamientos restantes, $n^{\circ} 1,2,4,17,19,22$.

En cada emplazamiento, se han seleccionado, con carácter general, 9 ventanas temporales de 30 minutos, en las cuales se ha comprobado la ausencia de terremotos con el catálogo sísmico del IGN (Instituto Geográfico Nacional, 2015b). Se ha procurado que las ventanas correspondan a horas nocturnas, a fin de evitar que el ruido procedente de las actividades industriales se encuentre registrado en las mismas. Debido a la operatividad de las estaciones centrales de los arrays sísmicos, consistente en la recepción y tratamiento de los datos procedentes del conjunto de sensores que constituyen el dispositivo en array, en los emplazamientos 2 y 17 la selección de las ventanas temporales ha estado condicionada a la disponibilidad de datos con una calidad mínima. En las Tablas 5.1 y 5.2 se especifica el comienzo de cada una de las ventanas temporales seleccionadas para tratar el ruido ambiental en cada estación.

Tabla 5.1: Comienzo de las ventanas de 30 minutos de duración y con ruido sísmico
ambiental, procedentes de las estaciones de registro de velocidad CCUM, CJUL,
CTAB, CTAN y CRST. Los tiempos están expresados en UTC (Universal Time
Coordinated).
\begin{tabular}{|c|l|}
\hline Comienzo de ventana & Estaciones \\
\hline $01 / 11 / 2013$ 03:00:00 & CCUM, CTAB, CTAN, CRST \\
\hline $03 / 11 / 2013$ 02:00:00 & CCUM, CTAB, CTAN, CRST \\
\hline $10 / 11 / 2013$ 03:00:00 & CCUM, CTAB, CTAN, CRST \\
\hline $17 / 11 / 2013$ 03:00:00 & CCUM, CTAB, CTAN, CRST \\
\hline $02 / 12 / 2013$ 03:00:00 & CCUM, CJUL, CTAB, CTAN, CRST \\
\hline $09 / 12 / 201304: 00: 00$ & CCUM, CJUL, CTAB, CTAN, CRST \\
\hline $13 / 12 / 2013$ 01:00:00 & CCUM, CTAB, CTAN, CRST \\
\hline $16 / 12 / 201303: 00: 00$ & CCUM, CTAB, CTAN, CRST \\
\hline $17 / 12 / 201302: 00: 00$ & CCUM, CTAB, CTAN, CRST \\
\hline
\end{tabular}

\subsection{Análisis de las curvas de la relación $\mathrm{H} / \mathrm{V}$ y de- terminación del periodo fundamental}

A continuación, los datos de las ventanas de ruido fueron procesados con el software GEOPSY, escrito por Marc Wathelet, del ISTerre (Francia). Como resultado, 
Tabla 5.2: Comienzo de las ventanas de $30 \mathrm{mi}-$ nutos de duración y con ruido sísmico ambiental, procedentes de la estación central HI01 del array de Tacorón, y de la estación central HV01 del array de El Verodal. Los tiempos están expresados en UTC (Universal Time Coordinated).

\begin{tabular}{|c|l|}
\hline Comienzo de ventana & Estación \\
\hline $01 / 11 / 2013$ 03:30:00 & HV01 \\
\hline $03 / 11 / 2013$ 02:00:00 & HV01 \\
\hline $05 / 11 / 2013$ 03:30:00 & HV01 \\
\hline $14 / 11 / 2013$ 02:00:00 & HI01 \\
\hline $17 / 11 / 201303: 30: 00$ & HV01 \\
\hline $18 / 11 / 201302: 00: 00$ & HI01 \\
\hline $21 / 11 / 201303: 00: 00$ & HI01 \\
\hline $22 / 11 / 201303: 00: 00$ & HI01 \\
\hline $25 / 11 / 201302: 00: 00$ & HI01 \\
\hline $26 / 11 / 201303: 00: 00$ & HI01 \\
\hline $27 / 11 / 201303: 00: 00$ & HI01 \\
\hline $02 / 12 / 201303: 00: 00$ & HI01 \\
\hline $02 / 12 / 201303: 30: 00$ & HV01 \\
\hline $03 / 12 / 201303: 00: 00$ & HI01 \\
\hline $09 / 12 / 201304: 00: 00$ & HV01 \\
\hline $13 / 12 / 201301: 00: 00$ & HV01 \\
\hline $16 / 12 / 201303: 30: 00$ & HV01 \\
\hline $17 / 12 / 201302: 00: 00$ & HV01 \\
\hline
\end{tabular}

en cada emplazamiento se han obtenido las curvas de amplificación espectral $\mathrm{H} / \mathrm{V}$, o de ratio espectral $\mathrm{H} / \mathrm{V}$, para cada ventana. También en cada ventana, se han generado los gráficos de la variación del ratio $\mathrm{H} / \mathrm{V}$ en función de la frecuencia y del acimut, para acimutes de $0^{\circ}$ a $180^{\circ}$.

En cada emplazamiento, se ha seleccionado la ventana temporal que presenta un máximo del ratio espectral $\mathrm{H} / \mathrm{V}$ en el intervalo de frecuencias comprendido entre $0 \mathrm{y}$ $10 \mathrm{~Hz}$. Como segundo criterio de selección, se ha tratado de asegurar, en la medida de lo posible, que este máximo se produce en todos los acimutes comprendidos entre $0^{\circ}$ a $180^{\circ}$, con el fin de descartar fuentes de ruido focalizadas y de posible origen industrial, y así garantizar la bondad de los resultados.

En la Figura 5.1 se presentan las curvas del ratio espectral H/V correspondientes a los emplazamientos 4, 17 y 22, junto con los gráficos del ratio $\mathrm{H} / \mathrm{V}$ en función de la frecuencia y el acimut. En el emplazamiento 4, se ha seleccionado la ventana temporal del registro de la estación CJUL que comienza el 09/12/2013 a las 04:00:00 UTC (Tabla 5.1). En el caso del emplazamiento 17, se ha seleccionado la ventana correspondiente a la estación central HV01 del array de El Verodal, con comienzo a las 03:30:00 UTC del 05/11/2013 (Tabla 5.2). Por último, en el emplazamiento 22 , se ha seleccionado la ventana temporal de la estación CCUM, con comienzo el 02/12/2013 a las 03:00:00 UTC (Tabla 5.1).

En el emplazamiento 17, el máximo ratio $\mathrm{H} / \mathrm{V}$ ocurre en todos los acimutes (Figura 5.1), lo que asegura que la fuente de ruido no se encuentra focalizada en una dirección en particular, y por tanto que no tiene un origen industrial. Este máximo se alcanza en la frecuencia de $4,5 \mathrm{~Hz}$, lo que equivale a un periodo fundamental de 0,2 s. Este valor del periodo fundamental coincide con el obtenido a partir de la curva de amplificación espectral (0,2 s) que se ha generado a partir del modelo de 

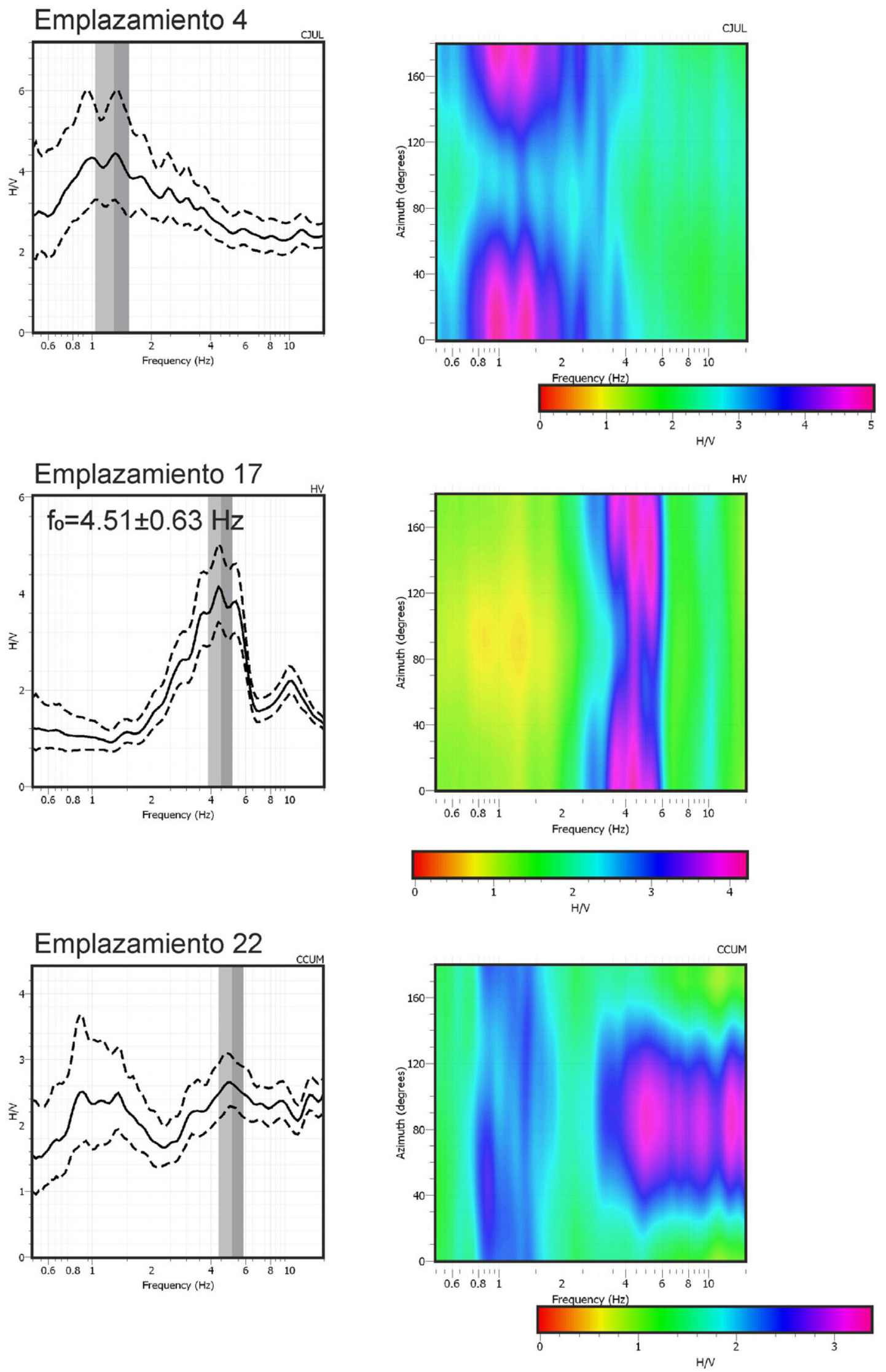

Figura 5.1: Curvas de la relación $\mathrm{H} / \mathrm{V}$ en función de la frecuencia (izquierda) obtenidas con ventana de ruido sísmico de 30 minutos en los emplazamientos $n^{\circ} 4,17$ y 22. En la parte derecha, análisis del ratio $\mathrm{H} / \mathrm{V}$ en función de la frecuencia para los acimutes $0^{\circ}$ a $180^{\circ}$. Se señala la frecuencia fundamental $\left(f_{0}\right)$ y su desviación estándar para el emplazamiento 17 , único en el que el máximo ratio $\mathrm{H} / \mathrm{V}$ no se encuentra focalizado. 
Vs procedente de la medida con ReMi (Tabla 4.2 y Figura 4.8).

En cambio, en el emplazamiento 22 el máximo ratio $\mathrm{H} / \mathrm{V}$ se encuentra claramente focalizado entre $40^{\circ}$ a $120^{\circ}$, como se observa en la Figura 5.1, y por este motivo no es representativo del ruido ambiental. No obstante, se ha encontrado un valor no focalizado del ratio $\mathrm{H} / \mathrm{V}$ en la frecuencia $1,4 \mathrm{~Hz}$, si bien el periodo que le corresponde $(0,7 \mathrm{~s})$ dobla el valor del periodo fundamental obtenido a partir de la curva de amplificación espectral de este emplazamiento (0,3 s), como se puede comprobar en la Tabla 4.2. Con respecto al emplazamiento 4, el máximo ratio $\mathrm{H} / \mathrm{V}$ está focalizado en acimutes menores que $60^{\circ}$ y mayores que $140^{\circ}$ (Figura 5.1). En este caso, no ha sido posible determinar un valor aceptable del periodo fundamental a partir de la curva de la relación $\mathrm{H} / \mathrm{V}$.

En el Apéndice F se pueden consultar las curvas del ratio espectral H/V obtenidas para cada uno de los 6 emplazamientos donde se ha aplicado el método $\mathrm{H} / \mathrm{V}$, así como los gráficos correspondientes de la variación del ratio $\mathrm{H} / \mathrm{V}$ en función de la frecuencia y del acimut, para los acimutes de $0^{\circ}$ a $180^{\circ}$. En referencia al emplazamiento 1 , se ha seleccionado la ventana temporal del registro de la estación CRST que comienza el 10/11/2013 a las 03:00:00 UTC (Tabla 5.1). En el caso del emplazamiento 2, se ha seleccionado la ventana correspondiente a la estación central HI01 del array de Tacorón, con comienzo en el día 27/11/2013 a las 03:00:00 UTC (Tabla 5.2). En cuanto al emplazamiento 19 , se han considerado las curvas del ratio espectral $\mathrm{H} / \mathrm{V}$ obtenidas con los registros de las estaciones CTAB y CTAN, pues ambas se sitúan en las proximidades del emplazamiento, a distancia similar. En ambas estaciones, se ha seleccionado la ventana temporal que comienza el 17/12/2013 a las 02:00:00 UTC (Tabla 5.1).

A la vista de los resultados obtenidos para el conjunto de los seis emplazamientos, el máximo ratio $\mathrm{H} / \mathrm{V}$ se encuentra focalizado en direcciones particulares, excepto en el emplazamiento 17. Por tanto, los registros de ruido que corresponden al resto de emplazamientos (1, 2, 4, 19 y 22) no son válidos para determinar experimentalmente el periodo fundamental. Sólo en el emplazamiento 17 se ha podido determinar experimentalmente un valor aceptable del periodo fundamental del suelo, que se ha podido comparar con el valor del periodo fundamental procedente de la curva de amplificación espectral generada a partir del modelo de Vs (Tabla 4.2). Al confirmar que los dos valores del periodo fundamental así calculados son coincidentes $(0,2$ s), se puede certificar la validez del periodo fundamental obtenido con la curva de amplificación espectral.

A pesar del hecho de que sólo se haya podido determinar de forma experimental el periodo fundamental del suelo en un emplazamiento en El Hierro, la aplicación del método $\mathrm{H} / \mathrm{V}$ que se ha llevado a cabo en esta tesis ha dado resultados satisfactorios en otros entornos, como se detalla en el trabajo de Rueda et al. (2015), quienes obtienen valores experimentales del periodo fundamental en determinados emplazamientos de medida ReMi en Torreperogil y Sabiote (Jaén). Estos valores han permitido certificar los periodos fundamentales calculados a partir de las curvas de amplificación espectral correspondientes a los mismos emplazamientos, y obtenidas por los autores a partir del modelo de Vs proporcionado por la aplicación del método ReMi. 


\section{Capítulo 6}

\section{ANÁLISIS Y SELECCIÓN DE LEYES DE ATENUACIÓN EN TÉRMINOS DE ACELERACIÓN MÁXIMA Y DETERMINACIÓN DE LA INTENSIDAD SINTÉTICA}

Una vez que se ha estimado la amplificación sísmica del terreno en la Península Ibérica y las Islas Baleares, y también en las Islas Canarias, se está en disposición de abordar la simulación de escenarios sísmicos en ambos territorios, tanto en términos de aceleración como de intensidad. En primera aproximación, se pretenden obtener valores sintéticos realistas de la aceleración máxima del terreno, suponiendo una fuente sísmica puntual, por medio de una ley de atenuación y de los factores de amplificación procedentes del mapa de amplificación sísmica. A partir de los valores sintéticos de aceleración máxima, se obtiene la intensidad sintética por medio de una correlación entre la aceleración máxima del terreno y la intensidad sísmica.

Por tanto, en este capítulo se presenta el análisis de las distintas leyes de atenuación en términos de aceleración máxima que se consideran susceptibles de aplicación en la Península Ibérica e Islas Baleares, y en las Islas Canarias. La calibración de las leyes de atenuación así preseleccionadas, en función de las registros de aceleración disponibles, permite seleccionar las leyes de atenuación óptimas en función de la escala de trabajo y de la magnitud del terremoto.

\subsection{Leyes de atenuación aplicables en la Península Ibérica y las Islas Baleares}

La falta de registros de aceleración de terremotos de elevada magnitud en España, por parte de la red de acelerómetros del IGN, no ha permitido la obtención de leyes de atenuación en aceleración que sean válidas en los distintos tipos de suelos aflorantes. Además, estos acelerómetros se encuentran instalados en el interior de edificios y no se conocen las propiedades del suelo en estos emplazamientos. En 
cambio, la existencia de observaciones de intensidad sísmica para terremotos de magnitud media ha permitido la producción de un número significativo de leyes de atenuación en términos de intensidad para la Península Ibérica. Pueden citarse a este respecto las relaciones de atenuación de Muñoz (1974), Martín Martín (1983, 1984) y López Casado et al. (2000). Las relaciones de atenuación de Martín Martín (1983), obtenidas para distintas zonas sismogenéticas de España, se encuentran implementadas en el programa SES 2002 (Dirección General de Protección Civil y Dirección General del Instituto Geográfico Nacional, 2002). Este programa lleva a cabo simulaciones de la intensidad sísmica que permitan estimar efectos sobre la población y las construcciones ante la ocurrencia de un sismo, para lo cual selecciona una de las relaciones de atenuación en función de la zona donde se localiza el epicentro del terremoto a simular. Partiendo de la intensidad epicentral del terremoto, el código SES 2002 obtiene la intensidad sintética en cualquier municipio, calculada su distancia epicentral, si bien no modifica la intensidad sintética en función de la amplificación sísmica del terreno. Por último, se incluye en esta revisión la relación obtenida por Mezcua et al. (2004) entre la intensidad, la magnitud Mw y la distancia epicentral en el contexto de su trabajo de reevaluación de terremotos históricos en España.

Se presenta a continuación una revisión somera de trabajos recientes en los cuales se han obtenido leyes de atenuación en términos de aceleración, a partir de registros de aceleración procedentes de la Península Ibérica. En este contexto, en esta tesis doctoral se analizan en profundidad las relaciones de Tapia (2006), Mezcua et al. (2008), García Blanco (2009) e Instituto Geográfico Nacional (2013), con el fin de proceder a su calibración en función de las intensidades observadas. Estas cuatro relaciones se han obtenido a partir de aceleraciones registradas por la red de acelerómetros del IGN, y que corresponden a emplazamientos en suelo duro (clase de emplazamiento 4 en Tabla 3.4) en la Península Ibérica.

Adicionalmente, cabe citar los trabajos de Cabañas et al. $(1999,2000)$ y de Benito et al. (2000), relacionados por Douglas (2011). De acuerdo con este último autor, estos trabajos determinan, de forma separada, relaciones de atenuación a partir de datos de la región mediterránea y relaciones basadas, exclusivamente, en datos de España. Las relaciones de atenuación así obtenidas discriminan cuatro categorías de emplazamiento: roca dura del basamento, roca sedimentaria y conglomerados, depósitos glaciales, y aluviales y sedimentos consolidados. Ambraseys et al. (2005) utilizan 6 registros de España, incluidos en un conjunto más amplio de 595 registros de movimiento fuerte distribuidos en Europa y Oriente Medio, para obtener una relación de atenuación que discrimina tres categorías de emplazamiento: suelo blando, suelo duro y roca. En esta tesis doctoral se analizan dos relaciones que emplean registros de España, en el marco de una selección más amplia de acelerogramas obtenidos en Europa (Berge-Thierry et al., 2003; Akkar y Bommer, 2010). Por último, Douglas (2011) relaciona el trabajo de Bommer et al. (2007), que también procesa acelerogramas registrados en España junto con otros registros de Europa y Oriente Medio. 


\subsubsection{Análisis de leyes de atenuación aplicables en el ámbito de la Península Ibérica y las Islas Baleares}

En el ámbito de la Península Ibérica y las Islas Baleares, se han analizado ocho relaciones empíricas de atenuación del movimiento del suelo, expresado en aceleración y velocidad máxima, que son susceptibles de aplicación en la Península Ibérica. A partir de este análisis, las relaciones han sido calibradas en función de las intensidades macrosísmicas disponibles, y, de esta forma, se han seleccionado las leyes de atenuación óptimas para el territorio.

El análisis de las relaciones de atenuación considera determinados parámetros en cada relación, con el fin de cuantificar la incertidumbre asociada a cada una, y consiguientemente valorar su aplicación en la Península Ibérica. Estas incertidumbres son cuantificadas por medio de la metodología del árbol lógico (Coppersmith y Youngs, 1986), asignando un peso normalizado a cada ley de atenuación. El peso otorgado a cada una depende del grado de cumplimiento de los diez criterios de Bommer et al. (2010) y de la calibración de la relación de atenuación con las intensidades observadas para los terremotos seleccionados a estos efectos.

Las relaciones de atenuación estudiadas son las de Berge-Thierry et al. (2003), Tapia (2006), Boore y Atkinson (2008), Cauzzi y Faccioli (2008), Cotton et al. (2008), Mezcua et al. (2008), García Blanco (2009), Akkar y Bommer (2010) e Instituto Geográfico Nacional (2013). Los parámetros examinados en cada una de estas relaciones de atenuación son los siguientes:

- Parámetros del movimiento del suelo: Las relaciones de atenuación permiten calcular valores de aceleración espectral (PSA), aceleración máxima (PGA) y velocidad máxima $(\mathrm{PGV})$

- Magnitud: La caracterización de los sismos es realizada por medio de la magnitud momento $(\mathrm{Mw})$, aunque también se utilizan la magnitud local $\left(M_{L}\right)$ y la magnitud de ondas superficiales $M_{S}$, convirtiéndolas a Mw con las correlaciones de Ristau et al. (2005) y de Wells y Coppersmith (1994), respectivamente.

- Distancia: Se consideran cuatro tipos de distancia (epicentral $\left(D_{e p i}\right)$, hipocentral $\left(D_{\text {hipo }}\right)$, Joyner y Boore $\left(R_{j b}\right)$, y de ruptura)).

- Profundidad del foco del terremoto (h)

- Efecto de sitio: Para calcular los valores de aceleración máxima y velocidad máxima con cada ley de atenuación, se ha considerado la expresión correspondiente a una clase de emplazamiento de roca dura o compacta $(760 \leq V s 30 \leq 1500)$ $\mathrm{m} / \mathrm{s}$.

- Movimiento de falla: Se considera que la falla responsable de los terremotos utilizados en la calibración tiene un mecanismo preponderante normal con componente de desgarre.

Como primera herramienta para obtener el peso normalizado de cada relación de atenuación, se utilizan los diez criterios de Bommer et al. (2010). Estos criterios son: 
1. El modelo de atenuación se ha obtenido a partir de un ambiente tectónico similar

2. El modelo está publicado en una revista de prestigio internacional del SCI (Science Citation Index)

3. El modelo no ha sido reemplazado por una publicación más reciente

4. Los datos se presentan en un formato accesible (lista de terremotos, sus características y el número de eventos)

5. El modelo proporciona predicciones espectrales para un periodo de respuesta de 0,0 a $2,0 \mathrm{~s}$

6. La expresión del modelo posee dependencia lineal con la magnitud y no hay una disminución importante de la magnitud con la distancia

7. Los coeficientes del modelo se determinan con un método que tiene en cuenta la variabilidad intra e inter-evento (análisis de regresión en una o dos fases)

8. El modelo utiliza definiciones apropiadas de las variables, como la magnitud y la distancia, y considera el efecto del suelo a partir del valor medio de la velocidad de propagación de la onda de corte (Vs) en los primeros $30 \mathrm{~m}$ de profundidad (Vs30).

9. El rango de aplicación del modelo, en cuanto a magnitud y distancia, ha de ser amplio. En principio, las magnitudes mínima $\left(M_{\min }\right)$ y máxima $\left(M_{\max }\right)$ deben cumplir que $M_{\min }>5$ y $M_{\max }<7$, respectivamente; la distancia máxima $\left(R_{\max }\right)$ debe ser tal que $R_{\max }<80 \mathrm{~km}$.

10. El modelo se ha construido con suficientes datos (más de 10 terremotos por unidad de magnitud y más de 100 por cada $100 \mathrm{~km}$ de distancia).

A continuación se resumen las características fundamentales de las relaciones empíricas analizadas. Tanto estas características, como las específicamente relacionadas con los criterios de Bommer et al. (2010) se pueden encontrar en la Tabla 6.1.

\section{Berge-Thierry et al. (2003)}

El modelo de Berge-Thierry et al. (2003) se obtiene a partir de 965 registros de componentes horizontales de acelerómetros y 485 de componentes verticales, procedentes de terremotos de magnitud moderada, ocurridos en Europa (cuatro terremotos ocurridos en España) y California. Se considera que este modelo es de aplicación en la Península Ibérica por haberse obtenido en un ambiente tectónico similar.

Las variables predictivas del modelo de Berge-Thierry et al. (2003) son la magnitud $M_{S}$ y la distancia $D_{\text {hipo }}$, con rangos de aplicación para la magnitud $4 \leq M \leq 7,9$, y para la distancia $4 \leq D_{\text {hipo }} \leq 330 \mathrm{~km}$. El modelo considera el efecto de sitio a partir de la Vs30, con roca para $V s 30 \geq 800 \mathrm{~m} / \mathrm{s}$ y suelo firme para $300<V s 30<800$ $\mathrm{m} / \mathrm{s}$, pero no incorpora parámetros relativos al mecanismo focal. El modelo de BergeThierry et al. (2003) tiene la siguiente expresión para la PGA en roca: 


$$
\log (P G A)=0,3118 M_{S}-0,0009303 D_{\text {hipo }}-\log \left(D_{\text {hipo }}\right)+1,537
$$

con PGA en $\mathrm{cm} / \mathrm{s}^{2}$ y $D_{\text {hipo }}$ en $\mathrm{km}$. La desviación estándar $\sigma$ del modelo es $\sigma=0,29$.

\section{Tapia (2006)}

El modelo de Tapia (2006) permite calcular valores de PGA. Se obtiene a partir del análisis de 280 registros de aceleración de 26 terremotos, que proceden de Francia, España, Argelia y Marruecos. Los terremotos tienen magnitudes locales comprendidas entre 3,8 y 5,2 y distancias epicentrales entre 7,5 y $542 \mathrm{~km}$. Se considera que el modelo es aplicable en la Península Ibérica para distancias epicentrales menores que $600 \mathrm{~km}$ y magnitudes comprendidas entre 3,8 y 5,2.

Como variables predictivas, el modelo considera la magnitud $M_{L}$ y la distancia $D_{\text {epi }}$, pero no incorpora ni la clase de emplazamiento ni el mecanismo focal. El modelo de Tapia (2006) tiene la siguiente expresión:

$$
\begin{aligned}
\log (P G A)= & -1,8+0,45(M w-0,6)-1,6 \log \left(\sqrt{D_{e p i}^{2}+10^{2}}\right) \\
& -0,0013 \sqrt{D_{e p i}^{2}+10^{2}}
\end{aligned}
$$

donde PGA se expresa en $\mathrm{g}\left(\mathrm{g}=9,8 \mathrm{~m} / \mathrm{s}^{2}\right)$ y $D_{\text {epi }}$ en $\mathrm{km}$. En esta tesis doctoral se utiliza la ecuación 6.2 previa conversión de la $M_{L}$ en Mw, según Ristau et al. (2005).

Se considera que, dado el origen de la mayor parte de los datos, la expresión del modelo es válida para suelo duro. Para obtener los valores de PGA referidos a roca $(760 \leq V s 30<1500 \mathrm{~m} / \mathrm{s})$, se realiza la suma de la ecuación6.2 y el valor resultante de la siguiente expresión de Campbell (1997):

$$
0,405-0,222 \ln \left(D_{\text {hipo }}\right)
$$

\section{Boore y Atkinson (2008)}

Boore y Atkinson (2008) desarrollan un modelo de atenuación para PGA, PGV y PSA (0,01-10 s) por medio de 1574 registros de 58 eventos ocurridos en todo el mundo. Estos eventos fueron registrados por el proyecto Pacific Earthquake Engineering Research Center's Next Generation Attenuation (PEER NGA), según Power et al. (2008) en Boore y Atkinson (2008). La PGA se calcula como la aceleración horizontal obtenida a partir de la media geométrica del percentil 50 de los valores de las medias geométricas calculadas a partir de todos los ángulos de rotación no redundantes.

Las variables predictivas son la magnitud Mw, la distancia $R_{j b}$, el mecanismo focal y la clase de emplazamiento. No obstante, Boore y Atkinson (2008) indican que $R_{j b}$ es aproximadamente igual a $D_{e p i}$ si $\mathrm{Mw}<6$. El modelo considera dos clases de emplazamiento en función de Vs30, con roca para $V s 30 \geq 960 \mathrm{~m} / \mathrm{s}$ y suelo firme para $490 \mathrm{~m} / \mathrm{s} \leq V s 30 \leq 960 \mathrm{~m} / \mathrm{s}$. El modelo es aplicable en la Península Ibérica 
para terremotos con magnitudes entre 4 y 6,5, distancias menores que $200 \mathrm{~km} \mathrm{y}$ emplazamientos con Vs30 tal que $180 \leq V s 30 \leq 1300 \mathrm{~m} / \mathrm{s}$.

En el caso de suelo firme, la expresión de la PGA es la siguiente, de acuerdo con Boore y Atkinson (2008):

$$
\begin{aligned}
\ln (P G A)= & -0,53804 U-0,50350 S S-0,75472 N S-0,50970 R S \\
& +0,28805(M w-6,75)-0,10164(M w-6,75)^{2}+[-0,66050 \\
& +0,11970(M w-4,5)] \ln \left(\sqrt{R_{j b}^{2}+1,35^{2}}\right) \\
& -0,01151\left(\sqrt{R_{j b}^{2}+1,35^{2}}-1\right)
\end{aligned}
$$

donde PGA se expresa en $\mathrm{g}\left(\mathrm{g}=9,8 \mathrm{~m} / \mathrm{s}^{2}\right)$ y $R_{j b}$ en $\mathrm{km}$. La desviación estándar $\sigma$ del modelo es $\sigma=0,502$. Los parámetros $S S, N S, R S$ y $U$ expresan el mecanismo de la falla. $S S=1$ si la falla es de desgarre, siendo entonces $N S=R S=U=0$, de acuerdo con Boore y Atkinson (2008). Análogamente, los parámetros $N S, R S$ y $U$ toman el valor 1 y anulan a los restantes para los casos de falla normal, falla inversa y mecanismo desconocido, respectivamente.

Para obtener la expresión de la PGA para emplazamientos en roca hay que sumar a la ecuación6.4 una función que considera el efecto de sitio, que a su vez es suma de un término lineal y otro no lineal, siendo de aplicación para $V s 30 \leq 1300 \mathrm{~m} / \mathrm{s}$. El término lineal se expresa como:

$$
F_{L I N(P G A)}=-0,36 \ln (V s 30 / 760)
$$

siendo Vs30 el valor medio de la velocidad de onda de corte en los primeros 30 $m$ de profundidad del emplazamiento donde se calcula la PGA. En este sentido, en esta tesis doctoral se toma Vs $30=1130 \mathrm{~m} / \mathrm{s}$ para emplazamientos en roca. En cuanto al término no lineal, se estima que toma un valor 0 siempre que $V s 30 \geq 760 \mathrm{~m} / \mathrm{s}$.

\section{Cauzzi y Faccioli (2008)}

Cauzzi y Faccioli (2008) analizan 1164 acelerogramas de 60 terremotos ocurridos en Japón, California, Europa e Irán, obteniendo la media geométrica de las dos componentes horizontales del acelerograma. Las variables predictivas son la magnitud $\mathrm{Mw}$, la distancia $D_{\text {hipo }}$, la clase de emplazamiento y el mecanismo focal. La expresión de la PGA, para magnitudes entre 5 y 7,2, es:

$$
\begin{aligned}
\log (P G A)= & -1,296+0,556 M w-1,582 \log \left(D_{\text {hipo }}\right)+0,22 S_{B} \\
& +0,304 S_{C}+0,332 S_{D}
\end{aligned}
$$

con PGA en $\mathrm{m} / \mathrm{s}^{2}, D_{\text {hipo }}$ en $\mathrm{km}$ y desviación estándar $\sigma$ tal que $\sigma=0,344$. Los parámetros de la clase de emplazamiento son $S_{B}, S_{C}$ y $S_{D}$, siendo $S_{B}=S_{C}=S_{D}=0$ para roca con $V s 30 \geq 800 \mathrm{~m} / \mathrm{s}$, y $S_{B}=1$ y $S_{C}=S_{D}=0$ para suelo firme con $360 \leq V s 30 \leq 800 \mathrm{~m} / \mathrm{s}$. En cuanto al mecanismo focal, no se considera esta variable a los efectos de la calibración y utilización del modelo. 


\section{Cotton et al. (2008)}

El modelo de Cotton et al. (2008) utiliza 3894 acelerogramas de 337 terremotos. Permite calcular valores de PSA para el rango de periodos $\mathrm{T}=0,01-3,3 \mathrm{~s}$. Las variables predictivas son la magnitud $\mathrm{Mw}$, la distancia $D_{\text {hipo }}$ y la clase de emplazamiento. El rango de aplicación abarca distancias menores que $100 \mathrm{~km}$ y magnitudes comprendidas entre 4 y 7,3 .

Las ecuaciones del modelo dependen de la consideración de la magnitud como variable dependiente o independiente del movimiento del suelo. Cotton et al. (2008) recomiendan no extrapolar las ecuaciones que consideran la magnitud constante a magnitudes y distancias diferentes a las correspondientes a los datos empleados en su obtención, con el fin de evitar la sobreestimación de las predicciones realizadas con estas ecuaciones.

En esta tesis doctoral se utiliza la siguiente ecuación para magnitud constante y emplazamiento en roca $V s 30>800 \mathrm{~m} / \mathrm{s}$ :

$$
\begin{aligned}
\log (P S A)= & -4,884+2,18080 M w-0,12964 M w^{2}-0,00397 D_{\text {hipo }} \\
& -\log \left(D_{\text {hipo }}+0,01226 \cdot 10^{0,42 M w}\right)
\end{aligned}
$$

donde PSA se expresa en $\mathrm{cm} / \mathrm{s}^{2}$ y $D_{\text {hipo }}$ en $\mathrm{km}$. La desviación estándar $\sigma$ del modelo es $\sigma=0,353$.

\section{Mezcua et al. (2008)}

El modelo de Mezcua et al. (2008) es aplicable a España, para un rango de magnitudes mayores o iguales a 3, de distancias epicentrales comprendidas entre 5 y $100 \mathrm{~km}$ y de profundidades de 1 a $16 \mathrm{~km}$. El modelo analiza 250 registros de acelerómetros correspondientes a 149 terremotos registrados por el IGN, tomando la aceleración máxima registrada en las componentes horizontales.

Las variables predictivas son la magnitud $\mathrm{Mw}$ y la distancia $D_{\text {hipo }}$. La expresión resultante del modelo es:

$$
\ln (P G A)=0,1248+1,2863 M w-1,1329 \ln \left(D_{\text {hipo }}\right)
$$

con PGA en $\mathrm{cm} / \mathrm{s}^{2}$ y $D_{\text {hipo }}$ en $\mathrm{km}$. La desviación estándar $\sigma$ del modelo es $\sigma=0,69$.

Dado que la mayor parte de los acelerómetros considerados por Mezcua et al. (2008) se encuentran instalados sobre suelo duro, con Vs30<400 m/s, la ecuación6.7 es aplicable en esta clase de emplazamiento. Para poder aplicarla a roca $(760 \leq$ $V s 30<1500) \mathrm{m} / \mathrm{s}$, se le suma el término de la ecuación6.3.

\section{García Blanco (2009)}

El modelo de García Blanco (2009) se basa en los registros del IGN para 149 terremotos con magnitudes mayores o iguales que 3 y distancias menores que $200 \mathrm{~km}$. 
El modelo consiste en una relación empírica para la PGA, que se obtiene a partir de la aceleración máxima registrada en las componentes horizontales del acelerograma.

Las variables predictivas son la magnitud Mw y la distancia $D_{e p i}$. En esta tesis doctoral se utiliza la siguiente expresión, para magnitudes no superiores a 6,5:

$$
\ln (P G A)=-0,2368+1,3285 M w-1,0749 \ln \left(D_{\text {epi }}\right)
$$

donde la PGA se expresa en $\mathrm{cm} / \mathrm{s}^{2}$ y la $D_{\text {epi }}$ en $\mathrm{km}$. La desviación estándar $\sigma$ del modelo es $\sigma=0,76$. Dado que la mayor parte de los acelerómetros considerados por García Blanco (2009) se encuentran instalados sobre suelo duro, con Vs30 < 400 $\mathrm{m} / \mathrm{s}$, la ecuación6.8 es aplicable en esta clase de emplazamiento. Para poder aplicarla a roca $(760 \leq V s 30<1500 \mathrm{~m} / \mathrm{s})$, se le suma el término de la ecuación6.3.

\section{Akkar y Bommer (2010)}

Akkar y Bommer (2010) presentan relaciones empíricas para PGA, PGV y PSA, incluyendo datos de Europa (2 terremotos ocurridos en España), Oriente Medio y la región mediterránea. Las relaciones de Akkar y Bommer (2010) cumplen con los diez criterios de Bommer et al. (2010) (Tabla 6.1).

Los datos de este modelo se han obtenido a partir de 532 acelerogramas situados a distancias de hasta $100 \mathrm{~km}$, procedentes de 131 terremotos con magnitudes comprendidas entre 5 y 7,6. La aceleración es la media geométrica de las dos componentes horizontales de cada acelerograma. Como variables predictivas, se toman la magnitud $\mathrm{Mw}$, la distancia $R_{j b}$, la clase de emplazamiento y el mecanismo de la falla. En esta tesis doctoral se considera que las relaciones de Akkar y Bommer (2010) son de aplicación en la Península Ibérica para magnitudes comprendidas entre 5 y 7,6.

Las estimaciones de PGA se obtienen por medio de la expresión siguiente de Akkar y Bommer (2010):

$$
\begin{aligned}
\log (P G A)= & 1,04159+0,91333 M w-0,08140 M w^{2}+(-2,92728 \\
& +0,28120 M w) \log \left(\sqrt{R_{j b}^{2}+7,86638^{2}}\right)+0,08753 S_{S} \\
& +0,01527 S_{A}-0,04189 F_{N}+0,08015 F_{R}
\end{aligned}
$$

con PGA en $\mathrm{cm} / \mathrm{s}^{2}$ y $R_{j b}$ en km. La desviación estándar $\sigma$ del modelo es $\sigma=0,278$. $S_{S}$ y $S_{A}$ son los parámetros de la clase de emplazamiento o suelo, que toman el valor de 1 en suelo blando $(V s 30<360 \mathrm{~m} / \mathrm{s})$ y 0 en roca $(V s 30>750 \mathrm{~m} / \mathrm{s})$. Los parámetros $F_{N}$ y $F_{R}$ expresan el mecanismo de la falla. $F_{N}$ toma el valor 1 si la falla es normal, siendo $F_{R}=0$ en ese caso. Si la falla es inversa, $F_{N}=0$ y $F_{R}=1$. Para falla de desgarre o de mecanismo desconocido, $F_{N}=F_{R}=0$.

\section{Instituto Geográfico Nacional (2013)}

El modelo de Instituto Geográfico Nacional (2013) se enmarca en la actualización de los mapas de peligrosidad sísmica de España de 2012, y se ha obtenido a partir 
de 140 registros acelerométricos del IGN. Estos registros corresponden a terremotos con magnitudes comprendidas entre 4 y 5,5 , y con distancias epicentrales entre 1 y $370 \mathrm{~km}$.

En el análisis de los registros se han utilizado las aceleraciones registradas en las dos componentes horizontales, considerando la media geométrica de ambas componentes. El modelo proporciona valores de PGA y PSA (0,1-2 s), en suelo duro. La relación empírica para la PGA es la siguiente:

$$
\begin{aligned}
\log (P G A)= & 2,745+0,409(M w-6)-\log \left(\sqrt{D_{e p i}{ }^{2}+h^{2}}\right) \\
& +0,00030 \sqrt{D_{e p i}{ }^{2}+h^{2}}
\end{aligned}
$$

donde $\mathrm{h}=3,921 \mathrm{~km}$. La PGA se expresa en $\mathrm{cm} / \mathrm{s}^{2}$ y la $D_{\text {epi }}$ en $\mathrm{km}$. La desviación estándar $\sigma$ del modelo es $\sigma=0,478$. Para poder aplicar la ecuación 6.10 a roca $(760 \leq V s 30<1500 \mathrm{~m} / \mathrm{s})$, se le suma el término de la ecuación6.3.

\subsubsection{Calibración de las leyes de atenuación}

Las relaciones de atenuación analizadas en cuanto a su adecuación a los diez criterios de Bommer et al. (2010), son calibradas en función de las intensidades sísmicas observadas para cuatro terremotos de calibración. Se hace por tanto necesario poder calcular las intensidades sintéticas que corresponden a las predicciones en aceleración máxima (PGA) que se pueden obtener a su vez con cada relación de atenuación.

La intensidad sísmica utilizada en esta tesis doctoral es la EMS-98. Para calcularla, se han seleccionado tres expresiones de correlación de PGA y PGV con la intensidad en las que la intensidad utilizada es la Intensidad Modificada de Mercalli $\left(I_{M M}\right)$, pues ésta puede considerarse equivalente a la EMS-98: Wald et al. (1999a), Atkinson y Kaka (2007), Worden et al. (2012).

Se considera que, dada la similaridad entre las atenuaciones en California y España, las correlaciones de Wald et al. (1999a) son de aplicación en la Península Ibérica. Para las intensidades comprendidas en el rango $V \leq I_{M M} \leq V I I I$, la expresión de correlación con la PGA $\left(\mathrm{cm} / \mathrm{s}^{2}\right)$ es la siguiente:

$$
I_{M M}=3,66 \log (P G A)-1,66
$$

siendo la desviación estándar $\sigma=1,08$. Wald et al. (1999a) también publica una ecuación obtenida a partir de terremotos con magnitudes comprendidas entre 3,5 y 5 , y que se considera válidas para intensidades comprendidas en el rango $I \leq I_{M M} \leq V$ :

$$
I_{M M}=2,20 \log (P G A)+1,00
$$

Wald et al. (1999a) recomiendan calcular las intensidades mayores que VI con las correlaciones que proponen para la PGV. No obstante, el uso de la PGA para 


\begin{tabular}{|c|c|c|c|c|c|c|c|c|c|c|c|c|c|c|c|}
\hline 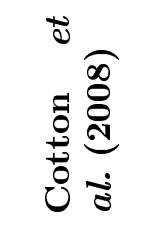 & $\tilde{\sigma}$ & $\ddot{n}$ & $\varpi$ & $\ddot{\sigma}$ & $\ddot{n}$ & $\sqrt{2}$ & $\bar{n}$ & $\sum$ & 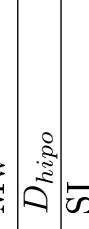 & $=0$ & $\begin{array}{l}m \\
⿱ 亠 䒑 \\
+\end{array}$ & $=\begin{array}{l}8 \\
-1 \\
i\end{array}$ & $\sqrt{n}$ & 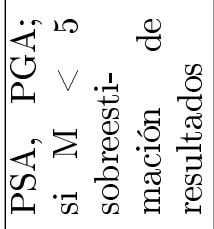 & 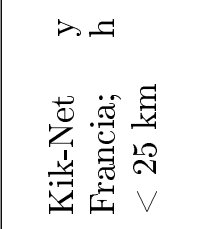 \\
\hline 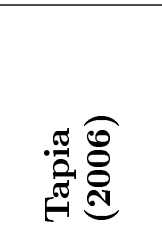 & $\sqrt[n]{2}$ & $\begin{array}{l}0 \\
Z\end{array}$ & $\vec{\omega}$ & $\begin{array}{l}0 \\
Z\end{array}$ & $\ddot{n}$ & $\begin{array}{l}0 \\
z\end{array}$ & $\varpi$ & 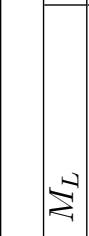 & $\begin{array}{c}0 \\
\vdots\end{array}$ & \begin{tabular}{ll|l}
0 & 0 \\
$z$ & $z$
\end{tabular} & $\begin{array}{l}\mathcal{N} \\
20 \\
\infty \\
\infty \\
\infty \\
\infty\end{array}$ & 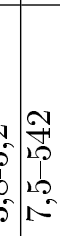 & 5 & 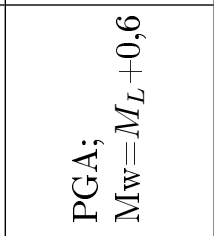 & 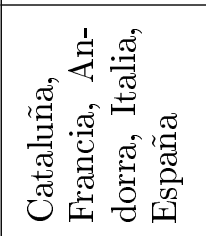 \\
\hline 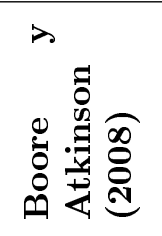 & 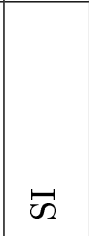 & $\ddot{n}$ & $\tilde{n}$ & $\tilde{s}$ & 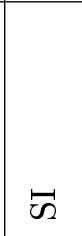 & $\vec{n}$ & $\ddot{\sigma}$ & $\sum$ & $=$ & $\vec{n} \vec{s}$ & $\mid \begin{array}{l}\infty \\
1 \\
1 \\
\infty \\
\infty\end{array}$ & $\begin{array}{ll}0 \\
b \\
b \\
b\end{array}$ & $\sqrt[n]{2}$ & 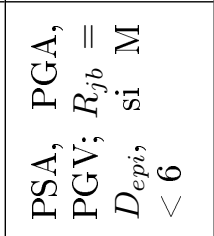 & 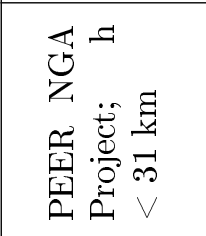 \\
\hline 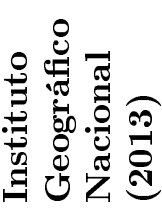 & $\ddot{n}$ & $\begin{array}{l}0 \\
z\end{array}$ & $\sqrt{2}$ & 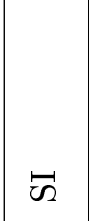 & 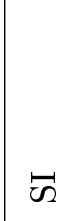 & $\bar{n}$ & $\ddot{\sigma}$ & $\frac{B}{2}$ & $\overbrace{}^{2}$ & $\begin{array}{lll}0 & 0 \\
z & 1\end{array}$ & $\begin{array}{c}10 \\
20 \\
4 \\
4\end{array}$ & $\left\{\begin{array}{l}R \\
\infty \\
v \\
V\end{array}\right.$ & 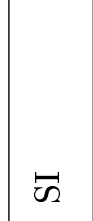 & 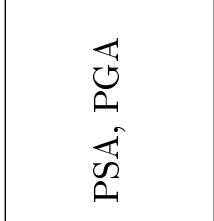 & 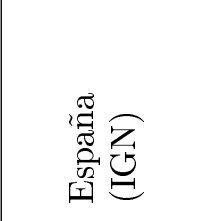 \\
\hline 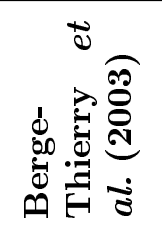 & 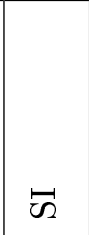 & $\ddot{n}$ & $\sqrt{2}$ & 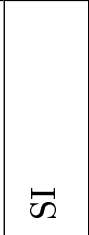 & $\begin{array}{l}0 \\
1 \\
1 \\
0 \\
0 \\
0 \\
0 \\
z\end{array}$ & $\vec{n}$ & $\ddot{\omega}$ & $\sum$ & | & $\bar{s}$ & $\begin{array}{l}3 \\
5 \\
4 \\
4\end{array}$ & 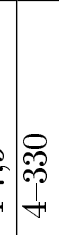 & $\varpi$ & 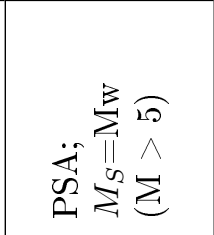 & 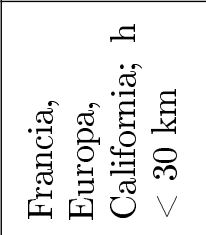 \\
\hline 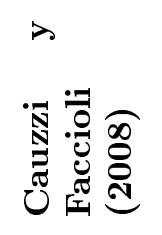 & $v$ & $\ddot{n}$ & $\tilde{\omega}$ & 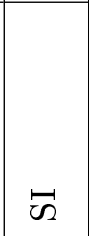 & $v$ & $\bar{\sigma}$ & $\ddot{n}$ & $\sum$ & | & $\vec{\omega}$ & \begin{tabular}{l}
2 \\
\multirow{2}{1}{} \\
1 \\
20
\end{tabular} & $=\frac{0}{20}$ & E & 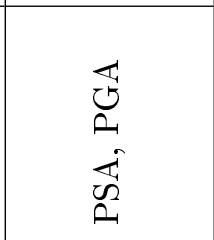 & 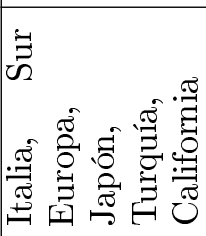 \\
\hline 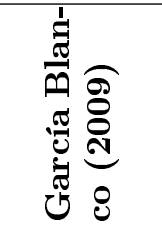 & $\bar{\Omega}$ & O & $\vec{\omega}$ & $\sqrt{2}$ & $\stackrel{0}{Z}$ & $\bar{s}$ & $v$ & $\sum$ & $\curvearrowright$ & $=0$ & $\stackrel{\infty}{\wedge}$ & 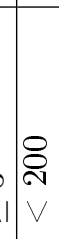 & $\ddot{n}$ & $\begin{array}{l}\mathbb{Ð} \\
2\end{array}$ & 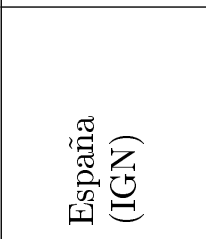 \\
\hline 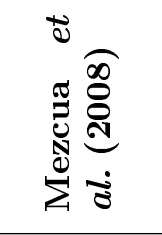 & $\sqrt{n}$ & $\ddot{n}$ & $\vec{\sigma}$ & $\ddot{n}$ & $\stackrel{0}{z}$ & $\vec{n}$ & $\ddot{\omega}$ & $\sum$ & 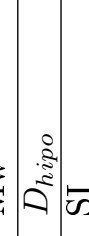 & $=0$ & $\stackrel{\infty}{\wedge}$ & $\frac{8}{11}$ & 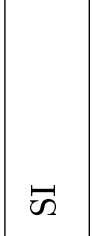 & $\begin{array}{l}\overleftrightarrow{\Xi} \\
2\end{array}$ & 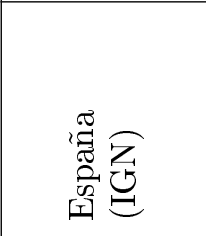 \\
\hline is & $\sqrt{n}$ & $\ddot{n}$ & $\tilde{\omega}$ & $\ddot{n}$ & 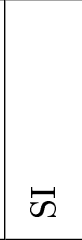 & $\tilde{\omega}$ & $\mathscr{\omega}$ & $\frac{B}{2}$ & |थ & $\vec{n} \mid \vec{s}$ & US & $=\frac{8}{9}$ & $\sqrt[n]{2}$ & 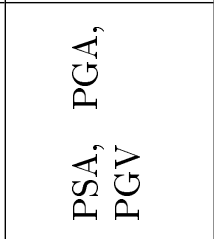 & 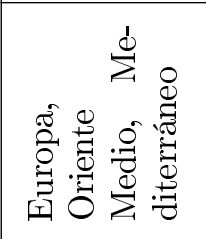 \\
\hline 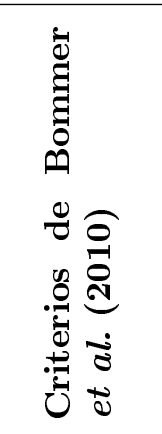 & 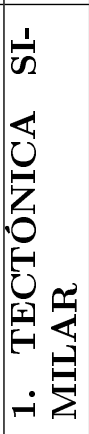 & 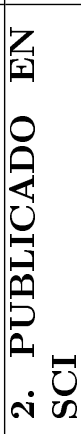 & & 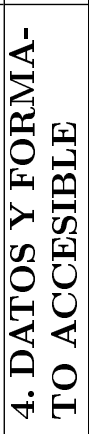 & 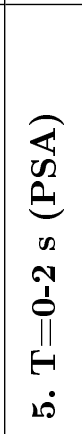 & 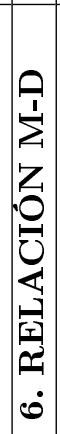 & 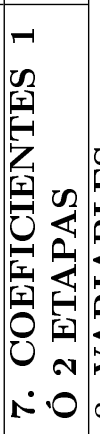 & 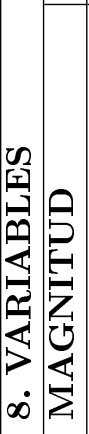 & 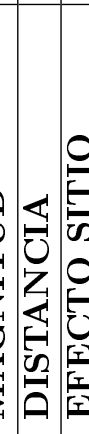 & 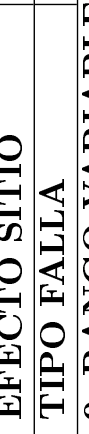 & 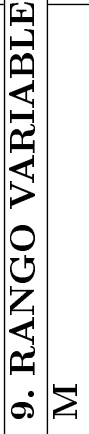 & 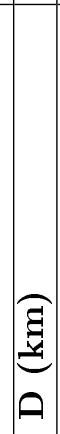 & 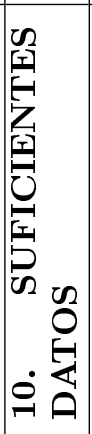 & 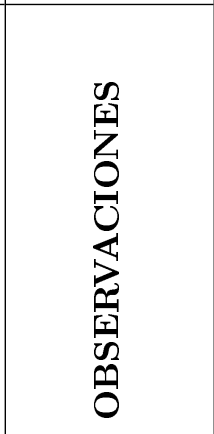 & $\begin{array}{l}02 \\
0 \\
E \\
0 \\
0\end{array}$ \\
\hline
\end{tabular}


estimar intensidades bajas $(<\mathrm{VI})$ es consistente con la idea de que las bajas intensidades se asignan basándose en la percepción del observador, que se encuentra más influenciada por la aceleración del suelo ante un terremoto que por la velocidad del mismo.

Atkinson y Kaka (2007) proponen las siguientes correlaciones de la intensidad $I_{M M}$ con la PGA, válidas para magnitudes entre 1,8 y 7,1 y para cualquier región, con PGA en $\mathrm{cm} / \mathrm{s}^{2}$ y desviación estándar $\sigma=1,01$ :

$$
\begin{aligned}
& I_{M M}=2,65+1,39 \log (P G A) \quad \text { si } \quad \log (P G A) \leq 1,69 \\
& I_{M M}=-1,91+4,09 \log (P G A) \quad \text { si } \quad \log (P G A) \geq 1,69
\end{aligned}
$$

Las correlaciones de Worden et al. (2012) entre la $I_{M M}$ y la PGA $\left(\mathrm{cm} / \mathrm{s}^{2}\right)$ son las siguientes, siendo la desviación estándar $\sigma=0,73$ :

$$
\begin{array}{crr}
I_{M M}=1,78+1,55 \log (P G A) & \text { si } & \log (P G A) \leq 1,57 \\
I_{M M}=-1,60+3,70 \log (P G A) & \text { si } & \log (P G A)>1,57
\end{array}
$$

En esta tesis doctoral se ha cuantificado la incertidumbre asociada a cada relación de atenuación analizada, por medio de la asignación de un peso normalizado a cada ley de atenuación. Como se ha explicado, este peso depende del cumplimiento de algunos de los diez criterios de Bommer et al. (2010), pero también del ajuste de las predicciones de cada ley, en términos de intensidad EMS-98, a las intensidades observadas para cuatro terremotos de calibración.

Los terremotos de calibración utilizados son los siguientes:

- Evid 123658: Mw 3,9

- Evid 1237104: Mw 4,2

- Evid 1237944: Mw 4,1

- Evid 1237976: Mw 4,1

En función de las intensidades observadas para estos terremotos, para proceder a realizar la calibración planteada se han calculado las intensidades sintéticas EMS-98 para dos rangos de distancias epicentrales $D_{e p i}, D_{e p i} \leq 100 \mathrm{~km}$ y $D_{e p i} \leq 180 \mathrm{~km}$.

Para llevar a cabo la calibración de las relaciones de atenuación con las observaciones de intensidad, se han convertido las PGA calculadas en intensidades EMS-98. En este sentido, es preciso recordar aquí que los valores de aceleración máxima calculados por cada relación de atenuación corresponden a un suelo tipo roca compacta, con $760 \leq V s 30 \leq 1500 \mathrm{~m} / \mathrm{s}$. Por tanto, las intensidades calculadas corresponden también a roca compacta. Se han utilizado las tres relaciones de conversión entre la PGA y la intensidad $I_{M M}$, de Wald et al. (1999a), Atkinson y Kaka (2007) y Worden et al. (2012), asumiendo la equivalencia entre esta escala de intensidad y la EMS-98 
(European Macroseismic Scale, 1998), que es la escala en la que se encuentran expresados los valores de intensidad observada. Por tanto, la calibración se ha llevado a cabo calculando la intensidad $I_{M M}$ a partir de la PGA calculada por cada ley de atenuación, y expresándola a posteriori según las tres relaciones de conversión.

Los resultados obtenidos en la calibración de las ochos relaciones de atenuación en aceleración analizadas muestran que, en primer lugar, las correlaciones $I_{M} M$ PGA de Worden et al. (2012) calculan valores de intensidad intermedios entre los proporcionados por las correlaciones de Wald et al. (1999a) y Atkinson y Kaka (2007). Las intensidades calculadas por medio de las correlaciones de Worden et al. (2012) ajustan mejor, en general, a las intensidades observadas, por lo que resultan recomendables para expresar los valores de PGA en términos de la intensidad sísmica.

Como segunda conclusión, y en cuanto a las relaciones de atenuación en términos de aceleración, se ha constatado que las expresiones que mejor se ajustan a los datos observados son las de Mezcua et al. (2008), García Blanco (2009), Instituto Geográfico Nacional (2013) y Tapia (2006). Sin embargo, el rango de aplicación en magnitudes de estas cuatro expresiones está limitado por la magnitud 5,5, lo que implica el incumplimiento del criterio de Bommer et al. (2010) correspondiente (Tabla 6.1). Por este motivo, se les asigna un peso menor que el que les correspondería en virtud de los resultados de la calibración.

En tercer lugar, los resultados obtenidos para las relaciones de Akkar y Bommer (2010), Boore y Atkinson (2008), Cauzzi y Faccioli (2008) y Cotton et al. (2008) predicen valores de PGA que, expresados en intensidad por medio de las correlaciones de Worden et al. (2012), presentan pequeñas diferencias con respecto a los valores observados en los cuatro terremotos de calibración. Por último, se ha comprobado que la relación de Berge-Thierry et al. (2003) es claramente la relación con peor ajuste respecto a las intensidades observadas.

\subsubsection{Selección de leyes de atenuación óptimas para distintas escalas de trabajo y en función de la magnitud del terremoto}

Examinados los resultados de los apartados anteriores, se ha procedido a seleccionar las relaciones de atenuación óptimas en la Península Ibérica y las Islas Baleares, en función de los rangos de distancias epicentrales y magnitudes correspondientes a los terremotos de calibración. En esta tesis doctoral, las relaciones así seleccionadas se utilizarán para calcular valores de aceleración sintética realistas en la Península Ibérica y las Islas Baleares, en una primera aproximación de fuente sísmica puntual.

Se han seleccionado 5 leyes de atenuación, en términos de aceleración máxima del terreno, de entre las 8 relaciones analizadas. En este contexto, se han elegido las relaciones de atenuación más recientes: Boore y Atkinson (2008), Mezcua et al. (2008), García Blanco (2009), Akkar y Bommer (2010) e Instituto Geográfico Nacional (2013). Además de la fecha de publicación de estas relaciones, se han valorado las conclusiones relativas a la bondad del ajuste de las intensidades calculadas a las intensidades observadas que se obtienen con las relaciones de Mezcua et al. (2008), 
García Blanco (2009) y Instituto Geográfico Nacional (2013). Atendiendo a los criterios de Bommer et al. (2010) (Tabla 6.1), resulta razonable escoger también las relaciones de Boore y Atkinson (2008) y Akkar y Bommer (2010), pues cumplen la totalidad de los 10 criterios.

Por último, se examinan los pesos normalizados que se han asignado a cada una de las relaciones que se han seleccionado para su empleo en esta tesis. La relación de atenuación de Akkar y Bommer (2010) recibe el mayor peso (0,25), y la de Boore y Atkinson (2008) el siguiente peso en orden decreciente $(0,20)$. En cuanto a las relaciones de Mezcua et al. (2008), García Blanco (2009) y Instituto Geográfico Nacional (2013), se les asignan pesos menores (0,10, 0,10 y 0,03, respectivamente). No obstante, estos pesos se deben a su falta de adecuación a los criterios de Bommer et al. (2010), como se puede observar en la (Tabla 6.1).

A los efectos de la implementación de las leyes seleccionadas en la herramienta plugin para la simulación de escenarios sísmicos por medio de las relaciones de atenuación (capítulo 9), a continuación se indica el factor de amplificación $F_{a}$ para altas frecuencias (2-10 Hz) de Borcherdt (1994), para la clase de emplazamiento en la que se expresan las aceleraciones calculadas con cada ley de atenuación, según la clasificación de síntesis de la Tabla 3.4. Siguiendo el procedimiento de la sección 2.5.2, esta clase de emplazamiento se entiende como la clase correspondiente al material geológico en el que se encuentran la mayor parte de las estaciones que han registrado los datos utilizados en la determinación de la relación de atenuación.

- Boore y Atkinson (2008): Clase de emplazamiento 3 (Rocas blandas a firmes/ Suelos muy densos), con $360 \mathrm{~m} / \mathrm{s}<V s 30 \leq 760 \mathrm{~m} / \mathrm{s} \mathrm{m} / \mathrm{s}$ y $F_{a}=1,25$. Razón: El modelo expresa aceleraciones en terreno con $490 \mathrm{~m} / \mathrm{s} \leq V \mathrm{~s} 30 \leq 960 \mathrm{~m} / \mathrm{s}$ (apartado 6.1.1).

- Mezcua et al. (2008): Clase de emplazamiento 4 (Suelos duros), con 180m/s $\leq$ $V s 30 \leq 360 \mathrm{~m} / \mathrm{s}$ y $F_{a}=1,61$. Razón: La mayor parte de los acelerómetros considerados en el modelo se encuentran instalados sobre suelo duro, con Vs30 $<400 \mathrm{~m} / \mathrm{s}$ (apartado 6.1.1).

- García Blanco (2009): Clase de emplazamiento 4 (Suelos duros), con 180m/s $\leq$ $V s 30 \leq 360 \mathrm{~m} / \mathrm{s}$ y $F_{a}=1,61$. Razón: Los valores de PGA observada que se han utilizado en el modelo corresponden a acelerómetros que, en su mayor parte, se encuentran en suelo duro, con Vs30 $<400 \mathrm{~m} / \mathrm{s}$ (apartado 6.1.1).

- Akkar y Bommer (2010): Clase de emplazamiento 2 (Rocas de resistencia media), con $760 \mathrm{~m} / \mathrm{s}<V s 30 \leq 1500 \mathrm{~m} / \mathrm{s}$ y $F_{a}=0,97$. Razón: Por medio de los parámetros $S_{S}$ y $S_{A}$ de la clase de emplazamiento, el modelo calcula aceleraciones en roca con $V s 30>750 \mathrm{~m} / \mathrm{s}$ (apartado 6.1.1).

- Instituto Geográfico Nacional (2013): Clase de emplazamiento 4 (Suelos duros), con $180 \mathrm{~m} / \mathrm{s} \leq V s 30 \leq 360 \mathrm{~m} / \mathrm{s}$ y $F_{a}=1,61$. Razón: Los valores de PGA calculada con esta relación de atenuación corresponden a un suelo duro (apartado 6.1.1). 


\subsection{Leyes de atenuación aplicables en las Islas Ca- narias}

\subsubsection{Investigación bibliográfica de leyes de atenuación sus- ceptibles de aplicación en las Islas Canarias}

Como primer paso en la selección de las leyes de atenuación en términos de aceleración máxima del terreno, que se van a utilizar en una primera aproximación a escenarios sísmicos realistas en las Islas Canarias, se ha llevado a cabo una investigación bibliográfica de las relaciones de atenuación que puedan ser susceptibles de aplicación en el archipiélago.

Como primer criterio de la investigación, se han examinado relaciones de atenuación obtenidas en ambientes tectónicos parecidos al correspondiente a las Islas Canarias. Estas relaciones, por tanto, se han determinado a partir de registros sísmicos procedentes de regiones volcánicas, y a ser posible en las que el volcanismo presente un carácter efusivo, como en las islas.

Como segundo criterio, se ha revisado el rango de aplicación de las relaciones de atenuación en magnitud Mw y en distancia, con el fin de comprobar su similitud con respecto a los intervalos de magnitudes de los terremotos seleccionados con este fin de entre los localizados en El Hierro, entre 2011 y 2013, y con las distancias epicentrales o hipocentrales de las estaciones acelerométricas del IGN que han proporcionado acelerograma para estos eventos (sección 2.5.1).

Como se ha indicado en el capítulo 4, el IGN ha proporcionado al doctorando el catálogo de acelerogramas registrados durante el periodo abarcado por la crisis sísmica de julio-octubre de 2011, previa a la erupción volcánica, y por las crisis sísmicas de junio de 2012 y marzo de 2013, así como los registros correspondientes. Se han seleccionado 36 acelerogramas correspondientes a 20 terremotos de magnitud Mw comprendida entre 4,1 y 4,9 (la máxima magnitud) y profundidad focal de 10 a $23 \mathrm{~km}$. Para estos acelerogramas, las distancias epicentrales e hipocentrales oscilan entre 3,4 y $37 \mathrm{~km}$. En la Tabla 6.2 se muestran los parámetros focales de cada terremoto, junto con la intensidad epicentral y las distancias epicentrales de los acelerómetros que han proporcionado acelerograma para cada evento. En la Figura 6.1 se representan los epicentros de estos eventos, en función de su magnitud Mw. En cuanto a la magnitud Mw de los terremotos seleccionados, ésta se ha obtenido en función de la magnitud calculada por el IGN a partir de la amplitud de la fase S de estos terremotos, que corresponde a la magnitud local $\left(M_{L}\right)$. El IGN emplea en el cálculo de esta magnitud una fórmula de magnitud a partir de la amplitud de la fase Lg (Instituto Geográfico Nacional, 2017a). Por este motivo, para obtener la Mw se ha utilizado la relación entre mbLg y Mw para la Península Ibérica de Rueda y Mezcua (2002). Esta relación ha sido obtenida a partir de terremotos de magnitudes similares al rango de magnitudes $M_{L}$ de los terremotos registrados en El Hierro, que corresponde a 4,2-4,9 (Tabla 6.2).

Las relaciones de atenuación susceptibles de aplicación deben cumplir las siguientes condiciones, en cuanto a su rango de aplicación en magnitud y distancia:

1. El rango de distancias epicentrales $\left(D_{e p i}\right)$ o de distancias hipocentrales $\left(D_{\text {hipo }}\right)$ 


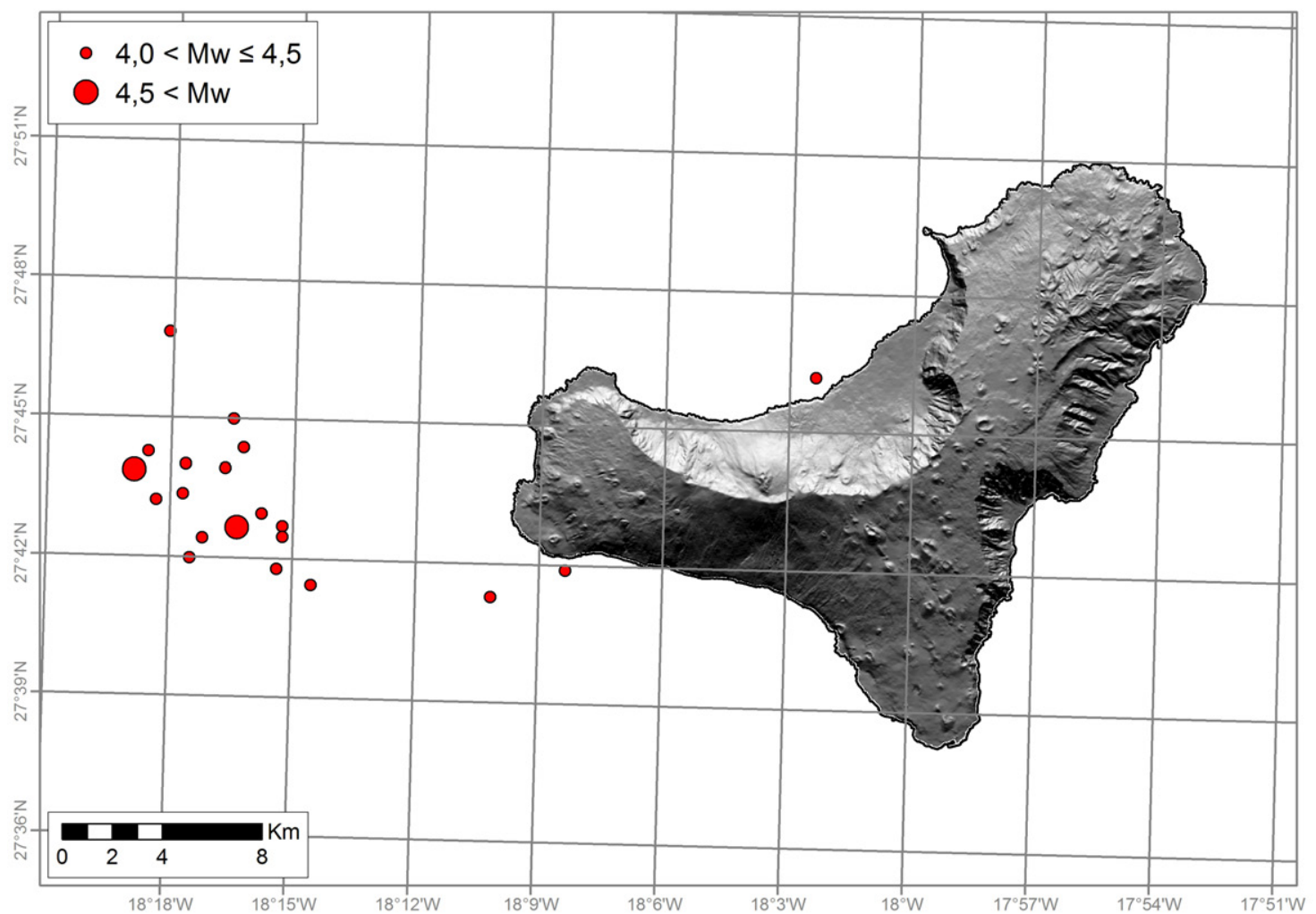

Figura 6.1: Terremotos de magnitud $4,1 \leq M w \leq 4,9$ ocurridos en El Hierro durante las crisis sísmicas de juliooctubre de 2011, junio de 2012 y marzo de 2013, para los cuales se han registrado acelerogramas en las estaciones acelerométricas del IGN. Estos eventos han sido utilizados en el análisis y calibración de las relaciones de atenuación en aceleración máxima que son aplicables en la isla. 
de los datos utilizados para obtener la relación debe contener los rangos de $D_{e p i}$ o $D_{\text {hipo }}$ de los terremotos de El Hierro para los que se dispone de acelerogramas

2. El rango de profundidades (prof) de los datos utilizados en la relación de atenuación debe contener al rango de profundidades de los terremotos de El Hierro sólo si la aplicabilidad de la relación se encuentra restringida al cálculo de aceleraciones de terremotos profundos

3. Si se ha cumplido la condición 1 y, en su caso, la condición 2, entonces los rangos de magnitudes $(\mathrm{M})$ de los datos utilizados para obtener la relación deben contener algunas de las $\mathrm{M}$ de los terremotos de El Hierro, de manera que no excluyan la totalidad de las $\mathrm{M}$ de estos terremotos

Considerando los dos criterios de investigación bibliográfica explicados, se han seleccionado 13 relaciones de atenuación para su calibración en función de las aceleraciones registradas en El Hierro. Estas leyes de atenuación en la Península Ibérica (2), Islandia (4), Italia (4), Hawái (1) y Guadalupe (1). A continuación se analiza cada ley separadamente en base a los dos criterios. Adicionalmente, se indica el factor de amplificación $F_{a}$ para altas frecuencias $(2-10 \mathrm{~Hz})$ de Borcherdt (1994) para la clase de emplazamiento en la que se expresan las aceleraciones calculadas con cada ley de atenuación. Esta clase de emplazamiento es la que le corresponde al material geológico en el que se encuentran la mayor parte de las estaciones que han registrado los datos utilizados en la determinación de la relación de atenuación (sección 2.5.2).

\section{Península Ibérica}

García Blanco (2009) La relación de atenuación de García Blanco (2009) ha sido analizada en el apartado 6.1.1. A los efectos de la presente investigación bibliográfica, se destaca que el rango de aplicación de esta relación en distancias epicentrales es tal que $D_{e p i}<200 \mathrm{~km}$, y que el rango de magnitudes es $3 \leq M w<6,5$, cumpliendo las condiciones del segundo criterio de la investigación.

Como se indica en el apartado 6.1.1, los valores de aceleración máxima (PGA) observada que se han utilizado para obtener esta ley de atenuación corresponden a acelerómetros que, en su mayor parte, se encuentran en suelo duro, con Vs30 < 400 $\mathrm{m} / \mathrm{s}$. De acuerdo con la clasificación de síntesis (Tabla 3.4), esta clase de suelo es equiparable a la clase de emplazamiento 4 (Suelos duros), con 180m/s $\leq V s 30 \leq$ $360 \mathrm{~m} / \mathrm{s}$. Según los resultados de la sección 3.5, a esta clase de emplazamiento le corresponde un factor de amplificación $F_{a}$ para altas frecuencias $(2-10 \mathrm{~Hz})$ tal que $F_{a}=1,61$. Por tanto, se asume que la ley de atenuación de García Blanco (2009) proporciona la aceleración máxima del terreno, PGA, en un emplazamiento de clase 4. Esta idea se va a expresar de una forma más resumida, indicando que la condición del terreno de referencia es la clase 4 .

Instituto Geográfico Nacional (2013) La relación de atenuación de Instituto Geográfico Nacional (2013) ha sido analizada en el apartado 6.1.1. A los efectos de la presente investigación bibliográfica, se destaca que el rango de aplicación de esta relación en distancias epicentrales es tal que $D_{e p i} \leq 370 \mathrm{~km}$, y que el rango de 
magnitudes es $4 \leq M w \leq 5,5$, cumpliendo las condiciones del segundo criterio de la investigación.

Con respecto a la condición del terreno de referencia, en el apartado 6.1.1 se ha indicado que los valores de aceleración máxima (PGA) calculada con esta relación de atenuación corresponden a un suelo duro. Por tanto, la relación de atenuación de Instituto Geográfico Nacional (2013) proporciona valores de PGA en un emplazamiento de clase $4(180 \mathrm{~m} / \mathrm{s} \leq V s 30 \leq 360 \mathrm{~m} / \mathrm{s})$, con $F_{a}=1,61$.

\section{Islandia}

Sigbjörnsson y Baldvinsson (1992) El modelo de Sigbjörnsson y Baldvinsson (1992) proporciona tres expresiones empíricas de atenuación para la aceleración máxima del terreno (PGA), a partir de registros acelerométricos obtenidos en Islandia, correspondientes a 39 eventos de magnitudes $\mathrm{M}$ comprendidas entre 2 y 6 . Definiendo la PGA como el mayor valor de las aceleraciones máximas de las dos componentes horizontales del acelerograma, la expresión de la atenuación es:

$$
\log (P G A)=-1,72+0,327 M-\log \left(\sqrt{R_{j b}^{2}+h^{2}}\right)-0,0043 \sqrt{R_{j b}^{2}+h^{2}}
$$

donde $\mathrm{h}$ es la profundidad del terremoto, en $\mathrm{km}$. La PGA se expresa en $\mathrm{g}$ ( $\mathrm{g}=9,8$ $\mathrm{m} / \mathrm{s}^{2}$ ) y la distancia $R_{j b}$ en $\mathrm{km}$. La desviación estándar $\sigma$ del modelo es tal que $\sigma=0,30$.

La expresión para la PGA, definida como la media de las aceleraciones máximas de las dos componentes horizontales del acelerograma es la siguiente:

$$
\log (P G A)=-1,98+0,365 M-\log \left(\sqrt{R_{j b}^{2}+h^{2}}\right)-0,0039 \sqrt{R_{j b}^{2}+h^{2}}
$$

donde PGA se expresa en g, y h y $R_{j b}$ en km. La desviación estándar $\sigma$ del modelo es tal que $\sigma=0,30$.

Los autores también determinan una tercera relación de atenuación para ambas componentes horizontales del acelerograma, definiendo en cada caso la PGA como la aceleración máxima:

$$
\log (P G A)=-2,28+0,386 M-\log \left(\sqrt{R_{j b}^{2}+h^{2}}\right)
$$

donde PGA se expresa en g, y h y $R_{j b}$ en $\mathrm{km}$. La desviación estándar $\sigma$ del modelo es tal que $\sigma=0,29$.

Sigbjörnsson y Baldvinsson (1992) utilizan registros acelerométricos para terremotos con magnitudes comprendidas entre 2 y 6 , si bien los autores no especifican la escala de magnitud. Los datos están asociados a distancias Joyner y Boore $R_{j b}$ 2-80 km. Para valorar la adecuación de los rangos de aplicación de la relación de atenuación a los datos observados en El Hierro, se asume que la escala de magnitud es la escala de Mw, y que $R_{j b}$ es aproximadamente igual a $D_{e p i}$ con $\mathrm{Mw}<6$ (Boore y 
Atkinson, 2008). Bajo estas consideraciones, la relación de atenuación de Sigbjörnsson y Baldvinsson (1992) puede aplicarse en la isla, pues los datos utilizados en su determinación tienen un rango de distancias epicentrales que comprende al intervalo de distancias de los registros de aceleración de El Hierro, y puesto que el rango de magnitudes empleado es compatible con las magnitudes Mw 4,1-4,9 observadas en El Hierro.

Se asume que la mayor parte de los registros de aceleración que se han utilizado proceden de estaciones acelerométricas intaladas en roca dura, con Vs30 > 1500 $\mathrm{m} / \mathrm{s}$, a partir de las referencias sobre la red de acelerómetros en la que se integran estas estaciones (Sigbjörnsson et al., 2014). Este tipo de roca pertenece a la clase de emplazamiento 1 de la clasificación de síntesis (Tabla 3.4). No obstante, del trabajo de Sigbjörnsson et al. (2014) se extrae que la mayoría de las estaciones se encuentran en terrenos de clase roca de acuerdo con el Eurocódigo-8 (Vs30 > 750 $\mathrm{m} / \mathrm{s}$ ) (European Committee for Standardization, 2004), si bien los autores advierten que la clasificación geológica de las estaciones es provisional, y que se encuentra pendiente de ser revisada. La información aportada por Sigbjörnsson et al. (2014) supondría que la mayor parte de las estaciones utilizadas por Sigbjörnsson y Baldvinsson (1992) se podrían encontrar en una roca de resistencia media, que pertenece a la clase 2, con $760 \mathrm{~m} / \mathrm{s}<V s 30 \leq 1500 \mathrm{~m} / \mathrm{s}$ (Tabla 3.4). En consecuencia, la clase de emplazamiento mayoritaria para las estaciones acelerométricas utilizadas no se encuentra plenamente identificada. En este contexto, se asume que ésta es la clase 1 (roca dura), caracterizada por un factor de amplificación $F_{a}=0,86$. Por tanto, se asume que la condición del terreno de referencia para la ley de atenuación de Sigbjörnsson y Baldvinsson (1992) es la clase 1.

A los efectos de la calibración de las tres expresiones del modelo de atenuación de Sigbjörnsson y Baldvinsson (1992) con los datos de El Hierro, se sustituirá $R_{j b}$ por $D_{e p i}$ (Boore y Atkinson, 2008).

Ólafsson y Sigbjörnsson (1999) Ólafsson y Sigbjörnsson (1999) proponen una relación de atenuación que proporciona valores sintéticos de la aceleración máxima del terreno (PGA), la cual es definida por los autores como el mayor valor de las aceleraciones máximas de las dos componentes horizontales del acelerograma. La expresión de la PGA en $\mathrm{cm} / \mathrm{s}^{2}$ es la siguiente:

$$
\log (P G A)=0,0451+0,3089 \log M_{0}-0,9642 \log R
$$

$M_{0}$ es el momento sísmico en $d y n \cdot \mathrm{cm}, \mathrm{y} R$ se define en función de la distancia epicentral y la profundidad, tal que $R=\sqrt{\left(D_{e p i}{ }^{2}+h^{2}\right)}(\mathrm{cm})$. La desviación estándar $\sigma$ del modelo es tal que $\sigma=0,3148$.

El modelo utiliza registros de aceleración para terremotos con magnitud $\mathrm{Mw}$ comprendida entre 3,4 y 5,9, situados a distancias epicentrales en el intervalo 2-112 $\mathrm{km}$. Considerando que las profundidades focales de los datos están comprendidas entre 1 y 11 km, se considera que la relación de atenuación de Ólafsson y Sigbjörnsson (1999) es válida para su aplicación en El Hierro, puesto que el rango de distancias epicentrales contiene al intervalo de distancias epicentrales de los acelerómetros de El Hierro que han registrado acelerograma. Además, el rango de magnitudes de los datos de los autores contiene al rango de magnitudes de los terremotos de El Hierro. 
Se asume que la relación de atenuación de Ólafsson y Sigbjörnsson(1999) ha sido obtenida con registros de aceleración correspondientes a roca dura, con Vs30 $>1500 \mathrm{~m} / \mathrm{s}$, ya que los autores indican que los acelerómetros utilizados se encuentran situados sobre roca o terreno muy duro (rock or very stiff ground). Dado que las rocas duras son plenamente clasificables en la clase de emplazamiento 1 de la clasificación de síntesis (Tabla 3.4), con factor de amplificación $F_{a}=0,86$, se asume que la condición del terreno de referencia para la ley de atenuación de Ólafsson y Sigbjörnsson(1999) es la clase 1.

A los efectos de la calibración del modelo de Ólafsson y Sigbjörnsson (1999) con los datos de El Hierro, se calculará el momento sísmico $M_{0}$ de cada terremoto a partir de su magnitud momento Mw, según la siguiente expresión de Hanks y Kanamori (1979):

$$
M_{0}=10^{(3 / 2)(M w+10,7)}
$$

Ágústsson et al. (2008) Ágústsson et al. (2008) desarrollan un modelo de atenuación para el sur de Islandia a partir de 1085 registros de aceleración pico del terreno, correspondientes a 64 terremotos. Los autores definen la aceleración pico (PGA) como el máximo de la raíz cuadrada de la suma de los cuadrados de las tres componentes del acelerograma (N-S, V, E-W) (máximo de la suma vectorial de las componentes). Además, proporcionan dos expresiones para la atenuación de la PGA en $\mathrm{m} / \mathrm{s}^{2}$, en función de la magnitud momento local $\left(M_{L w}\right)$ y de la distancia epicentral $\left(D_{e p i}\right)$, dependiendo de que la PGA se atenúe linealmente con la magnitud o con el logaritmo decimal de la misma:

$$
\log (P G A)=-1,95600 \log \left(D_{\text {epi }}\right)+9,59878 \log \left(M_{L w}\right)-4,87778 \quad \sigma=0,4591
$$

$$
\log (P G A)=-1,96297 \log \left(D_{\text {epi }}\right)+0,89343 M_{L w}-2,65660 \quad \sigma=0,4596
$$

donde la distancia $D_{e p i}$ se expresa en $\mathrm{km}$ y $\sigma$ es la desviación estándar del modelo.

El modelo utiliza registros de aceleración para terremotos con magnitud $M_{L w}$ comprendida entre 3,5 y 6,5 . Con respecto a la magnitud $M_{L w}$, se cumple que $M_{L w} \approx$ $M w+0,5$ para $M_{L w}=3,5$, y que $M_{L w}=M w$ para $M_{L w}=6,5$ (Pétursson $\mathrm{y}$ Vogfjörd, 2009). Por tanto, y en términos de Mw, la magnitud mínima $M_{\text {min }}$ de los datos es $M_{\min } \approx 3$, y la magnitud máxima $M_{\max }=6,5$. En cuanto a las distancias epicentrales de los datos, éstas toman valores en el intervalo comprendido entre 3 y $350 \mathrm{~km}$. Los rangos de distancias y magnitudes de las aceleraciones utilizadas para determinar el modelo cumplen con las condiciones planteadas en la investigación bibliográfica, en razón de su compatibilidad con intervalos correspondientes de los registros de aceleración de El Hierro.

Partiendo de la información proporcionada por Ágústsson et al. (2008), se entiende que los registros de aceleración máxima observada han sido derivados de registros de velocidad de estaciones sísmicas (sismógrafos). Es razonable suponer que estas 
estaciones de registro de velocidad se encuentran emplazadas sobre roca, a fin de optimizar la calidad de los registros de velocidad, y por este motivo las aceleraciones máximas pueden considerarse referidas a roca. Se asume, por tanto, que la condición del terreno de referencia para las relaciones de atenuación de Ágústsson et al. (2008) es la clase de emplazamiento 1 (Tabla 3.4), al definirse como rocoas duras, con Vs30 $>1500 \mathrm{~m} / \mathrm{s}$. Le corresponde un factor de amplificación $F_{a}=0,86$.

Dada la definición de PGA, y a los efectos de proceder a calibrar el modelo de atenuación, se aproxima la PGA de los registros de El Hierro como la raíz cuadrada de la suma de los cuadrados de las aceleraciones máximas de cada componente N-S, V, E-W: $P G A=\sqrt{(N-S)^{2}+V^{2}+(E-W)^{2}}$. Con objeto de calcular las PGA sintéticas con las ecuaciones 6.22 y 6.23 , se consideran las precisiones de Pétursson y Vogfjörd (2009) en cuanto a la correspondencia entre $M_{L w}$ y Mw: $M_{L w} \approx M w+$ 0,5 para el rango de $M_{L w} 3,5-5,5$. Por tanto, para el rango de magnitudes de los terremotos de El Hierro que participan en la calibración, es posible introducir en las citadas ecuaciones la variable $M_{L w} \approx M w+0,5$, pues este rango es $4,0<M w \leq 5,0$.

Pétursson y Vogfjörd (2009) La relación de atenuación de Pétursson y Vogfjörd (2009) ha sido obtenida a partir de los registros de velocidad correspondientes a 46 terremotos localizados en el SW de Islandia, en terrenos de naturaleza volcánica. La utilización de esta relación en las Islas Canarias se debe, primeramente, a su aplicabilidad a un volcanismo con actividad superficial, el cual es parecido al presente en el archipiélago canario.

La ley de atenuación ha sido determinada a partir de las formas de onda registradas en sismógrafos de 3 componentes, pertenecientes a la red sísmica nacional de Islandia (SIL). Los registros de aceleración correspondientes a los 46 terremotos estudiados han sido obtenidos por medio de la diferenciación de las componentes de los registros de velocidad. A partir de estos registros de aceleración, se han determinado las aceleraciones pico del terreno (PGA) correspondientes a cada estación. La PGA es definida por los autores como el máximo de la raíz cuadrada de la suma de los cuadrados (maximum of the squared sum) de las 3 componentes del registro de aceleración. A partir de 823 estimaciones de PGA, se ha obtenido la expresión de la ley de atenuación, que proporciona la PGA en $\mathrm{m} / \mathrm{s}^{2}$. De acuerdo a la ecuación D en Pétursson y Vogfjörd (2009), esta expresión es:

$$
\begin{aligned}
\log (P G A)= & -2,26 \log \left(D_{e p i}+0,0309 \cdot 10^{\left(0,569 M w-0,0194 M w^{2}\right)}\right) \\
& -0,0437 M w^{2}+1,28 M w-2,85
\end{aligned}
$$

donde $D_{e p i}$ se expresa en $\mathrm{km}$. La desviación estándar $\sigma$ del modelo es tal que $\sigma=$ 0,302 .

El rango de magnitudes para los datos empleados en la determinación de la ley de atenuación se expresa en magnitud momento local, $M_{L w}$ (Pétursson y Vogfjörd, 2009), siendo 3,3 $\leq M_{L w} \leq 6,5$. De acuerdo con los autores, $M_{L w} \approx M w+0,5$ para el rango de $M_{L w} 3,5-5,5$. En términos de Mw, el rango de aplicabilidad, por tanto, contiene al intervalo de $\mathrm{Mw}$ 3,0-5,0. La revisión de la magnitud $\mathrm{Mw}$ de los datos utilizados implica que Mw está comprendida entre 3,1 y 6,5, de acuerdo con la Tabla 
2 en Pétursson y Vogfjörd (2009). En consecuencia, el rango de magnitudes $\mathrm{Mw}$ en el que la relación de atenuación es aplicable contiene al rango de magnitudes de los terremotos de El Hierro. Con respecto a la distancia epicentral, los datos utilizados presentan $D_{\text {epi }} 3-380 \mathrm{~km}$, y profundidades h en el rango 0-9 km, por lo tanto la relación también es aplicable en el rango de distancias epicentrales de los registros acelerométricos de El Hierro.

La condición del terreno de referencia para los registros de la PGA observada es la clase de emplazamiento 1 , con Vs $30>1500 \mathrm{~m} / \mathrm{s}$ y $F_{a}=0,86$, porque los registros de aceleración asociados han sido obtenidos por diferenciación de las componentes de registros de velocidad, que se han obtenido en estaciones de registro de velocidad Pétursson y Vogfjörd (2009). Es razonable suponer que estas estaciones se encuentran emplazadas sobre roca, a fin de optimizar la calidad de los registros de velocidad.

Para calibrar esta relación de atenuación, se considera una primera aproximación a la definición de la PGA de Pétursson y Vogfjörd (2009), equiparando la PGA de los registros de aceleración de El Hierro al valor de la raíz cuadrada de la suma de los cuadrados de las aceleraciones máximas de cada componente. Aunque estos máximos de aceleración no tienen por qué ser simultáneos, puede considerarse que su composición es una aproximación válida al valor de PGA que se utiliza en la ley de atenuación (que es el máximo de la composición de las componentes del registro de aceleración).

\section{Italia}

Tento et al. (1992) La relación de atenuación de Tento et al. (1992) se obtiene a partir de 137 acelerogramas, correspondientes a 40 terremotos localizados en Italia. Define la PGA como el máximo valor de aceleración de la mayor de las dos componentes horizontales del acelerograma (E-W, N-S). La expresión de la PGA es:

$$
\ln (P G A)=4,73+0,52\left(M_{L}\right)-\ln (R)-0,00216 R
$$

con $R=\sqrt{R_{j b}^{2}+h^{2}}$, siendo $R_{j b}$ la distancia Joyner y Boore $(\mathrm{km})$ y h la profundidad hipocentral $(\mathrm{km})$. PGA se expresa en gales $\left(1 \mathrm{gal}=1 \mathrm{~cm} / \mathrm{s}^{2}\right)$, y la desviación estándar $\sigma$ del modelo es $\sigma=0,67$

Con respecto a los datos utilizados, el rango de magnitudes se expresa en magnitud local $M_{L}$, siendo la magnitud mínima $M_{\min }=4$, y la magnitud máxima $M_{\max }=6,6$ (Tento et al., 1992). Utilizando la conversión entre $M_{L} \mathrm{y} \mathrm{Mw}$ de Ristau et al. (2005), $M w=M_{L}+0,6$, el rango de magnitudes en términos de $\mathrm{Mw}$ es tal que $M_{\min }=4,6$ y $M_{\max }=7,2$. En cuanto al rango de distancias de los datos, se entiende que las distancias epicentrales están comprendidas entre 3,2 y $170 \mathrm{~km}$, considerando que Tento et al. (1992) utilizan este tipo de distancias para caracterizar los registros de los terremotos con $M_{L}<5,7$. En consecuencia, se considera que la relación de atenuación de Tento et al. (1992) es válida para su aplicación en El Hierro, pues, primeramente, el rango de distancias epicentrales contiene al intervalo de distancias epicentrales de los acelerómetros de El Hierro que han registrado acelerograma. En segundo lugar, el rango de magnitudes $\mathrm{Mw}$ contiene al menos a las magnitudes $\mathrm{Mw}$ 4,6-4,9 de los terremotos con acelerograma registrado en El Hierro. 
A partir de los registros de PGA normalizada en función de la distancia hipocentral utilizados por los autores, que se pueden consultar en la Figura 4 de Tento et al. (1992), se asume que la ley de atenuación ha sido obtenida con registros de aceleración que corresponden a estaciones instaladas en su mayoría sobre suelo. Los autores atribuyen a un emplazamiento tipo suelo un intervalo de velocidad de onda de corte, Vs, tal que $400 \mathrm{~m} / \mathrm{s}<V s \leq 800 \mathrm{~m} / \mathrm{s}$, siguiendo a Sabetta y Pugliese (1987). Existe una clara correspondencia entre este rango de velocidades y el correspondiente a la clase de emplazamiento 3 de la clasificación de síntesis (Tabla 3.4), con $360 \mathrm{~m} / \mathrm{s}<V s 30 \leq 760 \mathrm{~m} / \mathrm{s}$. Por tanto, se asume que la ley de atenuación de Tento et al. (1992) ha sido obtenida con registros de aceleración correspondientes a la clase 3. En consecuencia, la condición del terreno de referencia del modelo es la clase 3 (Rocas blandas a firmes/Suelos muy densos), con $F_{a}=1,25$.

Para calibrar esta relación de atenuación, se introduce en la ecuación 6.25 la variable $M_{L}=M w-0,6$, de acuerdo con la conversión de Ristau et al. (2005). También se sustituirá $R_{j b}$ por $D_{e p i}$, siguiendo a Boore y Atkinson (2008). Con respecto a las PGA observadas en El Hierro, se aproxima la definición de PGA de Tento et al. (1992) como el máximo de las aceleraciones pico de cada componente horizontal (E-W, N-S).

Bindi et al. (2009a) El modelo de atenuación de Bindi et al. (2009a) consta de tres expresiones que dependen de la definición de la PGA. Las variables predictivas, en todos los casos, son la magnitud momento Mw, la distancia Joyner y Boore $R_{j b}$ y la distancia hipocentral $D_{\text {hipo }}$, el efecto de sitio y el mecanismo focal. Los autores distinguen tres clases de emplazamiento con respecto al efecto de sitio, siguiendo a Sabetta y Pugliese $(1987,1996)$ : roca, aluvial somero $(400 \mathrm{~m} / \mathrm{s} \leq V s \leq 800 \mathrm{~m} / \mathrm{s})$ y aluvial profundo. Asimismo, distinguen tres posibles mecanismo focales: falla normal, de desgarre e inversa. Para calibrar este modelo en El Hierro, se estudiarán las expresiones correspondientes a un emplazamiento en roca y a un mecanismo focal de falla inversa. La razón de considerar una falla inversa reside en el mecanismo focal calculado para el terremoto de 08-10-2011, ocurrido a 1,5 km de la costa SW de El Hierro, con $M_{L} 4,3(\mathrm{Mw} 4,0)$ y $12 \mathrm{~km}$ de profundidad, único de la crisis sísmica de 2011 del que se dispone de mecanismo, de acuerdo con el cálculo automático del Tensor Momento Sísmico (Instituto Geográfico Nacional, 2016c) llevado a cabo por el IGN con el procedimiento descrito por Rueda y Mezcua (2005).

Si la PGA se define como el máximo valor de aceleración de la mayor de las dos componentes horizontales del acelerograma (E-W, N-S), el modelo predictivo de PGA $\left(\mathrm{cm} / \mathrm{s}^{2}\right)$ en roca y para mecanismo de falla inversa, en función de la $D_{e p i}$, es el siguiente:

$$
\begin{aligned}
\log (P G A)= & 3,0761+0,1587\left(M w-M_{r e f}\right)+0,0845\left(M w-M_{r e f}\right)^{2} \\
& +\left[-1,0504-0,0148\left(M w-M_{r e f}\right)\right] \log \left(\sqrt{R_{j b}+h^{2}}\right)+0,0168
\end{aligned}
$$

donde $M_{r e f}=5,5, h=7,3469$ y la desviación estándar $\sigma=0,2963$. La distancia $R_{j b}$ se expresa en $\mathrm{km}$.

Para la definición de la PGA como la aceleración pico de la media geométrica de las componentes horizontales del acelerograma, la expresión para PGA $\left(\mathrm{cm} / \mathrm{s}^{2}\right)$ en roca y mecanismo focal de falla inversa, en función de la $D_{e p i}$, es la siguiente: 


$$
\begin{aligned}
\log (P G A)= & 3,0191+0,1643\left(M w-M_{r e f}\right)+0,0674\left(M w-M_{r e f}\right)^{2} \\
& +\left[-1,0284-0,0041\left(M w-M_{r e f}\right)\right] \log \left(\sqrt{R_{j b}+h^{2}}\right)+0,0005
\end{aligned}
$$

donde $M_{r e f}=5,5, h=6,8963$ y la desviación estándar $\sigma=0,2930$. La distancia $R_{j b}$ se expresa en $\mathrm{km}$.

Para la definición de la PGA como la aceleración pico de la componente vertical (V) del acelerograma, la PGA $\left(\mathrm{cm} / \mathrm{s}^{2}\right)$ sintética en roca y ante un terremoto con mecanismo focal de falla inversa se calcula, en función de la $D_{e p i}$, por medio de la siguiente expresión:

$$
\begin{aligned}
\log (P G A)= & 3,0421+0,3762\left(M w-M_{r e f}\right)+0,0925\left(M w-M_{r e f}\right)^{2} \\
& +\left[-1,2350-0,0891\left(M w-M_{r e f}\right)\right] \log \left(\sqrt{R_{j b}+h^{2}}\right)+0,0222
\end{aligned}
$$

donde $M_{r e f}=5,5, h=9,3012$ y la desviación estándar $\sigma=0,2831$. La distancia $R_{j b}$ se expresa en $\mathrm{km}$.

Los autores también presentan una expresión para la PGA en función de la distancia hipocentral $D_{\text {hipo }}$. En este caso, la PGA se define como el máximo valor de aceleración de la mayor de las dos componentes horizontales del acelerograma (E-W, N-S), y no se considera el mecanismo focal. Por tanto, la expresión de esta PGA $\left(\mathrm{cm} / \mathrm{s}^{2}\right)$ sintética en roca y en función de la $D_{\text {hipo }}(\mathrm{km})$, es la siguiente:

$$
\begin{aligned}
\log (P G A)= & 3,4192+0,4672\left(M w-M_{r e f}\right)+0,1231 M w-M_{r e f}{ }^{2} \\
& +\left[-1,2221-0,1643\left(M w-M_{\text {ref }}\right)\right] \log \left(D_{\text {hipo }}\right)
\end{aligned}
$$

donde $M_{r e f}=5,5$.

El modelo de Bindi et al. (2009a) utiliza registros de aceleración para terremotos con magnitud Mw comprendida entre 4,6 y 6,9, situados a distancias Boore y Joyner menores o iguales que $190 \mathrm{~km}$. A este respecto, los autores consideran distancias epicentrales para los terremotos con acelerograma cuya magnitud Mw es menor que 5,5. Considerando que las profundidades focales de los datos están comprendidas entre 2 y $29 \mathrm{~km}$, se considera que el modelo es válido para ser aplicado en El Hierro. Por una parte, el rango de distancias epicentrales contiene al intervalo de distancias epicentrales de los acelerómetros de El Hierro que han registrado acelerograma. Además, el rango de magnitudes Mw contiene al menos a las magnitudes $\mathrm{Mw}$ 4,64,9 de los terremotos con acelerograma registrado en El Hierro.

$\mathrm{Al}$ haber seleccionado las expresiones de PGA que corresponden a roca, se considera que la condición del terreno de referencia para el modelo de Bindi et al. (2009a) es la clase de emplazamiento 1 (Tabla 3.4 ), con Vs30 $>1500 \mathrm{~m} / \mathrm{s}$ y $F_{a}=0,86$.

Con respecto a las PGA observadas en El Hierro, y para proceder a realizar la calibración del modelo de Bindi et al. (2009a) con las ecuaciones 6.26 y 6.29, se aproxima la definición de PGA como el máximo de las aceleraciones pico de cada componente horizontal del acelerograma (N-S, E-W). Para calibrar el modelo con la 
ecuación 6.27, se aproxima la PGA como la media geométrica de las aceleraciones máximas observadas en las dos componentes horizontales del acelerograma (N-S, EW). En las ecuaciones 6.26, 6.27 y 6.28 se introduce $D_{e p i}$ en vez de $R_{j b}$ pues se van a calcular PGA sintéticas para terremotos con $\mathrm{Mw}<5,5$, siguiendo a Bindi et al. (2009a), quienes emplean $D_{e p i}$ de terremotos con $\mathrm{Mw}<5,5$ para obtener el modelo de atenuación.

Bindi et al. (2009b) La relación de atenuación de Bindi et al. (2009b) permite obtener la PGA, en base a su definición como el máximo valor de aceleración de la mayor de las dos componentes horizontales del acelerograma (E-W, N-S). Las variables predictivas del modelo son la magnitud, la distancia y el efecto de sitio. En el caso de magnitudes menores que 5,5, la magnitud se expresa en la escala $M_{L}$ y la distancia en términos de distancia epicentral $D_{\text {epi }}$, en función de los datos empleados por Bindi et al. (2009b). Se utilizan asimismo las tres clases de emplazamiento con respecto al efecto de sitio que publican Sabetta y Pugliese $(1987,1996)$ : roca, aluvial somero $(400 \mathrm{~m} / \mathrm{s} \leq V s \leq 800 \mathrm{~m} / \mathrm{s})$ y aluvial profundo. Para calibrar este modelo en El Hierro, se estudiará la expresión correspondiente a un emplazamiento en roca, tomando también como variables predictivas la magnitud $M_{L}$ y la distancia $D_{e p i}$, pues los terremotos de El Hierro cumplen con la condición de tener magnitudes menores que 5,5 :

$$
\log (P G A)=1,344+0,328 M_{L}-1,09 \log \left(\sqrt{D_{e p i}^{2}+h^{2}}\right)+0,262
$$

siendo $\mathrm{h}=5$, y expresándose PGA en $\mathrm{cm} / \mathrm{s}^{2}$ y $D_{e p i}$ en $\mathrm{km}$. La desviación estándar $\sigma$ del modelo es tal que $\sigma=0,32$.

El modelo de Bindi et al. (2009b) utiliza datos correspondientes a terremotos con magnitud Mw comprendida entre 4,6 y 6,9, situados a distancias epicentrales tales que $0,4 \mathrm{~km} \leq D e p i \leq 183 \mathrm{~km}$. Los autores precisan que, para los datos acelerométricos de los terremotos con $M w \geq 5,5$, se han considerado distancias Joyner y Boore. Las profundidades focales de los datos están comprendidas entre 2 y 30 $\mathrm{km}$. El modelo resulta aplicable en El Hierro, atendiendo al rango de distancias epicentrales, que contiene al intervalo correspondiente de distancias epicentrales de los acelerómetros de El Hierro que han registrado acelerograma. Además, el rango de magnitudes $\mathrm{Mw}$ contiene al menos a las magnitudes $\mathrm{Mw}$ 4,6-4,9 de los terremotos con acelerograma registrado en El Hierro.

Seleccionada la expresión de PGA en roca, se considera que la condición del terreno de referencia para el modelo de Bindi et al. (2009b) es la clase de emplazamiento 1 (Tabla 3.4), con Vs30>1500 m/s y $F_{a}=0,86$.

Para calibrar el modelo de Bindi et al. (2009b) con las PGA observadas en El Hierro, se aproxima la definición de PGA como el máximo de las aceleraciones pico de cada componente horizontal del acelerograma (N-S, E-W). Para calcular las PGA sintéticas, se introduce como variable predictiva la magnitud $\mathrm{Mw}$, de acuerdo con la conversión entre $M_{L}$ y Mw de Ristau et al. (2005), $M w=M_{L}+0,6$. 
Bindi et al. (2010) El modelo de atenuación de Bindi et al. (2010) consta de dos expresiones que dependen de la definición de la PGA. Las variables predictivas, en todos los casos, son la magnitud momento Mw, la distancia y el efecto de sitio. En el caso de terremotos con $\mathrm{Mw}<5,5$, se toma como variable predictiva la distancia epicentral $D_{e p i}$. Los autores distinguen las tres clases de emplazamiento con respecto al efecto de sitio, consideradas por Sabetta y Pugliese (1987, 1996): roca, aluvial somero $(400 \mathrm{~m} / \mathrm{s} \leq V s \leq 800 \mathrm{~m} / \mathrm{s})$ y aluvial profundo. Para calibrar este modelo en El Hierro, se estudiarán las expresiones correspondientes a un emplazamiento en roca, tomando como variable predictiva la distancia epicentral pues los terremotos de El Hierro cumplen con el rango $\mathrm{Mw}<5,5$.

Para la definición de la PGA como el máximo valor de aceleración de la mayor de las dos componentes horizontales del acelerograma (E-W, N-S), el modelo predictivo de PGA $\left(\mathrm{cm} / \mathrm{s}^{2}\right)$ en roca y con $\mathrm{Mw}<5,5$ es el siguiente:

$$
\begin{aligned}
\log (P G A)= & 3,750+0,1180\left(M w-M_{r e f}\right)-0,1147\left(M w-M_{r e f}\right)^{2} \\
& +\left[-1,9267+0,4285\left(M w-M_{r e f}\right)\right] \log \left(\sqrt{D_{e p i}{ }^{2}+h^{2}}\right)
\end{aligned}
$$

con $M_{r e f}=4,5 \mathrm{y} \mathrm{h}=10,0497$ y la desviación estándar del modelo $\sigma=0,3555$. $D_{\text {epi }}$ se expresa en $\mathrm{km}$.

Para la definición de la PGA como la aceleración pico de la componente vertical del acelerograma, el modelo predictivo de PGA $\left(\mathrm{cm} / \mathrm{s}^{2}\right)$ en roca y con $\mathrm{Mw}<5,5$ es el siguiente:

$$
\begin{aligned}
\log (P G A)= & 3,2015+0,2482\left(M w-M_{r e f}\right)-0,0428\left(M w-M_{r e f}\right)^{2} \\
& +\left[-1,7514+0,2588\left(M w-M_{r e f}\right)\right] \log \left(\sqrt{D_{e p i}^{2}+h^{2}}\right)
\end{aligned}
$$

con $M_{r e f}=4,5 \mathrm{y} \mathrm{h}=9,1513$ y la desviación estándar del modelo $\sigma=0,3241$. $D_{\text {epi }}$ se expresa en $\mathrm{km}$.

El modelo de atenuación de Bindi et al. (2010) utiliza aceleraciones correspondientes a terremotos con magnitud $\mathrm{Mw}$ comprendida entre 4,0 y 6,9, situados a distancias $R_{j b}$ comprendidas entre 1 y $100 \mathrm{~km}$. Los autores precisan que, para los datos acelerométricos de los terremotos con $\mathrm{Mw}<5,5$, se han considerado distancias epicentrales. El modelo presenta un rango de distancias epicentrales que contiene al rango de distancias epicentrales de los datos de aceleración de El Hierro que se utilizan en la calibración del modelo (Mw 4,1-4,9). Asimismo, este intervalo de magnitudes está contenido en el rango de magnitudes de los datos del modelo de atenuación, por lo cuál éste es de total aplicación en El Hierro.

Como se han seleccionado las expresiones de PGA en roca, se considera que la condición del terreno de referencia para el modelo de Bindi et al. (2010) es la clase de emplazamiento 1 (Tabla 3.4), con Vs30 $>1500 \mathrm{~m} / \mathrm{s}$ y $F_{a}=0,86$.

Para realizar la calibración del modelo con respecto a las PGA observadas en El Hierro, y por medio la ecuación 6.31, se aproxima la definición de PGA como el máximo de las aceleraciones pico de cada componente horizontal del acelerograma $(\mathrm{N}-\mathrm{S}, \mathrm{E}-\mathrm{W})$. 


\section{Hawái}

Munson y Thurber (1997) La utilización del modelo de atenuación de Munson y Thurber (1997) en las Islas Canarias se debe, primeramente, a su aplicabilidad a un volcanismo con actividad superficial y carácter efusivo, el cual es parecido al presente en el archipiélago canario.

El modelo permite obtener la PGA, en base a su definición como el máximo valor de aceleración de la mayor de las dos componentes horizontales del acelerograma (EW, N-S). Las variables predictivas del modelo son la magnitud, la distancia Joyner y Boore $R_{j b}$ y el efecto de sitio. De acuerdo con Munson y Thurber (1997), la expresión de la PGA sintética es válida para magnitud local $M_{L}$, si se introduce una magnitud $M_{L}$ menor que 6,1. Con respecto al efecto de sitio, el modelo distingue dos clases de emplazamiento: lava y ceniza, con $60 \mathrm{~m} / \mathrm{s} \leq V s \leq 200 \mathrm{~m} / \mathrm{s}$. Para calibrar el modelo de Munson y Thurber (1997) respecto a los datos de aceleración de El Hierro, se estudia la expresión correspondiente a un emplazamiento en lava, entendida como roca dura, tomando como variable predictiva la magnitud $M_{L}$, pues los terremotos de El Hierro cumplen con la condición $M_{L}<6,1$. Esto se demuestra convirtiendo el rango de magnitudes de estos terremotos, $\mathrm{Mw} 4,1-4,9$, a $M_{L}$ con la relación de Ristau et al. (2005) $\left(M_{L}=M w-0,6\right)$, resultando un intervalo $M_{L} 3,5-4,3$. La expresión de la PGA es por tanto:

$$
\log (P G A)=0,518+0,387\left(M_{L}-6\right)-0,00256 r-\log (r)
$$

siendo $r=\sqrt{R_{j b}^{2}+h^{2}}$ y $h=11,29$. La PGA se expresa en $\mathrm{g}\left(\mathrm{g}=9,8 \mathrm{~m} / \mathrm{s}^{2}\right)$ y la distancia $R_{j b}$ en $\mathrm{km}$. La desviación estándar del modelo $\sigma$ es tal que $\sigma=0,237$.

De acuerdo con Munson y Thurber (1997), los datos de aceleración utilizados para determinar el modelo corresponden a magnitudes expresadas en términos de magnitud de ondas superficiales $M_{S}$ para $M_{S} \geq 6,1$, y en términos de $M_{L}$ para $M_{L}<6,1$. El intervalo de magnitudes de los datos está comprendido entre una magnitud mínima $M_{\min }$ tal que $M_{\min }=4,0\left(M_{L}\right)$ y una magnitud máxima $M_{\max }$ tal que $M_{\max }=7,2\left(M_{S}\right)$. Utilizando la conversión de Ristau et al. (2005), $M_{\min }=4,6$ (Mw), y por medio de la conversión de Wells y Coppersmith (1994) entre $M_{S}$ y Mw $\left(M_{S}=M w\right)$, válida para magnitudes mayores que 5,7 y menores que 8 , se obtiene $M_{\max }=7,2(\mathrm{Mw})$. En cuanto al rango de distancias Joyner y Boore, los registros de aceleración se encuentran situados en un intervalo de $R_{j b}$ comprendido entre 0 y 88 $\mathrm{km}$. Las profundidades hipocentrales de los terremotos cuyos registros de aceleración se han empleado en la determinación del modelo oscilan entre 4 y $14 \mathrm{~km}$. El modelo de Munson y Thurber (1997) resulta aplicable en El Hierro, atendiendo al rango de distancias epicentrales, que contiene al intervalo correspondiente de distancias epicentrales de los acelerómetros de El Hierro que han registrado acelerograma. Además, el rango de magnitudes $\mathrm{Mw}$ contiene al menos a las magnitudes $\mathrm{Mw}$ 4,64,9 de los terremotos con acelerograma registrado en El Hierro.

Se ha seleccionado la expresión del modelo para calcular la PGA en lava. Entendiendo que la lava es una roca dura, se clasifica en la clase de emplazamiento 1 de la clasificación de síntesis (Tabla 3.4). Por este motivo, se considera que la condición del terreno de referencia para el modelo de Munson y Thurber (1997) es la clase de emplazamiento 1 , con Vs30 $>1500 \mathrm{~m} / \mathrm{s}$ y $F_{a}=0,86$. 
Para calibrar el modelo en función de los registros de aceleración de El Hierro, se aproxima la definición de PGA como el máximo de las aceleraciones pico de cada componente horizontal del acelerograma (N-S, E-W). Asimismo, se introduce la variable $M_{L}=M w-0,6$ en la ecuación 6.33, utilizando la conversión de Ristau et al. (2005). En cuanto a la distancia, se sustituye $R_{j b}$ por $D_{e p i}$, siguiendo a Boore y Atkinson (2008).

\section{Guadalupe}

Beauducel et al. (2004) El modelo de atenuación de Beauducel et al. (2004) se ha obtenido a partir de 1430 valores de PGA que corresponden a 398 terremotos localizados en la región de la isla de Guadalupe (Mar Caribe). Atendiendo a la naturaleza volcánica de la isla, el modelo también resulta de aplicación en las Islas Canarias. Beauducel et al. (2004) obtienen la PGA a partir de las observaciones de aceleración como el máximo de la raíz cuadrada de la suma de los cuadrados de las componentes horizontales del acelerograma.

Las variables predictivas del modelo de atenuación son la magnitud y la distancia hipocentral $D_{\text {hipo. }}$. Con respecto a la magnitud, la relación de atenuación es válida para magnitudes por duración $M_{D}$ en el caso de simulaciones de aceleración pico para terremotos con magnitud menor que 4,5, de acuerdo con Beauducel et al. (2004). En el caso de terremotos con magnitudes superiores o iguales a 4,5, se estima que la relación de atenuación es válida para magnitudes momento $\mathrm{Mw}$ (Beauducel et al., 2004). Para calibrar esta relación con respecto a los terremotos con acelerograma registrado en El Hierro, con $\mathrm{Mw} 4,1-4,9$, se calculan las PGA sintéticas para una magnitud 4,5, por lo que puede tomarse como variable predictiva la magnitud $\mathrm{Mw}$. Por tanto, la expresión de la PGA sintética que se va a utilizar es la siguiente:

$$
\log (P G A)=0,611377 M w-0,00584334 D_{\text {hipo }}-\log \left(D_{\text {hipo }}\right)-3,216674
$$

con PGA en g $\left(\mathrm{g}=9,8 \mathrm{~m} / \mathrm{s}^{2}\right)$ y $D_{\text {hipo }}$ en $\mathrm{km}$. La desviación estándar del modelo $\sigma$ es tal que $\sigma=0,5$.

El modelo de atenuación de Beauducel et al. (2004) utiliza aceleraciones correspondientes a terremotos con magnitudes expresadas en distintas escalas: $M_{D}, \mathrm{mb}$ (magnitud de ondas internas) y Mw. Atendiendo al conjunto de los datos, la magnitud mínima es $M_{\min }=1,1\left(M_{D}\right)$ y la magnitud máxima es $M_{\max }=6,3(\mathrm{Mw}$ o mb). Para convertir el rango de magnitudes de los datos a magnitudes momento, y así poder valorar la aplicabilidad del modelo de atenuación en El Hierro, se adopta la expresión $M_{D}=M w-0,5$, determinada por Drouet et al. (2011) para $M_{D} 2-6$. La razón estriba en que esta expresión ha sido obtenida en una isla volcánica, Guadalupe, al igual que lo es El Hierro, y para un rango de $\mathrm{Mw}(2,5-6,5)$ que también es similar al rango de $\mathrm{Mw}$ de todos los terremotos de El Hierro con acelerograma registrado entre 2011 y $2013(2,4-4,9)$, de acuerdo con el catálogo de acelerogramas proporcionado por el IGN (subsección 2.5.1). Aplicando esta expresión, el rango de magnitudes $\mathrm{Mw}$ de los datos de Beauducel et al. (2004) está comprendido entre 1,6 y 6,3 . Con respecto al intervalo de distancias hipocentrales de estos datos, éste comprende distancias entre 1,7 y $450 \mathrm{~km}$. Este rango de distancias contiene al 
rango correspondiente para los acelerogramas de El Hierro $(3,4-37 \mathrm{~km})$. Por este motivo, el modelo de Beauducel et al. (2004) es aplicable en El Hierro, para todas las magnitudes correspondientes a los registros de aceleración de los que se dispone.

Beauducel et al. (2004) no tienen en cuenta el tipo de terreno sobre el cual se encuentran instaladas las estaciones acelerométricas, a los efectos del cálculo de la ley de atenuación, a pesar de que los registros de aceleración se han obtenido en suelo y en roca. Dado que los autores sugieren multiplicar por 3 las PGA calculadas a fin de obtener su valor sobre el suelo, como se observa en la Figura 4-d de su trabajo, se asume que las PGA calculadas con esta relación de atenuación están referidas a roca. Por este motivo, se considera que la condición del terreno de referencia para el modelo de Beauducel et al. (2004) es la clase de emplazamiento 1 (Rocas duras) (Tabla 3.4), con Vs30 $>1500 \mathrm{~m} / \mathrm{s}$ y $F_{a}=0,86$.

Para calibrar el modelo de atenuación con respecto a las aceleraciones registradas en El Hierro, se aproxima la PGA (máximo de la raíz cuadrada de la suma de los cuadrados de las componentes horizontales del acelerograma) por la raíz cuadrada de la suma de los cuadrados de las aceleraciones máximas horizontales. La distancia hipocentral se obtiene a partir de la distancia epicentral y de la profundidad hipocentral.

\subsubsection{Preparación de datos de aceleración máxima registra- da de los terremotos de $\mathrm{Mw}>4,0$ localizados en El Hierro}

Las relaciones de atenuación seleccionadas por su aplicabilidad en El Hierro se han calibrado en función de los acelerogramas de los terremotos registrados con $\mathrm{Mw}$ 4,1-4,9 en la isla. Como paso previo a la calibración de cada relación de atenuación, se han calculado las aceleraciones pico, PGA, en cada acelerómetro y para cada terremoto, a partir de las componentes del acelerograma correspondiente, utilizando la definición dada por el modelo de atenuación. Con este fin, ha sido necesario obtener la aceleración máxima de cada componente del acelerograma, ya que, como se ha explicado en la subsección 6.2.1, la definición de la PGA de cada relación de atenuación se ha aproximado a partir de las aceleraciones pico de las componentes.

La aceleración máxima de cada componente del acelerograma se ha obtenido por medio del programa SeismoSignal, versión 5.1, que permite el procesado de acelerogramas en formato ASCII. Al registro de cada componente se le ha aplicado una corrección lineal por línea de base y un filtro paso-banda Butterworth con frecuencias 0,50-25 Hz.

En la Tabla 6.2 se presentan los parámetros focales de cada uno de los terremotos utilizados en la calibración de las relaciones de atenuación, junto con la distancia epicentral de cada acelerómetro, su factor de amplificación $F_{a}$ para periodos cortos y las aceleraciones máximas de cada componente del acelerograma. Los epicentros de los terremotos se han representado en función de su magnitud Mw en la Figura 6.1. 


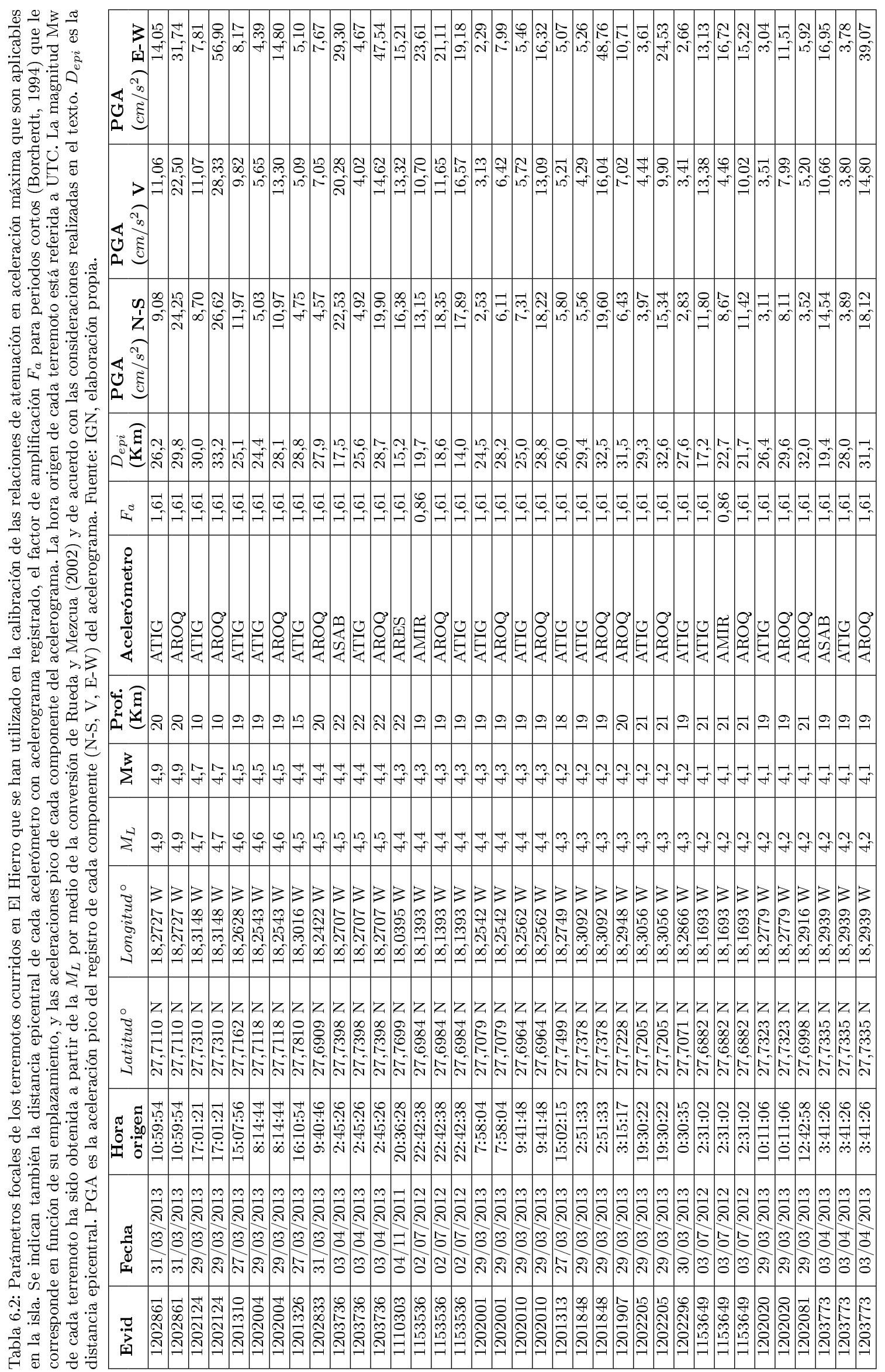




\subsubsection{Calibración de las relaciones de atenuación en acelera- ción máxima}

Para calibrar cada relación de atenuación, se ha obtenido la PGA observada que corresponde al acelerómetro que ha registrado cada terremoto de calibración (Tabla 6.2), aproximando la definición de PGA a partir de las aceleraciones máximas de cada componente del acelerograma. Todas las PGA resultantes se han corregido a la condición del terreno de referencia del modelo de atenuación. Esta corrección se realiza bajo la premisa de que, en acelerómetros instalados sobre la misma clase de emplazamiento (Tabla 3.4) que el terreno de referencia, la PGA observada no experimenta ninguna corrección. Para ello, se realiza un reescalamiento de todos los factores de amplificación $F_{a}$ de los acelerómetros en función del factor $F_{a}$ del terreno de referencia, de manera que el factor de amplificación modificado para el mismo sea igual a 1. Por tanto, este reescalamiento se realiza dividiendo los factores de amplificación entre el factor del terreno de referencia. Las PGA se corrigen a la condición del terreno de referencia dividiendo su valor por el factor $F_{a}$ reescalado. En definitiva, la operación de corrección implica dividir cada PGA por el factor $F_{a}$ del acelerómetro y multiplicar el resultado por el factor $F_{a}$ que corresponde al terreno de referencia de la relación de atenuación.

A continuación, se han calculado las PGA sintéticas para una magnitud intermedia al rango de magnitudes de los terremotos de calibración, y para un rango de distancias epicentrales coherente con el de los emplazamientos de los acelerómetros, teniendo en cuenta los datos de magnitud $\mathrm{Mw}$ y de distancia epicentral de la Tabla 6.2. Dado que los terremotos de calibración tienen $\mathrm{Mw}$ en el intervalo 4,1-4,9, los cálculos se han realizado para una $\mathrm{Mw}=4,5$. Atendiendo al rango de distancias epicentrales de los acelerómetros con registro de aceleración para los terremotos de calibración (3,4-37 km), las PGA sintéticas se han calculado para distancias epicentrales entre 1 y $100 \mathrm{~km}$, a intervalos de $1 \mathrm{~km}$. En el caso del modelo de Beauducel et al. (2004), las distancias hipocentrales necesarias se han calculado teniendo en cuenta la profundidad media de los terremotos de calibración, que es de $19 \mathrm{~km}$. En el cálculo de las PGA sintéticas con cada relación de atenuación, se han tenido en cuenta las conversiones de las distintas escalas de magnitud a Mw, y de las distintas métricas de distancias a distancia epicentral, según la subsección 6.2.1.

A continuación, se presenta, como ejemplo, el proceso de calibración de la relación de Pétursson y Vogfjörd (2009). En este caso, las PGA observadas se aproximan con el valor de la raíz cuadrada de la suma de los cuadrados de las aceleraciones máximas de cada componente. A continuación, se corrigen a roca dura (condición del terreno de referencia), con $F_{a}=0,86$. Para ello, se multiplica por este factor el resultado de dividir los valores de PGA observadas por el factor de amplificación $F_{a}$ del acelerómetro en cuestión. En la Figura 6.2 se representan las PGA registradas y corregidas a roca dura en función de la distancia epicentral para los terremotos de calibración $(4,0<M w \leq 5,0)$, por medio de puntos de color azul. En la Figura 6.2 también se puede observar la curva de atenuación de la PGA para magnitud Mw 4,5, según Pétursson y Vogfjörd (2009), representada por la línea roja. Se representan asimismo las dos curvas de la PGA sintética que incorporan la desviación estándar $\sigma$ del modelo $(0,302)$. En el caso de Pétursson y Vogfjörd (2009), estas curvas se han obtenido a partir de los resultados de $\log (P G A)+2 \sigma$ y $\log (P G A)-2 \sigma$. 


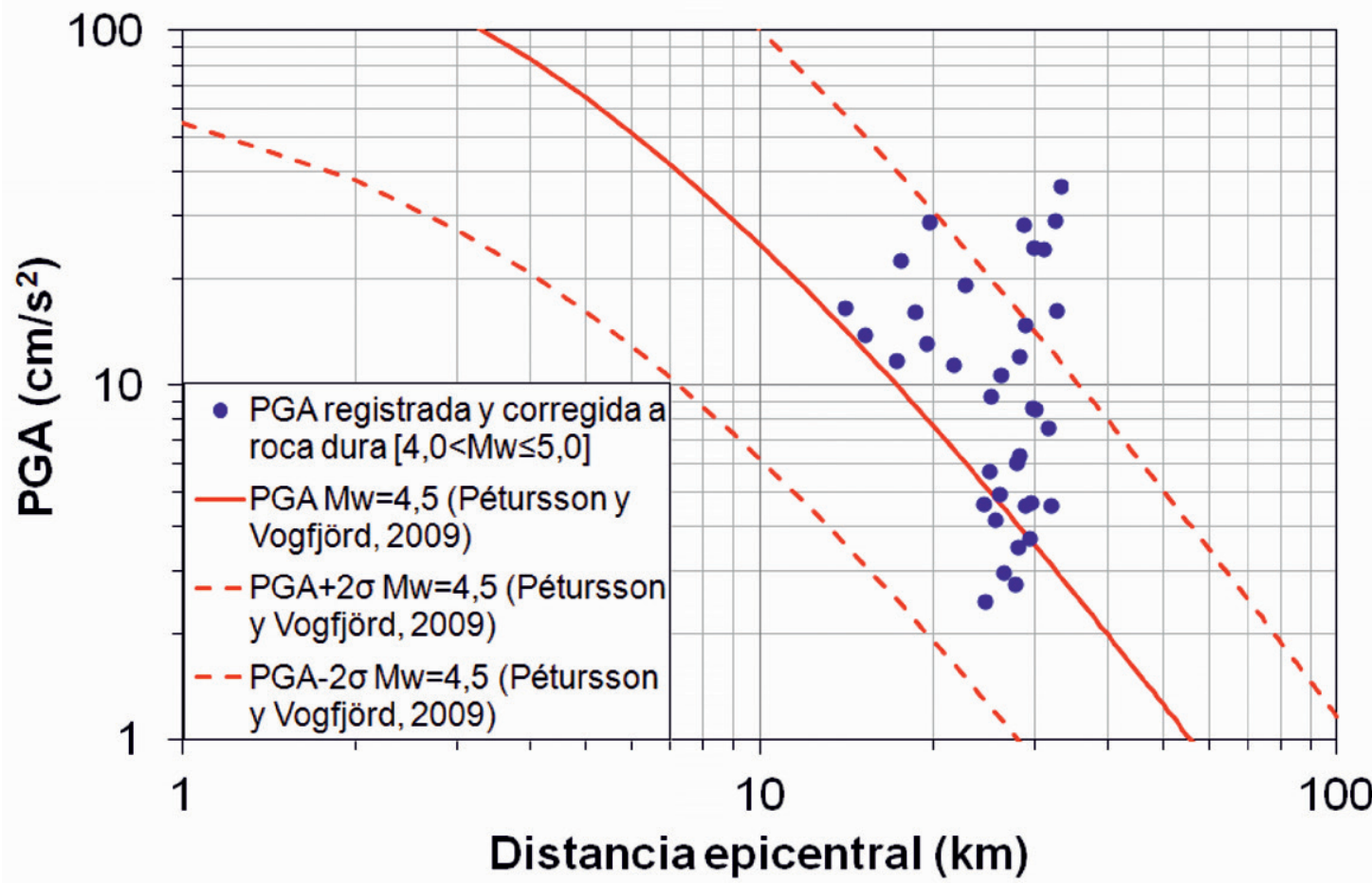

Figura 6.2: PGA registradas y corregidas a roca dura para los terremotos de calibración de El Hierro (2011-2013) con 4,0<Mw $\leq 5,0$, curva de atenuación de la PGA para un terremoto de Mw 4,5, de acuerdo al modelo de atenuación de Pétursson y Vogfjörd (2009), y curvas de atenuación para la PGA que incluyen la desviación estándar $\sigma$ del modelo y que han sido obtenidas a partir de $\log (P G A) \pm 2 \sigma)$.

Como se puede comprobar, la curva de atenuación ajusta razonablemente bien la distribución de las PGA registradas u observadas para los sismos de magnitud $4,0<$ $M w \leq 5,0$. Se puede visualizar también que la mayor parte de las PGA registradas quedan en el intervalo de PGA sintéticas establecido por la desviación estándar del modelo de atenuación. Por tanto, las diferencias entre PGA registradas y sintéticas pueden considerarse aceptables, dentro del rango de distancias epicentrales de los datos observados. La bondad de este ajuste debe entenderse, en todo caso, limitado al rango de magnitudes estudiado.

En la Figura 6.3 se representan las PGA registradas y corregidas a roca dura, en función de la distancia epicentral, para los cuatro terremotos de calibración con Mw $>4,5$, por medio de puntos de color azul. También se han representado las PGA calculadas con la relación de atenuación de Pétursson y Vogfjörd (2009) para las mismas magnitudes y distancias epicentrales de los terremotos en cuestión (puntos rojos). Se pretende por tanto particularizar el análisis de la bondad del ajuste a los eventos de mayor magnitud, comprobándose que las diferencias entre las PGA calculadas y observadas para cada terremoto oscilan entre 1 y $8 \mathrm{~cm} / \mathrm{s}^{2}$. Por medio de puntos sin relleno, se representan las PGA sintéticas que resultan de considerar la desviación estándar y que marcan los límites del intervalo $\log (P G A) \pm 2 \sigma$. Las PGA observadas para los terremotos de $\mathrm{Mw}>4,5$ quedan mayoritariamente contenidas en este intervalo, al igual que en el caso de los terremotos con $4,0<M w \leq 5,0$.

En el Apéndice G se incluyen los resultados de la calibración de las 12 relaciones de atenuación examinadas en la subsección 6.2.1 y que se pueden aplicar en El Hierro. Para cada modelo de atenuación se proporciona el ajuste de la curva de 


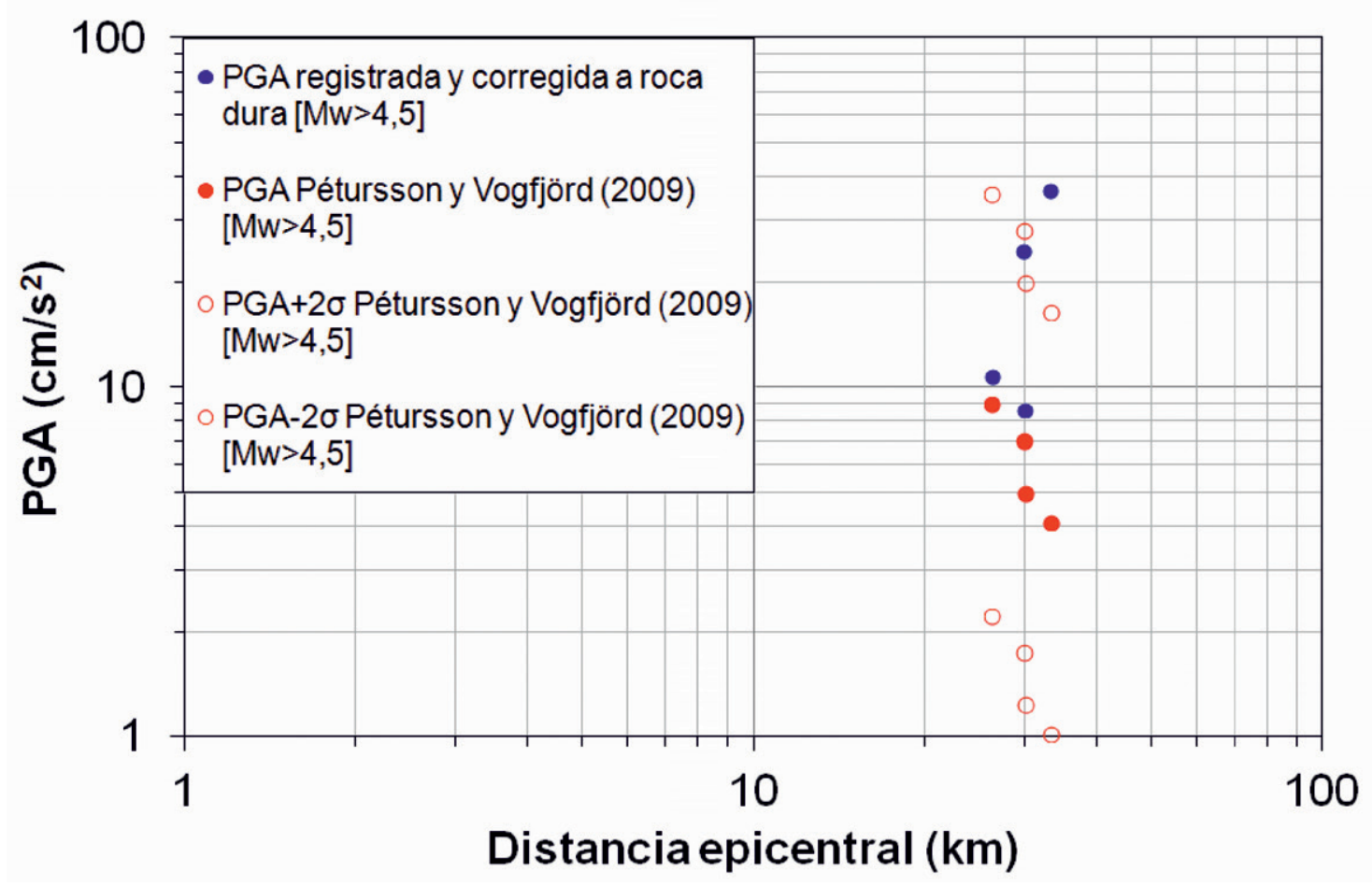

Figura 6.3: PGA registradas y corregidas a roca dura para los terremotos de calibración de El Hierro (2011-2013) con $\mathrm{Mw}>4,5$, PGA sintéticas obtenidas con el modelo de atenuación de Pétursson y Vogfjörd (2009) y PGA sintéticas obtenidas a partir de $\log (P G A) \pm 2 \sigma$, siendo $\sigma$ la desviación estándar del modelo.

atenuación de la PGA sintética a las PGA observadas y corregidas a la condición del terreno de referencia, tanto para los terremotos con 4,0<Mw $\leq 5,0$ como para los terremotos con $\mathrm{Mw}>4,5$.

\subsubsection{Selección de leyes de atenuación óptimas para distintas escalas de trabajo y en función de la magnitud del terremoto}

A partir de los resultados de la calibración de las relaciones de atenuación en aceleración máxima y aplicables en El Hierro, se ha procedido a seleccionar 3 relaciones de atenuación óptimas, en función de los rangos de distancias epicentrales y magnitudes correspondientes a los terremotos de calibración. En una primera fase, esta selección se ha llevado a cabo examinando la bondad del ajuste de las leyes de atenuación en función de las PGA registradas y reducidas al terreno de referencia correspondiente para los terremotos con $4,0<M w \leq 5,0$. Se ha atendido particularmente a dos criterios, por una parte la estimación cualitativa de la bondad del ajuste de cada modelo de atenuación al conjunto de PGA registradas, y por otra parte el número de PGA registradas que se sitúan sobre la curva de atenuación de la PGA, y que por tanto coinciden con la PGA sintética calculada para la misma distancia epicentral y para la misma $\mathrm{Mw}$ del terremoto.

En una segunda fase del proceso de selección, se han valorado el rango de distancias epicentrales o hipocentrales y el rango de magnitudes de los datos utilizados para determinar cada relación de atenuación. Este paso permite conocer la escala de 
trabajo en la cual la relación seleccionada es óptima, así como el rango de magnitudes de los terremotos para los cuales puede simular aceleraciones sintéticas realistas. En este paso, se preferirán aquellas relaciones con rangos de distancias y magnitudes lo más amplios posible.

En la Tabla 6.3 se presentan los dos criterios de examen de la bondad del ajuste para las 12 relaciones de atenuación aplicables en El Hierro, los cuales constituyen la primera fase del proceso de selección. También se indica si la condición del terreno de referencia de cada relación de atenuación ha podido ser confirmada en la investigación bibliográfica, o bien se ha tenido que suponer un terreno de referencia a partir de la información disponible sobre el modelo de atenuación, y por último el factor de amplificación $F_{a}$ de la condición del terreno de referencia finalmente adoptado.

Como resultado de la primera fase de selección, se han descartado las relaciones de atenuación de Tento et al. (1992), Munson y Thurber (1997), Bindi et al. (2009a) y Bindi et al. (2009b). Se estima que estos modelos de atenuación no se ajustan bien a las PGA observadas, pues se observan diferencias significativas entre las observaciones y las correspondientes PGA sintéticas. En el modelo de Tento et al. (1992), llegan al orden de $9 \mathrm{~cm} / \mathrm{s}^{2}$ (Apéndice G), y en los modelos de Munson y Thurber (1997) y de Bindi et al. (2009b), a $10 \mathrm{~cm} / \mathrm{s}^{2}$. Para el modelo de Bindi et al. (2009a) con PGA definida en función de la componente máxima horizontal del acelerograma, las diferencias llegan al orden de $100 \mathrm{~cm} / \mathrm{s}^{2}$. Además, en cada caso, el conjunto de observaciones tiene una dispersión que no permite una modelización adecuada por medio de la ley de atenuación. En cuanto al número de PGA registradas que se sitúan sobre la curva de atenuación, es 0 en los modelos de atenuación de Tento et al. (1992) y de Bindi et al. (2009b), y 1 en el modelo de Munson y Thurber (1997). En el modelo de Bindi et al. (2009a), sólo la expresión de la PGA (definida en función de la componente máxima horizontal) que se atenúa con la distancia hipocentral permite obtener 1 valor de PGA sintética coincidente con el valor correspondiente de PGA registrada (Apéndice $G$ ).

En cuanto a los modelos de atenuación con varias expresiones de atenuación de la PGA, se han seleccionado las expresiones con mejor ajuste a las PGA registradas, en función de los criterios mencionados. En el modelo de Sigbjörnsson y Baldvinsson (1992), se selecciona la curva de atenuación de la PGA definida como la aceleración pico de la componente horizontal E-W. Para el modelo de atenuación de Ágústsson et al. (2008), se selecciona la curva de atenuación de la PGA que se atenúa en función del logaritmo de la magnitud. Con respecto al modelo de atenuación de Bindi et al. (2010), se selecciona la curva de atenuación de la PGA definida como la aceleración pico de la componente vertical (V).

Las 8 relaciones de atenuación que se han conservado como resultado de la primera fase han pasado a la segunda fase de selección, en la cual se han valorado conjuntamente el rango de distancias epicentrales o hipocentrales y el rango de magnitudes de los datos utilizados en su determinación (Tabla 6.4). El modelo de atenuación de Sigbjörnsson y Baldvinsson (1992) se descarta, pues presenta los rangos de distancias epicentrales más pequeños (2-80 km y 0-80 km, respectivamente). Además, el modelo de Sigbjörnsson y Baldvinsson (1992) presenta la incertidumbre de no conocer la escala de la magnitud de los datos (se ha supuesto Mw). La relaciones de atenuación de Ólafsson y Sigbjörnsson (1999) y de Bindi et al. (2010) 
Tabla 6.3: Análisis de los resultados de la calibración de las relaciones de atenuación aplicables en El Hierro (sección 6.2.3 y Apéndice G). Se presenta la estimación cualitativa de la bondad del ajuste de cada relación a las PGA registradas y reducidas al terreno de referencia correspondiente para los terremotos con $4,0<M w<5,0$, el número de PGA registradas que coinciden con las correspondientes PGA sintéticas ( $\mathrm{N}^{\circ}$ PGA reg.= PGA sint.), la confirmación o no de la condición del terreno de referencia para cada modelo de atenuación (Terr. ref. confirmado) y el factor de amplificación $F_{a}$ finalmente considerado. En el caso del modelo de atenuación de Ágústsson et al. (2008), $\log (\mathrm{R})-\operatorname{lin}(\mathrm{M})$ se refiere a la expresión de la PGA que depende linealmente de la magnitud $M_{L w}$ (ecuación 6.23), y $\log (\mathrm{R})-\log (\mathrm{M})$ a la expresión de la PGA que depende del logaritmo de la magnitud $M_{L w}$ (ecuación 6.22).

\begin{tabular}{|c|c|c|c|c|}
\hline Modelo de atenuación & $\begin{array}{l}\text { Terr. ref. } \\
\text { confirmado }\end{array}$ & $F_{a}$ & $\begin{array}{l}\text { Estimación } \\
\text { ajuste }\end{array}$ & $\begin{array}{l}N^{\circ} \quad \text { PGA } \\
\text { reg. } \quad= \\
\text { PGA }\end{array}$ \\
\hline $\begin{array}{l}\text { Ágústsson et al. (2008) (Log(R)- } \\
\operatorname{lin}(\mathrm{M}))\end{array}$ & NO & 0,86 & Bueno & 5 \\
\hline $\begin{array}{l}\text { Ágústsson et al. (2008) (Log(R)- } \\
\log (\mathrm{M}))\end{array}$ & NO & 0,86 & Bueno & 4 \\
\hline García Blanco (2009) & SÎ & 1,61 & Bueno & 2 \\
\hline $\begin{array}{l}\text { Instituto Geográfico Nacional } \\
(2013)\end{array}$ & SÍ & 1,61 & Bueno & 3 \\
\hline Pétursson y Vogfjörd (2009) & Sİ & 0,86 & Bueno & 2 \\
\hline Tento et al. (1992) & ESTIMADO & 1,25 & Malo & 0 \\
\hline $\begin{array}{l}\text { Bindi } \text { et al. }(2009 \mathrm{a}) \text { (Componen- } \\
\text { te máxima horizontal) }\end{array}$ & SÍ & 0,86 & Malo & 0 \\
\hline $\begin{array}{l}\text { Bindi } \text { et al. (2009a) (Media geo- } \\
\text { métrica de componentes horizon- } \\
\text { tales) }\end{array}$ & Sí & 0,86 & Malo & 0 \\
\hline $\begin{array}{l}\text { Bindi } \text { et al. (2009a) (Componen- } \\
\text { te vertical) }\end{array}$ & Sí & 0,86 & Malo & 0 \\
\hline $\begin{array}{l}\text { Bindi et al. (2009a) (Componen- } \\
\text { te máxima horizontal, distancia } \\
\text { hipocentral) }\end{array}$ & Sí & 0,86 & Malo & 1 \\
\hline Bindi et al. $(2009 \mathrm{~b})$ & SÍ & 0,86 & Malo & 0 \\
\hline $\begin{array}{l}\text { Bindi et al. (2010) (Componente } \\
\text { máxima horizontal) }\end{array}$ & Sí & 0,86 & Bueno & 1 \\
\hline $\begin{array}{l}\text { Bindi et al. (2010) (Componente } \\
\text { vertical) }\end{array}$ & SÍ & 0,86 & Bueno & 1 \\
\hline $\begin{array}{l}\text { Sigbjörnsson y } \text { Baldvinsson } \\
\text { (1992) } \\
\text { E-W) }\end{array}$ & NO & 0,86 & Bueno & 1 \\
\hline $\begin{array}{l}\text { Sigbjörnsson y } \text { Baldvinsson } \\
\text { (1992) (Componente horizontal } \\
\text { N-S) }\end{array}$ & NO & 0,86 & Bueno & 0 \\
\hline $\begin{array}{l}\text { Sigbjörnsson y } \text { Baldvinsson } \\
\text { (1992) (Componente máxima } \\
\text { horizontal) }\end{array}$ & $\mathrm{NO}$ & 0,86 & Bueno & 1 \\
\hline 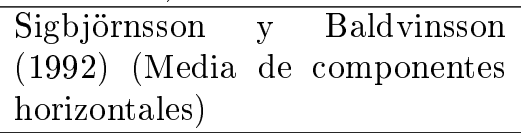 & NO & 0,86 & Bueno & 2 \\
\hline Ólafsson y Sigbjörnsson (1999) & ESTIMADO & 0,86 & Bueno & 4 \\
\hline Munson y Thurber (1997) & Sİ & 0,86 & Malo & 1 \\
\hline Beauducel et al. (2004) & ESTIMADO & 0,86 & Bueno & 3 \\
\hline
\end{tabular}


presentan los siguientes intervalos de distancia en orden creciente (1-100 km y 2-112 $\mathrm{km}$ ), por lo que se descartan también.

Las cinco relaciones de atenuación que se han retenido (Beauducel et al., 2004; Ágústsson et al., 2008; García Blanco, 2009; Pétursson y Vogfjörd, 2009; Instituto Geográfico Nacional, 2013) presentan rangos de magnitudes y distancias de al menos 1,5 y $200 \mathrm{~km}$ de amplitud, respectivamente. En función del ambiente tectónico en el cual se han registrado los datos acelerométricos, se considera que las relaciones de Beauducel et al. (2004), Ágústsson et al. (2008) y Pétursson y Vogfjörd (2009) son más adecuadas para su utilización en la simulación de escenarios sísmicos en El Hierro, y por extensión en Canarias. Estos modelos se han construido con aceleraciones observadas en la isla de Guadalupe y en Islandia, por lo cual están referidas a un ambiente volcánico equiparable al del archipiélago canario. Como conclusión, se han seleccionado las relaciones de Beauducel et al. (2004), Ágústsson et al. (2008) y Pétursson y Vogfjörd (2009) como relaciones de atenuación óptimas en Canarias, en función de la magnitud del terremoto y la distancia epicentral, con una aplicación conjunta para magnitudes $\mathrm{Mw}$ comprendidas entre 3,1 y 6,3, y distancias epicentrales comprendidas entre 3 y $350 \mathrm{~km}$.

Tabla 6.4: Rangos de distancias epicentrales $\left(D_{\text {epi }}\right)$ o hipocentrales $\left(D_{\text {hipo }}\right)$ y rangos de magnitudes Mw de las relaciones de atenuación seleccionadas en el análisis de los resultados de la calibración (Tabla 6.3). En la relación de Sigbjörnsson y Baldvinsson (1992), se ha supuesto que el rango de magnitudes se expresa en Mw.

\begin{tabular}{|l|c|c|}
\hline Modelo de atenuación & Rango de Mw & $\begin{array}{l}\text { Rango de } \\
\text { distancia (km) }\end{array}$ \\
\hline Ágústsson et al. $(2008)(\log (\mathrm{R})-\log (\mathrm{M}))$ & $3-6,5$ & $3-350\left(D_{\text {epi }}\right)$ \\
\hline García Blanco $(2009)$ & $3-6,5$ & $0-200\left(D_{\text {epi }}\right)$ \\
\hline Instituto Geográfico Nacional $(2013)$ & $4-5,5$ & $1-370\left(D_{\text {epi }}\right)$ \\
\hline Pétursson y Vogfjörd (2009) & $3,1-6,5$ & $3-380\left(D_{\text {epi }}\right)$ \\
\hline Bindi et al. (2010) (Componente vertical) & $4-5,5$ & $1-100\left(D_{\text {epi }}\right)$ \\
\hline $\begin{array}{l}\text { Sigbjörnsson y Baldvinsson (1992) (Com- } \\
\text { ponente horizontal E-W) }\end{array}$ & $2-6$ & $2-80\left(D_{\text {epi }}\right)$ \\
\hline Ólafsson y Sigbjörnsson (1999) & $3,4-5,9$ & $2-112\left(D_{\text {epi }}\right)$ \\
\hline Beauducel et al. $(2004)$ & $1,6-6,3$ & $1,7-450\left(D_{\text {hipo }}\right)$ \\
\hline
\end{tabular}

\subsection{Selección de leyes de atenuación de alcance mun- dial}

Se ha estimado conveniente, a los efectos de su programación en el código de la herramienta plugin (capítulo 9), la selección de 3 relaciones de atenuación en términos de aceleración, adicionales y con alcance mundial. Estas relaciones sin duda introducen una aplicabilidad en la herramienta que trasciende el ámbito español, y que podrían permitir su utilización en otras zonas del mundo. Con este fin, se han estudiado las leyes de atenuación seleccionadas para el proyecto Global Earthquake Model (Stewart et al., 2013), en función de los principales ambientes tectónicos.

El proyecto Global Earthquake Model desarrolla recomendaciones sobre las relaciones de atenuación o de predicción de los movimientos fuertes a utilizar cuando se llevan a cabo estudios de peligrosidad sísmica a escala global. En el contexto de este proyecto, se han compilado y revisado distintas relaciones de atenuación, con 
el fin de seleccionar un conjunto de las mismas para su aplicación global. Las recomendaciones realizadas por el proyecto se circunscriben a tres ambientes tectónicos determinados (Stewart et al., 2013):

- Regiones continentales estables: Se proponen los modelos de Toro et al. (1997), Atkinson y Boore (2006), Silva et al. (2002) y Pezeshk et al. (2011).

- Zonas de subducción: Se proponen los modelos de Atkinson y Boore (2003), Zhao et al. (2006) y Abrahamson et al. (2012).

- Regiones corticales activas: Se proponen los modelos de Akkar y Bommer (2010), Chiou y Youngs (2008) y Zhao et al. (2006).

Algunas de las relaciones de atenuación recomendadas por Stewart et al. (2013) no tienen término de efecto de sitio, o bien incluyen términos lineales de efecto de sitio. En ambos casos, se recomienda la aplicación de los modelos para un emplazamiento de referencia en roca, con la posiblidad de modificar las aceleraciones resultantes con un término no lineal de efecto de sitio. La razón estriba en que los términos lineales pueden sobreestimar significativamente los parámetros del movimiento fuerte (aceleración) para magnitudes relativamente grandes o distancias relativamente cortas.

En esta tesis doctoral se ha escogido un modelo de atenuación para cada uno de los ambientes tectónicos diferenciados por el proyecto Global Earthquake Model. El criterio de selección ha consistido en dar preferencia a modelos de atenuación obtenidos para un terreno de referencia en roca, de forma que no sea necesario modificar las aceleraciones máximas con un término no lineal de efecto de sitio. De esta forma, para las regiones continentales estables, se ha elegido el modelo de atenuación de Atkinson y Boore (2006), modificado por Atkinson y Boore (2011). En el caso de las regiones corticales activas se ha escogido la relación de atenuación de Chiou y Youngs (2008), y en el de zonas de subducción, el modelo de Zhao et al. (2006).

\subsubsection{Regiones continentales estables: Atkinson y Boore (2006), modificada por Atkinson y Boore (2011)}

Atkinson y Boore (2006) obtienen su modelo de atenuación a partir de simulaciones de acelerogramas realizadas por medio del código EXSIM, de forma que realmente utilizan datos para un intervalo de magnitudes momento 3,5-8,0 y de distancias respecto a la falla 1-1000 km. El modelo de atenuación toma como variables predictivas la magnitud momento $\mathrm{Mw}$, la profundidad $\mathrm{h}$ en $\mathrm{km}$ y la distancia más cercana a la falla $\left(R_{c d}\right)$ en $\mathrm{km}$. La expresión del modelo para PGA $\left(\mathrm{cm} / \mathrm{s}^{2}\right)$ en emplazamientos en roca es:

$$
\begin{aligned}
\log (P G A)= & 0,907+0,983 M w-0,0660 M w^{2}+(-2,70+0,159 M w) f_{1} \\
& +(-2,80+0,212 M w) f_{2}+(-0,301-0,0653 M w) f_{0}-0,000448 R_{c d}
\end{aligned}
$$


donde $f_{0}=\operatorname{máx}\left(\log \left(R_{0} / R_{c d}\right), 0\right)$ y $f_{1}=\operatorname{mín}\left(\log \left(R_{c d}\right), \log \left(R_{1}\right)\right)$. También se tiene que $f_{2}=\operatorname{máx}\left(\log \left(R_{c d} / R_{2}\right), 0\right), R_{0}=10, R_{1}=70$ y $R_{2}=140$.

Esta relación de atenuación ha sido obtenida para un parámetro de esfuerzos de 140 bares, pero los autores reconocen que la incertidumbre asociada a este valor es del orden de un factor de 1,5 a 2. Por este motivo, para modelizar el efecto de parámetros de esfuerzos distintos en la amplitud de la PGA, es necesario sumar un término de ajuste de este parámetro. Atkinson y Boore (2011) recomiendan considerar un parámetro de esfuerzos dependiente de la magnitud, sugiriendo por tanto sumar a la ecuación 6.35 el término $\log (\triangle \sigma)=3,45-0,2 \mathrm{Mw}$, válido para $M w \geq 5$. Para $M w<5$, Atkinson y Boore (2011) recomiendan tomar directamente una caída de esfuerzos fija $\triangle \sigma=280$ bares.

De acuerdo con Atkinson y Boore (2006), la relación de atenuación de la ecuación 6.35 se aplica en emplazamientos de roca dura, con $V s \geq 2000 \mathrm{~m} / \mathrm{s}$. Por este motivo, se considera que la condición del terreno de referencia para el modelo de Atkinson y Boore $(2006,2011)$ es la clase de emplazamiento 1 (Rocas duras) de la Tabla 3.4, pues esta clase se caracteriza por presentar Vs30 $>1500 \mathrm{~m} / \mathrm{s}$. Por tanto, el valor del factor de amplificación para altas frecuencias que le corresponde a este modelo es $F_{a}=0,86$.

A los efectos de la programación de la relación de atenuación expresada por la ecuación 6.35 en el plugin (capítulo 9), se considera que la distancia más próxima a la falla es la distancia hipocentral, y por eso se introduce la variable $R_{c d}=D_{\text {hipo }}$.

\subsubsection{Regiones corticales activas: Chiou y Youngs (2008)}

Las variables predictivas del modelo de atenuación de Chiou y Youngs (2008) son las siguientes:

- Mw: Magnitud momento

- $R_{R U P}$ : Distancia más corta al plano de ruptura $(\mathrm{km})$

- $R_{j b}$ : Distancia Joyner y Boore $(\mathrm{km})$

- $R_{X}$ : Coordenada del sitio, medida perpendicularmente a la dirección de la falla desde la proyección en superficie del lado de la ruptura situado en sentido contrario al del buzamiento $(\mathrm{km})$

- $F_{H W}$ : Parámetro del labio de la falla colgante (hanging-wall flag), 1 para $R_{X} \geq$ 0 y 0 para $R_{X}<0$

- $d^{\circ}$ : Ángulo de buzamiento de la falla

- $\lambda$ : Ángulo de deslizamiento de la falla (rake angle)

- $Z_{T O R}$ : Profundidad del techo de la ruptura $(\mathrm{km})$

- $F_{R V}$ : Parámetro de falla inversa, 1 para $30^{\circ} \leq \lambda \leq 150^{\circ}$, 0 para el resto de valores de $\lambda$ 
- $F_{N M}$ : Parámetro de falla normal, 1 para $-120^{\circ} \leq \lambda \leq-60^{\circ}, 0$ para el resto de valores de $\lambda$

- Parámetro de réplica: 1 si el evento es una réplica, 0 en otro caso

El modelo de atenuación proporciona los valores de PGA en $\mathrm{g}\left(\mathrm{g}=9,8 \mathrm{~m} / \mathrm{s}^{2}\right)$. Para valores de PGA en roca, se tiene la siguiente expresión:

$$
\begin{aligned}
\ln (P G A)= & 1,2687+\left[0,1 F_{R V}-0,2550 F_{N M}\right. \\
& \left.+0,0512\left(Z_{T O R}-4\right)\right]\left(1-A S_{i}\right)+\left[0,1+0,0512\left(Z_{T O R}-4\right)\right]\left(A S_{i}\right) \\
& +1,06(M w-6)+[(1,06-3,45) / 2,996] \ln \left(1+e^{2,996(4,1840-M w)}\right) \\
& -2,1 \ln \left[R_{R U P}+6,1600 \cosh (0,4893 \operatorname{máx}(M w-3,0))\right] \\
& +(-0,5+2,1) \ln \left(\sqrt{R_{R U P}{ }^{2}+50^{2}}\right) \\
& +[-0,00804-0,00785 / \cosh (\operatorname{máx}(M w-4,0))] R_{R U P} \\
& +0,7900 F_{H W} \tanh \left(\left(R_{X} \cos d^{2}\right) / 1,5005\right) \\
& {\left[1-\left(\sqrt{{R_{j b}}^{2}+Z_{T O R}^{2}}\right) /\left(R_{R U P}+0,001\right)\right] }
\end{aligned}
$$

De acuerdo con Chiou y Youngs (2008), la relación de atenuación de la ecuación 6.36 se aplica en emplazamientos de roca, con Vs30=1130 m/s. Por este motivo, se considera que la condición del terreno de referencia para el modelo de Chiou y Youngs (2008) es la clase de emplazamiento 2 (Rocas de resistencia media) de la Tabla 3.4, pues esta clase se caracteriza por presentar $760<V s 30 \leq 1500$. Por tanto, el valor del factor de amplificación para altas frecuencias que le corresponde a este modelo es $F_{a}=0,97$.

A los efectos de la programación de la relación de atenuación expresada por la ecuación 6.36 en el plugin (capítulo 9), se realizan las siguientes aproximaciones, para considerar una fuente puntual:

- $R_{R U P}:$ Distancia hipocentral, $D_{\text {hipo }}(\mathrm{km})$

- $R_{j b}$ : Distancia epicentral, $D_{e p i}(\mathrm{~km})$

- $R_{X}: D_{e p i}(\mathrm{~km})$

- $F_{H W}: 1$, pues $R_{X} \approx D_{e p i}$ siempre es mayor que 0

- $Z_{T O R}$ : Profundidad hipocentral $(\mathrm{km})$

\subsubsection{Zonas de subducción: Zhao et al. (2006)}

El modelo de atenuación de Zhao et al. (2006) permite obtener la PGA a partir de las variables predictivas siguientes: magnitud momento Mw, profundidad h $(\mathrm{km})$, distancia $\mathrm{x}$ a la fuente $(\mathrm{km})$, mecanismo focal y tipo de fuente sísmica. La expresión del modelo para la PGA $\left(\mathrm{cm} / \mathrm{s}^{2}\right)$ en roca es la siguiente: 


$$
\begin{aligned}
\ln (P G A)= & 1,101 M w-0,00564 x-\ln (r)+0,01412\left(h-h_{c}\right) \delta_{h} \\
& +F_{R}+S_{I}+S_{S}+S_{S L} \ln (x)
\end{aligned}
$$

donde $r=x+0,0055 e^{1,080 M w}, \delta_{h}=1$ si $h \geq h_{c}$ y 0 en otro caso, y $h_{c}=15 \mathrm{~km}$. La variable h está limitada a $125 \mathrm{~km}$ (Zhao et al., 2006).

En función del tipo de fuente, el modelo distingue tres tipos de eventos, y los siguientes parámetros toman valores en la ecuación 6.37:

- Cortical (Crustal)

- En interfaz de subducción (Interface): $S_{I}=0,000$

- En corteza subducida $(S l a b): S_{S}=2,607, S_{S L}=-0,528$

En función del mecanismo focal, el modelo distingue cuatro posibles mecanismos, y los siguientes parámetros toman valores en la ecuación 6.37:

- Falla inversa: $F_{R}=0,251$, pero sólo si el evento es cortical

- Falla de desgarre

- Falla normal

- Mecanismo desconocido

Dado que Zhao et al. (2006) caracterizan los emplazamientos con Vs30 >1100 $\mathrm{m} / \mathrm{s}$ como roca dura, se considera que la condición del terreno de referencia para el modelo de atenuación es la clase de emplazamiento 2 (Rocas de resistencia media) de la Tabla 3.4, descrita por el intervalo $760<V s 30 \leq 1500$. Por tanto, el valor del factor de amplificación para altas frecuencias que le corresponde a este modelo es $F_{a}=0,97$.

A los efectos de la programación de la relación de atenuación expresada por la ecuación 6.37 en el plugin (capítulo 9), se considera una aproximación a fuente puntual. Por tanto, se considera que la distancia a la fuente es equiparable a la distancia hipocentral, y se introduce la variable $x=D_{\text {hipo }}$.

\subsection{Cálculo de intensidades sintéticas a partir de parámetros del movimiento fuerte}

Finalmente, se ha escogido una correlación entre la aceleración y la intensidad sísmicas, que se utilizará en el capítulo 9 con objeto de obtener las intensidades sintéticas a partir de los valores de aceleración máxima sintética calculados con las leyes de atenuación óptimas en la Península Ibérica y las Islas Baleares, las Islas Canarias y un contexto mundial. A estos efectos, se han estudiado las correlaciones entre la PGA, la PGV y la Intensidad Modificada de Mercalli $\left(I_{M M}\right)$ de Wald et al. 
(1999a) y de Atkinson y Kaka (2007). También pueden citarse las correlaciones de la PGA y la PGV con respecto a la intensidad de Caprio et al. (2015).

Se han elegido las correlaciones entre PGA y la Intensidad Modificada de Mercalli de Wald et al. (1999a), obtenidas para distintos rangos de intensidad, considerando la equivalencia entre la escala modificada de Mercalli y la Escala Macrosísmica Europea (EMS-98). También es preciso destacar su utilización en el cálculo de intensidades sintéticas realizado por Wald et al. (1999b) a partir de los parámetros máximos del movimiento fuerte obtenidos en el sur de California, y la aplicación ya efectuada en la calibración de las relaciones de atenuación potencialmente aplicables en la Península Ibérica.

Como se ha explicado en la subsección 6.1.2, las correlaciones de Wald et al. (1999a) consideran dos rangos de intensidades para el cálculo de la $I_{M M}$ a partir de la PGA, en $\mathrm{cm} / \mathrm{s}^{2}$ :

- $I \leq I_{M M} \leq V$ : Ecuación 6.12, $I_{M M}=2,20 \log (P G A)+1,00$

- $V \leq I_{M M} \leq V I I I$ : Ecuación $6.11, I_{M M}=3,66 \log (P G A)-1,66$

Ante un valor de PGA calculado con cualquier relación de atenuación, es preciso convertirlo en primer lugar utilizando la ecuación 6.12. Siguiendo a Wald et al. (1999a), el resultado se redondea al número entero inmediatamente superior en caso de que el primer decimal sea 5 o superior, y al número entero inmediatamente inferior en caso contrario. Si este valor redondeado de $I_{M M}$ es menor que $\mathrm{V}$, se considera válido pues cumple con el rango de intensidades de la ecuación 6.12. Si es mayor o igual que $\mathrm{V}$, pero aún se encuentra en el intervalo $V \leq I_{M M} \leq V I I I$, será necesario convertir de nuevo la PGA en $I_{M M}$, por medio de la ecuación 6.11, y redondear de nuevo el resultado. 


\section{Capítulo 7}

\section{ESTIMACIÓN DE LA ATENUACIÓN ANELÁSTICA DE LA LITOSFERA EN CANARIAS}

El conocimiento del factor de calidad Q en Canarias reviste gran importancia al permitir la medida de la atenuación anelástica de la litosfera regional, y además reflejar la evolución de la tectónica en esta región. En este capítulo, se pretende llevar a cabo una estimación nueva y precisa de la atenuación anelástica de la litosfera en el archipiélago canario. Por medio de la aplicación de la metodología desarrollada por Havskov et al. (1989), se ha calculado el factor de calidad Q a partir del análisis, por primera vez, de registros digitales procedentes de estaciones de banda ancha, desplegadas por el IGN en el archipiélago durante los últimos 20 años. Se han obtenido los mapas de la regionalización espacial de los parámetros que expresan la dependencia frecuencial de $\mathrm{Q}, Q_{0}$ y $\nu$. Estos resultados son comparados con los procedentes de estudios previos de la atenuación en Canarias, que utilizan registros analógicos y un número más reducido de terremotos y estaciones que el empleado en esta tesis (Canas et al., 1995). De esta forma, con la nueva estimación de la atenuación que se plantea se busca superar las limitaciones de estos trabajos previos, al menos en cuanto a la dependencia de la precisión de los resultados con respecto al número de datos utilizados.

Ante el proceso eruptivo ocurrido en El Hierro entre octubre de 2011 y marzo de 2012, se ha estudiado la evolución temporal de los parámetros $Q_{0}$ y $\nu$ en la isla a lo largo de la crisis sísmica precedente, entre julio y octubre de 2011, y durante la erupción, considerando su posible carácter predictivo. Dada la gran cantidad de datos geofísicos, geoquímicos y geodésicos obtenidos por el IGN durante este periodo de tiempo en El Hierro (López et al., 2012), se han correlacionado las variaciones temporales de los parámetros de la dependencia frecuencial de Q con cambios significativos de estas variables, particularmente la sismicidad y las deformaciones. 


\subsection{Determinación de $\mathrm{Q}$ y de su dependencia fre- cuencial en las Islas Canarias}

La metodología de Havskov et al. (1989) permite obtener el factor de calidad Q por medio del análisis de las ondas de coda del sismograma. Como se ha explicado en la sección 2.6.2, se aplica un filtro paso-banda de frecuencia central f conocida sobre la señal de la onda de coda, y se modeliza la amplitud $\mathrm{A}(\mathrm{f}, \mathrm{t})$ de la señal filtrada por medio del ajuste de una envolvente decreciente con el tiempo t, medido desde el tiempo origen del terremoto. Puesto que esta envolvente cumple la ecuación 2.12, en cuanto a su dependencia con t, para obtener $\mathrm{Q}$ es preciso realizar una regresión lineal de la función $\ln (A(f, t))+\beta \ln (t)$ respecto de $\mathrm{t}$, considerando $\mathrm{f}$ constante (ecuación 2.12).

Los registros sísmicos que se van a utilizar para estimar el factor Q en Canarias proceden de la red sísmica desplegada por el IGN en el archipiélago, destinada a monitorizar permanentemente la sismicidad en esta región. Actualmente, la red está constituida por 32 estaciones de registro de velocidad, de banda ancha y de corto periodo. La Figura 7.1 muestra la distribución de las 24 estaciones sismográficas cuyos registros se han utilizado en la tesis doctoral para obtener el factor Q. Se incluyen 4 estaciones que se encuentran fuera de servicio a fecha de octubre de 2015, de acuerdo con Instituto Geográfico Nacional (2015a). En una ampliación específica para El Hierro (esquina inferior derecha), la Figura 7.1 muestra también la distribución de las estaciones localizadas en la isla. Asimismo, se diferencian los instrumentos de banda ancha (triángulos) de los de corto periodo (cuadrados).

La metodología de Havskov et al. (1989) se ha aplicado para procesar los registros de onda de coda de las estaciones sísmicas de la Figura 7.1, por medio de su implementación en el programa CODAQ, incluido en el sistema de análisis sísmico SEISAN (Havskov y Ottemöller, 1999). Por medio de este programa, en el sismograma correspondiente a cada trayectoria evento-estación se aplica un filtro paso-banda sobre una ventana que contiene a la onda de coda (ventana de coda, coda window). De esta forma, se ajusta una envolvente a la señal filtrada y se calcula el factor de calidad Q que corresponde a la banda frecuencial de filtro, o a su frecuencia central. Para cada una de las frecuencias, los valores individuales de Q que se han obtenido para distintos sismogramas se utilizan para calcular el valor medio de $\mathrm{Q}$ que corresponde a la frecuencia. Dados los valores medios de Q para las distintas frecuencias, el programa CODAQ ajusta a continuación una curva de Q en función de la frecuencia $\mathrm{f}$ a este conjunto de valores medios, la cual se expresa de acuerdo con la ecuación 2.9 y considerando $f_{0}=1 \mathrm{~Hz}$ :

$$
Q(f)=Q_{0} f^{\nu}
$$

Como primer paso, se han seleccionado los terremotos localizados en las Islas Canarias, cuyos registros de onda de coda se van a analizar. Con este fin, se ha realizado una explotación del catálogo sísmico del IGN (Instituto Geográfico Nacional, $2015 \mathrm{~b}$ ), en la que se han limitado las latitudes de los epicentros entre $26^{\circ} \mathrm{N} \mathrm{y} 31^{\circ} \mathrm{N}$, y las longitudes entre $12^{\circ} \mathrm{W}$ y $20^{\circ} \mathrm{W}$. Con respecto a las fechas de los eventos, la búsqueda de los mismos se ha realizado entre 2003 y 2013. A nivel de magnitud, sólo se han seleccionado los terremotos con magnitud $M_{L}$ mayor o igual que 3,0 , a 


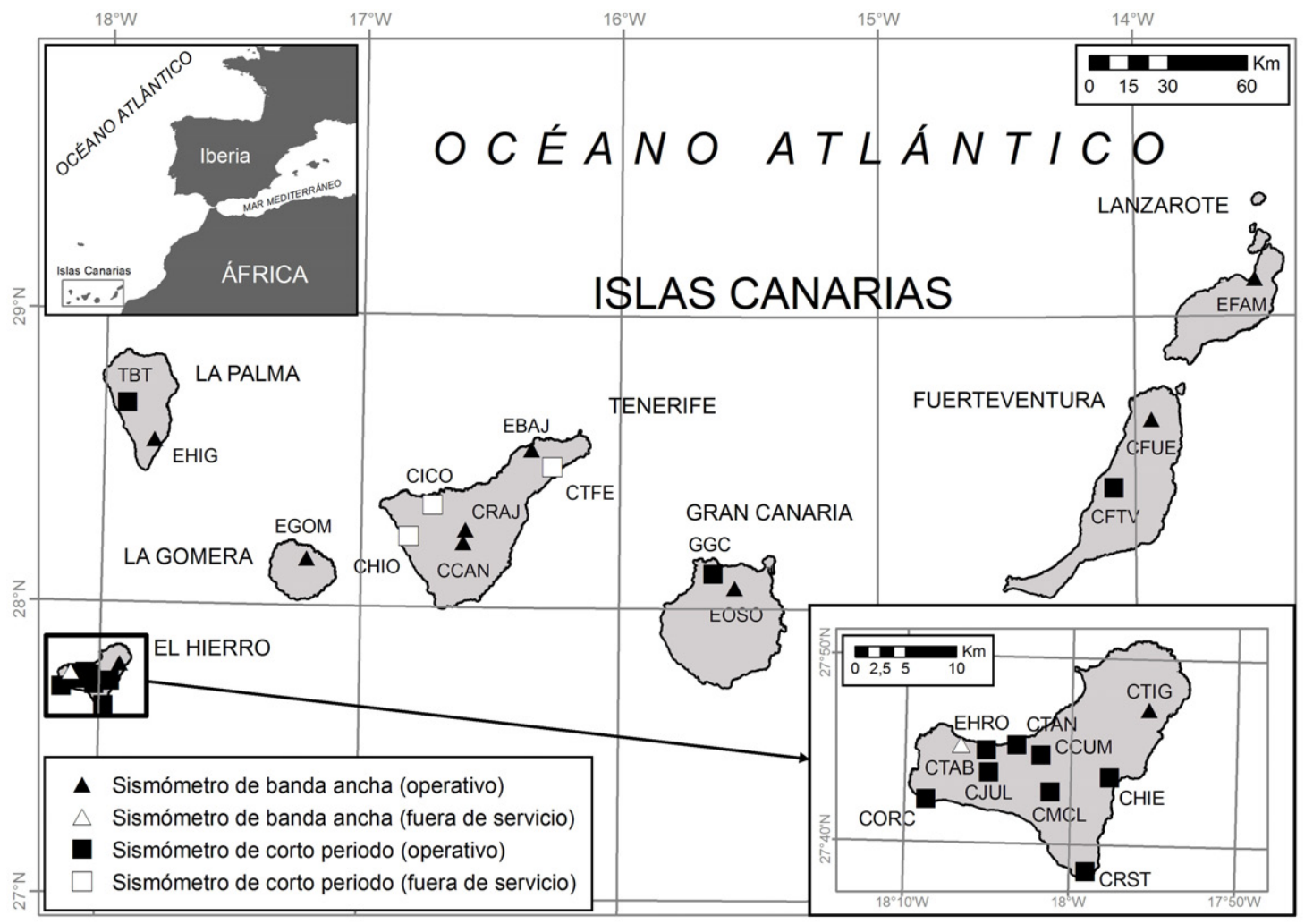

Figura 7.1: Distribución espacial de las estaciones de registro de velocidad del IGN en las Islas Canarias y que han sido utilizadas en esta tesis. Se muestra también una ampliación específica para la isla de El Hierro (esquina inferior derecha). La información sobre el estado operativo de cada estación está actualizada a fecha de octubre de 2015. 


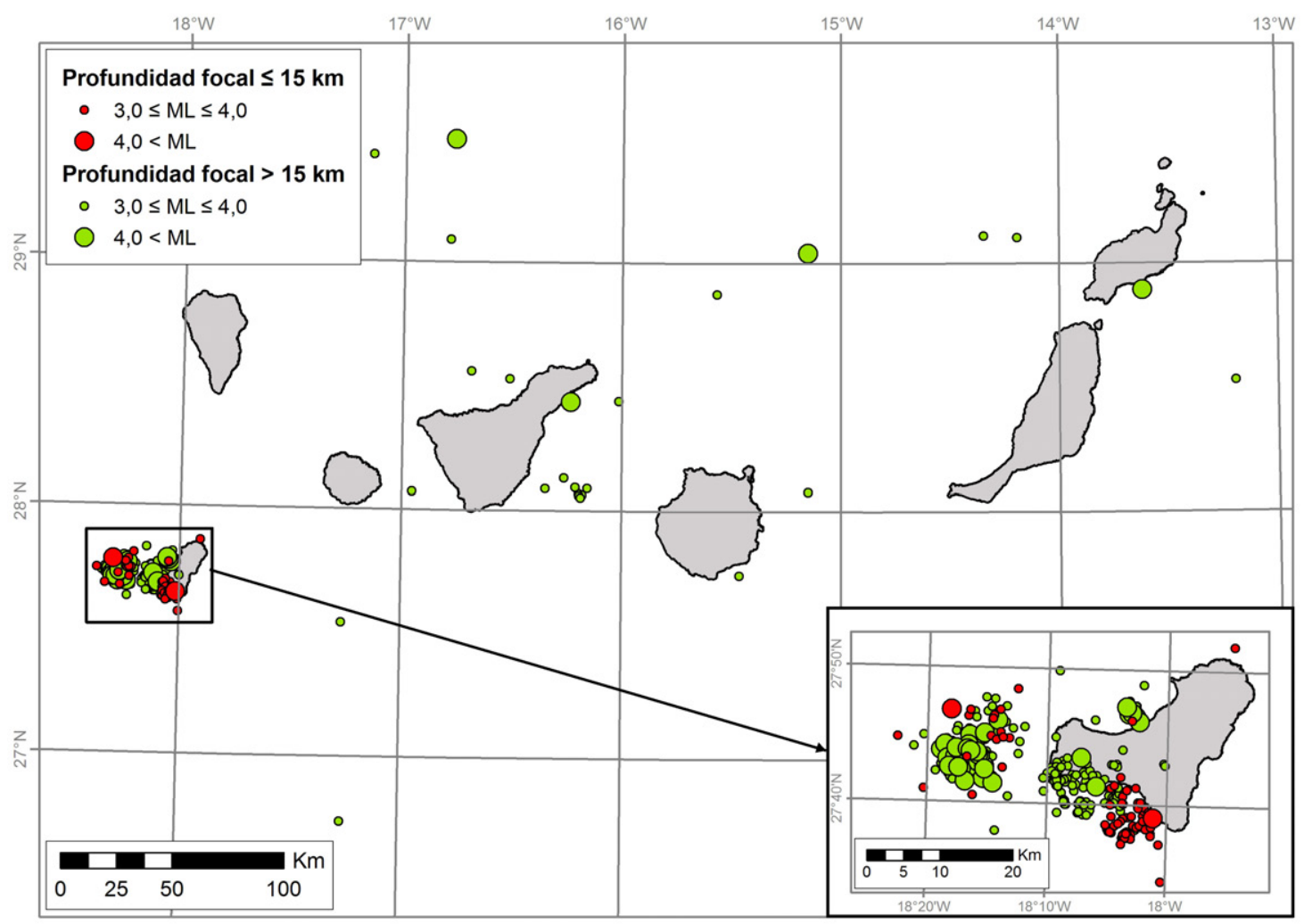

Figura 7.2: Terremotos de magnitud $M_{L} \geq 3$ para los que se ha obtenido al menos una observación de Q, y que han sido registrados por el IGN entre mayo de 2003 y agosto de 2013. En la esquina inferior derecha se muestra una ampliación específica para la isla de El Hierro.

partir de todos los eventos localizados en el área de explotación y en el intervalo temporal especificado. Como resultado de la explotación, se han considerado 799 terremotos con una profundidad hipocentral máxima de $79 \mathrm{~km}$, ocurridos entre mayo de 2003 y agosto de 2013. De este conjunto de eventos, la metodología de Havskov et al. (1989) ha permitido obtener valores de Q sólo en 371 sismos. En la Figura 7.2 se representan los epicentros y magnitudes de este subconjunto de 371 terremotos, mostrándose en una ampliación específica para El Hierro (esquina inferior derecha) su distribución en la isla.

Para cada trayectoria evento-estación, se ha seleccionado el sismograma que presenta el registro de onda de coda con la mayor calidad. Dado que el correcto procesado de cada onda de coda exige su visualización en toda su extensión, la selección de la forma de onda adecuada se ha realizado tras llevar a cabo una inspección visual rigurosa de todas las trazas correspondientes al terremoto. Se ha procurado especialmente que el registro de coda no fuese interrumpido por las primeras fases sísmicas del evento siguiente. Siguiendo a Havskov y Ottemöller (2010), las trazas se han examinado visualmente tras filtrarlas por medio de un filtro paso-banda de 5-10 $\mathrm{Hz}$, de forma que las lecturas de la coda se realizaran utilizando un filtro consistente. Las trazas seleccionadas han sido filtradas por medio de un filtro Butterworth de 6 polos, centrado en 5 frecuencias centrales Havskov et al. (1989), con un ancho de banda variable. Tomando como referencia el trabajo de Canas et al. (1998), se han seleccionado las bandas frecuenciales $1,5 \pm 0,5,3,0 \pm 1,0,5,0 \pm 1,0$ y 7,0 $\pm 1,0 \mathrm{~Hz}$. Siguiendo a Jain et al. (2015), se ha seleccionado también la banda 9,0 $\pm 3,0 \mathrm{~Hz}$. 


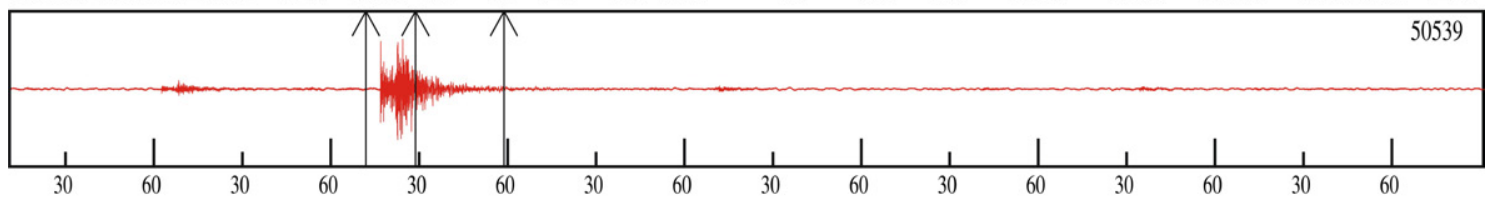

$\mathrm{F}=1.5 \quad \mathrm{Q}=93 \quad \mathrm{CO}=-.77 \quad \mathrm{~S} / \mathrm{N}=22$

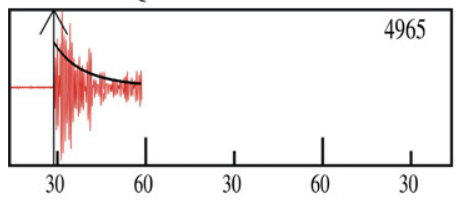

$\mathrm{F}=7.0 \quad \mathrm{Q}=280 \quad \mathrm{CO}=-.90 \quad \mathrm{~S} / \mathrm{N}=3$

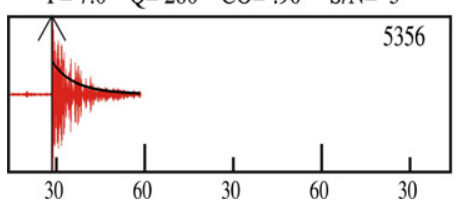

$\mathrm{F}=3.0 \quad \mathrm{Q}=172 \quad \mathrm{CO}=-83 \quad \mathrm{~S} / \mathrm{N}=10$

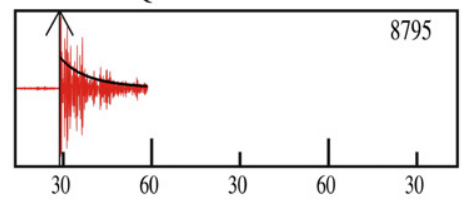

$\mathrm{F}=9.0 \quad \mathrm{Q}=360 \quad \mathrm{CO}=-.92 \quad \mathrm{~S} / \mathrm{N}=3$

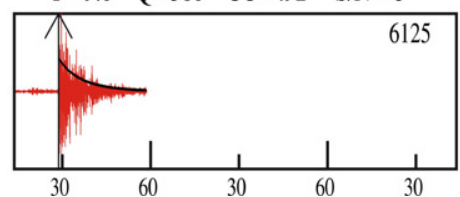

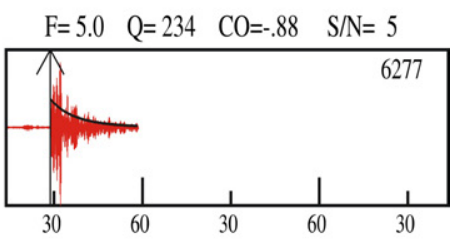

Figura 7.3: Traza original del registro de la componente vertical de la estación CTIG (arriba) y ventanas de coda filtradas con filtro paso-banda (abajo), para el terremoto de $M_{L}$ 3,3 ocurrido el 26-03-2013 a las 05:52:11 UTC en la isla de El Hierro, a $23 \mathrm{~km}$ de distancia, tal y como son presentadas por CODAQ. En la traza original (arriba), las flechas verticales indican (de izquierda a derecha), el tiempo origen del terremoto, el inicio de la ventana de coda y el final de la misma. Encima de esta traza, $\mathrm{H}$ es la profundidad del hipocentro (km), M es la magnitud $M_{L}$, TP es el tiempo de viaje de la onda P (s), TC es el tiempo inicial de la ventana de coda medido desde el origen (s), WIN es la longitud de la ventana de coda (s) y ST el tiempo inicial de la ventana de coda en términos del tiempo de viaje de la onda S. En cada ventana de coda filtrada se dan los valores Q: observación de Q de coda, CO: coeficiente de correlación, S/N: relación señal/ruido. El ajuste de la envolvente a la señal filtrada se representa como una curva decreciente con el tiempo. UTC es Tiempo Universal Coordinado.

Con el fin de ilustrar los parámetros del procesado de los registros de onda de coda, en la Figura 7.3 (arriba), se presenta la traza original del registro de la componente vertical de la estación CTIG, correspondiente al terremoto de magnitud $M_{L} 3,3$ y $20 \mathrm{~km}$ de profundidad hipocentral, ocurrido el 26-03-2013 a las 05:52:11 UTC en El Hierro, a una distancia epicentral de $23 \mathrm{~km}$. En la Figura 7.3 (abajo) también se muestran las ventanas de coda, que han sido filtradas de acuerdo con las bandas frecuenciales seleccionadas. De acuerdo con Havskov y Ottemöller (2010), se ha establecido $2 t_{S}$ como tiempo inicial de la ventana de coda, siendo $t_{S}$ el tiempo de viaje de la onda $\mathrm{S}$, medido a partir del tiempo origen del terremoto. El tiempo de viaje de la onda $\mathrm{S}$ se ha calculado por medio del tiempo de la onda $\mathrm{P}$ y de la relación entre las velocidades de propagación de la onda $\mathrm{P}(V p)$ y la onda $\mathrm{S}(V s)$, $V p / V s=1,75$, publicada por Instituto Geográfico Nacional (1998) para la corteza regional en las Islas Canarias. En la Figura 7.3, la segunda flecha (empezando por la izquierda) situada sobre la traza original marca el tiempo inicial de la ventana de coda, el cual también es indicado por el valor TC que figura encima de esta traza. Para cada ventana de coda, se ha considerado una longitud estándar de $30 \mathrm{~s}$. En la Figura 7.3, la longitud de la ventana de coda está limitada por la segunda y tercera flecha (empezando por la izquierda) situadas sobre la traza original. Esta longitud también es indicada por el valor WIN que figura encima de esta traza.

Para calcular la relación señal-ruido de cada una de las señales filtradas de la coda se han considerado para la ventana de ruido los primeros $5 \mathrm{~s}$ de la traza y los últimos $5 \mathrm{~s}$ de la ventana de la coda. Se ha considerado como aceptable una relación 
señal ruido de 5 para que el valor de Q obtenido por el ajuste a la señal filtrada sea considerado en el promedio de Q para la correspondiente banda de frecuencia (Havskov y Ottemöller, 2010). En la Figura 7.3 se presenta la relación señal/ruido de las ventanas de coda filtradas con filtro paso-banda como el valor $\mathrm{S} / \mathrm{N}$ en la parte superior de las trazas filtradas. En cuanto al ajuste de Q a las señales filtradas de la coda, se requiere un coeficiente de correlación mínimo de 0,5 para que el valor correspondiente entre en el cálculo del valor promedio de Q. También se presentan en la Figura 7.3 los coeficientes de correlación (CO) correspondientes a los valores de $Q$ obtenidos en cada ventana de coda considerada.

El procedimiento seguido en esta tesis para procesar las ondas de coda ha permitido considerar 3994 trayectorias evento-estación con un valor aceptable de Q. El error cuadrático medio (RMS) de estas observaciones oscila entre 0,09 y 0,73 , lo que indica un ajuste razonable. De acuerdo con Havskov et al. (1989), se han agrupado las trayectorias en función de la profundidad del sismo. De esta forma se han considerado 1331 trayectorias correspondientes a sismos de profundidad igual o inferior a $15 \mathrm{~km}$ y 2663 trayectorias correspondientes a eventos de profundidad superior a 15 $\mathrm{km}$. En cada grupo, sin embargo, se han desechado los valores no razonables de Q. Así, se ha obtenido la regionalización tanto de $Q_{0}$ como de $\nu$ para las Islas Canarias, que es presentada en mapas de isolíneas que muestran la distribución espacial de estos parámetros. Estos mapas se han obtenido para cada grupo de trayectorias según la profundidad, y la Q obtenida para cada trayectoria se ha asociado al punto medio de la misma. Usando el método Kriging, se han interpolado en una malla los valores de Q correspondientes a cada frecuencia, para ambos grupos de profundidades, mayores que $15 \mathrm{~km}$ o menores o iguales que $15 \mathrm{~km}$. Para el grupo de profundidades mayores que $15 \mathrm{~km}$ el paso de malla es de $7 \mathrm{~km}$, mientras que el paso es de $1 \mathrm{~km}$ para el grupo de eventos superficiales, con profundidades menores o iguales que 15 $\mathrm{km}$.

Los valores de $\mathrm{Q}$ obtenidos para cada grupo de profundidades se han procesado siguiendo el método de Havskov et al. (1989). A cada punto de la malla se le han asociado los cinco valores correspondientes a las cinco frecuencias centrales de cada filtro, y con esos valores se ha calculado un valor de $Q_{0}$ y de $\nu$, así como los correspondientes valores de su desviación típica. Estos errores se tuvieron posteriormente en cuenta para considerar o no los valores correspondientes a cada punto de la malla, conservando sólo los valores de $Q_{0}$ y de $\nu$ con errores aceptables. Finalmente, se ha procedido con los valores finales a una interpolación y a un curveado de estos resultados. En las Figuras 7.4 y 7.5 se muestran los mapas de $Q_{0}$ y de $\nu$ para el grupo de profundidades mayores que $15 \mathrm{~km}$. En estos mapas se han ocultado las zonas en las que no se dispone de observaciones de Q. En las Figuras 7.6 y 7.7 se presentan los resultados para el grupo de eventos superficiales, con profundidades menores o iguales que $15 \mathrm{~km}$, realizándose igualmente la ocultación de aquellas zonas del mapa que no disponen de valores de $\mathrm{Q}$.

Puede observarse en la Figura 7.4 que la tendencia de $Q_{0}$ para profundidades $>15 \mathrm{~km}$ se corresponde con cuatro máximos marcados con isolíneas de altos valores de $Q_{0}$ (240). Dos de estos máximos se encuentran entre El Hierro y La Gomera, estando el tercero sobre Tenerife con un valor de $Q_{0}=220$. Más hacia el este, se aprecia el mínimo de $Q_{0}$ (60) entre Gran Canaria y Fuerteventura, con un ligero ascenso de $Q_{0}$ hacia Lanzarote. Si se observa en cambio el valor de $\nu$ (Figura 7.5), 


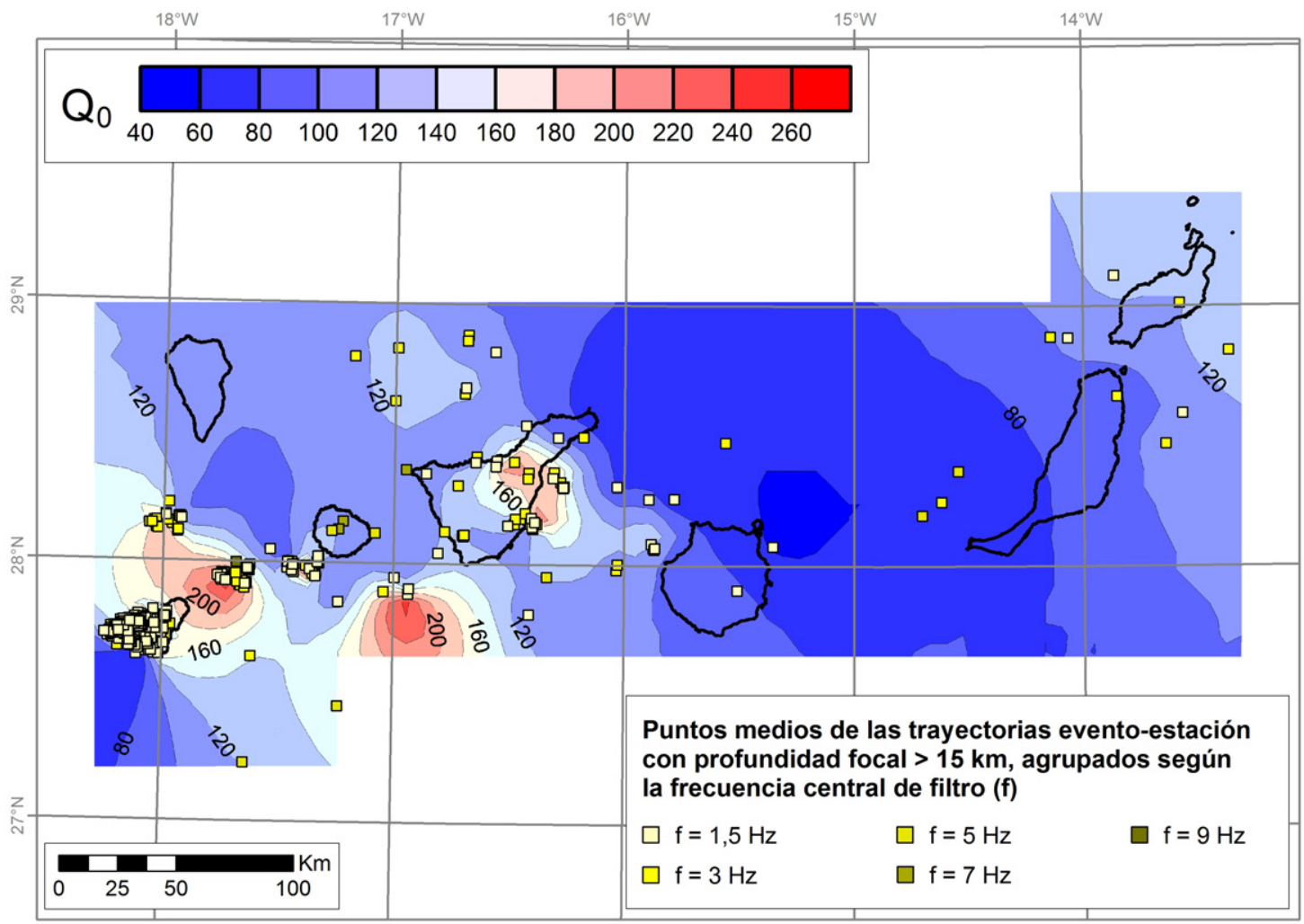

Figura 7.4: Mapa de isolíneas de $Q_{0}$ para las trayectorias evento-estación con profundidad focal $>15 \mathrm{~km}$. Los puntos medios de las trayectorias se encuentran también representados.

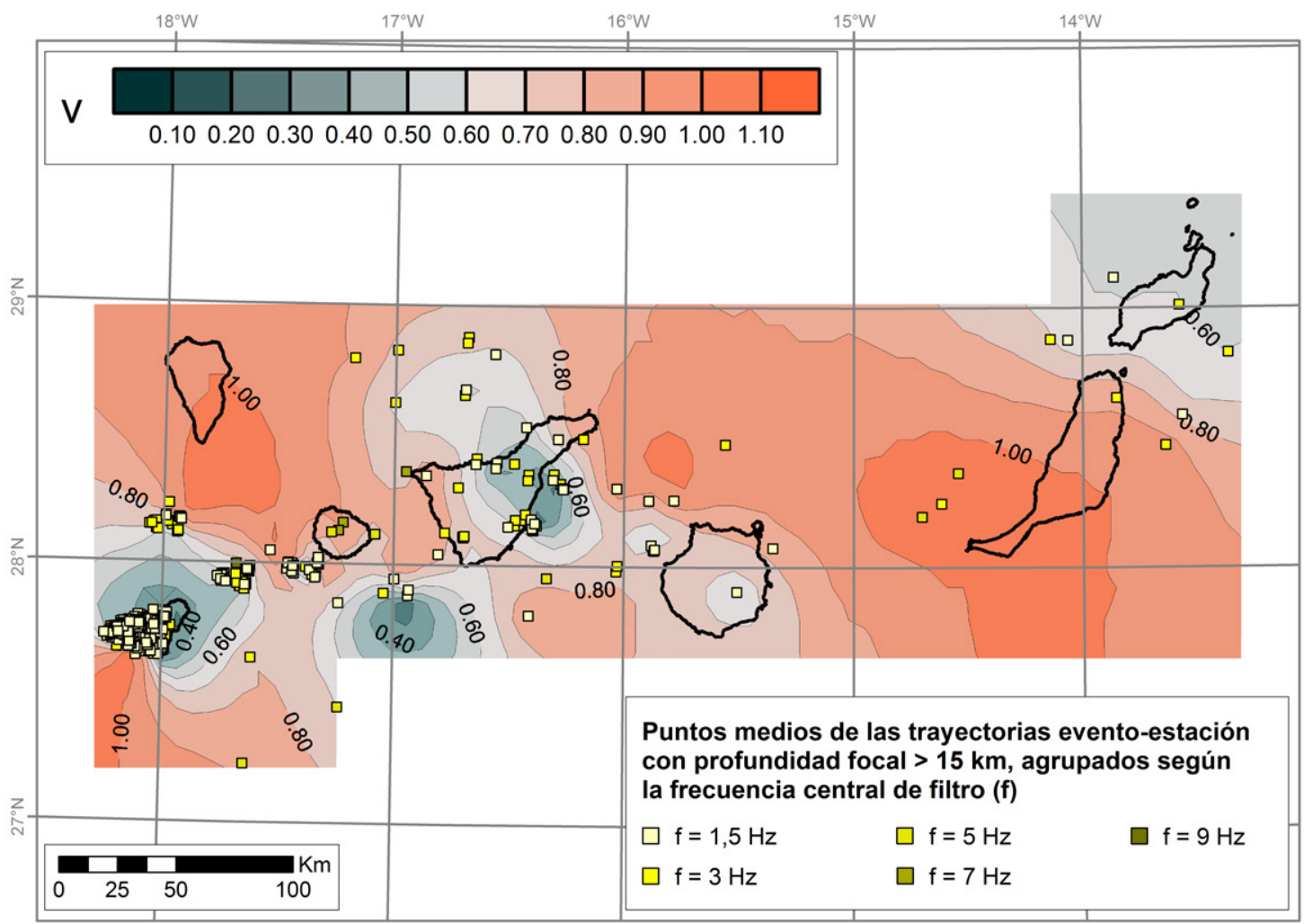

Figura 7.5: Mapa de isolíneas de $\nu$ para las trayectorias evento-estación con profundidad focal $>15 \mathrm{~km}$. Los puntos medios de las trayectorias se encuentran también representados. 


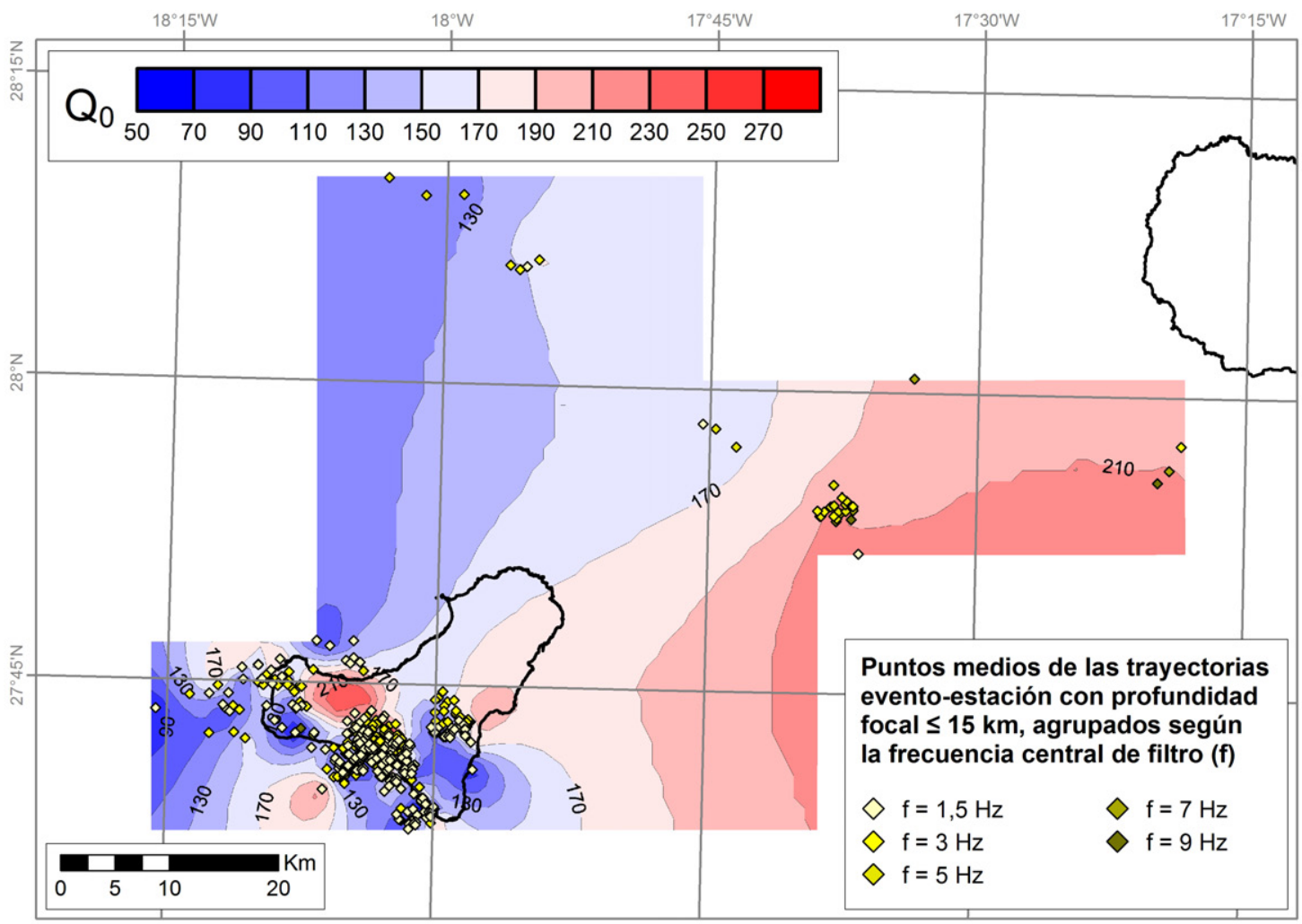

Figura 7.6: Mapa de isolíneas de $Q_{0}$ para las trayectorias evento-estación con profundidad focal $\leq 15 \mathrm{~km}$. Los puntos medios de las trayectorias se encuentran también representados.

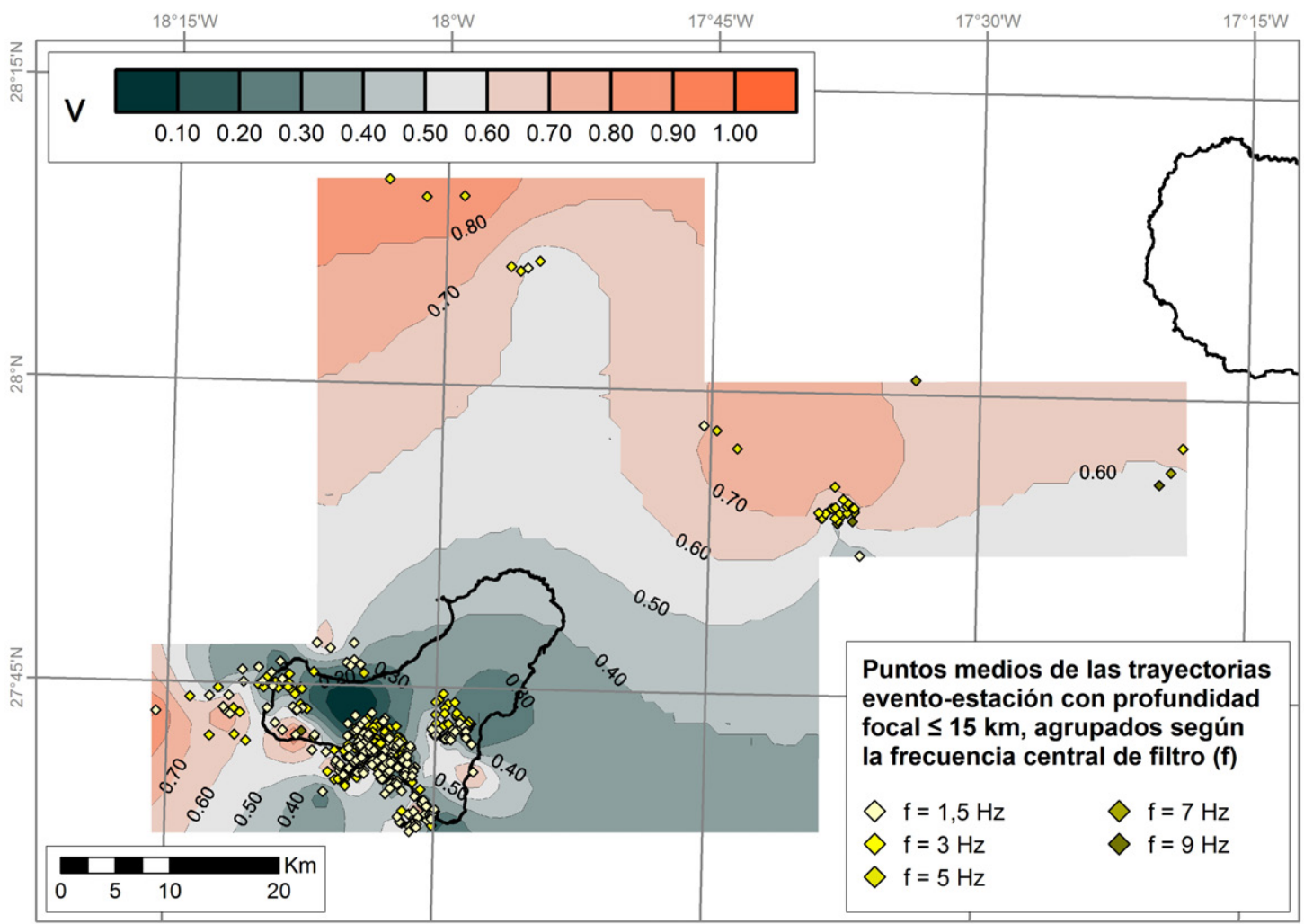

Figura 7.7: Mapa de isolíneas de $\nu$ para las trayectorias evento-estación con profundidad focal $\leq 15 \mathrm{~km}$. Los puntos medios de las trayectorias se encuentran también representados. 
este parámetro muestra una oscilación entre 0,30 y 1,00 en todo el archipiélago, observándose un comportamiento contrario al de $Q_{0}$. Esto es, se observan valores mínimos de $\nu$ próximos a los máximos de $Q_{0}$ (Figura 7.4). Como ejemplo, se indica el valor de $\nu=0,30$ próximo a Tenerife y a El Hierro. En el otro extremo, se observa cómo el máximo de $\nu=1,00$ está al suroeste de Fuerteventura, próximo al valor de la isolínea de $Q_{0}=60$. En cuanto al grupo de eventos con profundidades $\leq 15 \mathrm{~km}$ (Figura 7.6), el parámetro $Q_{0}$ oscila entre 90 y 120 en la dirección hacia el Este. Sin embargo, este parámetro muestra cambios notables sobre El Hierro, oscilando entre valores máximos y mínimos en unos pocos kilómetros. Por ejemplo, se observa un máximo de $Q_{0}=210$ en la parte noroeste de la isla, limitando con bajos valores de $Q_{0}=70$ en la parte sur. En cuanto al valor de $\nu$, éste aumenta de forma general hacia el norte (Figura 7.7) desde 0,30 a 0,80. Sin embargo, sobre El Hierro este parámetro experimenta cambios contrarios a los de $Q_{0}$. Es decir, se encuentran bajos valores de las isolíneas de $\nu$ en puntos próximos a máximos de $Q_{0}$ y viceversa. Por ejemplo, el máximo de $Q_{0}=210$ en el noroeste (Figura 7.6) se puede correlacionar con el mínimo $\nu=0,10$ sobre la misma área. De forma similar, los valores cercanos de $Q_{0}$ de 90 y 70 (Figura 7.6) están situados a su vez próximos a las isolíneas de $\nu$ con valores más altos de 0,60 y 0,90 (Figura 7.7).

\subsection{Variación temporal de $Q_{0}$ y $\nu$ en la isla de El Hierro durante la crisis sísmica y el proceso eruptivo de 2011-2012}

Aunque se ha realizado un estudio de regionalización de la atenuación anelástica de las Islas Canarias mediante el cálculo y distribución espacial de $Q_{0}$ y $\nu$, se pretende completar este estudio con el comportamiento temporal de estos parámetros y su relación con la erupción de El Hierro ocurrida en el periodo de octubre de 2011-marzo de 2012 (Figura 7.8). Dado que las Islas Canarias constituyen una región volcánica activa, esta investigación es especialmente pertinente pues el desarrollo de un proceso eruptivo presenta una oportunidad única para estudiar su comportamiento y establecer relaciones con los posibles cambios de los parámetros de atenuación.

Se han calculado los valores $Q_{0}$ y $\nu$ en la estación CTIG situada en El Hierro (Figura 7.1) durante el periodo de crisis sísmica de julio-octubre de 2011 y la posterior erupción, hasta su culminación en marzo de 2012. Para comenzar, se ha seleccionado una fuente específica y una ventana temporal en la que obtener los sismos necesarios para realizar el cálculo. La fuente seleccionada fue limitada entre las latitudes $27,55^{\circ} \mathrm{N}$ y $27,85^{\circ} \mathrm{N}$, y entre las longitudes $17,85^{\circ} \mathrm{W}$ y $18,20^{\circ} \mathrm{W}$, que abarcan la isla de El Hierro. En cuanto a la ventana temporal, se han seleccionado los sismos ocurridos entre el 1 de enero de 2011 y el 31 de mayo de 2012 para poder examinar todo el proceso de crisis sísmica y la erupción, así como los intervalos de tiempo anteriores y posteriores. Los sismos seleccionados son 180, y sus características y localizaciones se muestran en la Figura 7.8. La estadística de esta serie, representada por el número de sismos/día así como por su magnitud, se presenta en la Figura 7.9. Estos sismos ocurrieron entre el 22 marzo de 2011 y el 16 de abril de 2012, de los cuales sólo 165 con magnitud $M_{L} \geq 3$ fueron registrados en la estación CTIG. 


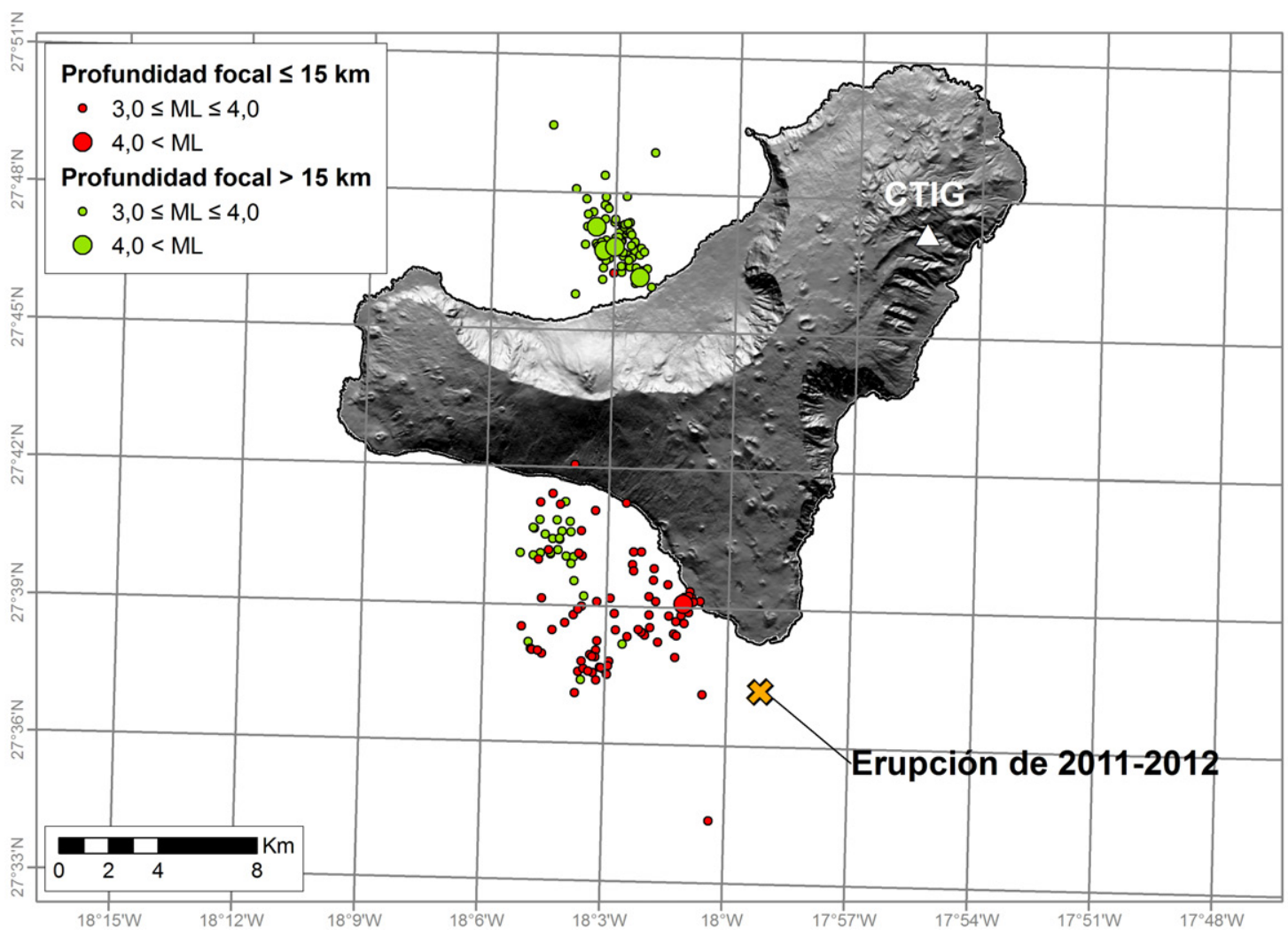

Figura 7.8: Sismicidad con $M_{L} \geq 3$ registrada por el IGN y localizada en una zona fuente constreñida entre $27,55^{\circ} \mathrm{N}$ y $27,85^{\circ} \mathrm{N}$, y entre $17,85^{\circ} \mathrm{W}$ y $18,20^{\circ} \mathrm{W}$. Los terremotos ocurrieron en una ventana temporal que se extiende desde el 1 de enero de 2011 al 31 de mayo de 2012. También se muestra la situación de la estación CTIG y la erupción de El Hierro de 2011-2012.

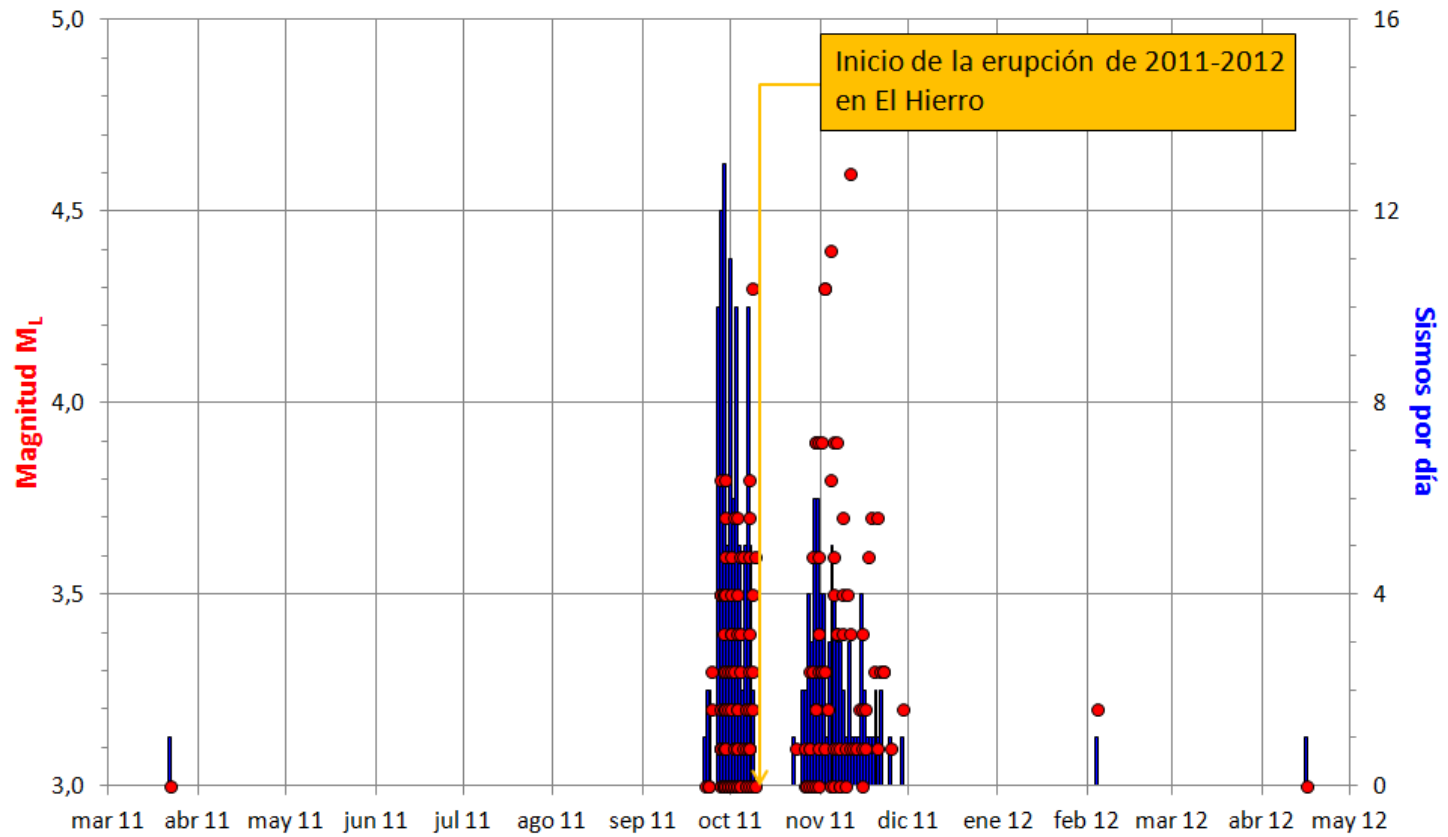

Figura 7.9: Evolución temporal de la sismicidad representada en la Figura 7.8, mostrando las magnitudes $M_{L}$ de los terremotos y el número de sismos por día. Se señala también el comienzo de la erupción de 2011-2012 en El Hierro. 
Se seleccionaron los registros diarios de CTIG de aquellos días en los que se produjo alguno de los 165 sismos y se procedió a realizar el cálculo del valor medio de Q y de sus desviaciones estándar para cada uno de los intervalos de frecuencia seleccionados con frecuencia central entre 1,5-9 Hz. Con el fin de reducir los errores en el cálculo del valor medio de $\mathrm{Q}$, se seleccionaron previamente todos los valores individuales de Q que cumpliesen el criterio de Chauvenet, y se rechazaron los valores que no lo cumpliesen. Posteriormente, se seleccionaron los valores promedio de $\mathrm{Q}$ que procediesen al menos dos valores de tal forma que los valores promedio y sus desviaciones estándar fuesen confiables. Finalmente, se realizaron los cálculos de $Q_{0}$ y $\nu$ mediante el ajuste de la ecuación 7.1 a los valores medios, siempre que hubiesen disponibles valores para al menos tres frecuencias. De esta forma se ha asegurado que los valores de $Q_{0}$ y $\nu$ son aceptables.

Siguiendo los criterios arriba mencionados, se realizó el cálculo de $Q_{0}$ y $\nu$ para un intervalo de 18 días entre el 24 de septiembre y el 5 de noviembre de 2011. En la Tabla 7.1 se presentan los resultados de $Q_{0}$ y $\nu$ y el número de sismos por día, el número de observaciones de Q por intervalo de frecuencia y el número total de observaciones. En la Figura 7.10 se representan de forma temporal los resultados, mostrando con círculos sin relleno los valores de $Q_{0}$ en la parte superior, y de $\nu$ en la inferior. Se observa a primera vista que, antes del comienzo de la erupción el 10 de octubre de 2011, el valor de $Q_{0}$ varía entre los valores de 75 y de 176 (Figura 7.10, parte superior) mientras que $\nu$ oscila entre 0,20 y 0,76 (Figura 7.10, parte inferior). Sin embargo, una vez comienza la erupción los valores de $Q_{0}$ claramente sufren un descenso hasta valores de 41-81. Por el contrario, $\nu$ comienza a aumentar desde esta fecha hasta alcanzar el valor de 0,99. En cuanto al número de eventos y a la magnitud $M_{L}$ alcanzada (Figura 7.9), puede observarse que el número de eventos con $M_{L} \geq 3$ por día disminuye cuando comienza la erupción, aunque el nivel de magnitud permanece estable antes y después de ésta. Así pues, los valores relativamente altos de $Q_{0}$ y bajos de $\nu$ encontrados antes de la erupción se asocian a esta sismicidad pre-eruptiva, mientras que el descenso en el número de eventos observados después puede ser el origen del descenso en los valores de $Q_{0}$ y del incremento de $\nu$.

Se propone en esta parte del capítulo establecer la correlación entre la variación temporal de los valores de $Q_{0}$ y $\nu$ observada en la estación de CTIG y los cambios en sismicidad y los diferentes parámetros geofísicos, geodésicos y geoquímicos registrados por la instrumentación instalada por el IGN en El Hierro entre julio de 2011 y el comienzo de la erupción López et al. (2012). Además, se intentará ver la relación existente entre los valores de $Q_{0}$ y $\nu$ durante el desarrollo de la erupción y la señal de tremor volcánico observada en las estaciones sísmicas del IGN desde el comienzo de la erupción el 10 de octubre de 2011 hasta finales de febrero de 2012 (Instituto Geográfico Nacional, 2016b).

A comienzos de julio de 2011 el IGN desplegó sobre la isla de El Hierro una red de observación que incluía estaciones sísmicas y GPS (López et al., 2012). Considerando la evolución de todos los parámetros observados entre julio y octubre de 2011, estos autores distinguen cinco fases pre-eruptivas ( I a V). Puesto que el intervalo temporal en que se ha calculado $Q_{0}$ y $\nu$ se extiende entre el 24 de septiembre y el 5 de noviembre de 2011, el estudio se centrará sólo en las fases III (4-26 de septiembre de 2011), IV(27 de septiembre-7 de octubre de 2011) y V(8-10 de octubre de 2011).

$\mathrm{Al}$ comienzo de la Fase III los valores de deformación obtenidos por GPS mues- 

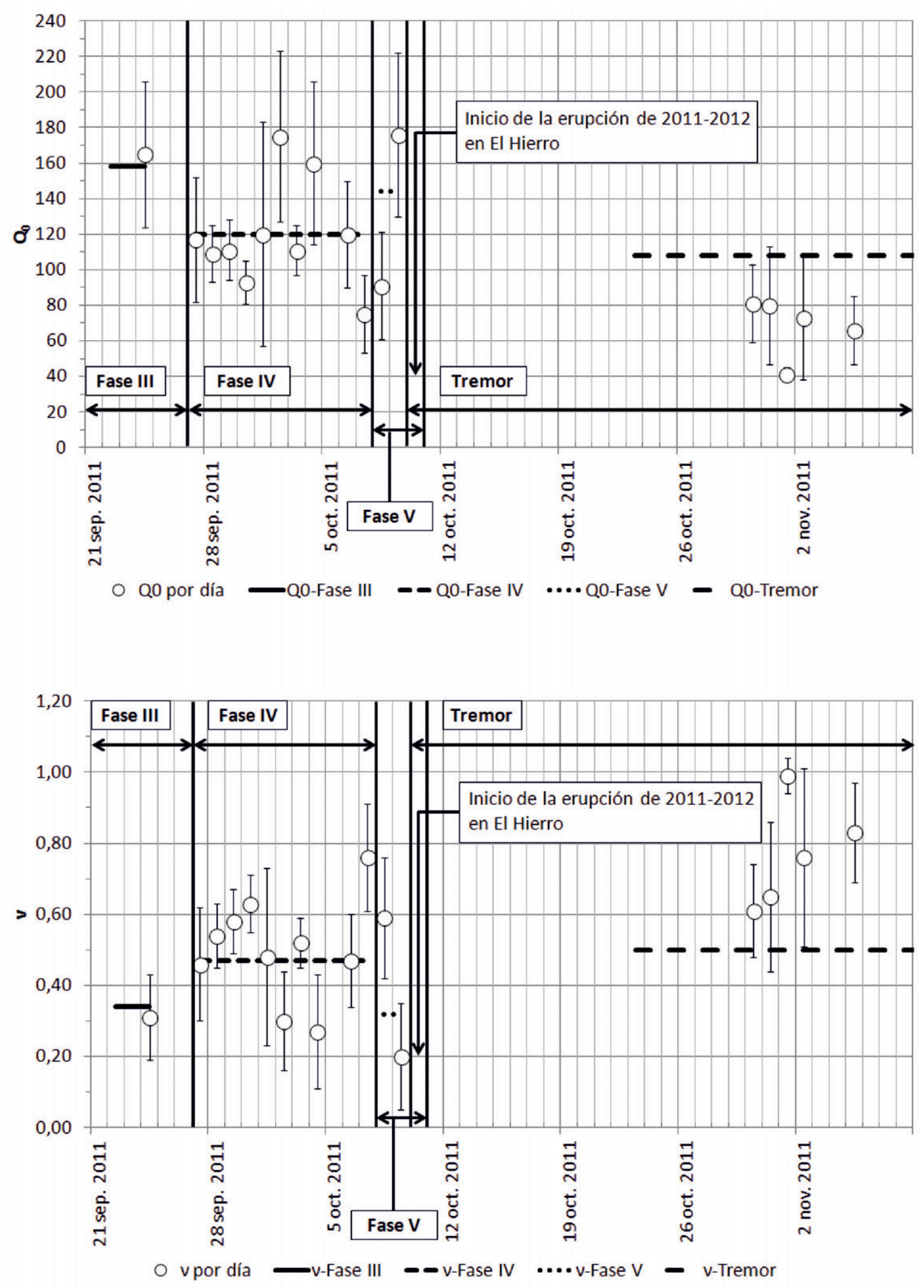

Figura 7.10: Evolución temporal de los valores de $Q_{0}$ (parte superior) y $\nu$ (parte inferior), obtenidos en la estación CTIG (El Hierro), para cada uno de los días de la Tabla 7.1 (círculos sin relleno). Las desviaciones estándar de cada parámetro son representadas por medio de barras de error. Se simbolizan también los valores de $Q_{0}$ y $\nu$ calculados en esta estación para cada una de las fases de López et al. (2012), así como los valores calculados durante el periodo del tremor registrado por el IGN (líneas continuas o discontinuas), ver la Tabla 7.2. Se indica también el comienzo de la erupción de 2011-2012 en El Hierro. 
tran un claro movimiento hacia el norte de cada estación de la red GPS, acompañada de una migración hacia el sur de la sismicidad que parece indicar un desplazamiento magmático en esa dirección. Durante la Fase IV tiene lugar un aumento drástico de la sismicidad que corresponde al 69,7\% del total de la energía liberada en el proceso. Durante el periodo del 1 al 6 de octubre se produce un proceso de deflación y de reinflación que aparece indicado en la deformación observada en todas las estaciones GPS. López et al. (2012) interpretan estos datos como resultado de una sobrepresión causada por una acumulación magmática en la parte sur de la isla. La Fase V comienza el 8 de octubre de 2011 con un sismo de 4,3 de magnitud $M_{L}$ y $12 \mathrm{~km}$ de profundidad, localizado a $1,5 \mathrm{~km}$ de la costa suroeste de la isla. Al día siguiente comienza un enjambre de terremotos en el océano, también cerca del sur de la isla, seguido de un tremor volcánico que comienza el día 10 de octubre, registrándose en todas las estaciones de El Hierro. Estos datos sugieren, de acuerdo con López et al. (2012), el comienzo de la erupción, que fue debido al sismo de magnitud $M_{L} 4,3$, que disparó el ascenso de magma a la superficie.

En la Figura 7.10 se presenta el desarrollo temporal de las Fases III, IV y V, así como el tiempo de registro del tremor volcánico, cubriendo el periodo total de cálculo diario de $Q_{0}$ y $\nu$ (24 de septiembre-5 de noviembre de 2011). Los límites de las fases están representadas por líneas verticales, pudiendo apreciarse que la Fase V finaliza el 10 de octubre de 2011 mientras que la señal del tremor comienza a ser registrada desde ese día en adelante. Por tanto, dos líneas consecutivas marcan el comienzo y el final del día 10 de octubre.

Los valores diarios de $Q_{0}$ y $\nu$ (Tabla 7.1) presentan importantes oscilaciones desde el 30 de septiembre hasta el 7 de octubre (Fase IV). $Q_{0}$ aumenta en dos días desde 93 (30 de septiembre) a 175 (2 de octubre), disminuyendo a 111 el día después. Posteriormente, este parámetro asciende a 160 el 4 de octubre, desplomándose a 75 el 7 de octubre. En cuanto al parámetro $\nu$ disminuye desde 0,63 (30 de septiembre) a 0,30 (2 de octubre), incrementándose a 0,52 el 3 de octubre, descendiendo a 0,27 el 4 de octubre para sufrir a continuación un ascenso a 0,76 el 7 de octubre. Estos cambios tan rápidos de $Q_{0}$ y $\nu$ están claramente relacionados con la aceleración que la deformación experimentó en el mismo periodo (1 al 6 de octubre), como se observa en las estaciones GPS de FRON y HI03 (Figura 4 en López et al. (2012)). Durante la Fase V, el valor de $Q_{0}$ se incrementa rápidamente desde 91 (8 de octubre) a 176 (9 de octubre), mientras que $\nu$ muestra la tendencia opuesta durante el mismo periodo, disminuyendo de 0,59 a 0,20 (Tabla 7.1). Estos cambios significativos coinciden con la ocurrencia del terremoto de magnitud $M_{L} 4,3$ del 8 de octubre.

Desde el principio del tremor el 10 de octubre, $Q_{0}$ parece disminuir gradualmente con el tiempo, alcanzando el valor de 81 el 30 de octubre y de 80 el 31 de octubre. Sin embargo, $Q_{0}$ disminuye a 41 el 1 de noviembre y asciende a 73 un día después. Estos cambios drásticos coinciden con la ocurrencia de un sismo de magnitud 3,9 ocurrido el 1 de noviembre, seguido por dos sismos de mayor magnitud $\left(4,3 M_{L}\right)$ ocurridos el día 2 (Figura 7.9). El parámetro $\nu$ aumenta de forma monótona desde el comienzo del tremor, cambiando la tendencia entre el 31 de octubre y el 2 de noviembre. Presenta pues un incremento desde 0,65 (31 de octubre) hasta 0,99 (1 de noviembre), disminuyendo a 0,76 un día más tarde.

Con el objeto de profundizar en la caracterización de la evolución temporal de $Q_{0}$ y $\nu$ durante las diferentes fases pre-eruptivas y durante el periodo de tremor se 
han calculado estos parámetros de forma específica en cada periodo de tiempo. Para ello, sólo se han considerado sismos de $M_{L} \geq 3$ registrados en CTIG durante cada periodo, de tal forma que las formas de onda de esos terremotos se han procesado de forma idéntica a la explicada anteriormente. Los resultados de $Q_{0}$ y $\nu$ se presentan en la Tabla 7.2 para cada fase y el periodo de tremor. Se indica el intervalo de tiempo en que ocurrieron los terremotos de magnitud $M_{L} \geq 3$ utilizados en el cálculo. En la misma tabla se proporcionan también el número de eventos, el número de observaciones de Q para cada banda de frecuencia y el número total de observaciones.

En la Figura 7.10 (parte superior e inferior) se muestran los valores calculados de $Q_{0}$ y $\nu$ para las distintas fases y el periodo de tremor, diferenciándose con líneas continuas y discontinuas, tal y como se explica en el pie de figura. Puede apreciarse que los valores de $Q_{0}$ tienden a disminuir durante el proceso eruptivo desde un valor de 183 en la Fase III a 108 durante el tremor (Figura 7.10, parte superior). Este comportamiento es semejante al observado para los valores de $Q_{0}$ calculados anteriormente para cada uno de los 18 días de la Tabla 7.1. Por el contrario, $\nu$ tiene tendencia a aumentar durante el proceso de desarrollo de las fases, desde un valor de 0,30 en la Fase III hasta 0,50 durante el tremor (Figura 7.10, parte inferior). La misma tendencia se observa en los valores de $\nu$ que fueron calculados para cada uno de los días de la Tabla 7.1.

En la Fase V, las tendencias observadas para $Q_{0}$ y $\nu$ se interrumpen. Así, para $Q_{0}$ la tendencia general de disminución de las fases anteriores se interrumpe por el alto valor de 144 en esta fase, como puede apreciarse en la Tabla 7.2. De forma similar, el bajo valor de $\nu$ alcanzado en esta Fase $\mathrm{V}(0,32)$ se separa de la tendencia global de aumento observado en las fases anteriores. El valor de $Q_{0}$ observado en la Fase $\mathrm{V}$ se refleja en el aumento observado en los días incluidos en esta fase ( 8 y 9 de octubre de 2011), como puede observarse en la Tabla 7.1. De la misma forma, el valor de $\nu$ en la Fase $\mathrm{V}$ está relacionado con la disminución observada en estos días (Tabla 7.1). Por esta razón, ambos valores de $Q_{0}$ y $\nu$ observados en la Fase $\mathrm{V}$ pueden estar asociados a la ocurrencia del sismo de 8 de octubre y magnitud $M_{L}$ 4,3 .

Puede observarse que el valor de $Q_{0}$ de 108 para el periodo del tremor es significativamente más alto que los valores de $Q_{0}$ obtenidos en los 5 días incluidos en el periodo (30 y 31 de octubre, y 1, 2 y 5 de noviembre de 2011), para los cuales los valores de $Q_{0}$ y $\nu$ han sido calculados. Como se ha señalado, estos valores diarios comprenden desde 41 hasta 81 (Tabla 7.1). En el caso de $\nu$, se ha observado la tendencia contraria, siendo el valor para el periodo de tremor $(0,50)$ menor que los valores calculados por día, que oscilan entre 0,61 y 0,99. Esta divergencia en las tendencias puede explicarse, por una parte, por el diferente número de observaciones de Q consideradas para cada día en relación al número de observaciones consideradas durante el tremor. Por otra parte, se ha comparado el número de bandas de frecuencia utilizadas para el cálculo de $Q_{0}$ y $\nu$ por día con el número de bandas frecuenciales utilizadas para calcular los mismos parámetros para el periodo de tremor. Como resultado final se puede decir que:

- El número total de observaciones de Q durante el periodo de tremor (76) es sensiblemente superior al número total de observaciones diarias de Q (42) correspondientes a cada uno de los días de este periodo, como puede comprobarse 
Tabla 7.1: Parámetros $Q_{0}$ y $\nu$ obtenidos en la estación CTIG (El Hierro) desde el 24 de septiembre hasta el 5 de noviembre de 2011 , según se explica en el texto. Se presentan también las desviaciones estándar de $Q_{0}$ y $\nu$ (SD), el número d eventos por día, el número de observaciones de Q para cada intervalo de frecuencias y el total de las observaciones que han sido analizadas. El valor medio de Q para cada intervalo de frecuencias de filtrado ha sido calculado tras seleccionar las observaciones de $\mathrm{Q}$ correspondientes por medio del criterio de Chauvenet.

\begin{tabular}{|c|c|c|c|c|c|c|c|c|c|c|c|}
\hline \multirow{2}{*}{ Fecha } & \multirow{2}{*}{$\begin{array}{l}\text { Núm. } \\
\text { even- } \\
\text { tos }\end{array}$} & \multicolumn{5}{|c|}{ Núm. observaciones de Q } & \multirow{2}{*}{$\begin{array}{l}\text { Total de ob- } \\
\text { servaciones } \\
\text { Q analizadas }\end{array}$} & \multirow{2}{*}{$Q_{0}$} & \multirow{2}{*}{ SD } & \multirow{2}{*}{$\nu$} & \multirow{2}{*}{ SD } \\
\hline & & $\begin{array}{l}\mathbf{1 , 5} \\
\mathbf{H z}\end{array}$ & $3 \mathbf{H z}$ & $5 \mathbf{H z}$ & $7 \mathrm{~Hz}$ & $9 \mathrm{~Hz}$ & & & & & \\
\hline 24 Septiembre 2011 & 2 & 0 & 2 & 2 & 2 & 2 & 8 & 165 & 41 & 0,31 & 0,12 \\
\hline 27 Septiembre 2011 & 9 & 4 & 8 & 8 & 7 & 7 & 34 & 117 & 35 & 0,46 & 0,16 \\
\hline 28 Septiembre 2011 & 10 & 3 & 5 & 4 & 4 & 3 & 19 & 109 & 16 & 0,54 & 0,09 \\
\hline 29 Septiembre 2011 & 12 & 5 & 10 & 9 & 10 & 9 & 43 & 111 & 17 & 0,58 & 0,09 \\
\hline 30 Septiembre 2011 & 5 & 3 & 5 & 3 & 3 & 3 & 17 & 93 & 12 & 0,63 & 0,08 \\
\hline 1 Octubre 2011 & 10 & 1 & 4 & 3 & 3 & 2 & 12 & 120 & 63 & 0,48 & 0,25 \\
\hline 2 Octubre 2011 & 6 & 2 & 5 & 5 & 5 & 5 & 22 & 175 & 48 & 0,30 & 0,14 \\
\hline 3 Octubre 2011 & 8 & 4 & 7 & 5 & 4 & 5 & 25 & 111 & 14 & 0,52 & 0,10 \\
\hline 4 Octubre 2011 & 4 & 2 & 4 & 4 & 3 & 3 & 16 & 160 & 46 & 0,27 & 0,20 \\
\hline 6 Octubre 2011 & 4 & 0 & 3 & 2 & 2 & 2 & 9 & 120 & 30 & 0,47 & 0,10 \\
\hline 7 Octubre 2011 & 8 & 1 & 5 & 4 & 4 & 4 & 17 & 75 & 22 & 0,76 & 0,20 \\
\hline 8 Octubre 2011 & 4 & 0 & 4 & 3 & 2 & 2 & 11 & 91 & 30 & 0,59 & 0,20 \\
\hline 9 Octubre 2011 & 2 & 2 & 2 & 2 & 2 & 2 & 10 & 176 & 46 & 0,20 & 0,20 \\
\hline 30 Octubre 2011 & 6 & 0 & 3 & 3 & 3 & 3 & 12 & 81 & 22 & 0,61 & 0,10 \\
\hline 31 Octubre 2011 & 6 & 1 & 3 & 3 & 2 & 2 & 10 & 80 & 33 & 0,65 & 0,21 \\
\hline 1 Noviembre 2011 & 4 & 0 & 1 & 2 & 2 & 2 & 6 & 41 & 4 & 0,99 & 0,05 \\
\hline 2 Noviembre 2011 & 4 & 1 & 2 & 2 & 2 & 1 & 6 & 73 & 35 & 0,76 & 0,25 \\
\hline 5 Noviembre 2011 & 5 & 0 & 2 & 2 & 2 & 2 & 8 & 66 & 19 & 0,83 & 0,14 \\
\hline
\end{tabular}

Tabla 7.2: Parámetros $Q_{0}$ y $\nu$ obtenidos en la estación CTIG (El Hierro) para cada fase pre-eruptiva de López et al. (2012), y para el periodo de registro de la señal de tremor (Instituto Geográfico Nacional, 2016b), según se explica en el texto. Se presentan también las desviaciones estándar de $Q_{0}$ y $\nu$ (SD), el número de eventos para cada periodo, el intervalo de tiempo en el cual ocurrieron los eventos, el número de observaciones de Q para cada intervalo de frecuencias y el total de las observaciones que han sido analizadas. El valor medio de Q para cada intervalo de frecuencias de filtrado ha sido calculado tras seleccionar las observaciones de Q correspondientes por medio del criterio de Chauvenet.

\begin{tabular}{|c|c|c|c|c|c|c|c|c|c|c|c|c|}
\hline \multirow{2}{*}{$\begin{array}{l}\text { Fase/ } \\
\text { Tremor }\end{array}$} & \multirow{2}{*}{$\begin{array}{l}\text { Núm. } \\
\text { even- } \\
\text { tos }\end{array}$} & \multirow{2}{*}{$\begin{array}{l}\text { Intervalo } \\
\text { tiempo }\end{array}$} & \multicolumn{5}{|c|}{ Núm. observaciones $\mathbf{Q}$} & \multirow{2}{*}{$\begin{array}{l}\text { Total ob- } \\
\text { servaciones } \\
\text { Q } \\
\text { analizadas }\end{array}$} & \multirow[b]{2}{*}{$Q_{0}$} & \multirow[b]{2}{*}{ SD } & \multirow[b]{2}{*}{$\nu$} & \multirow[b]{2}{*}{ SD } \\
\hline & & & $\begin{array}{l}\mathbf{1 , 5} \\
\mathbf{H z}\end{array}$ & $3 \mathbf{H z}$ & $5 \mathbf{H z}$ & $7 \mathrm{~Hz}$ & $9 \mathrm{~Hz}$ & & & & & \\
\hline Fase III & 5 & $\begin{array}{l}22-24 \text { Septiem- } \\
\text { bre } 2011\end{array}$ & 0 & 4 & 4 & 3 & 3 & 14 & 158 & 44 & 0,34 & 0,14 \\
\hline Fase IV & 78 & $\begin{array}{l}27 \text { Septiembre- } \\
7 \text { Octubre } 2011\end{array}$ & 26 & 57 & 44 & 42 & 41 & 210 & 120 & 21 & 0,47 & 0,10 \\
\hline Fase V & 6 & $\begin{array}{ll}8-9 & \text { Octubre } \\
2011 & \end{array}$ & 2 & 6 & 5 & 4 & 4 & 21 & 144 & 47 & 0,32 & 0,17 \\
\hline Tremor & 75 & $\begin{array}{lr}23 & \text { Octubre } \\
2011-4 & \text { Febrero } \\
2012 & \end{array}$ & 5 & 18 & 19 & 18 & 16 & 76 & 108 & 27 & 0,50 & 0,13 \\
\hline
\end{tabular}


en las Tablas 7.1 y 7.2. Esto significa que se han introducido en el cálculo de $Q_{0}$ para el periodo de tremor 34 observaciones más que en el cálculo diario. Según se ha visto, el periodo de tremor presenta la mayor diferencia entre el total de observaciones de Q utilizadas para todo el periodo y el total de observaciones diarias, dado que para las fases III,IV y $\mathrm{V}$ esta diferencia es menor que 6 (Tablas 7.1 y 7.2 ).

En cuanto a las bandas de frecuencia individuales, se observa el gran número de observaciones de Q para el periodo de tremor en comparación con el número de observaciones de $\mathrm{Q}$ para los días aislados. Como consecuencia, el valor promedio de Q obtenido para una determinada banda de frecuencia durante el periodo de tremor es diferente al valor promedio calculado con las observaciones correspondientes a un día del periodo. Por ejemplo, el valor promedio de Q para la banda de frecuencia $7 \mathrm{~Hz}$ para el periodo de tremor es de 289 , siendo obtenido con 18 observaciones (Tabla 7.2). Por el contrario, el cálculo del valor promedio de $\mathrm{Q}$ correspondiente al día 2 de noviembre, para la misma banda de frecuencia, se ha efectuado solamente con 2 observaciones, obteniéndose un valor superior de 343 (Tabla 7.1). Además, el valor promedio de Q para el periodo de tremor que corresponde a la frecuencia de $9 \mathrm{~Hz}$ se realizó con 16 observaciones (Tabla 7.2), arrojando un valor de 361, mientras que del cálculo realizado el día 5 de noviembre se obtiene un valor superior (456), utilizando solamente 2 observaciones (Tabla 7.1).

- Los valores de $Q_{0}$ para el periodo de tremor se han obtenido usando las cinco bandas de frecuencia propuestas para este estudio (Tabla 7.2), mientras que los valores correspondientes a los 5 días incluidos en este periodo se han obtenido con un máximo de 4 bandas (Tabla 7.1). En algunos días (1 y 2 de noviembre), únicamente se han utilizado 3 bandas de frecuencia. Por ejemplo, los valores de $Q_{0}$ para el 2 de noviembre (73) y para el 5 de noviembre (66) fueron obtenidos con tres y cuatro bandas respectivamente (Tabla 7.1). Estos resultados son claramente inferiores al valor calculado para el periodo de tremor (108) usando las 5 bandas de frecuencia, como puede observarse en la Tabla 7.2.

Puede deducirse por tanto que las diferencias observadas entre los valores de $Q_{0}$ obtenidos para el tremor y los valores correspondientes al cálculo diario están relacionadas con el número de bandas utilizadas y con el número de observaciones de $\mathrm{Q}$ que intervienen en cada caso en el cálculo. Consecuentemente, el mayor número de bandas de frecuencia utilizadas en el cálculo de $Q_{0}$ para el periodo de tremor, y el mayor número de observaciones de Q empleadas en la determinación de los promedios de $\mathrm{Q}$ para cada banda frecuencial, conducen a unos valores de $Q_{0}$ superiores a los resultados correspondientes a cada uno de los días incluidos en este periodo.

\subsection{Discusión}

Los valores del factor de calidad Q para una determinada área, calculados utilizando las ondas de coda y terremotos regionales, han puesto de manifiesto la estrecha relación entre éstos y la actividad tectónica. Se ha encontrado una relación entre regiones de alta actividad sísmica o volcánica con bajos valores de $Q_{0}$ cercanos a 100 
y altos valores de $\nu$ (mayores que 0,50 e inferiores a 1,0). Por el contrario, los altos valores de $Q_{0}$ son usuales en zonas sin actividad tectónica. Los resultados obtenidos en este capítulo para las Islas Canarias son los esperados para zonas activas, ya que el valor de $Q_{0}$ para profundidades focales $>15 \mathrm{Km}$ está en el rango de 60-240, mientras que para profundidades $\leq 15 \mathrm{~km}$ este parámetro toma valores en el rango 70-210 (Figuras 7.4 y 7.6, respectivamente). Por otro lado, $\nu$ oscila entre $0,3-1,0$ para profundidades $>15 \mathrm{Km}$ (Figura 7.5) y entre $0,3-0,8$ para profundidades $\leq 15 \mathrm{~km}$ (Figura 7.7).

A escala local, se pueden explicar las variaciones laterales de $Q_{0}$ y $\nu$ encontradas en esta tesis mediante las bien conocidas geología y tectónica de las Islas Canarias. Si se consideran las conclusiones de Mezcua et al. (1992) sobre la existencia de una falla inversa de dirección NE-SO entre Gran Canaria y Tenerife se puede esperar que esta zona es una zona frágil en la que se espera un alto valor de $Q_{0}$. En el mapa de isolíneas de $Q_{0}$ de la Figura 7.4 se observa un incremento constante hacia el sur alcanzando un valor de 140. De forma contraria, el parámetro $\nu$ presenta una disminución desde el valor de 1,00 hasta 0,70 (Figura 7.5). En cambio, en El Hierro se observa una concentración de epicentros de magnitud $M_{L} \geq 3$ en el sur-suroeste de la isla (Figura 7.2). Este hecho, junto con el comienzo de la erupción de 2011 en las proximidades de la costa sur, permite suponer que la zona es más dúctil, y que por este motivo tendría que presentar valores más bajos de $Q_{0}$ y más altos de $\nu$. En el mapa de isoíneas de la Figura 7.6 se puede apreciar que en el sur de El Hierro los valores de $Q_{0}$ disminuyen desde 130 a 70, mientras que $\nu$ sufre un incremento brusco de 0,50 a 0,90 (Figura 7.7).

Se ha buscado una correlación entre la regionalización de $Q_{0}$ y $\nu$ obtenida en esta tesis y la de otros trabajos sobre Q para las Islas Canarias, como el de Canas et al. (1995). En el trabajo de Canas et al. (1995), se utilizaron 148 registros correspondientes a 48 terremotos registrados entre 1989 y 1990. La regionalización de $Q_{0}$ obtenida por estos autores para un valor constante de $\nu=0,90$, comparada con la regionalización obtenida en la presente tesis doctoral para sismos con profundidad $>15 \mathrm{Km}$ (Figura 7.4), muestra que el mínimo valor de $Q_{0}=60$ entre Gran Canaria y Fuerteventura obtenido en esta tesis puede compararse con el valor de 100 que aparece en Canas et al. (1995) pero en la zona entre Gran Canaria y Tenerife, dada su posición central en el archipiélago (Figura 9 de Canas et al. (1995)). Hacia el norte, estos autores presentan un claro incremento de $Q_{0}$ hasta alcanzar el valor de 320 , que se corresponde con el valor máximo de 240 de la Figura 7.4. Se encuentra otra similitud en la zona de Fuerteventura y Lanzarote, entre el incremento de $Q_{0}$ hacia el noreste obtenido en esta tesis y el incremento observado con la misma tendencia en Canas et al. (1995). En La Palma, Canas et al. (1995) presentan valores cercanos a 100 que son similares al valor obtenido en esta tesis, $Q_{0}=120$. Además, en la Figura 7.4 se observa, coincidiendo con Canas et al. (1995), una fuerte disminución de $Q_{0}$ en El Hierro, pasando de 180 a 80, mientras que para estos autores $Q_{0}$ disminuye de 140 a 80 (Figura 9 de Canas et al. (1995)). Esta coincidencia se corresponde con un área de alta concentración de puntos medios de trayectorias empleadas para el cálculo de Q (Figura 7.4), lo que ha permitido obtener mejores resultados. Podemos por tanto afirmar que la tendencia de las isolíneas de $Q_{0}$ para sismos con profundidad $>15 \mathrm{Km}$ coincide con la tendencia de la regionalización de $Q_{0}$ de Canas et al. (1995), aunque los valores de estos autores son generalmente más altos. Finalmente, si se comparan los resultados obtenidos en esta tesis con los valores de Canas et al. 
(1998) para todo el archipiélago $\left(Q_{0} \approx 103\right.$ y $\left.\nu \approx 0,57\right)$, se observa que son similares ya que en esta tesis se han obtenido valores mínimos de 60 para $Q_{0}$ y de 0,10 para $\nu$.

Finalmente, se han investigado las posibles relaciones entre los valores regionales de $Q_{0}$ y $\nu$ que se han obtenido en esta tesis y los resultados para otras zonas activas. Por ejemplo, se han comparado los resultados con los valores obtenidos por Havskov et al. (1989) en el Monte Santa Elena (Washington), aplicando la misma metodología. En su trabajo, los valores de $Q_{0}$ y $\nu$ oscilan entre 54 y 66, y entre 0,39 y 0,98, respectivamente. Estos valores están ligeramente por debajo de los valores mínimos obtenidos, $Q_{0}=70$, para profundidades $\leq 15 \mathrm{~km}$ en la parte sur de El Hierro (Figura 7.6), que es un área volcánica. Al mismo tiempo, el valor máximo de $\nu$ calculado por Havskov et al. (1989) es mayor que el máximo valor de 0,90 encontrado para esta parte de El Hierro (Figura 7.7). Para el noroeste de Colombia, Vargas et al. (2004) han encontrado un valor mínimo de $Q_{0}$ (cercano a 42) que está por debajo del valor mínimo de $Q_{0}$ obtenido en El Hierro, y un valor de $\nu=1,01$ superior al alcanzado en la isla. La comparación de los resultados aquí obtenidos para Canarias con los de Havskov et al. (1989) para el Monte Santa Elena y Vargas et al. (2004) para Colombia sugieren que las diferencias encontradas son debidas quizá al diferente origen del volcanismo, de tipo subducción para los estudios comparados y de punto caliente para las Islas Canarias. En este contexto, los valores relativamente altos de $Q_{0}$ estimados en El Hierro indican niveles de atenuación más bajos para el volcanismo de punto caliente. 